\title{
Supporting information \\ NHC-Catalyzed Enantioselective Dearomatizing Hydroacylation of Benzofurans and Benzothiophenes for the Synthesis of Spirocycles
}

Daniel Janssen-Müller ${ }^{\dagger}$, Mirco Fleige ${ }^{\dagger}$, Danny Schlüns ${ }^{\dagger, \ddagger}$, Marco Wollenburg ${ }^{\dagger}$, Constantin G. Daniliuc ${ }^{\dagger}$, Johannes Neugebauer ${ }^{\dagger, \ddagger, *}$ and Frank Glorius ${ }^{\dagger, *}$

†Organisch-Chemisches Institut, Westfälische Wilhelms-Universität Münster, Corrensstraße 40, 48149

Münster, Germany

${ }_{\ddagger}$ Center for Multiscale Theory and Computation, Westfälische Wilhelms-Universität Münster,

Corrensstraße 40,

48149 Münster, Germany

Email:

glorius@uni-muenster.de

j.neugebauer@uni-muenster.de

\section{Contents}

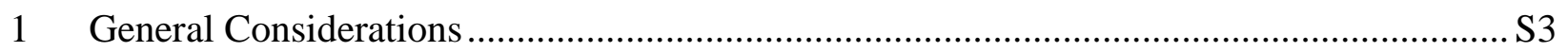

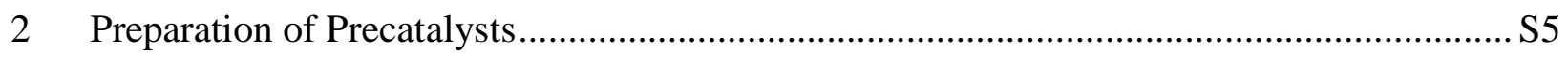

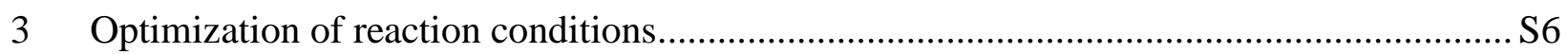

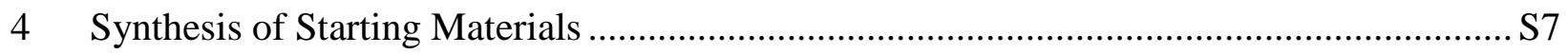

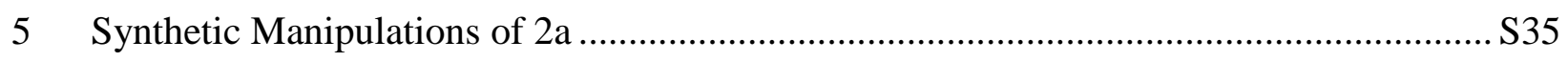

6 Synthesis of Hydroacylation Products .......................................................................... S38

6.1 General procedure for the racemic hydroacylation ................................................. S38

6.2 Enantioselective hydroacylation of benzofurans ....................................................... S38

6.3 Enantioselective hydroacylation of benzothiophenes ............................................... S55

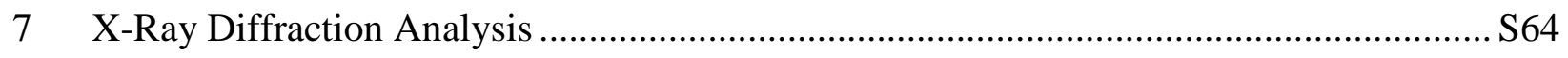

$8 \quad 3-D e u t e r i u m$ and 3-Methyl substituted Substrates................................................................ S66

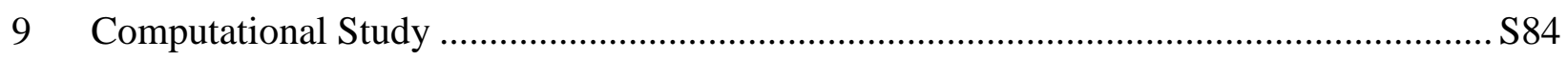




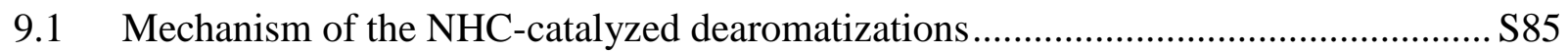

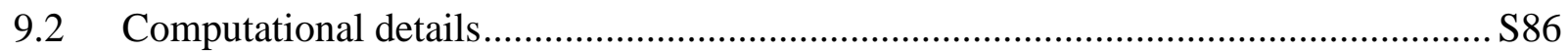

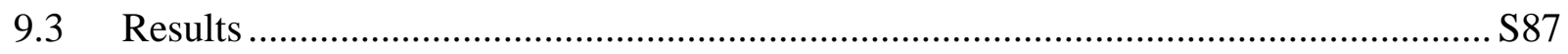

9.3.1 Attack on the sterically less demanding side .......................................... S87

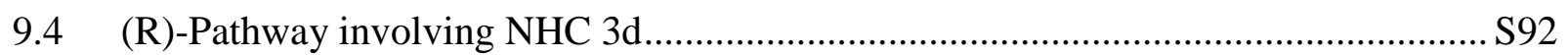

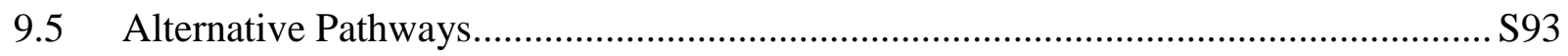

9.6 Attack on the sterically more demanding side ................................................. 997

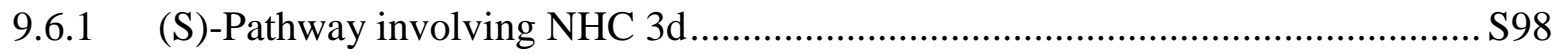

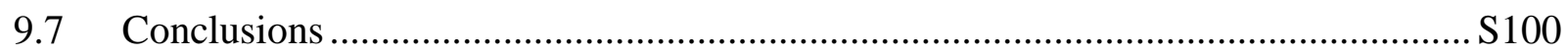

10 NMR Spectra Hydroacylation Products............................................................ S101

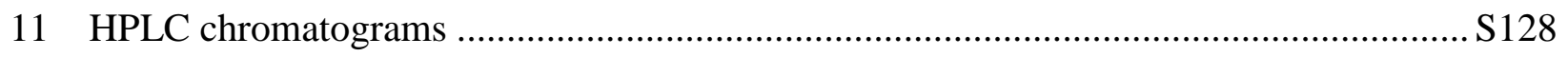

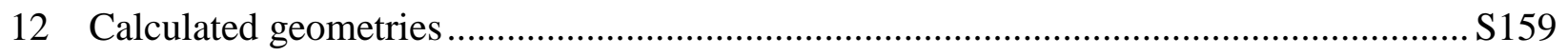

12.1 Optimized Geometries for presented reaction pathways ................................... S159

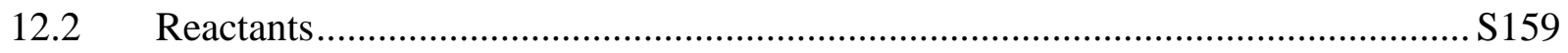

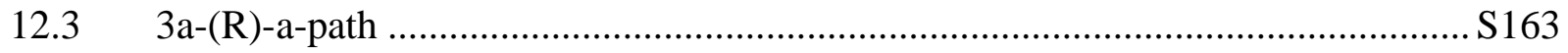

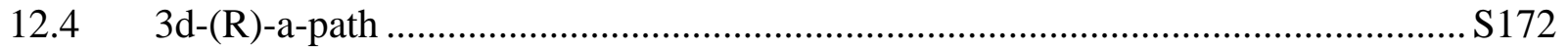

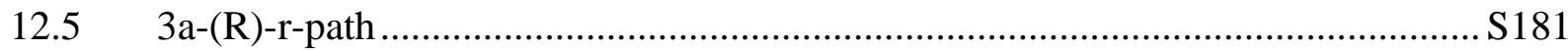

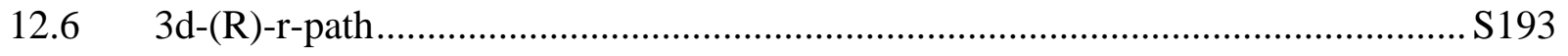

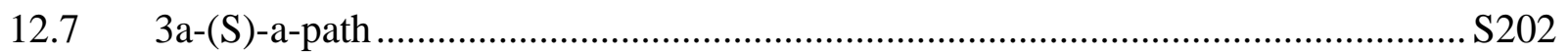

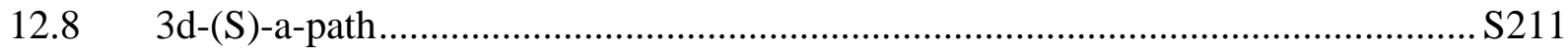

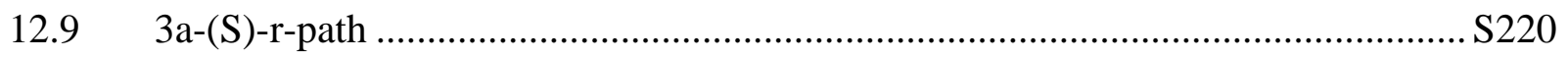

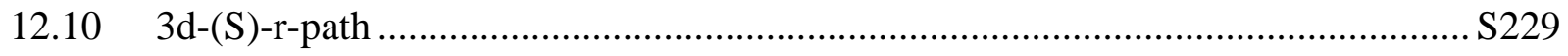

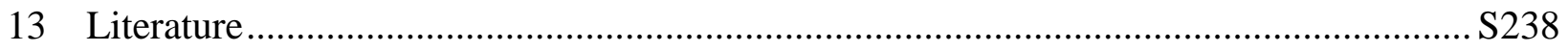




\section{General Considerations}

Unless otherwise noted, all reactions were carried out in oven-dried glassware and under argon atmosphere. Reaction temperatures are reported as the temperature of the oil bath surrounding the vessel.

The following solvents were dried by distillation over the drying agents indicated in parentheses: THF (Na/benzophenone), toluene $\left(\mathrm{CaH}_{2}\right), \mathrm{Et}_{2} \mathrm{O}\left(\mathrm{Na} /\right.$ benzophenone), $\mathrm{CH}_{2} \mathrm{Cl}_{2}\left(\mathrm{CaH}_{2}\right)$. Additional anhydrous solvents (<50 ppm water) were purchased from Acros Organics, Sigma-Aldrich or Carl Roth and stored over molecular sieves under an argon atmosphere.

Commercially available chemicals were obtained from Acros Organics, Sigma-Aldrich, Alfa Aesar, ABCR, TCI Europe, Fluorochem, Carbolution Chemicals and Combi-Blocks and used as received unless otherwise stated.

Analytical thin layer chromatography (TLC) was performed on silica gel $60 \mathrm{~F}_{254}$ aluminum plates (Merck). TLC plates were visualized by exposure to short wave ultraviolet light (254 nm, $366 \mathrm{~nm}$ ) and were dipped into a solution of $\mathrm{KMnO}_{4}\left(1 \mathrm{~g} \mathrm{KMnO}_{4}, 6 \mathrm{~g} \mathrm{~K} \mathrm{KO}_{3}\right.$ and $0.1 \mathrm{~g} \mathrm{KOH}$ in $100 \mathrm{~mL}$ water) or vanillin ( $2 \mathrm{~g}$ vanillin and $4 \mathrm{~mL}$ concentrated $\mathrm{H}_{2} \mathrm{SO}_{4}$ in $100 \mathrm{~mL}$ EtOH) followed by heating. Flash column chromatography was performed on Acros silica gel (35-70 $\mu \mathrm{m}$ mesh) with a positive overpressure of argon by 0.5 bar.

GC-MS spectra were recorded on an Agilent Technologies 7890A GC-system with Agilent 5975 inert Mass Selective Detector (EI) and a HP-5MS column (0.25 mm x 30 m, Film: $0.25 \mu \mathrm{m})$. The method used starts with the injection temperature of $50{ }^{\circ} \mathrm{C}$, after holding this temperature for 3 min, the column is heated to $290{ }^{\circ} \mathrm{C}$ with a gradient of $40{ }^{\circ} \mathrm{C} / \mathrm{min}$ and holds the final temperature for $3 \mathrm{~min}$. The major signals are quoted in $\mathrm{m} / \mathrm{z}$ with the relative intensity in parentheses.

${ }^{1} \mathrm{H}-,{ }^{13} \mathrm{C}$ - and ${ }^{19} \mathrm{~F}$ NMR spectra were recorded at room temperature on a Bruker AV 300 or AV 400 or Agilent $600 \mathrm{MHz}$ INOVA. Chemical shifts $(\delta)$ are given in ppm. The residual solvent signals were used as references and the chemical shifts converted to the TMS scale $\left(\mathrm{CDCl}_{3}: \delta \mathrm{H}=7.26\right.$ ppm, $\left.\delta \mathrm{C}=77.16 \mathrm{ppm} ; \mathrm{CD}_{2} \mathrm{Cl}_{2}: \delta \mathrm{H}=5.32 \mathrm{ppm}, \delta \mathrm{C}=53.84 \mathrm{ppm}\right) .{ }^{19} \mathrm{~F}$ NMR spectra are not calibrated and $\delta(\mathrm{ppm})$ is given relative to $\mathrm{CCl}_{3} \mathrm{~F}$. Coupling constants $(J)$ are quoted in $\mathrm{Hz}$.

High resolution ESI mass spectra were recorded on a Thermo-Fisher Scientific Orbitrap LTQ XL or on a Bruker Daltonics MicroTof. 
Infrared spectra were recorded on a Varian Associates FT-IR 3100 Excalibur with ATR unit. The wave numbers $(\tilde{v})$ of recorded IR-signals are quoted in $\mathrm{cm}^{-1}$.

Optical rotations were recorded on a Perkin Elmer 341 Polarimeter.

The enantiomeric excess (ee) was determined by HPLC analysis using chiral columns AD-H, AS$\mathrm{H}, \mathrm{OD}-\mathrm{H}$ and $\mathrm{OJ}-\mathrm{H}$. 


\section{Preparation of Precatalysts}

Azolium salts $\mathbf{3 a},{ }^{[\mathrm{S} 1]} \mathbf{3 b},{ }^{[\mathrm{S} 2]} \mathbf{3 c},{ }^{[\mathrm{S} 2]}, \mathbf{3 d},{ }^{[\mathrm{S} 3]} \mathbf{3 e},{ }^{[\mathrm{S} 4]} \mathbf{3 f},{ }^{[\mathrm{S} 4]} \mathbf{3 g},{ }^{[\mathrm{S} 5]} \mathbf{3 h},{ }^{[\mathrm{S} 6]} \mathbf{3 i} \mathbf{-} \mathbf{k}^{[\mathrm{S} 7]}$ and $\mathbf{3 \mathbf { I } ^ { [ \mathrm { S } 8 ] }}$ were prepared according to known procedures.

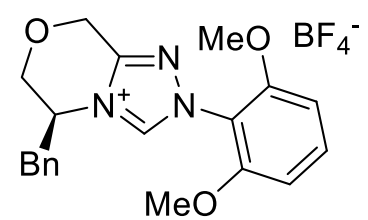

3a<smiles>Cc1cc(C)c(-n2c[n+]3c(n2)COCC3Cc2ccccc2)c(C)c1</smiles>

3b

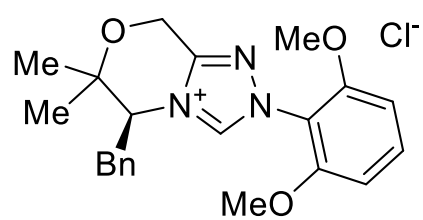

$3 c$<smiles>Cc1cc(C)c(-n2c[n+]3c(n2)CCC3[B]c2ccccc2)c(C)c1</smiles>

3d<smiles>COc1cccc(OC)c1-n1c[n+]2c(n1)CO[C@H]1Cc3ccccc3[C@@H]12</smiles>

$3 e$<smiles>COc1cccc(OC)c1-n1c[n+]2c(n1)CCC2</smiles><smiles>CCc1cccc(CC)c1-n1c[n+]2c(n1)CCC2</smiles>

3i<smiles>Cc1cc(C)c(-n2c[n+]3c(n2)CCC3)c(C)c1</smiles>

$3 g$<smiles>c1ccc(-n2c[n+]3c(n2)CCC3)cc1</smiles>

3j<smiles>C[n+]1csc2c1CCCCC2</smiles>

$3 \mathrm{~h}$<smiles>CCCc1nn(C(F)(F)F)cc1C(F)(F)F</smiles>

3k<smiles>ClC(Cl)(Cl)c1cn(-c2ccccc2)c[n+]1-c1ccccc1</smiles>

31

Figure S1. NHC precatalysts. 


\section{Optimization of reaction conditions}

Reactions for optimization were tested on a $0.1 \mathrm{mmol}$ scale and the yield was determined as an NMR yield. After the reaction was performed, the solution was filtered through a short pad of silica with EtOAc to remove the catalyst and any salts. The filtrate was concentrated under reduced pressure. The residue was dissolved in $\mathrm{CDCl}_{3}$ or $\mathrm{CD}_{2} \mathrm{Cl}_{2}$ and $14 \mu \mathrm{L} \mathrm{CH}_{2} \mathrm{Br}_{2}$ (0.20 mmol, 2.0 equiv.) was added as internal standard. The amount of product was determined by integration of characteristic peaks in respect to the signal of $\mathrm{CH}_{2} \mathrm{Br}_{2}$. The ee value was determined by HPLC using a chiral stationary phase. DBU = 1,8-diazabicyclo [5.4.0] undec-7-ene, n.d. = not determined.

Table S1. Optimization of reaction conditions.

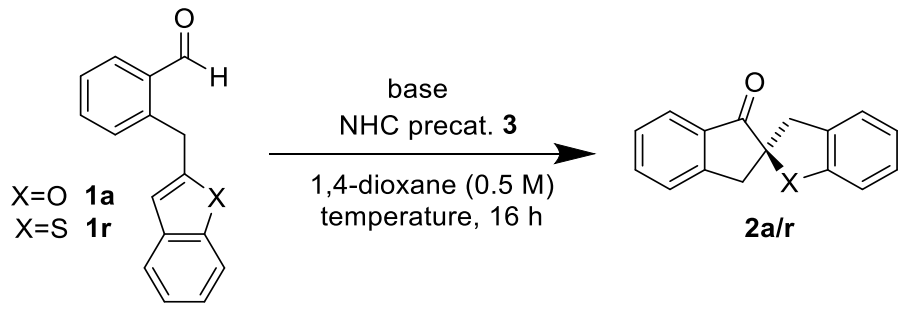

\begin{tabular}{|c|c|c|c|c|c|}
\hline Substrate & Base & NHC precat. & $T\left[{ }^{\circ} \mathbf{C}\right]$ & Yield [\% $]^{\mathrm{b}}$ & $e e[\%]^{c}$ \\
\hline 1a & DBU (40 mol\%) & $3 \mathbf{a}(20 \mathrm{~mol} \%)$ & 80 & 53 & 98 \\
\hline 1a & DBU (40 mol\%) & $3 \mathbf{a}(20 \mathrm{~mol} \%)$ & 100 & 25 & 97 \\
\hline 1a & DBU (40 mol\%) & $3 \mathbf{a}(20 \mathrm{~mol} \%)$ & 140 & $<1$ & n.d. \\
\hline 1a & $\mathrm{Cs}_{2} \mathrm{CO}_{3}(100 \mathrm{~mol} \%)$ & $3 \mathbf{a}(20 \mathrm{~mol} \%)$ & 80 & 8 & n.d. \\
\hline 1a & $\mathrm{K}_{2} \mathrm{CO}_{3}(100 \mathrm{~mol} \%)$ & 3a $(20 \mathrm{~mol} \%)$ & 80 & $>95$ & 98 \\
\hline $1 \mathrm{a}$ & $\mathrm{K}_{2} \mathrm{CO}_{3}(100 \mathrm{~mol} \%)$ & $3 \mathbf{a}(20 \mathrm{~mol} \%)$ & 70 & $>95$ & 98 \\
\hline $1 \mathrm{a}$ & $\mathrm{K}_{2} \mathrm{CO}_{3}(100 \mathrm{~mol} \%)$ & $3 \mathbf{a}(20 \mathrm{~mol} \%)$ & 60 & $>95$ & 98 \\
\hline $1 \mathrm{a}$ & $\mathrm{K}_{2} \mathrm{CO}_{3}(100 \mathrm{~mol} \%)$ & $3 \mathbf{a}(20 \mathrm{~mol} \%)$ & 50 & 52 & 98 \\
\hline $1 \mathrm{a}$ & $\mathrm{K}_{2} \mathrm{CO}_{3}(100 \mathrm{~mol} \%)$ & $3 \mathbf{a}(20 \mathrm{~mol} \%)$ & 25 & 22 & 99 \\
\hline $1 \mathrm{a}$ & $\mathrm{K}_{2} \mathrm{CO}_{3}(20 \mathrm{~mol} \%)$ & $3 \mathbf{a}(20 \mathrm{~mol} \%)$ & 80 & $>95$ & 98 \\
\hline $1 \mathrm{a}$ & $\mathrm{K}_{2} \mathrm{CO}_{3}(10 \mathrm{~mol} \%)$ & 3a (10 mol\%) & 80 & $>95$ & 98 \\
\hline 1a & $\mathrm{K}_{2} \mathrm{CO}_{3}(5 \mathrm{~mol} \%)$ & 3a $(5 \mathrm{~mol} \%)$ & 80 & 42 & 98 \\
\hline 1a & $\mathrm{K}_{2} \mathrm{CO}_{3}(100 \mathrm{~mol} \%)$ & $\mathbf{3 b}(20 \mathrm{~mol} \%)$ & 80 & 50 & 93 \\
\hline 1a & $\mathrm{K}_{2} \mathrm{CO}_{3}(100 \mathrm{~mol} \%)$ & $3 \mathbf{c}(20 \mathrm{~mol} \%)$ & 80 & 18 & 66 \\
\hline $1 \mathrm{a}$ & $\mathrm{K}_{2} \mathrm{CO}_{3}(100 \mathrm{~mol} \%)$ & $3 \mathbf{e}(20 \mathrm{~mol} \%)$ & 80 & 52 & 96 \\
\hline 1a & $\mathrm{K}_{2} \mathrm{CO}_{3}(100 \mathrm{~mol} \%)$ & 3f $(20 \mathrm{~mol} \%)$ & 80 & $>95$ & rac \\
\hline 1a & $\mathrm{K}_{2} \mathrm{CO}_{3}(100 \mathrm{~mol} \%)$ & $3 g(20 \mathrm{~mol} \%)$ & 80 & 53 & rac \\
\hline 1a & $\mathrm{K}_{2} \mathrm{CO}_{3}(100 \mathrm{~mol} \%)$ & $\mathbf{3 h}(20 \mathrm{~mol} \%)$ & 80 & $<1$ & rac \\
\hline 1a & $\mathrm{K}_{2} \mathrm{CO}_{3}(100 \mathrm{~mol} \%)$ & $3 \mathbf{i}(10 \mathrm{~mol} \%)$ & 80 & 13 & rac \\
\hline 1a & $\mathrm{K}_{2} \mathrm{CO}_{3}(100 \mathrm{~mol} \%)$ & $\mathbf{3 j - 1}(10 \mathrm{~mol} \%)$ & 80 & $<1$ & rac \\
\hline $\mathbf{1 r}$ & $\mathrm{K}_{2} \mathrm{CO}_{3}(100 \mathrm{~mol} \%)$ & 3a $(20 \mathrm{~mol} \%)$ & 80 & 82 & 51 \\
\hline $1 \mathbf{r}$ & $\mathrm{K}_{2} \mathrm{CO}_{3}(20 \mathrm{~mol} \%)$ & 3a $(20 \mathrm{~mol} \%)$ & 80 & 80 & 51 \\
\hline $1 \mathbf{r}$ & $\mathrm{K}_{2} \mathrm{CO}_{3}(10 \mathrm{~mol} \%)$ & 3a $(10 \mathrm{~mol} \%)$ & 80 & 28 & 50 \\
\hline $1 \mathbf{r}$ & $\mathrm{K}_{2} \mathrm{CO}_{3}(100 \mathrm{~mol} \%)$ & $\mathbf{3 b}(20 \mathrm{~mol} \%)$ & 80 & $<1$ & n. d. \\
\hline $1 \mathbf{r}$ & $\mathrm{K}_{2} \mathrm{CO}_{3}(100 \mathrm{~mol} \%)$ & $3 c(20 \mathrm{~mol} \%)$ & 80 & $<1$ & n. d. \\
\hline $1 \mathbf{r}$ & $\mathrm{K}_{2} \mathrm{CO}_{3}(100 \mathrm{~mol} \%)$ & 3d (20 mol\%) & 80 & 56 & 80 \\
\hline $\mathbf{1 r}$ & $\mathrm{K}_{2} \mathrm{CO}_{3}(100 \mathrm{~mol} \%)$ & $3 \mathbf{e}(20 \mathrm{~mol} \%)$ & 80 & 18 & 89 \\
\hline $1 \mathbf{r}$ & $\mathrm{K}_{2} \mathrm{CO}_{3}(100 \mathrm{~mol} \%)$ & $3 f(20 \mathrm{~mol} \%)$ & 80 & 86 & rac \\
\hline
\end{tabular}




\section{Synthesis of Starting Materials}

\section{2-(2-Bromobenzyl)benzofuran (S1)}

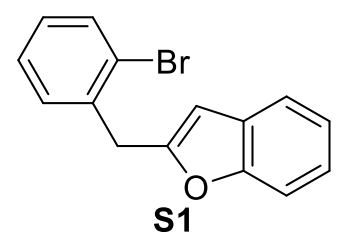

Benzofuran (1.08 mL, $10.0 \mathrm{mmol}, \quad 1.0$ equiv.) was dissolved in THF $(20 \mathrm{~mL})$ and cooled to $0{ }^{\circ} \mathrm{C}$. Then $n$-BuLi solution in hexanes (1.6 M, $10 \mathrm{~mL}, 16.0 \mathrm{mmol}, 1.6$ equiv.) was added dropwise to the reaction mixture. The resulting suspension was stirred for $30 \mathrm{~min}$. before 2-bromobenzyl bromide (6.23 g, $25.0 \mathrm{mmol}, 2.5$ equiv.) was added as a solution in $6.3 \mathrm{~mL}$ THF. The reaction was stirred and allowed to warm up to room temperature overnight. The reaction was stopped by dropwise addition of water. After extraction with EtOAc the organic layer was dried over $\mathrm{Na}_{2} \mathrm{SO}_{4}$. Purification by column chromatography on silica (n-pentane) afforded 2-(2bromobenzyl)benzofuran (S1) as a colorless oil (818 $\mathrm{mg}, 2.85 \mathrm{mmol}, 29 \%)$.

$\mathbf{R}_{f}$ (n-Pentane): 0.21;

${ }^{1}$ H NMR $\left(400 \mathrm{MHz}, \mathrm{CDCl}_{3}\right): \delta / \mathrm{ppm}=7.60(\mathrm{dd}, J=7.8,1.1 \mathrm{~Hz}, 1 \mathrm{H}), 7.51-7.47(\mathrm{~m}, 1 \mathrm{H}), 7.43$ $(\mathrm{dq}, J=8.2,0.9 \mathrm{~Hz}, 1 \mathrm{H}), 7.32-7.11(\mathrm{~m}, 5 \mathrm{H}), 6.39(\mathrm{~d}, J=1.0 \mathrm{~Hz}, 1 \mathrm{H}), 4.26(\mathrm{~d}, J=1.0 \mathrm{~Hz}, 2 \mathrm{H})$; ${ }^{13} \mathrm{C}$ NMR $\left(101 \mathrm{MHz}, \mathrm{CDCl}_{3}\right): \delta / \mathrm{ppm}=156.2,155.1,136.9,133.1,131.0,128.9,128.7,127.8$, $124.8,123.6,122.7,120.6,111.1,104.2,35.4$;

APCI-MS: calculated $\left[\mathrm{C}_{15} \mathrm{H}_{11} \mathrm{BrO}\right]^{+}:$285.99878, found: 285.99881;

ATR-FTIR $\left(\mathrm{cm}^{-1}\right): 3055,1579,1566,1438,1346,1250,1188,1161,1103,1026,953,918,829$, 795, 737, 660, 633.

\section{2-(Benzofuran-2-ylmethyl)benzaldehyde (1a)}

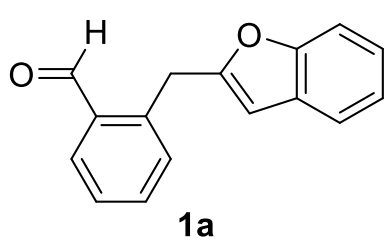

2-(2-Bromobenzyl)benzofuran (S1) $(574 \mathrm{mg}, 2.00 \mathrm{mmol}, 1.0$ equiv.) was dissolved in $\mathrm{THF}(8 \mathrm{~mL})$ and cooled to $-78^{\circ} \mathrm{C}$. Then $n$-BuLi solution in hexanes (1.6 M, $1.4 \mathrm{~mL}, 2.2 \mathrm{mmol}, 1.1$ equiv.) was added dropwise to the reaction mixture. After $1 \mathrm{~h}$ at $-78^{\circ} \mathrm{C}, \mathrm{DMF}(0.19 \mathrm{~mL}$, 2.4 mmol, 1.2 equiv.) was added dropwise. The reaction was stirred for further $15 \mathrm{~min}$ at $-78^{\circ} \mathrm{C}$ and was then allowed to warm to room temperature overnight. The reaction was quenched by addition of saturated aqueous $\mathrm{NH}_{4} \mathrm{Cl}$ solution. After extraction with $\mathrm{Et}_{2} \mathrm{O}$ the organic layer was dried over $\mathrm{Na}_{2} \mathrm{SO}_{4}$. Purification by column chromatography on silica $(n$-pentane/EtOAc $=95 / 5)$ 
afforded 2-(benzofuran-2-ylmethyl)benzaldehyde (1a) as a colorless oil (347 mg, $1.40 \mathrm{mmol}$, $70 \%)$.

$\mathbf{R}_{f}(n$-Pentane/EtOAc $=90 / 10): 0.31 ;$

${ }^{1} \mathbf{H}$ NMR (400 MHz, $\left.\mathrm{CDCl}_{3}\right): \delta / \mathrm{ppm}=10.28(\mathrm{~s}, 1 \mathrm{H}), 7.88(\mathrm{dd}, J=7.6,1.5 \mathrm{~Hz}, 1 \mathrm{H}), 7.56$ (td, $J=7.5,1.6 \mathrm{~Hz}, 1 \mathrm{H}), 7.51-7.44(\mathrm{~m}, 2 \mathrm{H}), 7.42-7.37(\mathrm{~m}, 2 \mathrm{H}), 7.22(\mathrm{td}, J=7.7,1.7 \mathrm{~Hz}, 1 \mathrm{H})$, $7.17(\mathrm{td}, J=7.4,1.4 \mathrm{~Hz}, 1 \mathrm{H}), 6.36(\mathrm{q}, J=1.0 \mathrm{~Hz}, 1 \mathrm{H}), 4.59$ (s, 2H);

${ }^{13} \mathrm{C}$ NMR $\left(101 \mathrm{MHz}, \mathrm{CDCl}_{3}\right): \delta / \mathrm{ppm}=192.6,156.9,155.0,139.3,134.1,134.0,133.1,131.6$, $128.8,127.7,123.7,122.7,120.6,111.0,104.0,31.8$

ESI-MS: calculated $\left[\mathrm{C}_{16} \mathrm{H}_{12} \mathrm{O}_{2} \mathrm{Na}\right]^{+}$: 259.0730, found: 259.0733;

ATR-FTIR $\left(\mathrm{cm}^{-1}\right):$ 3063, 3032, 2850, 2742, 1694, 1597, 1454, 1296, 1254, 1196, 1161, 1103, $1007,949,914,868,802,748,702,660,637,613$.

\section{2-(Bromomethyl)-4-fluorobenzonitrile (S2)}

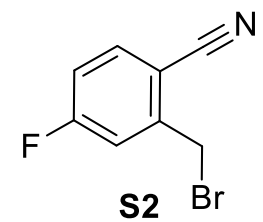

A solution of 4-fluoro-2-methylbenzonitrile (3.50 g, $26.0 \mathrm{mmol}, 1.00$ equiv.) in dry $\mathrm{CHCl}_{3}(22 \mathrm{~mL})$ was prepared under argon and $\mathrm{N}$-bromosuccinimide $(5.30 \mathrm{~g}$, $29.8 \mathrm{mmol}, 1.15$ equiv.) and a catalytic amount of benzoyl peroxide were added. The reaction mixture was stirred for $24 \mathrm{~h}$ at $85^{\circ} \mathrm{C}$. After that, the reaction mixture was cooled to ambient temperature and the precipitate was removed by filtration and washed with EtOAc $(40 \mathrm{~mL})$. The solvent was evaporated under reduced pressure. Purification by column chromatography on silica (n-pentane/EtOAc $=10 / 0.25)$ afforded 2-(bromomethyl)-4fluorobenzonitrile $\mathbf{S 2}$ as a yellow solid (3.20 g, $15.0 \mathrm{mmol}, 58 \%)$.

$\mathbf{R}_{\boldsymbol{f}}(n$-Pentane/EtOAc $=10 / 0.25): 0.25$

${ }^{1} \mathbf{H}$ NMR $\left(300 \mathrm{MHz}, \mathrm{CDCl}_{3}\right): \delta / \mathrm{ppm}=7.68(\mathrm{dd}, J=8.6,5.3 \mathrm{~Hz}, 1 \mathrm{H}), 7.22(\mathrm{dd}, J=8.6,2.6 \mathrm{~Hz}$, 1H), 7.13 (m, 1H), 4.60 (s, 2H);

${ }^{13} \mathrm{C}$ NMR $\left(75 \mathrm{MHz}, \mathrm{CDCl}_{3}\right): \delta / \mathrm{ppm}=165.0(\mathrm{~d}, J=260 \mathrm{~Hz}), 144.4(\mathrm{~d}, J=9 \mathrm{~Hz}), 135.6(\mathrm{~d}$, $J=10 \mathrm{~Hz}), 118.1(\mathrm{~d}, J=24 \mathrm{~Hz}), 116.8(\mathrm{~d}, J=22 \mathrm{~Hz}), 116.2,108.6(\mathrm{~d}, J=4 \mathrm{~Hz}), 28.5(\mathrm{~d}$, $J=2 \mathrm{~Hz})$;

GC-MS: $t_{R}=7.20 \mathrm{~min},(\mathrm{EI}) \mathrm{m} / z(\%): 213.0$ (5), $135.1(11), 134.1$ (100), 108.1 (6), 107.1 (20); ATR-FTIR $\left(\mathrm{cm}^{-1}\right):$ 2225, 1608, 1586, 1496, 1423, 1281, 1250, 1219, 1188, 1157, 1126, 1084, $964,875,829,748,733,687,644,613$. 


\section{2-(Bromomethyl)-4-fluorobenzaldehyde (S3)}<smiles>O=Cc1ccc(F)cc1CBr</smiles>

S3

According to the literature, ${ }^{[\mathrm{S} 9]}$ a solution of 2-(bromomethyl)-4fluorobenzonitrile (3.00 g, $14.0 \mathrm{mmol}, 1.0$ equiv.) in dry $\mathrm{CH}_{2} \mathrm{Cl}_{2}(45 \mathrm{~mL})$ was prepared under argon. A solution of DIBAL-H in hexane $(1.0 \mathrm{M}, 15.4 \mathrm{~mL}$, 15.4 mmol, 1.10 equiv.) was added dropwise over $30 \mathrm{~min}$ at $0{ }^{\circ} \mathrm{C}$. The resulting solution was allowed to warm up to ambient temperature for $3 \mathrm{~h}$ by removing the ice bath. The mixture was cooled to $0{ }^{\circ} \mathrm{C}$ again and poured into a solution of aqueous $\mathrm{HBr}(6 \mathrm{M}, 50 \mathrm{~mL})$ and ice (50 g). The mixture was stirred for $1 \mathrm{~h}$ and was extracted with $\mathrm{CH}_{2} \mathrm{Cl}_{2}(40 \mathrm{~mL})$. The organic phase was washed with aqueous $\mathrm{NaHCO}_{3}(1 \mathrm{M}, 2 \times 50 \mathrm{~mL})$ and water $(2 \times 50 \mathrm{~mL})$, then dried over $\mathrm{MgSO}_{4}$ and concentrated under reduced pressure. Compound S3 was afforded as a dark solid (2.90 g, $13.2 \mathrm{mmol}, 94 \%)$.

$\mathbf{R}_{f}\left(\mathrm{CH}_{2} \mathrm{Cl}_{2}\right): 0.60$;

${ }^{\mathbf{1}} \mathbf{H}$ NMR $\left(400 \mathrm{MHz}, \mathrm{CDCl}_{3}\right): \delta / \mathrm{ppm}=10.18(\mathrm{~s}, 1 \mathrm{H}), 7.87(\mathrm{dd}, J=8.5,5.7 \mathrm{~Hz}, 1 \mathrm{H}), 7.25-7.11$ (m, 2H), $4.92(\mathrm{~s}, 2 \mathrm{H})$;

${ }^{13} \mathrm{C} \mathrm{NMR}\left(100 \mathrm{MHz}, \mathrm{CDCl}_{3}\right): \delta / \mathrm{ppm}=190.5,165.6(\mathrm{~d}, J=258 \mathrm{~Hz}), 142.6(\mathrm{~d}, J=9 \mathrm{~Hz}), 136.7$ (d, $J=10 \mathrm{~Hz}), 129.9$ (d, $J=3 \mathrm{~Hz}), 119.1(\mathrm{~d}, J=23 \mathrm{~Hz}), 116.2(\mathrm{~d}, J=22 \mathrm{~Hz}), 28.6(\mathrm{~d}, J=1 \mathrm{~Hz})$; EI-MS: calculated for [ $\left.\mathrm{C}_{8} \mathrm{H}_{8} \mathrm{BrFO}\right]: 216.0$, found: 216.0; ATR-FTIR $\left(\mathbf{c m}^{-1}\right):$ 2758, 1697, 1582, 1497, 1427, 1397, 1312, 1277, 1242, 1196, 1157, 1126, 1088, 968, 883, 829, 783, 745, 721, 644, 617.

\section{2-(Benzofuran-2-ylmethyl)-4-fluorobenzaldehyde (1b)}

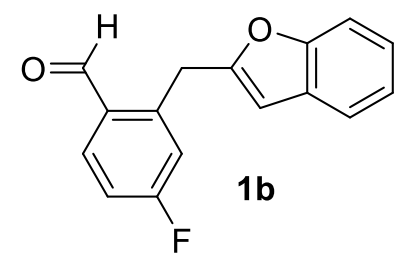

A schlenk-flask was filled with 2-benzofuranylboronic acid (364 mg, 2.25 mmol, 1.5 equiv.), 2-(bromomethyl)-4-fluorobenzaldehyde (S3) (326 mg, $\quad 1.50 \mathrm{mmol}, \quad 1.0$ equiv.), tetrakis(triphenylphosphine)palladium(0) (87 mg, $0.075 \mathrm{mmol}, 0.05$ equiv.), $1.65 \mathrm{~mL}$ aqueous $\mathrm{Na}_{2} \mathrm{CO}_{3}$ solution $(1 \mathrm{M}), 2.5 \mathrm{~mL}$ toluene, $2.0 \mathrm{~mL}$ ethanol and $0.5 \mathrm{~mL} \mathrm{H} \mathrm{H}_{2} \mathrm{O}$. The flask was evacuated and backfilled with argon three times and stirred at $80{ }^{\circ} \mathrm{C}$ overnight. The reaction was quenched with $10 \mathrm{~mL} \mathrm{H}_{2} \mathrm{O}$ and extracted with $3 \times 10 \mathrm{~mL} \mathrm{CH}_{2} \mathrm{Cl}_{2}$, over $\mathrm{MgSO}_{4}$ and concentrated under reduced pressure. Purification by column chromatography on silica $(n$-pentane/EtOAc $=95 / 5)$ 
afforded 2-(benzofuran-2-ylmethyl)-4-fluorobenzaldehyde (1b) as a yellow oil (157 mg, $0.62 \mathrm{mmol}, 41 \%)$.

$\mathbf{R}_{f}(n$-Pentane/EtOAc $=9 / 1): 0.38 ;$

${ }^{1} \mathbf{H}$ NMR $\left(300 \mathrm{MHz}, \mathrm{CD}_{2} \mathrm{Cl}_{2}\right): \delta / \mathrm{ppm}=10.21(\mathrm{~s}, 1 \mathrm{H}), 7.90(\mathrm{dd}, J=8.6,5.9 \mathrm{~Hz}, 1 \mathrm{H}), 7.51-7.47$ (m, 1H), $7.45-7.38(\mathrm{~m}, 1 \mathrm{H}), 7.32-7.04(\mathrm{~m}, 4 \mathrm{H}), 6.43(\mathrm{dd}, J=1.0,1.0 \mathrm{~Hz}, 1 \mathrm{H}), 4.59(\mathrm{~s}, 2 \mathrm{H})$; ${ }^{13}$ C NMR (100 MHz, $\left.\mathrm{CDCl}_{3}\right): \delta / \mathrm{ppm}=191.0,166.1(\mathrm{~d}, J=256 \mathrm{~Hz}), 156.4,155.3,143.0(\mathrm{~d}$, $J=9 \mathrm{~Hz}), 136.0(\mathrm{~d}, J=10 \mathrm{~Hz}), 131.1$ (d, $J=3 \mathrm{~Hz}), 129.0,124.1,123.1,120.9,118.8$ (d, $J=23 \mathrm{~Hz}), 115.0$ (d, $J=22 \mathrm{~Hz}), 111.2,104.5,31.7$ (d, $J=1 \mathrm{~Hz})$;

ESI-MS: calculated for $\left[\mathrm{C}_{16} \mathrm{H}_{11} \mathrm{FO}_{2} \mathrm{Na}\right]^{+}:$: 277.0635, found: 277.0633;

ATR-FTIR $\left(\mathrm{cm}^{-1}\right):$ 3063, 2851, 1694, 1605, 1582, 1493, 1454, 1431, 1242, 1200, 1153, 1088, 1007, 968, 952, 880, 818, 802, 748, 613.

\section{2-(Bromomethyl)-4-chlorobenzonitrile (S4)}<smiles>N#Cc1ccc(Cl)cc1CBr</smiles>

mixture was cooled to ambient temperature and the precipitate was removed by filtration and washed with EtOAc $(40 \mathrm{~mL})$. The solvent was evaporated under reduced pressure. Purification by column chromatography on silica $(n$-pentane/EtOAc $=10 / 0.1)$ afforded 2-(bromomethyl)-4chlorobenzonitrile (S4) as a yellow solid (2.90 g, $13.0 \mathrm{mmol}, 57 \%)$.

$\mathbf{R}_{f}(n$-Pentane/EtOAc $=10 / 0.1): 0.25 ;$

${ }^{1} \mathbf{H}$ NMR $\left(300 \mathrm{MHz}, \mathrm{CDCl}_{3}\right): \delta / \mathrm{ppm}=7.61(\mathrm{~d}, J=8.3 \mathrm{~Hz}, 1 \mathrm{H}), 7.56(\mathrm{~d}, J=2.1 \mathrm{~Hz}, 1 \mathrm{H}), 7.40$ $(\mathrm{dd}, J=8.3,2.1 \mathrm{~Hz}, 1 \mathrm{H}), 4.58(\mathrm{~s}, 2 \mathrm{H})$;

${ }^{13} \mathrm{C}$ NMR (100 MHz, $\left.\mathrm{CDCl}_{3}\right): \delta / \mathrm{ppm}=143.0,139.9,134.4,130.9,129.5,116.2,110.9,28.4$;

GC-MS: $t_{R}=7.84 \mathrm{~min},(\mathrm{EI}) \mathrm{m} / z$ (\%): 231.0 (7), 229.0 (6), 152.0 (33), 151.0 (11), 150.0 (100), 123.0 (12), 115.1 (5), 114.1 (9), 88.1 (6), 63.1 (5), 62.0 (5);

ATR-FTIR $\left(\mathrm{cm}^{-1}\right):$ 2361, 2226, 1693, 1562, 1481, 1404, 1284, 1227, 1027, 1180, 1107, 1080, $902,825,729,644$. 


\section{2-(Bromomethyl)-4-chlorobenzaldehyde (S5)}<smiles>O=Cc1ccc(Cl)cc1CBr</smiles>

According to the literature, ${ }^{[\mathrm{S} 9]}$ a solution of 2-(bromomethyl)-4chlorobenzonitrile (2.70 g, $12.0 \mathrm{mmol}, 1.00$ equiv.) in dry $\mathrm{CH}_{2} \mathrm{Cl}_{2}(40 \mathrm{~mL})$ was prepared under argon. A solution of DIBAL-H in hexane $(1.0 \mathrm{M}, 13.2 \mathrm{~mL}$, $13.2 \mathrm{mmol}, 1.10$ equiv.) was added dropwise over $30 \mathrm{~min}$ at $0{ }^{\circ} \mathrm{C}$. The resulting solution was allowed to warm up to ambient temperature for $3 \mathrm{~h}$ by removing the ice bath. The mixture was cooled to $0{ }^{\circ} \mathrm{C}$ again and poured into a solution containing aqueous $\mathrm{HBr}(6 \mathrm{M}, 50 \mathrm{~mL})$ and ice $(50 \mathrm{~g})$. The mixture was stirred for $1 \mathrm{~h}$ and was extracted with $\mathrm{CH}_{2} \mathrm{Cl}_{2}$. The organic phases were washed with aqueous $\mathrm{NaHCO}_{3}(1 \mathrm{M}, 2 \times 50 \mathrm{~mL})$ and water $(2 \times 50 \mathrm{~mL})$, dried over $\mathrm{MgSO}_{4}$ and concentrated under reduced pressure. Compound $\mathbf{S 5}$ was afforded as a dark solid (2.70 g, $11.4 \mathrm{mmol}, 95 \%)$.

$\mathbf{R}_{f}\left(\mathrm{CH}_{2} \mathrm{Cl}_{2}\right): 0.69$;

${ }^{1} \mathbf{H}$ NMR $\left(400 \mathrm{MHz}, \mathrm{CDCl}_{3}\right): \delta / \mathrm{ppm}=10.20(\mathrm{~s}, 1 \mathrm{H}), 7.86-7.73(\mathrm{~m}, 1 \mathrm{H}), 7.54-7.44(\mathrm{~m}, 2 \mathrm{H})$, $4.88(\mathrm{~s}, 2 \mathrm{H})$;

${ }^{13} \mathrm{C}$ NMR (101 MHz, $\left.\mathrm{CDCl}_{3}\right): \delta / \mathrm{ppm}=190.8,141.0,140.4,135.0,131.9,131.6,129.4,28.5$;

ESI-MS: calculated for $\left[\mathrm{C}_{8} \mathrm{H}_{5} \mathrm{OBrNa}\right]^{+}: 253.1983$, found: 253.1983;

ATR-FTIR $\left(\mathrm{cm}^{-1}\right):$ 2855, 2754, 2229, 1694, 1589, 1562, 1485, 1431, 1385, 1282, 1276, 1227 , 1196, 1150, 1111, 1080, 1003, 907, 821, 760, 729, 694, 648, 629.

\section{2-(Benzofuran-2-ylmethyl)-4-chlorobenzaldehyde (1c)}

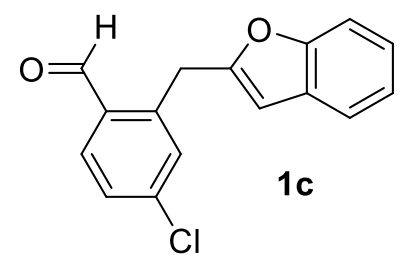

A schlenk-flask was filled with 2-benzofuranylboronic acid (364 mg, 2.25 mmol, 1.5 equiv.), 2-(bromomethyl)-4-chlorobenzaldehyde (S5) (350 mg, $\quad 1.50 \mathrm{mmol}, \quad 1.0$ equiv.), tetrakis(triphenylphosphine)palladium(0) (87 $\mathrm{mg}, \quad 0.075 \mathrm{mmol}, 0.05$ equiv.), $1.65 \mathrm{~mL}$ aqueous $\mathrm{Na}_{2} \mathrm{CO}_{3}$ solution $(1 \mathrm{M}), 2.5 \mathrm{~mL}$ toluene, $2.0 \mathrm{~mL}$ ethanol and $0.5 \mathrm{~mL} \mathrm{H}_{2} \mathrm{O}$. The flask was evacuated and backfilled with argon three times and stirred at $80{ }^{\circ} \mathrm{C}$ overnight. The reaction was quenched with $10 \mathrm{~mL} \mathrm{H}_{2} \mathrm{O}$ and extracted with 3 x $10 \mathrm{~mL} \mathrm{CH}_{2} \mathrm{Cl}_{2}$, dried over $\mathrm{MgSO}_{4}$ and concentrated under reduced pressure. Purification by column chromatography on silica $(n$-pentane/EtOAc $=95 / 5)$ 
afforded 2-(benzofuran-2-ylmethyl)-4-chlorobenzaldehyde (1c) as a yellow solid (161 mg, $0.54 \mathrm{mmol}, 36 \%)$.

$\mathbf{R}_{f}(n$-Pentane/EtOAc $=9 / 1): 0.42 ;$

${ }^{1} \mathbf{H}$ NMR $\left(300 \mathrm{MHz}, \mathrm{CD}_{2} \mathrm{Cl}_{2}\right): \delta / \mathrm{ppm}=10.23(\mathrm{~s}, 1 \mathrm{H}), 7.83(\mathrm{~d}, J=8.3 \mathrm{~Hz}, 1 \mathrm{H}), 7.52-7.44(\mathrm{~m}$, 2H), $7.44-7.37(\mathrm{~m}, 2 \mathrm{H}), 7.26-7.14(\mathrm{~m}, 2 \mathrm{H}), 6.42(\mathrm{dd}, J=1.0,1.0 \mathrm{~Hz}, 1 \mathrm{H}), 4.56(\mathrm{~s}, 2 \mathrm{H})$;

${ }^{13} \mathrm{C}$ NMR $\left(75 \mathrm{MHz}, \mathrm{CD}_{2} \mathrm{Cl}_{2}\right): \delta / \mathrm{ppm}=191.5,156.6,155.5,141.5,140.6,134.5,133.0,132.0$, $129.2,128.4,124.3,123.3,121.1,111.4,104.6,31.8$

ESI-MS: calculated for $\left[\mathrm{C}_{16} \mathrm{H}_{11} \mathrm{ClO}_{2} \mathrm{Na}\right]^{+}$: 293.0340, found: 293.0343;

ATR-FTIR $\left(\mathrm{cm}^{-1}\right)$ : 1694, 1589, 1562, 1451, 1424, 1389, 1296, 1254, 1196, 1088, 1007, 953, 910, 891, 802, 748, 606 .

\section{3-Methyl-[1,1'-biphenyl]-4-carbonitrile (S6)}

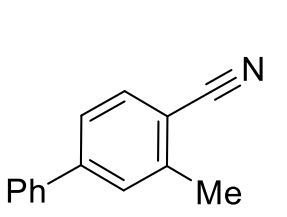

S6

According to the literature, ${ }^{[\mathrm{S} 10]}$ a flask was charged with $\mathrm{Pd}(\mathrm{OAc})_{2}(57 \mathrm{mg}$, 0.26 mmol, $\quad 0.01$ equiv.), $\quad \mathrm{PPh}_{3} \quad(134 \mathrm{mg}, \quad 0.52 \mathrm{mmol}, \quad 0.02$ equiv.), phenylboronic acid $\left(4.70 \mathrm{~g}, 38.25 \mathrm{mmol}, 1.50\right.$ equiv.) and $\mathrm{K}_{3} \mathrm{PO}_{4}$ (10.6 g, $51.0 \mathrm{mmol}, 2.00$ equiv.). 4-Bromo-2-methylbenzonitrile (5.00 g, $25.5 \mathrm{mmol}$, 1.00 equiv.) and dry toluene $(60 \mathrm{~mL})$ were added. The reaction mixture was stirred for $12 \mathrm{~h}$ at $80{ }^{\circ} \mathrm{C}$. After that, the reaction mixture was cooled to ambient temperature and quenched with water $(50 \mathrm{~mL})$. The aqueous phase was extracted with $\mathrm{Et}_{2} \mathrm{O}(4 \times 50 \mathrm{~mL})$. The combined organic layers were washed with water $(50 \mathrm{~mL}), \mathrm{NaOH}(1 \mathrm{M}, 50 \mathrm{~mL})$, brine $(50 \mathrm{~mL})$ and were dried over $\mathrm{MgSO}_{4}$. The solvent was evaporated under reduced pressure. Purification by column chromatography on silica $(n$-pentane/EtOAc $=10 / 0.15)$ afforded compound $\mathbf{S 6}$ as a yellow solid (3.20 g, $16.0 \mathrm{mmol}, 63 \%)$.

$\mathbf{R}_{f}(n$-Pentane/EtOAc $=10 / 0.1): 0.1 ;$

${ }^{1} \mathbf{H}$ NMR (400 MHz, $\left.\mathrm{CDCl}_{3}\right): \delta / \mathrm{ppm}=7.67(\mathrm{dd}, J=8.1 \mathrm{~Hz}, 3.0 \mathrm{~Hz}, 1 \mathrm{H}), 7.63-7.56(\mathrm{~m}, 2 \mathrm{H})$, $7.56-7.37(\mathrm{~m}, 5 \mathrm{H}), 2.62(\mathrm{~d}, J=3.0 \mathrm{~Hz}, 3 \mathrm{H})$;

${ }^{13} \mathrm{C}$ NMR $\left(101 \mathrm{MHz}, \mathrm{CDCl}_{3}\right): \delta / \mathrm{ppm}=145.7,142.5,139.5,133.1,129.2,129.0,128.6,127.4$, 125.1, 118.4, 111.5, 20.8;

GC-MS: $t_{R}=8.67 \mathrm{~min},(\mathrm{EI}) \mathrm{m} / \mathrm{z}(\%): 194.1$ (16), 193.1 (100), 192.2 ( 29), 191.1 (7), 190.1 (11), 
178.1 (7), 177.1 (7), 166.1 (6), 165.1 (25), 164.1 (5), 82.3 (7);

ATR-FTIR $\left(\mathrm{cm}^{-1}\right):$ 3032, 2222, 1609, 1559, 1481, 1447, 1397, 1269, 1184, 1157, 1119, 1076, 1026, 1003, 953, 918, 887, 833, 780, 725, 694, 606.

\section{3-(Bromomethyl)-[1,1'-biphenyl]-4-carbonitrile (S7)}

A solution of 3-methyl-[1,1'-biphenyl]-4-carbonitrile (S6) (2.90 g, $15.0 \mathrm{mmol}, 1.00$ equiv.) in dry<smiles>N#Cc1ccc(-c2ccccc2)cc1CBr</smiles>
$\mathrm{CHCl}_{3}(22 \mathrm{~mL})$ was prepared under argon and $\mathrm{N}$-bromosuccinimide $(3.07 \mathrm{~g}$, 17.3 mmol, 1.15 equiv.) and a catalytic amount of dibenzoylperoxide were added. The reaction mixture was stirred for $24 \mathrm{~h}$ at $85^{\circ} \mathrm{C}$. After that, the reaction mixture was cooled to ambient temperature and the precipitate was removed by filtration and washed with EtOAc. The solvent was evaporated under reduced pressure. Purification by column chromatography on silica ( $n$-pentane/EtOAc $=10 / 0.15)$ afforded 3(bromomethyl)-[1,1'-biphenyl]-4-carbonitrile) (S7) as a yellow solid (2.70 g, $10.0 \mathrm{mmol}, 67 \%)$.

$\mathbf{R}_{f}(n$-pentane/EtOAc $\left.)=10 / 0.15\right): 0.20 ;$

${ }^{1} \mathbf{H}$ NMR $\left(400 \mathrm{MHz}, \mathrm{CDCl}_{3}\right): \delta / \mathrm{ppm}=7.82-7.68(\mathrm{~m}, 2 \mathrm{H}), 7.68-7.55(\mathrm{~m}, 3 \mathrm{H}), 7.55-7.39(\mathrm{~m}$, $3 \mathrm{H}), 4.70$ (s, 2H);

${ }^{13} \mathrm{C}$ NMR (101 MHz, $\left.\mathrm{CDCl}_{3}\right): \delta / \mathrm{ppm}=146.4,141.7,138.7,133.8,129.3,129.2,129.1,127.6$, 127.4, 117.0, 111.0, 29.6;

GC-MS: $t_{R}=9.52 \mathrm{~min},(\mathrm{EI}) \mathrm{m} / z(\%): 273.0$ (13), 271.0 (14), 193.1 (18), 192.1 (100), 191.1 (9), 190.1 (25), 177.1 (6), 165.1 (18), 96.0 (11), 82.5 (8);

ATR-FTIR $\left(\mathrm{cm}^{-1}\right)$ : 3032, 2222, 1604, 1481, 1481, 1450, 1404, 1307, 1219, 1067, 1022, 907, $837,764,729,694,652,610$.

\section{3-(Bromomethyl)-[1,1'-biphenyl]-4-carbaldehyde (S8)}<smiles>O=Cc1ccc(-c2ccccc2)cc1CBr</smiles>

S8
According to the literature ${ }^{[\mathrm{S} 9]}$ a solution of 4-(bromomethyl)-[1,1'-biphenyl]-3carbonitrile $\mathbf{S 7}$ (2.40 g, $8.20 \mathrm{mmol}, 1.0$ equiv.) in dry $\mathrm{CH}_{2} \mathrm{Cl}_{2}(30 \mathrm{~mL})$ was prepared under argon. A solution of DIBAL-H in hexane $(1.0 \mathrm{M}, 9.0 \mathrm{~mL}$, $9.0 \mathrm{mmol}, 1.1$ equiv.) was added dropwise over $30 \mathrm{~min}$. The resulting solution was allowed to warm up to ambient temperature over $3 \mathrm{~h}$. The mixture was 
cooled to $0{ }^{\circ} \mathrm{C}$ again and poured into a solution containing aqueous $\mathrm{HBr}(6 \mathrm{M}, 40 \mathrm{~mL})$ and ice (40 g). The mixture was stirred for $1 \mathrm{~h}$ and was extracted with $\mathrm{CH}_{2} \mathrm{Cl}_{2}$. The organic phases were washed with aqueous $\mathrm{NaHCO}_{3}(1 \mathrm{M}, 2 \times 50 \mathrm{~mL})$, water $(2 \times 50 \mathrm{~mL})$, dried over $\mathrm{MgSO}_{4}$ and concentrated under reduced pressure to afford compound $\mathbf{S 8}$ as a brown solid (1.40 g, 5.20 mmol, $63 \%)$.

$\mathbf{R}_{f}\left(\mathrm{CH}_{2} \mathrm{Cl}_{2}\right): 0.63$;

${ }^{1} \mathbf{H}$ NMR $\left(300 \mathrm{MHz}, \mathrm{CDCl}_{3}\right): \delta / \mathrm{ppm}=10.28(\mathrm{~s}, 1 \mathrm{H}), 7.92(\mathrm{~d}, J=7.8 \mathrm{~Hz}, 1 \mathrm{H}), 7.77-7.61(\mathrm{~m}$, 4H), $7.56-7.39(\mathrm{~m}, 3 \mathrm{H}), 5.01(\mathrm{~s}, 2 \mathrm{H})$;

${ }^{13} \mathrm{C}$ NMR (75 MHz, $\left.\mathrm{CDCl}_{3}\right): \delta / \mathrm{ppm}=191.8,146.9,139.8,139.1,134.6$ 132.0, 130.6, 129.2, $128.9,127.7,127.5,29.8$;

ESI-MS: calculated for $\left[\mathrm{C}_{14} \mathrm{H}_{11} \mathrm{BrONa}\right]^{+}$: 296.9885, found: 296.9885;

ATR-FTIR $\left(\mathrm{cm}^{-1}\right):$ 3032, 2222, 1682, 1601, 1559, 1451, 1389, 1207, 1180, 1026, 907, 841, 802, $750,730,703,655$.

\section{3-(Benzofuran-2-ylmethyl)-[1,1'-biphenyl]-4-carbaldehyde (1d)}

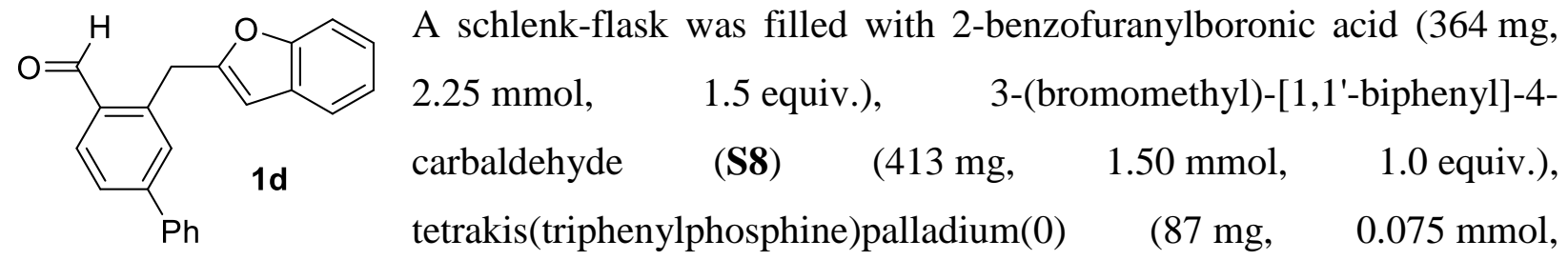

0.05 equiv.), $1.65 \mathrm{~mL}$ aqueous $\mathrm{Na}_{2} \mathrm{CO}_{3}$ solution $(1 \mathrm{M}), 2.5 \mathrm{~mL}$ toluene, $2.0 \mathrm{~mL}$ ethanol and $0.5 \mathrm{~mL}$ $\mathrm{H}_{2} \mathrm{O}$. The flask was evacuated and backfilled with argon three times and stirred at $80{ }^{\circ} \mathrm{C}$ overnight. The reaction was quenched with $10 \mathrm{~mL} \mathrm{H}_{2} \mathrm{O}$ and extracted with $3 \times 10 \mathrm{~mL} \mathrm{CH}_{2} \mathrm{Cl}_{2}$, then dried over $\mathrm{MgSO}_{4}$ and concentrated under reduced pressure. Purification by column chromatography on silica $\quad(n$-pentane/EtOAc $=$ 95/5) afforded 3-(benzofuran-2-ylmethyl)-[1,1'-biphenyl]-4carbaldehyde (1d) as a yellow oil (271 $\mathrm{mg}, 0.87 \mathrm{mmol}, 58 \%)$.

$\mathbf{R}_{f}(n$-Pentane/EtOAc $=9 / 1): 0.47$;

${ }^{1} \mathbf{H}$ NMR $\left(300 \mathrm{MHz}, \mathrm{CD}_{2} \mathrm{Cl}_{2}\right): \delta / \mathrm{ppm}=10.30(\mathrm{~s}, 1 \mathrm{H}), 7.96(\mathrm{~d}, J=8.0 \mathrm{~Hz}, 1 \mathrm{H}), 7.73(\mathrm{dd}, J=8.0$, $1.8 \mathrm{~Hz}, 1 \mathrm{H}), 7.69-7.63(\mathrm{~m}, 3 \mathrm{H}), 7.54-7.37(\mathrm{~m}, 5 \mathrm{H}), 7.28-7.11(\mathrm{~m}, 2 \mathrm{H}), 6.40(\mathrm{dd}, J=1.0$, $1.0 \mathrm{~Hz}, 1 \mathrm{H}), 4.79-4.54(\mathrm{~m}, 2 \mathrm{H})$; 
${ }^{13} \mathrm{C}$ NMR $\left(75 \mathrm{MHz}, \mathrm{CD}_{2} \mathrm{Cl}_{2}\right): \delta / \mathrm{ppm}=192.3,157.7,155.4,146.9,140.1,139.9,133.9,133.4$, $130.9,129.5,129.3,129.1,127.9,126.7,124.1,123.1,121.0,111.3,104.2,32.3$;

ESI-MS: calculated for $\left[\mathrm{C}_{22} \mathrm{H}_{16} \mathrm{O}_{2} \mathrm{Na}\right]^{+}: 335.1043$, found: 335.1040 ;

ATR-FTIR $\left(\mathrm{cm}^{-1}\right):$ 3059, 3032, 2851, 2739, 1686, 1601, 1562, 1508, 1478, 1454, 1397, 1249, 1200, 1103, 1076, 1007, 953, 895, 829, 802, 748, 694.

\section{2-(2-Bromobenzyl)-5-methylbenzofuran (S9)}

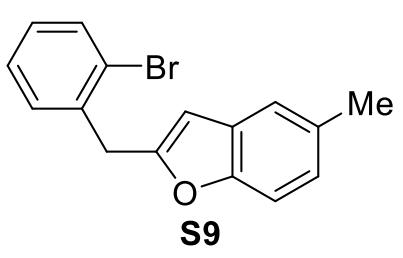

5-Methylbenzofuran ${ }^{[\mathrm{S11}]} \quad(1.32 \mathrm{~mL}, \quad 10.0 \mathrm{mmol}, \quad 1.0$ equiv. $) \quad$ was dissolved in THF $(20 \mathrm{~mL})$ and cooled to $0{ }^{\circ} \mathrm{C}$. Then $n$-BuLi solution in hexane (1.6 M, $6.3 \mathrm{~mL}, 10.0 \mathrm{mmol}, 1.0$ equiv.) was added dropwise to the reaction mixture. The resulting suspension was stirred for $30 \mathrm{~min}$. before 2-bromobenzyl bromide (6.23 g, $25.0 \mathrm{mmol}, 2.5$ equiv.) was added as a solution in $6.3 \mathrm{~mL}$ THF. The reaction was stirred and allowed to warm up to room temperature overnight. The reaction was stopped by dropwise addition of water. After extraction with EtOAc the organic layer was dried over $\mathrm{Na}_{2} \mathrm{SO}_{4}$. Purification by column chromatography on silica ( $n$-pentane) afforded 2(2-bromobenzyl)-5-methylbenzofuran (S9) as a colorless oil (770 mg, $2.65 \mathrm{mmol}, 27 \%$ ).

$\mathbf{R}_{f}(n$-Pentane): 0.36;

${ }^{1}$ H NMR (300 MHz, CDCl3): $\delta / \mathrm{ppm}=7.65$ - $7.52(\mathrm{~m}, 1 \mathrm{H}), 7.35$ - $7.21(\mathrm{~m}, 4 \mathrm{H}), 7.19-7.06(\mathrm{~m}$, 1H), $7.03(\mathrm{dd}, J=8.4,1.8 \mathrm{~Hz}, 1 \mathrm{H}), 6.31(\mathrm{~d}, J=1.0 \mathrm{~Hz}, 1 \mathrm{H}), 4.23(\mathrm{~s}, 2 \mathrm{H}), 2.41(\mathrm{~s}, 3 \mathrm{H})$;

${ }^{13}$ C NMR (75 MHz, CDCl3): $\delta / \mathrm{ppm}=156.2$, 153.5, 137.1, 133.0, 132.1, 131.0, 129.0, 128.6, $127.7,124.8,124.7,120.5,110.6,104.0,35.4,21.5$.

\section{2-((5-Methylbenzofuran-2-yl)methyl)benzaldehyde (1e)}

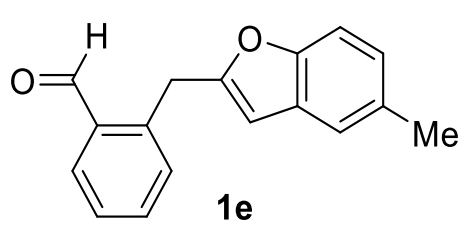

Following a procedure by Ashfield and Barnard, ${ }^{[\mathrm{S} 12]}$ 2-(2bromobenzyl)-5-methylbenzofuran (S9) $\quad(500 \mathrm{mg}, 1.66 \mathrm{mmol}$, 1.0 equiv.) together with $\mathrm{Pd}(\mathrm{dppf}) \mathrm{Cl}_{2} \quad(24.5 \mathrm{mg}, \quad 41 \mu \mathrm{mol}$, $2.5 \mathrm{~mol} \%), \mathrm{Et}_{3} \mathrm{SiH}\left(530 \mu \mathrm{L}, 3.32 \mathrm{mmol}, 2.0\right.$ equiv.) and $\mathrm{Na}_{2} \mathrm{CO}_{3}$

(176 mg, $1.66 \mathrm{mmol}, 1.0$ equiv.) was dissolved in DMF (3 mL) and filled with CO (1 bar). After heating to $90{ }^{\circ} \mathrm{C}$, the pressure was increased to 3 bar. The reaction was stirred for $18 \mathrm{~h}$ and then 
diluted with $10 \mathrm{~mL} \mathrm{CH}_{2} \mathrm{Cl}_{2}$. The mixture was washed with aqueous saturated $\mathrm{NH}_{4} \mathrm{Cl}$-solution and dried over $\mathrm{Na}_{2} \mathrm{SO}_{4}$. Purification by column chromatography on silica ( $n$-pentane/EtOAc $\left.=95 / 5\right)$ afforded 2-((5-methylbenzofuran-2-yl)methyl)benzaldehyde (1e) as a colorless oil (151 mg, $0.60 \mathrm{mmol}, 36 \%)$.

$\mathbf{R}_{f}(n$-Pentane/EtOAc $=90 / 10): 0.36 ;$

${ }^{1} \mathbf{H}$ NMR (400 MHz, $\left.\mathrm{CDCl}_{3}\right): \delta / \mathrm{ppm}=10.28(\mathrm{~s}, 1 \mathrm{H}), 7.88(\mathrm{dd}, J=7.6,1.6 \mathrm{~Hz}, 1 \mathrm{H}), 7.55$ (td, $J=7.5,1.6 \mathrm{~Hz}, 1 \mathrm{H}), 7.47(\mathrm{td}, J=7.5,1.3 \mathrm{~Hz}, 1 \mathrm{H}), 7.37(\mathrm{dd}, J=7.6,1.2 \mathrm{~Hz}, 1 \mathrm{H}), 7.28(\mathrm{~d}$, $J=8.4 \mathrm{~Hz}, 1 \mathrm{H}), 7.24(\mathrm{~s}, 1 \mathrm{H}), 7.02(\mathrm{dd}, J=8.4,1.8 \mathrm{~Hz}, 1 \mathrm{H}), 6.29$ (q, $J=1.0 \mathrm{~Hz}, 1 \mathrm{H}), 4.56(\mathrm{~s}$, $2 \mathrm{H}), 2.41(\mathrm{~s}, 3 \mathrm{H})$;

${ }^{13} \mathrm{C}$ NMR (101 MHz, $\left.\mathrm{CDCl}_{3}\right): \delta / \mathrm{ppm}=192.5,156.9,153.4,139.4,134.1,134.0,132.9,132.1$, $131.6,128.9,127.6,124.9,120.5,110.5,103.8,31.8,21.4$;

ESI-MS: calculated $\left[\mathrm{C}_{17} \mathrm{H}_{14} \mathrm{O}_{2} \mathrm{Na}\right]^{+}$: 273.0886, found: 273.0888;

ATR-FTIR $\left(\mathrm{cm}^{-1}\right):$ 2916, 2862, 2735, 1694, 1597, 1574, 1474, 1451, 1400, 1288, 1261, 1196, $1146,1119,949,910,868,799,752,664,637$.

\section{2-(Bromomethyl)benzaldehyde (S10)}

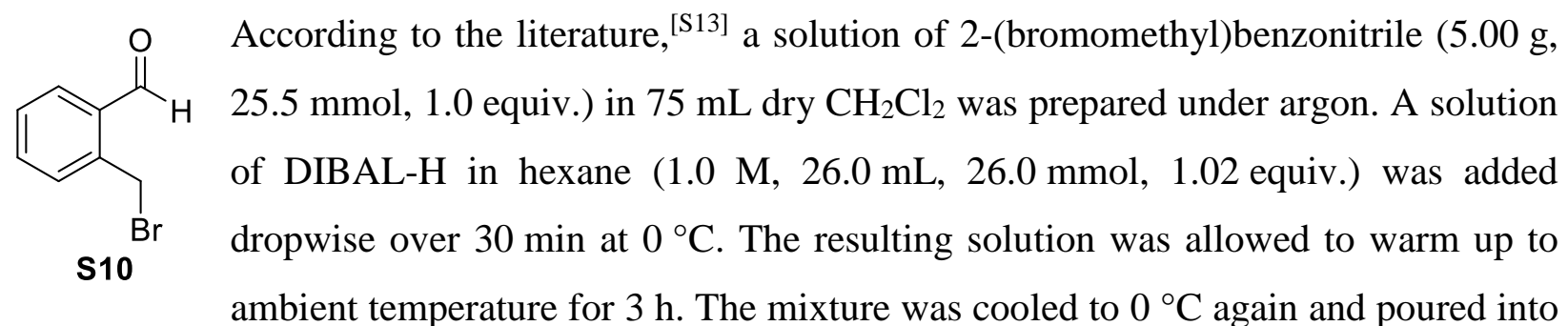
a solution containing $100 \mathrm{~mL}$ aqueous $\mathrm{HBr}(6 \mathrm{M})$ and $100 \mathrm{~g}$ ice. The mixture was stirred for $1 \mathrm{~h}$ at $0{ }^{\circ} \mathrm{C}$ and then extracted with $\mathrm{CH}_{2} \mathrm{Cl}_{2}$. The organic phases were washed with aqueous $\mathrm{NaHCO}_{3}$ (1M) and brine, then dried over $\mathrm{MgSO}_{4}$ and concentrated under reduced pressure. Purification by column chromatography on silica ( $n$-pentane/ $\mathrm{CH}_{2} \mathrm{Cl}_{2}=1 / 1$ ) afforded 2-(bromomethyl)benzaldehyde (S10) as a dark solid (4.90 g, $24.5 \mathrm{mmol}, 96 \%)$.

$\mathbf{R}_{f}\left(\mathrm{CH}_{2} \mathrm{Cl}_{2}\right): 0.65$;

${ }^{1} \mathbf{H}$ NMR $\left(300 \mathrm{MHz}, \mathrm{CDCl}_{3}\right): \delta / \mathrm{ppm}=10.25(\mathrm{~s}, 1 \mathrm{H}), 7.90-7.79(\mathrm{~m}, 1 \mathrm{H}), 7.65-7.52(\mathrm{~m}, 1 \mathrm{H})$, $7.53(\mathrm{dd}, J=7.3,1.7 \mathrm{~Hz}, 1 \mathrm{H}), 7.48(\mathrm{dd}, J=7.3,1.7 \mathrm{~Hz}, 1 \mathrm{H}), 4.95$ (s, 2H);

${ }^{13} \mathrm{C}$ NMR $\left(100 \mathrm{MHz}, \mathrm{CDCl}_{3}\right): \delta / \mathrm{ppm}=192.2,139.3,134.1,133.9,133.2,131.8,129.3,29.7$; 
GC-MS: $t_{\mathrm{R}}=7.31 \mathrm{~min} ;(\mathrm{EI}) \mathrm{m} / z(\%)=199.9(15), 197.9(16), 120.1$ (8), $119.0(87), 118.0$ (14), 92.0 (8), 91.0 (100), 90.1 (33), 89.1 (39), 65.0 (28), 64.0 (7), 63.0 (29), 62.0 (12), 61.1 (5), 51.0 (9.0), $50.1(8.0), 39.0(13), 38.0(5)$.

\section{(5-Methoxybenzofuran-2-yl)boronic acid (S11)}

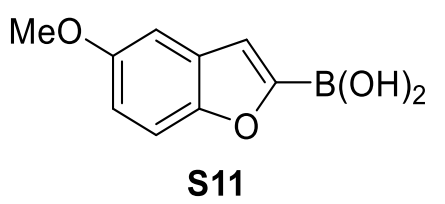

A flame-dried schlenk-flask was filled with 5-methoxybenzofuran ${ }^{[\mathrm{S} 12]}$ (370 mg, $2.5 \mathrm{mmol}, 1.0$ equiv.) under argon. $5 \mathrm{~mL}$ THF was added and cooled to $-78^{\circ} \mathrm{C}$. $n$-BuLi solution in hexanes $(1.6 \mathrm{M}, 2.4 \mathrm{~mL}$,

$3.75 \mathrm{mmol}, 1.5$ equiv.) was added dropwise. The resulting solution was stirred for $1 \mathrm{~h}$ at $-78{ }^{\circ} \mathrm{C}$. Triisopropyl borate $(0.87 \mathrm{~mL}, 3.8 \mathrm{mmol}, 1.5$ equiv. $)$ was added dropwise. The reaction was warmed to rt and stirred overnight. The solution was acidified to $\mathrm{pH} 3 \mathrm{using}$ aqueous $\mathrm{HCl}$ solution $(2 \mathrm{~N})$. The mixture was extracted with EtOAc $(3 \times 20 \mathrm{~mL})$ and dried over $\mathrm{Na}_{2} \mathrm{SO}_{4}$. The solvent was reduced to $5 \mathrm{~mL}$ under reduced pressure. $50 \mathrm{~mL} n$-pentane was added to precipitate the product. The solid was filtered, washed with $n$-pentane and dried in an air-stream to yield (5methoxybenzofuran-2-yl)boronic acid (S11) as a white powder (140 mg, $0.73 \mathrm{mmol}, 29 \%)$.

${ }^{11} \mathbf{B}$ NMR $\left(128 \mathrm{MHz}, \mathrm{CDCl}_{3}\right): \delta / \mathrm{ppm}=26.1$.

\section{2-((5-Methoxybenzofuran-2-yl)methyl)benzaldehyde (1f)}

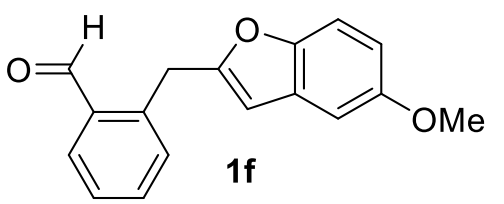

A schlenk-flask was filled with (5-methoxybenzofuran-2yl)boronic acid (S11) (192 mg, $1.00 \mathrm{mmol}, 1.0$ equiv.), 2-(bromomethyl)benzaldehyde (S10) (298 mg, $1.50 \mathrm{mmol}, 1.5$ equiv.), tetrakis(triphenylphosphine)palladium(0) $(58 \mathrm{mg}, 0.05 \mathrm{mmol}, 0.05$ equiv.), $1.1 \mathrm{~mL}$ aqueous $\mathrm{Na}_{2} \mathrm{CO}_{3}$ solution $(1 \mathrm{M}), 3 \mathrm{~mL}$ toluene, $2 \mathrm{~mL}$ ethanol and $0.5 \mathrm{~mL} \mathrm{H}_{2} \mathrm{O}$. The flask was evacuated and backfilled with argon three times and stirred at $80{ }^{\circ} \mathrm{C}$ overnight. The reaction was quenched with $10 \mathrm{~mL} \mathrm{H} \mathrm{H}_{2} \mathrm{O}$ and extracted with $\mathrm{CH}_{2} \mathrm{Cl}_{2}(3 \times 10 \mathrm{~mL})$, dried over $\mathrm{MgSO}_{4}$ and concentrated under reduced pressure. Purification by column chromatography on silica $(n$-pentane/EtOAc $=9 / 1)$ afforded 2-((5-methoxybenzofuran-2-yl)methyl)benzaldehyde (1f) as a yellow oil (147 mg, $0.55 \mathrm{mmol}, 55 \%$ ). 
$\mathbf{R}_{f}(n$-Pentane/EtOAc $=80 / 20): 0.67$;

${ }^{1} \mathrm{H}$ NMR $\left(400 \mathrm{MHz}, \mathrm{CD}_{2} \mathrm{Cl}_{2}\right): \delta / \mathrm{ppm}=10.26(\mathrm{~s}, 1 \mathrm{H}), 7.88(\mathrm{dd}, J=7.6,1.6 \mathrm{~Hz}, 1 \mathrm{H}), 7.58(\mathrm{td}$, $J=7.5,1.6 \mathrm{~Hz}, 1 \mathrm{H}), 7.49(\mathrm{td}, J=7.5,1.3 \mathrm{~Hz}, 1 \mathrm{H}), 7.39(\mathrm{dd}, J=7.6,1.3 \mathrm{~Hz}, 1 \mathrm{H}), 7.27(\mathrm{~d}, J=$ $8.9 \mathrm{~Hz}, 1 \mathrm{H}), 6.93$ (d, $J=2.6 \mathrm{~Hz}, 1 \mathrm{H}), 6.79$ (dd, $J=8.9,2.6 \mathrm{~Hz}, 1 \mathrm{H}), 6.29$ (dd, $J=1.0,1.0 \mathrm{~Hz}$, $1 \mathrm{H}), 4.55(\mathrm{~s}, 2 \mathrm{H}), 3.79(\mathrm{~s}, 3 \mathrm{H})$;

${ }^{13} \mathrm{C}$ NMR $\left(75 \mathrm{MHz}, \mathrm{CD}_{2} \mathrm{Cl}_{2}\right): \delta / \mathrm{ppm}=192.8,158.5,156.5,150.4,139.7,134.6,134.4,133.2$, $132.1,129.9,128.2,112.5,111.7,104.4,103.8,56.3,32.2$;

ESI-MS: calculated $\left[\mathrm{C}_{17} \mathrm{H}_{14} \mathrm{O}_{3} \mathrm{Na}\right]^{+}$: 289.0835, found: 289.0847;

ATR-FTIR $\left(\mathrm{cm}^{-1}\right):$ 282, 1694, 1616, 1597, 1574, 1474, 1447, 1288, 1261, 1204, 1165, 1126, 1030, 953, 910, 841, 791, 752, 691, 664.

\section{(5-Bromobenzofuran-2-yl)boronic acid (S12)}

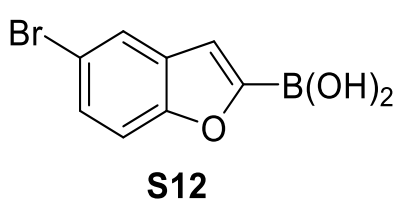

A flame-dried schlenk-flask was filled with diisopropylamine $(16.4 \mathrm{~mL}$,

$116 \mathrm{mmol}, 1.55$ equiv.) under argon. $100 \mathrm{~mL}$ THF was added and cooled to $-10{ }^{\circ} \mathrm{C}$. $n$-BuLi solution in hexanes $(1.6 \mathrm{M}, 70 \mathrm{~mL}$, 113 mmol, 1.5 equiv.) was added dropwise. The resulting solution was stirred for $30 \mathrm{~min}$ at $-10{ }^{\circ} \mathrm{C}$. A separate flame-dried schlenk-flask was filled under argon with 5bromobenzofuran ${ }^{[\mathrm{S} 12]}$ (14.8 g, $75.0 \mathrm{mmol}, 1.0$ equiv.), $115 \mathrm{~mL}$ THF and cooled to $-78{ }^{\circ} \mathrm{C}$. The LDA-solution from the first schlenk-flask was added dropwise to this solution via cannula. After stirring for $30 \mathrm{~min}$ at $-78{ }^{\circ} \mathrm{C}$, triisopropyl borate $(26 \mathrm{~mL}, 113 \mathrm{mmol}, 1.5$ equiv.) was added dropwise. The reaction was allowed to warm up to room temperature and stirred for $2 \mathrm{~h}$. The solution was acidified to $\mathrm{pH} 2$ using aqueous $\mathrm{HCl}$ solution $(2 \mathrm{~N})$. The mixture was extracted with EtOAc $(5 \times 200 \mathrm{~mL})$ and dried over $\mathrm{Na}_{2} \mathrm{SO}_{4}$. The solvent was reduced to approximately $20 \mathrm{~mL}$ under reduced pressure. $100 \mathrm{~mL} n$-pentane was added to precipitate the product. The solid was filtered, washed with $n$-pentane and dried in an air-stream to yield (5-bromobenzofuran-2yl)boronic acid (S12) as a beige powder (11.4 g, $47.3 \mathrm{mmol}, 63 \%)$.

${ }^{1}$ H NMR (400 MHz, MeOH-d4): $\delta / \mathrm{ppm}=7.76(\mathrm{t}, J=1.3 \mathrm{~Hz}, 1 \mathrm{H}), 7.44-7.40(\mathrm{~m}, 2 \mathrm{H}), 7.27$ (s, $1 \mathrm{H})$. 


\section{2-((5-Bromobenzofuran-2-yl)methyl)benzaldehyde (1g)}

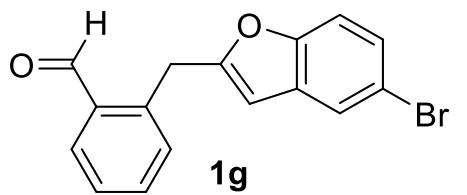

A schlenk-flask was filled with (5-bromobenzofuran-2-yl)boronic acid (S12) (4.8 g, 20.0 mmol, 1.0 equiv.), 2-(bromomethyl)benz-

aldehyde (S10) (4.0 g, $20.0 \mathrm{mmol}, 1.0$ equiv.), tetrakis(triphenylphosphine)palladium(0) (480 mg, $0.40 \mathrm{mmol}, 0.02$ equiv.), $30 \mathrm{~mL}$ aqueous $\mathrm{Na}_{2} \mathrm{CO}_{3}$ solution (1M), $40 \mathrm{~mL}$ toluene, $32 \mathrm{~mL}$ ethanol and $9 \mathrm{~mL} \mathrm{H}_{2} \mathrm{O}$. The flask was evacuated and backfilled with argon three times and stirred at room temperature overnight. The reaction was quenched with $30 \mathrm{~mL} \mathrm{H} \mathrm{H}_{2} \mathrm{O}$ and extracted with $\mathrm{CH}_{2} \mathrm{Cl}_{2}(3 \times 50 \mathrm{~mL})$, dried over $\mathrm{MgSO}_{4}$ and concentrated under reduced pressure. Purification by column chromatography on silica $(n$-pentane/EtOAc $=95 / 5)$ afforded 2-((5-bromobenzofuran-2-yl)methyl)benzaldehyde (1g) as a white solid (5.08 g, $16.1 \mathrm{mmol}, 81 \%)$.

$\mathbf{R}_{f}(n$-Pentane/EtOAc $=90 / 10): 0.49 ;$

${ }^{1} \mathbf{H}$ NMR $\left(300 \mathrm{MHz} \mathrm{CDCl}_{3}\right): \delta / \mathrm{ppm}=10.23(\mathrm{~s}, 1 \mathrm{H}), 7.88(\mathrm{dd}, J=7.5,1.6 \mathrm{~Hz}, 1 \mathrm{H}), 7.62-7.54$ $(\mathrm{m}, 2 \mathrm{H}), 7.50(\mathrm{td}, J=7.5,1.4 \mathrm{~Hz}, 1 \mathrm{H}), 7.38(\mathrm{~d}, J=7.5 \mathrm{~Hz}, 1 \mathrm{H}), 7.33-7.23(\mathrm{~m}, 2 \mathrm{H}), 6.30(\mathrm{q}$, $J=0.9 \mathrm{~Hz}, 1 \mathrm{H}), 4.58(\mathrm{~s}, 2 \mathrm{H})$;

${ }^{13} \mathrm{C}$ NMR $\left(75 \mathrm{MHz}, \mathrm{CDCl}_{3}\right): \delta / \mathrm{ppm}=192.6,158.4,153.7,138.7,134.1,134.0,133.7,131.7$, 130.8, 127.9, 126.5, 123.3, 115.8, 112.5, 103.5, 31.8;

ESI-MS: calculated $\left[\mathrm{C}_{16} \mathrm{H}_{11} \mathrm{O}_{2} \mathrm{BrNa}\right]^{+}:$336.9835, found: 336.9838 ;

ATR-FTIR $\left(\mathrm{cm}^{-1}\right):$ 3106, 5835, 2735, 1697, 1578, 1485, 1443, 1312, 1292, 1258, 1196, 1153, 1045, 961, 953, 926, 899, 864, 826, 810, 786, 756, 741, 679, 664.

\section{2-(2-Bromobenzyl)-5-fluorobenzofuran (S13)}

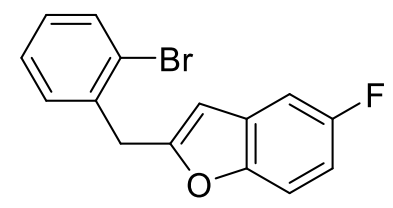

S13
5-Fluorobenzofuran ${ }^{[\mathrm{S} 11]}(1.18 \mathrm{~g}, 8.70 \mathrm{mmol}, 1.0$ equiv.) was dissolved in THF $(17 \mathrm{~mL})$ and cooled to $-45^{\circ} \mathrm{C}$. Then $n$-BuLi solution in hexanes (1.6 M, $5.4 \mathrm{~mL}, 8.70 \mathrm{mmol}, 1.0$ equiv.) was added dropwise to the reaction mixture. The resulting suspension was stirred for $10 \mathrm{~min}$ before 2-bromobenzyl bromide (5.41 g, $21.7 \mathrm{mmol}, 2.5$ equiv.) was added as a solution in $8 \mathrm{~mL}$ THF. The reaction was stirred and allowed to warm up to room temperature and stirred for $30 \mathrm{~min}$. The 
reaction was stopped by dropwise addition of water and saturated aqueous $\mathrm{NH}_{4} \mathrm{Cl}$ solution. After extraction with $\mathrm{CH}_{2} \mathrm{Cl}_{2}$ the organic layer was dried over $\mathrm{MgSO}_{4}$. Purification by column chromatography on silica (n-pentane) afforded impure 2-(2-bromobenzyl)-5-fluorobenzofuran (S13) as a colorless oil (764 mg) which was used without further purification.

$\mathbf{R}_{f}$ (n-Pentane): 0.23;

${ }^{19}$ F NMR (282 MHz, CDCl 3$): \delta / p p m=-121.5(\mathrm{ddd}, J=9.3,8.6,4.2 \mathrm{~Hz})$.

\section{2-((5-Fluorobenzofuran-2-yl)methyl)benzaldehyde (1h)}

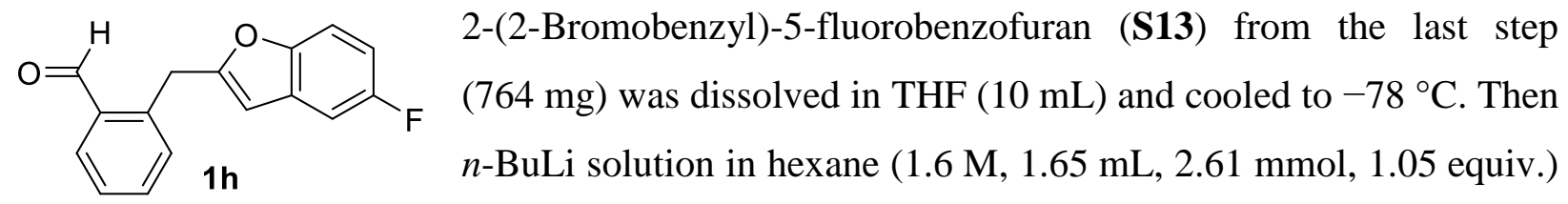
was added dropwise to the reaction mixture. After $1 \mathrm{~h}$ at $-78^{\circ} \mathrm{C}, \mathrm{DMF}(0.21 \mathrm{~mL}, 2.75 \mathrm{mmol}$, 1.1 equiv.) was added dropwise. The reaction was stirred and allowed to warm up to room temperature overnight. The reaction was stopped by addition of water. After extraction with EtOAc the organic layer was dried over $\mathrm{Na}_{2} \mathrm{SO}_{4}$. Purification by column chromatography on silica ( $n$ pentane/EtOAc = 95/5) afforded 2-((5-fluorobenzofuran-2-yl)methyl)benzaldehyde (1h) as a colorless oil (142 mg, $0.56 \mathrm{mmol}, 6 \%$ over 2 steps).

$\mathbf{R}_{f}(n$-Pentane/EtOAc $=95 / 5): 0.18 ;$

${ }^{1} \mathbf{H}$ NMR (300 MHz, $\left.\mathrm{CDCl}_{3}\right): \delta / \mathrm{ppm}=10.26(\mathrm{~s}, 1 \mathrm{H}), 7.89$ (dd, $\left.J=7.6,1.5 \mathrm{~Hz}, 1 \mathrm{H}\right), 7.59$ (td, $J=7.5,1.6 \mathrm{~Hz}, 1 \mathrm{H}), 7.50(\mathrm{td}, J=7.5,1.4 \mathrm{~Hz}, 1 \mathrm{H}), 7.42-7.37(\mathrm{~m}, 1 \mathrm{H}), 7.39-7.24(\mathrm{~m}, 1 \mathrm{H})$,

$7.11(\mathrm{dd}, J=8.6,2.5 \mathrm{~Hz}, 1 \mathrm{H}), 6.93(\mathrm{td}, J=9.1,2.6 \mathrm{~Hz}, 1 \mathrm{H}), 6.33(\mathrm{dd}, J=1.0,1.0 \mathrm{~Hz}, 1 \mathrm{H}), 4.59$ $(\mathrm{s}, 2 \mathrm{H})$

${ }^{13} \mathrm{C}$ NMR (75 MHz, $\left.\mathrm{CDCl}_{3}\right): \delta / \mathrm{ppm}=192.5,159.1(\mathrm{~d}, J=238 \mathrm{~Hz}), 158.7,151.1,138.7,134.0$, 133.9, 133.4, 131.6, 129.5 (d, $J=11 \mathrm{~Hz}), 127.7,111.4(\mathrm{~d}, J=10 \mathrm{~Hz}), 111.1$ (d, $J=26 \mathrm{~Hz}), 106.1$ $(\mathrm{d}, J=25 \mathrm{~Hz}), 104.1(\mathrm{~d}, J=4 \mathrm{~Hz}), 31.8$;

${ }^{19}$ F NMR (282 MHz, $\left.\mathrm{CDCl}_{3}\right): \delta / \mathrm{ppm}=-121.46(\mathrm{ddd}, J=9.3,8.6,4.2 \mathrm{~Hz})$;

ESI-MS: calculated $\left[\mathrm{C}_{16} \mathrm{H}_{11} \mathrm{FO}_{2} \mathrm{Na}\right]^{+}:$277.0635, found: 277.0653;

ATR-FTIR $\left(\mathrm{cm}^{-1}\right):$ 2739, 1694, 1624, 1597, 1574, 1470, 1447, 1288, 1250, 1184, 1165, 1115, $1026,949,910,856,795,752,802,660$. 
General procedure for Suzuki coupling with 2-((5-bromobenzofuran-2-yl)methyl)benzaldehyde (1g) or 2-((5-Bromobenzo[b]thiophen-2-yl)methyl)benzaldehyde (1s) to boronic acids

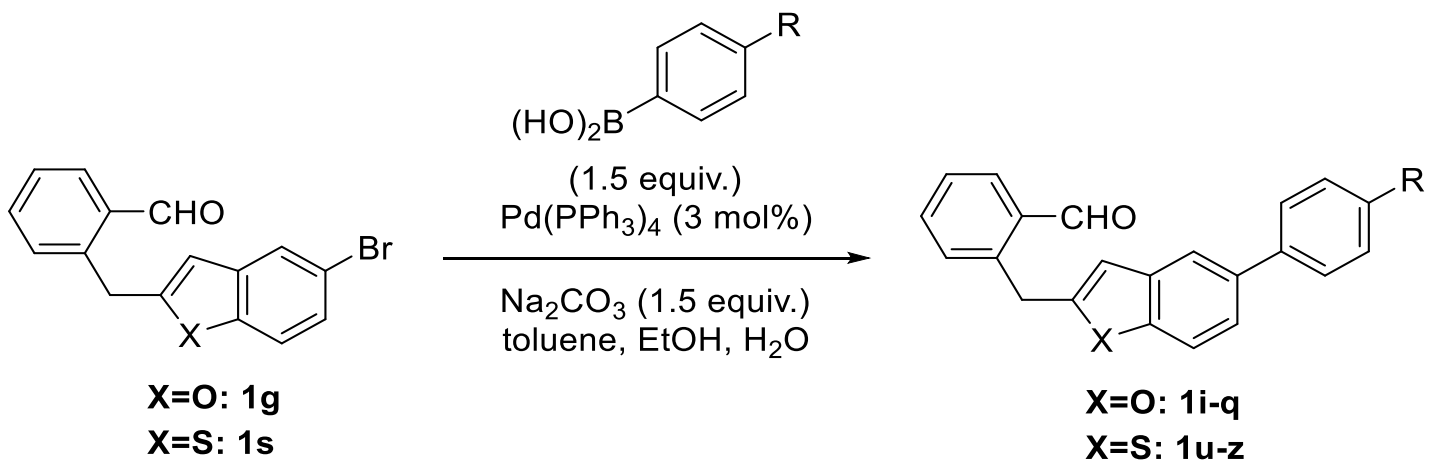

A flame-dried schlenk-tube was charged with either 2-((5-bromobenzofuran-2yl)methyl)benzaldehyde (1g) (315 mg, $1.00 \mathrm{mmol}, 1.0$ equiv.) or 2-((5-bromobenzo[b]thiophen2-yl)methyl)benzaldehyde (1s), the corresponding boronic acid (1.50 mmol, 1.5 equiv.) and tetrakis(triphenylphosphine)palladium( 0$) \quad(35 \mathrm{mg}, 0.03 \mathrm{mmol}, 0.03$ equiv.). Toluene ( $2 \mathrm{~mL})$, EtOH $(1.5 \mathrm{~mL}), \mathrm{H}_{2} \mathrm{O}(0.5 \mathrm{~mL})$ and aqueous $\mathrm{Na}_{2} \mathrm{CO}_{3}$ solution $(1 \mathrm{M}, 1.5 \mathrm{~mL})$ were added, the flask was filled with argon and stirred at room temperature overnight. The solution was stirred for additional $20 \mathrm{~h}$ at $80{ }^{\circ} \mathrm{C}$. The reaction was quenched with water $(5 \mathrm{~mL})$ and extracted with $\mathrm{CH}_{2} \mathrm{Cl}_{2}$ $\left(3 \times 10 \mathrm{~mL}\right.$ ). The organic phases were dried over $\mathrm{Na}_{2} \mathrm{SO}_{4}$, concentrated under reduced pressure and purified by column chromatography on silica.

\section{2-((5-(4-Methoxyphenyl)benzofuran-2-yl)methyl)benzaldehyde (1i)}

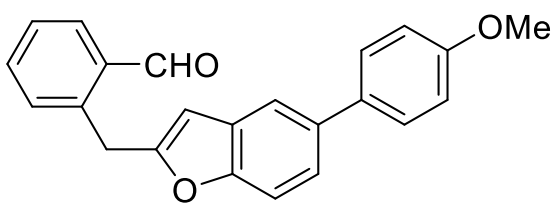

$1 \mathrm{i}$

Synthesized according to the general procedure from (4-methoxyphenyl)boronic acid (228 $\mathrm{mg}, \quad 1.50 \mathrm{mmol}$, 1.5 equiv.). Purification by column chromatography on silica $(n$-pentane/EtOAc $=90 / 10)$ afforded 2-((5-(4-methoxyphenyl)benzofuran-2-yl)methyl)benzaldehyde (1i) as a white solid (198 mg, $0.58 \mathrm{mmol}, 58 \%$ ).

$\mathbf{R}_{f}(n$-Pentane/EtOAc $=90 / 10): 0.39 ;$

${ }^{1} \mathbf{H}$ NMR (400 MHz, $\left.\mathrm{CD}_{2} \mathrm{Cl}_{2}\right): \delta / \mathrm{ppm}=10.28(\mathrm{~s}, 1 \mathrm{H}), 7.89(\mathrm{dd}, J=7.6,1.6 \mathrm{~Hz}, 1 \mathrm{H}), 7.63-7.60$ $(\mathrm{m}, 1 \mathrm{H}), 7.58(\mathrm{dd}, J=7.5,1.6 \mathrm{~Hz}, 1 \mathrm{H}), 7.55-7.47(\mathrm{~m}, 3 \mathrm{H}), 7.46-7.36(\mathrm{~m}, 3 \mathrm{H}), 7.00-6.93(\mathrm{~m}$, 2H), 6.39 (td, $J=1.0,1.0 \mathrm{~Hz}, 1 \mathrm{H}), 4.60(\mathrm{~s}, 2 \mathrm{H}), 3.83(\mathrm{~s}, 3 \mathrm{H})$; 
${ }^{13} \mathrm{C}$ NMR (101 MHz, $\left.\mathrm{CD}_{2} \mathrm{Cl}_{2}\right): \delta / \mathrm{ppm}=192.9,159.5,158.4,154.8,139.6,136.5,134.6,134.6$, $134.5,133.4,132.1,129.9,128.8,128.2,123.4,119.0,114.7,111.4,104.4,55.9,32.2$;

ESI-MS: calculated $\left[\mathrm{C}_{23} \mathrm{H}_{18} \mathrm{O}_{3} \mathrm{Na}\right]^{+}: 365.1148$, found: 365.1158 ;

ATR-FTIR $\left(\mathrm{cm}^{-1}\right):$ 2839, 2735, 1694, 1601, 1574, 1516, 1462, 1277, 1250, 1231, 1180, 1153, 1115, 1038, 1015, 945, 883, 864, 841, 822, 814, 795, 748, 683, 664, 637.

\section{2-((5-(4-(tert-Butyl)phenyl)benzofuran-2-yl)methyl)benzaldehyde (1j)}

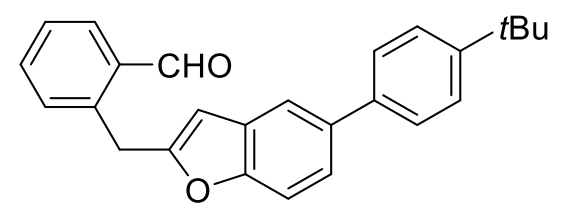

$1 \mathrm{j}$

Synthesized according to the general procedure from (4-(tertbutyl)phenyl)boronic acid (267 mg, $1.50 \mathrm{mmol}, 1.5$ equiv.). Purification by column chromatography on silica (n-pentane/EtOAc = 90/10) afforded 2-((5-(4-(tert-butyl)phenyl)benzofuran-2-yl)methyl)benzaldehyde (1j) as a white solid (293 mg, $0.80 \mathrm{mmol}, 80 \%$ ).

$\mathbf{R}_{f}(n$-Pentane/EtOAc $=90 / 10): 0.47$;

${ }^{1} \mathbf{H}$ NMR $\left(400 \mathrm{MHz}, \mathrm{CD}_{2} \mathrm{Cl}_{2}\right): \delta / \mathrm{ppm}=10.28(\mathrm{~s}, 1 \mathrm{H}), 7.89(\mathrm{dd}, J=7.6,1.6 \mathrm{~Hz}, 1 \mathrm{H}), 7.66(\mathrm{t}, J=$ $1.3 \mathrm{~Hz}, 1 \mathrm{H}), 7.60(\mathrm{td}, J=7.5,1.6 \mathrm{~Hz}, 1 \mathrm{H}), 7.56-7.38(\mathrm{~m}, 8 \mathrm{H}), 6.40(\mathrm{td}, J=0.8,0.8 \mathrm{~Hz}, 1 \mathrm{H})$, $4.60(\mathrm{~s}, 2 \mathrm{H}), 1.36(\mathrm{~s}, 9 \mathrm{H})$;

${ }^{13} \mathrm{C}$ NMR (101 MHz, $\left.\mathrm{CD}_{2} \mathrm{Cl}_{2}\right): \delta / \mathrm{ppm}=192.9,158.4,154.9,150.4,139.5,139.1,136.6,134.6$, $134.5,133.4$, 132.1, 129.8, 128.2, 127.4, 126.2, 123.6, 119.3, 111.4, 104.4, 34.9, 32.2, 31.7;

ESI-MS: calculated $\left[\mathrm{C}_{26} \mathrm{H}_{24} \mathrm{O}_{2} \mathrm{Na}\right]^{+}: 391.1669$, found: 391.1676 ;

ATR-FTIR $\left(\mathrm{cm}^{-1}\right):$ 2959, 2905, 2866, 2727, 1697, 1597, 1574, 1462, 1404, 1366, 1265, 1231, 1192, 1153, 1126, 1026, 949, 926, 887, 864, 818, 806, 783, 752, 687, 664, 637.

\section{2-((5-Phenylbenzofuran-2-yl)methyl)benzaldehyde (1k)}

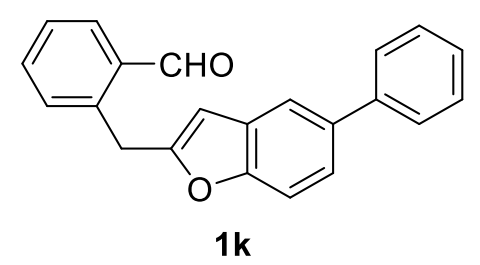

(278 mg, $0.89 \mathrm{mmol}, 89 \%)$.
Synthesized according to the general procedure from phenylboronic acid (183 mg, $1.5 \mathrm{mmol}, 1.5$ equiv.). Purification by column chromatography on silica ( $n$-pentane/EtOAc $=90 / 10)$ afforded 2-((5phenylbenzofuran-2-yl)methyl)benzaldehyde (1k) as a white solid 
$\mathbf{R}_{f}(n$-Pentane/EtOAc $=90 / 10): 0.40 ;$

${ }^{1} \mathbf{H}$ NMR (400 MHz, $\left.\mathrm{CD}_{2} \mathrm{Cl}_{2}\right): \delta / \mathrm{ppm}=10.28(\mathrm{~s}, 1 \mathrm{H}), 7.89(\mathrm{dd}, J=7.6,1.5 \mathrm{~Hz}, 1 \mathrm{H}), 7.70-7.65$ (m, 1H), $7.64-7.55(\mathrm{~m}, 3 \mathrm{H}), 7.51(\mathrm{td}, J=7.5,1.3 \mathrm{~Hz}, 1 \mathrm{H}), 7.47-7.39(\mathrm{~m}, 5 \mathrm{H}), 7.36-7.29(\mathrm{~m}$, $1 \mathrm{H}), 6.41(\mathrm{td}, J=1.1,1.1 \mathrm{~Hz}, 1 \mathrm{H}), 4.60(\mathrm{~s}, 2 \mathrm{H})$;

${ }^{13} \mathrm{C}$ NMR (101 MHz, $\left.\mathrm{CD}_{2} \mathrm{Cl}_{2}\right): \delta / \mathrm{ppm}=192.9,158.5,155.1,142.2,139.5,136.8,134.6,134.5$, $133.4,132.1,129.9,129.3,128.2,127.8,127.4,123.7,119.5,111.4,104.5,32.2$;

ESI-MS: calculated $\left[\mathrm{C}_{22} \mathrm{H}_{16} \mathrm{O}_{2} \mathrm{Na}\right]^{+}: 335.1043$, found: 335.1046;

ATR-FTIR $\left(\mathrm{cm}^{-1}\right):$ 1694, 1597, 1574, 1462, 1424, 1400, 1269, 1234, 1196, 1153, 1126, 945, $883,864,833,799,764,698,664,629$.

2-((5-(4-(Hydroxymethyl)phenyl)benzofuran-2-yl)methyl)benzaldehyde (11)

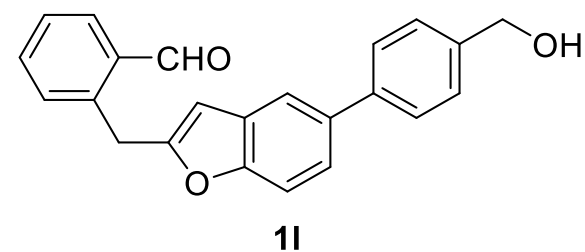

Synthesized according to the general procedure from (4-(hydroxymethyl)phenyl)boronic acid (228 mg, $1.50 \mathrm{mmol}$, 1.5 equiv.). Purification by column chromatography on silica $(n$-pentane/EtOAc $=70 / 30)$ afforded 2- $((5-$ (4-(hydroxymethyl)phenyl)benzofuran-2-yl)methyl)benzaldehyde (11) as a white solid (225 mg, $0.66 \mathrm{mmol}, 66 \%)$.

$\mathbf{R}_{f}(n$-Pentane/EtOAc $=70 / 30): 0.27$;

${ }^{1} \mathbf{H}$ NMR $\left(400 \mathrm{MHz}, \mathrm{CD}_{2} \mathrm{Cl}_{2}\right): \delta / \mathrm{ppm}=10.27(\mathrm{~s}, 1 \mathrm{H}), 7.89(\mathrm{dd}, J=7.6,1.5 \mathrm{~Hz}, 1 \mathrm{H}), 7.69-7.65$ $(\mathrm{m}, 1 \mathrm{H}), 7.64-7.56(\mathrm{~m}, 3 \mathrm{H}), 7.51(\mathrm{td}, J=7.6,1.3 \mathrm{~Hz}, 1 \mathrm{H}), 7.47-7.37(\mathrm{~m}, 6 \mathrm{H}), 6.40(\mathrm{~s}, 1 \mathrm{H})$, $4.71(\mathrm{~s}, 2 \mathrm{H}), 4.60(\mathrm{~s}, 2 \mathrm{H})$;

${ }^{13} \mathrm{C}$ NMR (101 MHz, $\left.\mathrm{CD}_{2} \mathrm{Cl}_{2}\right): \delta / \mathrm{ppm}=192.9,158.5,155.1,141.3,140.5,139.5,136.4,134.6$, 134.5, 133.4, 132.1, 129.9, 128.2, 127.9, 127.9, 123.6, 119.4, 111.5, 104.5, 65.4, 32.2;

ESI-MS: calculated $\left[\mathrm{C}_{23} \mathrm{H}_{18} \mathrm{O}_{3} \mathrm{Na}\right]^{+}: 365.1148$, found: 365.1148 ;

ATR-FTIR $\left(\mathrm{cm}^{-1}\right):$ 3360, 3271, 2866, 2754, 1694, 1601, 1574, 1466, 1412, 1316, 1296, 1265, 1234, 1192, 1123, 1038, 999, 949, 864, 799, 759, 702, 663, 625. 
2-((5-(4-(Trifluoromethoxy)phenyl)benzofuran-2-yl)methyl)benzaldehyde (1m)

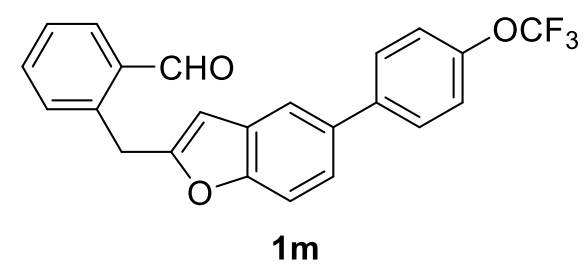

Synthesized according to the general procedure from (4-(trifluoromethoxy)phenyl)boronic acid (309 mg, $1.50 \mathrm{mmol}$, 1.5 equiv.). Purification by column chromatography on silica ( $n$-pentane/EtOAc $=90 / 10)$

afforded 2-((5-(4-(trifluoromethoxyl)phenyl)benzofuran-2-yl)methyl)benzaldehyde (1m) as a white solid (287 mg, $0.72 \mathrm{mmol}, 72 \%$ ).

$\mathbf{R}_{f}(n$-Pentane/EtOAc $=90 / 10): 0.38 ;$

${ }^{1} \mathbf{H}$ NMR $\left(400 \mathrm{MHz}, \mathrm{CD}_{2} \mathrm{Cl}_{2}\right): \delta / \mathrm{ppm}=10.27(\mathrm{~s}, 1 \mathrm{H}), 7.89(\mathrm{dd}, J=7.6,1.5 \mathrm{~Hz}, 1 \mathrm{H}), 7.67-7.56$ $(\mathrm{m}, 4 \mathrm{H}), 7.51(\mathrm{td}, J=7.5,1.3 \mathrm{~Hz}, 1 \mathrm{H}), 7.48-7.40(\mathrm{~m}, 3 \mathrm{H}), 7.35-7.23(\mathrm{~m}, 2 \mathrm{H}), 6.41(\mathrm{td}$, $J=1.0,1.0 \mathrm{~Hz}, 1 \mathrm{H}), 4.60(\mathrm{~s}, 2 \mathrm{H})$;

${ }^{13}$ C NMR (126 MHz, $\mathrm{CD}_{2} \mathrm{Cl}_{2}$ ): $\delta / \mathrm{ppm}=192.9,158.8,155.2,148.9$ (q, $J=1.9 \mathrm{~Hz}$ ), 141.2, 139.4, $135.4,134.7,134.5,133.6,132.2$, 130.0, 129.2, 128.3, 123.7, 121.8, 121.2 (q, J = 256 Hz), 119.6, 111.6, 104.4, 32.2;

${ }^{19}$ F NMR $\left(470 \mathrm{MHz}, \mathrm{CD}_{2} \mathrm{Cl}_{2}\right): \delta / \mathrm{ppm}=-58.25(\mathrm{t}, J=1.0 \mathrm{~Hz})$;

ESI-MS: calculated $\left[\mathrm{C}_{23} \mathrm{H}_{15} \mathrm{~F}_{3} \mathrm{O}_{3} \mathrm{Na}\right]^{+}:$419.0866, found: 419.0856;

ATR-FTIR (cm $\left.{ }^{-1}\right):$ 2735, 1697, 1605, 1574, 1508, 1466, 1258, 1231, 1200, 1150, 1015, 961, 949, 899, 868, 856, 806, 775, 752, 664, 625.

\section{2-((5-(4-Chlorophenyl)benzofuran-2-yl)methyl)benzaldehyde (1n)}

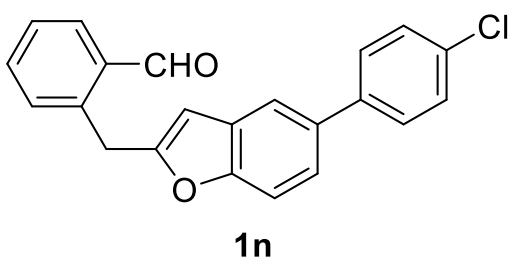

Synthesized according to the general procedure from (4-chlorophenyl)boronic acid (235 mg, $1.5 \mathrm{mmol}, 1.5$ equiv.). Purification by column chromatography on silica $(n$-pentane/EtOAc $=80 / 20)$ afforded 2-((5-(4-chlorophenyl $)-$ benzofuran-2-yl)methyl)benzaldehyde (1n) as a white solid (217 mg, $0.63 \mathrm{mmol}, 63 \%)$.

$\mathbf{R}_{f}(n$-Pentane/EtOAc $=80 / 20): 0.67$;

${ }^{1} \mathbf{H}$ NMR (400 MHz, $\left.\mathrm{CD}_{2} \mathrm{Cl}_{2}\right): \delta / \mathrm{ppm}=10.27(\mathrm{~s}, 1 \mathrm{H}), 7.89(\mathrm{dd}, J=7.6,1.6 \mathrm{~Hz}, 1 \mathrm{H}), 7.66-7.62$ $(\mathrm{m}, 1 \mathrm{H}), 7.59(\mathrm{td}, J=7.5,1.6 \mathrm{~Hz}, 1 \mathrm{H}), 7.56-7.36(\mathrm{~m}, 8 \mathrm{H}), 6.40(\mathrm{td}, J=1.0,1.0 \mathrm{~Hz}, 1 \mathrm{H}), 4.60$ (s, 2H); 
${ }^{13} \mathrm{C}$ NMR $\left(101 \mathrm{MHz}, \mathrm{CD}_{2} \mathrm{Cl}_{2}\right): \delta / \mathrm{ppm}=192.9,158.7,155.2,140.7,139.4,135.5,134.6,134.5$, 133.5, 133.3, 132.1, 123.0, 129.3, 129.2, 128.2, 123.5, 119.4, 111.6, 104.4, 32.2;

ESI-MS: calculated $\left[\mathrm{C}_{22} \mathrm{H}_{15} \mathrm{ClO}_{2} \mathrm{Na}\right]^{+}: 369.0653$, found: 369.0644 ;

ATR-FTIR $\left(\mathrm{cm}^{-1}\right):$ 1694, 1597, 1574, 1462, 1404, 1308, 1273, 1227, 1196, 1157, 1088, 1011, $957,872,845,810,791,779,748,660$.

\section{2-((5-(4-(Trifluoromethyl)phenyl)benzofuran-2-yl)methyl)benzaldehyde (10)}

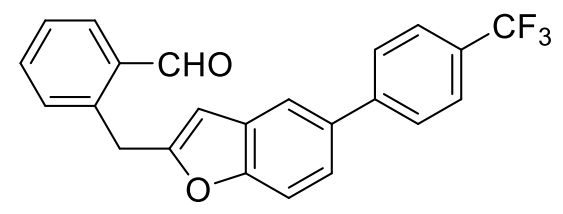

10

Synthesized according to the general procedure from (4-(trifluoromethyl)phenyl)boronic acid $(235 \mathrm{mg}, 1.50 \mathrm{mmol}$, 1.5 equiv.). Purification by column chromatography on silica $(n$-pentane/EtOAc $\quad=\quad 90 / 10) \quad$ afforded $2-((5-(4-$

(trifluoromethyl)phenyl)benzofuran-2-yl)methyl)benzaldehyde (10) as a white solid (243 mg, $0.64 \mathrm{mmol}, 64 \%)$.

$\mathbf{R}_{f}(n$-Pentane/EtOAc $=90 / 10): 0.38 ;$

${ }^{1} \mathbf{H}$ NMR (400 MHz, $\mathrm{CD}_{2} \mathrm{Cl}_{2}$ ): $\delta / \mathrm{ppm}=10.27(\mathrm{~s}, 1 \mathrm{H}), 7.89(\mathrm{dd}, J=7.6,1.5 \mathrm{~Hz}, 1 \mathrm{H}), 7.78-7.65$ $(\mathrm{m}, 5 \mathrm{H}), 7.60(\mathrm{td}, J=7.5,1.5 \mathrm{~Hz}, 1 \mathrm{H}), 7.55-7.39(\mathrm{~m}, 4 \mathrm{H}), 6.42(\mathrm{td}, J=1.0,1.0 \mathrm{~Hz}, 1 \mathrm{H}), 4.61$ $(\mathrm{s}, 2 \mathrm{H})$;

${ }^{13} \mathrm{C}$ NMR $\left(126 \mathrm{MHz}, \mathrm{CD}_{2} \mathrm{Cl}_{2}\right): \delta / \mathrm{ppm}=192.9,158.9,155.5,145.9,139.4,135.3,134.7,134.5$, 133.6, 132.2, 130.1, $129.2(\mathrm{q}, J=32 \mathrm{~Hz}), 128.3,128.2,126.2(\mathrm{q}, J=4 \mathrm{~Hz}), 124.7$ (q, $J=273 \mathrm{~Hz}), 123.8,119.9,111.7,104.4,32.2$;

${ }^{19}$ F NMR $\left(470 \mathrm{MHz}, \mathrm{CD}_{2} \mathrm{Cl}_{2}\right): \delta / \mathrm{ppm}=-62.67(\mathrm{~s})$;

ESI-MS: calculated $\left[\mathrm{C}_{23} \mathrm{H}_{15} \mathrm{~F}_{3} \mathrm{O}_{2} \mathrm{Na}\right]^{+}$: 403.0916, found: 403.0912;

ATR-FTIR (cm $\left.{ }^{-1}\right):$ 1686, 1616, 1601, 1574, 1466, 1412, 1323, 1120, 1161, 1107, 1069, 1015, 949, 891, 848, 818, 760, 725, 660, 633. 
2-((5-(4-Acetylphenyl)benzofuran-2-yl)methyl)benzaldehyde (1p)

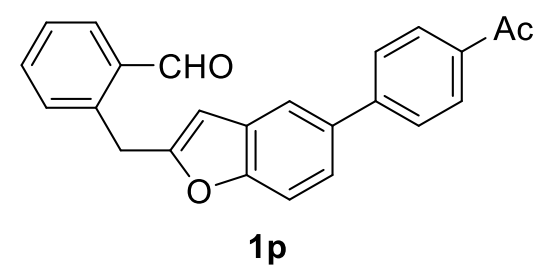

Synthesized according to the general procedure from (4-acetylphenyl)boronic acid (246 mg, $1.50 \mathrm{mmol}, 1.5$ equiv.). Purification by column chromatography on silica $(n$-pentane/EtOAc $\quad=\quad 70 / 30) \quad$ afforded $\quad 2-((5-(4-$ acetylphenyl)benzofuran-2-yl)methyl)benzaldehyde (1p) as a white solid (275 mg, $0.78 \mathrm{mmol}$, $78 \%)$.

$\mathbf{R}_{f}(n$-pentane/EtOAc $=70 / 30): 0.12 ;$

${ }^{1} \mathbf{H}$ NMR $\left(400 \mathrm{MHz}, \mathrm{CD}_{2} \mathrm{Cl}_{2}\right): \delta / \mathrm{ppm}=10.26(\mathrm{~s}, 1 \mathrm{H}), 8.06-7.96(\mathrm{~m}, 2 \mathrm{H}), 7.89(\mathrm{dd}, J=7.6$, $1.5 \mathrm{~Hz}, 1 \mathrm{H}), 7.76-7.66(\mathrm{~m}, 3 \mathrm{H}), 7.60(\mathrm{td}, J=7.5,1.6 \mathrm{~Hz}, 1 \mathrm{H}), 7.55-7.39(\mathrm{~m}, 4 \mathrm{H}), 6.42(\mathrm{td}$, $J=0.9,0.9 \mathrm{~Hz}, 1 \mathrm{H}), 4.61(\mathrm{~s}, 2 \mathrm{H}), 2.60(\mathrm{~s}, 3 \mathrm{H})$;

${ }^{13}$ C NMR (101 MHz, $\left.\mathrm{CD}_{2} \mathrm{Cl}_{2}\right): \delta / \mathrm{ppm}=197.9,192.9,158.8,155.5,146.6,139.3,136.1,135.4$, 134.6, 134.5, 133.6, 132.1, 130.0, 129.3, 128.2, 127.8, 123.7, 119.8, 111.7, 104.4, 32.2, 27.0;

ESI-MS: calculated $\left[\mathrm{C}_{24} \mathrm{H}_{18} \mathrm{O}_{3} \mathrm{Na}\right]^{+}: 377.1148$, found: 377.1150 ;

ATR-FTIR $\left(\mathrm{cm}^{-1}\right):$ 1694, 1670, 1601, 1574, 1466, 1404, 1358, 1269, 1192, 1157, 1123, 1018, $949,883,864,841,806,791,764,729,691,679,664$.

\section{4-(2-(2-Formylbenzyl)benzofuran-5-yl)benzonitrile (1q)}

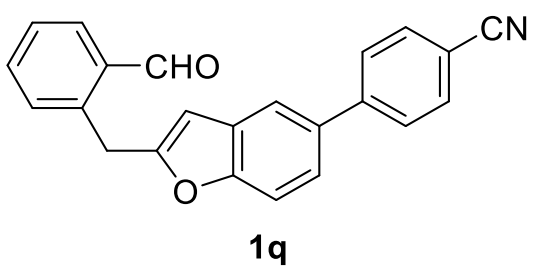

Synthesized according to the general procedure from (4-cyanophenyl)boronic acid (220 mg, $1.50 \mathrm{mmol}, 1.5$ equiv.). Purification by column chromatography on silica $(n$-pentane/EtOAc $=90 / 10)$ afforded 4-(2-(2-formylbenzyl)benzofuran-5-yl)benzonitrile (1q) as a white solid (270 $\mathrm{mg}, 0.80 \mathrm{mmol}, 80 \%)$.

$\mathbf{R}_{f}(n$-Pentane/EtOAc $=90 / 10): 0.17 ;$

${ }^{1} \mathbf{H}$ NMR $\left(400 \mathrm{MHz}, \mathrm{CD}_{2} \mathrm{Cl}_{2}\right): \delta / \mathrm{ppm}=10.26(\mathrm{~s}, 1 \mathrm{H}), 7.89(\mathrm{dd}, J=7.6,1.6 \mathrm{~Hz}, 1 \mathrm{H}), 7.82-7.65$ (m, 5H), 7.60 (td, $J=7.5,1.6 \mathrm{~Hz}, 1 \mathrm{H}), 7.56-7.30(\mathrm{~m}, 4 \mathrm{H}), 6.42$ (td, $J=1.1,1.1 \mathrm{~Hz}, 1 \mathrm{H}), 4.61$ $(\mathrm{s}, 2 \mathrm{H})$;

${ }^{13}$ C NMR (101 MHz, $\left.\mathrm{CD}_{2} \mathrm{Cl}_{2}\right): \delta / \mathrm{ppm}=192.9,159.1,155.7,146.6,139.2,134.8,134.6,134.5$, 133.7, 133.1, 132.2, 130.2, 128.4, 128.3, 123.7, 119.9, 119.5, 111.8, 111.0, 104.4, 32.2; 
ESI-MS: calculated $\left[\mathrm{C}_{23} \mathrm{H}_{15} \mathrm{NO}_{2} \mathrm{Na}\right]^{+}:$360.0995, found: 360.0998 ;

ATR-FTIR $\left(\mathrm{cm}^{-1}\right):$ 2747, 2234, 1697, 1601, 1574, 1404, 1312, 1273, 1231, 1196, 1153, 1119 , $953,872,853,814,791,779,756,679,660$.

\section{2-(2-Bromobenzyl)benzo[b]thiophene (S14)}

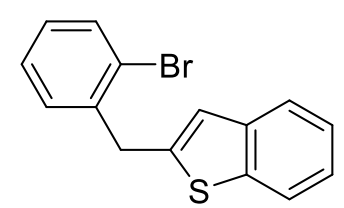

S14

In analogy to the literature, ${ }^{[\mathrm{S} 14]}$ benzo[b]thiophene $(1.34 \mathrm{~g}, 10.0 \mathrm{mmol}$, 1.0 equiv.) was dissolved in THF $(10 \mathrm{~mL})$ and cooled to $-78^{\circ} \mathrm{C}$. Then

$t$-BuLi solution in $n$-pentane $(1.7 \mathrm{M}, 9.4 \mathrm{~mL}, 16.0 \mathrm{mmol}, 1.6$ equiv.) was added dropwise to the reaction mixture. The resulting suspension was stirred for $30 \mathrm{~min}$ before 2-bromobenzyl bromide $(5.0 \mathrm{~g}, 20.0 \mathrm{mmol}, 2.0$ equiv.) was added as a solution in $5.0 \mathrm{~mL} \mathrm{THF}$. The reaction was stirred for further $15 \mathrm{~min}$. at $-78{ }^{\circ} \mathrm{C}$ and was allowed to warm up to room temperature. After $16 \mathrm{~h}$ the reaction was stopped by dropwise addition of water. After extraction with EtOAc the organic layer was dried over $\mathrm{Na}_{2} \mathrm{SO}_{4}$. Purification by column chromatography on silica (n-pentane) afforded 2-(2-bromobenzyl)benzo[b]thiophene (S14) as a white solid (1.49 g, $4.90 \mathrm{mmol}, 49 \%)$.

$\mathbf{R}_{f}$ (n-Pentane): 0.35;

${ }^{1} \mathbf{H}$ NMR (300 MHz, $\left.\mathrm{CDCl}_{3}\right): \delta / \mathrm{ppm}=7.81-7.70(\mathrm{~m}, 1 \mathrm{H}), 7.70-7.64(\mathrm{~m}, 1 \mathrm{H}), 7.60(\mathrm{dd}$, $J=7.9,1.2 \mathrm{~Hz}, 1 \mathrm{H}), 7.38-7.20(\mathrm{~m}, 4 \mathrm{H}), 7.20-7.08(\mathrm{~m}, 1 \mathrm{H}), 7.02(\mathrm{dt}, J=1.0,1.0 \mathrm{~Hz}, 1 \mathrm{H})$, $4.37(\mathrm{~d}, J=1.0 \mathrm{~Hz}, 2 \mathrm{H})$;

${ }^{13} \mathrm{C}$ NMR $\left(75 \mathrm{MHz}, \mathrm{CDCl}_{3}\right): \delta / \mathrm{ppm}=143.2,140.0,139.8,139.0,133.0,130.8,128.5,127.7$, $124.5,124.2,123.7,123.0,122.2,122.2,37.1$;

ESI-MS: calculated $\left[\mathrm{C}_{15} \mathrm{H}_{11} \mathrm{BrSAg}\right]^{+}:$408.8810, found: 408.8798;

ATR-FTIR $\left(\mathrm{cm}^{-1}\right): 3056,1797,1585,1567,1460,1437,1411,1339,1311,1260,1234,1152$, 1132, 1101, 1062, 1046, 1027, 948, 936, 864, 830, 795, 751, 740, 725, 670, 655, 603, 561.

\section{2-(Benzo $[b]$ thiophen-2-ylmethyl)benzaldehyde (1r)}<smiles>O=Cc1ccccc1Cc1cc2ccccc2s1</smiles>

In analogy to the literature, ${ }^{[\mathrm{S} 15]}$ 2-(2-bromobenzyl)benzo[ $[b]$ thiophene (S14) (606 mg, $2.0 \mathrm{mmol}, 1.0$ equiv.) was dissolved in THF (8 mL) and cooled to $-78{ }^{\circ} \mathrm{C}$. Then $n$-BuLi solution in hexane $(1.6 \mathrm{M}, 1.4 \mathrm{~mL}$, 
$2.20 \mathrm{mmol}, 1.1$ equiv.) was added dropwise to the reaction mixture. After $1 \mathrm{~h}$ at $-78{ }^{\circ} \mathrm{C}$, DMF (0.19 mL, $2.40 \mathrm{mmol}, 1.2$ equiv.) was added dropwise. The reaction was stirred for further $15 \mathrm{~min}$ at $-78{ }^{\circ} \mathrm{C}$ and was allowed to warm up to room temperature. By addition of saturated aqueous $\mathrm{NH}_{4} \mathrm{Cl}$ solution the reaction was stopped. After extraction with $\mathrm{Et}_{2} \mathrm{O}$ the organic layer was dried over $\mathrm{Na}_{2} \mathrm{SO}_{4}$. Purification by column chromatography on silica ( $n$-pentane/EtOAc $=$ 95/5) afforded 2-(benzo[b]thiophen-2-ylmethyl)benzaldehyde (1r) as a colorless oil (347 mg, $1.40 \mathrm{mmol}, 70 \%)$.

$\mathbf{R}_{f}($-Pentane/EtOAc $=90 / 10): 0.42 ;$

${ }^{1} \mathbf{H}$ NMR (300 MHz, $\left.\mathrm{CDCl}_{3}\right): \delta / \mathrm{ppm}=10.25(\mathrm{~s}, 1 \mathrm{H}), 7.88(\mathrm{dd}, J=7.6,1.6 \mathrm{~Hz}, 1 \mathrm{H}), 7.72(\mathrm{dd}$, $J=7.9,1.2 \mathrm{~Hz}, 1 \mathrm{H}), 7.63(\mathrm{~d}, J=7.3 \mathrm{~Hz}, 1 \mathrm{H}), 7.57(\mathrm{dt}, J=7.5,1.5 \mathrm{~Hz}, 1 \mathrm{H}), 7.48$ (dt, $J=7.5$, $1.3 \mathrm{~Hz}, 1 \mathrm{H}), 7.41(\mathrm{~d}, J=7.2 \mathrm{~Hz}, 1 \mathrm{H}), 7.31-7.22(\mathrm{~m}, 2 \mathrm{H}), 6.96$ (s, 1H), 4.69 (s, 2H);

${ }^{13} \mathrm{C}$ NMR $\left(75 \mathrm{MHz}, \mathrm{CDCl}_{3}\right): \delta / \mathrm{ppm}=192.4,144.2,141.4,139.9,139.7,134.0,133.7,133.0$, 131.4, 127.6, 124.2, 123.8, 123.0, 122.1, 122.0, 33.5;

ESI-MS: calculated: $\left[\mathrm{C}_{16} \mathrm{H}_{12} \mathrm{OSNa}\right]^{+}=275.0501$, found: 275.0497 ;

ATR-FTIR $\left(\mathrm{cm}^{-1}\right):$ 2770, 1692, 1595, 1483, 1453, 1438, 1403, 1301, 1272, 1212, 1150, 1066, 1015, 942, 870, 829, 802, 766, 753, 728, 708, 668, 642.

\section{2-((5-Bromobenzo[b]thiophen-2-yl)methyl)benzaldehyde (1s)}

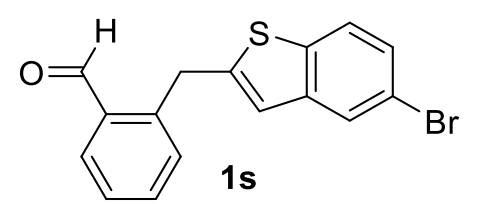

A schlenk-flask was filled with 5-bromobenzo[b]thiophene-2boronic acid (3.85 g, $15.0 \mathrm{mmol}, 1.5$ equiv.), 2-(bromomethyl)benzaldehyde (S10) (2.0 g, $10.0 \mathrm{mmol}, 1.0$ equiv.), tetrakis(triphenylphosphine)palladium(0) (290 mg, $0.25 \mathrm{mmol}, 0.025$ equiv.), $18 \mathrm{~mL}$ aqueous $\mathrm{Na}_{2} \mathrm{CO}_{3}$ solution (1M), $20 \mathrm{~mL}$ toluene, $19 \mathrm{~mL}$ ethanol and $5 \mathrm{~mL} \mathrm{H} \mathrm{H}_{2} \mathrm{O}$. The flask was evacuated and backfilled with argon three times and stirred at $80{ }^{\circ} \mathrm{C}$ overnight. The reaction was quenched with $15 \mathrm{~mL} \mathrm{H}_{2} \mathrm{O}$ and extracted with $\mathrm{CH}_{2} \mathrm{Cl}_{2}(3 \times 25 \mathrm{~mL})$, dried over $\mathrm{MgSO}_{4}$ and concentrated under reduced pressure. Purification by column chromatography on silica $(n$-pentane/EtOAc $=95 / 5)$ afforded 2-((5bromobenzo[b]thiophen-2-yl)methyl)benzaldehyde (1s) as a yellow solid $(2.15 \mathrm{~g}, 6.49 \mathrm{mmol}$, $65 \%)$.

$\mathbf{R}_{f}($ n-Pentane/EtOAc $=90 / 10): 0.68 ;$

${ }^{1} \mathbf{H}$ NMR (400 MHz, $\left.\mathrm{CD}_{2} \mathrm{Cl}_{2}\right): \delta / \mathrm{ppm}=10.21(\mathrm{~s}, 1 \mathrm{H}), 7.87(\mathrm{dd}, J=7.6,1.6 \mathrm{~Hz}, 1 \mathrm{H}), 7.78(\mathrm{~d}$, 
$J=2.0 \mathrm{~Hz}, 1 \mathrm{H}), 7.63-7.56(\mathrm{~m}, 2 \mathrm{H}), 7.50(\mathrm{td}, J=7.5,1.4 \mathrm{~Hz}, 1 \mathrm{H}), 7.41(\mathrm{dd}, J=7.6,1.2 \mathrm{~Hz}$, 1H), 7.34 (dd, $J=8.5,1.9 \mathrm{~Hz}, 1 \mathrm{H}), 6.91$ (s, 1H), 4.68 (s, 2H);

${ }^{13} \mathrm{C}$ NMR (101 MHz, $\left.\mathrm{CD}_{2} \mathrm{Cl}_{2}\right): \delta / \mathrm{ppm}=192.9,147.4,142.2,141.4,138.9,134.5,134.4,133.9$, 132.0, 128.3, 127.2, 126.1, 124.0, 121.6, 118.6, 34.0;

ESI-MS: calculated $\left[\mathrm{C}_{16} \mathrm{H}_{11} \mathrm{BrOSNa}\right]^{+}:$352.9606, found: 352.9612;

ATR-FTIR $\left(\mathrm{cm}^{-1}\right): 1686,1597,1578,1481,1451,1431,1404,1285,1207,1150,1111,1069$, $887,845,799,764,741,721,656,633,613,583$.

\section{Thieno[3,2-c]pyridin-2-ylboronic acid trihydrogen phosphate (S15)}<smiles>OCc1cc2cnccc2s1</smiles>

A flame-dried schlenk-flask was filled with thieno[3,2-c]pyridine ${ }^{[\mathrm{S} 16]}$ ( $2.0 \mathrm{~g}, 14.8 \mathrm{mmol}, 1.0$ equiv.) under argon. $25 \mathrm{~mL}$ THF was added and cooled to $-40{ }^{\circ} \mathrm{C}$. $n$-BuLi solution in hexanes $(1.6 \mathrm{M}, 10 \mathrm{~mL}, 16 \mathrm{mmol}$, 1.08 equiv.) was added dropwise. The resulting solution was stirred for $10 \mathrm{~min}$ at $-40{ }^{\circ} \mathrm{C}$. Triisopropyl borate (4.2 mL, $18.2 \mathrm{mmol}, 1.23$ equiv.) was added dropwise. The reaction was warmed to room temperature and stirred for $3 \mathrm{~h}$. $\mathrm{H}_{3} \mathrm{PO}_{4}(85 \%$ in water, $1.2 \mathrm{~mL}$ ) was added, followed by $110 \mathrm{~mL}$ water and $100 \mathrm{~mL} \mathrm{Et}_{2} \mathrm{O}$. The suspension was filtered and the solid was dried under vacuum to afford the product $(\mathbf{S 1 5})$ as a white powder (2.07 $\mathrm{g}, 7.46 \mathrm{mmol}, 50 \%)$. Spectral data was in accordance with the literature. ${ }^{[\mathrm{S} 17]}$

\section{2-(Thieno[3,2-c]pyridin-2-ylmethyl)benzaldehyde (1t)}

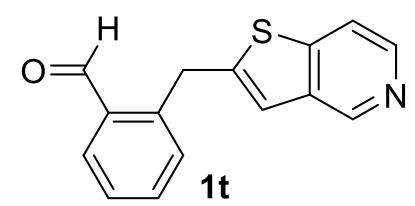

A schlenk-flask was filled with thieno[3,2-c]pyridin-2-ylboronic acid trihydrogen phosphate (S15) (554 mg, $2.00 \mathrm{mmol}, 1.0$ equiv.), 2-(bromomethyl)benzaldehyde (S10) (398 mg, $2.00 \mathrm{mmol}, 1.0$ equiv.), tetrakis(triphenylphosphine)palladium(0) (115 mg, $0.1 \mathrm{mmol}, 0.05$ equiv.), $3.0 \mathrm{~mL}$ aqueous $\mathrm{Na}_{2} \mathrm{CO}_{3}$ solution $(1 \mathrm{M}), 5 \mathrm{~mL}$ toluene, $4 \mathrm{~mL}$ ethanol and $1.2 \mathrm{~mL} \mathrm{H}_{2} \mathrm{O}$. The flask was evacuated and backfilled with argon three times and stirred at $60{ }^{\circ} \mathrm{C}$ overnight. The reaction was quenched with $10 \mathrm{~mL} \mathrm{H} \mathrm{H}_{2} \mathrm{O}$ and extracted with $\mathrm{CH}_{2} \mathrm{Cl}_{2}(3 \times 10 \mathrm{~mL})$, dried over $\mathrm{MgSO}_{4}$ and concentrated under reduced pressure. Purification by column chromatography on silica $(n$-pentane/EtOAc $=7 / 3$ ) 
afforded 2-(thieno[3,2-c]pyridin-2-ylmethyl)benzaldehyde (1t) as a yellow solid (205 mg, $0.81 \mathrm{mmol}, 27 \%)$.

$\mathbf{R}_{f}(n$-Pentane/EtOAc $=70 / 30): 0.15$;

${ }^{1} \mathbf{H}$ NMR (400 MHz, $\left.\mathrm{CD}_{2} \mathrm{Cl}_{2}\right): \delta / \mathrm{ppm}=10.19(\mathrm{~s}, 1 \mathrm{H}), 8.96(\mathrm{~s}, 1 \mathrm{H}), 8.39(\mathrm{~d}, J=5.5 \mathrm{~Hz}, 1 \mathrm{H}), 7.88$ $(\mathrm{dd}, J=7.6,1.6 \mathrm{~Hz}, 1 \mathrm{H}), 7.61(\mathrm{td}, J=7.5,1.6 \mathrm{~Hz}, 1 \mathrm{H}), 7.56-7.49(\mathrm{~m}, 2 \mathrm{H}), 7.43(\mathrm{~d}, J=7.6 \mathrm{~Hz}$, $1 \mathrm{H}), 7.01(\mathrm{dd}, J=1.1,1.1 \mathrm{~Hz}, 1 \mathrm{H}), 4.75(\mathrm{~d}, J=1.2 \mathrm{~Hz}, 2 \mathrm{H})$;

${ }^{13} \mathrm{C}$ NMR $\left(75 \mathrm{MHz}, \mathrm{CD}_{2} \mathrm{Cl}_{2}\right): \delta / \mathrm{ppm}=193.1,151.6,145.7,144.6,143.8,140.9,136.9,134.6$, $134.4,134.4,132.2,128.5,121.5,117.6,34.3$;

ESI-MS: calculated $\left[\mathrm{C}_{15} \mathrm{H}_{11} \mathrm{NOSNa}\right]^{+}:$276.0454, found: 276.0458 ;

ATR-FTIR $\left(\mathrm{cm}^{-1}\right): 1690,1601,1574,1555,1508,1451,1408,1261,1204,1096,1080,1022$, $864,810,787,748,702,664,637,579$.

\section{2-((5-(4-Methoxyphenyl)benzo[b]thiophen-2-yl)methyl)benzaldehyde (1u)}

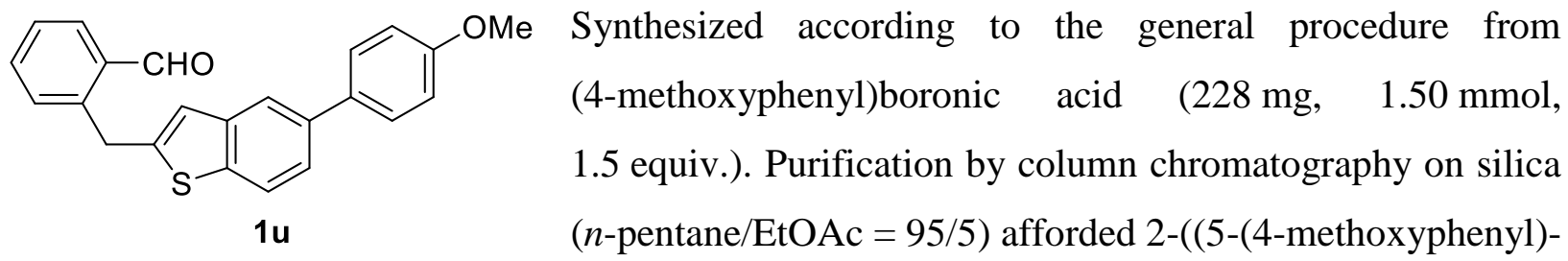

benzo[b]thiophen-2-yl)methyl)benzaldehyde (1u) as a yellow solid (350 mg, $0.98 \mathrm{mmol}, 98 \%$ ).

$\mathbf{R}_{f}(n$-Pentane/EtOAc $=90 / 10): 0.29 ;$

${ }^{1} \mathbf{H}$ NMR (400 MHz, $\left.\mathrm{CD}_{2} \mathrm{Cl}_{2}\right): \delta / \mathrm{ppm}=10.26(\mathrm{~s}, 1 \mathrm{H}), 7.88(\mathrm{dd}, J=7.7,1.5 \mathrm{~Hz}, 1 \mathrm{H}), 7.81(\mathrm{~d}$, $J=1.8 \mathrm{~Hz}, 1 \mathrm{H}), 7.77(\mathrm{~d}, J=8.5 \mathrm{~Hz}, 1 \mathrm{H}), 7.62-7.54(\mathrm{~m}, 3 \mathrm{H}), 7.53-7.42(\mathrm{~m}, 3 \mathrm{H}), 7.04-6.94$ $(\mathrm{m}, 3 \mathrm{H}), 4.70(\mathrm{~d}, J=1.2 \mathrm{~Hz}, 2 \mathrm{H}), 3.84(\mathrm{~s}, 3 \mathrm{H})$;

${ }^{13} \mathrm{C}$ NMR (101 MHz, $\left.\mathrm{CD}_{2} \mathrm{Cl}_{2}\right): \delta / \mathrm{ppm}=192.8,159.7,145.8,141.8,141.2,138.7,137.8,134.5$, 134.4, 134.2, 133.4, 132.0, 128.7, 128.2, 123.6, 122.8, 122.7, 121.3, 114.8, 55.9, 34.0;

ESI-MS: calculated $\left[\mathrm{C}_{23} \mathrm{H}_{18} \mathrm{O}_{2} \mathrm{SNa}\right]^{+}:$381.0920, found: 381.0924 ;

ATR-FTIR $\left(\mathrm{cm}^{-1}\right):$ 2743, 1694, 1597, 1574, 1539, 1512, 1451, 1273, 1250, 1238, 1196, 1111, 1072, 1030, 1015, 883, 868, 822, 802, 783, 756, 737, 664, 602. 


\section{2-((5-(p-Tolyl)benzo[b]thiophen-2-yl)methyl)benzaldehyde (1v)}

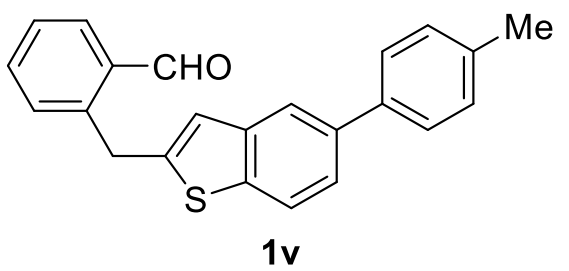

Synthesized according to the general procedure from p-tolylboronic acid (204 mg, $1.50 \mathrm{mmol}, \quad 1.5$ equiv.). Purification by column chromatography on silica $(n$-pentane/EtOAc $=95 / 5)$ afforded $2-((5-(p$-tolyl $)-$

benzo[b]thiophen-2-yl)methyl)benzaldehyde (1v) as a yellow solid (342 mg, 0.99 mmol, 99\%).

$\mathbf{R}_{f}($ n-Pentane/EtOAc $=90 / 10): 0.58 ;$

${ }^{1} \mathbf{H}$ NMR $\left(400 \mathrm{MHz}, \mathrm{CD}_{2} \mathrm{Cl}_{2}\right): \delta / \mathrm{ppm}=10.26(\mathrm{~s}, 1 \mathrm{H}), 7.88(\mathrm{dd}, J=7.6,1.6 \mathrm{~Hz}, 1 \mathrm{H}), 7.84(\mathrm{~d}$, $J=1.8 \mathrm{~Hz}, 1 \mathrm{H}), 7.78(\mathrm{~d}, J=8.4 \mathrm{~Hz}, 1 \mathrm{H}), 7.60(\mathrm{td}, J=7.5,1.6 \mathrm{~Hz}, 1 \mathrm{H}), 7.55-7.47(\mathrm{~m}, 4 \mathrm{H})$, $7.45(\mathrm{~d}, J=8.2 \mathrm{~Hz}, 1 \mathrm{H}), 7.26(\mathrm{~d}, J=7.8 \mathrm{~Hz}, 2 \mathrm{H}), 7.01(\mathrm{~s}, 1 \mathrm{H}), 4.71(\mathrm{~s}, 2 \mathrm{H}), 2.39$ (s, 3H);

${ }^{13} \mathrm{C}$ NMR (101 MHz, $\left.\mathrm{CD}_{2} \mathrm{Cl}_{2}\right): \delta / \mathrm{ppm}=192.8,145.8,141.8,141.2,139.0,138.8,138.1,137.6$, 134.5, 134.4, 133.4, 132.0, 130.0, 128.2, 127.5, 123.7, 122.8, 122.7, 121.6, 34.0, 21.3;

ESI-MS: calculated $\left[\mathrm{C}_{23} \mathrm{H}_{18} \mathrm{OSNa}\right]^{+}: 365.0971$, found: 365.0971 ;

ATR-FTIR $\left(\mathrm{cm}^{-1}\right):$ 2747, 1690, 1601, 1574, 1512, 1485, 1447, 1404, 1288, 1261, 1204, 1157, $1111,1067,887,860,826,810,775,745,733,664$.

\section{2-((5-Phenylbenzo[b]thiophen-2-yl)methyl)benzaldehyde (1w)}

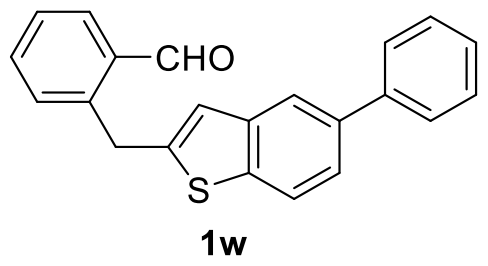

Synthesized according to the general procedure from phenylboronic acid (183 mg, $1.5 \mathrm{mmol}, 1.5$ equiv.). Purification by column chromatography on silica $(n$-pentane/EtOAc $=95 / 5)$ afforded 2-((5-phenylbenzo[b]thiophen-2-yl)methyl)benzaldehyde (1w) as a yellow solid (301 $\mathrm{mg}, 0.92 \mathrm{mmol}, 92 \%)$.

$\mathbf{R}_{f}(n$-Pentane/EtOAc $=90 / 10): 0.56$;

${ }^{1} \mathbf{H}$ NMR $\left(400 \mathrm{MHz}, \mathrm{CD}_{2} \mathrm{Cl}_{2}\right): \delta / \mathrm{ppm}=10.26(\mathrm{~s}, 1 \mathrm{H}), 7.93-7.84(\mathrm{~m}, 2 \mathrm{H}), 7.80(\mathrm{~d}, J=8.4 \mathrm{~Hz}$, $1 \mathrm{H}), 7.68-7.57(\mathrm{~m}, 3 \mathrm{H}), 7.52(\mathrm{dd}, J=8.3,1.7 \mathrm{~Hz}, 2 \mathrm{H}), 7.49-7.42(\mathrm{~m}, 3 \mathrm{H}), 7.38-7.32(\mathrm{~m}$, $1 \mathrm{H}), 7.03(\mathrm{~s}, 1 \mathrm{H}), 4.71(\mathrm{~s}, 2 \mathrm{H})$;

${ }^{13} \mathrm{C}$ NMR (101 MHz, $\left.\mathrm{CD}_{2} \mathrm{Cl}_{2}\right): \delta / \mathrm{ppm}=192.9,146.0,141.8,141.2,139.3,138.2,134.5,134.4$, $133.5,132.0,129.3,128.2,127.8,127.7,123.8,122.9,122.7,121.9,34.0$;

ESI-MS: calculated $\left[\mathrm{C}_{22} \mathrm{H}_{16} \mathrm{OSNa}\right]^{+}:$351.0814, found: 351.0820 ; 
ATR-FTIR $\left(\mathrm{cm}^{-1}\right): 1686,1597,1447,1400,1200,1107,1072,1038,887,849,822,795,756$, $687,652,606$.

\section{2-((5-(4-(Trifluoromethoxy)phenyl)benzo[b]thiophen-2-yl)methyl)benzaldehyde (1x)}

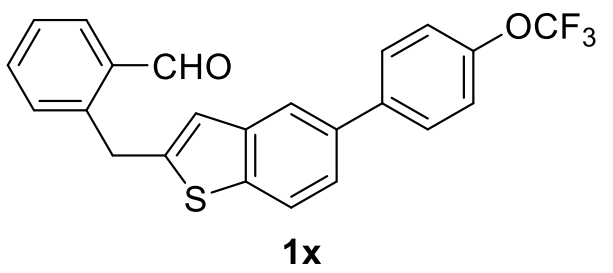

Synthesized according to the general procedure from (4(trifluoromethoxy)phenyl)boronic acid (309 mg, $1.50 \mathrm{mmol}$, 1.5 equiv.). Purification by column chromatography on silica $(n$-pentane/EtOAc $=95 / 5)$

afforded 2-((5-(4-(trifluoromethoxy)phenyl)benzo[b]thiophen-2-yl)methyl)benzaldehyde (1x) as a yellow oil (255 mg, $0.62 \mathrm{mmol}, 62 \%)$.

$\mathbf{R}_{f}(n$-Pentane/EtOAc $=90 / 10): 0.41 ;$

${ }^{1} \mathbf{H}$ NMR (400 MHz, $\left.\mathrm{CD}_{2} \mathrm{Cl}_{2}\right): \delta / \mathrm{ppm}=10.24(\mathrm{~s}, 1 \mathrm{H}), 7.88(\mathrm{dd}, J=7.5,1.6 \mathrm{~Hz}, 1 \mathrm{H}), 7.83(\mathrm{~d}$, $J=1.5 \mathrm{~Hz}, 1 \mathrm{H}), 7.81(\mathrm{dt}, J=8.4,0.7 \mathrm{~Hz}, 1 \mathrm{H}), 7.69-7.64(\mathrm{~m}, 2 \mathrm{H}), 7.60(\mathrm{td}, J=7.5,1.6 \mathrm{~Hz}$, 1H), $7.52(\mathrm{dd}, J=7.5,1.4 \mathrm{~Hz}, 1 \mathrm{H}), 7.50-7.42(\mathrm{~m}, 2 \mathrm{H}), 7.35-7.28(\mathrm{~m}, 2 \mathrm{H}), 7.03(\mathrm{dd}, J=1.0$, $1.0 \mathrm{~Hz}, 1 \mathrm{H}), 4.71(\mathrm{~d}, J=1.1 \mathrm{~Hz}, 2 \mathrm{H})$;

${ }^{13} \mathrm{C}$ NMR (101 MHz, $\left.\mathrm{CD}_{2} \mathrm{Cl}_{2}\right): \delta / \mathrm{ppm}=192.9,149.0$ (q, J = $\left.2 \mathrm{~Hz}\right), 146.3,141.7,141.1,140.8$, 139.7, 136.7, 134.5, 134.4, 133.6, 132.0, 129.1, 128.2, 123.6, 123.0, 122.6, 121.9, 121.8, 121.1 $(\mathrm{d}, \mathrm{J}=257 \mathrm{~Hz}), 34.1$;

${ }^{19}$ F NMR $\left(282 \mathrm{MHz}, \mathrm{CD}_{2} \mathrm{Cl}_{2}\right): \delta / \mathrm{ppm}=-58.20(\mathrm{t}, J=1.0 \mathrm{~Hz})$;

ESI-MS: calculated $\left[\mathrm{C}_{23} \mathrm{H}_{15} \mathrm{~F}_{3} \mathrm{O}_{2} \mathrm{SNa}\right]^{+}$: 435.0637, found: 435.0638;

ATR-FTIR $\left(\mathrm{cm}^{-1}\right):$ 1694, 1597, 1508, 1447, 1250, 1204, 1153, 1072, 1015, 922, 887, 853, 802, $752,660,613$.

\section{2-((5-(4-(Trifluoromethyl)phenyl)benzo[b]thiophen-2-yl)methyl)benzaldehyde (1y)}

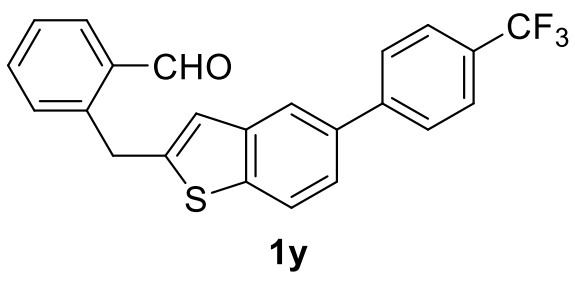

Synthesized according to the general procedure from (4-(trifluoromethyl)phenyl)boronic acid (235 mg, $1.50 \mathrm{mmol}$, 1.5 equiv.). Purification by column chromatography on silica $(n$-pentane/EtOAc $=95 / 5)$ afforded 
2-((5-(4-(trifluoromethyl)phenyl)benzo[b]thiophen-2-yl)methyl)benzaldehyde (1y) as a yellow solid (328 mg, $0.83 \mathrm{mmol}, 83 \%)$.

$\mathbf{R}_{f}(n$-Pentane/EtOAc $=90 / 10): 0.45 ;$

${ }^{1} \mathbf{H}$ NMR $\left(400 \mathrm{MHz}, \mathrm{CD}_{2} \mathrm{Cl}_{2}\right): \delta / \mathrm{ppm}=10.25(\mathrm{~s}, 1 \mathrm{H}), 7.92-7.86(\mathrm{~m}, 2 \mathrm{H}), 7.83(\mathrm{~d}, J=8.4 \mathrm{~Hz}$, 1H), $7.77-7.69(\mathrm{~m}, 4 \mathrm{H}), 7.61(\mathrm{td}, J=7.5,1.6 \mathrm{~Hz}, 1 \mathrm{H}), 7.54-7.48(\mathrm{~m}, 2 \mathrm{H}), 7.45(\mathrm{~d}, J=8.1 \mathrm{~Hz}$, $1 \mathrm{H}), 7.05(\mathrm{~s}, 1 \mathrm{H}), 4.72(\mathrm{~s}, 2 \mathrm{H})$;

${ }^{13} \mathrm{C}$ NMR (101 MHz, $\left.\mathrm{CD}_{2} \mathrm{Cl}_{2}\right): \delta / \mathrm{ppm}=192.9,146.5,145.5(\mathrm{q}, J=2 \mathrm{~Hz}), 141.7,141.2,140.2$, 136.6, 134.6, 134.4, 133.7, 132.1, 129.4 (q, $J=32$ Hz), 128.2, 128.1, 126.2 (q, $J=4 \mathrm{~Hz}), 125.1$ $(\mathrm{q}, J=272 \mathrm{~Hz}), 123.7,123.2,122.6,122.1,34.1$;

${ }^{19}$ F NMR $\left(282 \mathrm{MHz}, \mathrm{CD}_{2} \mathrm{Cl}_{2}\right): \delta / \mathrm{ppm}=-62.58(\mathrm{t}, J=0.7 \mathrm{~Hz})$;

ESI-MS: calculated $\left[\mathrm{C}_{23} \mathrm{H}_{15} \mathrm{~F}_{3} \mathrm{OSNa}\right]^{+}:$419.0688, found: 419.0690;

ATR-FTIR $\left(\mathrm{cm}^{-1}\right):$ 2727, 1701, 1613, 1601, 1574, 1451, 1431, 1404, 1323, 1265, 1204, 1153, 1107, 1072, 1011, 953, 891, 849, 814, 775, 752, 664, 613, 590.

\section{4-(2-(2-Formylbenzyl)benzo[b]thiophen-5-yl)benzonitrile (1z)}

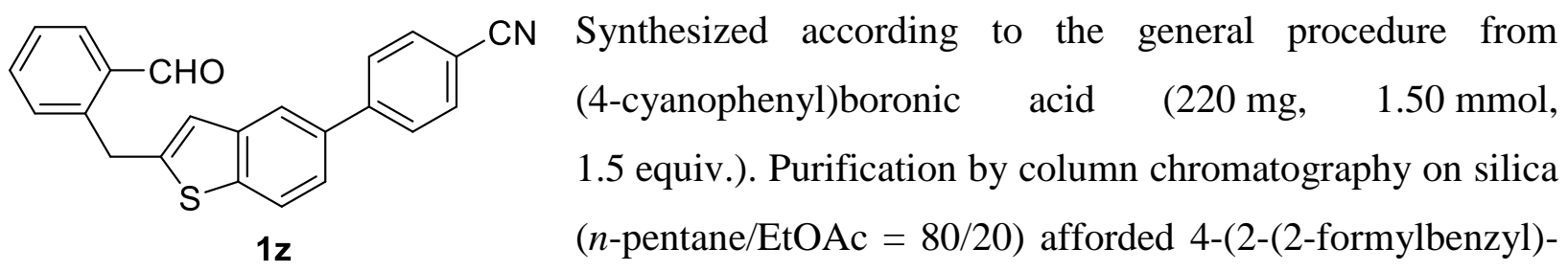
benzo[b]thiophen-5-yl)benzonitrile (1z) as a yellow solid (269 $\mathrm{mg}, 0.76 \mathrm{mmol}, 76 \%$ ).

$\mathbf{R}_{f}($-Pentane/EtOAc $=90 / 10): 0.12 ;$

${ }^{1} \mathbf{H}$ NMR $\left(400 \mathrm{MHz}, \mathrm{CD}_{2} \mathrm{Cl}_{2}\right): \delta / \mathrm{ppm}=10.24(\mathrm{~s}, 1 \mathrm{H}), 7.88(\mathrm{dd}, J=7.7,1.7 \mathrm{~Hz}, 1 \mathrm{H}), 7.86(\mathrm{~d}$, $J=1.6 \mathrm{~Hz}, 1 \mathrm{H}), 7.82(\mathrm{dt}, J=8.4,0.8 \mathrm{~Hz}, 1 \mathrm{H}), 7.72(\mathrm{~s}, 4 \mathrm{H}), 7.60(\mathrm{td}, J=7.5,1.5 \mathrm{~Hz}, 1 \mathrm{H}), 7.53-$ $7.47(\mathrm{~m}, 2 \mathrm{H}), 7.44$ (d, J = 7.6 Hz, 1H), $7.04(\mathrm{~s}, 1 \mathrm{H}), 4.71(\mathrm{~s}, 2 \mathrm{H})$;

${ }^{13} \mathrm{C}$ NMR (101 MHz, $\left.\mathrm{CD}_{2} \mathrm{Cl}_{2}\right): \delta / \mathrm{ppm}=192.9,146.7,146.2,141.5,141.2,140.6,136.1,134.5$, $134.4,133.8,133.1,132.0,128.3,128.2,123.4,123.2,122.6,122.1,119.5,111.2,34.1$;

ESI-MS: calculated $\left[\mathrm{C}_{23} \mathrm{H}_{15} \mathrm{NOSNa}\right]^{+}: 376.0767$, found: 376.0764 ;

ATR-FTIR $\left(\mathrm{cm}^{-1}\right):$ 2222, 1694, 1601, 1574, 1447, 1404, 1288, 1204, 1157, 1072, 891, 845, 806, $752,733,660,671,556$. 
tert-Butyl 2-(2-formylbenzyl)-1H-indole-1-carboxylate (14)

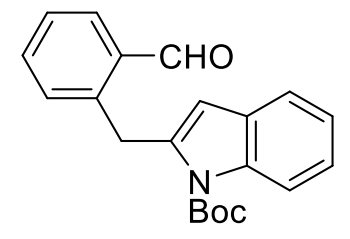

14

A schlenk-flask was filled with $\mathrm{N}$-Boc-indole-2-boronic acid (785 mg, 3.0 mmol, 1.0 equiv.), 2-(bromomethyl)benzaldehyde (S10) (597 mg, $3.0 \mathrm{mmol}, 1.0$ equiv.), tetrakis(triphenylphosphine)palladium(0) (173 mg, $0.15 \mathrm{mmol}, 0.05$ equiv.), $3.3 \mathrm{~mL}$ aqueous $\mathrm{Na}_{2} \mathrm{CO}_{3}$ solution (1M), $6 \mathrm{~mL}$ toluene, $5 \mathrm{~mL}$ ethanol and $1.5 \mathrm{~mL} \mathrm{H} \mathrm{H}_{2} \mathrm{O}$. The flask was evacuated and backfilled with argon three times and stirred at $60{ }^{\circ} \mathrm{C}$ overnight. The reaction was quenched with $20 \mathrm{~mL} \mathrm{H}_{2} \mathrm{O}$ and extracted with EtOAc, dried over $\mathrm{Na}_{2} \mathrm{SO}_{4}$ and concentrated under reduced pressure. Purification by column chromatography on silica ( $n$-pentane/EtOAc = from 98/2 to 94/6) afforded tert-butyl 2-(2formylbenzyl)-1 $H$-indole-1-carboxylate (14) as a yellow solid (417 mg, $1.13 \mathrm{mmol}, 38 \%$ ).

$\mathbf{R}_{f}(n$-Pentane/EtOAc $=10 / 1): 0.45 ;$

${ }^{1} \mathbf{H}$ NMR $\left(300 \mathrm{MHz}, \mathrm{CD}_{2} \mathrm{Cl}_{2}\right): \delta / \mathrm{ppm}=10.23(\mathrm{~s}, 1 \mathrm{H}), 8.17-8.11(\mathrm{~m}, 1 \mathrm{H}), 7.92(\mathrm{dd}, J=7.6$, $1.6 \mathrm{~Hz}, 1 \mathrm{H}), 7.55(\mathrm{td}, J=7.5,1.7 \mathrm{~Hz}, 1 \mathrm{H}), 7.48(\mathrm{td}, J=7.5,1.4 \mathrm{~Hz}, 1 \mathrm{H}), 7.41-7.35(\mathrm{~m}, 1 \mathrm{H})$, 7.29-7.14 (m, 1H), 5.98 (dd, $J=1.1,1.1 \mathrm{~Hz}, 1 \mathrm{H}), 4.78$ (s, 2H), 1.56 (s, 9H);

${ }^{13} \mathrm{C}$ NMR $\left(75 \mathrm{MHz}, \mathrm{CD}_{2} \mathrm{Cl}_{2}\right): \delta / \mathrm{ppm}=192.6,150.8,141.9,140.4,137.2,134.4,134.3,132.1$, $131.2,129.4,127.6,124.0,123.0,120.2,116.0,109.8,84.5,33.8,28.2$;

ESI-MS: calculated $\left[\mathrm{C}_{21} \mathrm{H}_{21} \mathrm{NO}_{3} \mathrm{H}\right]^{+}: 336.1594$, found: 336.1595 ;

ATR-FTIR $\left(\mathrm{cm}^{-1}\right):$ 3099, 3068, 3010, 2981, 2936, 2834, 2736, 1726, 1689, 1590, 1570, 1473, 1452, 1396, 1369, 1328, 1257, 1196, 1156, 1146, 1113, 1087, 958, 941, 872, 845, 830, 771, 751, 699, 661, 644, 622, 589, 557. 


\section{Synthetic Manipulations of 2a}

\section{(R)-3H-3H-Spiro[benzofuran-2,2'-inden]-1'(3'H)-one oxime (11)}

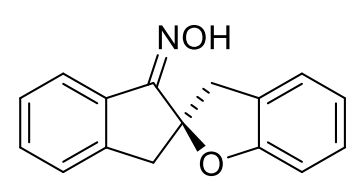

11

Following a modified procedure,${ }^{[\mathrm{S} 18]}(R)-3 H$-spiro[benzofuran-2,2'-inden]-

1'(3'H)-one (2a) $\quad(71 \mathrm{mg}, \quad 0.30 \mathrm{mmol}, \quad 1.0$ equiv.), hydroxylamine hydrochloride (104 mg, $1.5 \mathrm{mmol}, 5.0$ equiv.) and sodium acetate (98 mg, $1.2 \mathrm{mmol}, 4.0$ equiv.) were stirred in $\mathrm{EtOH}(5 \mathrm{~mL})$ for $16 \mathrm{~h}$ at room temperature. The reaction was stopped by addition of water, extracted with EtOAc $(3 \mathrm{x})$ and washed with brine. Removal of solvent afforded the crude product which was purified by column chromatography on silica $(n$-pentane/EtOAc $=85 / 15)$. The oxime 11 was yielded as a white solid in a 1:1 mixture of $E / Z$ isomers $(73 \mathrm{mg}, 0.29 \mathrm{mmol}, 97 \%)$. $E / Z$ isomers were assigned via 2DNMR spectroscopy. The racemic compound was synthesized by using racemic $\mathbf{2 a}$.

$\mathbf{R}_{f}(n$-Pentane/EtOAc $=85 / 15): Z$ isomer: $0.55 ; E$ isomer: 0.43 ;

Z-Isomer: $[\alpha]_{D}{ }^{20}=-204.7\left(c=0.37, \mathrm{CH}_{2} \mathrm{Cl}_{2}\right)$;

E-Isomer: $[\alpha]_{D} \mathbf{2 0}=-92.0\left(c=0.03, \mathrm{CH}_{2} \mathrm{Cl}_{2}\right)$;

Z-Isomer: ${ }^{1} \mathrm{H}$ NMR (600 MHz, $\mathrm{CD}_{2} \mathrm{Cl}_{2}$ ): $\delta / \mathrm{ppm}=8.29$ (d, $\left.J=7.8 \mathrm{~Hz}, 1 \mathrm{H}\right), 8.25(\mathrm{~s}, 1 \mathrm{H}), 7.47$ (td, $J=7.5,1.2 \mathrm{~Hz}, 1 \mathrm{H}), 7.41-7.33(\mathrm{~m}, 2 \mathrm{H}), 7.23(\mathrm{dd}, J=7.4,1.3 \mathrm{~Hz}, 1 \mathrm{H}), 7.14(\mathrm{td}, J=7.7,1.2 \mathrm{~Hz}$, 1H), 3.72 (d, $J=15.8 \mathrm{~Hz}, 1 \mathrm{H}), 3.57$ (d, $J=17.1 \mathrm{~Hz}, 1 \mathrm{H}), 3.40-3.33(\mathrm{~m}, 2 \mathrm{H})$;

${ }^{13} \mathrm{C}$ NMR $\left(150 \mathrm{MHz}, \mathrm{CD}_{2} \mathrm{Cl}_{2}\right): \delta / \mathrm{ppm}=160.2,158.9,144.5,132.0,131.9,129.8,128.6,127.9$, 127.2, 125.7, 125.1, 121.1, 109.8, 92.0, 45.2, 39.8;

E-Isomer: ${ }^{1} \mathbf{H}$ NMR $\left(400 \mathrm{MHz}, \mathrm{CD}_{2} \mathrm{Cl}_{2}\right): \delta / \mathrm{ppm}=7.62(\mathrm{~d}, J=8.0 \mathrm{~Hz}, 1 \mathrm{H}), 7.43-7.37(\mathrm{~m}, 1 \mathrm{H})$, $7.35-7.25(\mathrm{~m}, 3 \mathrm{H}), 7.20(\mathrm{~d}, J=7.4 \mathrm{~Hz}, 1 \mathrm{H}), 7.14(\mathrm{t}, J=7.8 \mathrm{~Hz}, 1 \mathrm{H}), 6.88(\mathrm{td}, J=7.5,1.1 \mathrm{~Hz}$, $1 \mathrm{H}), 6.76(\mathrm{~d}, J=8.0 \mathrm{~Hz}, 1 \mathrm{H}), 4.07(\mathrm{~d}, J=15.7 \mathrm{~Hz}, 1 \mathrm{H}), 3.59(\mathrm{~d}, J=17.5 \mathrm{~Hz}, 1 \mathrm{H}), 3.34(\mathrm{~d}, J=$ $17.5 \mathrm{~Hz}, 1 \mathrm{H}), 3.29$ (d, $J=15.7 \mathrm{~Hz}, 1 \mathrm{H})$;

${ }^{13} \mathrm{C}$ NMR (100 MHz, $\left.\mathrm{CD}_{2} \mathrm{Cl}_{2}\right): \delta / \mathrm{ppm}=161.3,159.5,143.9$, 135.0, 131.5, 128.3, 128.0, 127.0, 125.7, 124.9, 122.0, 120. 9, 109.3, 90.3, 48.6, 41.3;

ESI-MS: calculated $\left[\mathrm{C}_{16} \mathrm{H}_{13} \mathrm{NO}_{2} \mathrm{Na}\right]^{+}:$274.0838; found: 274.0837;

Z-Isomer:ATR-FTIR (cm $\left.{ }^{-1}\right)$ : 1477, 1458, 1312, 1246, 1223, 1096, 1011, 988, 949, 872, 853, $745,702,617$

E-Isomer:ATR-FTIR (cm $\left.{ }^{-1}\right):$ 2920, 2851, 1597, 1477, 1458, 1246, 1223, 1204, 1030, 1018, 976, 934, 872, 745, 664;

Z-Isomer: HPLC: 99\% ee (Chiralcel AS-H, $n$-hexane/ $i$-PrOH = 95/5, $1.0 \mathrm{~mL} / \mathrm{min}, 254 \mathrm{~nm}$, S35 
$t_{R}=11.13 \min ($ major $), t_{R}=19.40 \min ($ minor $)$;

E-Isomer: HPLC: 99\% ee (Chiralcel AS-H, $n$-hexane/ $i$-PrOH = 95/5, $1.0 \mathrm{~mL} / \mathrm{min}, 210 \mathrm{~nm}$, $t_{R}=15.98 \min ($ major $), t_{R}=30.89 \min ($ minor $)$;

Racemate HPLC: E/Z mixture (Chiralcel AS-H, $n$-hexane/i-PrOH = 95/5, $1.0 \mathrm{~mL} / \mathrm{min}, 210 \mathrm{~nm}$, $\left.t_{R}=11.13 \min (\mathrm{Z}), t_{R}=16.06 \min (\mathrm{E}), t_{R}=19.35 \min (\mathrm{Z}), t_{R}=30.98 \min (\mathrm{E})\right)$.

\section{(2R)-1',3'-Dihydro-3H-spiro[benzofuran-2,2'-inden]-1'-ol (12)}

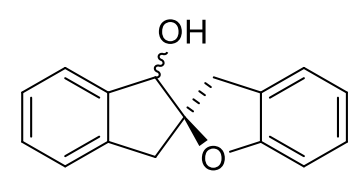

12

Following a modified procedure, ${ }^{[\mathrm{S} 18]}(R)-3 H$-spiro[benzofuran-2,2'-inden]$1^{\prime}\left(3^{\prime} H\right)$-one (2a) $(71 \mathrm{mg}, \quad 0.30 \mathrm{mmol}, \quad 1.0$ equiv. $) \quad$ was dissolved in $\mathrm{MeOH}(2 \mathrm{~mL})$ and cooled to $0{ }^{\circ} \mathrm{C}$. After addition of $\mathrm{NaBH}_{4}(17 \mathrm{mg}$, $0.45 \mathrm{mmol}, 1.5$ equiv.) the reaction mixture was stirred for $1 \mathrm{~h}$ at $0{ }^{\circ} \mathrm{C}$. The reaction was stopped by addition of water, extracted with EtOAc (3x) and washed with brine. Removal of solvent afforded the crude product which was purified by column chromatography on silica $(n$-pentane/EtOAc $=85 / 15)$. The alcohol 12 was afforded as a white solid $(62 \mathrm{mg}$, $0.26 \mathrm{mmol}, 87 \%$, diastereomeric ratio (d.r.) determined by NMR: 6:1). The racemic compound was synthesized by using racemic $\mathbf{2 a}$.

$\mathbf{R}_{f}(n$-Pentane/EtOAc $=85 / 15): 0.49 ;$

$[\alpha]_{D}^{\mathbf{2 0}}=+52.0\left(c=0.99, \mathrm{CHCl}_{3}\right)$;

${ }^{1} \mathbf{H}$ NMR (major diastereomer, $\left.400 \mathrm{MHz}, \mathrm{CDCl}_{3}\right): \delta / \mathrm{ppm}=7.51-7.48(\mathrm{~m}, 1 \mathrm{H}), 7.32-7.29(\mathrm{~m}$, 2H), 7.25-7.20 (m, 2H), $7.13(\mathrm{t}, J=7.8 \mathrm{~Hz}, 1 \mathrm{H}), 6.90(\mathrm{t}, J=7.5 \mathrm{~Hz}, 1 \mathrm{H}), 6.78(\mathrm{~d}, J=8.0 \mathrm{~Hz}$, 1H), $5.01(\mathrm{~s}, 1 \mathrm{H}), 3.57(\mathrm{~d}, J=15.9 \mathrm{~Hz}, 1 \mathrm{H}), 3.44(\mathrm{~d}, J=16.2 \mathrm{~Hz}, 1 \mathrm{H}), 3.32(\mathrm{~d}, J=16.1 \mathrm{~Hz}, 1 \mathrm{H})$, $3.04(\mathrm{~d}, J=16.1 \mathrm{~Hz}, 1 \mathrm{H})$;

${ }^{13} \mathrm{C}$ NMR (major diastereomer, $101 \mathrm{MHz}, \mathrm{CDCl}_{3}$ ): $\delta / \mathrm{ppm}=158.7,142.8,139.4,128.8,128.3$, $127.5,126.9,125.2,125.1,124.6,121.1,109.9,96.4,81.0,43.3,37.2$

ESI-MS: calculated $\left[\mathrm{C}_{16} \mathrm{H}_{14} \mathrm{O}_{2} \mathrm{Na}\right]^{+}=261.0886$; found: 261.0887;

ATR-FTIR $\left(\mathrm{cm}^{-1}\right):$ 1597, 1478, 1462, 1397, 1331, 1234, 1207, 1177, 1049, 1015, 907, 868, 752, 694;

HPLC: 99\% ee (major diastereomer, Chiralcel AS-H, $n$-hexane $/ i-\mathrm{PrOH}=98 / 2,1.0 \mathrm{~mL} / \mathrm{min}$, $210 \mathrm{~nm}, t_{R}=13.99 \min ($ major $), t_{R}=18.93 \min ($ minor $)$ ); 
Racemate HPLC: (major diastereomer, Chiralcel AS-H, $n$-hexane $/ i$-PrOH = 98/2, $1.0 \mathrm{~mL} / \mathrm{min}$, $\left.210 \mathrm{~nm}, t_{R}=14.10 \mathrm{~min}, t_{R}=18.91 \mathrm{~min}\right)$.

\section{(S)-3H-Spiro[benzofuran-2,3'-isochroman]-1'-one (13)}

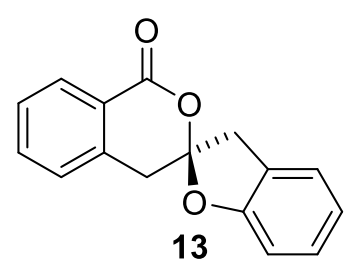

Following a modified procedure ${ }^{[\mathrm{S} 18]}(R)-3 H$-spiro[benzofuran-2,2'-inden]1'(3'H)-one (2a) (71 $\mathrm{mg}, \quad 0.30 \mathrm{mmol}, \quad 1.0$ equiv.) and magnesium monoperoxyphthalate hexahydrate $(490 \mathrm{mg}, 0.99 \mathrm{mmol}, 3.3$ equiv.) was dissolved in $\mathrm{DMF} / \mathrm{H}_{2} \mathrm{O}(1 \mathrm{~mL} ; 3 / 1)$ and stirred for $19 \mathrm{~h}$ at $\mathrm{rt}$. The reaction

was stopped by addition of sat. aq. $\mathrm{Na}_{2} \mathrm{~S}_{2} \mathrm{O}_{3}$, followed by sat. aq. $\mathrm{NaHCO}_{3}$. After extraction with EtOAc (3x) the combined organic layers were washed with brine. Removing the solvent afforded the crude product which was purified by column chromatography on silica $(n$-pentane/EtOAc $=$ 85/15). The product 13 was isolated as a white solid (62 $\mathrm{mg}, 0.25 \mathrm{mmol}, 82 \%)$.

$\mathbf{R}_{f}(n$-Pentane/EtOAc $=85 / 15): 0.23$;

$[\alpha]_{D}^{\mathbf{2 0}}=-206.2\left(c=1.01, \mathrm{CHCl}_{3}\right)$;

${ }^{1} \mathbf{H}$ NMR $\left(300 \mathrm{MHz}, \mathrm{CDCl}_{3}\right): \delta / \mathrm{ppm}=8.16(\mathrm{dd}, J=7.7,1.4 \mathrm{~Hz}, 1 \mathrm{H}), 7.61(\mathrm{td}, J=7.6,1.4 \mathrm{~Hz}$, 1H), $7.52-7.39(\mathrm{~m}, 1 \mathrm{H}), 7.36-7.30(\mathrm{~m}, 1 \mathrm{H}), 7.28-7.18(\mathrm{~m}, 1 \mathrm{H}), 7.21-7.08(\mathrm{~m}, 1 \mathrm{H}), 6.95$ $(\mathrm{td}, J=7.5,1.0 \mathrm{~Hz}, 1 \mathrm{H}), 6.75(\mathrm{~d}, J=8.0 \mathrm{~Hz}, 1 \mathrm{H}), 3.64$ (d, $J=13.1 \mathrm{~Hz}, 1 \mathrm{H}), 3.59$ (d, $J=13.2 \mathrm{~Hz}$, 1H), 3.42 (d, $J=10.4 \mathrm{~Hz}, 1 \mathrm{H}), 3.37$ (d, $J=10.1 \mathrm{~Hz}, 1 \mathrm{H})$;

${ }^{13} \mathrm{C} \mathrm{NMR}\left(75 \mathrm{MHz}, \mathrm{CDCl}_{3}\right): \delta / \mathrm{ppm}=163.7,157.5,136.5,134.3,130.3,128.5,128.1,125.0$, 124.2(7), 124.2(5), 122.1, 112.2, 110.2, 42.0, 36.8;

ESI-MS: calculated $\left[\mathrm{C}_{16} \mathrm{H}_{12} \mathrm{O}_{3} \mathrm{Na}\right]^{+}=275.0679$; found: 275.0680;

ATR-FTIR $\left(\mathrm{cm}^{-1}\right):$ 1736, 1694, 1601, 1481, 1462, 1370, 1288, 1234, 1207, 1184, 1138, 1115, 1069, 991, 930, 872, 826, 799, 748, 706, 683, 610, 590;

HPLC: $98 \%$ ee (Chiralcel AS-H, $n$-hexane $/ i$-PrOH = 95/5, $1 \mathrm{~mL} / \mathrm{min}, 210 \mathrm{~nm}, t_{R}=30.26 \mathrm{~min}$ (major), $t_{R}=47.42 \min ($ minor));

HPLC: $r a c$ (Chiralcel AS-H, $n$-hexane $/ i$-PrOH =95/5, $1 \mathrm{~mL} / \mathrm{min}, 210 \mathrm{~nm}, t_{R}=30.64 \mathrm{~min}$ (major), $t_{R}=47.18 \min$ (minor)). 


\section{Synthesis of Hydroacylation Products}

\subsection{General procedure for the racemic hydroacylation}

The racemic products were synthesized on a $0.05 \mathrm{mmol}$ scale under similar conditions as the enantioselective reactions, using NHC precursor $3 f(2.8 \mathrm{mg}, 0.01 \mathrm{mmol}, 20 \mathrm{~mol} \%)$ and $\mathrm{K}_{2} \mathrm{CO}_{3}$ (6.9 mg , $0.05 \mathrm{mmol}, 1.0$ equiv.) in dry 1,4-dioxane $(0.10 \mathrm{~mL})$.

\subsection{Enantioselective hydroacylation of benzofurans}

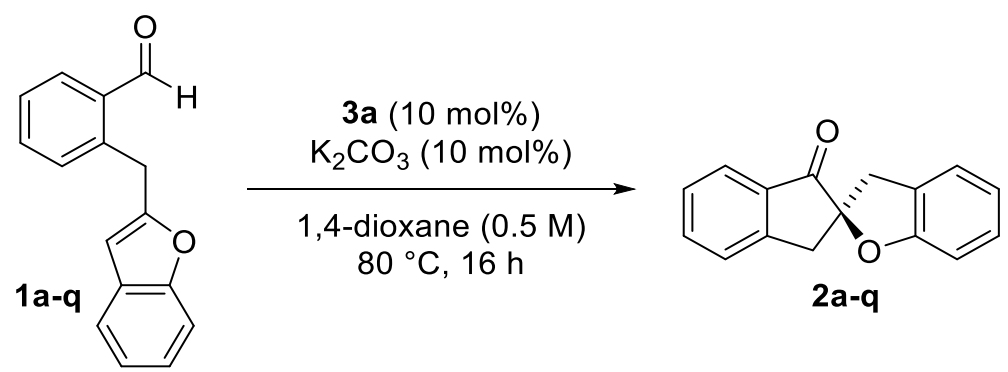

To a flame-dried screw-capped test tube equipped with a magnetic stir bar was added $\mathrm{K}_{2} \mathrm{CO}_{3}$ (4.2 mg, $0.03 \mathrm{mmol}, 10 \mathrm{~mol} \%)$, NHC precursor 3a (13.2 mg, $0.03 \mathrm{mmol}, 10 \mathrm{~mol} \%)$ and aldehyde (0.30 mmol, 1.0 equiv.). Dry 1,4-dioxane $(0.60 \mathrm{~mL})$ was added and the resulting mixture was then stirred in a pre-heated oil bath at $80^{\circ} \mathrm{C}$ for $16 \mathrm{~h}$. The reaction was cooled to ambient temperature, pre-absorbed on silica gel and purified by column chromatography on silica gel.

\section{(R)-3H-Spiro[benzofuran-2,2'-inden]-1'(3'H)-one (2a)}

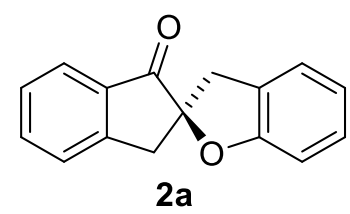

Synthesized according to the general procedure but on a $2.00 \mathrm{mmol}$ scale using 2-(benzofuran-2-ylmethyl)benzaldehyde (1a) (472 mg, $2.00 \mathrm{mmol})$. Isolated as colorless solid, yield: $385 \mathrm{mg}, 1.63 \mathrm{mmol}, 81 \%$.

$\mathbf{R}_{f}(n$-Pentane/EtOAc $=70 / 30): 0.76$;

$[\alpha]_{D}^{20}=-161.1\left(c=1.15, \mathrm{CHCl}_{3}\right)$;

${ }^{1} \mathbf{H}$ NMR $\left(300 \mathrm{MHz}, \mathrm{CDCl}_{3}\right): \delta / \mathrm{ppm}=7.86(\mathrm{~d}, J=7.6 \mathrm{~Hz}, 1 \mathrm{H}), 7.68(\mathrm{td}, J=7.5,1.3 \mathrm{~Hz}, 1 \mathrm{H})$, $7.54-7.39$ (m, 2H), $7.24-7.10(\mathrm{~m}, 2 \mathrm{H}), 7.00-6.77$ (m, 2H), $3.71-3.51(\mathrm{~m}, 2 \mathrm{H}), 3.39(\mathrm{~d}, J=$ 
$17.3 \mathrm{~Hz}, 1 \mathrm{H}), 3.22(\mathrm{~d}, J=15.7 \mathrm{~Hz}, 1 \mathrm{H})$;

${ }^{13} \mathrm{C}$ NMR $\left(75 \mathrm{MHz}, \mathrm{CDCl}_{3}\right): \delta / \mathrm{ppm}=202.3,159.1,150.8,136.2,134.3,128.5,128.3,126.8$, $125.7,125.3,125.0,121.2,109.8,89.7,42.2,39.2$

ESI-MS: calculated $\left[\mathrm{C}_{16} \mathrm{H}_{12} \mathrm{O}_{2} \mathrm{Na}\right]^{+}:$259.0730, found: 259.0747;

ATR-FTIR $\left(\mathrm{cm}^{-1}\right):$ 1721, 1605, 1589, 1478, 1454, 1427, 1300, 1238, 1211, 1153, 1103, 1072, $1018,949,926,883,802,752,737,702,621$;

HPLC: 99\% ee (Chiralcel AS-H, $n$-hexane $/ i$-PrOH = 95/5, $1.0 \mathrm{~mL} / \mathrm{min}, 210 \mathrm{~nm}, t_{R}=12.48 \mathrm{~min}$ (major), $t_{R}=18.11 \mathrm{~min}$ (minor)).

\section{( \pm -3H-Spiro[benzofuran-2,2'-inden]-1'(3'H)-one}

Synthesized from 2-(benzofuran-2-ylmethyl)benzaldehyde (1a) (11.8 mg, 0.05 mmol).

$\mathbf{R}_{f}$ and ${ }^{1} \mathbf{H}$ NMR data were in accordance with the one obtained with catalyst $\mathbf{3 a}$.

HPLC: $r a c$ (Chiralcel AS-H, $n$-Hexane $/ i-\mathrm{PrOH}=95 / 5,1.0 \mathrm{~mL} / \mathrm{min}, 210 \mathrm{~nm}, t_{R}=12.72 \mathrm{~min}, t_{R}=$ $18.45 \mathrm{~min})$.

\section{(R)-5'-Fluoro-3H-spiro[benzofuran-2,2'-inden]-1'(3'H)-one (2b)}

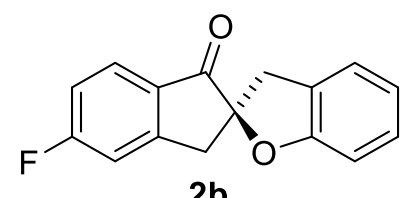

Synthesized according to the general procedure but using $20 \mathrm{~mol} \% \mathrm{NHC}$ precursor and $20 \mathrm{~mol} \% \mathrm{~K}_{2} \mathrm{CO}_{3}$ from 2-(benzofuran-2-ylmethyl)-4fluorobenzaldehyde (1b) $(76.3 \mathrm{mg}, 0.30 \mathrm{mmol})$. Isolated as colorless solid, yield: $49 \mathrm{mg}, 0.19 \mathrm{mmol}, 64 \%$.

$\mathbf{R}_{f}(n$-Pentane/EtOAc $=90 / 10): 0.24 ;$

$[\alpha]_{D}^{20}=-132.5\left(c=0.54, \mathrm{CHCl}_{3}\right)$;

${ }^{1} \mathbf{H}$ NMR $\left(300 \mathrm{MHz}, \mathrm{CDCl}_{3}\right): \delta / \mathrm{ppm}=7.79(\mathrm{dd}, J=9.1,5.3 \mathrm{~Hz}, 1 \mathrm{H}), 7.16-7.02(\mathrm{~m}, 4 \mathrm{H}), 6.85$ $(\mathrm{td}, J=7.5,1.0 \mathrm{~Hz}, 1 \mathrm{H}), 6.75(\mathrm{~d}, J=8.0 \mathrm{~Hz}, 1 \mathrm{H}), 3.56(\mathrm{~d}, J=15.6 \mathrm{~Hz}, 1 \mathrm{H}), 3.50(\mathrm{~d}, J=17.1 \mathrm{~Hz}$, 1H), $3.29(\mathrm{~d}, J=17.5 \mathrm{~Hz}, 1 \mathrm{H}), 3.14(\mathrm{~d}, J=15.7 \mathrm{~Hz}, 1 \mathrm{H})$;

${ }^{13} \mathrm{C}$ NMR $\left(75 \mathrm{MHz}, \mathrm{CDCl}_{3}\right): \delta / \mathrm{ppm}=200.4,168.1(\mathrm{~d}, J=258 \mathrm{~Hz}), 158.9,153.9(\mathrm{~d}, J=10 \mathrm{~Hz})$, $130.7(\mathrm{~d}, J=2 \mathrm{~Hz}), 128.6,127.8(\mathrm{~d}, J=11 \mathrm{~Hz}), 125.5,125.0,121.4,116.7$ (d, $J=24 \mathrm{~Hz})$, $113.6(\mathrm{~d}, J=23 \mathrm{~Hz}), 109.8,89.7,42.4(\mathrm{~d}, J=2 \mathrm{~Hz}), 39.1$; 
${ }^{19}$ F NMR $\left(282 \mathrm{MHz}, \mathrm{CDCl}_{3}\right): \delta / \mathrm{ppm}=-100.0(\mathrm{td}, \mathrm{J}=8.6,5.3 \mathrm{~Hz})$;

ESI-MS: calculated $\left[\mathrm{C}_{16} \mathrm{H}_{11} \mathrm{FO}_{2} \mathrm{Na}\right]^{+}:$277.0635, found: 277.0643;

ATR-FTIR $\left(\mathrm{cm}^{-1}\right): 1721,1613,1589,1478,1454,1420,1339,1258,1211,1169,1126,1084$, $1072,1026,1015,953,937,883,856,841,799,772,752,687,671$;

HPLC: $93 \%$ ee (Chiralcel AS-H, $n$-hexane $/ i-\mathrm{PrOH}=90 / 10,1.0 \mathrm{~mL} / \mathrm{min}, 210 \mathrm{~nm}, t_{R}=10.82 \mathrm{~min}$ (major), $t_{R}=16.88 \mathrm{~min}($ minor $)$ ).

\section{( \pm )-5'-Fluoro-3H-spiro[benzofuran-2,2'-inden]-1'(3'H)-one}

Synthesized from 2-(benzofuran-2-ylmethyl)-4-fluorobenzaldehyde (1b) (12.7 mg, 0.05 mmol).

$\mathbf{R}_{f}$ and ${ }^{1} \mathbf{H}$ NMR data were in accordance with the one obtained with catalyst $3 \mathbf{a}$.

HPLC: $r a c$ (Chiralcel AS-H, $n$-Hexane $/ i-\mathrm{PrOH}=90 / 10,1.0 \mathrm{~mL} / \mathrm{min}, 210 \mathrm{~nm}, t_{R}=10.87 \mathrm{~min}$, $\left.t_{R}=16.82 \mathrm{~min}\right)$.

(R)-5'-Chloro-3H-spiro[benzofuran-2,2'-inden]-1'(3'H)-one (2c)

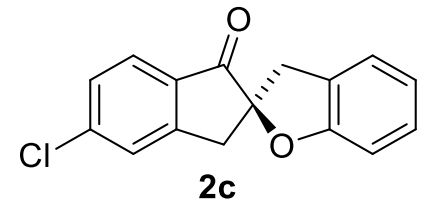

Synthesized according to the general procedure from 2-(benzofuran-2ylmethyl)-4-chlorobenzaldehyde (1c) $(83.1 \mathrm{mg}, 0.30 \mathrm{mmol})$. Isolated as colorless solid, yield: $58 \mathrm{mg}, 0.21 \mathrm{mmol}, 71 \%$.

$\mathbf{R}_{f}(n$-Pentane/EtOAc $=90 / 10): 0.41 ;$

$[\alpha]_{D^{20}}=-138.9\left(c=1.04, \mathrm{CHCl}_{3}\right)$;

${ }^{1} \mathbf{H}$ NMR $\left(300 \mathrm{MHz}, \mathrm{CD}_{2} \mathrm{Cl}_{2}\right): \delta / \mathrm{ppm}=7.66(\mathrm{~d}, J=8.2 \mathrm{~Hz}, 1 \mathrm{H}), 7.46-7.31(\mathrm{~m}, 2 \mathrm{H}), 7.17-$ $7.03(\mathrm{~m}, 2 \mathrm{H}), 6.83(\mathrm{td}, J=7.4,1.0 \mathrm{~Hz}, 1 \mathrm{H}), 6.70(\mathrm{~d}, J=8.0 \mathrm{~Hz}, 1 \mathrm{H}), 3.48(\mathrm{~d}, J=16.9 \mathrm{~Hz}, 2 \mathrm{H})$, $3.30(\mathrm{~d}, J=17.6 \mathrm{~Hz}, 1 \mathrm{H}), 3.17(\mathrm{~d}, J=15.9 \mathrm{~Hz}, 1 \mathrm{H})$;

${ }^{13} \mathrm{C}$ NMR $\left(75 \mathrm{MHz}, \mathrm{CD}_{2} \mathrm{Cl}_{2}\right): \delta / \mathrm{ppm}=201.3,159.4,153.0,143.0,133.1,129.5,128.9,127.5$, 126.6, 126.3, 125.5, 121.7, 109.9, 90.1, 42.3, 39.3;

ESI-MS: calculated $\left[\mathrm{C}_{16} \mathrm{H}_{11} \mathrm{O}_{2} \mathrm{ClNa}\right]^{+}:$293.0340, found: 293.0339;

ATR-FTIR $\left(\mathrm{cm}^{-1}\right):$ 3082, 2920, 1721, 1597, 1574, 1478, 1454, 1420, 1327, 1308, 1242, 1211, $1169,1099,1069,1018,941,922,883,849,748,683,640$; 
HPLC: $97 \%$ ee (Chiralcel AD-H, $n$-hexane $/ i$-PrOH = 80/20, $1.0 \mathrm{~mL} / \mathrm{min}, 210 \mathrm{~nm}, t_{R}=7.15 \mathrm{~min}$ (major), $t_{R}=8.09 \min ($ minor $)$ ).

\section{( \pm )-5'-Chloro-3H-spiro[benzofuran-2,2'-inden]-1'(3' H)-one}

Synthesized from 2-(benzofuran-2-ylmethyl)-4-chlorobenzaldehyde (1c) (27.1 mg, $0.1 \mathrm{mmol}$ ).

$\mathbf{R}_{f}$ and ${ }^{1} \mathbf{H}$ NMR data were in accordance with the one obtained with catalyst $3 \mathbf{a}$.

HPLC: $r a c$ (Chiralcel AD-H, $n$-hexane $/ i$-PrOH = 80/20, $1.0 \mathrm{~mL} / \mathrm{min}, 210 \mathrm{~nm}, t_{R}=7.15 \mathrm{~min}$, $\left.t_{R}=8.09 \mathrm{~min}\right)$.

\section{(R)-5'-Phenyl-3H-spiro[benzofuran-2,2'-inden]-1'(3'H)-one (2d)}

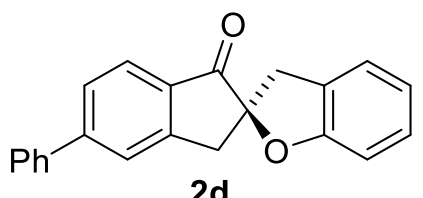

Synthesized according to the general procedure but using $20 \mathrm{~mol} \%$ NHC precursor and $20 \mathrm{~mol} \% \mathrm{~K}_{2} \mathrm{CO}_{3}$ from 3-(benzofuran-2-ylmethyl)[1,1'-biphenyl]-4-carbaldehyde (1d) $(93.7 \mathrm{mg}, 0.30 \mathrm{mmol})$. Isolated as colorless solid, yield: $52 \mathrm{mg}, 0.17 \mathrm{mmol}, 55 \%$.

$\mathbf{R}_{f}(n$-Pentane/EtOAc $=90 / 10): 0.16 ;$

$[\alpha]_{D}{ }^{\mathbf{2 0}}=-144.9\left(c=1.05, \mathrm{CHCl}_{3}\right)$;

${ }^{1} \mathbf{H}$ NMR $\left(300 \mathrm{MHz}, \mathrm{CDCl}_{3}\right): \delta / \mathrm{ppm}=7.83(\mathrm{~d}, J=8.5 \mathrm{~Hz}, 1 \mathrm{H}), 7.62-7.52(\mathrm{~m}, 4 \mathrm{H}), 7.46-7.31$ (m, 3H), $7.16-7.04(\mathrm{~m}, 2 \mathrm{H}), 6.84(\mathrm{td}, J=7.4,1.0 \mathrm{~Hz}, 1 \mathrm{H}), 6.76(\mathrm{~d}, J=8.0 \mathrm{~Hz}, 1 \mathrm{H}), 3.65-3.48$ $(\mathrm{m}, 2 \mathrm{H}), 3.34(\mathrm{~d}, J=17.3 \mathrm{~Hz}, 1 \mathrm{H}), 3.16(\mathrm{~d}, J=15.7 \mathrm{~Hz}, 1 \mathrm{H})$;

${ }^{13} \mathrm{C}$ NMR (75 MHz, $\left.\mathrm{CDCl}_{3}\right): \delta / \mathrm{ppm}=201.8,159.1,151.5,149.3,140.0,133.1,129.2,128.8$, $128.5,127.7,127.6,125.7,125.7,125.2,125.0,121.2,109.8,90.0,42.5,39.2$;

ESI-MS: calculated $\left[\mathrm{C}_{22} \mathrm{H}_{16} \mathrm{O}_{2} \mathrm{Na}\right]^{+}: 335.1043$, found: 335.1042 ;

ATR-FTIR $\left(\mathrm{cm}^{-1}\right): 1717,1605,1508,1478,1454,1424,1331,1308,1281,1238,1211,1150$, 1107, 1076, 1018, 949, 930, 887, 841, 799, 783, 752, 725, 694, 629;

HPLC: $98 \%$ ee (Chiralcel AS-H, $n$-hexane $/ i$-PrOH = 90/10, $1.0 \mathrm{~mL} / \mathrm{min}, 210 \mathrm{~nm}, t_{R}=9.04 \mathrm{~min}$ (major), $t_{R}=11.96 \min ($ minor $)$ ). 


\section{( \pm )-5'-Phenyl-3H-spiro[benzofuran-2,2'-inden]-1'(3' $H)$-one}

Synthesized from 3-(benzofuran-2-ylmethyl)-[1,1'-biphenyl]-4-carbaldehyde (1d) (31.2 mg, $0.1 \mathrm{mmol})$.

$\mathbf{R}_{f}$ and ${ }^{1} \mathbf{H}$ NMR data were in accordance with the one obtained with catalyst $3 \mathbf{a}$.

HPLC: $r a c$ (Chiralcel AS-H, $n$-Hexane $/ i-\mathrm{PrOH}=90 / 10,1.0 \mathrm{~mL} / \mathrm{min}, 210 \mathrm{~nm}, t_{R}=9.05 \mathrm{~min}$, $\left.t_{R}=11.96 \mathrm{~min}\right)$.

(R)-5-Methyl-3H-spiro[benzofuran-2,2'-inden]-1'(3'H)-one (2e)

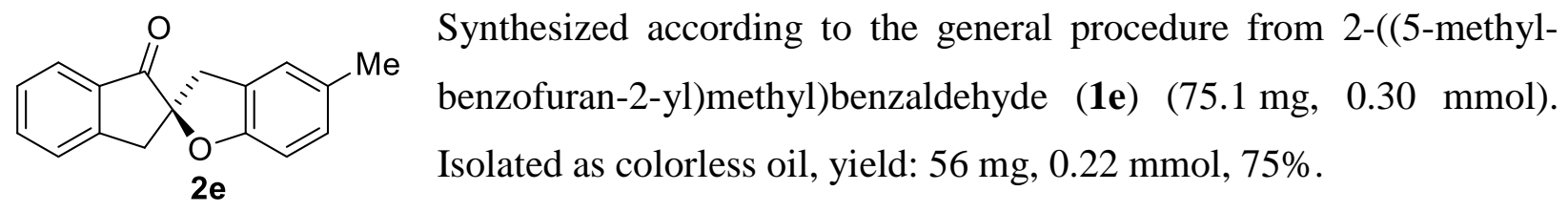

$\mathbf{R}_{f}(n$-Pentane/EtOAc $=90 / 10): 0.47$;

$[\boldsymbol{\alpha}]_{D^{\mathbf{2 0}}}=-159.8\left(c=0.89, \mathrm{CHCl}_{3}\right)$;

${ }^{1}$ H NMR (300 MHz, CDCl3): $\delta / \mathrm{ppm}=7.85(\mathrm{~d}, J=7.7 \mathrm{~Hz}, 1 \mathrm{H}), 7.67(\mathrm{td}, J=7.5,1.3 \mathrm{~Hz}, 1 \mathrm{H})$, $7.52-7.40(\mathrm{~m}, 2 \mathrm{H}), 7.02(\mathrm{~s}, 1 \mathrm{H}), 6.99-6.92(\mathrm{~m}, 1 \mathrm{H}), 6.72(\mathrm{~d}, J=8.1 \mathrm{~Hz}, 1 \mathrm{H}), 3.63-3.53(\mathrm{~m}$, 2H), 3.37 (d, $J=17.3 \mathrm{~Hz}, 1 \mathrm{H}), 3.18(\mathrm{~d}, J=15.6 \mathrm{~Hz}, 1 \mathrm{H}), 2.30(\mathrm{~s}, 3 \mathrm{H})$;

${ }^{13}$ C NMR (75 MHz, CDCl3): $\delta / \mathrm{ppm}=202.4,157.0,150.9,136.1,134.4,130.6,128.8,128.3$, 126.8, 125.7, 125.5, 125.3, 109.3, 89.8, 42.4, 39.2, 20.9;

ESI-MS: calculated $\left[\mathrm{C}_{17} \mathrm{H}_{14} \mathrm{O}_{2} \mathrm{Na}\right]^{+}$: 273.0886, found: 273.0892;

ATR-FTIR $\left(\mathrm{cm}^{-1}\right):$ 3036, 2924, 1721, 1593, 1478, 1454, 1424, 1327, 1300, 1238, 1211, 1153, 1103, 1072, 1018, 949, 926, 883, 802, 737, 702, 621;

HPLC 96\% ee (Chiralcel AS-H, $n$-hexane/ $i$-PrOH = 90/10, $1.0 \mathrm{~mL} / \mathrm{min}, 210 \mathrm{~nm}, t_{R}=6.51 \mathrm{~min}$ (major), $t_{R}=7.45 \min ($ minor $)$ ). 


\section{( \pm )-5-Methyl-3H-spiro[benzofuran-2,2'-inden]-1'(3'H)-one}

Synthesized from 2-((5-methylbenzofuran-2-yl)methyl)benzaldehyde (1e) (25.0 mg, $0.1 \mathrm{mmol})$.

$\mathbf{R}_{\boldsymbol{f}}$ and ${ }^{\mathbf{1}} \mathbf{H}$ NMR data were in accordance with the one obtained with catalyst $\mathbf{3 a}$.

HPLC: $r a c$ (Chiralcel AS-H, $n$-hexane $/ i-\mathrm{PrOH}=90 / 10,1.0 \mathrm{~mL} / \mathrm{min}, 210 \mathrm{~nm}, t_{R}=6.55 \mathrm{~min}$, $\left.t_{R}=7.47 \mathrm{~min}\right)$.

\section{(R)-5-Methoxy-3H-spiro[benzofuran-2,2'-inden]-1'(3'H)-one (2f)}<smiles></smiles>

$\mathbf{R}_{f}(n$-Pentane/EtOAc $=80 / 20): 0.35$

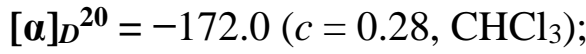
$7.51-7.39(\mathrm{~m}, 2 \mathrm{H}), 6.81-6.77(\mathrm{~m}, 1 \mathrm{H}), 6.76-6.66(\mathrm{~m}, 2 \mathrm{H}), 3.77(\mathrm{~s}, 3 \mathrm{H}), 3.65-3.52(\mathrm{~m}, 2 \mathrm{H})$,

$3.37(\mathrm{~d}, J=17.4 \mathrm{~Hz}, 1 \mathrm{H}), 3.19(\mathrm{~d}, J=16.0 \mathrm{~Hz}, 1 \mathrm{H})$;

${ }^{13}$ C NMR (101 MHz, CDCl3): $\delta / p p m=202.3,154.8,153.2,150.8,136.1,134.3,128.3,126.8$, 126.7, 125.3, 113.4, 111.2, 109.7, 90.0, 56.2, 42.3, 39.5;

ESI-MS: calculated $\left[\mathrm{C}_{17} \mathrm{H}_{14} \mathrm{O}_{3} \mathrm{Na}\right]^{+}: 289.0835$, found: 289.0841 ;

ATR-FTIR $\left(\mathrm{cm}^{-1}\right):$ 2994, 2959, 2920, 2821, 2835, 1717, 1605, 1485, 1462, 1424, 1296, 1261, $1246,1211,1173,1153,1034,1015,945,918,887,849,806,741,698,613$;

HPLC: $96 \%$ ee (Chiralcel AS-H, $n$-hexane $/ i$-PrOH = 90/10, $1.0 \mathrm{~mL} / \mathrm{min}, 210 \mathrm{~nm}, t_{R}=9.31 \mathrm{~min}$ (major), $t_{R}=10.53 \mathrm{~min}$ (minor)).

\section{( \pm )-5-Methoxy-3H-spiro[benzofuran-2,2'-inden]-1'(3'H)-one}

Synthesized from 2-((5-methoxybenzofuran-2-yl)methyl)benzaldehyde (1f) (26.6 mg, $0.1 \mathrm{mmol}$ ). 
$\mathbf{R}_{f}$ and ${ }^{1} \mathbf{H}$ NMR data were in accordance with the one obtained with catalyst $3 \mathbf{a}$.

HPLC: $r a c$ (Chiralcel AS-H, $n$-hexane $/ i$-PrOH =90/10, $1.0 \mathrm{~mL} / \mathrm{min}, 210 \mathrm{~nm}, t_{R}=9.34 \mathrm{~min}, t_{R}=$ $10.53 \mathrm{~min})$.

\section{(R)-5-Bromo-3H-spiro[benzofuran-2,2'-inden]-1'(3'H)-one (2g)}<smiles>O=C1c2ccccc2C[C@]12Cc1cc(Br)ccc1O2</smiles>

2g
Synthesized according to the general procedure from 2-((5bromobenzofuran-2-yl)methyl)benzaldehyde (1g) (94.6 mg, $0.30 \mathrm{mmol})$. Isolated as grey solid (77.3 $\mathrm{mg}, 0.25 \mathrm{mmol}, 82 \%)$.

$\mathbf{R}_{f}(n$-Pentane/EtOAc $=90 / 10): 0.29 ;$

$[\alpha]_{D^{20}}=-138.7\left(c=0.45, \mathrm{CHCl}_{3}\right)$;

${ }^{1} \mathbf{H}$ NMR $\left(300 \mathrm{MHz}, \mathrm{CDCl}_{3}\right): \delta / \mathrm{ppm}=7.80(\mathrm{~d}, J=7.6 \mathrm{~Hz}, 1 \mathrm{H}), 7.63(\mathrm{td}, J=7.5,1.3 \mathrm{~Hz}, 1 \mathrm{H})$, $7.48-7.33(\mathrm{~m}, 2 \mathrm{H}), 7.29-7.12(\mathrm{~m}, 2 \mathrm{H}), 6.65(\mathrm{~d}, J=8.5 \mathrm{~Hz}, 1 \mathrm{H}), 3.66-3.44(\mathrm{~m}, 2 \mathrm{H}), 3.33(\mathrm{~d}$, $J=17.3 \mathrm{~Hz}, 1 \mathrm{H}), 3.16(\mathrm{~d}, J=15.9 \mathrm{~Hz}, 1 \mathrm{H})$;

${ }^{13} \mathrm{C} \mathrm{NMR}\left(75 \mathrm{MHz}, \mathrm{CDCl}_{3}\right): \delta / \mathrm{ppm}=201.6,158.3,150.7,136.3,134.0,131.3,128.5,128.3$, $127.9,126.8,125.4,113.1,111.3,90.3,42.1,38.8$;

ESI-MS: calculated $\left[\mathrm{C}_{16} \mathrm{H}_{11} \mathrm{BrO}_{2} \mathrm{Na}\right]^{+}:$336.9835, found: 336.9845 ;

ATR-FTIR $\left(\mathrm{cm}^{-1}\right): 1717,1605,1586,1466,1420,1300,1246,1211,1169,1107,1026,937$, $891,880,864,818,810,741,698,656,633$;

HPLC: $96 \%$ ee (Chiralcel AS-H, $n$-hexane $/ i-\mathrm{PrOH}=90 / 10,1.0 \mathrm{~mL} / \mathrm{min}, 254 \mathrm{~nm}, t_{R}=14.40 \mathrm{~min}$ (major), $t_{R}=37.52 \mathrm{~min}$ (minor)).

\section{( \pm )-5-Bromo-3H-spiro[benzofuran-2,2'-inden]-1'(3' $H)$-one}

Synthesized from 2-((5-bromobenzofuran-2-yl)methyl)benzaldehyde (1g) (31.5 mg, $0.1 \mathrm{mmol})$.

$\mathbf{R}_{f}$ and ${ }^{1} \mathbf{H}$ NMR data were in accordance with the one obtained with catalyst $3 \mathbf{a}$.

HPLC: $r a c$ (Chiralcel AS-H, $n$-hexane $/ i-\mathrm{PrOH}=90 / 10,1.0 \mathrm{~mL} / \mathrm{min}, 254 \mathrm{~nm}, t_{R}=14.73 \mathrm{~min}$, $\left.t_{R}=38.00 \mathrm{~min}\right)$. 
(R)-5-Fluoro-3H -spiro[benzofuran-2,2'-inden]-1'(3'H)-one (2h)

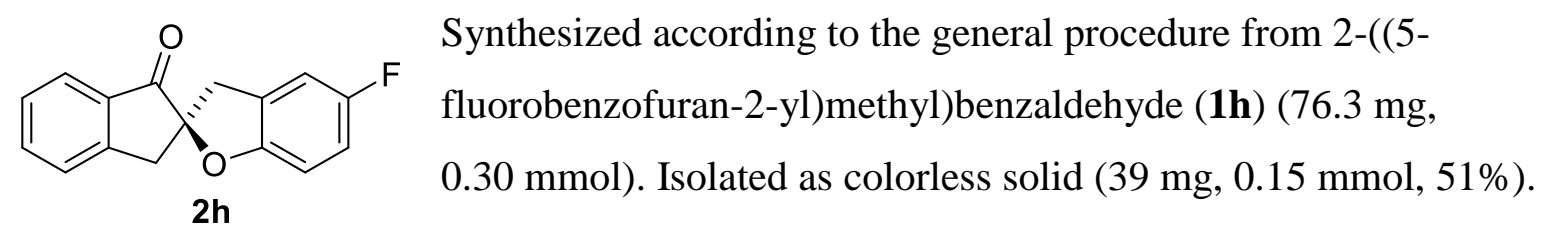

$\mathbf{R}_{f}(n-\mathrm{Pentane} / \mathrm{EtOAc}=90 / 10): 0.26 ;$

$[\alpha]_{D}{ }^{20}=-159.8\left(c=1.00, \mathrm{CHCl}_{3}\right)$;

${ }^{1} \mathbf{H}$ NMR $\left(300 \mathrm{MHz}, \mathrm{CD}_{2} \mathrm{Cl}_{2}\right): \delta / \mathrm{ppm}=7.81(\mathrm{dt}, J=7.7,1.0 \mathrm{~Hz}, 1 \mathrm{H}), 7.70(\mathrm{td}, J=7.5,1.3 \mathrm{~Hz}$,

$1 \mathrm{H}), 7.53-7.43(\mathrm{~m}, 2 \mathrm{H}), 6.98-6.91(\mathrm{~m}, 1 \mathrm{H}), 6.90-6.81(\mathrm{~m}, 1 \mathrm{H}), 6.71(\mathrm{dd}, J=8.7,4.2 \mathrm{~Hz}$,

$1 \mathrm{H}), 3.63-3.52(\mathrm{~m}, 2 \mathrm{H}), 3.41(\mathrm{~d}, J=17.4 \mathrm{~Hz}, 1 \mathrm{H}), 3.25(\mathrm{dd}, J=16.2,1.1 \mathrm{~Hz}, 1 \mathrm{H})$;

${ }^{13}$ C NMR (75 MHz, $\left.\mathrm{CD}_{2} \mathrm{Cl}_{2}\right): \delta / \mathrm{ppm}=202.2,158.4(\mathrm{~d}, J=237 \mathrm{~Hz}), 155.6(\mathrm{~d}, J=1 \mathrm{~Hz}), 151.4$,

136.7, 134.5, 128.8, 128.1 (d, $J=9 \mathrm{~Hz}), 127.3,125.4,114.8$ (d, $J=24 \mathrm{~Hz}), 112.5$ (d, $J=25 \mathrm{~Hz})$, $109.9(\mathrm{~d}, J=9 \mathrm{~Hz}), 90.8,42.4,39.4(\mathrm{~d}, J=2 \mathrm{~Hz})$;

${ }^{19} \mathbf{F}$ NMR $\left(282 \mathrm{MHz}, \mathrm{CD}_{2} \mathrm{Cl}_{2}\right): \delta / \mathrm{ppm}=-124.76(\mathrm{dddt}, J=9.1,8.1,4.3,1.0 \mathrm{~Hz})$;

ESI-MS: calculated $\left[\mathrm{C}_{16} \mathrm{H}_{11} \mathrm{O}_{2} \mathrm{FNa}\right]^{+}$: 277.0635, found: 277.0636;

ATR-FTIR $\left(\mathrm{cm}^{-1}\right): 3067,2924,2851,1717,1605,1481,1304,1234,1204,1173,1123,1026$,

941, 891, 864, 802, 737, 702;

HPLC: $96 \%$ ee (Chiralcel AS-H, $n$-hexane $/ i-\mathrm{PrOH}=90 / 10,1.0 \mathrm{~mL} / \mathrm{min}, 254 \mathrm{~nm}, t_{R}=9.65 \mathrm{~min}$ (major), $t_{R}=17.72 \min ($ minor)).

\section{( \pm )-5-Fluoro-3H-spiro[benzofuran-2,2'-inden]-1'(3' $H)$-one}

Synthesized from 2-((5-fluorobenzofuran-2-yl)methyl)benzaldehyde (1h) $(25.4 \mathrm{mg}, 0.1 \mathrm{mmol})$.

$\mathbf{R}_{f}$ and ${ }^{1} \mathbf{H}$ NMR data were in accordance with the one obtained with catalyst $\mathbf{3 a}$.

HPLC: $r a c$ (Chiralcel AS-H, $n$-hexane $/ i-\mathrm{PrOH}=90 / 10,1.0 \mathrm{~mL} / \mathrm{min}, 254 \mathrm{~nm}, t_{R}=9.81 \mathrm{~min}, t_{R}=$ $17.75 \mathrm{~min})$. 
(R)-5-(4-Methoxyphenyl)-3H-spiro[benzofuran-2,2'-inden]-1'(3'H)-one (2i)

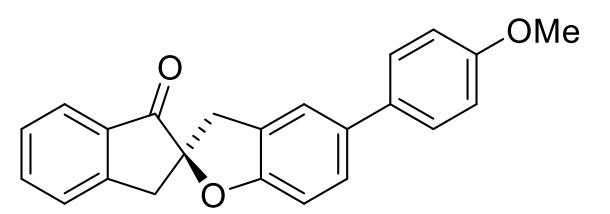

$2 \mathbf{i}$

Synthesized according to the general procedure from

2-((5-(4-methoxyphenyl)benzofuran-2-yl)methyl)-benzaldehyde (1i) $(102.7 \mathrm{mg}, 0.30 \mathrm{mmol})$. Isolated as green solid (92.8 mg, $0.27 \mathrm{mmol}, 90 \%)$.

$\mathbf{R}_{f}(n$-Pentane/EtOAc $=80 / 20): 0.29 ;$

$[\alpha]_{D^{20}}=-141.0\left(c=1.00, \mathrm{CHCl}_{3}\right)$;

${ }^{1} \mathbf{H}$ NMR $\left(300 \mathrm{MHz}, \mathrm{CDCl}_{3}\right): \delta / \mathrm{ppm}=7.87(\mathrm{~d}, J=7.6 \mathrm{~Hz}, 1 \mathrm{H}), 7.68(\mathrm{td}, J=7.5,1.2 \mathrm{~Hz}, 1 \mathrm{H})$, $7.52-7.41(\mathrm{~m}, 4 \mathrm{H}), 7.41-7.30(\mathrm{~m}, 2 \mathrm{H}), 7.01-6.91(\mathrm{~m}, 2 \mathrm{H}), 6.87(\mathrm{~d}, \mathrm{~J}=8.3 \mathrm{~Hz}, 1 \mathrm{H}), 3.85(\mathrm{~s}$, $3 \mathrm{H}), 3.69(\mathrm{~d}, J=15.8 \mathrm{~Hz}, 1 \mathrm{H}), 3.62(\mathrm{~d}, J=17.1 \mathrm{~Hz}, 1 \mathrm{H}), 3.42(\mathrm{~d}, J=17.3 \mathrm{~Hz}, 1 \mathrm{H}), 3.27(\mathrm{~d}, J=$ $15.8 \mathrm{~Hz}, 1 \mathrm{H})$;

${ }^{13} \mathrm{C}$ NMR $\left(75 \mathrm{MHz}, \mathrm{CDCl}_{3}\right): \delta / \mathrm{ppm}=202.2,158.8,158.3,150.8,136.2,134.6,134.3,134.0$, 128.4, 128.0, 127.2, 126.8, 126.4, 125.3, 123.5, 114.3, 109.8, 90.1, 55.5, 42.4, 39.2;

ESI-MS: calculated $\left[\mathrm{C}_{23} \mathrm{H}_{18} \mathrm{O}_{3} \mathrm{Na}\right]^{+}: 365.1148$, found: 365.1153 ;

ATR-FTIR $\left(\mathrm{cm}^{-1}\right): 1721,1605,1520,1485,1466,1296,1269,1238,1184,1157,1115,1072$, $1034,1015,937,895,853,810,745,698,625$;

HPLC: $96 \%$ ee (Chiralcel AS-H, $n$-hexane $/ i$-PrOH = 70/30, $1.5 \mathrm{~mL} / \mathrm{min}, 210 \mathrm{~nm}, t_{R}=7.49 \mathrm{~min}$ (major), $t_{R}=8.43 \mathrm{~min}$ (minor)).

\section{( \pm )-5-(4-Methoxyphenyl)-3H-spiro[benzofuran-2,2'-inden]-1'(3'H)-one}

Synthesized from 2-((5-(4-methoxyphenyl)benzofuran-2-yl)methyl)-benzaldehyde (1i) (17.1 mg, $0.05 \mathrm{mmol})$.

$\mathbf{R}_{\boldsymbol{f}}$ and ${ }^{1} \mathbf{H}$ NMR data were in accordance with the one obtained with catalyst $3 \mathbf{a}$.

HPLC: $r a c$ (Chiralcel AS-H, $n$-hexane $/ i$-PrOH = 70/30, $1.5 \mathrm{~mL} / \mathrm{min}, 210 \mathrm{~nm}, t_{R}=7.49 \mathrm{~min}$, $t_{R}=8.41 \mathrm{~min}$ ). 
(R)-5-(4-(tert-Butyl)phenyl)-3H-spiro[benzofuran-2,2'-inden]-1'(3'H)-one (2j)

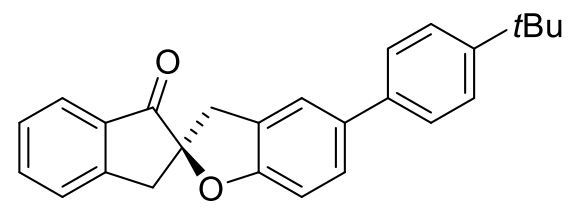

2j

Synthesized according to the general procedure from

2-((5-(4-(tert-butyl)phenyl)benzofuran-2-yl)methyl)-benzaldehyde (1j) (110.5 mg, $0.30 \mathrm{mmol})$. Isolated as green solid (97.6 mg, $0.26 \mathrm{mmol}, 88 \%)$.

$\mathbf{R}_{f}(n$-Pentane/EtOAc $=90 / 10): 0.37$;

$[\alpha]_{D^{20}}=-131.0\left(c=1.00, \mathrm{CHCl}_{3}\right)$;

${ }^{1} \mathbf{H}$ NMR $\left(300 \mathrm{MHz}, \mathrm{CDCl}_{3}\right): \delta / \mathrm{ppm}=7.87(\mathrm{~d}, J=7.6 \mathrm{~Hz}, 1 \mathrm{H}), 7.69(\mathrm{td}, J=7.5,1.3 \mathrm{~Hz}, 1 \mathrm{H})$, $7.54-7.32(\mathrm{~m}, 8 \mathrm{H}), 6.88(\mathrm{~d}, J=8.2 \mathrm{~Hz}, 1 \mathrm{H}), 3.69(\mathrm{~d}, J=15.8 \mathrm{~Hz}, 1 \mathrm{H}), 3.62(\mathrm{~d}, J=17.4 \mathrm{~Hz}$,

1H), 3.42 (d, $J=17.3 \mathrm{~Hz}, 1 \mathrm{H}), 3.28$ (d, $J=15.7 \mathrm{~Hz}, 1 \mathrm{H}), 1.37$ (s, 9H);

${ }^{13}$ C NMR $\left(75 \mathrm{MHz}, \mathrm{CDCl}_{3}\right): \delta / \mathrm{ppm}=202.2,158.5,150.9,149.7,138.4,136.2,134.8,134.3$, 128.4, 127.5, 126.8, 126.6, 126.3, 125.8, 125.4, 123.7, 109.9, 90.1, 42.4, 39.2, 34.6, 31.5;

ESI-MS: calculated $\left[\mathrm{C}_{26} \mathrm{H}_{24} \mathrm{O}_{2} \mathrm{Na}\right]^{+}: 391.1669$, found: 391.1670 ;

ATR-FTIR $\left(\mathrm{cm}^{-1}\right):$ 2955, 1717, 1609, 1481, 1427, 1296, 1269, 1238, 1211, 1157, 1119, 1072, $1015,949,895,853,837,814,745,702,664,617$;

HPLC: $97 \%$ ee (Chiralcel AS-H, $n$-hexane $/ i-\mathrm{PrOH}=90 / 10,1.0 \mathrm{~mL} / \mathrm{min}, 254 \mathrm{~nm}, t_{R}=10.27 \mathrm{~min}$ (major), $t_{R}=20.07 \mathrm{~min}($ minor $)$ ).

\section{( \pm )-5-(4-(tert-Butyl)phenyl)-3H-spiro[benzofuran-2,2'-inden]-1'(3'H)-one}

Synthesized from 2-((5-(4-(tert-butyl)phenyl)benzofuran-2-yl)methyl)-benzaldehyde (1j) (18.4 mg, $0.05 \mathrm{mmol})$.

$\mathbf{R}_{\boldsymbol{f}}$ and ${ }^{1} \mathbf{H}$ NMR data were in accordance with the one obtained with catalyst 3a.

HPLC: $r a c$ (Chiralcel AS-H, $n$-hexane $/ i$-PrOH = 90/10, $1.0 \mathrm{~mL} / \mathrm{min}, 254 \mathrm{~nm}, t_{R}=10.52 \mathrm{~min}$, $\left.t_{R}=20.52 \mathrm{~min}\right)$. 
(R)-5-Phenyl-3H-spiro[benzofuran-2,2'-inden]-1'(3'H)-one (2k)<smiles>O=C1c2ccccc2C[C@]12Cc1cc(-c3ccccc3)ccc1O2</smiles>

2k

Synthesized according to the general procedure from 2-((5-

phenylbenzofuran-2-yl)methyl)benzaldehyde (1k) $\quad(93.7 \mathrm{mg}$, $0.30 \mathrm{mmol})$. Isolated as green solid (91.0 mg, $0.29 \mathrm{mmol}, 97 \%)$.

$\mathbf{R}_{f}(n$-Pentane/EtOAc $=90 / 10): 0.29$;

$[\alpha]_{D}{ }^{\mathbf{2 0}}=-160.2\left(c=1.01, \mathrm{CHCl}_{3}\right)$;

${ }^{1} \mathbf{H}$ NMR $\left(300 \mathrm{MHz}, \mathrm{CDCl}_{3}\right): \delta / \mathrm{ppm}=7.87(\mathrm{~d}, J=7.5 \mathrm{~Hz}, 1 \mathrm{H}), 7.69(\mathrm{td}, J=7.5,1.3 \mathrm{~Hz}, 1 \mathrm{H})$, $7.59-7.35(\mathrm{~m}, 8 \mathrm{H}), 7.35-7.27(\mathrm{~m}, 1 \mathrm{H}), 6.90(\mathrm{~d}, J=8.2 \mathrm{~Hz}, 1 \mathrm{H}), 3.70(\mathrm{~d}, J=15.7 \mathrm{~Hz}, 1 \mathrm{H})$, $3.63(\mathrm{~d}, J=17.4 \mathrm{~Hz}, 1 \mathrm{H}), 3.43(\mathrm{~d}, J=17.3 \mathrm{~Hz}, 1 \mathrm{H}), 3.28(\mathrm{~d}, J=15.7 \mathrm{~Hz}, 1 \mathrm{H})$;

${ }^{13} \mathrm{C} \mathrm{NMR}\left(75 \mathrm{MHz}, \mathrm{CDCl}_{3}\right): \delta / \mathrm{ppm}=202.2,158.7,150.8,141.3,136.2,134.9,134.2,128.82$, $128.4,127.7,127.0,126.8,126.8,126.4,125.3,123.9,109.9,90.2,42.4,39.1$;

ESI-MS: calculated $\left[\mathrm{C}_{22} \mathrm{H}_{16} \mathrm{O}_{2} \mathrm{Na}\right]^{+}: 335.1043$, found: 335.1043 ;

ATR-FTIR $\left(\mathrm{cm}^{-1}\right): 1717,1605,1474,1416,1312,1296,1246,1227,1211,1153,1115,1076$, $1011,945,895,849,818,760,741,694,621$;

HPLC: 97\% ee (Chiralcel AS-H, $n$-hexane $/ i-\mathrm{PrOH}=85 / 15,1.0 \mathrm{~mL} / \mathrm{min}, 210 \mathrm{~nm}, t_{R}=10.35 \mathrm{~min}$ (major), $t_{R}=20.43 \mathrm{~min}$ (minor)).

\section{( \pm )-5-Phenyl-3H-spiro[benzofuran-2,2'-inden]-1'(3'H)-one}

Synthesized from 2-((5-phenylbenzofuran-2-yl)methyl)benzaldehyde (1k) (15.6 mg, 0.05 mmol).

$\mathbf{R}_{f}$ and ${ }^{1} \mathbf{H}$ NMR data were in accordance with the one obtained with catalyst $\mathbf{3 a}$.

HPLC: $r a c$ (Chiralcel AS-H, $n$-hexane $/ i$-PrOH = 85/15, $1.0 \mathrm{~mL} / \mathrm{min}, 210 \mathrm{~nm}, t_{R}=10.62 \mathrm{~min}$, $\left.t_{R}=21.19 \mathrm{~min}\right)$.

\section{(R)-5-(4-(Hydroxymethyl)phenyl)-3H-spiro[benzofuran-2,2'-inden]-1'(3'H)-one (2l)}<smiles>O=C1c2ccccc2C[C@]12Cc1cc(-c3ccc(CO)cc3)ccc1O2</smiles>

2I

Synthesized according to the general procedure from 2-((5-

(4-(hydroxymethyl)phenyl)-benzofuran-2-yl)methyl)benzaldehyde (11) (102.7 mg, $0.30 \mathrm{mmol})$. Isolated as green solid (99.6 mg, $0.29 \mathrm{mmol}, 97 \%)$. 
$\mathbf{R}_{f}(n$-Pentane/EtOAc $=80 / 20): 0.03$;

$[\alpha]_{D}{ }^{20}=-142.6\left(c=1.00, \mathrm{CHCl}_{3}\right)$;

${ }^{1} \mathbf{H}$ NMR (300 MHz, $\left.\mathrm{CDCl}_{3}\right): \delta / \mathrm{ppm}=7.86(\mathrm{~d}, J=7.6 \mathrm{~Hz}, 1 \mathrm{H}), 7.69(\mathrm{td}, J=7.5,1.3 \mathrm{~Hz}, 1 \mathrm{H})$, $7.58-7.34(\mathrm{~m}, 9 \mathrm{H}), 6.89(\mathrm{~d}, J=8.2 \mathrm{~Hz}, 1 \mathrm{H}), 4.72(\mathrm{~s}, 2 \mathrm{H}), 3.74-3.56(\mathrm{~m}, 2 \mathrm{H}), 3.42(\mathrm{~d}$,

$J=17.3 \mathrm{~Hz}, 1 \mathrm{H}), 3.28(\mathrm{~d}, J=15.8 \mathrm{~Hz}, 1 \mathrm{H})$;

${ }^{13} \mathrm{C}$ NMR $\left(75 \mathrm{MHz}, \mathrm{CDCl}_{3}\right): \delta / \mathrm{ppm}=202.2,158.8,150.8,140.7,139.4,136.3,134.5,134.2$,

128.4, 127.6, 127.1, 126.8, 126.5, 125.3, 123.8, 109.9, 90.2, 77.4, 65.2, 42.3, 39.1;

ESI-MS: calculated $\left[\mathrm{C}_{23} \mathrm{H}_{18} \mathrm{O}_{3} \mathrm{Na}\right]^{+}: 365.1148$, found: 365.1150 ;

ATR-FTIR $\left(\mathrm{cm}^{-1}\right): 1713,1601,1481,1431,1416,1300,1242,1219,1157,1115,1042,1018$, 937, 899, 860, 814, 799, 737, 694;

HPLC: $96 \%$ ee (Chiralcel AS-H, $n$-hexane $/ i$-PrOH = 65/35, $1.1 \mathrm{~mL} / \mathrm{min}, 210 \mathrm{~nm}, t_{R}=11.29 \mathrm{~min}$ (major), $t_{R}=32.14 \mathrm{~min}($ minor) $)$.

\section{(士)-5-(4-(Hydroxymethyl)phenyl)-3H-spiro[benzofuran-2,2'-inden]-1'(3'H)-one}

Synthesized from 2-((5-(4-(hydroxymethyl)phenyl)-benzofuran-2-yl)methyl)benzaldehyde (11) (17.1 mg, $0.05 \mathrm{mmol})$.

$\mathbf{R}_{f}$ and ${ }^{1} \mathbf{H}$ NMR data were in accordance with the one obtained with catalyst $3 \mathbf{a}$.

HPLC: $r a c$ (Chiralcel AS-H, $n$-hexane $/ i$-PrOH $=65 / 35,1.1 \mathrm{~mL} / \mathrm{min}, 210 \mathrm{~nm}, t_{R}=11.39 \mathrm{~min}$, $\left.t_{R}=32.08 \mathrm{~min}\right)$.

\section{(R)-5-(4-(Trifluoromethoxy)phenyl)-3H-spiro[benzofuran-2,2'-inden]-1'(3'H)-one (2m)}

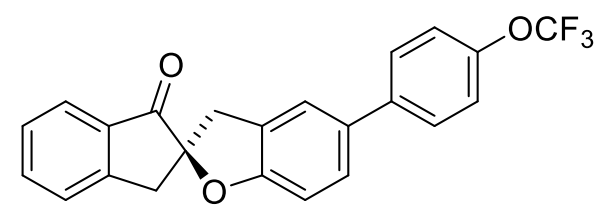

$2 m$

Synthesized according to the general procedure from 2-((5-4-(trifluoromethoxyl)phenyl)benzofuran-2-yl)methyl)benzaldehyde (1m) (118.9 $\mathrm{mg}, 0.30 \mathrm{mmol})$. Isolated as grey solid (107.4 mg, $0.27 \mathrm{mmol}, 90 \%)$.

$\mathbf{R}_{f}(n$-Pentane/EtOAc $=85 / 15): 0.43$;

$[\alpha]_{D^{20}}=-130.6\left(c=1.00, \mathrm{CHCl}_{3}\right)$;

${ }^{1} \mathbf{H}$ NMR $\left(600 \mathrm{MHz}, \mathrm{CDCl}_{3}\right): \delta / \mathrm{ppm}=7.88(\mathrm{~d}, J=7.7 \mathrm{~Hz}, 1 \mathrm{H}), 7.70(\mathrm{td}, J=7.6,0.9 \mathrm{~Hz}, 1 \mathrm{H})$, $7.57-7.52(\mathrm{~m}, 2 \mathrm{H}), 7.52-7.45(\mathrm{~m}, 2 \mathrm{H}), 7.40(\mathrm{~d}, J=1.8 \mathrm{~Hz}, 1 \mathrm{H}), 7.37(\mathrm{dd}, J=8.3,2.0 \mathrm{~Hz}, 1 \mathrm{H})$, $7.27(\mathrm{~d}, J=1.6 \mathrm{~Hz}, 2 \mathrm{H}), 6.90(\mathrm{~d}, J=8.3 \mathrm{~Hz}, 1 \mathrm{H}), 3.70(\mathrm{~d}, J=15.7 \mathrm{~Hz}, 1 \mathrm{H}), 3.64$ (d, $J=17.2 \mathrm{~Hz}$, 
1H), $3.44(\mathrm{~d}, J=17.3 \mathrm{~Hz}, 1 \mathrm{H}), 3.30(\mathrm{~d}, J=15.7 \mathrm{~Hz}, 1 \mathrm{H})$;

${ }^{13} \mathrm{C}$ NMR $\left(151 \mathrm{MHz}, \mathrm{CDCl}_{3}\right): \delta / \mathrm{ppm}=202.0,159.1,150.8,148.3,140.2,136.3,134.2,133.6$, 128.5, 128.2, 127.7, 126.8, 126.7, 125.4, 123.9, 121.3, 120.7 (q, $J=256 \mathrm{~Hz}$ ), 110.0, 90.3, 42.3, 39.0 ;

${ }^{19}$ F NMR (564 MHz, $\left.\mathrm{CDCl}_{3}\right): \delta / \mathrm{ppm}=-57.85(\mathrm{~s}) ;$

ESI-MS: calculated $\left[\mathrm{C}_{23} \mathrm{H}_{15} \mathrm{~F}_{3} \mathrm{O}_{3} \mathrm{Na}\right]^{+}$: 419.0866, found: 419.0868;

ATR-FTIR $\left(\mathrm{cm}^{-1}\right): 1721,1605,1481,1258,1242,1207,1153,1115,1072,1015,945,934,899$, 849, 806, 737, 694, 671, 625;

HPLC: $93 \%$ ee (Chiralcel AS-H, $n$-hexane $/ i$-PrOH = 90/10, $1.0 \mathrm{~mL} / \mathrm{min}, 210 \mathrm{~nm}, t_{R}=13.23 \mathrm{~min}$ (major), $t_{R}=26.44 \min ($ minor $)$ ).

\section{( \pm )-5-(4-(Trifluoromethoxy)phenyl)-3H-spiro[benzofuran-2,2'-inden]-1'(3'H)-one}

Synthesized from 2-((5-(4-(trifluoromethoxyl)phenyl)benzofuran-2-yl)methyl)benzaldehyde (1m) (19.8 mg, $0.05 \mathrm{mmol})$.

$\mathbf{R}_{f}$ and ${ }^{1} \mathbf{H}$ NMR data were in accordance with the one obtained with catalyst 3a.

HPLC: $r a c$ (Chiralcel AS-H, $n$-hexane $/ i-\mathrm{PrOH}=90 / 10,1.0 \mathrm{~mL} / \mathrm{min}, 210 \mathrm{~nm}, t_{R}=13.59 \mathrm{~min}$, $\left.t_{R}=27.24 \mathrm{~min}\right)$.

\section{(R)-5-(4-Chlorophenyl)-3H-spiro[benzofuran-2,2'-inden]-1' (3' H)-one (2n)}

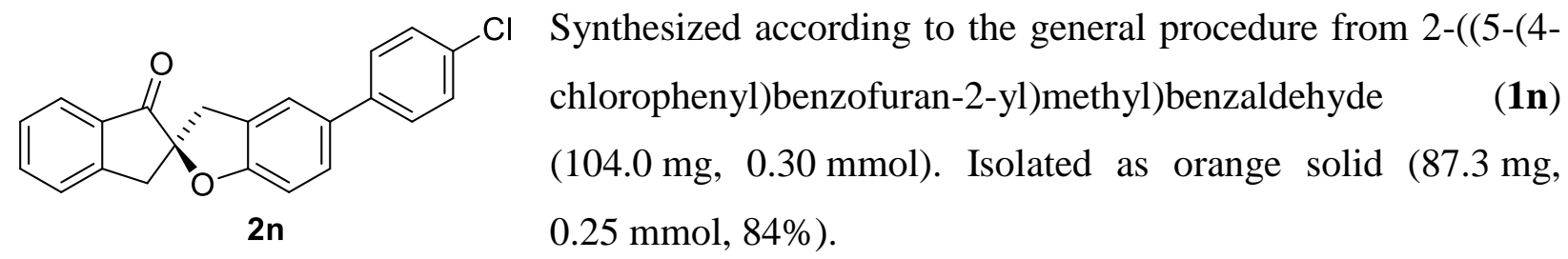

$\mathbf{R}_{f}\left(n-\right.$ Pentane $\left./ \mathrm{CH}_{2} \mathrm{Cl}_{2}=50 / 50\right): 0.88$;

$[\alpha]_{D}^{20}=-140.4\left(c=0.99, \mathrm{CHCl}_{3}\right)$;

${ }^{1} \mathbf{H}$ NMR $\left(300 \mathrm{MHz}, \mathrm{CDCl}_{3}\right): \delta / \mathrm{ppm}=7.87(\mathrm{~d}, J=7.6 \mathrm{~Hz}, 1 \mathrm{H}), 7.69(\mathrm{td}, J=7.5,1.3 \mathrm{~Hz}, 1 \mathrm{H})$, $7.53-7.42(\mathrm{~m}, 4 \mathrm{H}), 7.41-7.30(\mathrm{~m}, 4 \mathrm{H}), 6.89(\mathrm{~d}, J=8.2 \mathrm{~Hz}, 1 \mathrm{H}), 3.74-3.56(\mathrm{~m}, 2 \mathrm{H}), 3.42(\mathrm{~d}$, $J=17.3 \mathrm{~Hz}, 1 \mathrm{H}), 3.28(\mathrm{~d}, J=15.8 \mathrm{~Hz}, 1 \mathrm{H})$; 
${ }^{13} \mathrm{C}$ NMR (75 MHz, $\left.\mathrm{CDCl}_{3}\right): \delta / \mathrm{ppm}=202.0,159.0,150.8,139.8,136.3,134.2,133.7,132.8$, 129.0, 128.4, 128.2 127.5, 126.8, 126.7, 125.4, 123.7, 110.0, 90.2, 42.3, 39.0;

ESI-MS: calculated $\left[\mathrm{C}_{22} \mathrm{H}_{15} \mathrm{ClO}_{2} \mathrm{Na}\right]^{+}:$369.0653, found: 369.0653 ;

ATR-FTIR $\left(\mathrm{cm}^{-1}\right): 1717,1605,1478,1420,1308,1296,1242,1211,1188,1153,1115,1011$, 945, 934, 895, 833, 810, 737, 698, 621;

HPLC: $95 \%$ ee (Chiralcel AS-H, $n$-hexane $/ i$-PrOH = 70/30, $1.0 \mathrm{~mL} / \mathrm{min}, 230 \mathrm{~nm}, t_{R}=8.52 \mathrm{~min}$ (major), $t_{R}=15.95 \min ($ minor $)$ ).

( \pm )-5-(4-Chlorophenyl)-3H-spiro[benzofuran-2,2'-inden]-1'(3'H)-one

Synthesized from 2-((5-(4-chlorophenyl)benzofuran-2-yl)methyl)benzaldehyde (1n) (17.3 mg, $0.05 \mathrm{mmol})$.

$\mathbf{R}_{\boldsymbol{f}}$ and ${ }^{1} \mathbf{H}$ NMR data were in accordance with the one obtained with catalyst 3a.

HPLC: $r a c$ (Chiralcel AS-H, $n$-hexane $/ i-\mathrm{PrOH}=70 / 30,1.0 \mathrm{~mL} / \mathrm{min}, 230 \mathrm{~nm}, t_{R}=8.52 \mathrm{~min}$, $\left.t_{R}=15.95 \mathrm{~min}\right)$.

\section{(R)-5-(4-(Trifluoromethyl)phenyl)-3H-spiro[benzofuran-2,2'-inden]-1'(3'H)-one (2o)}

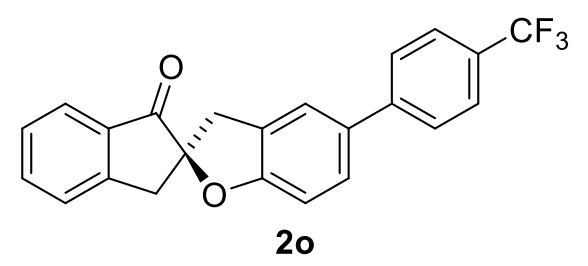

Synthesized according to the general procedure from 2-((5-)(4-

(trifluoromethyl)phenyl)benzofuran-2-yl)methyl)benzaldehyde (1o) $(114.1 \mathrm{mg}, 0.30 \mathrm{mmol})$. Isolated as green solid (94 mg, $0.25 \mathrm{mmol}, 83 \%$ ).

$\mathbf{R}_{f}(n$-Pentane/EtOAc $=85 / 15): 0.40 ;$

$[\alpha]_{D}{ }^{\mathbf{2 0}}=-138.1\left(c=1.01, \mathrm{CHCl}_{3}\right)$;

${ }^{1} \mathbf{H}$ NMR $\left(600 \mathrm{MHz}, \mathrm{CDCl}_{3}\right): \delta / \mathrm{ppm}=7.87(\mathrm{~d}, J=7.7 \mathrm{~Hz}, 1 \mathrm{H}), 7.72-7.61(\mathrm{~m}, 5 \mathrm{H}), 7.51-7.43$ $(\mathrm{m}, 3 \mathrm{H}), 7.43-7.39(\mathrm{~m}, 1 \mathrm{H}), 6.91(\mathrm{~d}, J=8.3 \mathrm{~Hz}, 1 \mathrm{H}), 3.70(\mathrm{~d}, J=15.7 \mathrm{~Hz}, 1 \mathrm{H}), 3.64(\mathrm{~d}, J=$ $17.2 \mathrm{~Hz}, 1 \mathrm{H}), 3.43(\mathrm{~d}, J=17.2 \mathrm{~Hz}, 1 \mathrm{H}), 3.30(\mathrm{~d}, J=15.7 \mathrm{~Hz}, 1 \mathrm{H})$;

${ }^{13} \mathrm{C}$ NMR (151 MHz, $\left.\mathrm{CDCl}_{3}\right): \delta / \mathrm{ppm}=201.9,159.5,150.8,144.8,136.3,134.2,133.4,128.8(\mathrm{q}$, $J=33 \mathrm{~Hz}), 128.5,127.9,127.2,126.9,126.8,125.8$ (q, $\mathrm{J}=4 \mathrm{~Hz}), 125.4,124.5(\mathrm{q}, J=272 \mathrm{~Hz})$, 124.0, 110.2, 90.3, 42.3, 39.0; 
${ }^{19}$ F NMR (564 MHz, $\left.\mathrm{CDCl}_{3}\right): \delta / \mathrm{ppm}=-62.33(\mathrm{~s})$;

ESI-MS: calculated $\left[\mathrm{C}_{23} \mathrm{H}_{15} \mathrm{~F}_{3} \mathrm{O}_{2} \mathrm{Na}\right]^{+}:$403.0916, found: 403.0921;

ATR-FTIR $\left(\mathrm{cm}^{-1}\right): 1717,1605,1485,1323,1281,1246,1211,1161,1107,1069,1011,945$, 899, 845, 814, 741, 714, 698;

HPLC: 97\% ee (Chiralcel AS-H, $n$-hexane $/ i-\mathrm{PrOH}=90 / 10,1.0 \mathrm{~mL} / \mathrm{min}, 210 \mathrm{~nm}, t_{R}=14.47 \mathrm{~min}$ (major), $t_{R}=25.91 \mathrm{~min}($ minor $)$ ).

\section{( \pm )-5-(4-(Trifluoromethyl)phenyl)-3H-spiro[benzofuran-2,2'-inden]-1'(3'H)-one}

Synthesized from 2-((5-(4-(trifluoromethyl)phenyl)benzofuran-2-yl)methyl)benzaldehyde (1o) (19.0 mg, $0.05 \mathrm{mmol})$.

$\mathbf{R}_{f}$ and ${ }^{1} \mathbf{H}$ NMR data were in accordance with the one obtained with catalyst 3a.

HPLC: $r a c$ (Chiralcel AS-H, $n$-hexane $/ i-\mathrm{PrOH}=90 / 10,1.0 \mathrm{~mL} / \mathrm{min}, 210 \mathrm{~nm}, t_{R}=14.87 \mathrm{~min}$, $\left.t_{R}=26.88 \mathrm{~min}\right)$.

\section{(R)-5-(4-Acetylphenyl)-3H-spiro[benzofuran-2,2'-inden]-1'(3'H)-one (2p)}

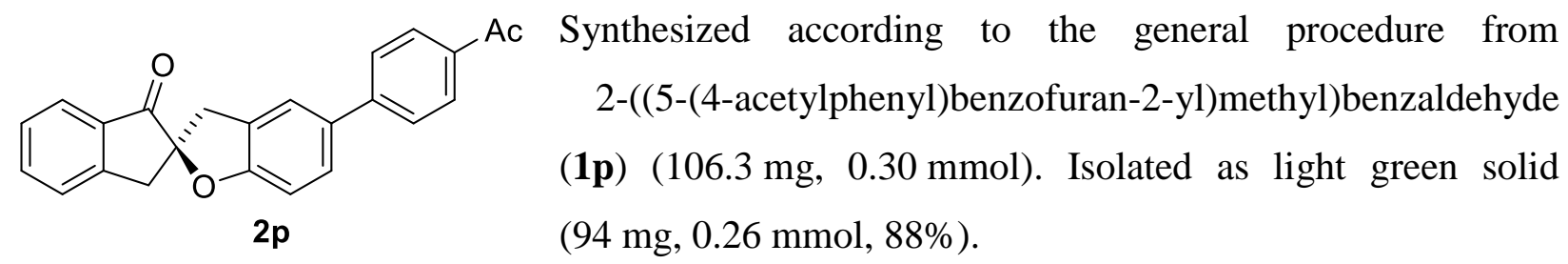

$\mathbf{R}_{f}(n$-Pentane/EtOAc $=80 / 20): 0.12 ;$

$[\alpha]_{D^{20}}=-161.8\left(c=1.00, \mathrm{CHCl}_{3}\right)$;

${ }^{1} \mathbf{H}$ NMR $\left(300 \mathrm{MHz}, \mathrm{CDCl}_{3}\right): \delta / \mathrm{ppm}=8.01(\mathrm{~d}, J=8.5 \mathrm{~Hz}, 2 \mathrm{H}), 7.86(\mathrm{~d}, J=7.7 \mathrm{~Hz}, 1 \mathrm{H}), 7.69$

$(\mathrm{td}, J=7.5,1.3 \mathrm{~Hz}, 1 \mathrm{H}), 7.65-7.58(\mathrm{~m}, 2 \mathrm{H}), 7.55-7.36(\mathrm{~m}, 4 \mathrm{H}), 6.91(\mathrm{~d}, J=8.3 \mathrm{~Hz}, 1 \mathrm{H})$,

$3.76-3.57(\mathrm{~m}, 2 \mathrm{H}), 3.43(\mathrm{~d}, J=17.3 \mathrm{~Hz}, 1 \mathrm{H}), 3.30(\mathrm{~d}, J=15.8 \mathrm{~Hz}, 1 \mathrm{H}), 2.63(\mathrm{~s}, 3 \mathrm{H})$;

${ }^{13} \mathrm{C}$ NMR $\left(75 \mathrm{MHz}, \mathrm{CDCl}_{3}\right): \delta / \mathrm{ppm}=201.9,197.9,159.6,150.8,145.9,136.3,135.4,134.1$, $133.4,129.1,128.5,127.9,126.9,126.8,125.4,124.0,110.1,90.3,42.3,38.9,26.8$; One aromatic $\mathrm{C}-\mathrm{H}^{13} \mathrm{C}$-Signal does overlap and could not be identified.

ESI-MS: calculated $\left[\mathrm{C}_{24} \mathrm{H}_{18} \mathrm{O}_{3} \mathrm{Na}\right]^{+}$: 377.1148 , found: 377.1152 ; 
ATR-FTIR $\left(\mathrm{cm}^{-1}\right): 1721,1670,1597,1481,1404,1362,1269,1246,1192,1157,1115,1072$, $1015,945,899,849,814,741,698,671$;

HPLC: $92 \%$ ee (Chiralcel AD-H, $n$-hexane $/ i$-PrOH = 70/30, $1.5 \mathrm{~mL} / \mathrm{min}, 210 \mathrm{~nm}$, $t_{R}=11.18 \mathrm{~min}$ (major), $t_{R}=13.04 \mathrm{~min}($ minor $)$ ).

( \pm )-5-(4-Acetylphenyl)-3H-spiro[benzofuran-2,2'-inden]-1'(3'H)-one

Synthesized from 2-((5-(4-acetylphenyl)benzofuran-2-yl)methyl)benzaldehyde (1p) (17.7 mg, $0.05 \mathrm{mmol})$.

$\mathbf{R}_{f}$ and ${ }^{1} \mathbf{H}$ NMR data were in accordance with the one obtained with catalyst 3a.

HPLC: $r a c$ (Chiralcel AD-H, $n$-hexane $/ i-\mathrm{PrOH}=70 / 30,1.5 \mathrm{~mL} / \mathrm{min}, 210 \mathrm{~nm}, t_{R}=11.18 \mathrm{~min}$, $\left.t_{R}=13.04 \mathrm{~min}\right)$.

\section{(R)-4-(1'-Oxo-1',3'-dihydro-3H-spiro[benzofuran-2,2'-inden]-5-yl)benzonitrile (2q)}

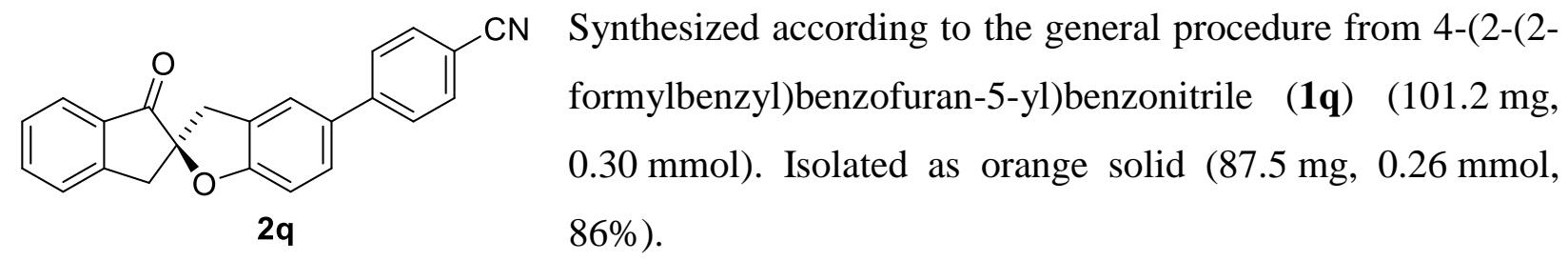

$\mathbf{R}_{f}(n$-Pentane/EtOAc $=90 / 10): 0.08 ;$

$[\alpha]_{D^{20}}=-166.0\left(c=1.01, \mathrm{CHCl}_{3}\right)$;

${ }^{1} \mathbf{H}$ NMR $\left(300 \mathrm{MHz}, \mathrm{CDCl}_{3}\right): \delta / \mathrm{ppm}=7.87(\mathrm{~d}, J=7.7 \mathrm{~Hz}, 1 \mathrm{H}), 7.77-7.57(\mathrm{~m}, 5 \mathrm{H}), 7.54-7.35$

(m, 4H), $6.92(\mathrm{~d}, J=8.3 \mathrm{~Hz}, 1 \mathrm{H}), 3.76-3.56(\mathrm{~m}, 2 \mathrm{H}), 3.43(\mathrm{~d}, J=17.4 \mathrm{~Hz}, 1 \mathrm{H}), 3.31(\mathrm{~d}$, $J=15.9 \mathrm{~Hz}, 1 \mathrm{H})$;

${ }^{13} \mathrm{C}$ NMR $\left(75 \mathrm{MHz}, \mathrm{CDCl}_{3}\right): \delta / \mathrm{ppm}=201.8,159.9,150.7,145.7,136.4,134.1,132.7,132.7$, 128.5, 128.0, 127.4, 127.1, 126.8, 125.4, 124.0, 119.3, 110.3, 110.2, 90.4, 42.2, 38.8;

ESI-MS: calculated $\left[\mathrm{C}_{23} \mathrm{H}_{15} \mathrm{NO}_{2} \mathrm{Na}\right]^{+}:$360.0995, found: 360.0999 ;

ATR-FTIR $\left(\mathrm{cm}^{-1}\right):$ 2226, 1717, 1597, 1474, 1466, 1416, 1308, 1292, 1246, 1207, 1150, 1115, 1080, 101, 953, 891, 841, 810, 748, 702, 613; 
HPLC: 85\% ee (Chiralcel AD-H, $n$-hexane/ $i$-PrOH = 70/30, 1.5 mL/min, 254 nm, $t_{R}=11.95 \min$ (major), $t_{R}=13.65 \min ($ minor $)$ ).

\section{( \pm )-4-(1'-Oxo-1',3'-dihydro-3H-spiro[benzofuran-2,2'-inden]-5-yl)benzonitrile}

Synthesized from 4-(2-(2-formylbenzyl)benzofuran-5-yl)benzonitrile (1q) (16.9 mg, 0.05 mmol).

$\mathbf{R}_{f}$ and ${ }^{1} \mathbf{H}$ NMR data were in accordance with the one obtained with catalyst 3a.

HPLC: $r a c$ (Chiralcel AD-H, $n$-hexane $/ i-\mathrm{PrOH}=70 / 30,1.5 \mathrm{~mL} / \mathrm{min}, 254 \mathrm{~nm}, t_{R}=11.97 \mathrm{~min}$, $\left.t_{R}=13.67 \mathrm{~min}\right)$. 


\subsection{Enantioselective hydroacylation of benzothiophenes}

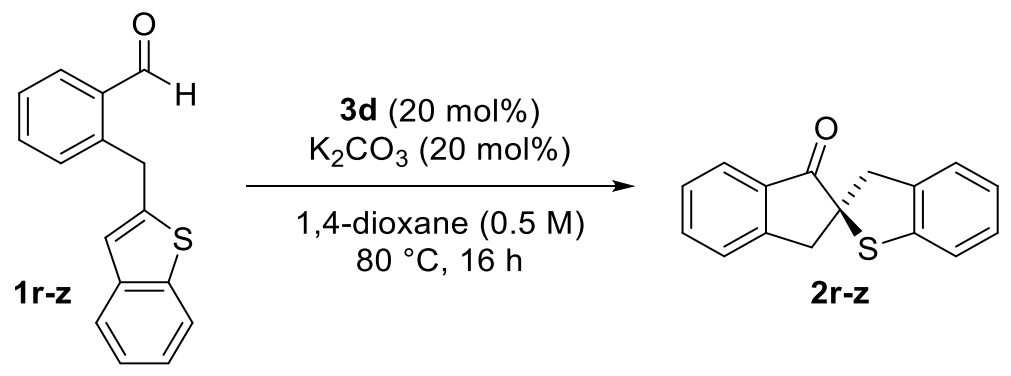

To a flame-dried screw-capped test tube equipped with a magnetic stir bar was added $\mathrm{K}_{2} \mathrm{CO}_{3}$ (8.4 mg, $0.06 \mathrm{mmol}, 20 \mathrm{~mol} \%$ ), NHC precursor 3d (21.1 mg, $0.06 \mathrm{mmol}, 20 \mathrm{~mol} \%$ ) and aldehyde (0.30 mmol, 1.0 equiv.). Dry 1,4-dioxane $(0.60 \mathrm{~mL})$ was added and the resulting mixture was then stirred in a pre-heated oil bath at $80{ }^{\circ} \mathrm{C}$ for $16 \mathrm{~h}$. The reaction was cooled to ambient temperature, pre-absorbed on silica gel and purified by column chromatography on silica gel.

\section{(R)-3H-Spiro[benzo[b]thiophene-2,2'-inden]-1'(3'H)-one (2r)}

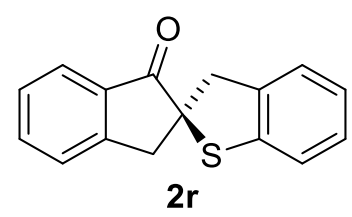

Synthesized according to the general procedure 2-(benzo[b]thiophen-2ylmethyl)benzaldehyde (1r) $(75.6 \mathrm{mg}, 0.30 \mathrm{mmol})$. Isolated as colorless solid (45 mg, $0.18 \mathrm{mmol}, 59 \%$ ).

$\mathbf{R}_{f}(n$-Pentane/EtOAc $=9 / 1): 0.37 ;$

$[\alpha]_{D}^{20}=-294.2\left(c=1.02, \mathrm{CHCl}_{3}\right)$;

${ }^{1} \mathbf{H}$ NMR $\left(300 \mathrm{MHz}, \mathrm{CDCl}_{3}\right): \delta / \mathrm{ppm}=7.85(\mathrm{~d}, J=7.9 \mathrm{~Hz}, 1 \mathrm{H}), 7.65(\mathrm{td}, J=7.5,1.2 \mathrm{~Hz}, 1 \mathrm{H})$, $7.46-7.42(\mathrm{~m}, 2 \mathrm{H}), 7.20-7.16(\mathrm{~m}, 3 \mathrm{H}), 7.11-7.06(\mathrm{~m}, 1 \mathrm{H}), 3.82(\mathrm{~d}, J=15.6 \mathrm{~Hz}, 1 \mathrm{H}), 3.68(\mathrm{~d}$, $J=17.5 \mathrm{~Hz}, 1 \mathrm{H}), 3.57(\mathrm{~d}, J=17.5 \mathrm{~Hz}, 1 \mathrm{H}), 3.34(\mathrm{~d}, J=15.7 \mathrm{~Hz}, 1 \mathrm{H})$;

${ }^{13}$ C NMR (100 MHz, $\left.\mathrm{CDCl}_{3}\right): \delta / \mathrm{ppm}=202.9,150.9,139.7,138.2,135.7,134.8,128.3,128.0$, $126.5,125.3,125.0,124.7,122.1,64.8,45.6,44.7$;

ESI-MS: calculated $\left[\mathrm{C}_{16} \mathrm{H}_{12} \mathrm{OSNa}\right]^{+}: 275.0501$, found: 275.0507;

ATR-FTIR $\left(\mathrm{cm}^{-1}\right): 3053,2921,2851,2361,2343,1710,1680,1600,1463,1446,1427,1329$, 1278, 1208, 1188, 1150, 1129, 1057, 1026, 992, 946, 924, 909, 877, 799, 749, 733, 697, 667, 630, 591;

HPLC: $78 \%$ ee (Chiralcel AS-H, $n$-hexane $/ i-\mathrm{PrOH}=95 / 5,1.0 \mathrm{~mL} / \mathrm{min}, 210 \mathrm{~nm}, t_{R}=10.23 \mathrm{~min}$ (major), $t_{R}=12.10 \min ($ minor)). 


\section{( \pm )-3H-Spiro[benzo[b]thiophene-2,2'-inden]-1'(3'H)-one}

Synthesized from 2-(benzo[b]thiophen-2-ylmethyl)benzaldehyde (1r) (25.2 mg, $0.10 \mathrm{mmol})$.

$\mathbf{R}_{f}$ and ${ }^{1} \mathbf{H}$ NMR data were in accordance with the one obtained with catalyst $\mathbf{3 a}$.

HPLC: $r a c$ (Chiralcel AS-H, $n-H e x a n e / i-\mathrm{PrOH}=95 / 5,1.0 \mathrm{~mL} / \mathrm{min}, 210 \mathrm{~nm}, t_{R}=10.07 \mathrm{~min}$, $\left.t_{R}=11.91 \mathrm{~min}\right)$.

\section{(R)-5-Bromo-3H-spiro[benzo[b]thiophene-2,2'-inden]-1'(3'H)-one (2s)}

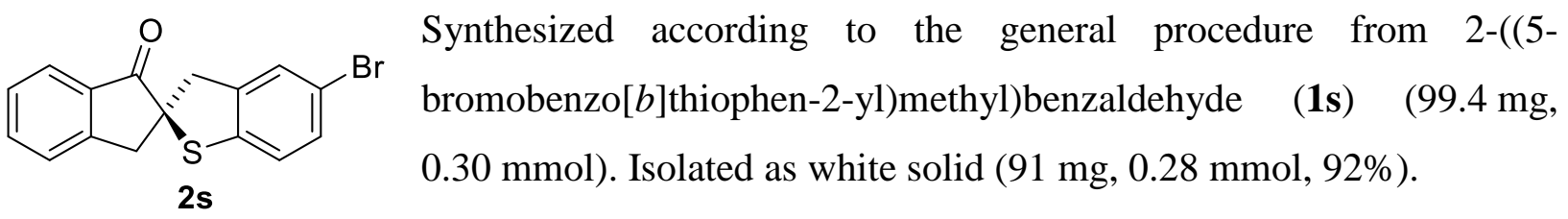

$\mathbf{R}_{f}(n$-Pentane/EtOAc $=90 / 10): 0.42 ;$

$[\alpha]_{D^{20}}=-249.0\left(c=1.08, \mathrm{CHCl}_{3}\right)$;

${ }^{1} \mathbf{H}$ NMR $\left(400 \mathrm{MHz}, \mathrm{CDCl}_{3}\right): \delta / \mathrm{ppm}=7.87-7.80(\mathrm{~m}, 1 \mathrm{H}), 7.65(\mathrm{td}, J=7.5,1.2 \mathrm{~Hz}, 1 \mathrm{H}), 7.48-$ $7.40(\mathrm{~m}, 2 \mathrm{H}), 7.33-7.25(\mathrm{~m}, 2 \mathrm{H}), 7.02(\mathrm{~d}, J=8.2 \mathrm{~Hz}, 1 \mathrm{H}), 3.77(\mathrm{~d}, J=15.8 \mathrm{~Hz}, 1 \mathrm{H}), 3.67$ (d, $J=17.6 \mathrm{~Hz}, 1 \mathrm{H}), 3.55(\mathrm{~d}, J=17.6 \mathrm{~Hz}, 1 \mathrm{H}), 3.33(\mathrm{~d}, J=15.9 \mathrm{~Hz}, 1 \mathrm{H})$;

${ }^{13} \mathrm{C}$ NMR (101 MHz, $\left.\mathrm{CDCl}_{3}\right): \delta / \mathrm{ppm}=202.3,150.7,140.6,139.1,135.8,134.5,130.8,128.4$, $127.8,126.5,125.4,123.3,118.4,65.0,45.0,44.1$;

ESI-MS: calculated $\left[\mathrm{C}_{16} \mathrm{H}_{11} \mathrm{BrOSNa}\right]^{+}:$352.9606, found: 352.9634;

ATR-FTIR (cm $\left.{ }^{-1}\right): 1713,1609,1458,1424,1400,1331,1288,1207,1188,1138,1069,991$, $945,934,910,876,856,814,752,733,675,633,578,548$;

HPLC: $73 \%$ ee (Chiralcel AS-H, $n$-hexane $/ i$-PrOH = 70/30, $1.0 \mathrm{~mL} / \mathrm{min}, 254 \mathrm{~nm}, t_{R}=7.66 \mathrm{~min}$ (major), $t_{R}=11.04 \min ($ minor $)$ ).

\section{( \pm -5-Bromo-3H-spiro[benzo[b]thiophene-2,2'-inden]-1'(3'H)-one}

Synthesized from 2-((5-bromobenzo[b]thiophen-2-yl)methyl)benzaldehyde (1s) (16.6 mg, $0.05 \mathrm{mmol})$. 
$\mathbf{R}_{f}$ and ${ }^{1} \mathbf{H}$ NMR data were in accordance with the one obtained with catalyst 3a.

HPLC: $r a c$ (Chiralcel AS-H, $n$-hexane $/ i-\mathrm{PrOH}=70 / 30,1.0 \mathrm{~mL} / \mathrm{min}, 254 \mathrm{~nm}, t_{R}=7.51 \mathrm{~min}$, $\left.t_{R}=10.65 \mathrm{~min}\right)$.

\section{3' $H$-Spiro[indene-2,2'-thieno[3,2-c]pyridin]-1(3H)-one (2t)}

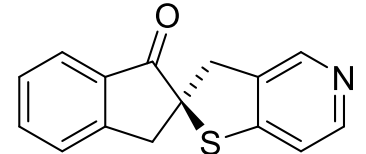

$2 t$

Synthesized according to the general procedure from 2-(thieno[3,2-c]pyridin-2-ylmethyl)benzaldehyde (1t) $(76.0 \mathrm{mg}, 0.30 \mathrm{mmol})$. Isolated as beige solid (71 mg, $0.28 \mathrm{mmol}, 94 \%)$.

$\mathbf{R}_{f}(n$-Pentane/EtOAc $=50 / 50): 0.28 ;$

$[\alpha]_{D^{20}}=-143.1\left(c=1.02, \mathrm{CHCl}_{3}\right)$;

${ }^{1} \mathbf{H}$ NMR $\left(400 \mathrm{MHz}, \mathrm{CDCl}_{3}\right): \delta / \mathrm{ppm}=8.37(\mathrm{~s}, 1 \mathrm{H}), 8.32(\mathrm{~d}, J=4.9 \mathrm{~Hz}, 1 \mathrm{H}), 7.83(\mathrm{dd}, J=7.9$, $1.1 \mathrm{~Hz}, 1 \mathrm{H}), 7.66(\mathrm{td}, J=7.5,1.3 \mathrm{~Hz}, 1 \mathrm{H}), 7.47-7.40(\mathrm{~m}, 2 \mathrm{H}), 7.13(\mathrm{dq}, J=4.9,1.0 \mathrm{~Hz}, 1 \mathrm{H})$, $3.77(\mathrm{dd}, J=16.5,1.1 \mathrm{~Hz}, 2 \mathrm{H}), 3.68(\mathrm{~d}, J=17.6 \mathrm{~Hz}, 1 \mathrm{H}), 3.55(\mathrm{~d}, J=17.6 \mathrm{~Hz}, 1 \mathrm{H}), 3.36(\mathrm{dd}$, $J=16.6,1.0 \mathrm{~Hz}, 1 \mathrm{H})$;

${ }^{13} \mathrm{C}$ NMR (101 MHz, $\left.\mathrm{CDCl}_{3}\right): \delta / \mathrm{ppm}=202.1,150.5,147.7,146.2,142.6,137.7,135.9,134.3$, $128.5,126.5,125.4,119.8,64.4,44.7,43.8$;

ESI-MS: calculated $\left[\mathrm{C}_{15} \mathrm{H}_{11} \mathrm{NOSNa}\right]^{+}:$276.0454, found: 276.0461;

ATR-FTIR $\left(\mathrm{cm}^{-1}\right): 1709,1601,1466,1408,1331,1281,1258,1184,1099,1065,1030,999$, 968, 930, 914, 802, 756, 737, 710, 671, 629, 590, 541;

HPLC: $38 \%$ ee (Chiralcel AS-H, $n$-hexane $/ i$-PrOH = 70/30, $1.0 \mathrm{~mL} / \mathrm{min}, 254 \mathrm{~nm}, t_{R}=18.95 \mathrm{~min}$ (major), $t_{R}=36.05 \min ($ minor $)$ ).

\section{( \pm )-3' $H$-Spiro[indene-2,2'-thieno[3,2-c $]$ pyridin]-1(3H)-one}

Synthesized from 2-(thieno[3,2-c]pyridin-2-ylmethyl)benzaldehyde (1t) (12.7 mg, $0.05 \mathrm{mmol})$.

$\mathbf{R}_{\boldsymbol{f}}$ and ${ }^{1} \mathbf{H}$ NMR data were in accordance with the one obtained with catalyst $3 \mathbf{a}$.

HPLC: $r a c$ (Chiralcel AS-H, $n$-hexane $/ i$-PrOH = 70/30, $1.0 \mathrm{~mL} / \mathrm{min}, 254 \mathrm{~nm}, t_{R}=18.72 \mathrm{~min}$, $\left.t_{R}=33.68 \mathrm{~min}\right)$. 
(R)-5-(4-Methoxyphenyl)-3H-spiro[benzo[b]thiophene-2,2'-inden]-1'(3'H)-one (2u)

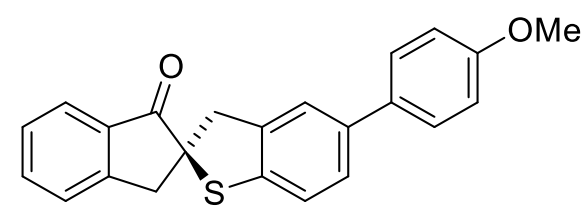

$2 \mathrm{u}$

Synthesized according to the general procedure from 2-5-(4-methoxyphenyl)benzo[ $b]$ thiophen-2-yl)methyl)benzaldehyde (1u) $(107.5 \mathrm{mg}, 0.30 \mathrm{mmol})$. Isolated as yellow solid (66 mg, $0.18 \mathrm{mmol}, 61 \%)$.

$\mathbf{R}_{f}(n$-Pentane/EtOAc $=90 / 10): 0.27$;

$[\alpha]_{D^{20}}=-502.2\left(c=1.01, \mathrm{CHCl}_{3}\right)$;

${ }^{1} \mathbf{H}$ NMR $\left(400 \mathrm{MHz}, \mathrm{CDCl}_{3}\right): \delta / \mathrm{ppm}=7.89-7.83(\mathrm{~m}, 1 \mathrm{H}), 7.66(\mathrm{td}, J=7.4,1.2 \mathrm{~Hz}, 1 \mathrm{H}), 7.54-$ $7.41(\mathrm{~m}, 4 \mathrm{H}), 7.39-7.34(\mathrm{~m}, 2 \mathrm{H}), 7.23-7.17(\mathrm{~m}, 1 \mathrm{H}), 7.00-6.93(\mathrm{~m}, 2 \mathrm{H}), 3.91-3.81(\mathrm{~m}$,

4H), $3.71(\mathrm{~d}, J=17.6 \mathrm{~Hz}, 1 \mathrm{H}), 3.60(\mathrm{~d}, J=17.6 \mathrm{~Hz}, 1 \mathrm{H}), 3.39(\mathrm{~d}, J=15.7 \mathrm{~Hz}, 1 \mathrm{H})$;

${ }^{13} \mathrm{C}$ NMR $\left(101 \mathrm{MHz}, \mathrm{CDCl}_{3}\right): \delta / \mathrm{ppm}=202.8,159.2,150.9,139.0,138.2,135.7,134.8,133.5$, 128.3, 128.0, 126.6, 126.5, 125.3, 123.1, 122.2, 114.4, 65.0, 55.5, 45.5, 44.6;

ESI-MS: calculated $\left[\mathrm{C}_{23} \mathrm{H}_{18} \mathrm{O}_{2} \mathrm{SNa}\right]^{+}: 381.0920$, found: 381.0925 ;

ATR-FTIR $\left(\mathrm{cm}^{-1}\right): 1713,1605,1516,1462,1327,1285,1246,1188,1153,1115,1061,1018$, $991,937,910,880,810,760,721,679,633,598$;

HPLC: $78 \%$ ee (Chiralcel AS-H, $n$-hexane $/ i-\mathrm{PrOH}=70 / 30,1.0 \mathrm{~mL} / \mathrm{min}, 210 \mathrm{~nm}, t_{R}=13.50 \mathrm{~min}$ (major), $t_{R}=25.50 \mathrm{~min}($ minor $)$ ).

\section{( \pm )-5-(4-Methoxyphenyl)-3H-spiro[benzo[b]thiophene-2,2'-inden]-1'(3'H)-one}

Synthesized from 2-5-(4-methoxyphenyl)benzo[b]thiophen-2-yl)methyl)benzaldehyde (1u) (16.9 mg, $0.05 \mathrm{mmol})$.

$\mathbf{R}_{f}$ and ${ }^{1} \mathbf{H}$ NMR data were in accordance with the one obtained with catalyst 3a.

HPLC: $r a c$ (Chiralcel AS-H, $n$-hexane $/ i$-PrOH = 70/30, $1.0 \mathrm{~mL} / \mathrm{min}, 210 \mathrm{~nm}, t_{R}=12.94 \mathrm{~min}$, $\left.t_{R}=23.89 \mathrm{~min}\right)$. 
(R)-5-(p-Tolyl)-3H-spiro[benzo[b]thiophene-2,2'-inden]-1'(3'H)-one (2v)

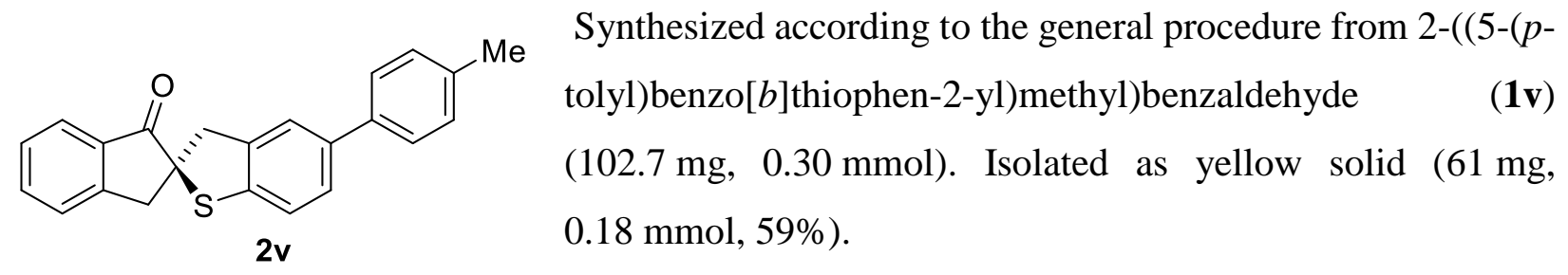

$\mathbf{R}_{f}(n$-Pentane/EtOAc $=90 / 10): 0.41 ;$

$[\alpha]_{D^{20}}=-558.9\left(c=0.81, \mathrm{CHCl}_{3}\right)$;

${ }^{1} \mathbf{H}$ NMR $\left(400 \mathrm{MHz}, \mathrm{CDCl}_{3}\right): \delta / \mathrm{ppm}=7.87(\mathrm{dd}, J=8.0,1.3 \mathrm{~Hz}, 1 \mathrm{H}), 7.66(\mathrm{td}, J=7.5,1.3 \mathrm{~Hz}$, 1H), $7.49-7.38(\mathrm{~m}, 6 \mathrm{H}), 7.26-7.20(\mathrm{~m}, 3 \mathrm{H}), 3.87$ (d, $J=15.7 \mathrm{~Hz}, 1 \mathrm{H}), 3.72$ (d, $J=17.5 \mathrm{~Hz}$,

$1 \mathrm{H}), 3.60$ (d, $J=17.6 \mathrm{~Hz}, 1 \mathrm{H}), 3.39$ (d, $J=15.7 \mathrm{~Hz}, 1 \mathrm{H}), 2.40$ (s, 3H);

${ }^{13} \mathrm{C}$ NMR $\left(101 \mathrm{MHz}, \mathrm{CDCl}_{3}\right): \delta / \mathrm{ppm}=202.8,150.9,139.0,138.6,138.4,138.0,137.0,135.7$, 134.8, 129.6, 128.3, 126.8, 126.8, 126.5, 125.3, 123.3, 122.2, 65.0, 45.4, 44.6, 21.2;

ESI-MS: calculated $\left[\mathrm{C}_{23} \mathrm{H}_{18} \mathrm{OSNa}\right]^{+}: 365.0971$, found: 365.0970 ;

ATR-FTIR $\left(\mathrm{cm}^{-1}\right): 1717,1605,1516,1462,1424,1323,1285,1207,1184,1150,1061,1026$, $991,949,937,910,845,806,752,733,679,633,617,598$;

HPLC: $81 \%$ ee (Chiralcel AS-H, $n$-hexane $/ i-\mathrm{PrOH}=70 / 30,1.0 \mathrm{~mL} / \mathrm{min}, 210 \mathrm{~nm}, t_{R}=7.70 \mathrm{~min}$ (major), $t_{R}=10.43 \min ($ minor $)$ ).

\section{( \pm )-5-(p-Tolyl)-3H-spiro[benzo[b]thiophene-2,2'-inden]-1'(3'H)-one}

Synthesized from 2-((5-(p-tolyl)benzo[b]thiophen-2-yl)methyl)benzaldehyde (1v) (17.1 mg, $0.05 \mathrm{mmol})$.

$\mathbf{R}_{f}$ and ${ }^{1} \mathbf{H}$ NMR data were in accordance with the one obtained with catalyst 3a.

HPLC: $r a c$ (Chiralcel AS-H, $n$-hexane $/ i-\mathrm{PrOH}=70 / 30,1.0 \mathrm{~mL} / \mathrm{min}, 210 \mathrm{~nm}, t_{R}=7.35 \mathrm{~min}$, $\left.t_{R}=9.61 \mathrm{~min}\right)$. 
(R)-5-Phenyl-3H-spiro[benzo[b]thiophene-2,2'-inden]-1'(3'H)-one (2w)

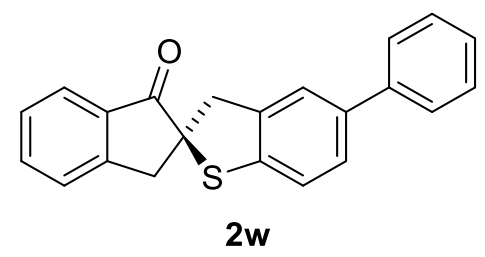

Synthesized according to the general procedure from 2-((5-phenylbenzo[b]thiophen-2-yl)methyl)benzaldehyde (1w) $\quad(98.5 \mathrm{mg}$, $0.30 \mathrm{mmol})$. Isolated as yellow solid (75 $\mathrm{mg}, 0.23 \mathrm{mmol}, 76 \%)$.

$\mathbf{R}_{f}($-Pentane/EtOAc $=90 / 10): 0.29 ;$

$[\alpha]_{D}^{20}=-282.1\left(c=0.98, \mathrm{CHCl}_{3}\right)$;

${ }^{1} \mathbf{H}$ NMR $\left(400 \mathrm{MHz}, \mathrm{CDCl}_{3}\right): \delta / \mathrm{ppm}=7.89-7.85(\mathrm{~m}, 1 \mathrm{H}), 7.66(\mathrm{td}, J=7.5,1.3 \mathrm{~Hz}, 1 \mathrm{H}), 7.61-$ $7.54(\mathrm{~m}, 2 \mathrm{H}), 7.50-7.38(\mathrm{~m}, 5 \mathrm{H}), 7.39-7.29(\mathrm{~m}, 1 \mathrm{H}), 7.27-7.20(\mathrm{~m}, 1 \mathrm{H}), 3.87$ (d, J= 15.7 $\mathrm{Hz}, 1 \mathrm{H}), 3.72$ (d, $J=17.6 \mathrm{~Hz}, 1 \mathrm{H}), 3.60$ (d, $J=17.6 \mathrm{~Hz}, 1 \mathrm{H}), 3.40$ (d, $J=15.7 \mathrm{~Hz}, 1 \mathrm{H})$; ${ }^{13} \mathrm{C}$ NMR (101 MHz, $\left.\mathrm{CDCl}_{3}\right): \delta / \mathrm{ppm}=202.8,150.9,140.9,139.0,139.0,138.5,135.7,134.8$, $128.9,128.3,127.2,127.0,127.0,127.0,126.5,125.3,123.5,122.3,65.0,45.4,44.6$;

ESI-MS: calculated $\left[\mathrm{C}_{22} \mathrm{H}_{16} \mathrm{OSNa}\right]^{+}: 351.0814$, found: 351.0823 ;

ATR-FTIR $\left(\mathrm{cm}^{-1}\right): 1709,1609,1593,1462,1427,1327,1288,1211,1188,1150,1065,1038$, 991, 949, 937, 910, 822, 799, 764, 733, 698, 660, 633, 602, 579;

HPLC: 77\% ee (Chiralcel AS-H, $n$-hexane $/ i-\mathrm{PrOH}=70 / 30,1.0 \mathrm{~mL} / \mathrm{min}, 210 \mathrm{~nm}, t_{R}=8.81 \mathrm{~min}$ (major), $t_{R}=13.02 \min ($ minor $)$ ).

\section{( \pm )-5-Phenyl-3H-spiro[benzo[b]thiophene-2,2'-inden]-1'(3'H)-one}

Synthesized from 2-((5-phenylbenzo[b]thiophen-2-yl)methyl)benzaldehyde (1w) (16.4 mg, $0.05 \mathrm{mmol})$.

$\mathbf{R}_{f}$ and ${ }^{1} \mathbf{H}$ NMR data were in accordance with the one obtained with catalyst 3a.

HPLC: $r a c$ (Chiralcel AS-H, $n$-hexane $/ i-\mathrm{PrOH}=70 / 30,1.0 \mathrm{~mL} / \mathrm{min}, 210 \mathrm{~nm}, t_{R}=8.45 \mathrm{~min}$, $\left.t_{R}=11.56 \mathrm{~min}\right)$. 
(R)-5-(4-(Trifluoromethoxy)phenyl)-3H-spiro[benzo[b]thiophene-2,2'-inden]-1'(3'H)-one $(2 x)$

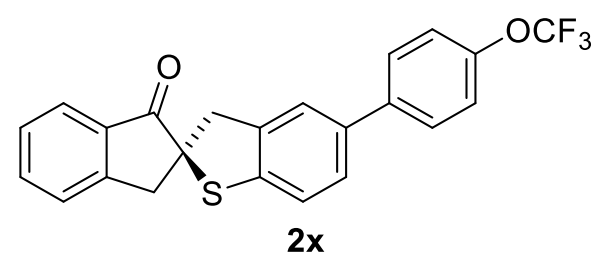

Synthesized according to the general procedure from 2-((5-

(4-(trifluoromethoxy)phenyl)benzo[b]thiophen-2-yl)methyl)benzaldehyde (1x) (123.7 $\mathrm{mg}, 0.30 \mathrm{mmol})$. Isolated as yellow solid (74 mg, $0.17 \mathrm{mmol}, 58 \%$ ).

$\mathbf{R}_{f}(n$-Pentane/EtOAc $=90 / 10): 0.28 ;$

$[\alpha]_{D^{20}}=-281.5\left(c=1.05, \mathrm{CHCl}_{3}\right)$;

${ }^{1} \mathbf{H}$ NMR $\left(600 \mathrm{MHz}, \mathrm{CDCl}_{3}\right): \delta / \mathrm{ppm}=7.92-7.82(\mathrm{~m}, 1 \mathrm{H}), 7.67(\mathrm{td}, \mathrm{J}=7.5,1.3 \mathrm{~Hz}, 1 \mathrm{H}), 7.60$ $7.53(\mathrm{~m}, 2 \mathrm{H}), 7.50-7.41(\mathrm{~m}, 2 \mathrm{H}), 7.39-7.35(\mathrm{~m}, 2 \mathrm{H}), 7.30-7.21(\mathrm{~m}, 3 \mathrm{H}), 3.86(\mathrm{~d}, \mathrm{~J}=15.8$

$\mathrm{Hz}, 1 \mathrm{H}), 3.72$ (d, J = 17.6 Hz, 1H), 3.60 (d, J = 17.6 Hz, 1H), 3.42 (d, J = 15.8 Hz, 1H);

${ }^{13} \mathrm{C}$ NMR $\left(151 \mathrm{MHz}, \mathrm{CDCl}_{3}\right): \delta / \mathrm{ppm}=202.7,150.8,148.7(\mathrm{q}, J=2 \mathrm{~Hz}), 139.7,139.7,139.3$,

$137.1,135.8,134.7,128.37,128.3,126.9,126.5,125.4,123.4,122.4,121.4,120.7$ (q,

$J=257 \mathrm{~Hz}), 65.0,45.3,44.4$;

${ }^{19}$ F NMR $\left(564 \mathrm{MHz}, \mathrm{CDCl}_{3}\right): \delta / \mathrm{ppm}=-57.81(\mathrm{t}, J=1.0 \mathrm{~Hz})$;

ESI-MS: calculated $\left[\mathrm{C}_{23} \mathrm{H}_{15} \mathrm{~F}_{3} \mathrm{O}_{2} \mathrm{SNa}\right]^{+}$: 435.0637, found: 435.0643;

ATR-FTIR $\left(\mathrm{cm}^{-1}\right): 1709,1609,1516,1466,1427,1327,1265,1211,1146,1061,1015,941$, 910, 849, 802, 752, 733, 683, 656, 633;

HPLC: $83 \%$ ee (Chiralcel AS-H, $n$-hexane $/ i$-PrOH = 70/30, $1.0 \mathrm{~mL} / \mathrm{min}, 254 \mathrm{~nm}, t_{R}=6.98 \mathrm{~min}$ (major), $t_{R}=7.73 \mathrm{~min}$ (minor)).

( \pm )-5-(4-(Trifluoromethoxy)phenyl)-3H-spiro[benzo[b]thiophene-2,2'-inden]-1'(3'H)-one

Synthesized from 2-((5-(4-(trifluoromethoxy)phenyl)benzo[b]thiophen-2-yl)methyl)benzaldehyde (1x) (20.6 mg, $0.05 \mathrm{mmol})$.

$\mathbf{R}_{f}$ and ${ }^{1} \mathbf{H}$ NMR data were in accordance with the one obtained with catalyst 3a.

HPLC: $r a c$ (Chiralcel AS-H, $n$-hexane $/ i-\mathrm{PrOH}=70 / 30,1.0 \mathrm{~mL} / \mathrm{min}, 254 \mathrm{~nm}, t_{R}=6.76 \mathrm{~min}$, $\left.t_{R}=7.46 \mathrm{~min}\right)$. 


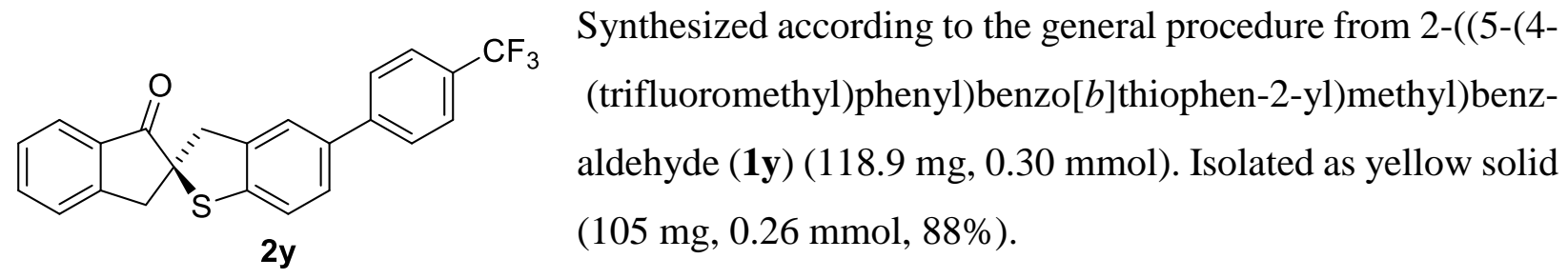

$\mathbf{R}_{f}(n$-Pentane/EtOAc $=90 / 10): 0.32 ;$

$[\alpha]_{D^{20}}=-236.4\left(c=0.54, \mathrm{CHCl}_{3}\right)$;

${ }^{1} \mathbf{H}$ NMR $\left(400 \mathrm{MHz}, \mathrm{CDCl}_{3}\right): \delta / \mathrm{ppm}=7.89-7.84(\mathrm{~m}, 1 \mathrm{H}), 7.72-7.63(\mathrm{~m}, 5 \mathrm{H}), 7.50-7.39(\mathrm{~m}$, 4H), $7.30-7.22(\mathrm{~m}, 1 \mathrm{H}), 3.87(\mathrm{~d}, J=15.8 \mathrm{~Hz}, 1 \mathrm{H}), 3.72(\mathrm{~d}, J=17.6 \mathrm{~Hz}, 1 \mathrm{H}), 3.60$ (d,

$J=17.6 \mathrm{~Hz}, 1 \mathrm{H}), 3.43(\mathrm{~d}, J=15.8 \mathrm{~Hz}, 1 \mathrm{H})$;

${ }^{13} \mathrm{C}$ NMR (101 MHz, $\left.\mathrm{CDCl}_{3}\right): \delta / \mathrm{ppm}=202.6,150.8,144.4,140.4,139.4,136.9,135.8,134.7$, $129.3(\mathrm{q}, J=32 \mathrm{~Hz}), 128.4,127.2,127.1,126.5,125.9$ (q, $J=4 \mathrm{~Hz}), 125.4,124.4$ (q,

$J=272 \mathrm{~Hz}), 123.5,122.5,64.9,45.3,44.3$;

${ }^{19}$ F NMR $\left(282 \mathrm{MHz}, \mathrm{CDCl}_{3}\right): \delta / \mathrm{ppm}=-62.33(\mathrm{~s})$;

ESI-MS: calculated $\left[\mathrm{C}_{23} \mathrm{H}_{15} \mathrm{~F}_{3} \mathrm{OFNa}\right]^{+}:$419.0688, found: 419.0680;

ATR-FTIR $\left(\mathrm{cm}^{-1}\right): 1709,1613,1562,1470,1420,1400,1323,1285,1211,1150,1111,1069$, $1015,941,910,891,849,822,752,737,671,714,633,602$;

HPLC: $70 \%$ ee (Chiralcel AS-H, $n$-hexane $/ i-\mathrm{PrOH}=70 / 30,1.0 \mathrm{~mL} / \mathrm{min}, 210 \mathrm{~nm}, t_{R}=7.42 \mathrm{~min}$ (major), $t_{R}=8.45 \mathrm{~min}$ (minor)).

\section{( \pm )-5-(4-(Trifluoromethyl)phenyl)-3H-spiro[benzo[b]thiophene-2,2'-inden]-1'(3'H)-one}

Synthesized from 2-((5-(4-(trifluoromethyl)phenyl)benzo[b]thiophen-2-yl)methyl)benzaldehyde (1y) (19.8 mg, $0.05 \mathrm{mmol})$.

$\mathbf{R}_{\boldsymbol{f}}$ and ${ }^{1} \mathbf{H}$ NMR data were in accordance with the one obtained with catalyst 3a.

HPLC: $r a c$ (Chiralcel AS-H, $n$-hexane $/ i-\mathrm{PrOH}=70 / 30,1.0 \mathrm{~mL} / \mathrm{min}, 230 \mathrm{~nm}, t_{R}=7.35 \mathrm{~min}$, $\left.t_{R}=8.30 \mathrm{~min}\right)$. 


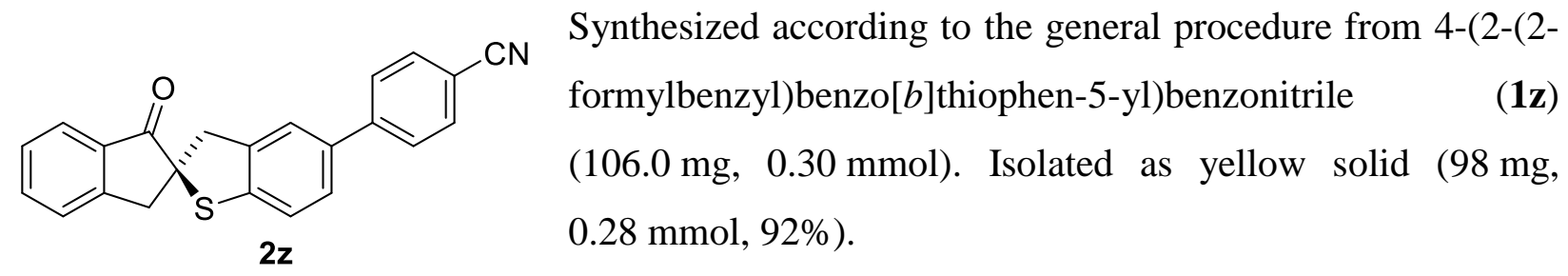

$\mathbf{R}_{f}(n-$ Pentane/EtOAc $=90 / 10): 0.16 ;$

$[\alpha]_{D}{ }^{20}=-185.2\left(c=0.81, \mathrm{CHCl}_{3}\right)$;

${ }^{1} \mathbf{H}$ NMR $\left(400 \mathrm{MHz}, \mathrm{CDCl}_{3}\right): \delta / \mathrm{ppm}=7.88-7.84(\mathrm{~m}, 1 \mathrm{H}), 7.73-7.63(\mathrm{~m}, 5 \mathrm{H}), 7.53-7.37(\mathrm{~m}$, 4H), $7.29-7.23(\mathrm{~m}, 1 \mathrm{H}), 3.85(\mathrm{~d}, J=15.8 \mathrm{~Hz}, 1 \mathrm{H}), 3.71(\mathrm{~d}, J=17.6 \mathrm{~Hz}, 1 \mathrm{H}), 3.60(\mathrm{~d}$,

$J=17.6 \mathrm{~Hz}, 1 \mathrm{H}), 3.44(\mathrm{~d}, J=15.8 \mathrm{~Hz}, 1 \mathrm{H})$;

${ }^{13}$ C NMR (101 MHz, $\left.\mathrm{CDCl}_{3}\right): \delta / \mathrm{ppm}=202.5,150.7,145.3,141.2,139.6,136.3,135.8,134.6$, 132.7, 128.4, 127.5, 127.0, 126.5, 125.4, 123.4, 122.6, 119.1, 110.8, 64.9, 45.2, 44.1;

ESI-MS: calculated $\left[\mathrm{C}_{23} \mathrm{H}_{15} \mathrm{NOSNa}\right]^{+}: 376.0767$, found: 376.0775 ;

ATR-FTIR $\left(\mathrm{cm}^{-1}\right):$ 2222, 1709, 1601, 1462, 1427, 1400, 1331, 1285, 1207, 1184, 1153, 1061, 1022, 995, 953, 941, 910, 845, 814, 756, 733, 718, 679, 633, 602;

HPLC: $84 \%$ ee (Chiralcel AS-H, $n$-hexane $/ i-\mathrm{PrOH}=70 / 30,1.0 \mathrm{~mL} / \mathrm{min}, 230 \mathrm{~nm}, t_{R}=27.06 \mathrm{~min}$ (major), $t_{R}=31.08 \mathrm{~min}$ (minor)).

\section{( \pm )-4-(1'-Oxo-1',3'-dihydro-3H-spiro[benzo[b]thiophene-2,2'-inden]-5-yl)benzonitrile}

Synthesized from 4-(2-(2-formylbenzyl)benzo[b]thiophen-5-yl)benzonitrile (1z) (17.7 mg, $0.05 \mathrm{mmol})$.

$\mathbf{R}_{f}$ and ${ }^{1} \mathbf{H}$ NMR data were in accordance with the one obtained with catalyst $\mathbf{3 a}$.

HPLC: $r a c$ (Chiralcel AS-H, $n$-hexane $/ i$-PrOH $=70 / 30,1.0 \mathrm{~mL} / \mathrm{min}, 210 \mathrm{~nm}, t_{R}=26.53 \mathrm{~min}$, $\left.t_{R}=29.86 \mathrm{~min}\right)$. 


\section{$7 \quad$ X-Ray Diffraction Analysis}

X-Ray diffraction: Data sets were collected with a Bruker APEX CCD diffractometer. Programs used: data collection: APEX2 V2014.5-0 (Bruker AXS Inc., 2014); ${ }^{[S 19]}$ cell refinement: SAINT V8.34A (Bruker AXS Inc., 2013); ${ }^{\left[{ }^{[19]}\right.}$ data reduction: SAINT V8.34A (Bruker AXS Inc.,

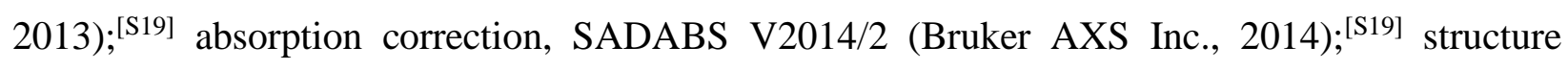
solution SHELXT-2014 (Sheldrick, 2014); ${ }^{[\text {S20] }}$ structure refinement SHELXL-2014 (Sheldrick, $2014)^{[\mathrm{S} 20]}$ and graphics, XP (Bruker AXS Inc., 2014) ${ }^{[\mathrm{S} 20]}$. $R$-values are given for observed reflections, and $w \mathrm{R}^{2}$ values are given for all reflections.

X-ray crystal structure analysis of 2e: A colorless prism-like specimen of $\mathrm{C}_{17} \mathrm{H}_{14} \mathrm{O}_{2}$, approximate dimensions $0.100 \mathrm{~mm} \times 0.140 \mathrm{~mm}$ x $0.200 \mathrm{~mm}$, was used for the X-ray crystallographic analysis. The X-ray intensity data were measured. A total of 1015 frames were collected. The total exposure time was 5.44 hours. The frames were integrated with the Bruker SAINT software package using a wide-frame algorithm. The integration of the data using an orthorhombic unit cell yielded a total of 13195 reflections to a maximum $\theta$ angle of $68.46^{\circ}(0.83$ $\AA$ resolution), of which 2284 were independent (average redundancy 5.777, completeness = $\left.99.3 \%, \mathrm{R}_{\text {int }}=4.38 \%, \mathrm{R}_{\text {sig }}=2.86 \%\right)$ and $2135(93.48 \%)$ were greater than $2 \sigma\left(\mathrm{F}^{2}\right)$. The final cell constants of $\underline{a}=8.6908(4) \AA, \underline{b}=10.3574(4) \AA, \underline{c}=13.9701(6) \AA$, volume $=1257.51(9) \AA^{3}$, are based upon the refinement of the XYZ-centroids of 4322 reflections above $20 \sigma(\mathrm{I})$ with $10.63^{\circ}<$ $2 \theta<136.9^{\circ}$. Data were corrected for absorption effects using the multi-scan method (SADABS). The ratio of minimum to maximum apparent transmission was 0.837 . The calculated minimum and maximum transmission coefficients (based on crystal size) are 0.8760 and 0.9350 . The structure was solved and refined using the Bruker SHELXTL Software Package, using the space group $P 2_{1} 2_{1} 2_{1}$, with $\mathrm{Z}=4$ for the formula unit, $\mathrm{C}_{17} \mathrm{H}_{14} \mathrm{O}_{2}$. The final anisotropic full-matrix leastsquares refinement on $\mathrm{F}^{2}$ with 173 variables converged at $\mathrm{R} 1=3.37 \%$, for the observed data and $\mathrm{wR} 2=8.21 \%$ for all data. The goodness-of-fit was 1.071 . The largest peak in the final difference electron density synthesis was $0.148 \mathrm{e}^{-} / \AA^{3}$ and the largest hole was $-0.167 \mathrm{e}^{-} / \AA^{3}$ with an RMS deviation of $0.039 \mathrm{e}^{-} / \AA^{3}$. On the basis of the final model, the calculated density was $1.322 \mathrm{~g} / \mathrm{cm}^{3}$ and $\mathrm{F}(000), 528 \mathrm{e}^{-}$. 


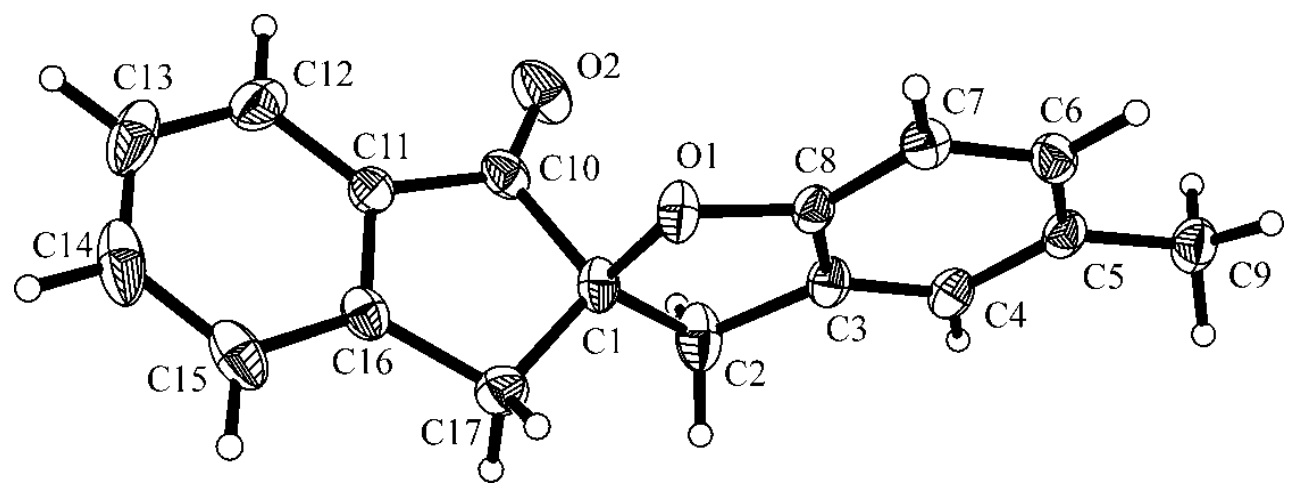

Figure S2. Crystal structure of compound 2e. (Thermals ellipsoids are shown with 50\% probability.) 


\section{3-Deuterium and 3-Methyl substituted Substrates}




\section{2-((Benzo[b]thiophen-2-yl-3- $d)$ methyl)benzaldehyde (7)}

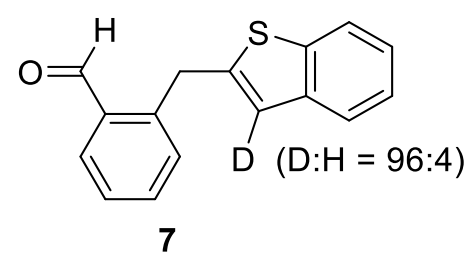

A solution of 3-deuteriobenzo[b]thiophene ${ }^{[\mathrm{S} 21]}(676 \mathrm{mg}, 5 \mathrm{mmol}$, 1.67 equiv.) in THF ( $15 \mathrm{~mL})$ was cooled to $-78{ }^{\circ} \mathrm{C}$. Then $n$-BuLi solution in hexanes (1.6 M, $3.44 \mathrm{~mL}, 5.5 \mathrm{mmol}, 1.83$ equiv.) was added dropwise to the reaction mixture. After $90 \mathrm{~min}$ at $-78^{\circ} \mathrm{C}$, triisopropyl borate $(1.38 \mathrm{~mL}, 6.0 \mathrm{mmol}, 2.0$ equiv.) was added dropwise. The reaction was stirred for further $30 \mathrm{~min}$ at $-78{ }^{\circ} \mathrm{C}$ and was allowed to warm up to room temperature for $2 \mathrm{~h}$. The solvent was removed under reduced pressure to give a yellow solid, which was used without further purification. 2-(Bromomethyl)benzaldehyde (S10) (597 mg, $3.0 \mathrm{mmol}, 1.0$ equiv.) was added together with tetrakis(triphenylphosphine)palladium(0) (69 mg, $0.060 \mathrm{mmol}, 0.05$ equiv.), $9.0 \mathrm{~mL}$ aqueous $\mathrm{Na}_{2} \mathrm{CO}_{3}$ solution $(1 \mathrm{M}), 10 \mathrm{~mL}$ toluene, $8.5 \mathrm{~mL}$ ethanol and $2.5 \mathrm{~mL} \mathrm{H}_{2} \mathrm{O}$. The flask was evacuated and backfilled with argon three times and stirred at $60{ }^{\circ} \mathrm{C}$ overnight. The reaction was quenched with $20 \mathrm{~mL} \mathrm{H}_{2} \mathrm{O}$ and extracted with $3 \times 20 \mathrm{~mL} \mathrm{CH}_{2} \mathrm{Cl}_{2}$, over $\mathrm{MgSO}_{4}$ and concentrated under reduced pressure. Purification by column chromatography on silica ( $n$-pentane/EtOAc $=$ 95/5) afforded 2-((benzo[b]thiophen-2-yl-3- $d$ )methyl)benzaldehyde (7) as a yellow solid (610 $\mathrm{mg}$, $2.41 \mathrm{mmol}, 80 \%)$.

$\mathbf{R}_{f}(n$-Pentane/EtOAc $=90 / 10): 0.41 ;$

${ }^{1} \mathrm{H}$ NMR $\left(600 \mathrm{MHz}, \mathrm{CD}_{2} \mathrm{Cl}_{2}\right): \delta / \mathrm{ppm}=10.25(\mathrm{~s}, 1 \mathrm{H}), 7.88(\mathrm{dd}, J=7.6,1.6 \mathrm{~Hz}, 1 \mathrm{H}), 7.72(\mathrm{dd}$, $J=7.9,1.2 \mathrm{~Hz}, 1 \mathrm{H}), 7.63(\mathrm{~d}, J=7.3 \mathrm{~Hz}, 1 \mathrm{H}), 7.57(\mathrm{dt}, J=7.5,1.5 \mathrm{~Hz}, 1 \mathrm{H}), 7.48(\mathrm{dt}, J=7.5$, $1.3 \mathrm{~Hz}, 1 \mathrm{H}), 7.41$ (d, $J=7.2 \mathrm{~Hz}, 1 \mathrm{H}), 7.31-7.22(\mathrm{~m}, 2 \mathrm{H}), 6.96$ (s, 0.04H), 4.69 (s, 2H);

ESI-MS: calculated: $\left[\mathrm{C}_{16} \mathrm{H}_{12} \mathrm{OSNa}\right]^{+}=275.0501$, found: 275.0506 , relative integration: $5.2 \%$; calculated: $\left[\mathrm{C}_{16} \mathrm{H}_{12} \mathrm{DOSNa}\right]^{+}=276.0564$, found: 276.0566 , relative integration: $100 \%$; 

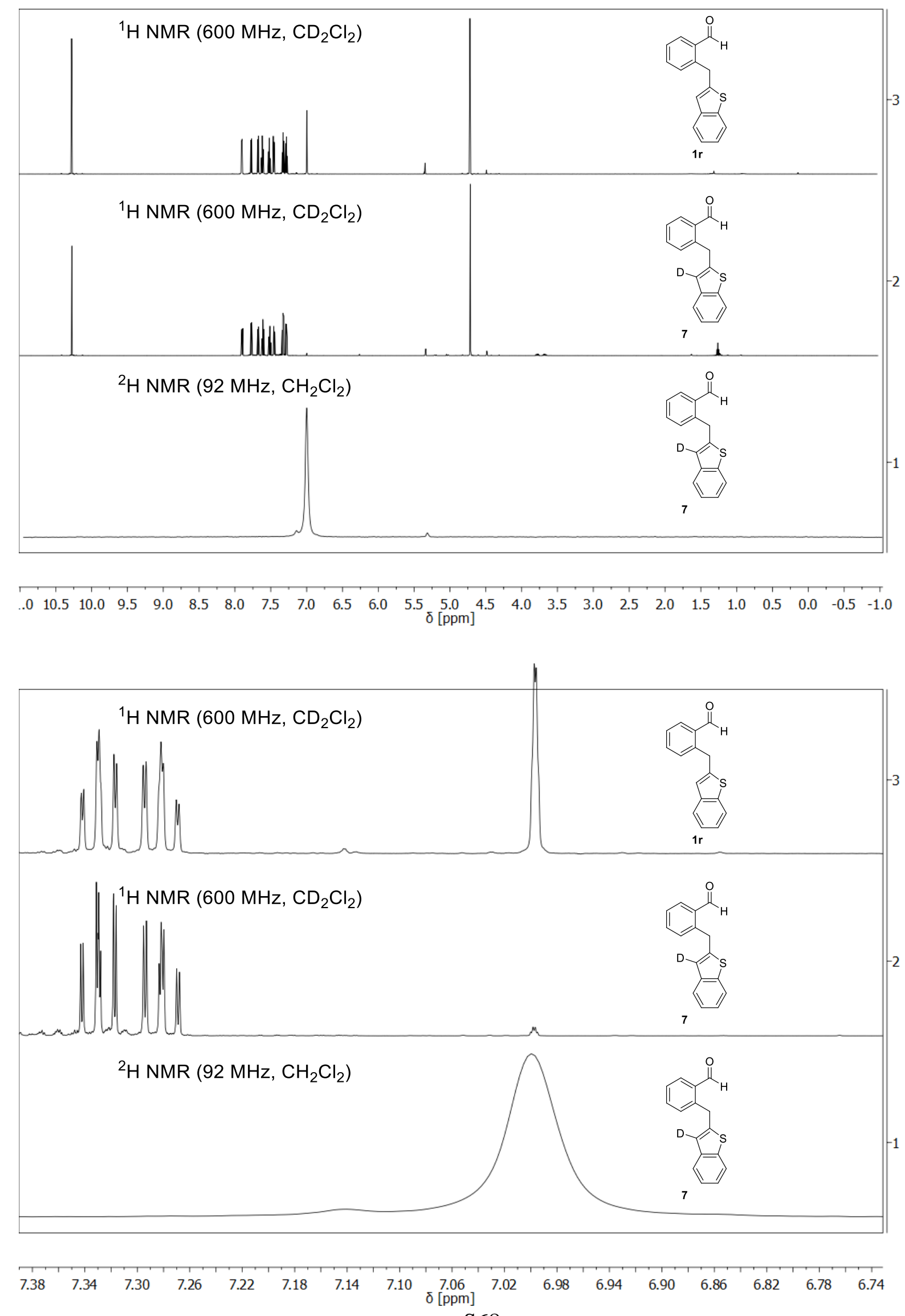


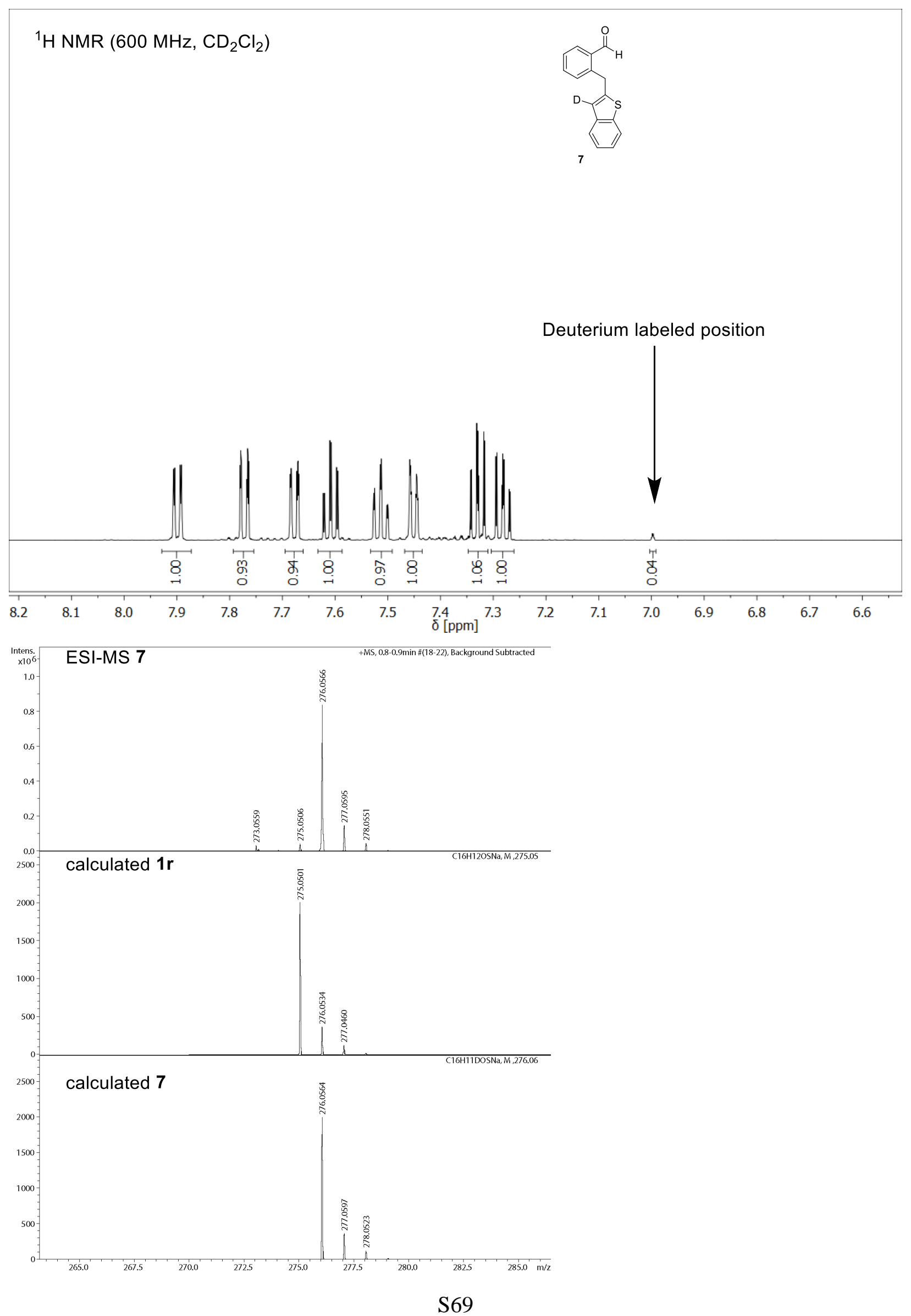




\section{(R)-3H-Spiro[benzo[b]thiophene-2,2'-inden]-1'(3'H)-one-(8)}<smiles>[2H]C12Cc3ccccc3C(=O)[C@@]1(C)Sc1ccccc12</smiles>

Synthesized according to the general procedure from 2-((benzo[b]thiophen2-yl-3- $d$ )methyl)benzaldehyde (7) $(76.0 \mathrm{mg}, 0.30 \mathrm{mmol}) .8$ isolated as yellow solid (57 mg, $0.22 \mathrm{mmol}, 74 \%, 53 \%$ ee) with catalyst 3a or using catalyst 3d (51 mg,0.20 mmol, 67\% ee).

ESI-MS: calculated: $\left[\mathrm{C}_{16} \mathrm{H}_{12} \mathrm{OSNa}\right]^{+}=275.0501$, found: 275.0500 , relative integration: $5.6 \%$; calculated: $\left[\mathrm{C}_{16} \mathrm{H}_{12} \mathrm{DOSNa}\right]^{+}=276.0564$, found: 276.0564 , relative integration: $100 \%$;

Table S2. Anti/syn ratios and e.r. of deuterated product samples with catalyst 3a and 3d before and after recrystallization for enantiomer-enrichment.

\begin{tabular}{|c|c|c|c|c|c|c|c|c|c|c|}
\hline & \multicolumn{2}{|c|}{$\begin{array}{l}{ }^{1} \mathrm{H} \text { NMR raw } \\
\text { integration }\end{array}$} & \multicolumn{2}{|c|}{$\begin{array}{l}5 \% \text { for non- } \\
\text { deuterated } 2 r \\
\text { substracted }\end{array}$} & \multicolumn{2}{|c|}{$\begin{array}{c}\text { anti/syn ratio } \\
\text { by } 1 \mathrm{H}\end{array}$} & \multicolumn{2}{|c|}{$\begin{array}{c}\text { anti/syn ratio } \\
\text { by } 2 \mathrm{H}\end{array}$} & \multicolumn{2}{|c|}{$\begin{array}{c}\text { "e.r." } \\
\text { by HPLC }\end{array}$} \\
\hline & $\begin{array}{c}\text { Integral } \\
3.40\end{array}$ & $\begin{array}{c}\text { Integral } \\
3.74\end{array}$ & $\begin{array}{c}\text { \% D at } \\
3.40\end{array}$ & $\begin{array}{c}\text { \% D at } \\
3.74\end{array}$ & anti & syn & anti & syn & $(R)-2 \mathrm{r}$ & $(S)-2 r$ \\
\hline Catalyst 3a & 24.6 & 69.7 & 19.6 & 64.7 & 76.8 & 23.2 & 77.4 & 22.6 & 76.7 & 23.3 \\
\hline recrystallized & 8.8 & 93.8 & 3.8 & 88.8 & 95.9 & 4.1 & 95.9 & 4.1 & 97.4 & 2.6 \\
\hline Catalyst 3d & 18.3 & 79.8 & 13.3 & 74.8 & 84.9 & 15.1 & 83.8 & 16.2 & 83.7 & 16.3 \\
\hline recrystallized & 6.0 & 87.9 & 1.0 & 82.9 & 98.8 & 1.2 & 100 & n.d. & 98.6 & 1.3 \\
\hline
\end{tabular}

\section{Recrystallization for enantiomer enrichment}

The deuterated products $(\mathbf{8})$ were recrystallized from hexanes. Using this procedure, the major enantiomer gets enriched. The products were then analyzed by HPLC, ${ }^{1} \mathrm{H}$ NMR and ${ }^{2} \mathrm{H}$ NMR again to detect any change in deuterium distribution (see Table S2).

\section{Determination of deuterium distribution in 8}

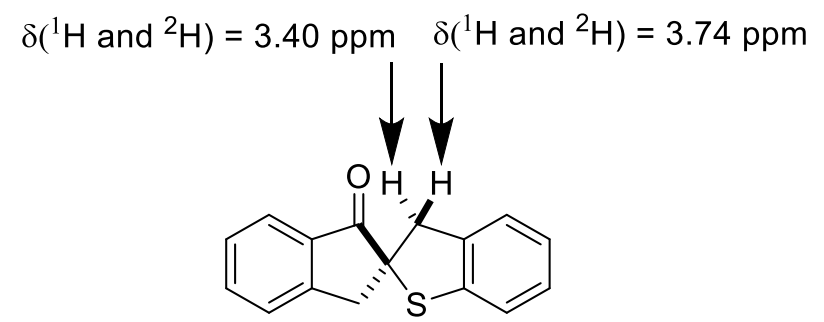

The position of the deuterium atom in respect to the quarternary sterocenter was determined by ${ }^{1} \mathrm{H}$ and ${ }^{2} \mathrm{H}$ NMR. Peaks at $\delta=3.74$ (anti-hydroacylation) ppm and $\delta=3.40$ (syn-hydroacylation) were integrated. Tthe deuterium distribution was separately determined as a deuterium ratio integration in the ${ }^{2} \mathrm{H}$ NMR and a deuteration grade by ${ }^{1} \mathrm{H}$ NMR integration in respect to integrals 
of signals from non-deuterated proton positions. Substracting the 5\% impurity of non-deuterated starting-material (determined by ESI-MS) gives the ratio of deuterium atoms. The occurance of naturally abundant deuterium is neglected in this analysis.

\section{8 synthesized with catalyst $3 a$ before recrystallization:}

\section{HPLC}

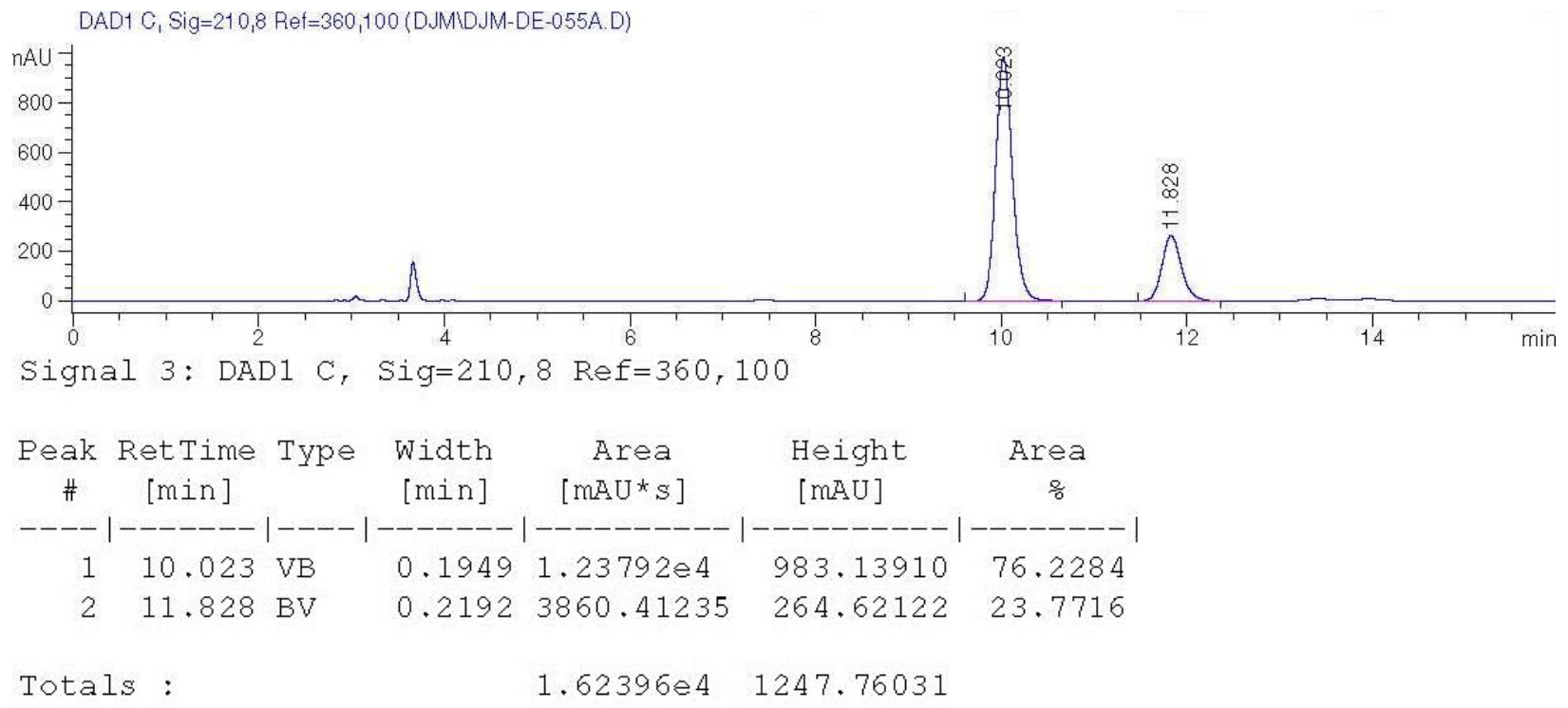


${ }^{1} \mathrm{H}$ NMR (600 MHz, $\left.\mathrm{CD}_{2} \mathrm{Cl}_{2}\right)$ :

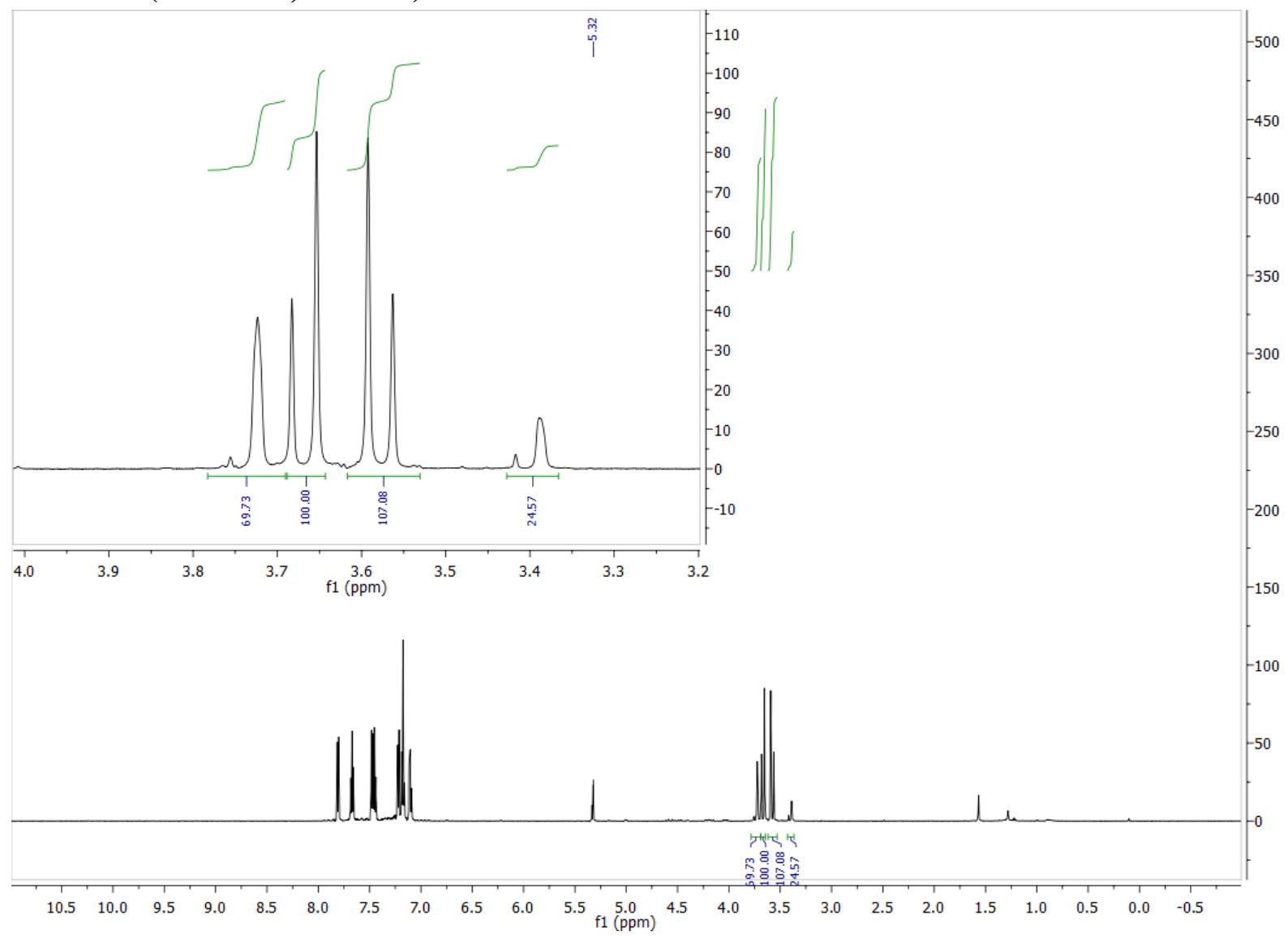


${ }^{2} \mathrm{H}$ NMR (92 MHz, $\left.\mathrm{CH}_{2} \mathrm{Cl}_{2}\right)$ :

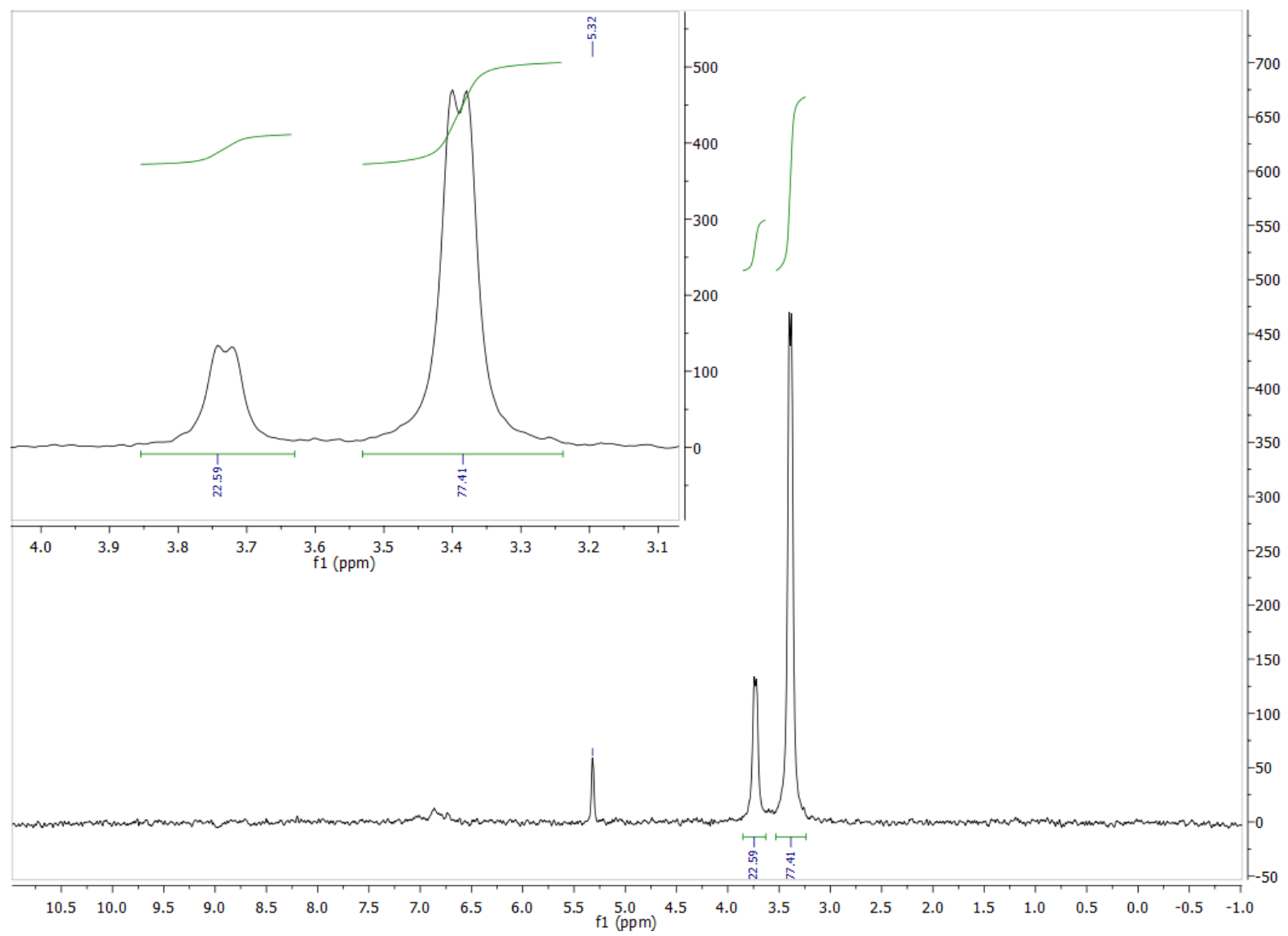

8 synthesized with catalyst $3 a$ after recrystallization:

\section{HPLC}

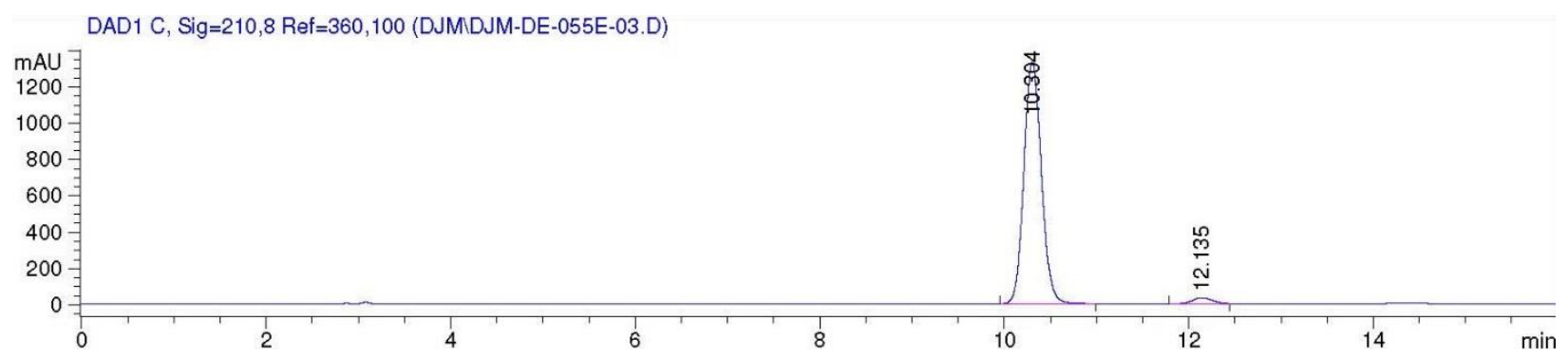

Signal 2: DAD1 B, Sig=254,16 Ref=360,100

\begin{tabular}{|c|c|c|c|c|c|c|}
\hline $\begin{array}{c}\text { Peak } \\
\#\end{array}$ & $\begin{array}{c}\text { RetTime } \\
\text { [min] }\end{array}$ & Type & $\begin{array}{l}\text { Width } \\
\text { [min] }\end{array}$ & $\begin{array}{c}\text { Area } \\
{\left[m A U^{*} s\right]}\end{array}$ & $\begin{array}{l}\text { Height } \\
\text { [mAU] }\end{array}$ & $\begin{array}{c}\text { Area } \\
\frac{\%}{\circ}\end{array}$ \\
\hline & & & 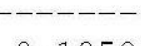 & $\mid----------$ & $----------\mid$ & $--------\mid$ \\
\hline 1 & 10.303 & $\mathrm{BB}$ & 0.1952 & 8010.30664 & 634.83136 & 97.4024 \\
\hline 2 & 12.144 & $\mathrm{BB}$ & 0.2237 & 213.62553 & 14.51575 & 2.5976 \\
\hline Total & : & & & 8223.93217 & 649.34711 & \\
\hline
\end{tabular}


${ }^{1} \mathrm{H}$ NMR (600 MHz, $\left.\mathrm{CD}_{2} \mathrm{Cl}_{2}\right)$ :

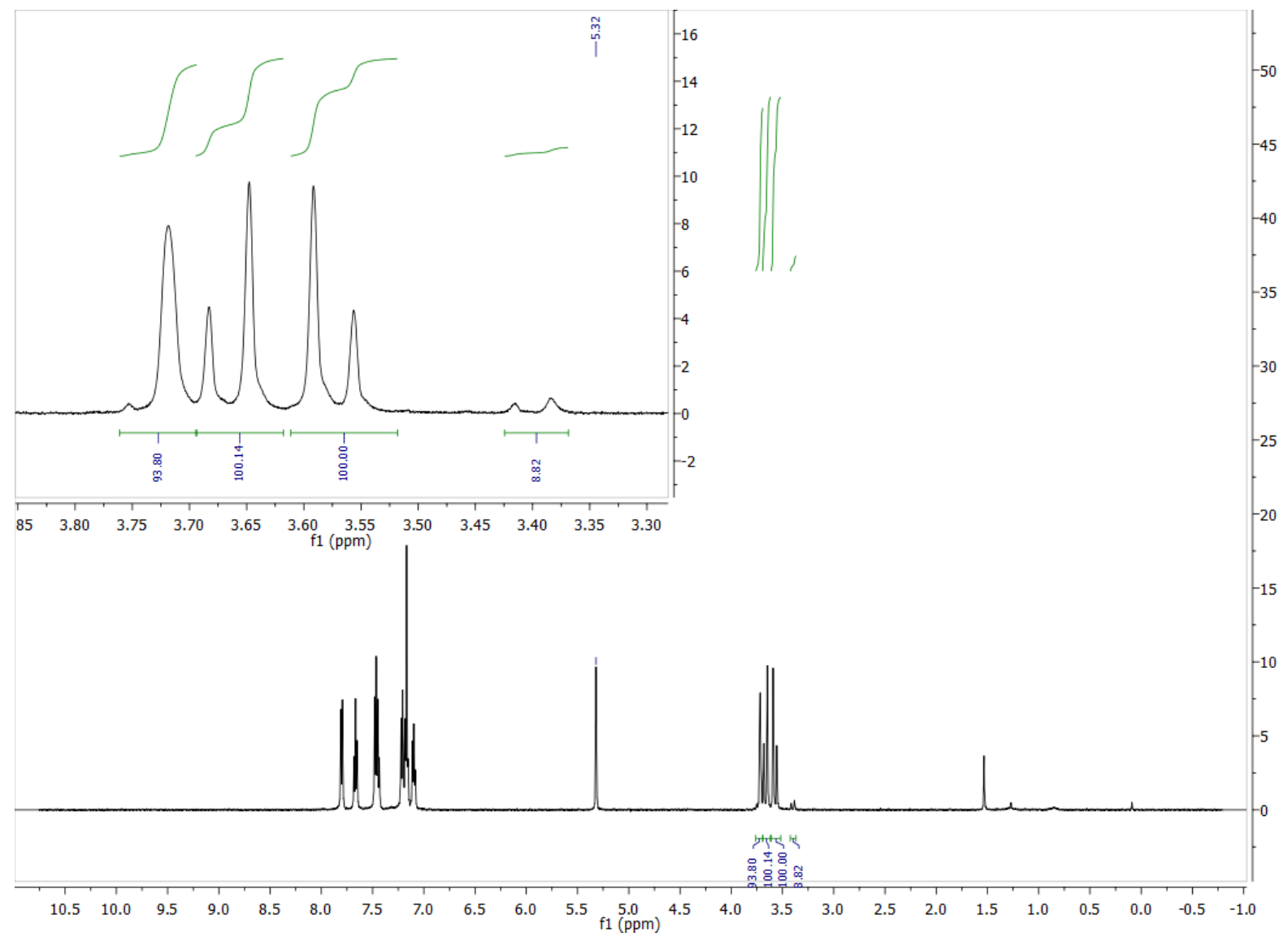


${ }^{2} \mathrm{H}$ NMR (92 MHz, $\left.\mathrm{CH}_{2} \mathrm{Cl}_{2}\right)$ :

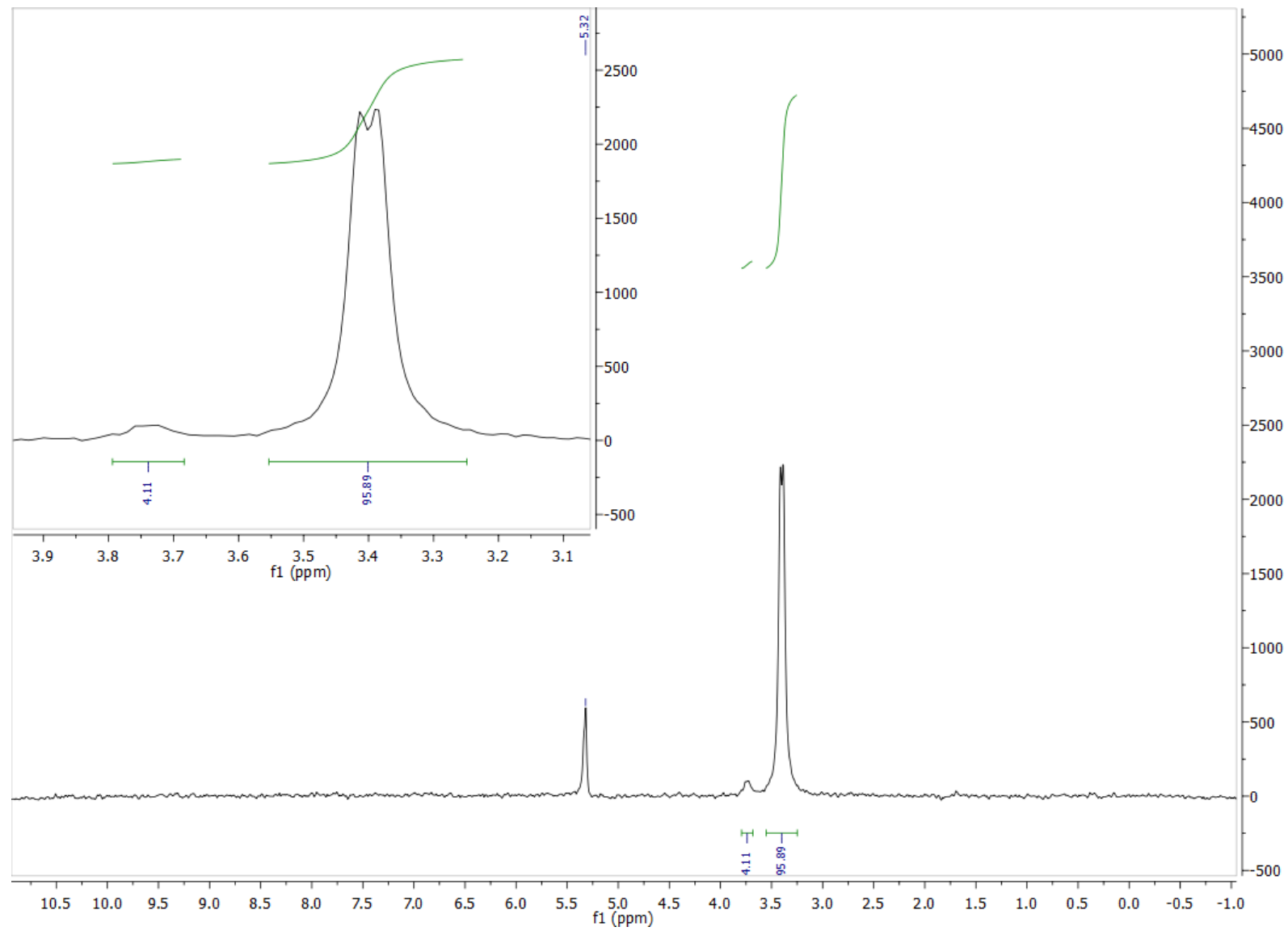

\section{8 synthesized with catalyst $3 d$ before recrystallization:}

\section{HPLC}

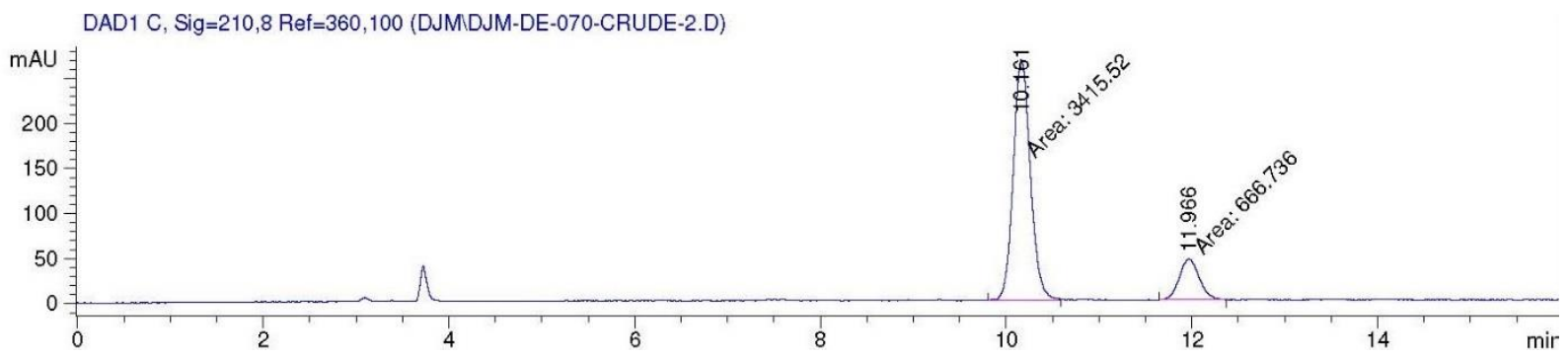


Signal 3: DAD1 C, $\operatorname{Sig}=210,8$ Ref $=360,100$

\begin{tabular}{|c|c|c|c|c|c|c|}
\hline $\begin{array}{c}\text { Peak } \\
\#\end{array}$ & $\begin{array}{c}\text { RetTime } \\
\text { [min] }\end{array}$ & Type & $\begin{array}{l}\text { Width } \\
\text { [min] }\end{array}$ & $\begin{array}{c}\text { Area } \\
{\left[\mathrm{mAU}^{*} \mathrm{~s}\right]}\end{array}$ & $\begin{array}{l}\text { Height } \\
\text { [mAU] }\end{array}$ & $\begin{array}{c}\text { Area } \\
\frac{\%}{\%}\end{array}$ \\
\hline & & & & & & \\
\hline 2 & 11. & 1 & 0.2457 & 666.73560 & 45.22977 & 16 \\
\hline
\end{tabular}

Totals :

$4082.25220 \quad 312.71021$

\section{${ }^{1} \mathrm{H}$ NMR (600 MHz, $\left.\mathrm{CD}_{2} \mathrm{Cl}_{2}\right)$ :}

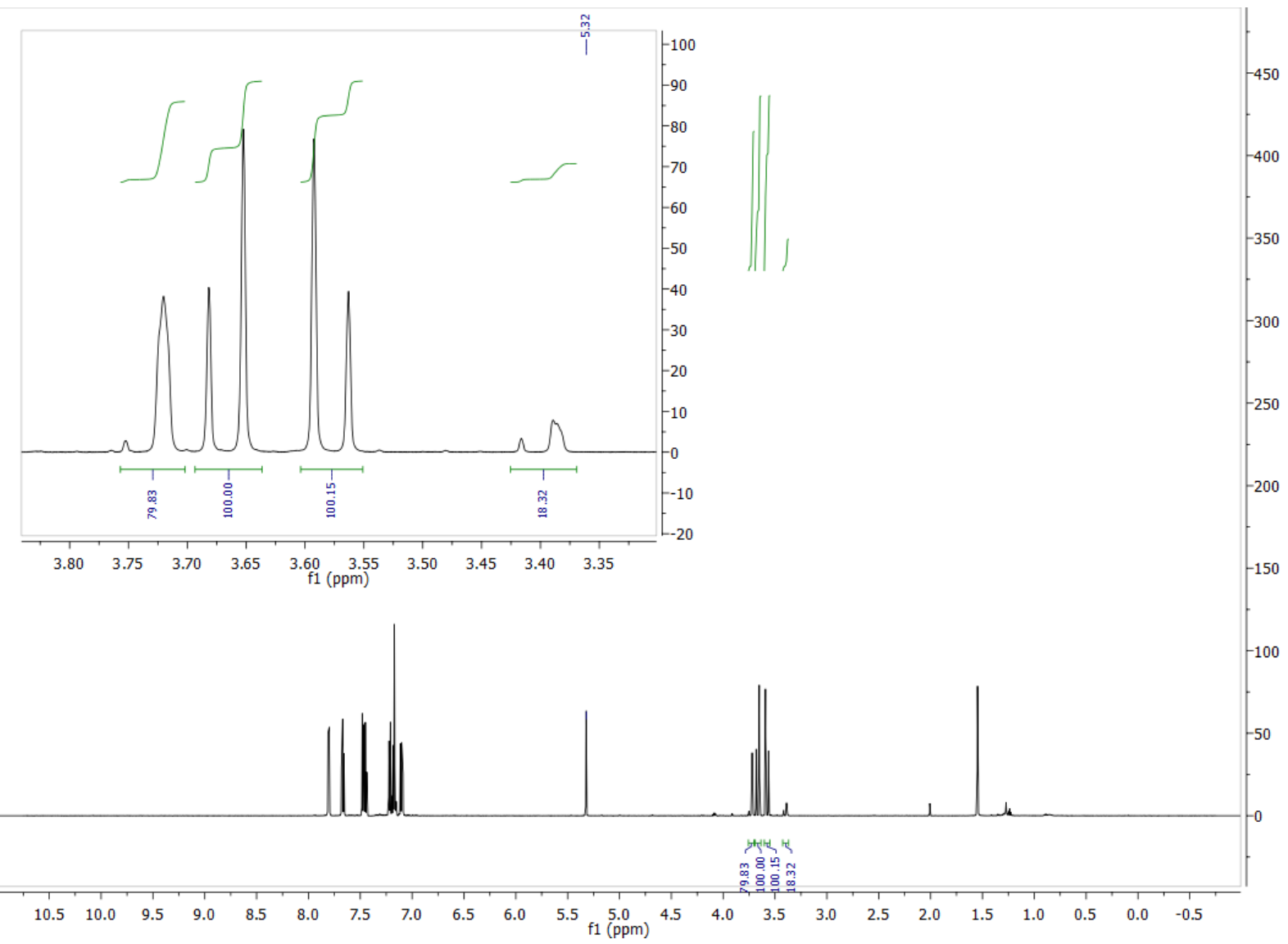


${ }^{2} \mathrm{H}$ NMR (92 MHz, $\left.\mathrm{CH}_{2} \mathrm{Cl}_{2}\right)$ :

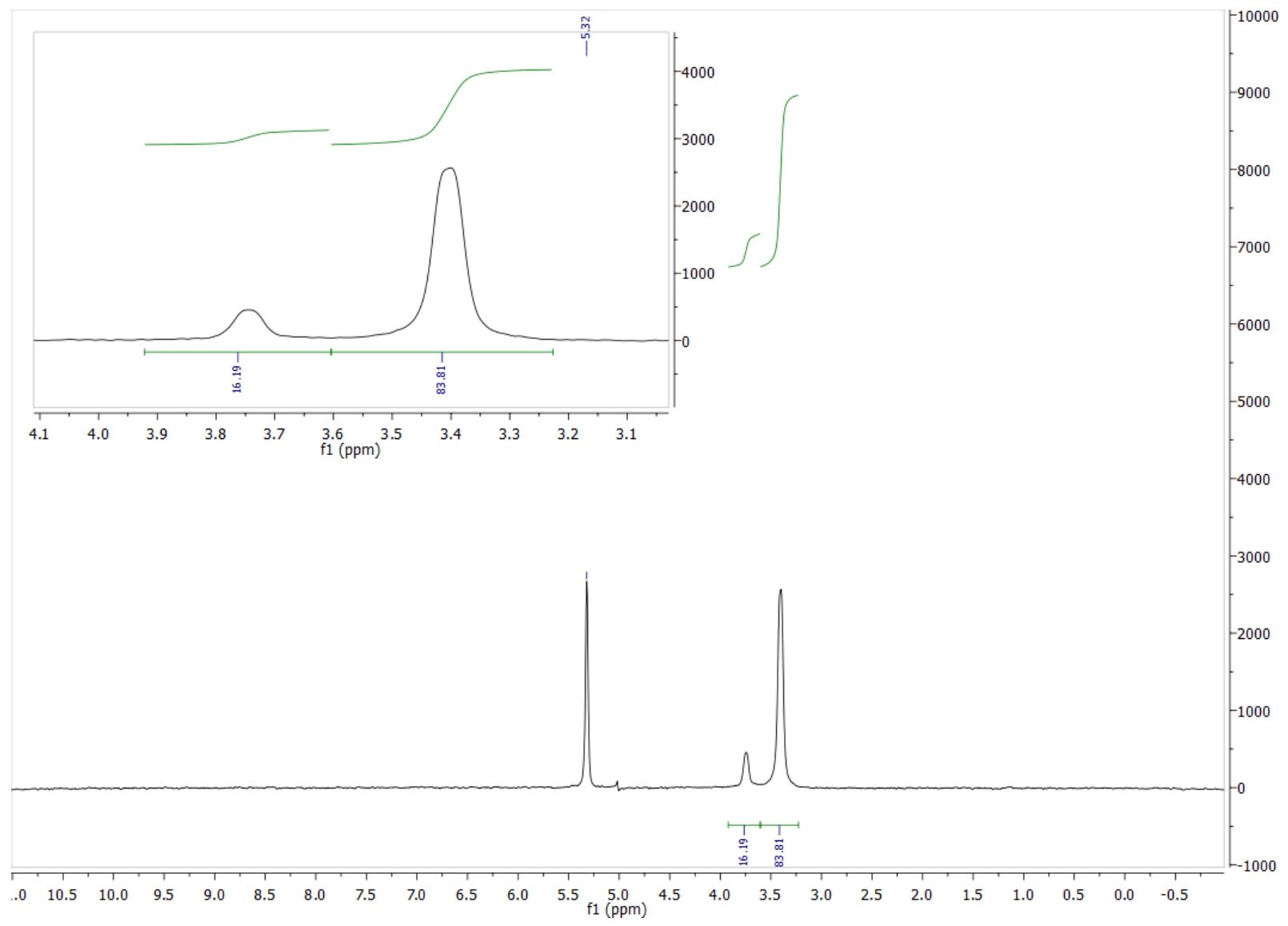

\section{8 synthesized with catalyst $3 d$ after recrystallization:}

\section{HPLC}

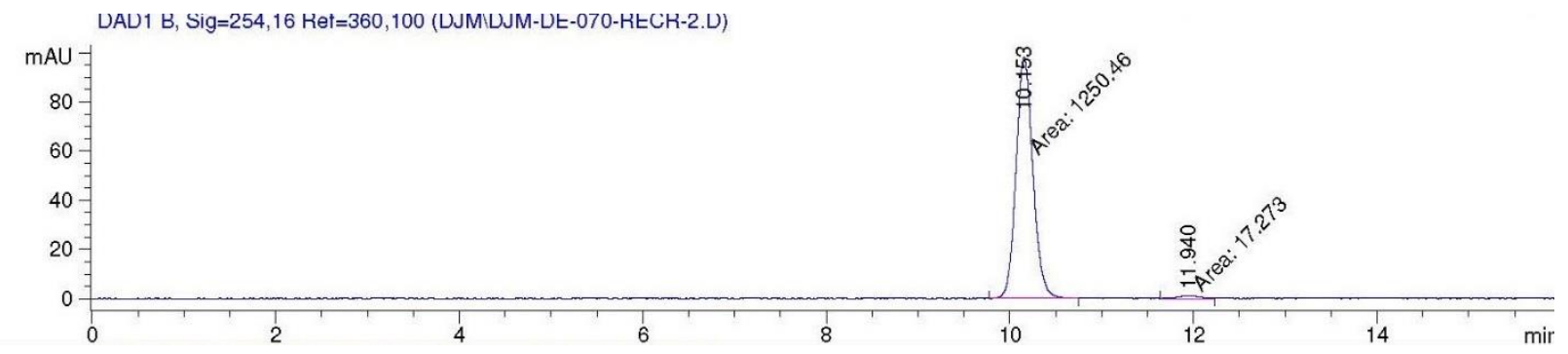

Signal 2: DAD1 B, Sig=254,16 Ref=360,100

\begin{tabular}{|c|c|c|c|c|c|c|}
\hline $\begin{array}{c}\text { Peak } \\
\#\end{array}$ & $\begin{array}{c}\text { RetTime } \\
\text { [min] }\end{array}$ & Type & $\begin{array}{l}\text { Width } \\
\text { [min] }\end{array}$ & $\begin{array}{c}\text { Area } \\
{\left[\mathrm{mAU}^{*} \mathrm{~s}\right]}\end{array}$ & $\begin{array}{l}\text { Height } \\
{[\mathrm{mAU}]}\end{array}$ & $\begin{array}{c}\text { Area } \\
\%\end{array}$ \\
\hline 1 & 10.153 & MM & 0.2126 & 1250.45959 & 98.02911 & 98.6375 \\
\hline 2 & 11.940 & $\mathrm{MM}$ & 0.2569 & 17.27295 & 1.12047 & 1.3625 \\
\hline otal & : & & & 1267.73255 & 99.14958 & \\
\hline
\end{tabular}


${ }^{1} \mathrm{H}$ NMR (600 MHz, $\left.\mathrm{CD}_{2} \mathrm{Cl}_{2}\right)$ :

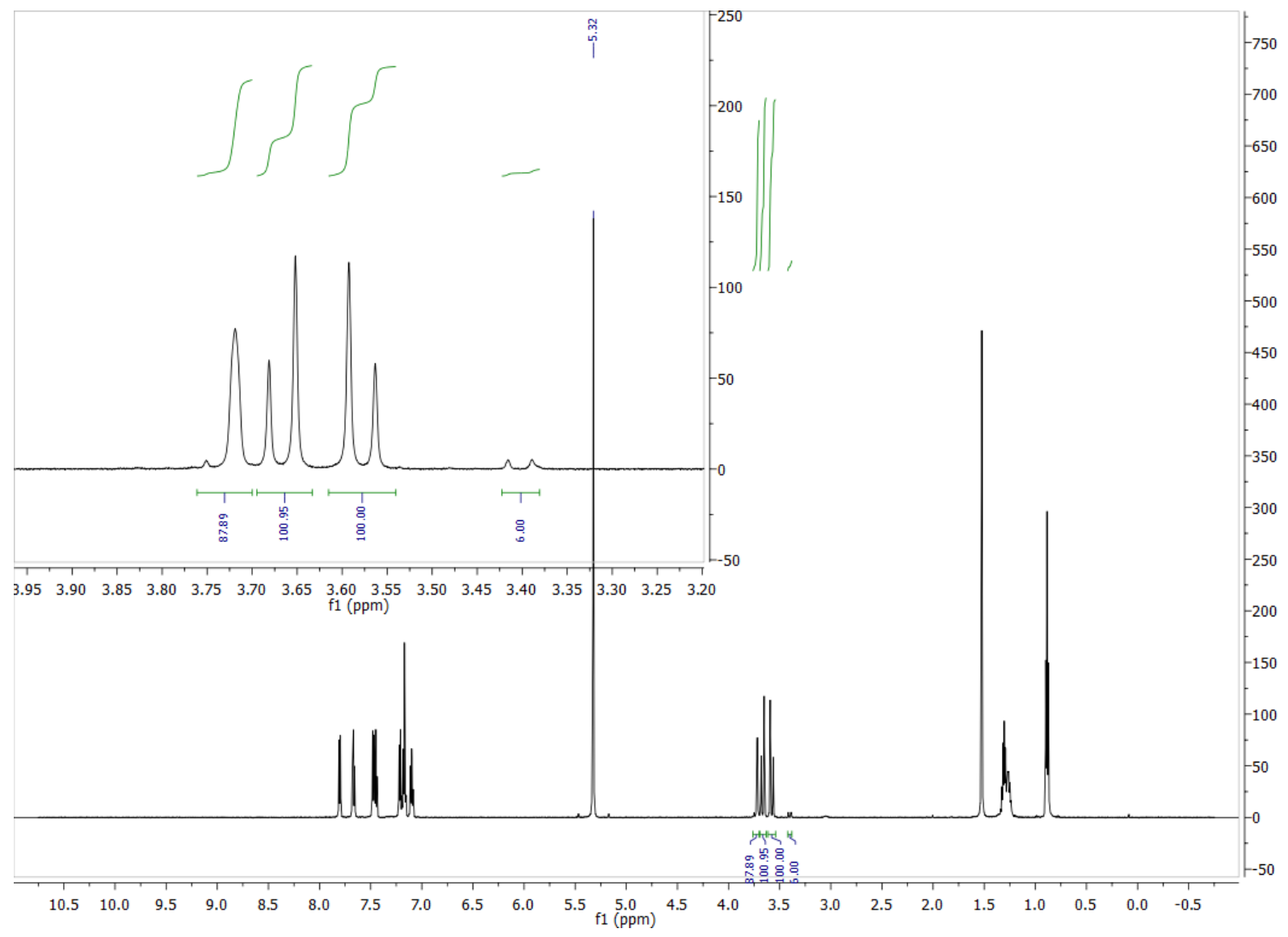


${ }^{2} \mathrm{H}$ NMR (92 MHz, $\left.\mathrm{CH}_{2} \mathrm{Cl}_{2}\right)$ :

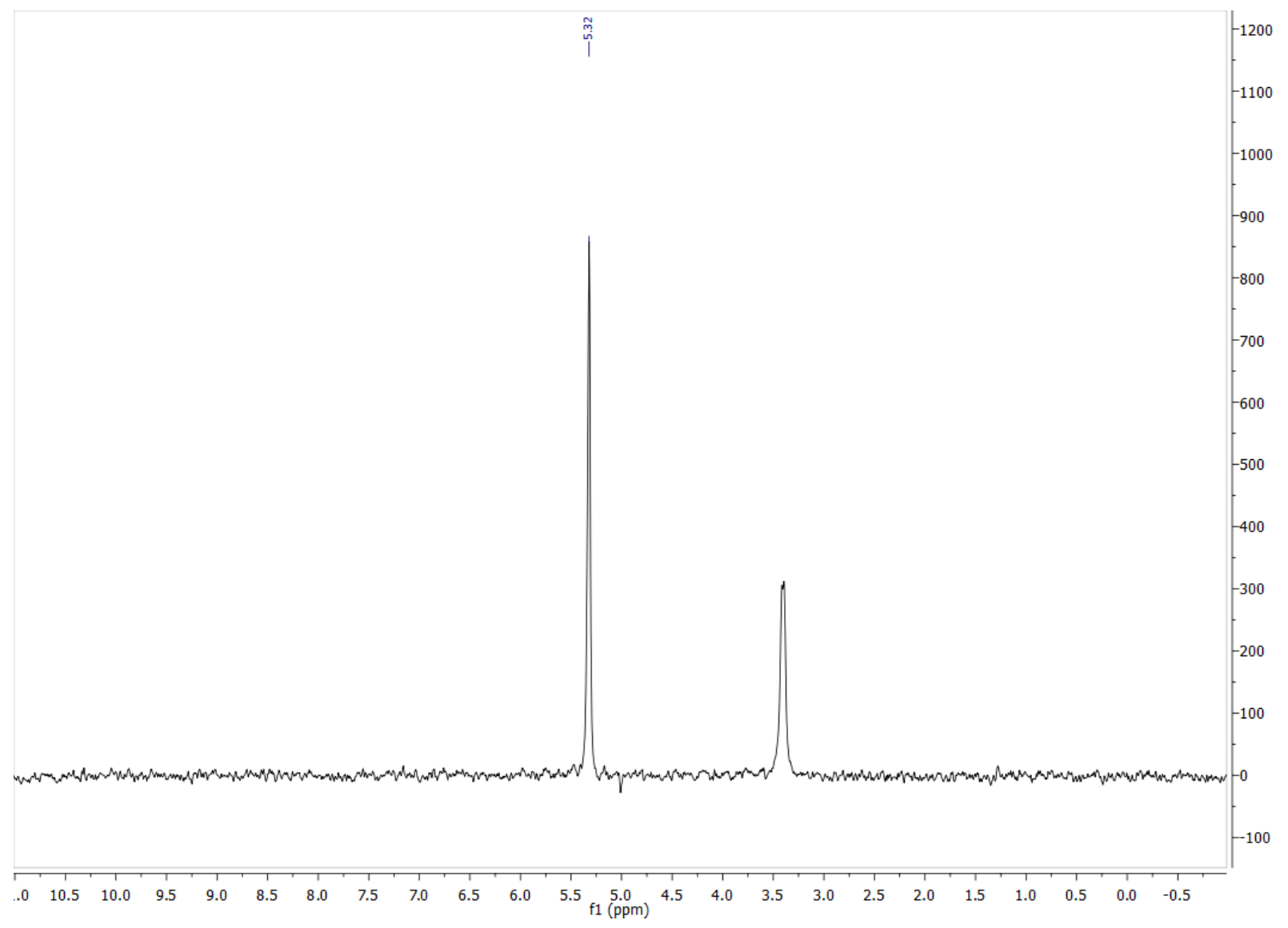




\section{2-((3-Methylbenzo[b]thiophen-2-yl)methyl)benzaldehyde (9)}

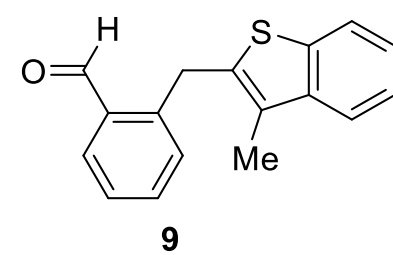

A solution of 3-methylbenzo[b]thiophene $(670 \mu \mathrm{L}, 5 \mathrm{mmol}, 1.67$ equiv. $)$ in THF $(15 \mathrm{~mL})$ was cooled to $-78^{\circ} \mathrm{C}$. Then $n$-BuLi solution in hexanes (1.6 M, $3.44 \mathrm{~mL}, 5.5 \mathrm{mmol}, 1.83$ equiv.) was added dropwise to the reaction mixture. After $90 \mathrm{~min}$ at $-78^{\circ} \mathrm{C}$, triisopropyl borate $(1.38 \mathrm{~mL}$,

$6.0 \mathrm{mmol}, 2.0$ equiv.) was added dropwise. The reaction was stirred for further $30 \mathrm{~min}$ at $-78{ }^{\circ} \mathrm{C}$ and was allowed to warm up to room temperature for $2 \mathrm{~h}$. The solvent was removed under reduced pressure to give a yellow solid, which was used without further purification. 2-(Bromomethyl)benzaldehyde (S10) (597 mg, $3.0 \mathrm{mmol}, 1.0$ equiv.) was added together with tetrakis(triphenylphosphine)palladium(0) (69 mg, $0.060 \mathrm{mmol}, 0.05$ equiv.), $9.0 \mathrm{~mL}$ aqueous $\mathrm{Na}_{2} \mathrm{CO}_{3}$ solution $(1 \mathrm{M}), 10 \mathrm{~mL}$ toluene, $8.5 \mathrm{~mL}$ ethanol and $2.5 \mathrm{~mL} \mathrm{H}_{2} \mathrm{O}$. The flask was evacuated and backfilled with argon three times and stirred at $60{ }^{\circ} \mathrm{C}$ overnight. The reaction was quenched with $20 \mathrm{~mL} \mathrm{H}_{2} \mathrm{O}$ and extracted with 3 x $20 \mathrm{~mL} \mathrm{CH}_{2} \mathrm{Cl}_{2}$, over $\mathrm{MgSO}_{4}$ and concentrated under reduced pressure. Purification by column chromatography on silica $(n$-pentane/EtOAc $=95 / 5)$ afforded 2-((3-methylbenzo[b]thiophen-2-yl)methyl)benzaldehyde (9) as a yellow oil (670 mg, $2.52 \mathrm{mmol}, 83 \%)$.

$\mathbf{R}_{f}(n$-Pentane/EtOAc $=90 / 10): 0.45 ;$

${ }^{1} \mathbf{H}$ NMR $\left(400 \mathrm{MHz}, \mathrm{CD}_{2} \mathrm{Cl}_{2}\right): \delta / \mathrm{ppm}=10.27(\mathrm{~s}, 1 \mathrm{H}), 7.87(\mathrm{dd}, J=7.5,1.6 \mathrm{~Hz}, 1 \mathrm{H}), 7.73(\mathrm{dt}$, $J=7.9,1.0 \mathrm{~Hz}, 1 \mathrm{H}), 7.69(\mathrm{dt}, J=8.1,1.0 \mathrm{~Hz}, 1 \mathrm{H}), 7.54(\mathrm{td}, J=7.5,1.7 \mathrm{~Hz}, 1 \mathrm{H}), 7.47(\mathrm{td}$, $J=7.5,1.4 \mathrm{~Hz}, 1 \mathrm{H}), 7.38(\mathrm{ddd}, J=8.1,7.1,1.2 \mathrm{~Hz}, 1 \mathrm{H}), 7.33-7.26(\mathrm{~m}, 2 \mathrm{H}), 4.70(\mathrm{~s}, 2 \mathrm{H}), 2.41$ $(\mathrm{s}, 3 \mathrm{H})$;

${ }^{13} \mathrm{C}$ NMR (400 MHz, $\mathrm{CD}_{2} \mathrm{Cl}_{2}$ ): $\delta / \mathrm{ppm}=193.02,142.17,141.35,139.19,137.62,134.41,133.56$, $131.31,128.62,127.85,124.50,124.36,122.63,122.06,31.62,12.08$;

ESI-MS: calculated: $\left[\mathrm{C}_{17} \mathrm{H}_{14} \mathrm{OSNa}\right]^{+}=289.0658$, found: 289.0655 ;

ATR-FTIR $\left(\mathrm{cm}^{-1}\right):$ 2570, 1697, 1601, 1578, 1489, 1458, 1435, 1408, 1381, 1304, 1285, 1204, $1130,1072,1057,1011,976,941,907,883,872,799,757,752,733,710,660,625$. 


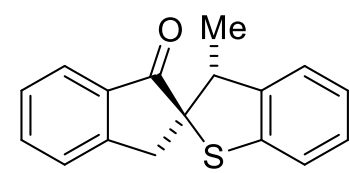

10

Synthesized according to the general procedure but using $100 \mathrm{~mol} \% \mathrm{NHC}$

precursor and $100 \mathrm{~mol}_{2} \mathrm{~K}_{2} \mathrm{CO}_{3}$ from 2-((3-methylbenzo[b]thiophen-2yl)methyl)benzaldehyde (10) $(79.8 \mathrm{mg}, 0.30 \mathrm{mmol})$. Isolated as yellow oil (8.4 mg, $0.03 \mathrm{mmol}, 11 \%)$.

$\mathbf{R}_{f}(n-$ Pentane/EtOAc $=9 / 1): 0.38 ;$

$[\alpha]_{D^{\mathbf{2 0}}}=-221.6\left(c=0.31, \mathrm{CHCl}_{3}\right)$;

${ }^{1} \mathbf{H}$ NMR $\left(300 \mathrm{MHz}, \mathrm{CDCl}_{3}\right): \delta / \mathrm{ppm}=7.81-7.75(\mathrm{~m}, 1 \mathrm{H}), 7.57(\mathrm{td}, \mathrm{J}=7.3,1.3 \mathrm{~Hz}, 1 \mathrm{H}), 7.41-$ $7.34(\mathrm{~m}, 2 \mathrm{H}), 7.16-7.00(\mathrm{~m}, 4 \mathrm{H}), 3.84(\mathrm{q}, \mathrm{J}=7.1 \mathrm{~Hz}, 1 \mathrm{H}), 3.64(\mathrm{~d}, \mathrm{~J}=17.8 \mathrm{~Hz}, 1 \mathrm{H}), 3.30(\mathrm{~d}$, $J=17.8 \mathrm{~Hz}, 1 \mathrm{H}), 1.23(\mathrm{~d}, \mathrm{~J}=7.1 \mathrm{~Hz}, 3 \mathrm{H})$;

${ }^{13} \mathrm{C}$ NMR $\left(126 \mathrm{MHz}, \mathrm{CDCl}_{3}\right): \delta / \mathrm{ppm}=202.7,151.2,143.5,138.6,135.6,135.4,128.1,127.9$, $126.5,125.4,125.1,123.9,122.4,70.0,47.6,38.0,14.6$;

ESI-MS: calculated: $\left[\mathrm{C}_{17} \mathrm{H}_{14} \mathrm{OSNa}\right]^{+}=289.0658$, found: 289.0653 ;

ATR-FTIR $\left(\mathrm{cm}^{-1}\right):$ 3059, 2967, 2924, 1709, 1605, 1586, 1495, 1462, 1443, 1373, 1327, 1292 , 1269, 1215, 1188, 1153, 1092, 1069, 1053, 1018, 968, 876, 822, 748, 737, 694, 660.

HPLC: $97 \%$ ee (Chiralcel AD-H, $n$-hexane $/ i$-PrOH = 70/30, $1.0 \mathrm{~mL} / \mathrm{min}, 254 \mathrm{~nm}, t_{R}=5.80 \mathrm{~min}$ (major), $t_{R}=6.77 \mathrm{~min}$ (minor)).

\section{( \pm )-3-Methyl-3H-spiro[benzo[b]thiophene-2,2'-inden]-1'(3'H)-one (10)}

Synthesized from 2-((3-methylbenzo[b]thiophen-2-yl)methyl)benzaldehyde (9) $(26.6 \mathrm{mg}$, $0.10 \mathrm{mmol})$.

$\mathbf{R}_{f}$ and ${ }^{1} \mathbf{H}$ NMR data were in accordance with the one obtained with catalyst $\mathbf{3 a}$.

HPLC: $r a c$ (Chiralcel AD-H, $n$-Hexane $/ i-\mathrm{PrOH}=70 / 30,1.0 \mathrm{~mL} / \mathrm{min}, 210 \mathrm{~nm}, t_{R}=5.81 \mathrm{~min}$, $\left.t_{R}=6.79 \mathrm{~min}\right)$. 
NMR spectra 3-Methyl-3H-spiro[benzo[b]thiophene-2,2'-inden]-1'(3' $H)$-one (10)
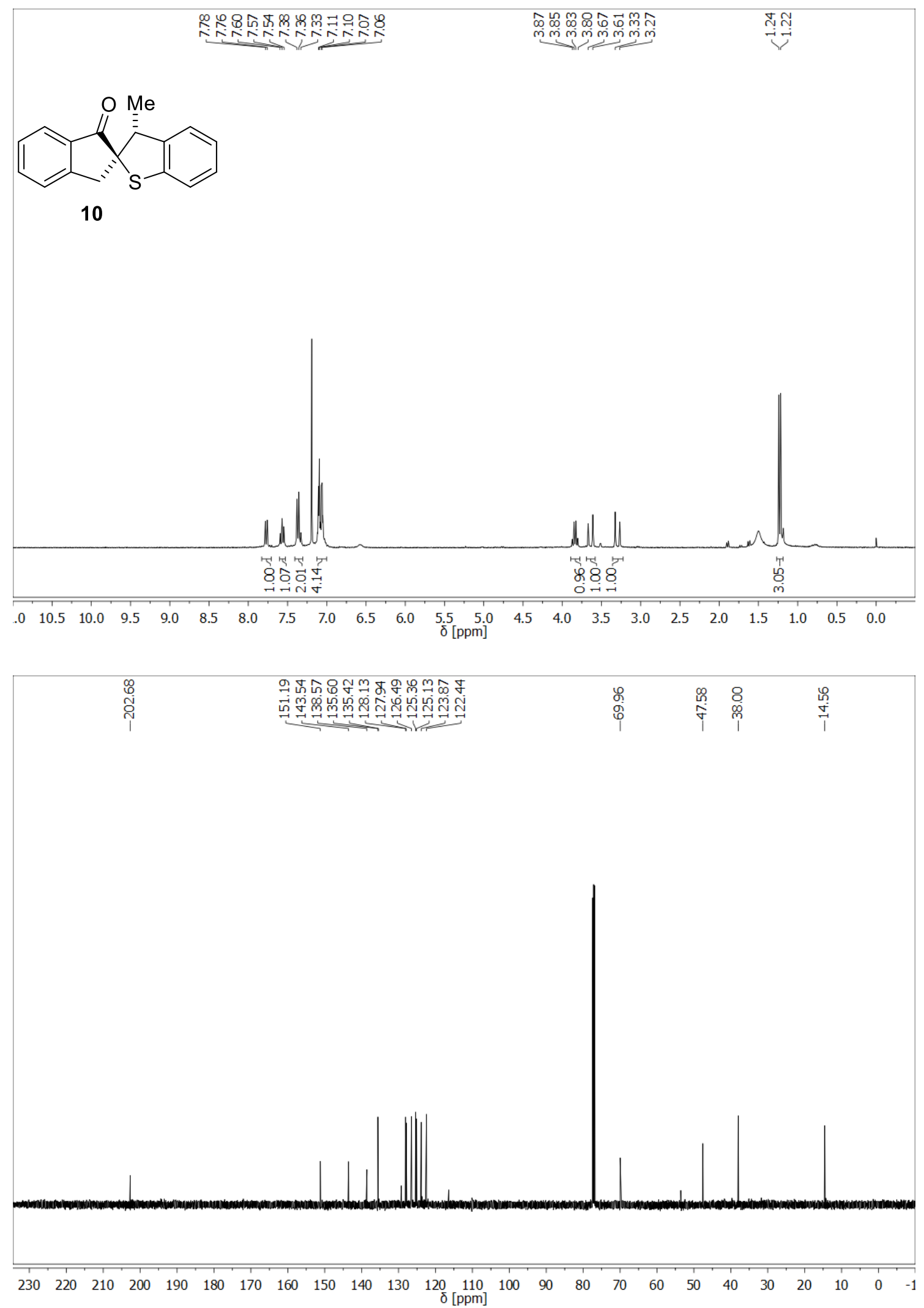


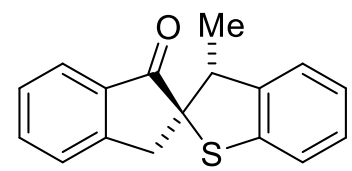

10

\section{Racemic compound}

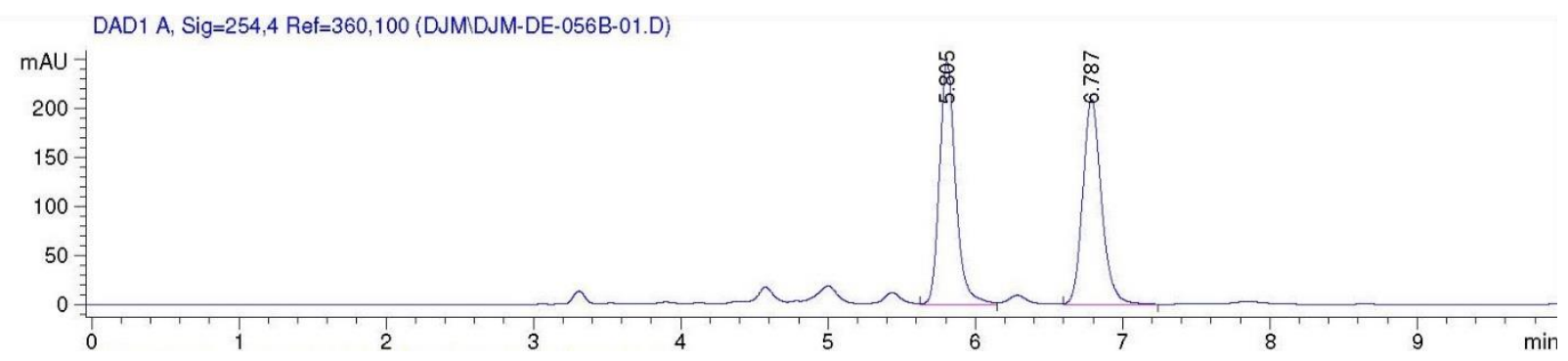

Signal 1: DAD1 A, Sig=254,4 Ref=360,100

\begin{tabular}{|c|c|c|c|c|c|c|}
\hline $\begin{array}{c}\text { Peak } \\
\#\end{array}$ & $\begin{array}{c}\text { RetTime } \\
\text { [min] }\end{array}$ & Type & $\begin{array}{l}\text { Width } \\
\text { [min] }\end{array}$ & $\begin{array}{c}\text { Area } \\
{\left[\mathrm{mAU}^{*} \mathrm{~S}\right]}\end{array}$ & $\begin{array}{l}\text { Height } \\
\text { [mAU] }\end{array}$ & $\begin{array}{c}\text { Area } \\
\%\end{array}$ \\
\hline & & V & & & & 66 \\
\hline 2 & 87 & סח & .1355 & 1857.63135 & 209.45268 & 49.6234 \\
\hline
\end{tabular}

Totals : $\quad 3743.45984 \quad 457.16968$

\section{Enantioenriched compound}

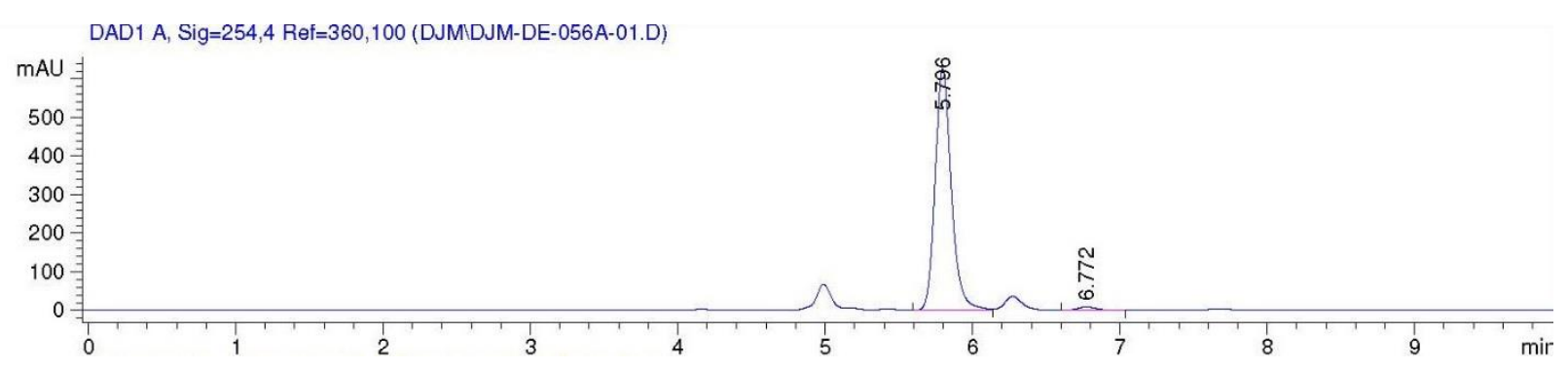

Signal 1: DAD1 A, $\mathrm{Sig}=254,4$ Ref $=360,100$

\begin{tabular}{|c|c|c|c|c|c|c|}
\hline $\begin{array}{c}\text { Peak } \\
\#\end{array}$ & $\begin{array}{c}\text { RetTime } \\
\text { [min] }\end{array}$ & Type & $\begin{array}{l}\text { Width } \\
\text { [min] }\end{array}$ & $\begin{array}{c}\text { Area } \\
{\left[\mathrm{mAU}^{*} \mathrm{~s}\right]}\end{array}$ & $\begin{array}{l}\text { Height } \\
{[\mathrm{mAU}]}\end{array}$ & $\begin{array}{c}\text { Area } \\
\%\end{array}$ \\
\hline 1 & & N & 0 & 4737. & 628. & 98 \\
\hline 2 & 6.772 & $\mathrm{BB}$ & .1352 & 76.41460 & 8.64035 & 1.5874 \\
\hline
\end{tabular}

Totals : $\quad 4813.96245 \quad 636.64712$ 
9 Computational Study 


\subsection{Mechanism of the NHC-catalyzed dearomatizations}

NHC-catalyzed hydroacylations have been computationally investigated previously and were described as "highly asymmetric" and concerted. ${ }^{[\mathrm{S2} 2 \mathrm{S22}]}$ The mechanism presented here is similar to the ones investigated in Refs. [S2,S22], but involves an additional dearomatization.

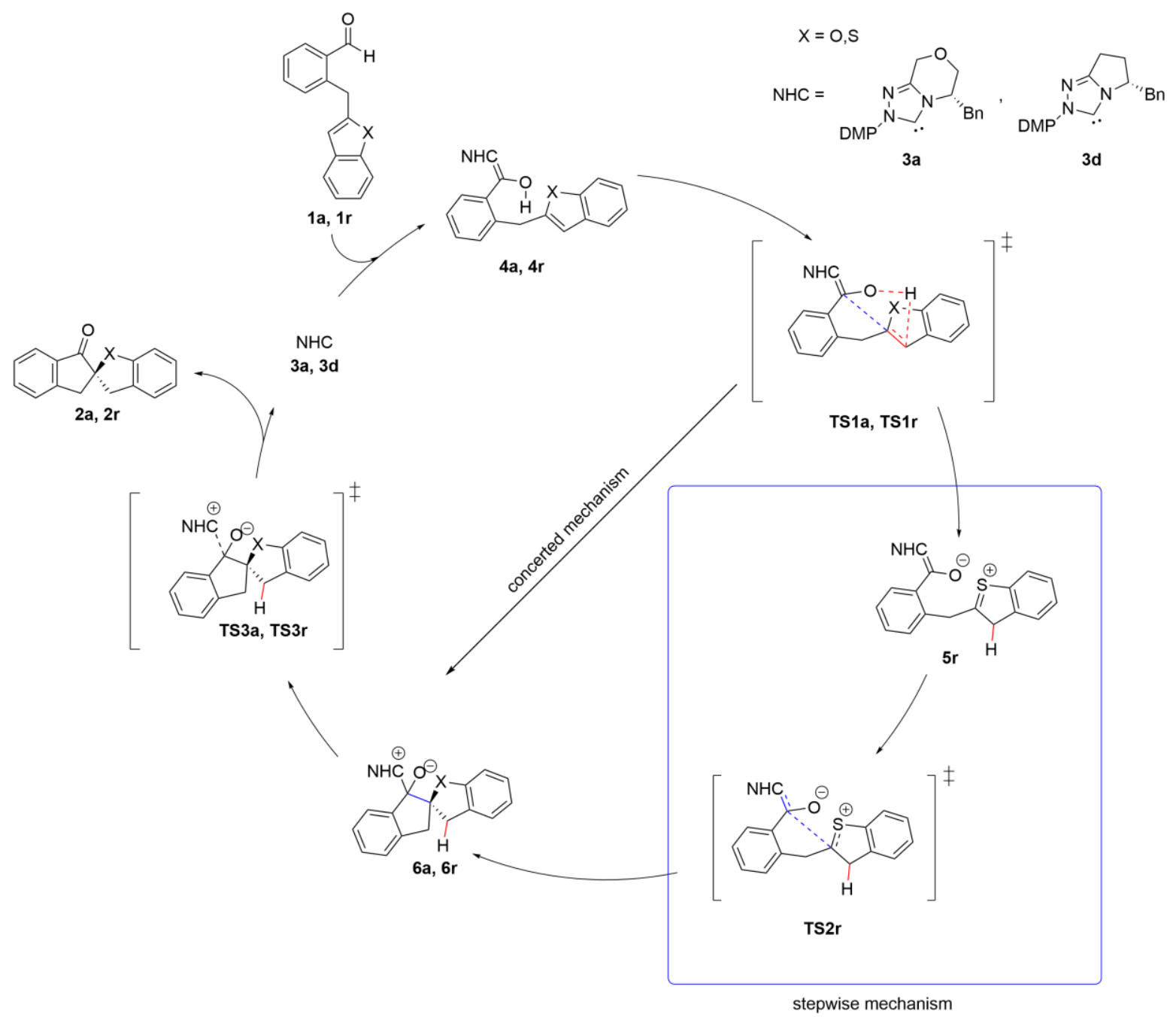

Scheme S1. Catalytic cycle describing the stepwise as well as the concerted mechanism. The experimentally found major product $(R)$-configuration was chosen to represent intermediates 6 to 2. 2,6-Dimethoxyphenyl is abbreviated as DMP.

In our study we follow the catalytic cycle depicted in Scheme 1 from reactants $1 \mathbf{a} / \mathbf{r}$ to the products 2a and 2r, respectively. Please note that in contrast to the main text 3a and $\mathbf{3 d}$ here are the free carbenes and not the respective tetrafluoroborate salts. As the formation of Breslow-intermediates $\mathbf{4}$ (formed by $\mathbf{1}$ and NHC 3a/3d) most likely follows an intermolecular mechanism, ${ }^{[\mathrm{S} 23]}$ it is 
not studied here. Additionally, there is evidence that Breslow-intermediates are predominantly formed in $(E)$-configuration. ${ }^{[\mathrm{S} 24]}$ Thus, we focus our investigations solely on $(E)$-Breslowintermediates. In our suggested mechanism (similar to Refs. [S2,S22]), a hydrogen is transferred from the enol group of the Breslow-intermediate 4 to the 3-position in the benzofuran/benzothiophene residue of 4 (cf. red bond in intermediates 5 through TS3 in Scheme S1). Two different mechanism are considered: (i) a concerted one, and (ii) a stepwise mechanism. During the concerted mechanism the benzylic carbon stereoselectively forms a new bond to the 2-position of the benzofuran/benzothiophene residue (cf. intermediate 6 in Scheme S1). During the stepwise mechanism a charge-separated intermediate $\mathbf{5}$ is formed, which enables the benzofuran/benzothio-phene residue to rotate before forming the carbon-carbon bond (thereby generating intermediate $\mathbf{6}$ ).

\subsection{Computational details}

All geometry optimizations and frequency analyses were carried out using the TURBOMOLE $6.5^{[\mathrm{S} 25]}$ program package. The dispersion-corrected meta-GGA TPSS-D3(BJ) ${ }^{[\mathrm{S} 26-28]}$ was chosen as density functional approximation. Energies were converged up to a change of $10^{-7}$ a.u., while structures were considered converged when the norm of the molecular gradient was smaller than $10^{-3}$ a.u.. The resolution of the identity (RI) approximation ${ }^{[\mathrm{S} 29]}$ together with the corresponding auxiliary basis sets ${ }^{[\mathrm{S} 30]}$ from the TURBOMOLE basis set library was employed for all TPSS-D3(BJ) calculations. Preparatory optimizations (partial optimizations, i.e., fixing some degrees of freedom to create guess structures for transition state searches) were partially performed using the double- $\zeta$ quality def2-SVP ${ }^{[\mathrm{S} 31]}$ basis set. Relaxed surface scans (with double- $\zeta$ basis sets) were executed using the ORCA $3.0 .3^{[\mathrm{S} 32]}$ program package. The resulting pre-optimized structures were then further optimized (without any structural restrictions) using a larger def2-TZVP ${ }^{[\mathrm{S} 33]}$ triple- $\zeta$ quality basis set which was also used for frequency analysis (using the aoforce ${ }^{[\mathrm{S} 34]}$ program from the TURBOMOLE suite) on optimized structures. Intrinsic reaction coordinate (IRC) calculations on the optimized transition states delivered starting structures for the optimization of the minima connected by the respective transition state. Solvent effects from 1,4-dioxane $\left(\mathrm{COSMO}^{[\mathrm{S} 35-36]}, \varepsilon=2.25\right.$, def2-TZVP) changed relative energies only by roughly $2 \mathrm{~kJ} / \mathrm{mol}$ and did not change the qualitative picture at all. Hence, solvent contributions are excluded from the discussion in this work. The final optimized RI-TPSS-D3(BJ)/def2-TZVP (vide supra) structures were used for single-point analysis with the M06-2 $\mathrm{X}^{[\mathrm{S} 37]}$ functional in a def2-TZVP ${ }^{[\mathrm{S} 33]}$ basis set 
(no RI used) using the TURBOMOLE $7.0^{[\mathrm{S} 38]}$ program package. Pictures from molecular 3D structures in this work were generated using VMD. ${ }^{[\mathrm{S} 39]}$

\subsection{Results}

There are four possibilities for the proton transfer to the aromatic C-C double bond in the (E)-Breslow-intermediates $\mathbf{4 a} / \mathbf{r}$. The carbenes $3 \mathbf{a}$ and $\mathbf{3 d}$ used in our investigations are chiral. Thus, the side of the carbene's triazolium plane without the benzyl group will be called "sterically less demanding side", while the other will be called "sterically more demanding side". For both cases, the proton transfer to the heteroaromatic residue can occur from the $r e$ - and si-face (cf. Figure S3). We checked for the proton transfer onto the $r e$ - and si-face only on the sterically less demanding side. This is due to our investigations (vide infra) showing that the orientation of the heteroaromatic residue is crucial upon formation of the transition state for the proton transfer. We will start our discussion with the catalytic cycle involving NHC 3a first and later discuss the most promising reaction pathway for the reaction involving NHC 3d.
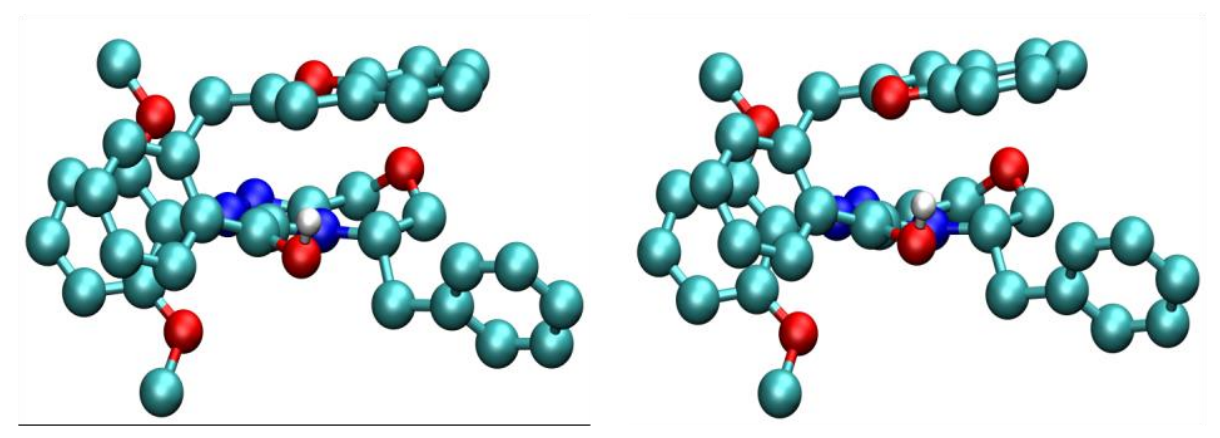

Figure S3. Structures of Breslow-intermediate 4a. Left: proton transfer would occur from the reface of the benzofuran. Right: benzofuran moiety is flipped, proton transfer would occur from siface of the bezofuran. All hydrogen atoms except the transferred one are omitted for clarity. Carbon, hydrogen, nitrogen, and oxygen are presented in turquoise, white, blue and red, respectively.

\subsubsection{Attack on the sterically less demanding side}

On the less sterically demanding side of the Breslow-intermediates $\mathbf{4 a} / \mathbf{r}$ proton transfer and the C-C-bond formation (enol-carbon and 3-position in benzofuran/benzothiophene residue) both happen from the re-face of the heteroaromatic benzofuran/benzothiophene residue. In the 
following we will call the reaction pathway involving benzofuran "a-path" and the pathway involving benzothiophene "r-path" (cf. Scheme S1). The rate-determining (vide infra) transition states $(R)$-TS1a and $(R)$-TS1r are presented in Figure S4. Both transition states are very similar and of first order, i.e., they contain one imaginary frequency of i 1119 and i $1096 \mathrm{~cm}^{-1}$, respectively. In both cases the imaginary mode is dominated by the movement of the hydrogen. This is in good agreement with previous studies for a similar kind of mechanism. ${ }^{[\mathrm{S} 2, \mathrm{~S} 22]}$ The orientation of the heteroaromatic residue is such that the $r e$-side is attacked. This allows for beneficial stacking of the carbons that will form the spirocycle: After the proton transfer only a small structural change is necessary in order to form the desired bond. We could not identify any transition state similar to $(R)$-TS1 (cf. Figure S4) resulting in an $(S)$ configuration just by flipping the benzofuran/benzothiophene residue on the less hindered side of the respective Breslowintermediate (for the meaning of flipping here, see Figure S3).

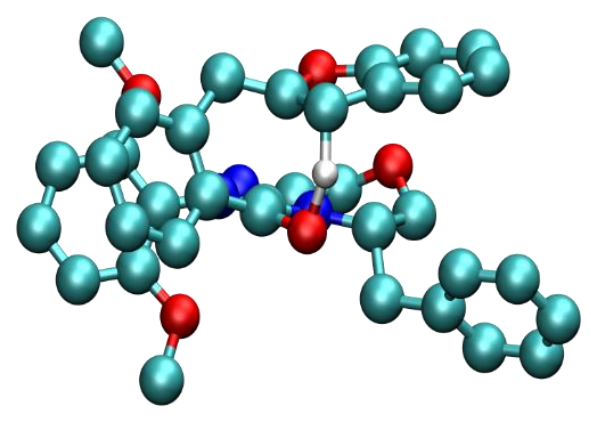

(a) $(R)$-TS1a

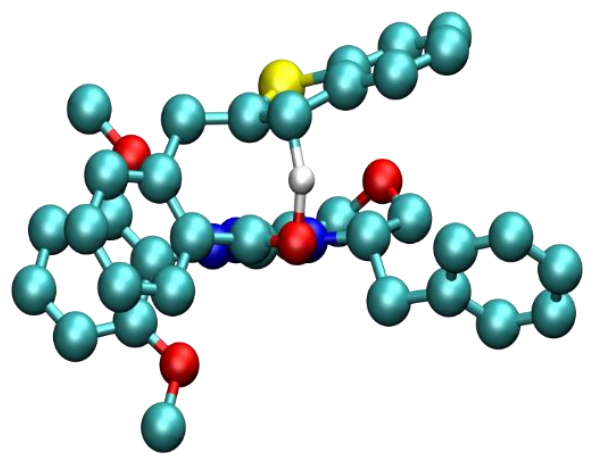

(b) $(R)-\mathbf{T S 1 r}$

Figure S4. First transition state for the a-path (TS1a) and r-path (TS1r). All hydrogen atoms except the transferred one are omitted for clarity. Carbon, hydrogen, nitrogen, oxygen and sulfur are presented in turquoise, white, blue, red and yellow, respectively.

In the flipped case, the carbon-carbon bond formation is hindered since it requires a larger conformational change in order to bring the groups together after the proton transfer started. This is corroborated by the deuterium experiments, which clearly revealed that the proton transfer is highly selective even for the r-path (with lower $e e$ ) and occurs from the re-side only. The main mechanistic difference between the a- and r-path becomes obvious from the $(R)$-TS1-products $(R)-6 \mathbf{a}$ and (R)-5r (see Figure S5). We assume that the reaction path a involves a concerted reaction since we could not identify any species $\mathbf{5 a}$ or TS2a (corresponding to $\mathbf{5 r}$ and TS2r) during our 
investigations [Here and in the rest of this section, we skip the " $(R)$ " label for the absolute configuration to keep our notation short]. Thus, the a-path follows the pattern $4 \mathbf{a} \rightarrow$ TS1a $\rightarrow \mathbf{6 a}$ $\rightarrow$ TS3a $\rightarrow$ 2a $+3 \mathbf{a}($ cf. Scheme S1).

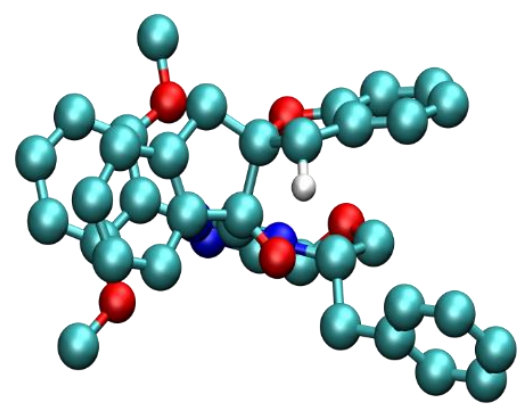

(a) $(R)-\mathbf{6 a}$

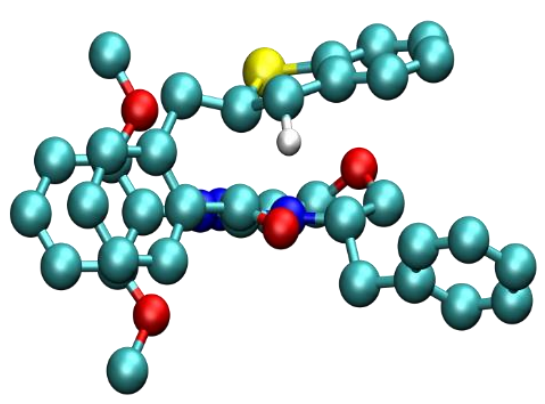

(b) $(R)-5 \mathbf{r}$

Figure S5. Products resulting from TS1a (6a, left) and TS1r (5r, right). All hydrogen atoms but the transferred one are omitted for clarity. Carbon, hydrogen, nitrogen, oxygen and sulfur are presented in turquoise, white, blue, red and yellow, respectively.

While the carbon-carbon bond is already formed in 6a/6r $\left(\mathrm{C}-\mathrm{C}\right.$-bond distances are $\mathrm{d}_{\mathrm{C}-\mathrm{C}}=1.640$ and $1.659 \AA$, respectively), there is no carbon-carbon bond yet in $\mathbf{5 r}\left(\mathrm{d}_{\mathrm{C}-\mathrm{C}}=2.126 \AA\right.$ ), which is the product of TS1r. We identified a first-order transition state TS2r (imag. frequency of i $77 \mathrm{~cm}^{-1}$ ) connecting intermediates $\mathbf{5 r}$ and $\mathbf{6 r}$. Both species $\mathbf{6 a} / \mathbf{r}$ release the carbene 3a through C-C-bond cleavage (TS3a/TS3r) and thereby form the spirocycles $2 \mathbf{a}$ and $\mathbf{2 r}$, respectively. From this we conclude that the $\mathbf{r}$-path is a stepwise mechanism, following the pattern $4 \mathbf{r} \rightarrow \mathbf{T S 1 r} \rightarrow \mathbf{5 r}$ $\rightarrow$ TS2r $\rightarrow$ 6r $\rightarrow$ TS3r $\rightarrow$ 2r $+3 \mathbf{r}($ cf. Scheme S1). All optimized structures of the r-path are presented in Figure S6. 


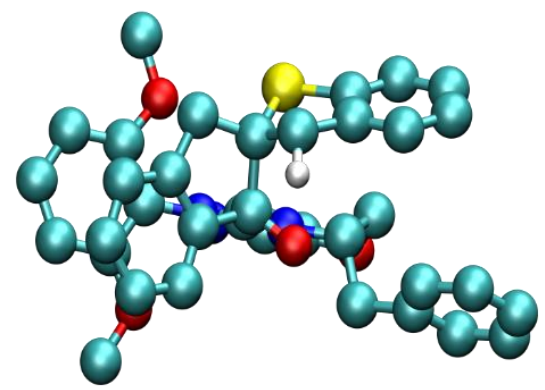

(a) $(R)-\mathbf{4 r}$

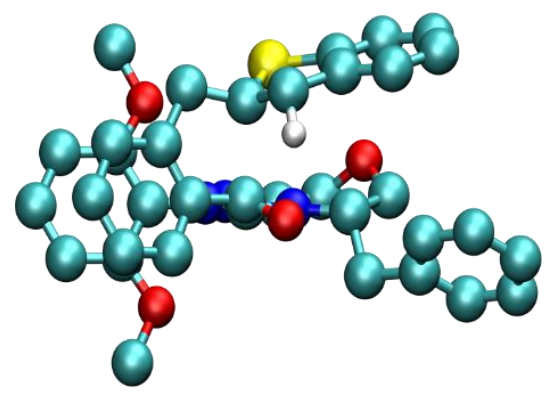

(c) $(R)-5 \mathbf{r}$

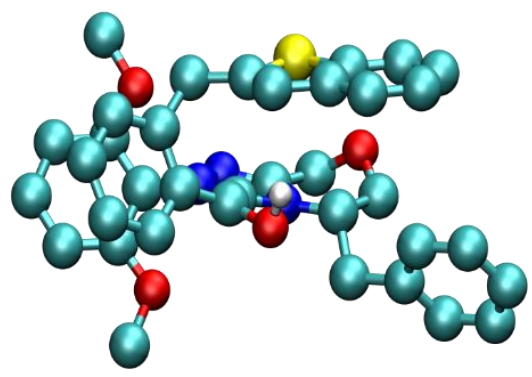

(e) $(R)-6 \mathbf{r}$

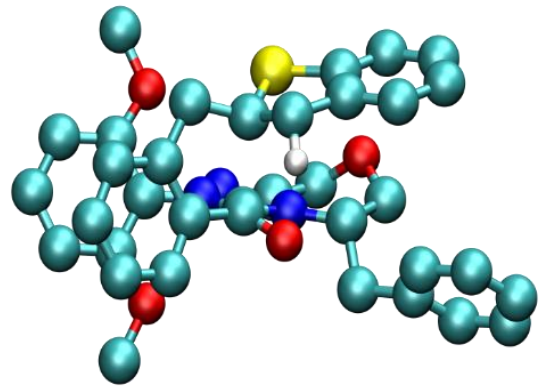

(b) $(R)$-TS1r

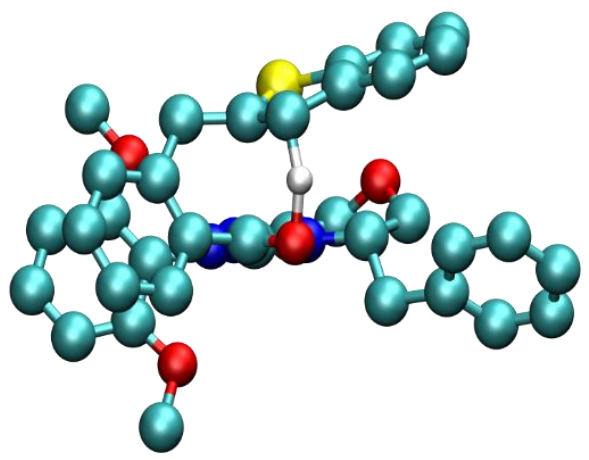

(d) $(R)-\mathbf{T S} 2 \mathbf{r}$

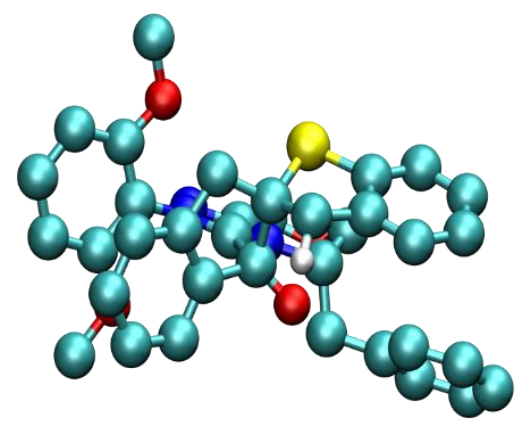

(f) $(R)-\mathbf{T S 3 r}$

Figure S6. Structures according to Scheme S1 and Figure S7. All hydrogen atoms but the transferred one are omitted for clarity. Carbon, hydrogen, nitrogen, oxygen and sulfur are presented in turquoise, white, blue, red and yellow, respectively.

The corresponding a-path structures look very similar (except for the non-existent structures $\mathbf{5 a}$ and TS2a) and are thus not presented here. The relative energies of both pathways are presented in Figure S7. The rate-determining barrier TS1 is of similar height ( $\Delta \mathrm{E} \approx 60 \mathrm{~kJ} / \mathrm{mol}$ ) for both substrates. We also tested for the application of the DFT-D3 ${ }^{[S 27]}$ (applying the "zero"-damping) correction onto the M06-2X energies [evaluated on TPSS-D3(BJ) geometries, cf. Computational details]. We denote these energies as M06-2X-D3(0). All relative M06-2X-D3(0) energies of 
intermediates $\mathbf{4 a} / \mathbf{r}$ through $\mathbf{2 a} / \mathbf{r}+\mathbf{3 a}$ are shifted by $-15 \pm 1 \mathrm{~kJ} / \mathrm{mol}$ (with respect to uncorrected M06-2X energies). The activation barrier $\Delta \mathrm{E}$ did not change upon application of the $\mathrm{D} 3(0)$ correction for the a- and r-pathways. This also holds for the qualitative picture of the resulting reaction pathways. The $\mathrm{D} 3(0)$ correction hence only shifts the energies relative to the separated reactants.

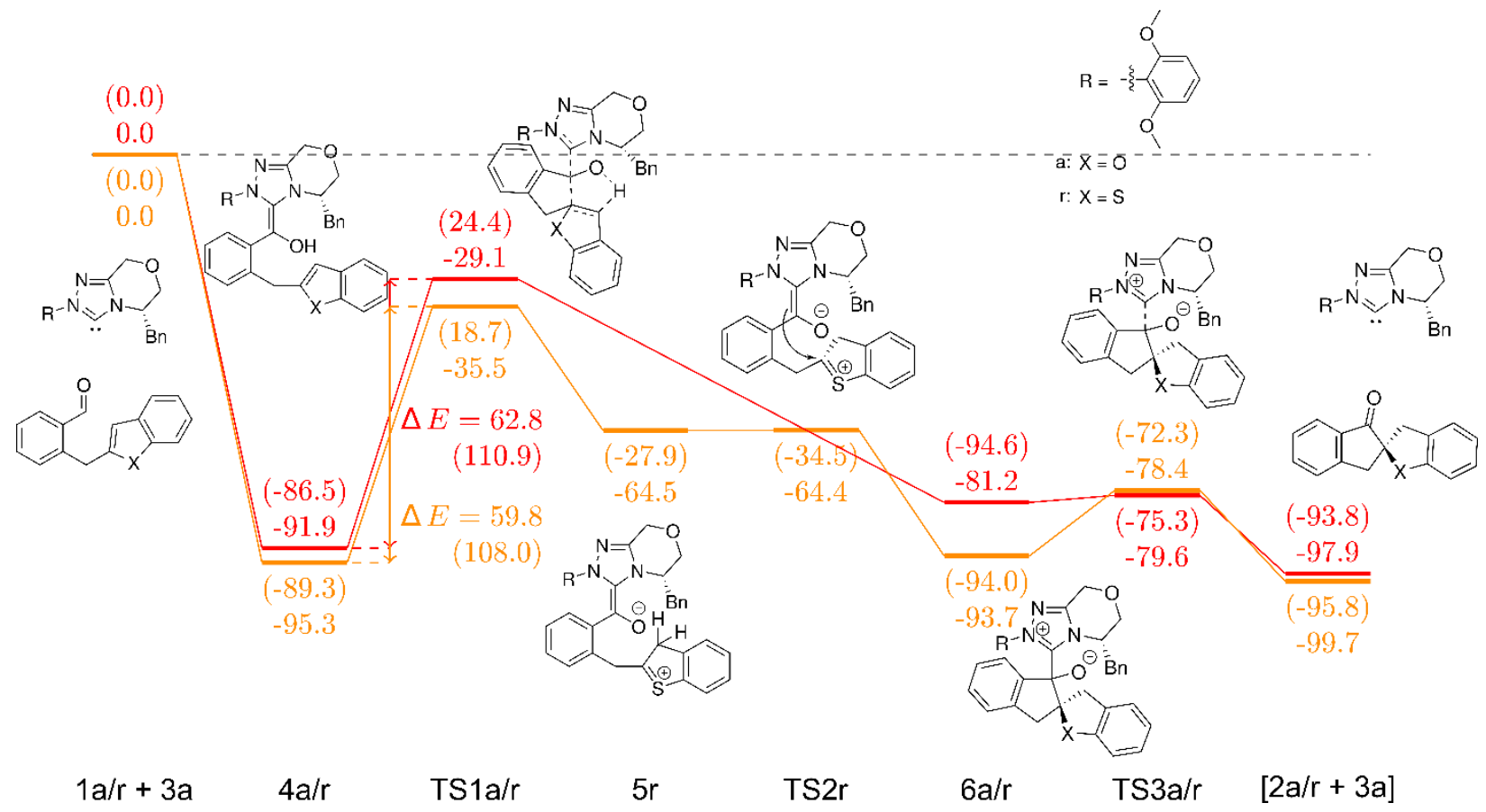

Figure S7. Energy profile of the (R)-reaction pathways for the a- and $\mathbf{r}$-path in red and orange, respectively. Relative RI-TPSS-D3(BJ)/def2-TZVP (M06-2X/def2-TZVP in parentheses) energies as well as activation barriers $\Delta \mathrm{E}$ are given in units of $\mathrm{kJ} / \mathrm{mol}$. All energies are given with respect to the separated reactants $\mathbf{1 a}+\mathbf{3 a}$ and $\mathbf{1 r}+\mathbf{3 a}$, respectively. $[\mathbf{2} \mathbf{a} / \mathbf{r}+\mathbf{3 a} \mathbf{a}]$ describes van-derWaals complexes of carbene $\mathbf{3 a}$ and the product spirocycle $\mathbf{2 a} / \mathbf{r}$.

For the benzofuran species we were able to identify a first order transition state (see Figure S8) that has a relative energy of $-59.4 \mathrm{~kJ} / \mathrm{mol}$. However, all attempts to optimize an intermediate corresponding to 5a (in analogy to 5r) as well as an IRC calculation and manual displacements along the mode with imaginary frequency $\left(\mathrm{i} 114 \mathrm{~cm}^{-1}\right)$ always led to product $\mathbf{6 a}$. Therefore, this transition state is not further considered. 


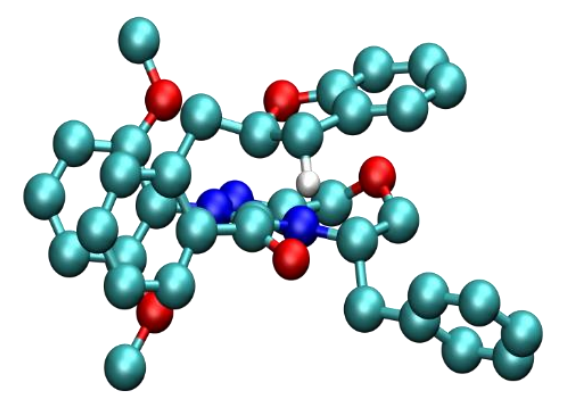

Figure S8. Structure of first-order transition state. All hydrogen atoms but the transferred one are omitted for clarity. Carbon, hydrogen, nitrogen, oxygen and sulfur are presented in turquoise, white, blue, red and yellow, respectively.

\section{4 (R)-Pathway involving NHC 3d}

We identified an analogous reaction pathway for the dearomatization reaction involving NHC 3d. The corresponding rate-determining structures are presented in Figure S9.
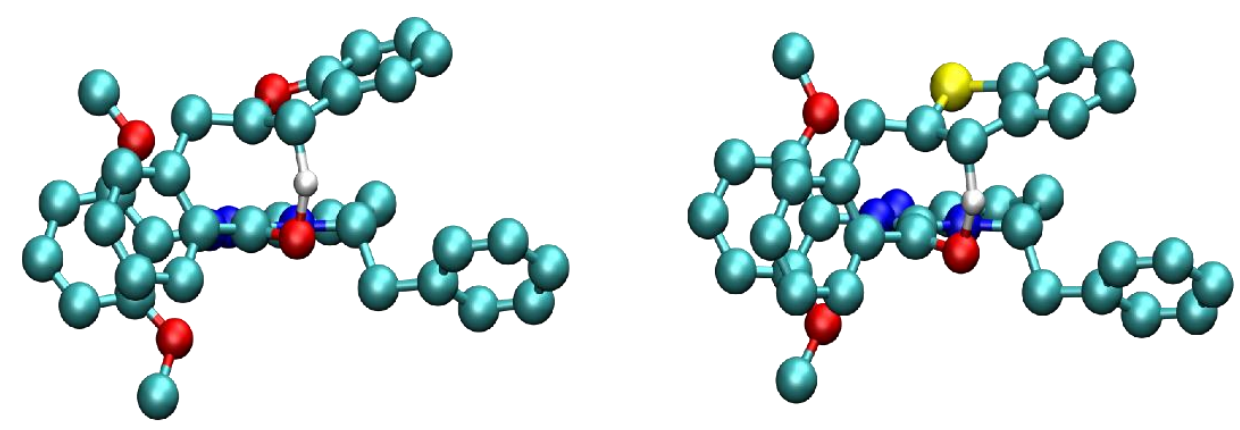

Figure S9. First transition state for the a-path (TS1a, left) and r-path (TS1r, right) involving NHC 3d. All hydrogen atoms except the transferred one are omitted for clarity. Carbon, hydrogen, nitrogen, oxygen and sulfur are presented in turquoise, white, blue, red and yellow, respectively.

The orientation of the heteroaromatic residues in the first-order transition states TS1a (imag. frequency $=\mathrm{i} 1210 \mathrm{~cm}^{-1}$ ) and $\mathbf{T S 1 r}$ (imag. frequency $=\mathrm{i} 1195 \mathrm{~cm}^{-1}$ ) are very similar to the transitions states involving NHC 3a (vide supra). The normal modes corresponding to the respective imaginary frequency are dominated by the movement of the transferred hydrogen. The resulting reaction pathways are presented in Figure S10. The activation barriers for the two different NHCs investigated here (3a and 3d) are very similar (roughly $60 \mathrm{~kJ} / \mathrm{mol}$ ). We could not identify any species $\mathbf{5 a} / \mathbf{r}$ for structures involving $\mathbf{3 d}$. 


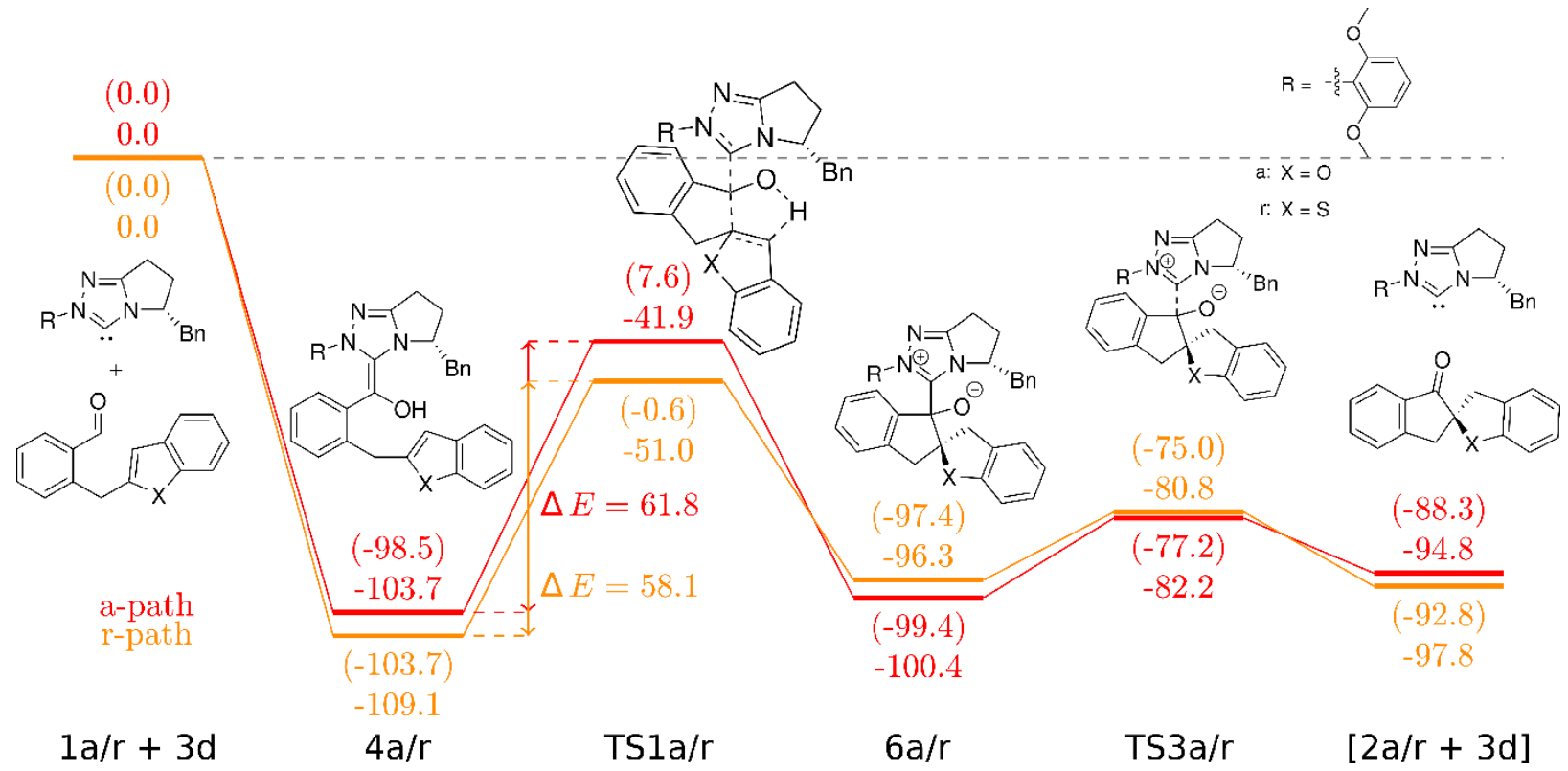

Figure S10. Energy profile of the $(R)$-reaction pathways for the a- and $\mathbf{r}$-path in red and orange, respectively. Relative RI-TPSS-D3(BJ)/def2-TZVP (M06-2X/def2-TZVP in parentheses) energies as well as activation barriers $\Delta \mathrm{E}$ are given in units of $\mathrm{kJ} / \mathrm{mol}$. All energies are given with respect to the separated reactants $1 \mathbf{a}+\mathbf{3 d}$ and $\mathbf{1 r}+\mathbf{3 d}$, respectively. $[\mathbf{2 a} / \mathbf{r}+\mathbf{3 d}]$ describes van-derWaals complexes of carbene $\mathbf{3 d}$ and the product spirocycle $\mathbf{2} \mathbf{a} / \mathbf{r}$.

\subsection{Alternative Pathways}

Apart from the reaction mechanisms discussed above, we also investigated alternative reaction pathways as described in the following for NHC 3a. An alternative pathway can be envisioned as shown in Scheme S2. The first transition (TS4a/r) corresponds to a concerted formation of the new C-C-bond and release of the NHC. Therefore, the proton transfer would have to take place in the products $\mathbf{7 a} / \mathbf{r}$. We were able to identify a transition state $\mathbf{T S 4 a}$ that is around $15 \mathrm{~kJ} / \mathrm{mol}$ lower in relative energy than the previously discussed TS1a. The normal mode corresponding to the imaginary frequency (i $348 \mathrm{~cm}^{-1}$ ) shows the movement of the desired carbons forming the spirocycle while also cleaving the NHC bond. 
<smiles>[X]c1ccccc1Cc1ccccc1C=O</smiles><smiles>[X]c1ccccc1C1(C)Cc2ccccc2C1=O</smiles>

$2 \mathrm{a}, 2 \mathrm{r}$<smiles>[Y]c1cc2ccccc2cc1Cc1ccccc1/C(O)=C1/N([Y6])N=C2COCC(Br)N21</smiles>

$4 a, 4 r$

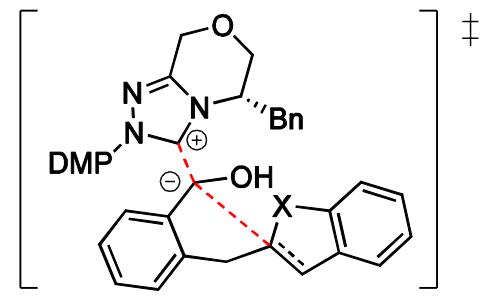

TS4a, TS4r

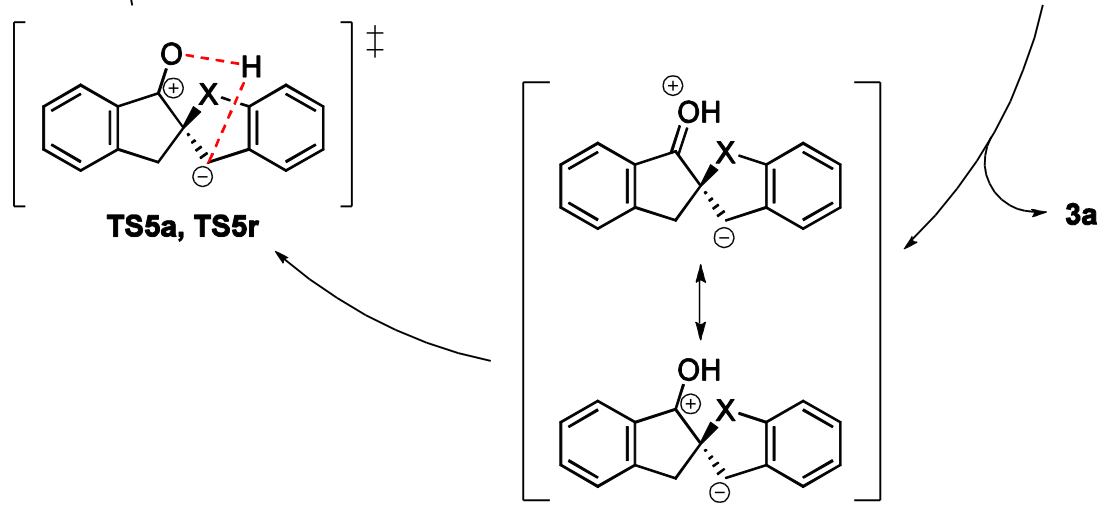

$7 a, 7 r$

Scheme S2. Alternative stepwise pathway for this reaction ivolving first the NHC cleavage followed by an intramolecular proton transfer.

However, this transition state could not be verified as a meaningful transition state connecting two different minima that are lower in energy than TS4a. By manually displacing very far along the normal mode corresponding to the imaginary frequency (and subsequent geometry optimization) we could identify another minimum on the PES, which was however $\sim 100 \mathrm{~kJ} / \mathrm{mol}$ higher in energy than TS4a and involved a tricyclic structure (see Figure S11). All other geometry optimizations starting from an IRC calculation or manual displacements converged to starting material 4a. No similar transition state could be identified for the benzothiophene analogue. 


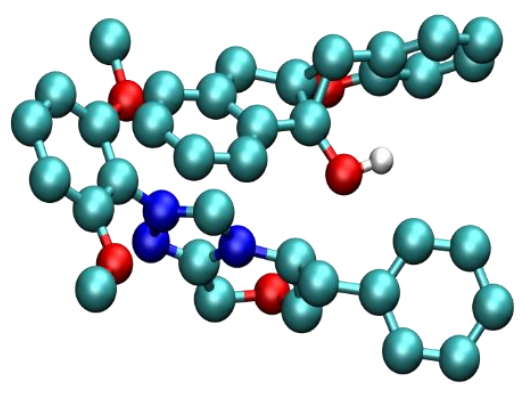

Figure S11. Tricyclic minimum-energy structure found by manually displacing very far along normal mode corresponding to the imaginary frequency of TS4a and subsequent geometry optimization. All hydrogen atoms but the transferred one are omitted for clarity. Carbon, hydrogen, nitrogen, oxygen and sulfur are presented in turquoise, white, blue, red and yellow, respectively.

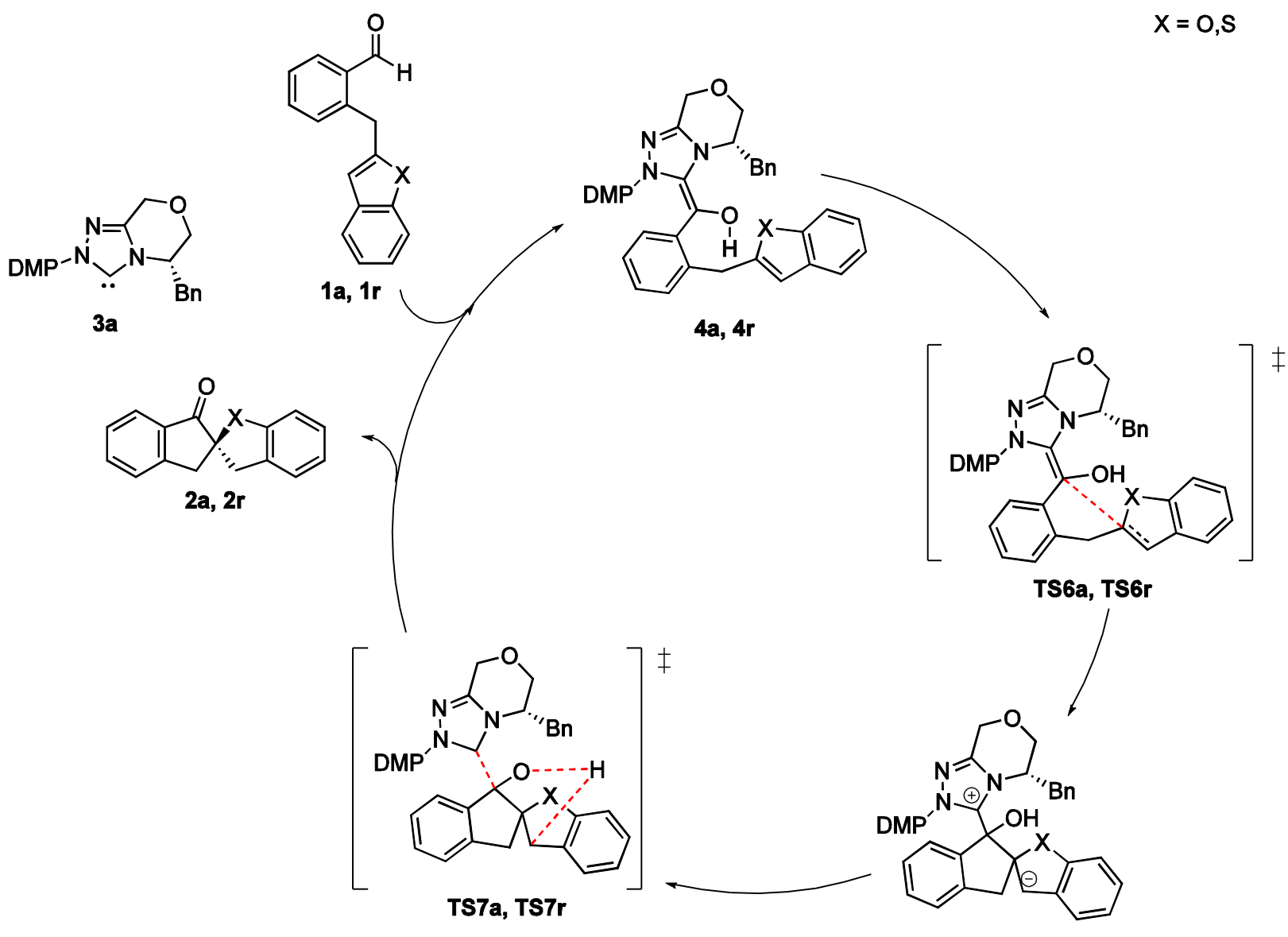

$8 a, 8 r$

Scheme S3. Alternative stepwise pathway for this reaction with C-C-bond formation as first step followed by an intramolecular proton transfer and cleavage of the C-C bond to the $\mathrm{NHC}$ residue. 
Additionally, the mechanism presented in Scheme S3 has been considered. Transition state TS6a/r solely involves the carbon-carbon bond formation leading to the spirocycle $\mathbf{8 a} / \mathbf{r}$ without any involvement of the previously discussed proton transfer. However, in both cases such a transition could not be identified. It was even impossible to optimize a stationary point corresponding to 8a/r: We generated pre-optimized spirocyclic geometries $8 \mathbf{a}$ and $8 \mathbf{r}$ by fixing the carbon-carbon distance. Unconstrained optimizations starting from these geometries always converged to structures $4 \mathrm{a}$ and $4 \mathrm{r}$, respectively. Thus, the proton transfer seems to play a major role in the initiation of this reaction and stabilization of the resulting spirocyclic structures. Finally, we considered a concerted mechanism that would combine all three steps in one, i.e., proton transfer, carbon-carbon bond formation and release of the NHC. The resulting catalytic cycle is described in Scheme S4. Since no transition state similar to TS8 could be identified, this type of mechanism is discarded here.

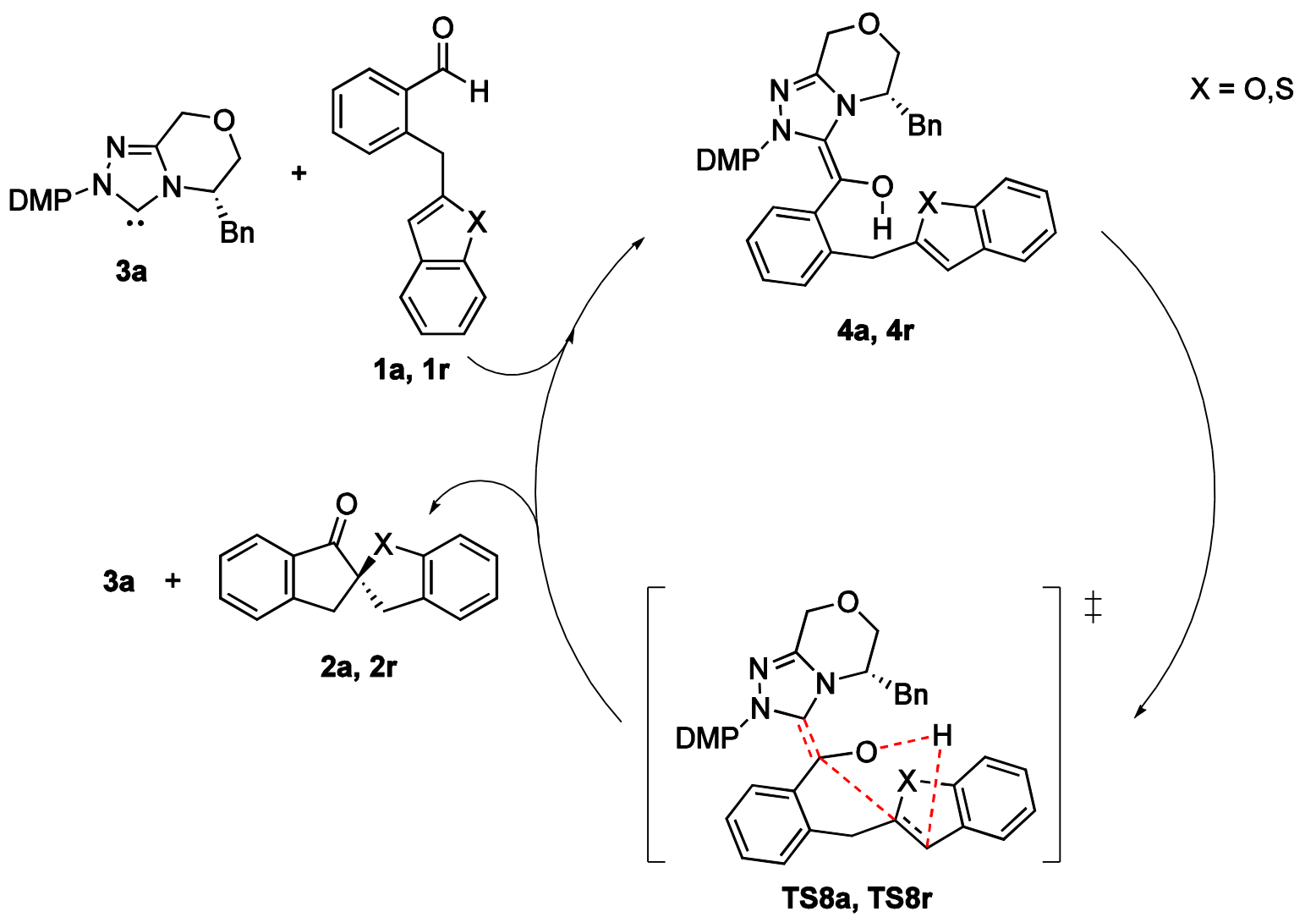

Scheme S4. Alternative concerted pathway for this reaction. 


\subsection{Attack on the sterically more demanding side}

A possible mechanism leading to the $(S)$-products is by a proton transfer on the sterically more demanding side. We assume that the mechanism for the respective $(S)$-Breslow intermediates is similar to Scheme S1. The most important first-order transition states are presented below for the a- and r-path in Figure S13. The transition states comprise one imaginary frequency of i 1279 and i $1310 \mathrm{~cm}^{-1}$, respectively. Similar to the transition states $(R)$-TS1a and $(R)$-TS1r the transitions are dominated by the movement of the transferred hydrogen. However, we could not identify any species corresponding to $(S)$-5a or $(S)$-5r resulting in concerted $\mathbf{a}$ - or $\mathbf{r}$-pathways. Compared to the $(R)$-pathways the activation barriers for the $(S)$-pathways are significantly higher due to the steric interaction of the benzyl group of the chiral NHC with the heteroaromatic residue.

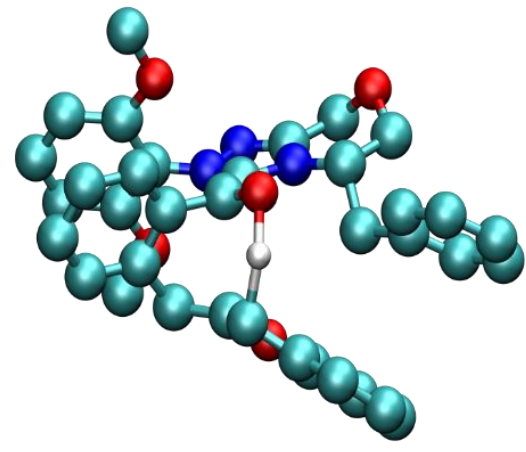

(a) (S)-TS1a

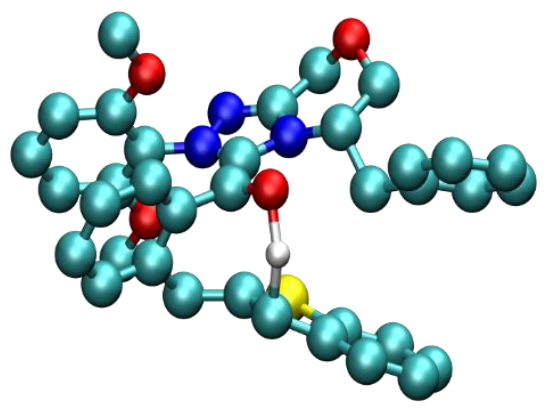

(b) (S)-TS1a

Figure S12. First transition state for the a-path [(S)-TS1a] and r-path [(S)-TS1r]. All hydrogen atoms but the transferred one are omitted for clarity. Carbon, hydrogen, nitrogen, oxygen and sulfur are presented in turquoise, white, blue, red and yellow, respectively. 


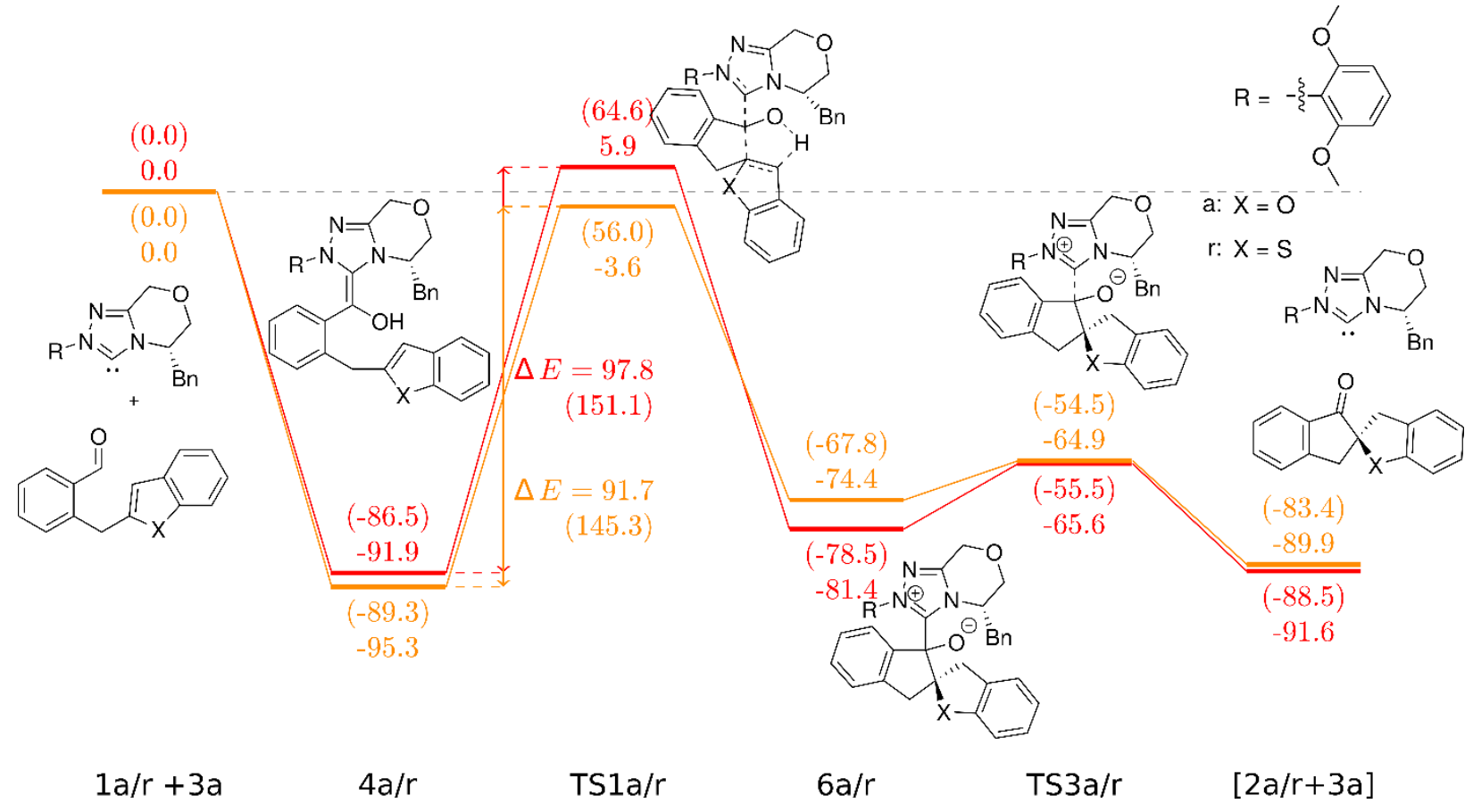

Figure S13. Energy profile of the $(S)$-reaction pathways for the a- and $\mathbf{r}$-path in red and orange, respectively. Relative RI-TPSS-D3(BJ)/def2-TZVP (M06-2X/def2-TZVP in parentheses) energies as well as activation barriers $\Delta \mathrm{E}$ are given in units of $\mathrm{kJ} / \mathrm{mol}$. All energies are given with respect to the separated reactants $\mathbf{1 a}+\mathbf{3 a}$ and $\mathbf{1 r}+\mathbf{3 a}$, respectively. $[\mathbf{2 a} / \mathbf{r}+\mathbf{3 a}]$ describes van-derWaals complexes of carbene $\mathbf{3 a}$ and the product spirocycle $\mathbf{2 a} / \mathbf{r}$.

\subsection{1 (S)-Pathway involving NHC 3d}

Also for the sterically more demanding site the reaction pathway according to Scheme S1 was identified with NHC 3d.
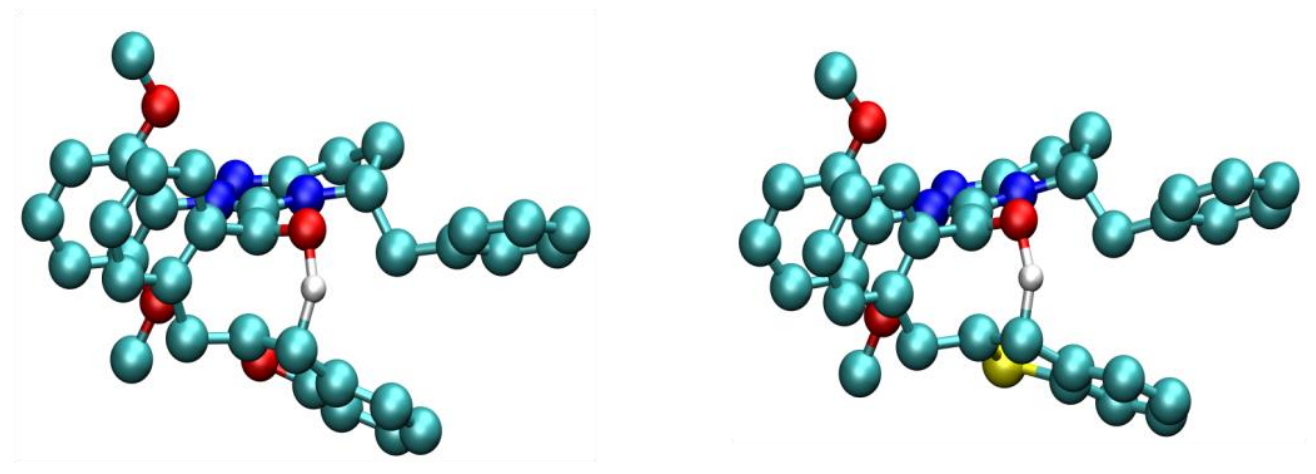

Figure S14. First transition state for the a-path [(S)-TS1a] and r-path [(S)-TS1r] with NHC 3d. All hydrogen atoms but the transferred one are omitted for clarity. Carbon, hydrogen, nitrogen, oxygen and sulfur are presented in turquoise, white, blue, red and yellow, respectively. 
The first-order transition states for the a- and r-pathways are presented in Figure S14. The imaginary frequencies of TS1a and TS1r are i 1310 and i $1244 \mathrm{~cm}^{-1}$, respectively. The corresponding normal modes are also dominated by the hydrogen movement. IRC calculations and structure optimizations starting therefrom did not reveal any species $\mathbf{5 a} / \mathbf{r}$ but always converged directly to species $\mathbf{6 a} / \mathbf{r}$. The resulting reactionpathways are presented in Figure S15.

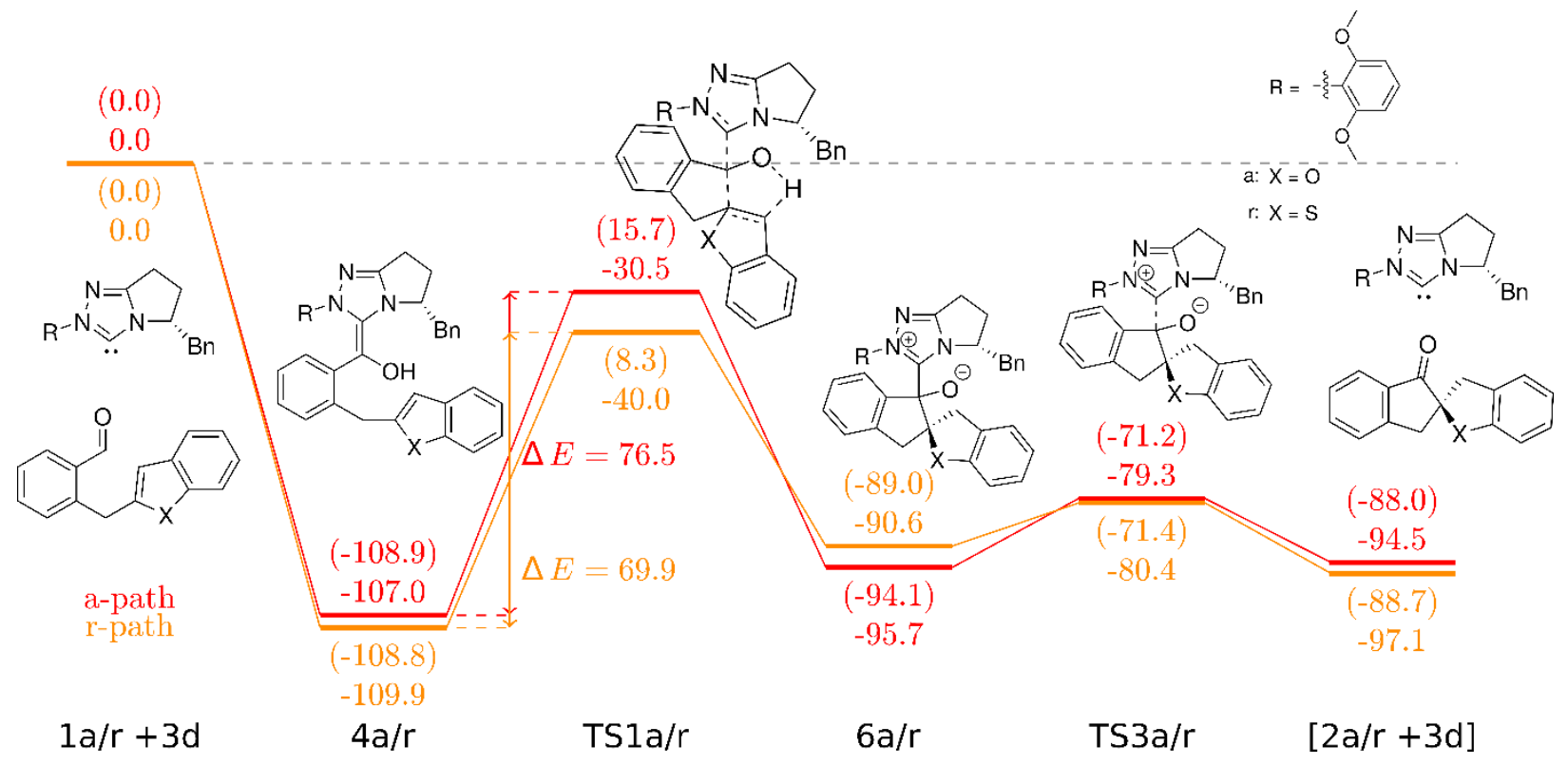

Figure S15. Energy profile of the $(S)$-reaction pathways involving NHC 3d for the a- and r-path in red and orange, respectively. Relative RI-TPSS-D3(BJ)/def2-TZVP (M06-2X/def2-TZVP in parentheses) energies as well as activation barriers $\Delta \mathrm{E}$ are given in units of $\mathrm{kJ} / \mathrm{mol}$. All energies are given with respect to the separated reactants $\mathbf{1 a}+\mathbf{3 d}$ and $\mathbf{1 r}+\mathbf{3 d}$, respectively. $[\mathbf{2 a} / \mathbf{r}+\mathbf{3 d}$ ] describes van-der-Waals complexes of carbene $\mathbf{3 d}$ and the product spirocycle $\mathbf{2} \mathbf{a} / \mathbf{r}$.

The activation barriers for this reaction (see Figure S15) are significantly lower to the analogous reaction involving NHC 3a (cf. Figure S13). 


\subsection{Conclusions}

We studied several pathways for the NHC-catalyzed dearomatization of benzofuran 1a and benzothiophene 1r. In both cases and independent of the NHC (3a or 3d) in use, the hydrogen transfer from the enol to the heteroaromatic residue initiates the reaction. This is in agreement with results from Refs. [S2, S22] for similar NHC-catalyzed reactions. The transition state structures of the hydrogen transfer for the $\mathbf{a}$ - and $\mathbf{r}$-pathways as well as the activation barriers are very similar. For the a-path we exclusively find a concerted one-step process with a highly asymmetric transition state (as in Refs. [S2, S22]), which explains the high enantiomeric excess observed in experiment.

In contrast, with NHC 3a we find a two-step mechanism for the r-path (on the sterically less demanding site) which allows for a rotation around the $\mathrm{C}$-C bond (connecting the 3-position of the benzothiophene to the benzaldehyde moiety). This rotation is possible after the proton transfer, but before the enol carbon forms a bond to the 3-position of the benzothiophene.

With NHC 3d, however, this possibility is not observed. Instead, the reaction barriers on the sterically more demanding side [which would result in $(S)-\mathbf{2 a} / \mathbf{r}$ ] with NHC $\mathbf{3 d}$ are just around $10 \mathrm{~kJ} / \mathrm{mol}$ higher than on the sterically less demanding side.

For NHC 3a other pathways were studied, but did not yield energetically competitive results. The computed reaction pathways nicely reproduce the experimental findings. 
10 NMR Spectra Hydroacylation Products 
(R)-3H-Spiro[benzofuran-2,2'-inden]-1'(3'H)-one (2a)
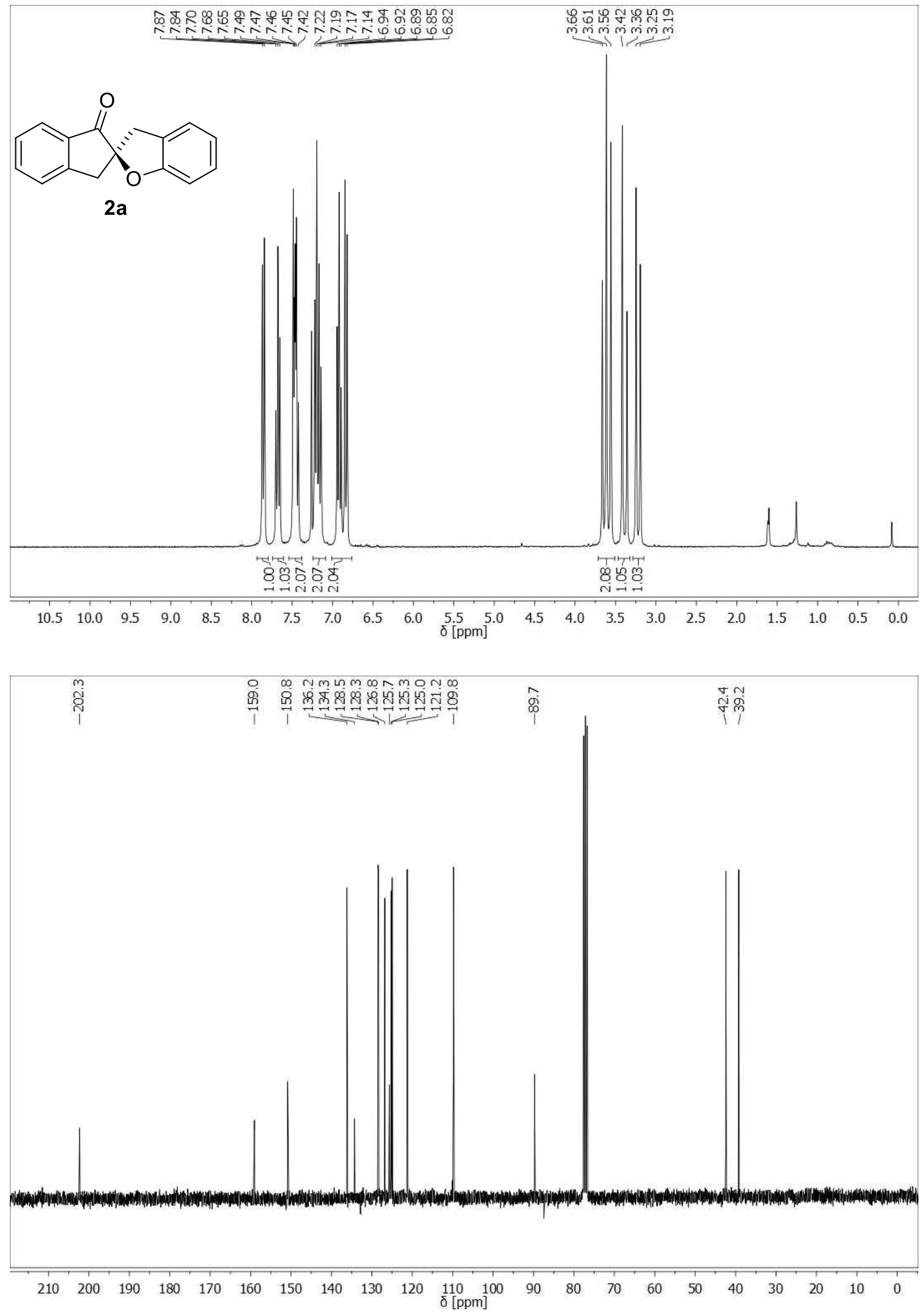
(R)-5'-Fluoro-3H-spiro[benzofuran-2,2'-inden]-1'(3'H)-one (2b)
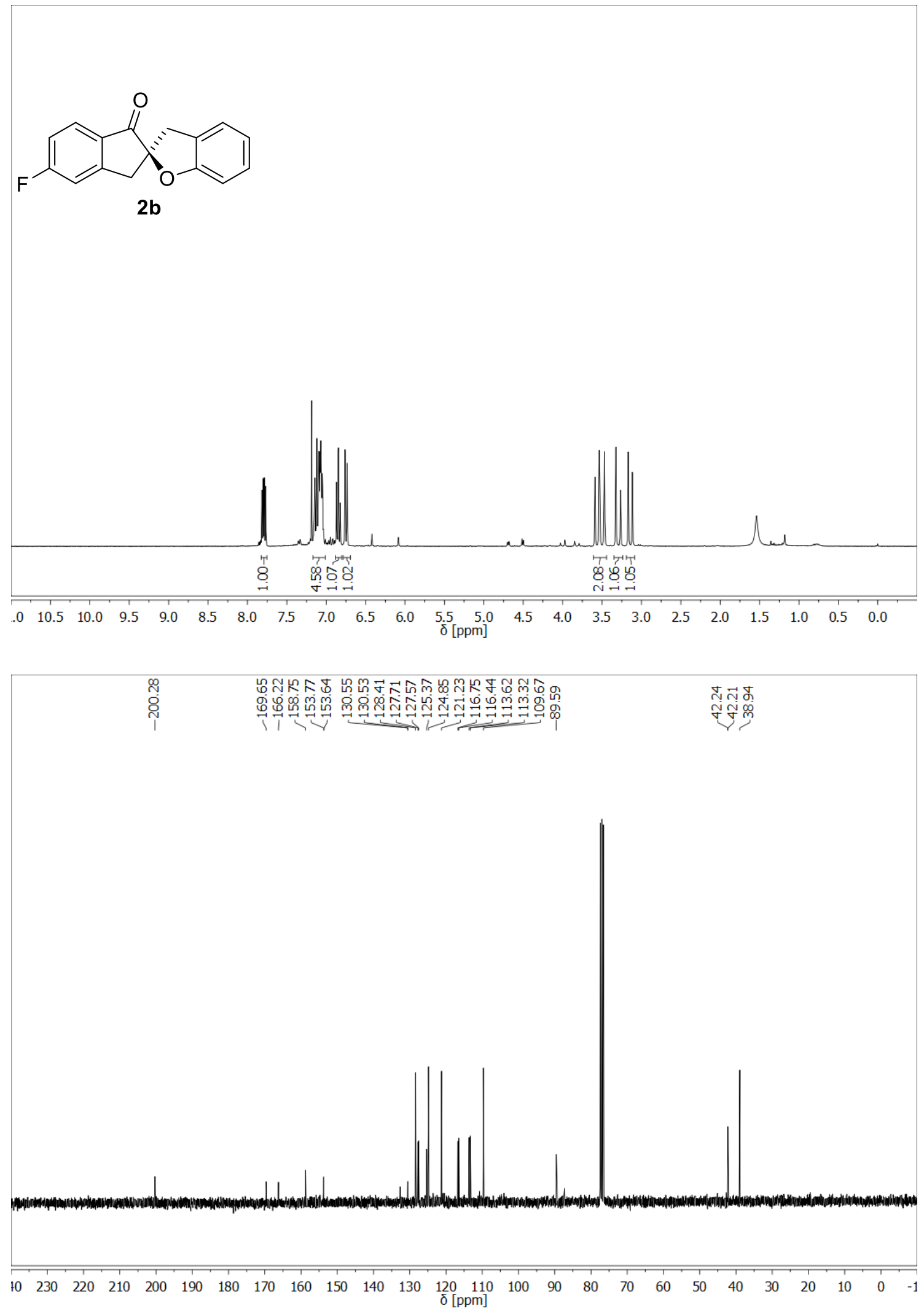
(R)-5'-Chloro-3H-spiro[benzofuran-2,2'-inden]-1' $\left(3^{\prime} \mathrm{H}\right)$-one (2c)
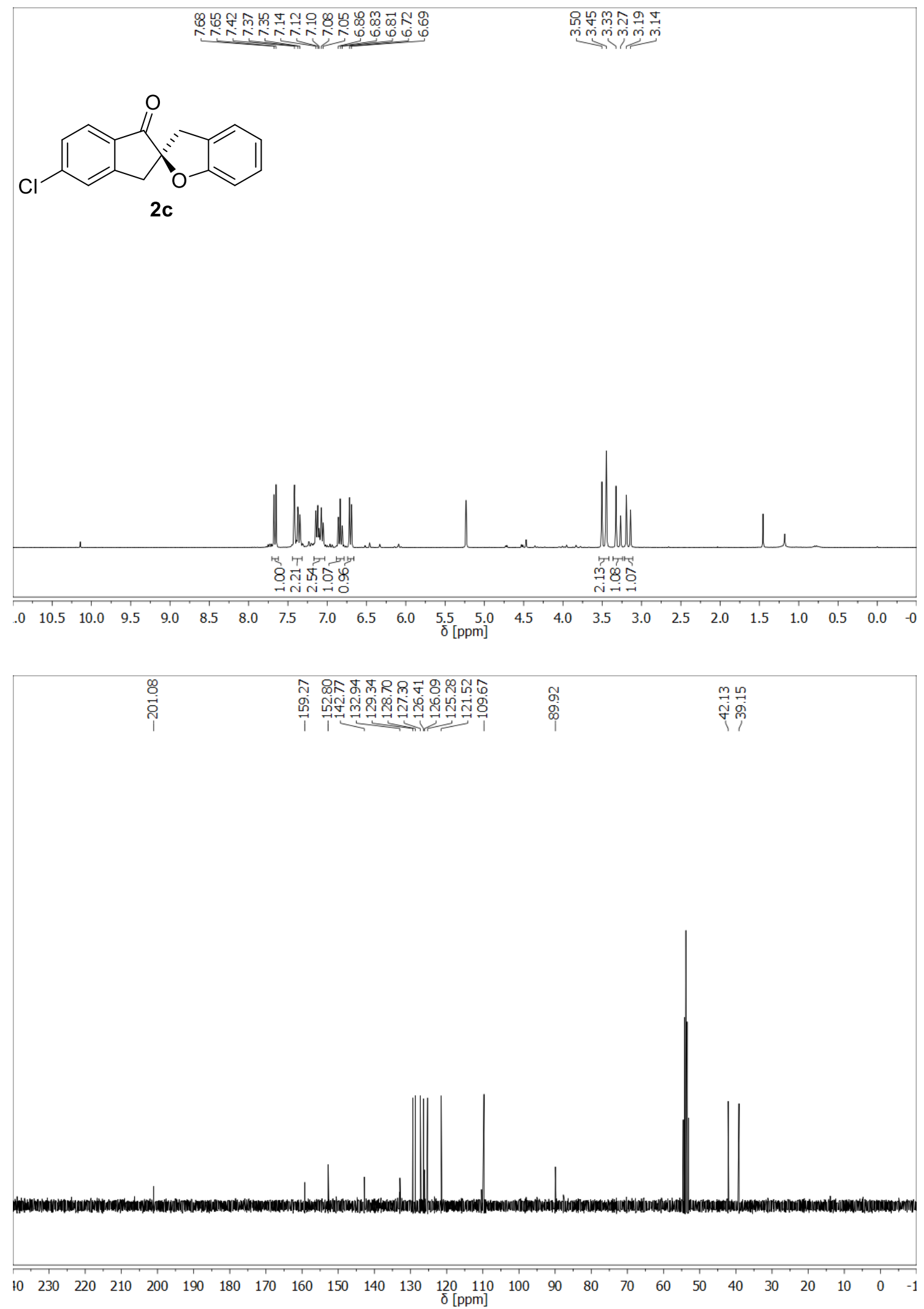
(R)-5'-Phenyl-3H -spiro[benzofuran-2,2'-inden]-1'(3'H)-one (2d)
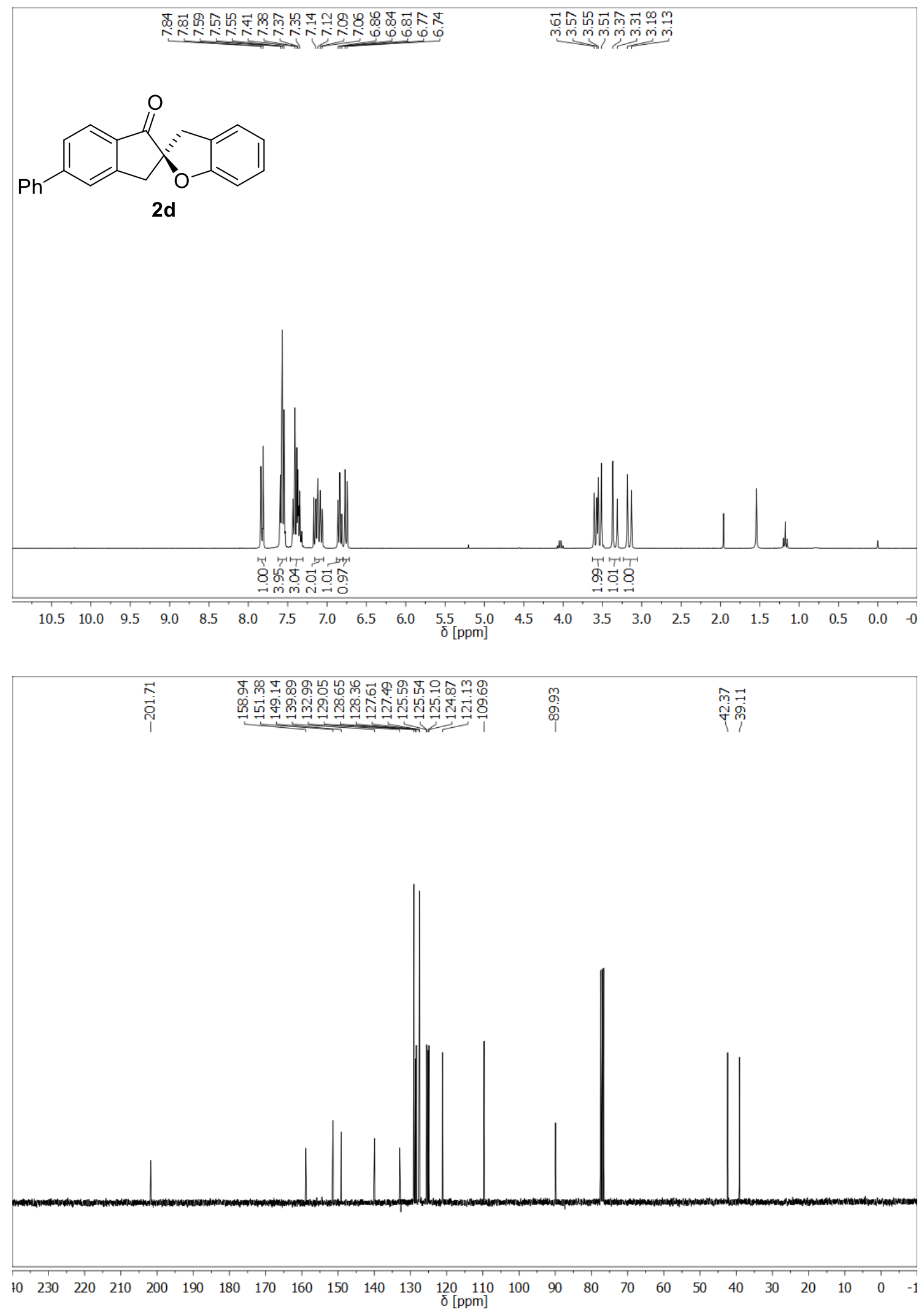
(R)-5-Methyl-3H-spiro[benzofuran-2,2'-inden]-1'(3'H)-one (2e)
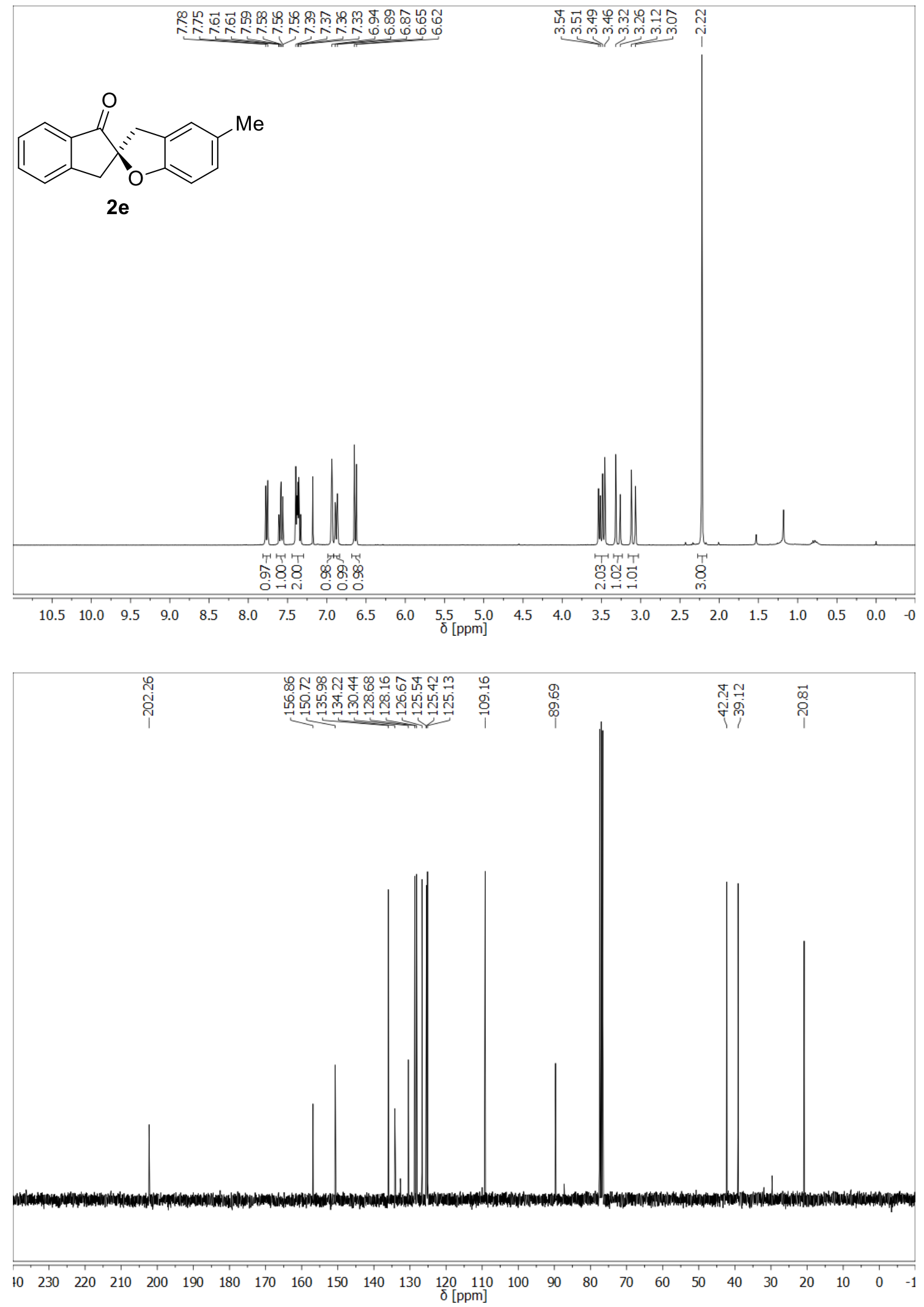
(R)-5-Methoxy-3H-spiro[benzofuran-2,2'-inden]-1'(3'H)-one (2f)
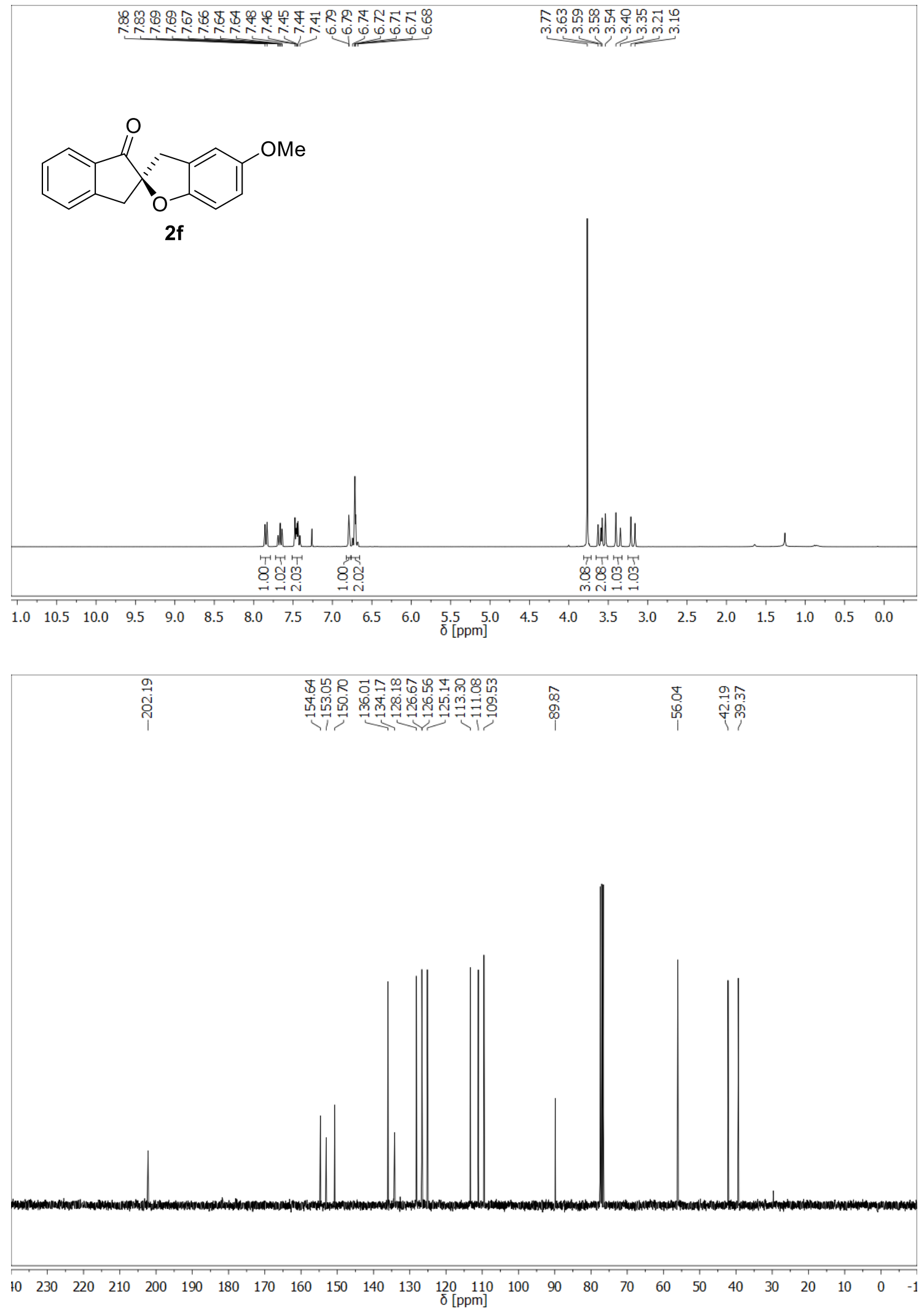
(R)-5-Bromo-3H-spiro[benzofuran-2,2'-inden]-1'(3'H)-one (2g)
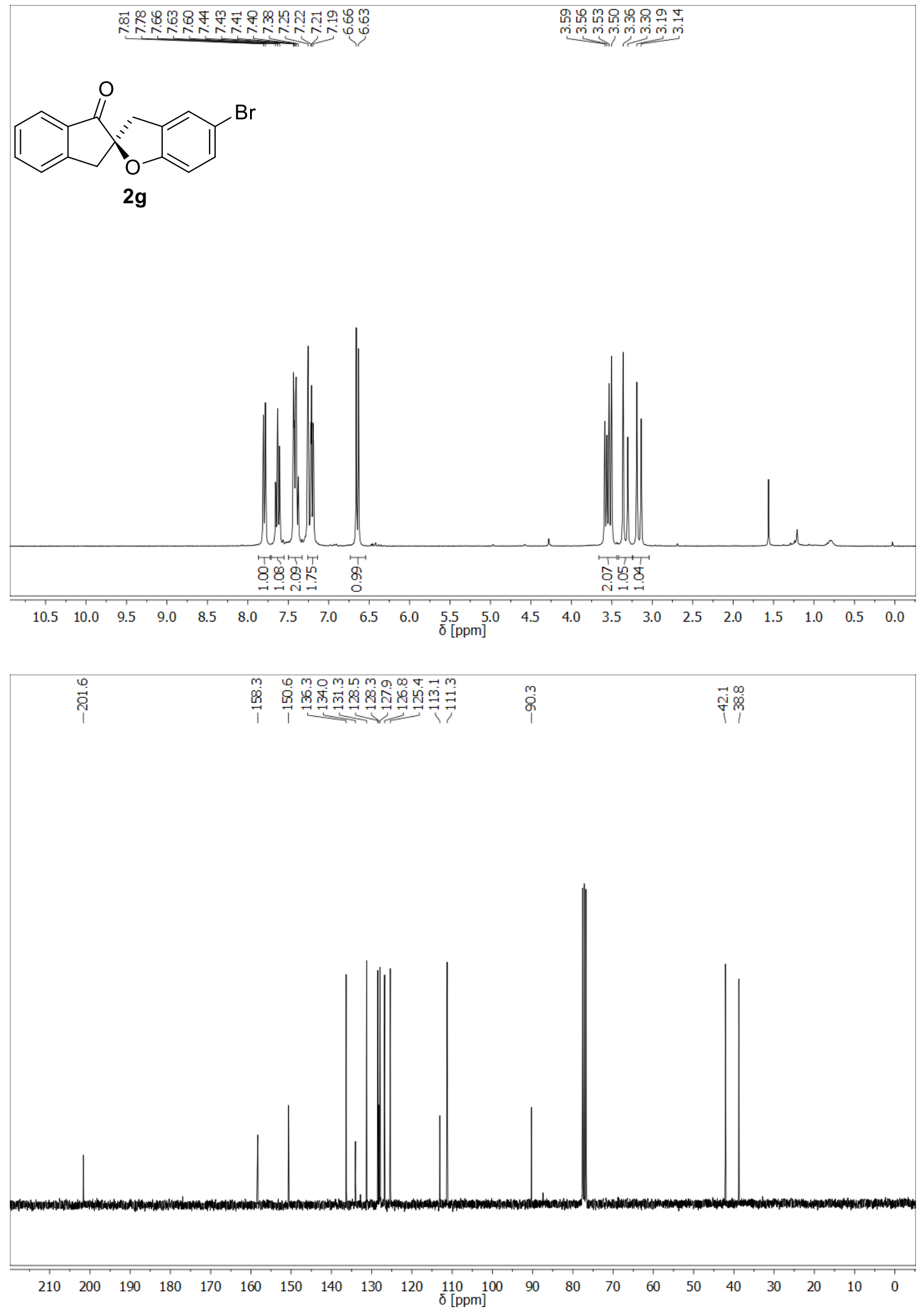


\section{(R)-5-Fluoro-3H-spiro[benzofuran-2,2'-inden]-1'(3'H)-one (2h)}
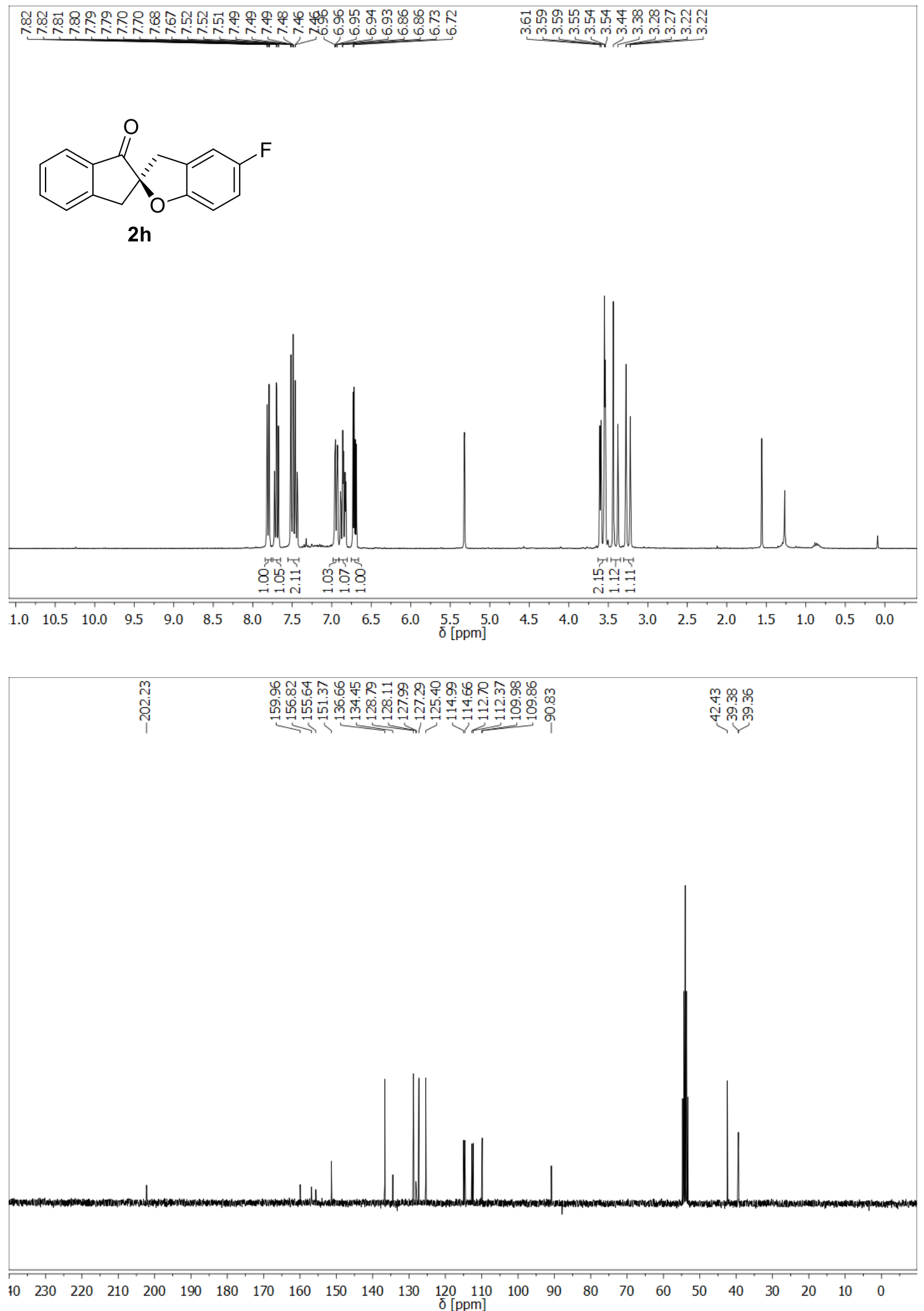
(R)-5-(4-Methoxyphenyl)-3H-spiro[benzofuran-2,2'-inden]-1'(3'H)-one (2i)
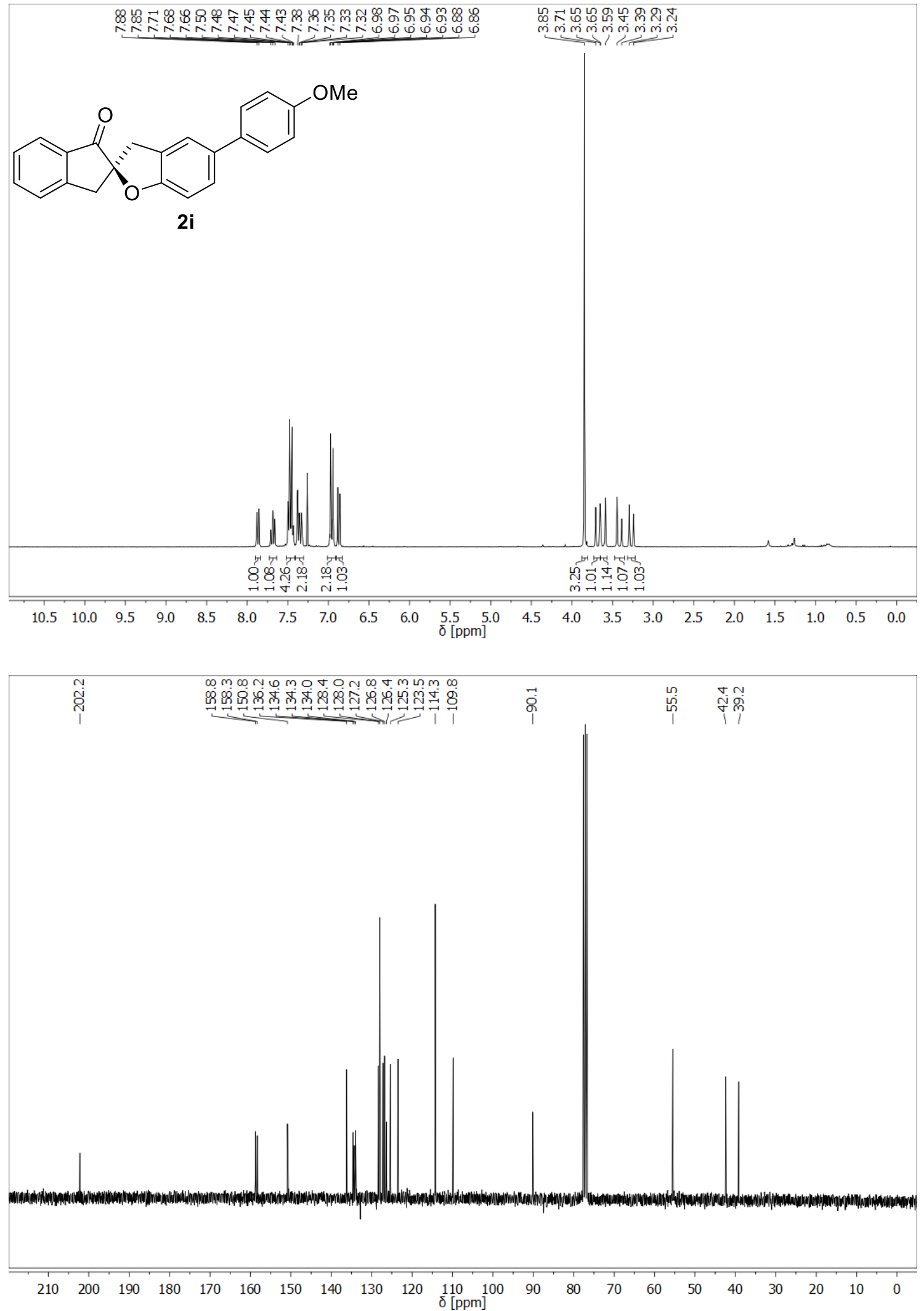
(R)-5-(4-(tert-Butyl)phenyl)-3H-spiro[benzofuran-2,2'-inden]-1'(3'H)-one (2j)
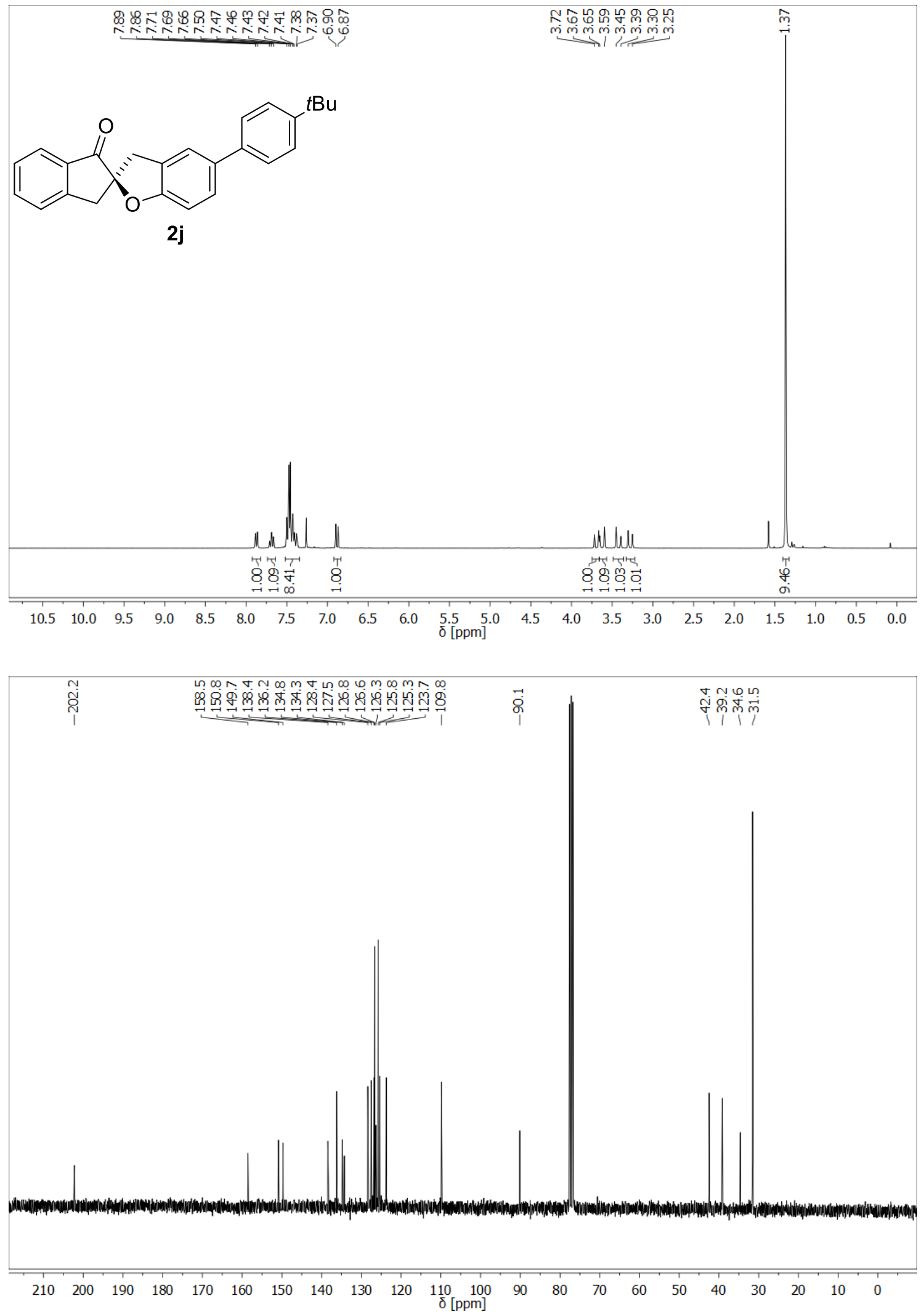
(R)-5-Phenyl-3H-spiro[benzofuran-2,2'-inden]-1'(3'H)-one (2k)
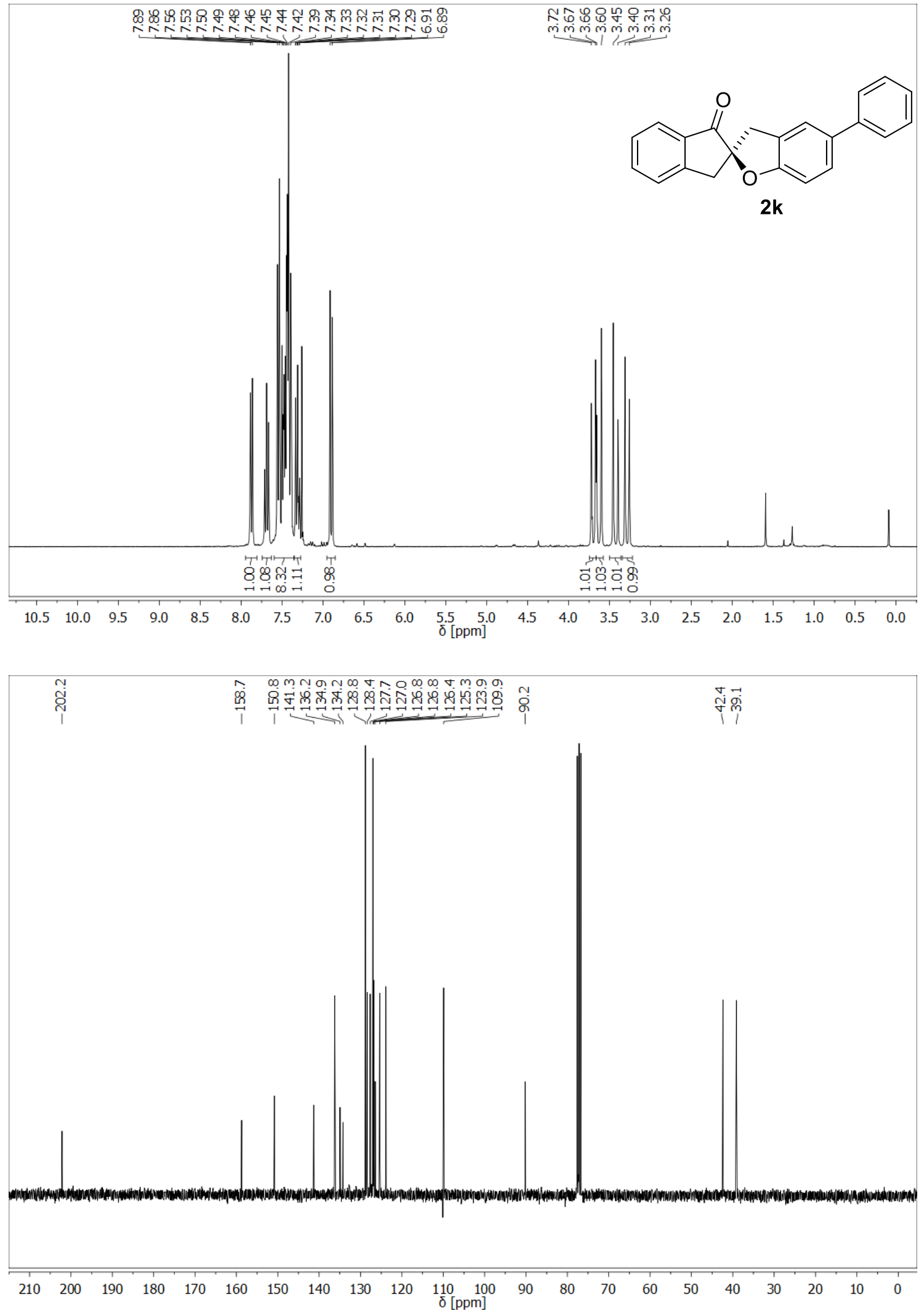
(R)-5-(4-(Hydroxymethyl)phenyl)-3H-spiro[benzofuran-2,2'-inden]-1'(3'H)-one (2I)
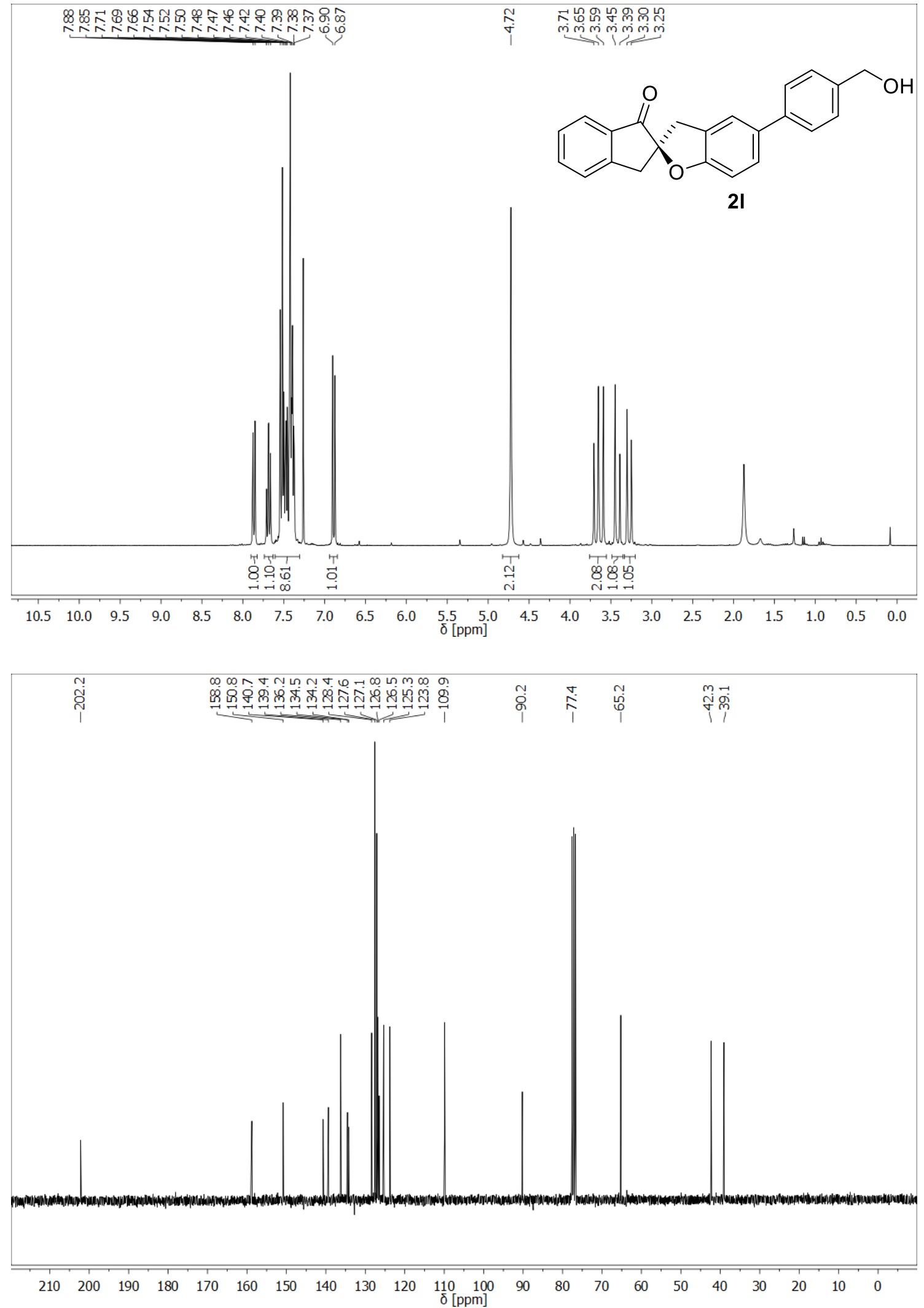
(R)-5-(4-(Trifluoromethoxy)phenyl)-3H-spiro[benzofuran-2,2'-inden]-1'(3'H)-one (2m)
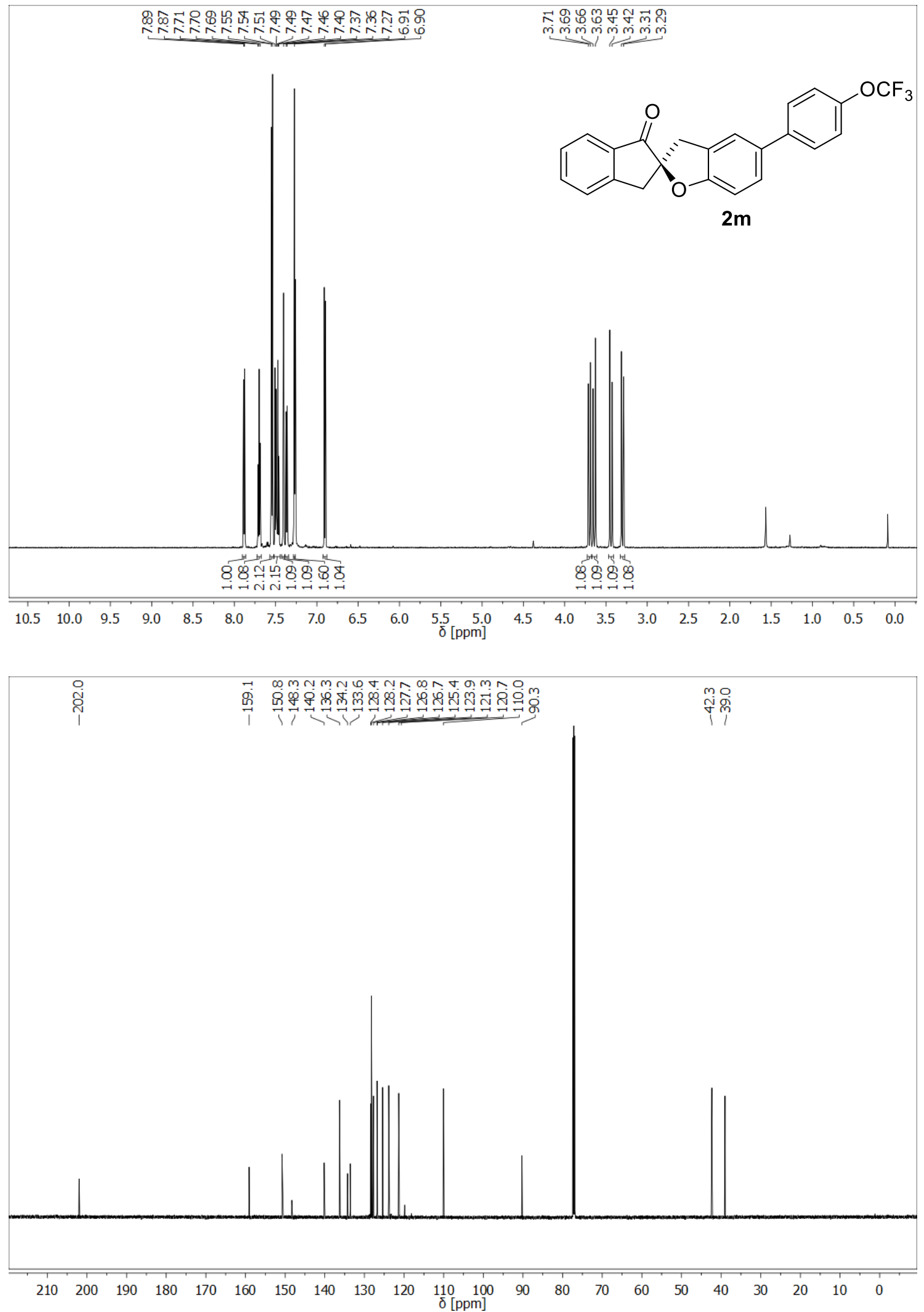
(R)-5-(4-Chlorophenyl)-3H-spiro[benzofuran-2,2'-inden]-1'(3'H)-one (2n)
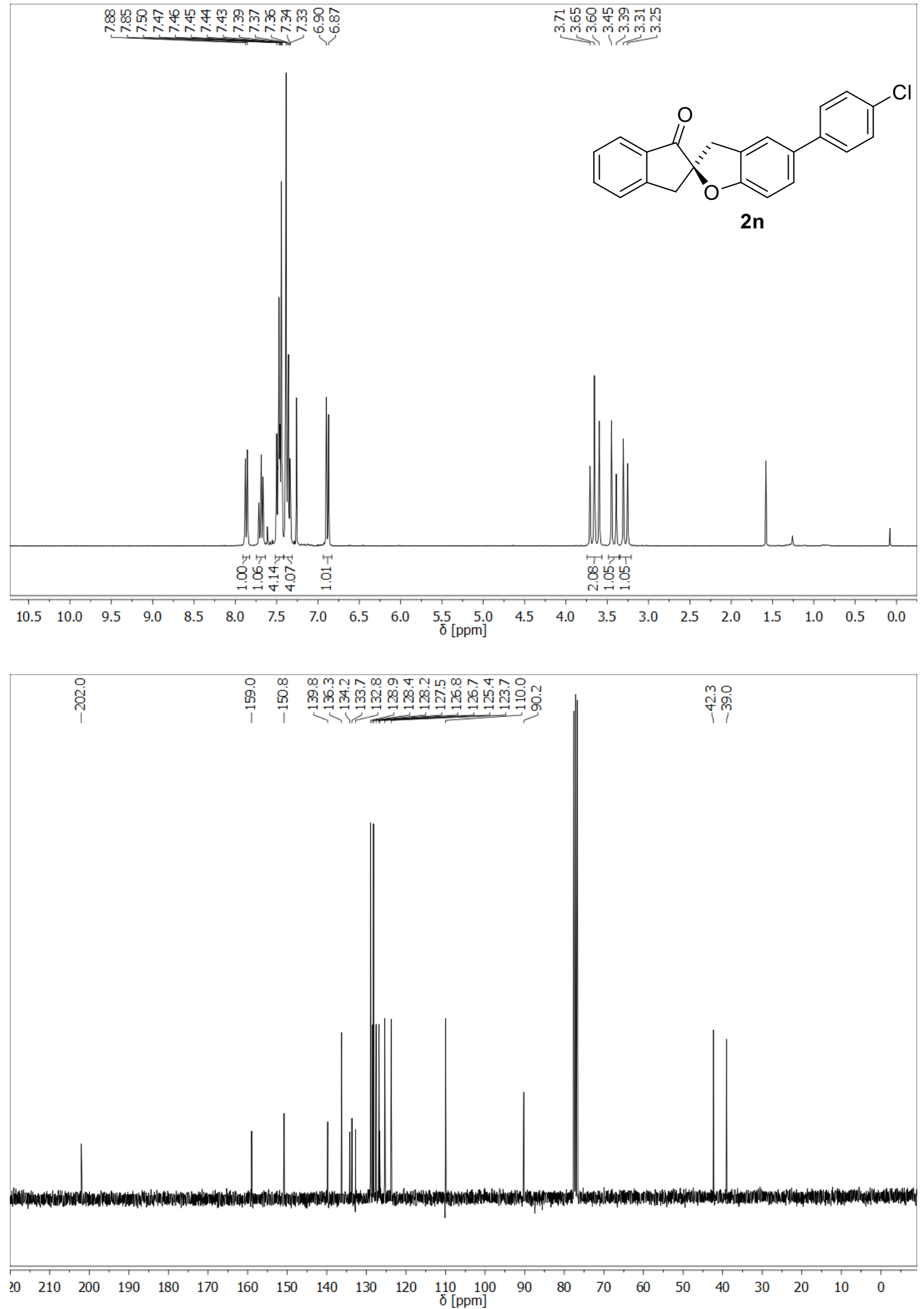
(R)-5-(4-(Trifluoromethyl)phenyl)-3H-spiro[benzofuran-2,2'-inden]-1'(3'H)-one (2o)
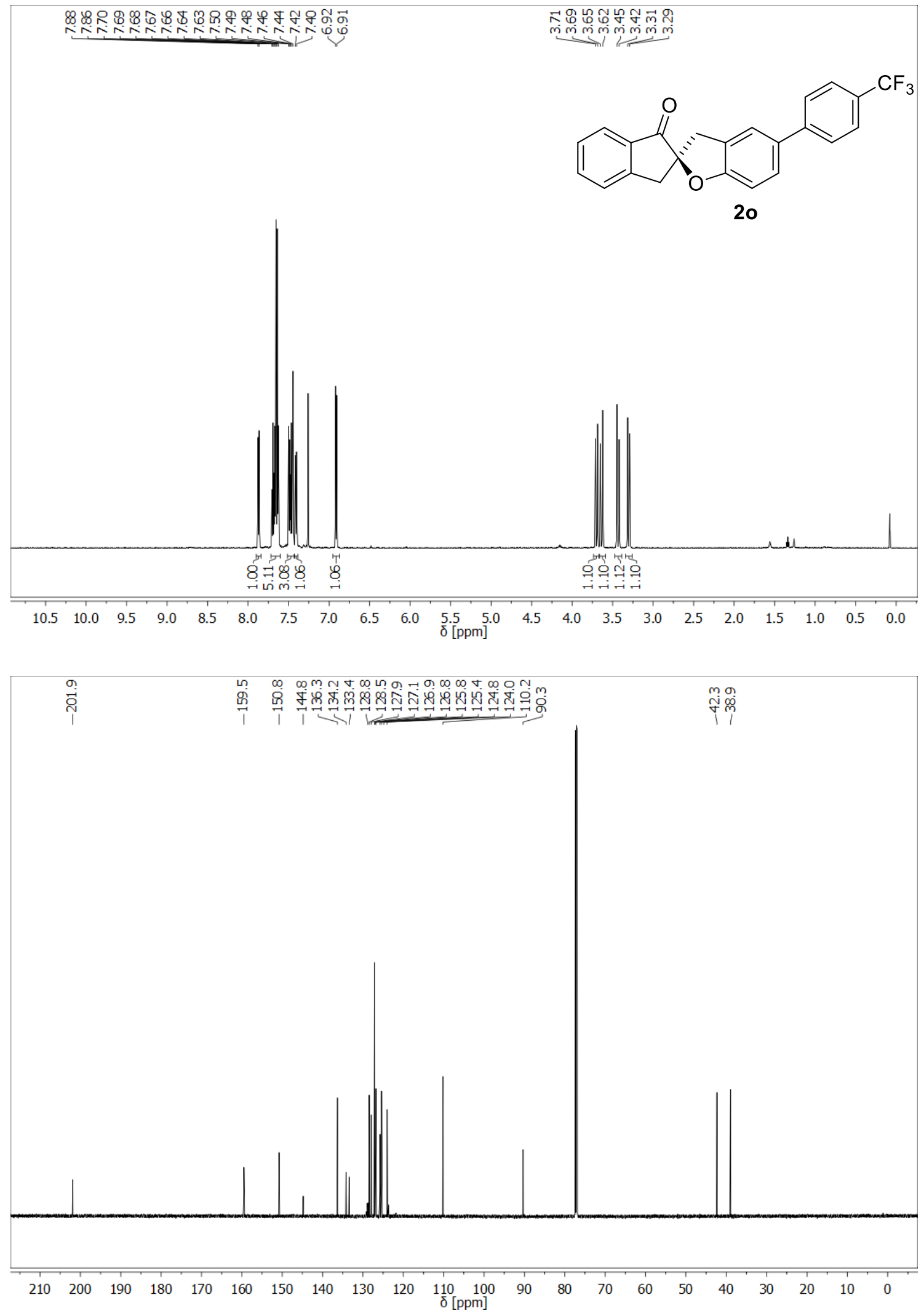
(R)-5-(4-Acetylphenyl)-3H-spiro[benzofuran-2,2'-inden]-1'(3'H)-one (2p)
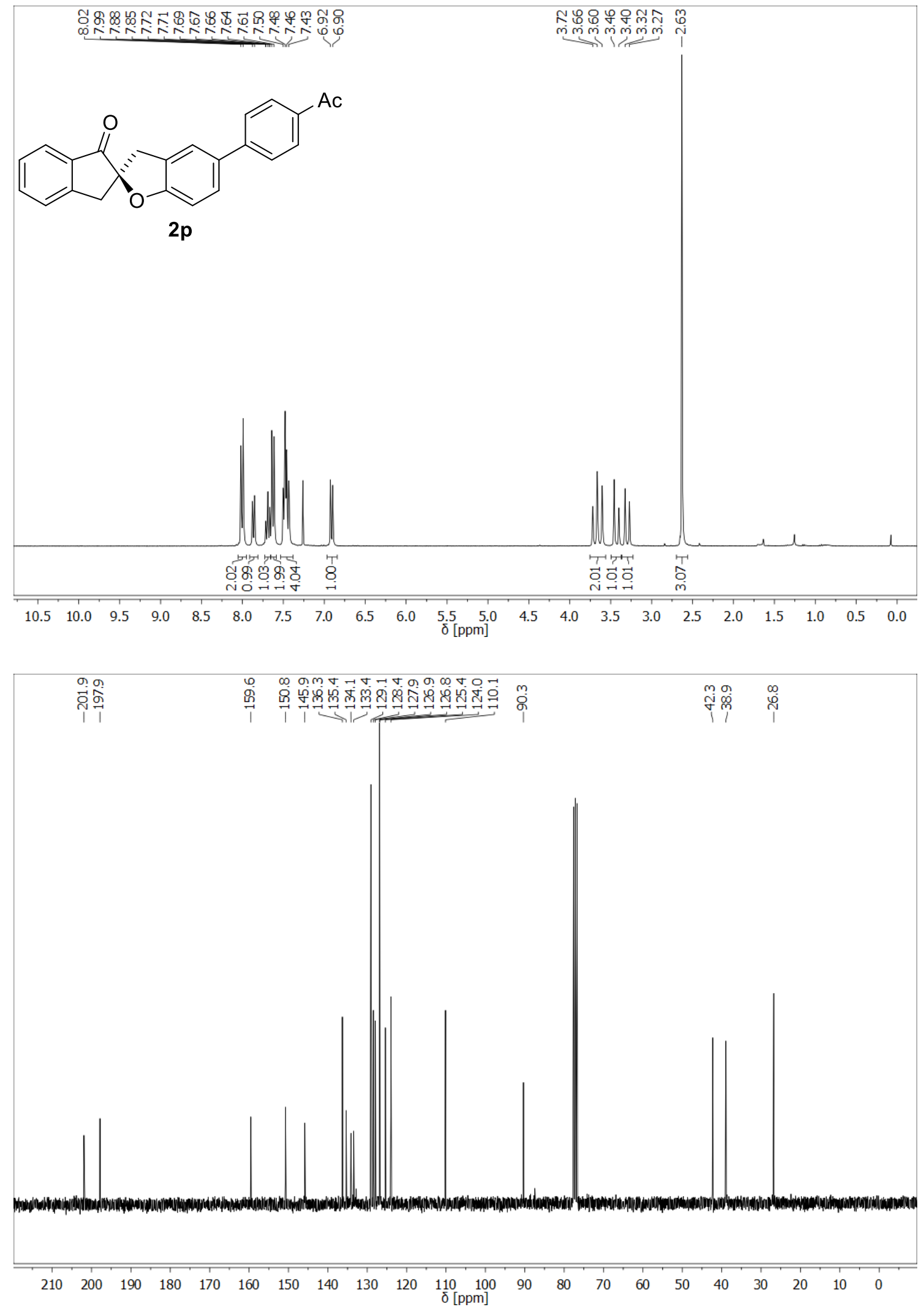
(R)-4-(1'-Oxo-1',3'-dihydro-3H-spiro[benzofuran-2,2'-inden]-5-yl)benzonitrile (2q)
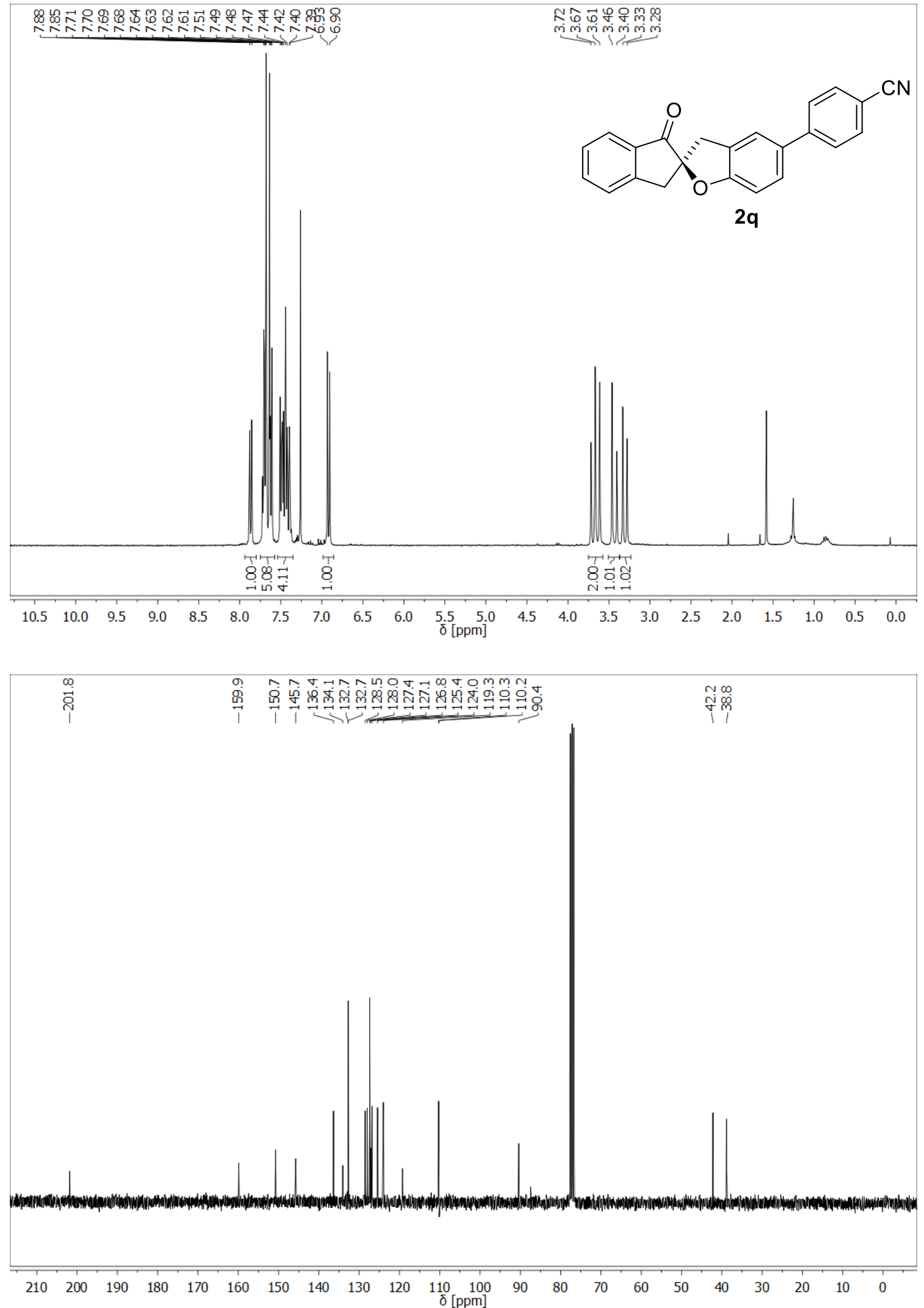
(R)-3H-spiro[benzo[b]thiophene-2,2'-inden]-1'(3'H)-one (2r)
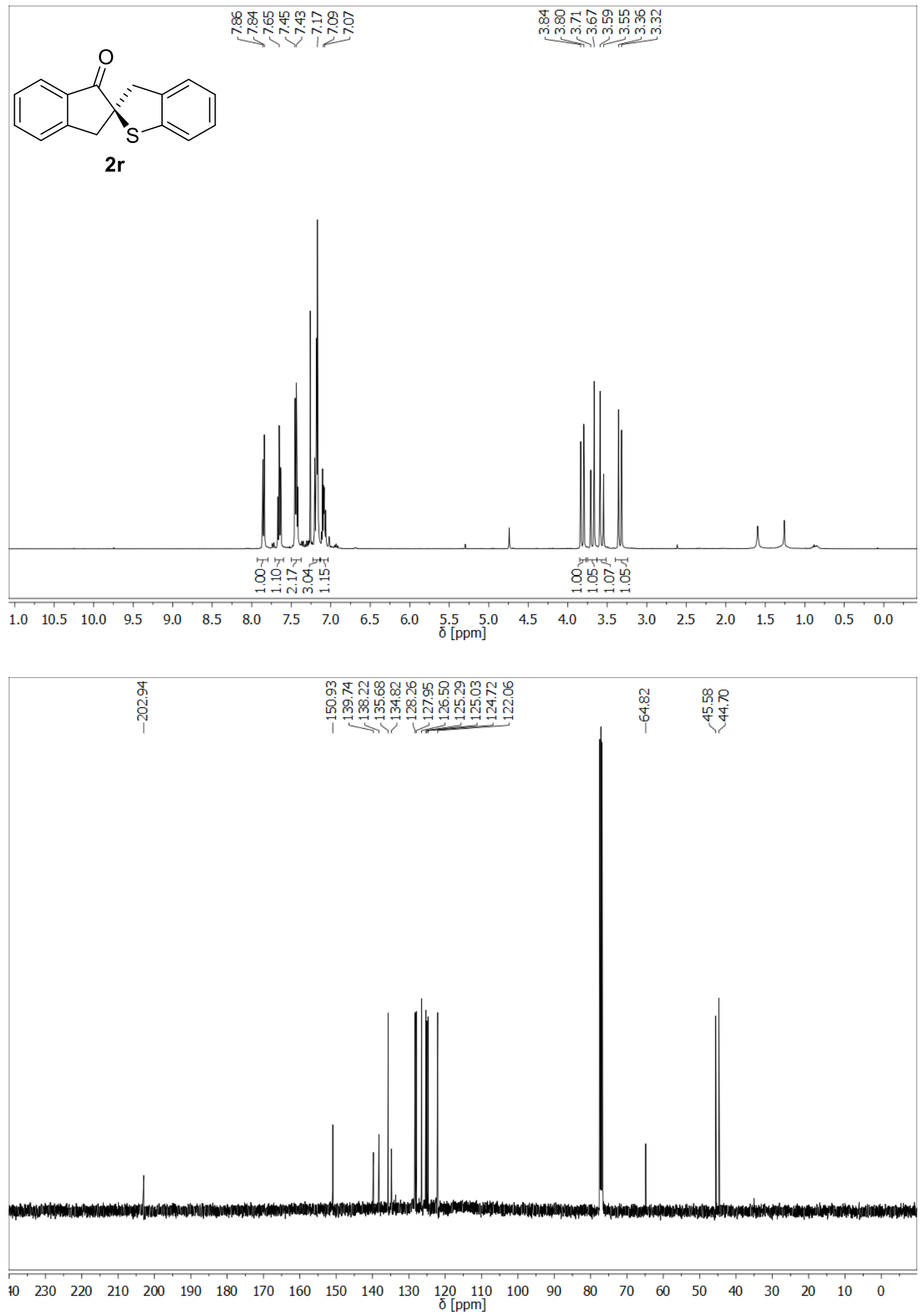
(R)-5-Bromo-3H-spiro[benzo[b]thiophene-2,2'-inden]-1'(3'H)-one (2s)
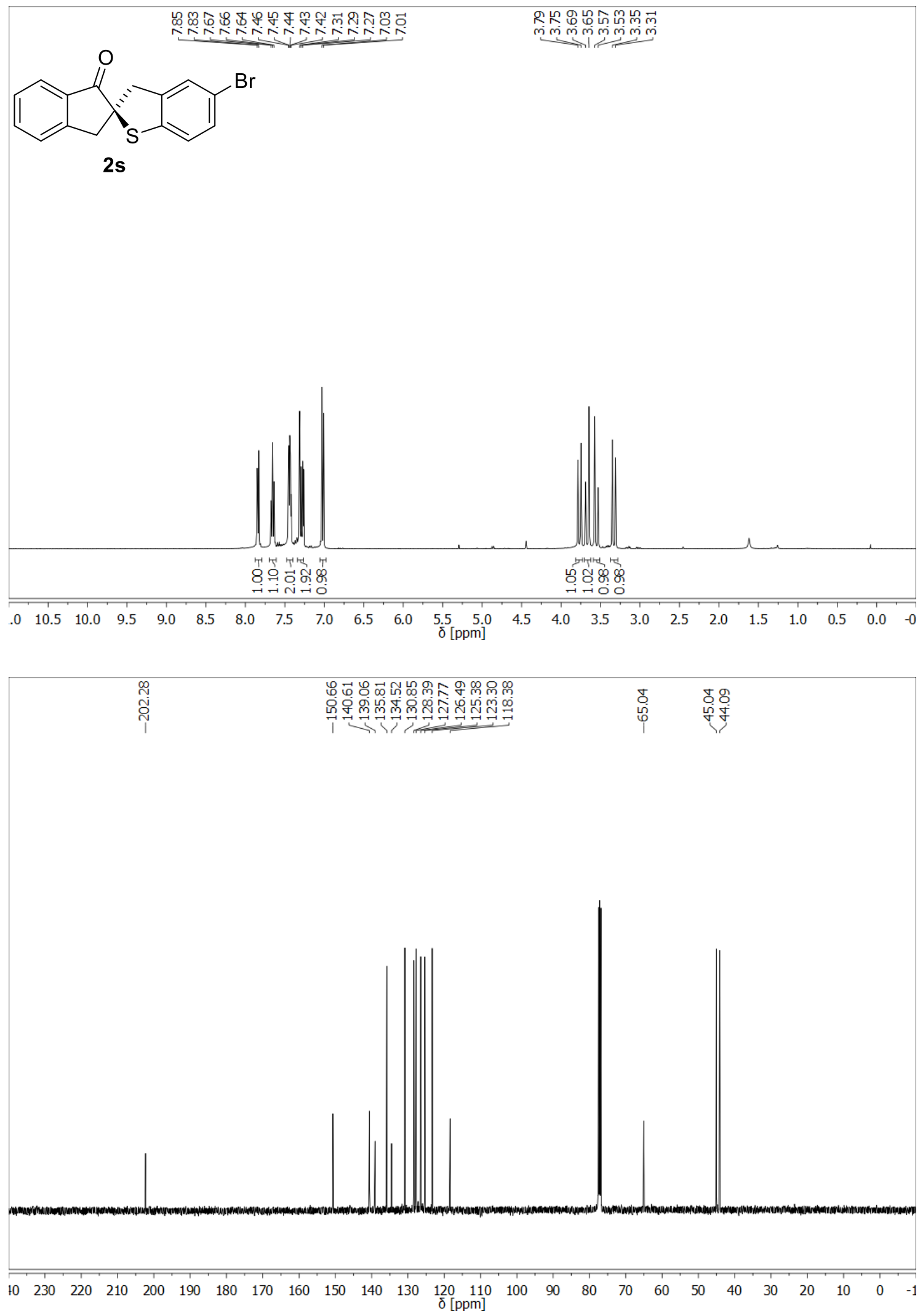
(R)-3'H-Spiro[indene-2,2'-thieno[3,2-c]pyridin]-1(3H)-one (2t)
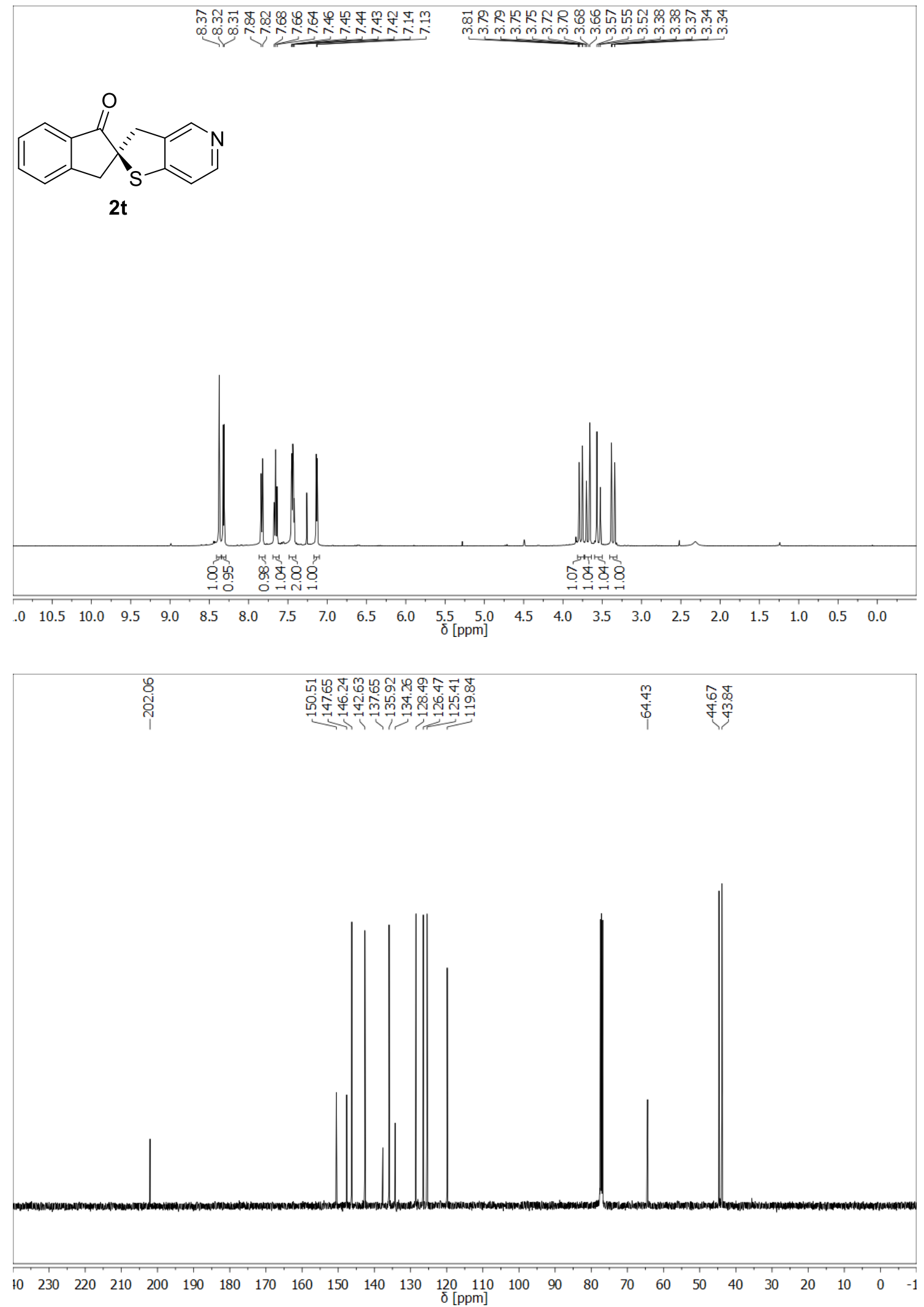
(R)-5-(4-Methoxyphenyl)-3H-spiro[benzo[b]thiophene-2,2'-inden]-1' (3' $H$ )-one (2u)
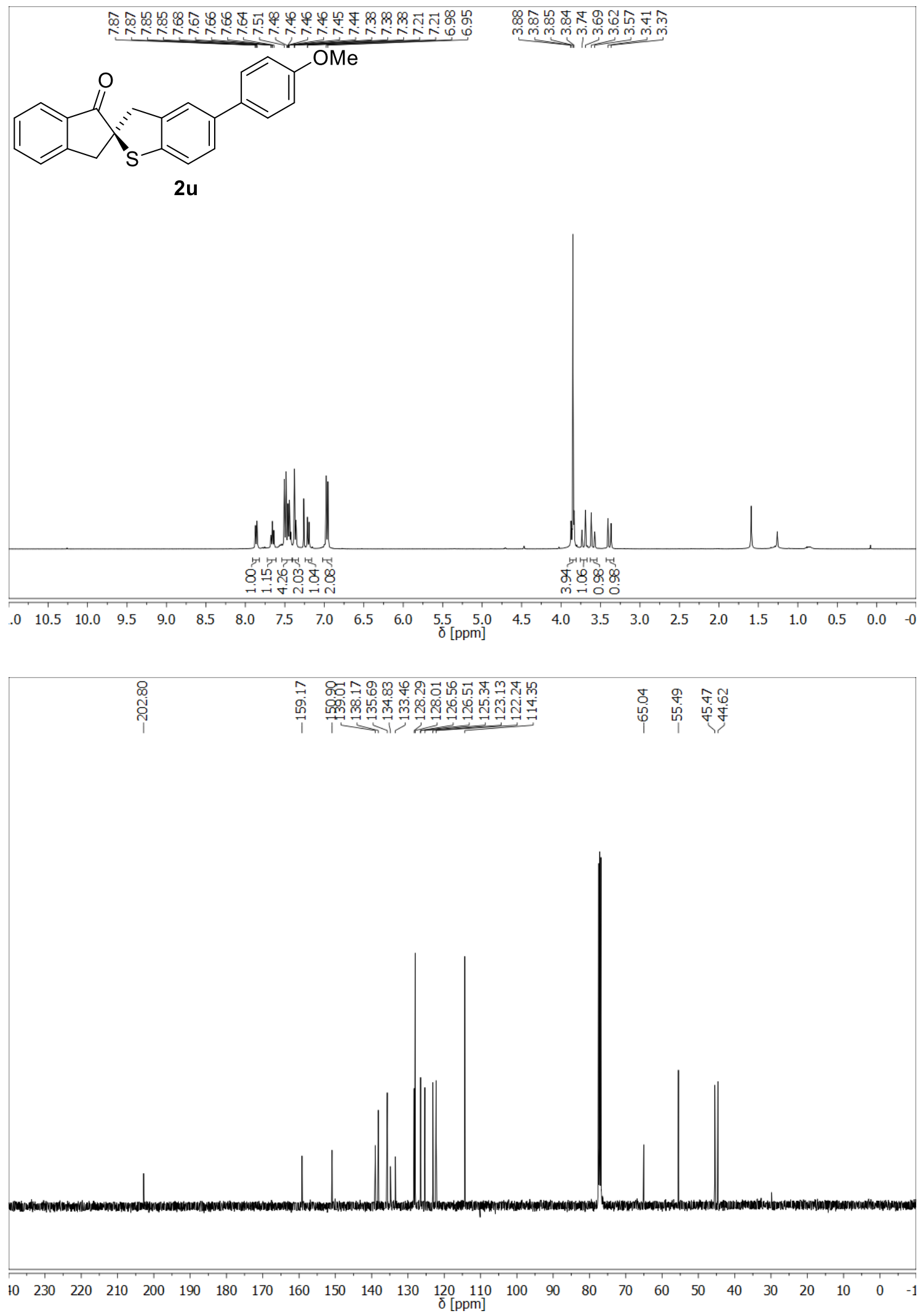
(R)-5-(p-Tolyl)-3H-spiro[benzo[b]thiophene-2,2'-inden]-1'(3'H)-one (2v)
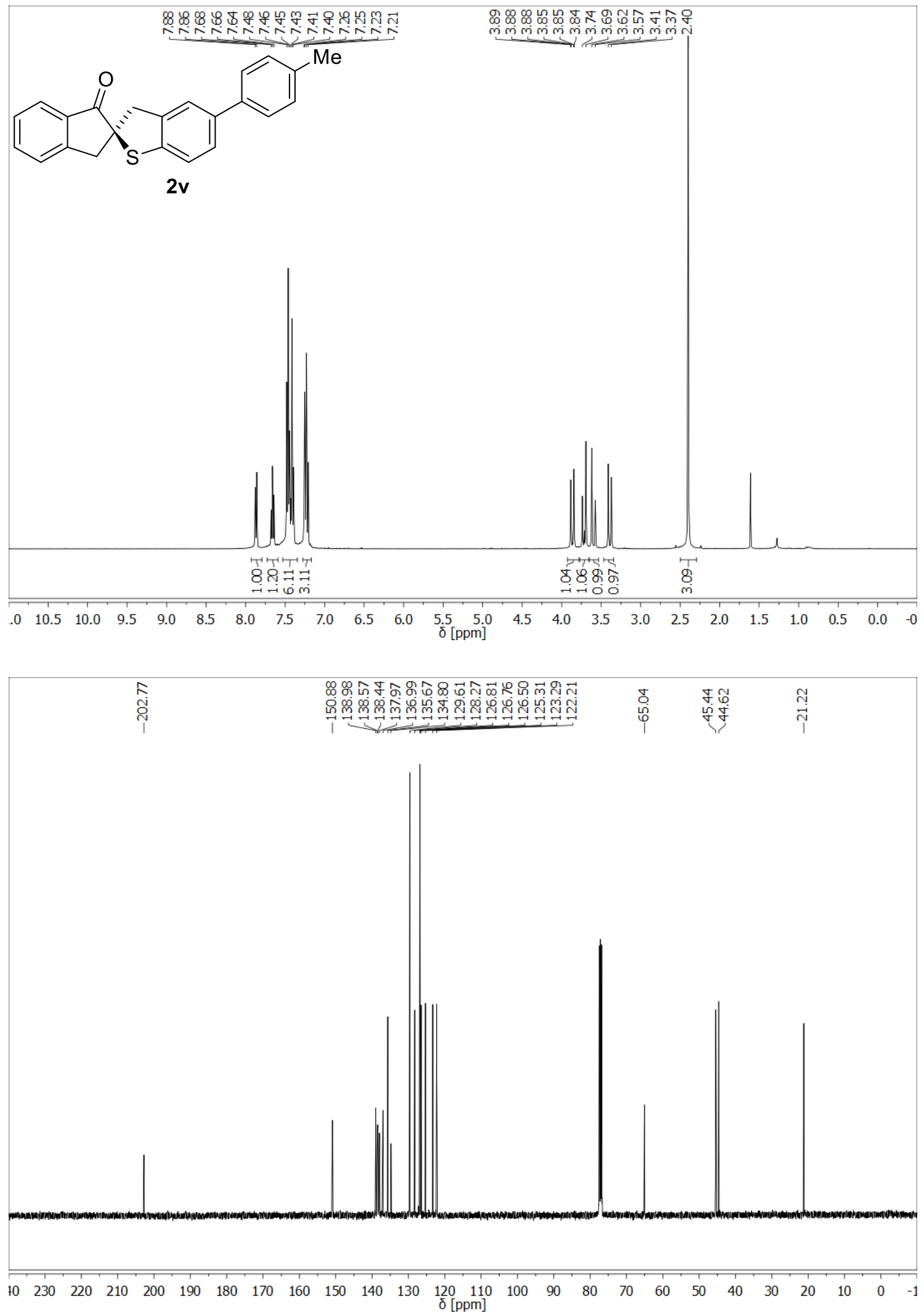
(R)-5-Phenyl-3H-spiro[benzo[b]thiophene-2,2'-inden]-1'(3'H)-one (2w)
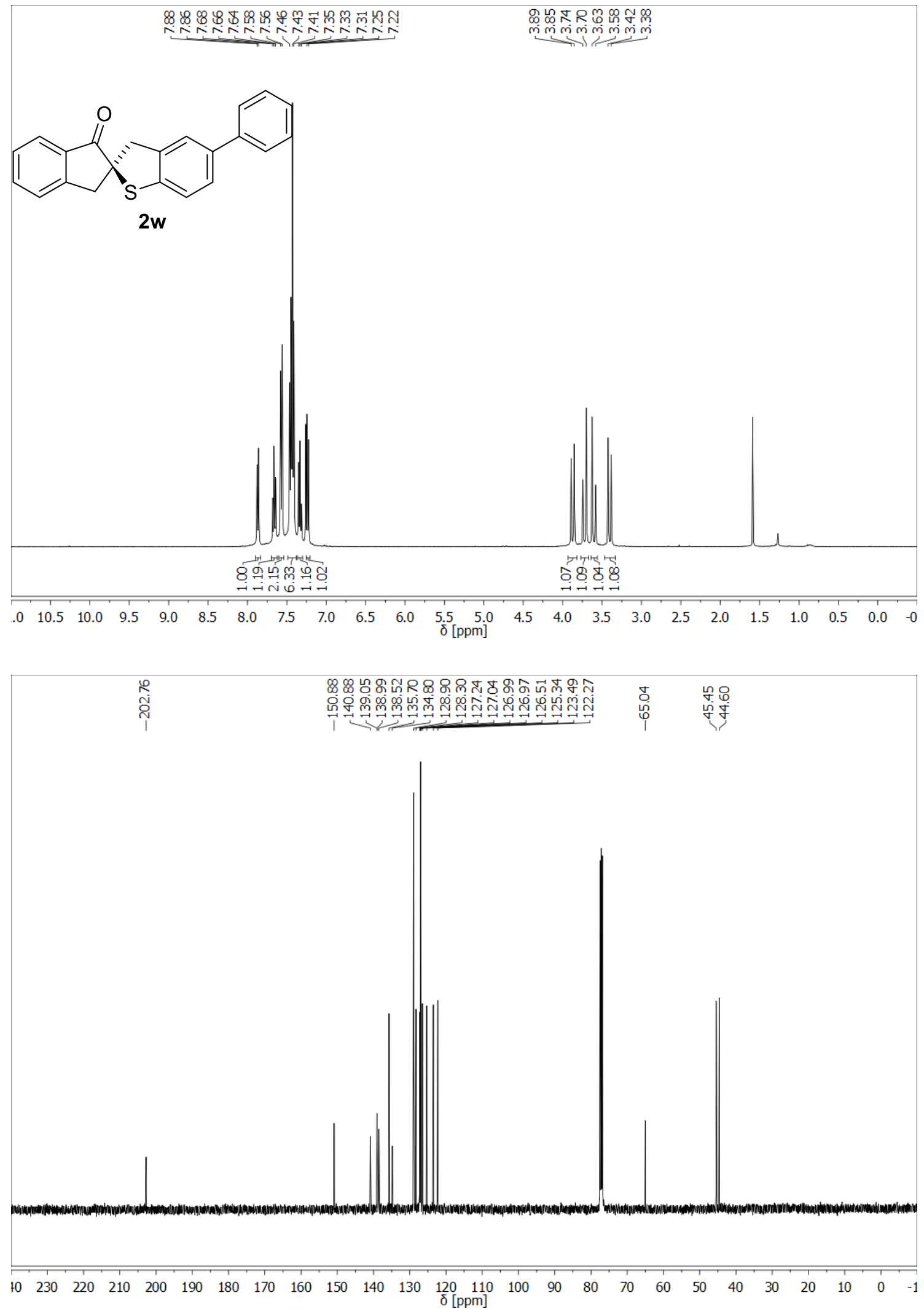
(R)-5-(4-(Trifluoromethoxy)phenyl)-3H-spiro[benzo[b]thiophene-2,2'-inden]-1'(3'H)-one (2x)
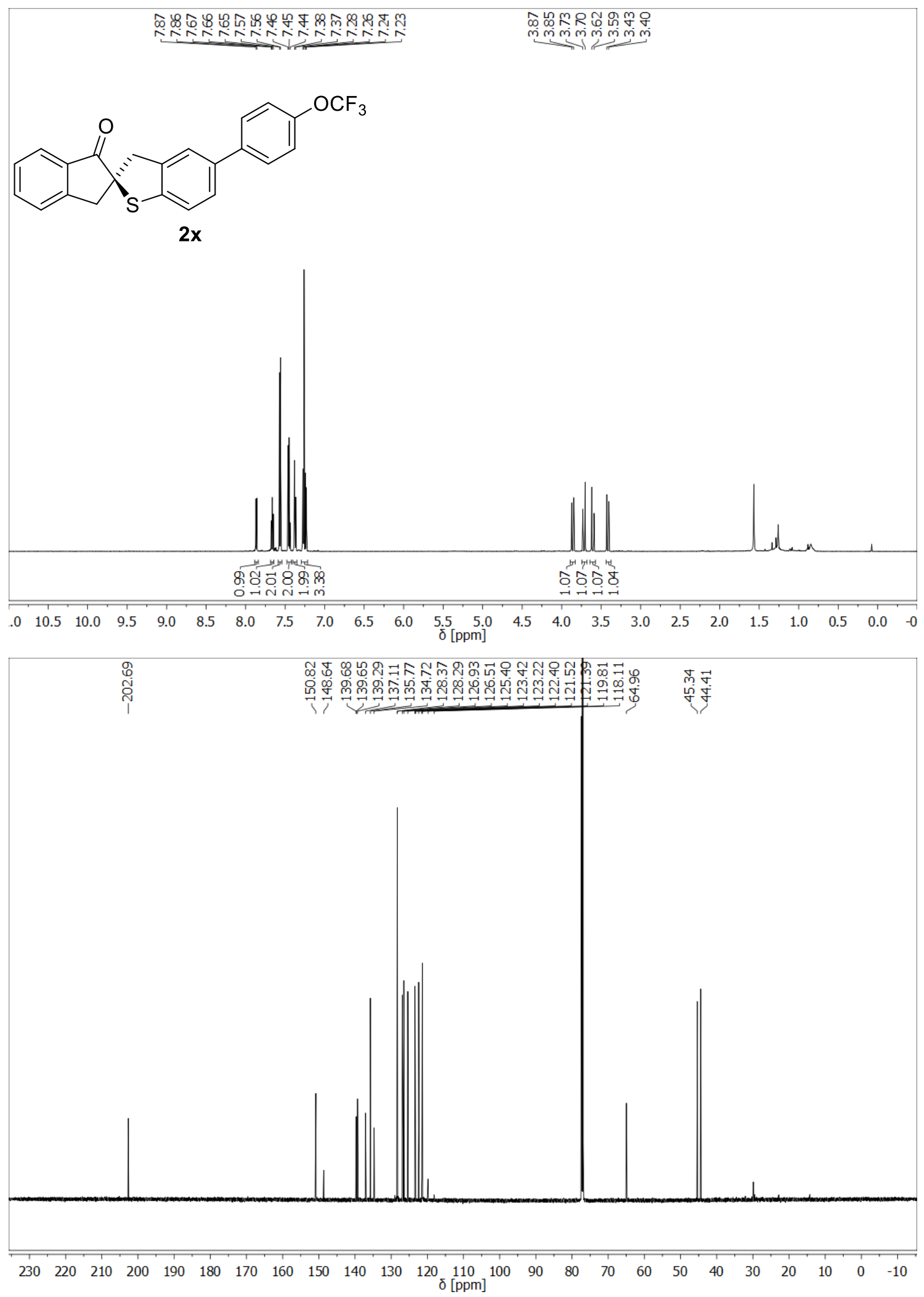
(R)-5-(4-(Trifluoromethyl)phenyl)-3H-spiro[benzo[b]thiophene-2,2'-inden]-1'(3'H)-one (2y)
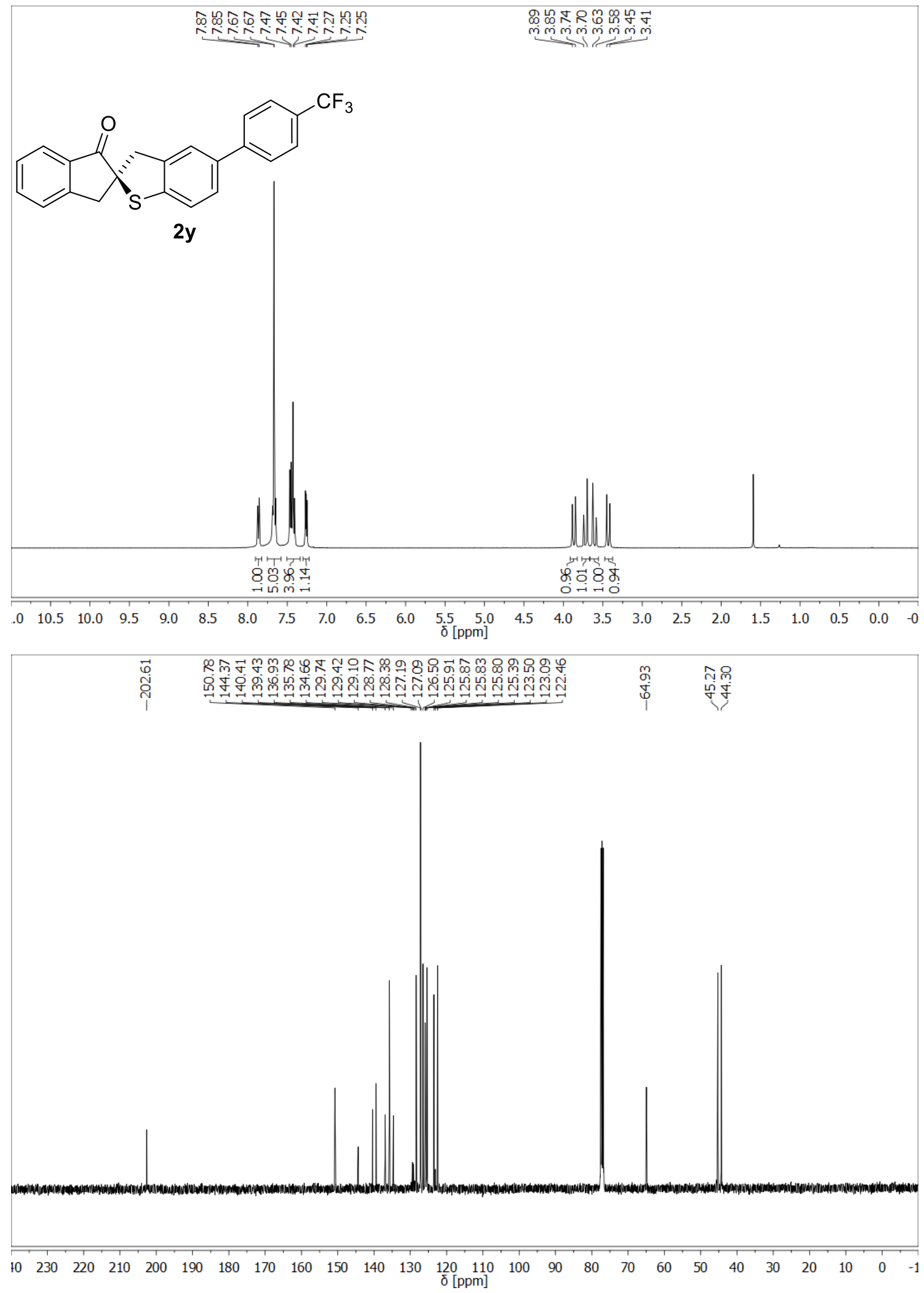
(R)-4-(1'-Oxo-1',3'-dihydro-3H-spiro[benzo[b]thiophene-2,2'-inden]-5-yl)benzonitrile (2z)
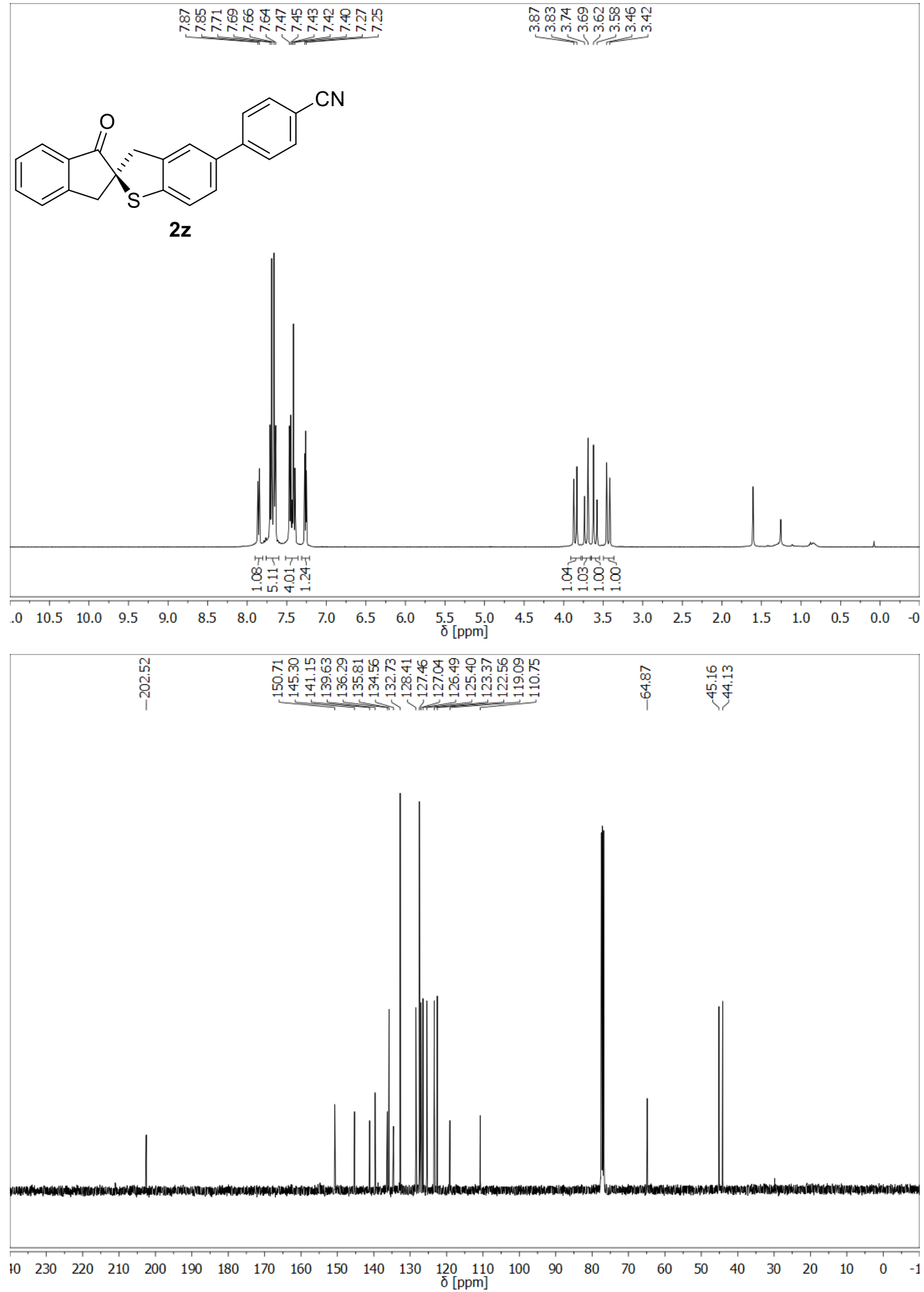


\section{HPLC chromatograms}




\section{(R)-3H-Spiro[benzofuran-2,2'-inden]-1'(3'H)-one (2a)}<smiles>O=C1c2ccccc2C[C@]12Cc1ccccc1O2</smiles>

\section{Racemic compound}

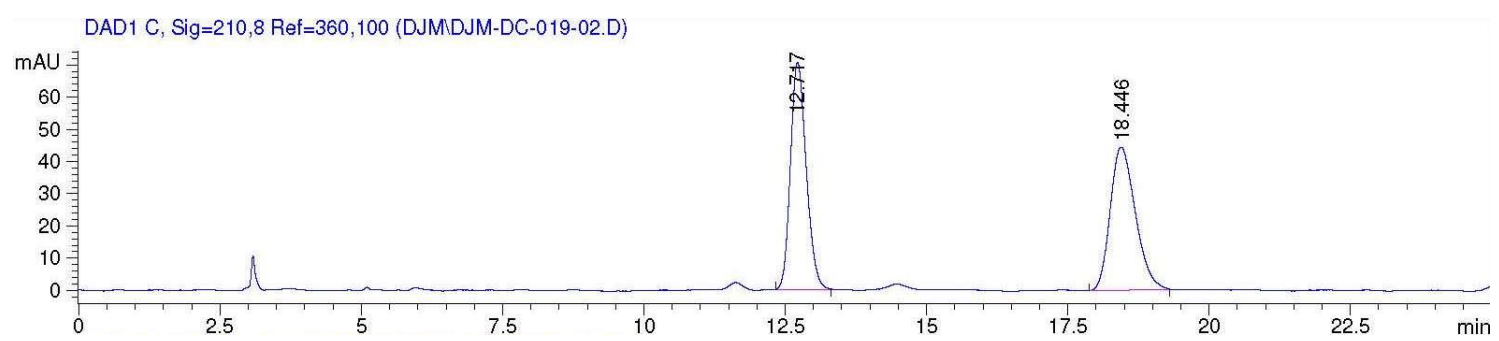

Signal 3: DAD1 C, Sig=210,8 Ref=360,100

\begin{tabular}{|c|c|c|c|c|c|c|}
\hline $\begin{array}{c}\text { Peak } \\
\#\end{array}$ & $\begin{array}{c}\text { RetTime } \\
\text { [min] }\end{array}$ & Type & $\begin{array}{l}\text { Width } \\
\text { [min] }\end{array}$ & $\begin{array}{c}\text { Area } \\
{\left[\mathrm{mAU}^{*} \mathrm{~s}\right]}\end{array}$ & $\begin{array}{l}\text { Height } \\
{[\mathrm{mAU}]}\end{array}$ & $\begin{array}{c}\text { Area } \\
\div\end{array}$ \\
\hline 1 & 12.717 & $\mathrm{BB}$ & 0.2995 & 1375.36633 & 70.54030 & 49.9182 \\
\hline 2 & 18.446 & $\mathrm{BB}$ & 0.4641 & 1379.87134 & 44.45324 & 50.0818 \\
\hline Tota & $:$ & & & 2755.23767 & 114.99353 & \\
\hline
\end{tabular}

\section{Enantioenriched compound}

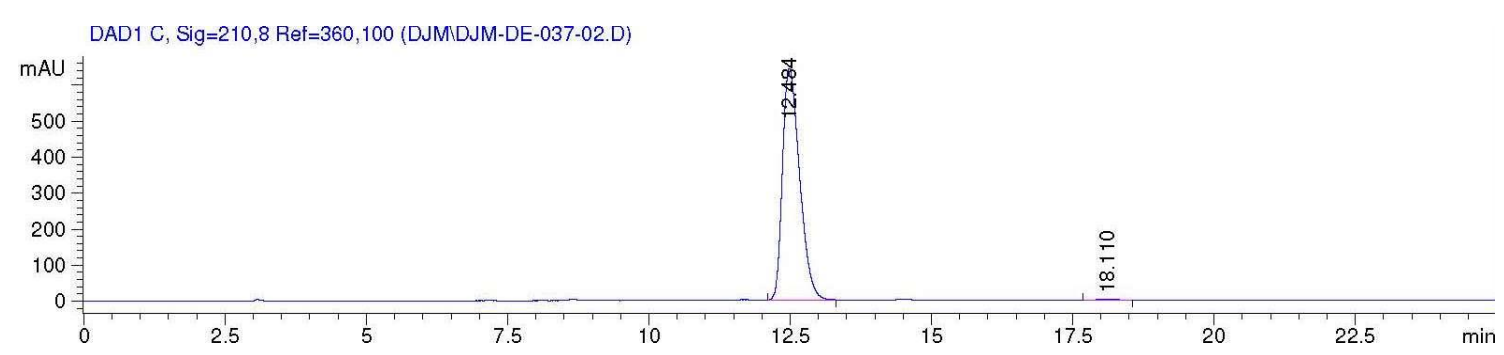

Signal 3: DAD1 C, $\operatorname{Sig}=210,8$ Ref $=360,100$

\begin{tabular}{|c|c|c|c|c|c|c|}
\hline $\begin{array}{c}\text { Peak } \\
\#\end{array}$ & $\begin{array}{c}\text { RetTime } \\
\text { [min] }\end{array}$ & Type & $\begin{array}{l}\text { Width } \\
\text { [min] }\end{array}$ & $\begin{array}{c}\text { Area } \\
{\left[\mathrm{mAU}^{*} \mathrm{~s}\right]}\end{array}$ & $\begin{array}{l}\text { Height } \\
\text { [mAU] }\end{array}$ & $\begin{array}{c}\text { Area } \\
\%\end{array}$ \\
\hline & & & & & & ------- \\
\hline 1 & 12 & BV & 216 & $1.35944 e 4$ & 643.12286 & 99.3998 \\
\hline 2 & 18.110 & VV & 216 & 82.08406 & 3.03410 & 0.6002 \\
\hline
\end{tabular}

Totals : $\quad 1.36765 e 4 \quad 646.15696$ 


\section{(R)-5'-Fluoro-3H-spiro[benzofuran-2,2'-inden]-1'(3'H)-one (2b)}<smiles>O=C1c2ccc(F)cc2C[C@]12Cc1ccccc1O2</smiles>

\section{Racemic compound}

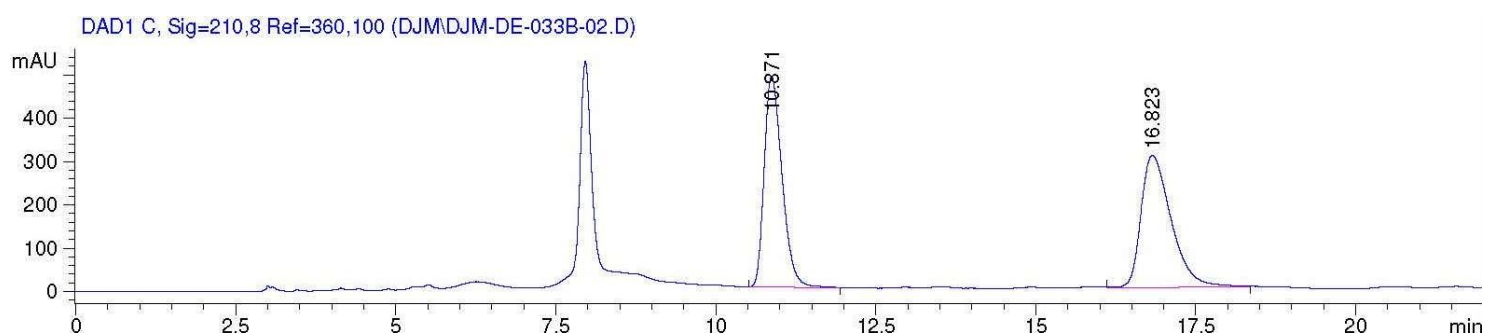

Signal 3: DAD1 C, $\mathrm{Sig}=210,8$ Ref $=360,100$

\begin{tabular}{|c|c|c|c|c|c|c|}
\hline $\begin{array}{c}\text { Peak } \\
\#\end{array}$ & $\begin{array}{c}\text { RetTime } \\
\text { [min] }\end{array}$ & Type & $\begin{array}{l}\text { Width } \\
\text { [min] }\end{array}$ & $\begin{array}{c}\text { Area } \\
{\left[\mathrm{mAU}^{*} \mathrm{~s}\right]}\end{array}$ & $\begin{array}{l}\text { Height } \\
\text { [mAU] }\end{array}$ & $\begin{array}{c}\text { Area } \\
\%\end{array}$ \\
\hline 1 & 1 & & & 94 & 21 & \\
\hline 2 & 16.823 & JD & 0.4796 & $1.00304 \mathrm{e} 4$ & 305.05072 & 51. \\
\hline
\end{tabular}

Totals :

$1.94963 e 4 \quad 791.00693$

\section{Enantioenriched compound}

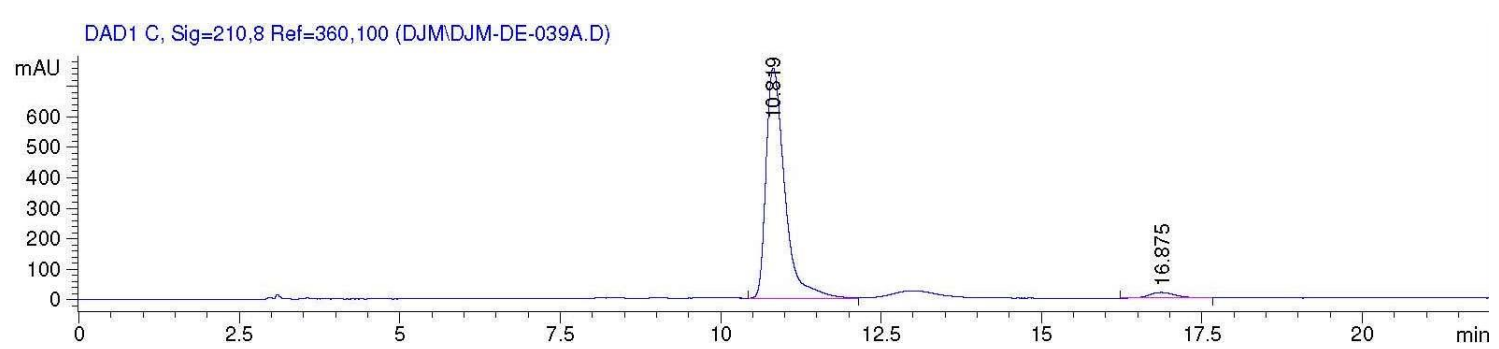

Signal 3: DAD1 C, $\operatorname{Sig}=210,8$ Ref $=360,100$

\begin{tabular}{|c|c|c|c|c|c|c|}
\hline $\begin{array}{c}\text { Peak } \\
\#\end{array}$ & $\begin{array}{c}\text { RetTime } \\
\text { [min] }\end{array}$ & Type & $\begin{array}{l}\text { Width } \\
\text { [min] }\end{array}$ & $\begin{array}{c}\text { Area } \\
{\left[\mathrm{mAU}^{*} \mathrm{~s}\right]}\end{array}$ & $\begin{array}{l}\text { Height } \\
{[\mathrm{mAU}]}\end{array}$ & $\begin{array}{c}\text { Area } \\
\%\end{array}$ \\
\hline 1 & & BB & & 1.5 & 75 & \\
\hline 2 & 16.875 & $\mathrm{BB}$ & 0.3489 & 546.48114 & 18.64909 & 3.4497 \\
\hline
\end{tabular}
Totals :

$$
1.58415 e 4 \quad 774.54210
$$




\section{(R)-5'-Chloro-3H-spiro[benzofuran-2,2'-inden]-1'(3'H)-one (2c)}<smiles>O=C1c2ccc(Cl)cc2C[C@]12Cc1ccccc1O2</smiles>

\section{Racemic compound}

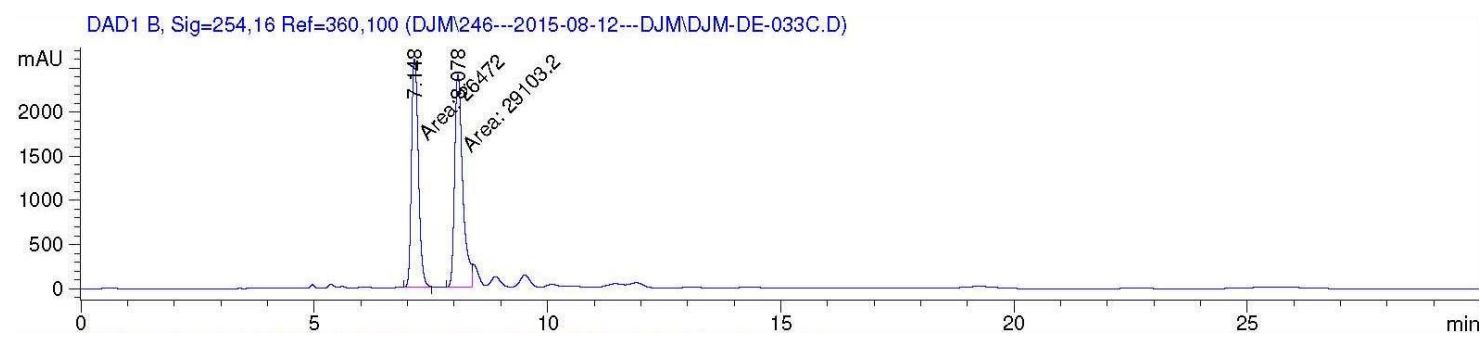

\begin{tabular}{|c|c|c|c|c|c|c|}
\hline $\begin{array}{c}\text { Peak } \\
\#\end{array}$ & $\begin{array}{c}\text { RetTime } \\
\text { [min] }\end{array}$ & Type & $\begin{array}{l}\text { Width } \\
\text { [min] }\end{array}$ & $\begin{array}{c}\text { Area } \\
{\left[\mathrm{mAU}^{*} \mathrm{~s}\right]}\end{array}$ & $\begin{array}{l}\text { Height } \\
{\left[\mathrm{mAU}^{-}\right.}\end{array}$ & $\begin{array}{c}\text { Area } \\
\frac{\circ}{\circ}\end{array}$ \\
\hline 1 & 7.148 & $\mathrm{MM}$ & 0.1707 & $2.64720 \mathrm{e} 4$ & 2583.94238 & 47.6328 \\
\hline 2 & 8.078 & MM & 0.2019 & $2.91032 \mathrm{e} 4$ & 2402.06250 & 52.3672 \\
\hline Tota & ls : & & & $5.55752 \mathrm{e} 4$ & 4986.00488 & \\
\hline
\end{tabular}

\section{Enantioenriched compound}

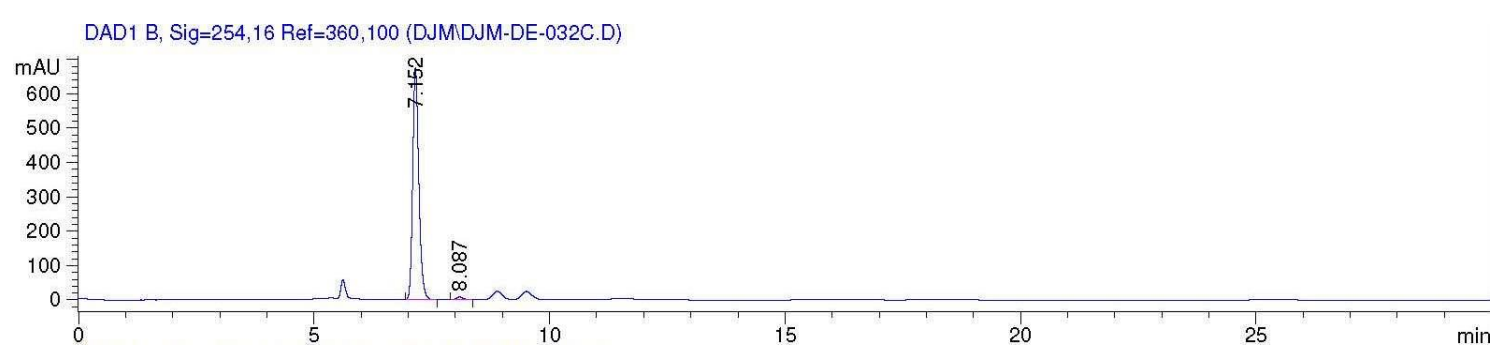

Signal 2: DAD1 B, Sig $=254,16$ Ref $=360,100$

\begin{tabular}{|c|c|c|c|c|c|c|}
\hline $\begin{array}{c}\text { Peak } \\
\#\end{array}$ & $\begin{array}{c}\text { RetTime } \\
\text { [min] }\end{array}$ & Type & $\begin{array}{l}\text { Width } \\
\text { [min] }\end{array}$ & $\begin{array}{c}\text { Area } \\
{\left[\mathrm{mAU}^{*} \mathrm{~s}\right]}\end{array}$ & $\begin{array}{l}\text { Height } \\
\text { [mAU] }\end{array}$ & $\begin{array}{c}\text { Area } \\
\quad \%\end{array}$ \\
\hline 1 & 7.1 & $B$ & & 6046 & 675 & \\
\hline 2 & 8.087 & $\mathrm{BB}$ & 0.1545 & 84.16341 & 8.35334 & 1.3729 \\
\hline
\end{tabular}

Totals : $\quad 6130.40755683 .90888$ 


\section{(R)-5'-Phenyl-3H-spiro[benzofuran-2,2'-inden]-1'(3'H)-one (2d)}<smiles>O=C1c2ccc(-c3ccccc3)cc2C[C@]12Cc1ccccc1O2</smiles>

\section{Racemic compound}

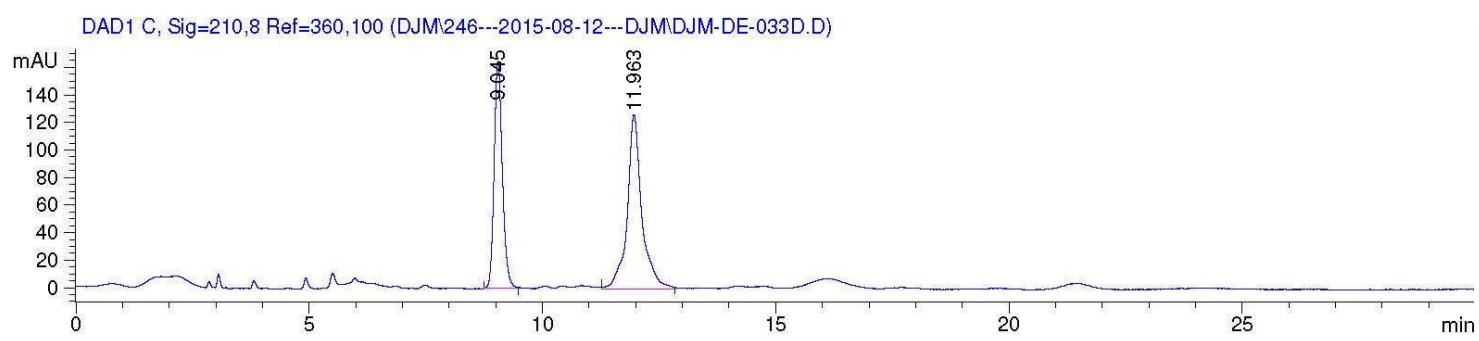

Signal 3: DAD1 C, $\operatorname{Sig}=210,8$ Ref $=360,100$

\begin{tabular}{|c|c|c|c|c|c|c|}
\hline $\begin{array}{c}\text { Peak } \\
\#\end{array}$ & $\begin{array}{c}\text { RetTime } \\
\text { [min] }\end{array}$ & Type & $\begin{array}{l}\text { Width } \\
\text { [min] }\end{array}$ & $\begin{array}{c}\text { Area } \\
{\left[\mathrm{mAU}^{*} \mathrm{~s}\right]}\end{array}$ & $\begin{array}{l}\text { Height } \\
{[\mathrm{mAU}]}\end{array}$ & $\begin{array}{c}\text { Area } \\
\div\end{array}$ \\
\hline 1 & 9.045 & BV & 0.1876 & 2024.90063 & 165.63608 & 42.5380 \\
\hline 2 & 11.963 & $\mathrm{BB}$ & 0.3084 & 2735.31152 & 126.44108 & 57.4620 \\
\hline rotal & $S:$ & & & 4760.21216 & 292.07716 & \\
\hline
\end{tabular}

\section{Enantioenriched compound}

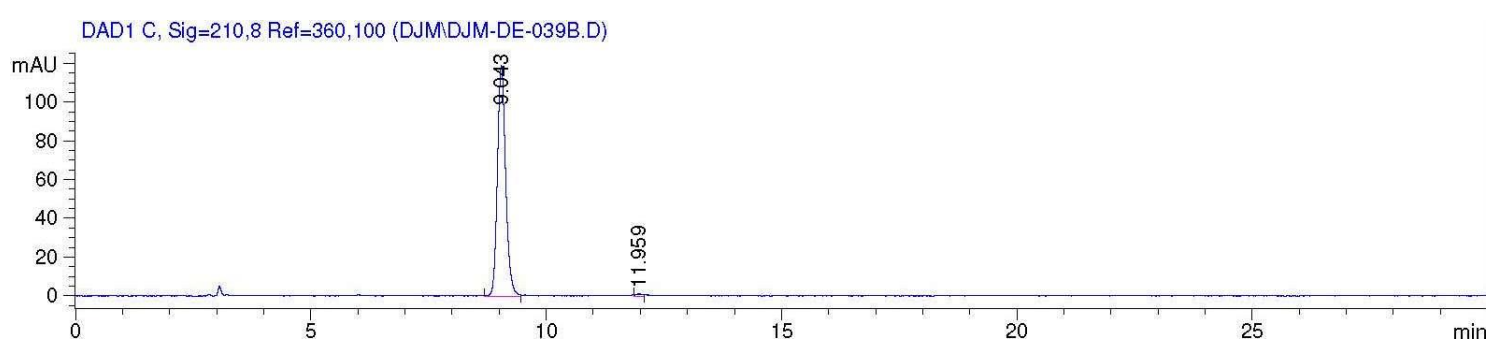

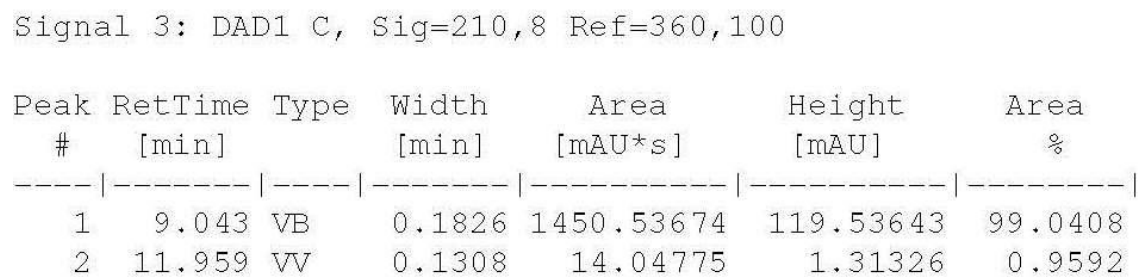

Totals :

$1464.58450 \quad 120.84969$ 
(R)-5-Methyl-3H-spiro[benzofuran-2,2'-inden]-1'(3'H)-one (2e)<smiles>Cc1ccc2c(c1)C[C@]1(Cc3ccccc3C1=O)O2</smiles>

\section{Racemic compound}

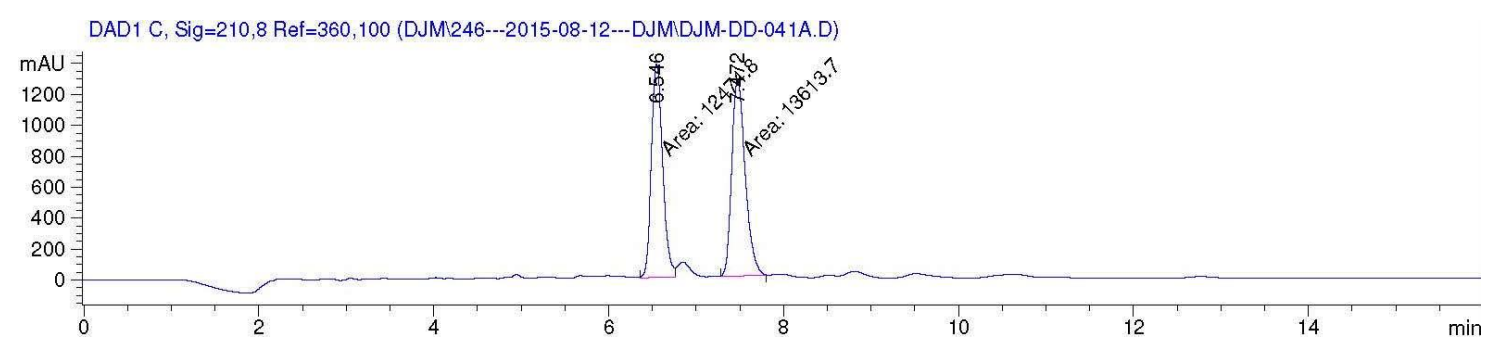

Signal 3: DAD1 C, $\operatorname{Sig}=210,8$ Ref $=360,100$

\begin{tabular}{|c|c|c|c|c|c|c|}
\hline $\begin{array}{c}\text { Peak } \\
\#\end{array}$ & $\begin{array}{c}\text { RetTime } \\
\text { [min] }\end{array}$ & Type & $\begin{array}{l}\text { Width } \\
\text { [min] }\end{array}$ & $\begin{array}{c}\text { Area } \\
{\left[\mathrm{mAU}^{*} \mathrm{~S}\right]}\end{array}$ & $\begin{array}{l}\text { Height } \\
{[\mathrm{mAU}]}\end{array}$ & $\begin{array}{c}\text { Area } \\
\frac{\%}{0}\end{array}$ \\
\hline 1 & & & & & & \\
\hline $\begin{array}{l}1 \\
2\end{array}$ & $\begin{array}{l}0.546 \\
7.472\end{array}$ & $\mathrm{MM}$ & $\begin{array}{l}0.1502 \\
0.1787\end{array}$ & $\begin{array}{l}1.24748 e 4 \\
1.36137 e 4\end{array}$ & $\begin{array}{l}1383.92334 \\
1269.70850\end{array}$ & $\begin{array}{l}47.8173 \\
52.1827\end{array}$ \\
\hline
\end{tabular}

Totals : $\quad 2.60884 e 4 \quad 2653.63184$

\section{Enantioenriched compound}

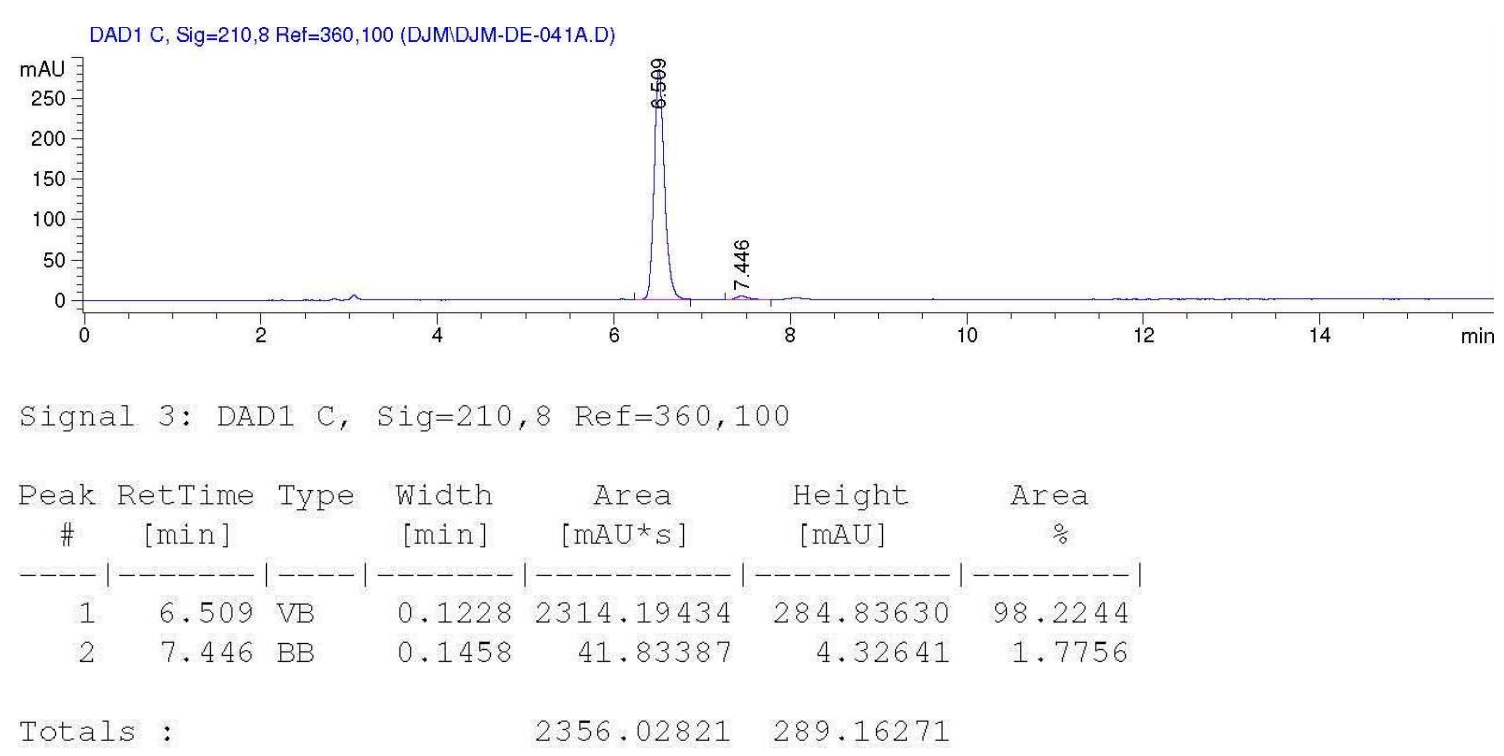


(R)-5-Methoxy-3H-spiro[benzofuran-2,2'-inden]-1'(3'H)-one (2f)<smiles></smiles>

\section{Racemic compound}

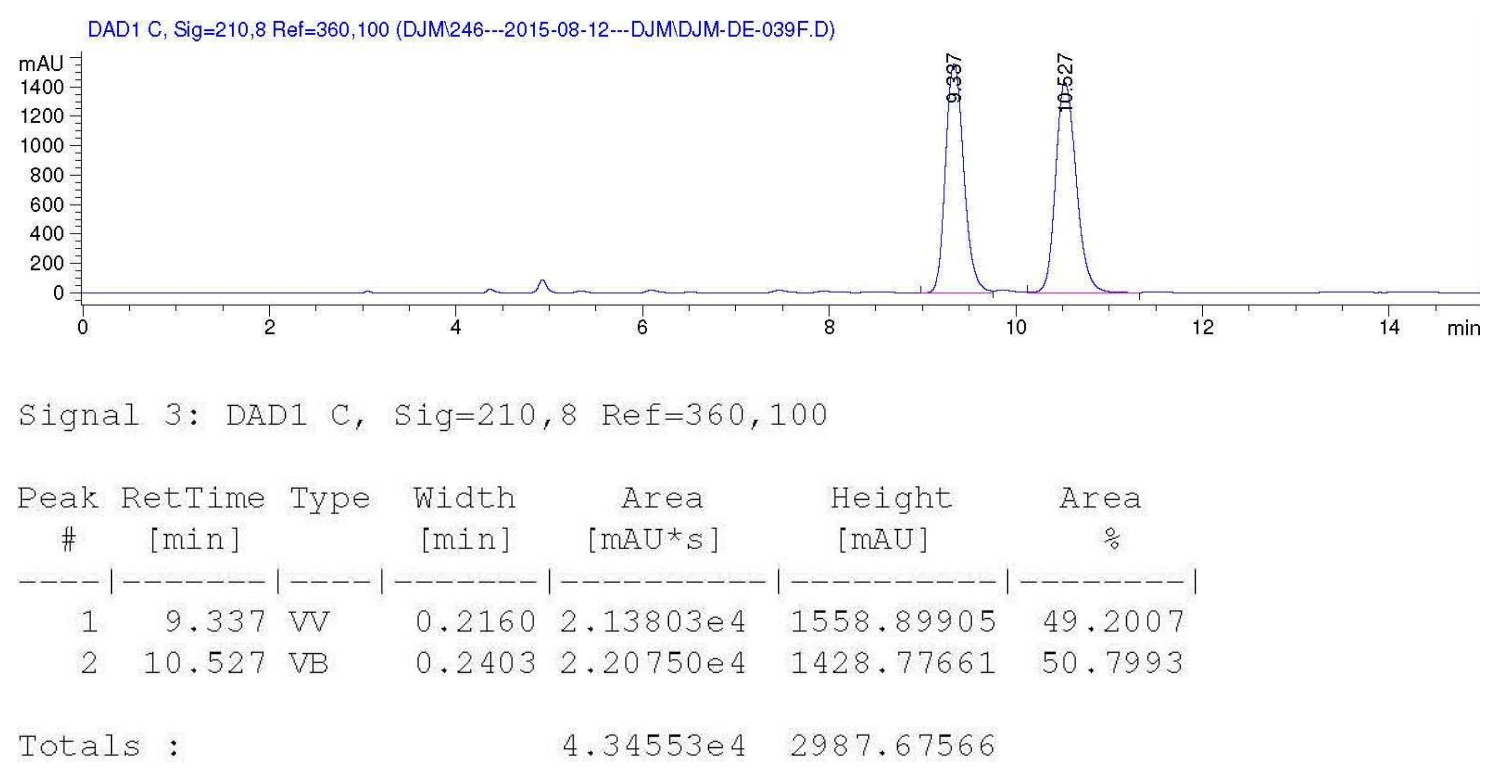

\section{Enantioenriched compound}

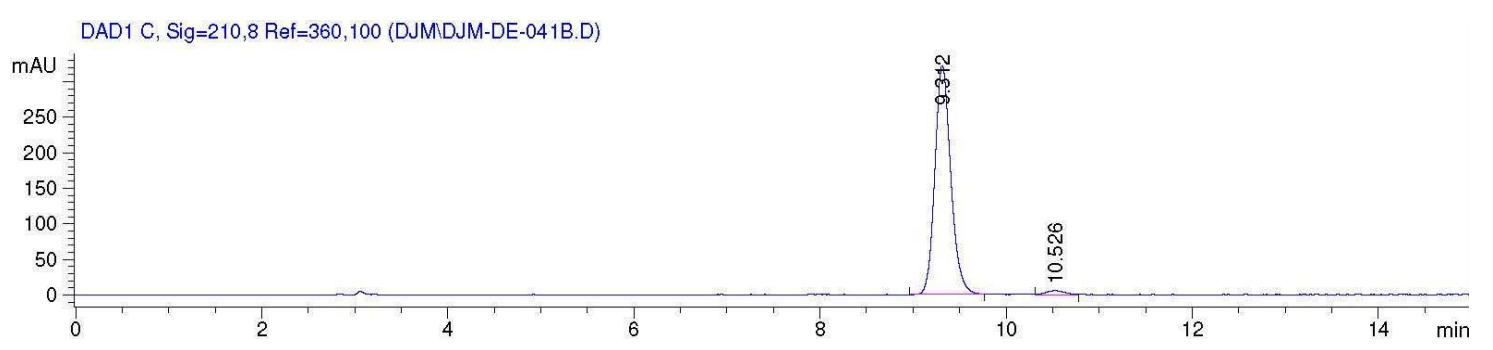

Signal 3: DAD1 C, $\operatorname{Sig}=210,8$ Ref $=360,100$

\begin{tabular}{|c|c|c|c|c|c|c|}
\hline $\begin{array}{c}\text { Peak } \\
\text { \# }\end{array}$ & $\begin{array}{c}\text { RetTime } \\
\text { [min] }\end{array}$ & Type & $\begin{array}{l}\text { Width } \\
\text { [min] }\end{array}$ & $\begin{array}{c}\text { Area } \\
{\left[\mathrm{mAU}^{*} \mathrm{~s}\right]}\end{array}$ & $\begin{array}{l}\text { Height } \\
{[\mathrm{mAU}]}\end{array}$ & $\begin{array}{c}\text { Area } \\
\%\end{array}$ \\
\hline 1 & & VB & & 3849 & 705 & 93 \\
\hline 2 & 10.526 & VV & 0.1620 & 77.79469 & 5.85446 & 1.9807 \\
\hline
\end{tabular}

Totals : $\quad 3927.66774 \quad 328.02151$ 
(R)-5-Bromo-3H-spiro[benzofuran-2,2'-inden]-1'(3'H)-one (2g)<smiles>O=C1c2ccccc2C[C@]12Cc1cc(Br)ccc1O2</smiles>

\section{Racemic compound}

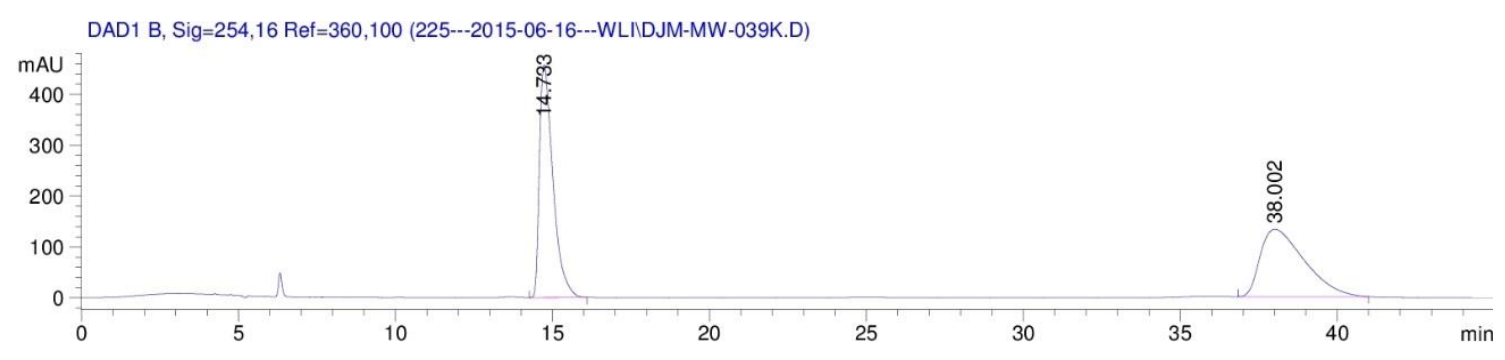

Signal 2: DAD1 B, Sig=254,16 Ref=360,100

\begin{tabular}{|c|c|c|c|c|c|c|}
\hline $\begin{array}{c}\text { Peak } \\
\#\end{array}$ & $\begin{array}{c}\text { RetTime } \\
\text { [min] }\end{array}$ & Type & $\begin{array}{l}\text { Width } \\
\text { [min] }\end{array}$ & $\begin{array}{c}\text { Area } \\
{\left[\mathrm{mAU}^{*} \mathrm{~S}\right]}\end{array}$ & $\begin{array}{l}\text { Height } \\
\text { [mAU] }\end{array}$ & $\begin{array}{c}\text { Area } \\
\frac{\circ}{0}\end{array}$ \\
\hline 1 & & & 3 & 1.370 & 25 & 51 \\
\hline 2 & 38.002 & $R$ & 68 & $1.29839 \mathrm{e} 4$ & 55040 & 48.652 \\
\hline
\end{tabular}

Totals : $\quad 2.66873 e 4 \quad 590.18565$

\section{Enantioenriched compound}

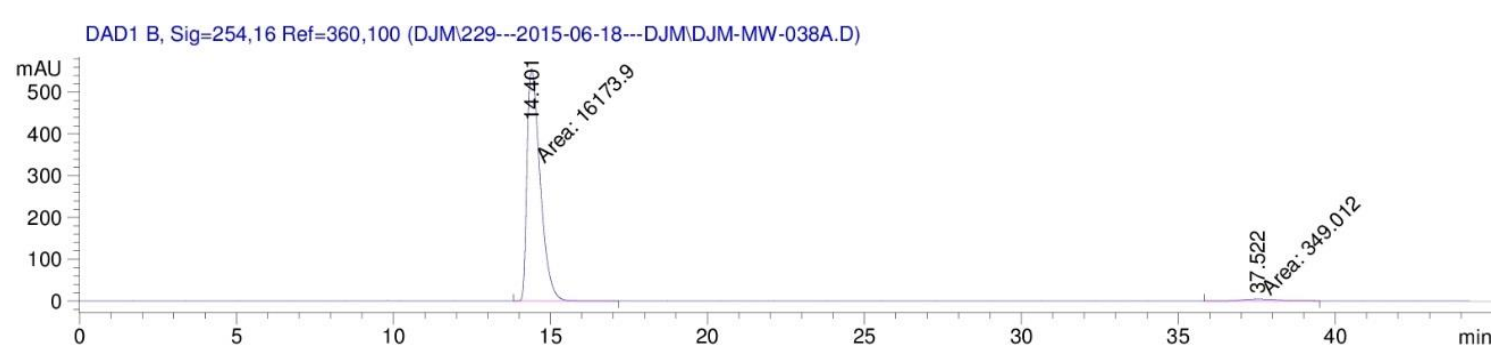

Signal 2: DAD1 B, Sig=254,16 Ref=360,100

\begin{tabular}{|c|c|c|c|c|c|c|}
\hline $\begin{array}{c}\text { Peak } \\
\#\end{array}$ & $\begin{array}{c}\text { RetTime } \\
\text { [min] }\end{array}$ & Type & $\begin{array}{l}\text { Width } \\
\text { [min] }\end{array}$ & $\begin{array}{c}\text { Area } \\
{\left[\mathrm{mAU}^{\star} \mathrm{S}\right]}\end{array}$ & $\begin{array}{l}\text { Height } \\
{[\mathrm{mAU}]}\end{array}$ & $\begin{array}{c}\text { Area } \\
\quad \%\end{array}$ \\
\hline 1 & & & & 1.61 & 556 & 77 \\
\hline 0 & 3752 & 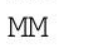 & 479 & 349.01245 & 4.31548 & 2.112 \\
\hline
\end{tabular}

Totals : $\quad 1.65229 \mathrm{e} 4 \quad 560.86480$ 


\section{(R)-5-Fluoro-3H-spiro[benzofuran-2,2'-inden]-1'(3'H)-one (2h)}<smiles>O=C1c2ccccc2C[C@]12Cc1cc(F)ccc1O2</smiles>

2h

\section{Racemic compound}

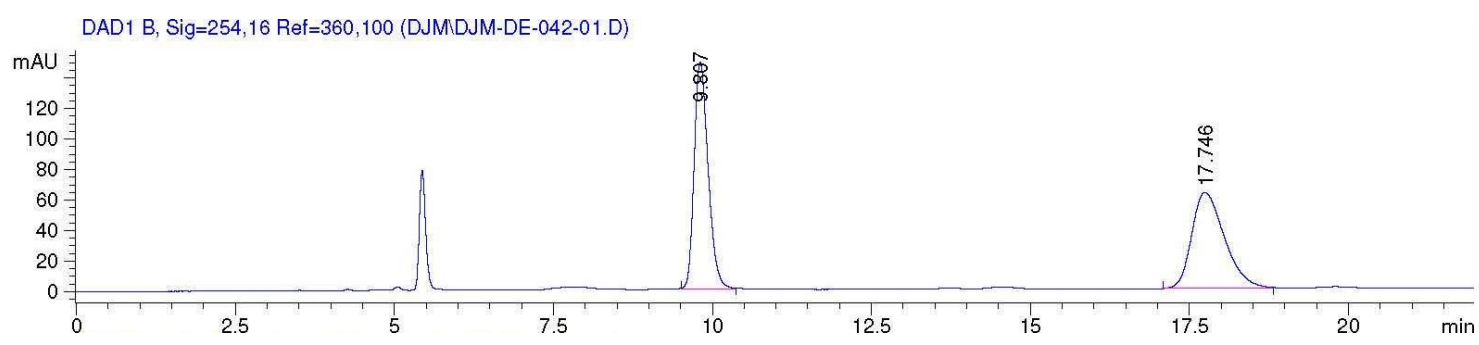

Signal 2: DAD1 B, Sig=254,16 Ref $=360,100$

\begin{tabular}{|c|c|c|c|c|c|c|}
\hline $\begin{array}{c}\text { Peak } \\
\#\end{array}$ & $\begin{array}{c}\text { RetTime } \\
\text { [min] }\end{array}$ & Type & $\begin{array}{l}\text { Width } \\
\text { [min] }\end{array}$ & $\begin{array}{c}\text { Area } \\
{\left[\mathrm{mAU}^{*} \mathrm{~s}\right]}\end{array}$ & $\begin{array}{l}\text { Height } \\
{[\mathrm{mAU}]}\end{array}$ & $\begin{array}{c}\text { Area } \\
\%\end{array}$ \\
\hline 1 & & $\mathrm{BB}$ & & 30601 & & \\
\hline 2 & 17.746 & B & 0.5462 & 2250.72974 & 62.93998 & 49.8959 \\
\hline
\end{tabular}

Totals : $\quad 4510.85303 \quad 211.28689$

\section{Enantioenriched compound}

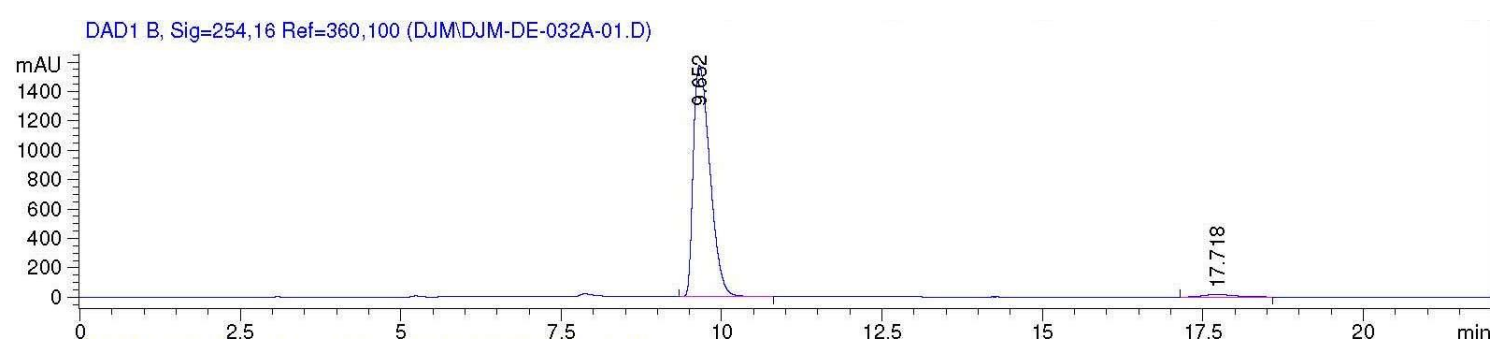

Signal 2: DAD1 B, Sig=254,16 Ref $=360,100$

\begin{tabular}{|c|c|c|c|c|c|c|}
\hline $\begin{array}{c}\text { Peak } \\
\#\end{array}$ & $\begin{array}{c}\text { RetTime } \\
\text { [min] }\end{array}$ & Type & $\begin{array}{l}\text { Width } \\
\text { [min] }\end{array}$ & $\begin{array}{c}\text { Area } \\
{\left[\mathrm{mAU}^{*} \mathrm{~s}\right]}\end{array}$ & $\begin{array}{l}\text { Height } \\
{\left[\mathrm{mAU}^{-}\right.}\end{array}$ & $\begin{array}{c}\text { Area } \\
\%\end{array}$ \\
\hline 1 & & & & & & 98 \\
\hline 2 & 17.718 & 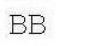 & 32 & 579.04120 & 17.00988 & 1.9826 \\
\hline
\end{tabular}

Totals : $\quad 2.92057 e 4 \quad 1591.95263$ 
(R)-5-(4-Methoxyphenyl)-3H-spiro[benzofuran-2,2'-inden]-1'(3'H)-one (2i)<smiles>COc1ccc(-c2ccc3c(c2)C[C@@]2(Cc4ccccc4C2=O)O3)cc1</smiles>

\section{Racemic compound}

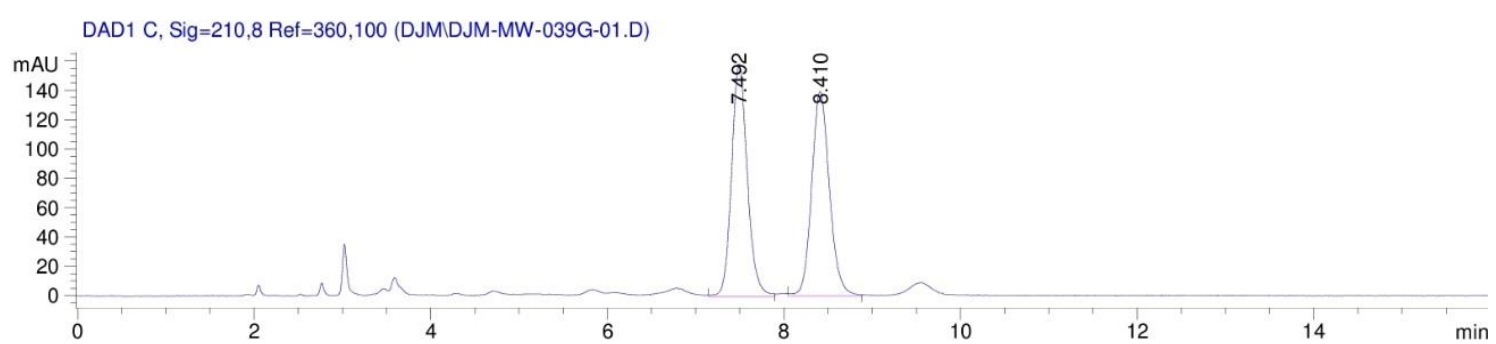

Signal 3: DAD1 C, Sig=210,8 $\operatorname{Ref}=360,100$

\begin{tabular}{|c|c|c|c|c|c|c|}
\hline $\begin{array}{c}\text { Peak } \\
\#\end{array}$ & $\begin{array}{c}\text { RetTime } \\
\text { [min] }\end{array}$ & Type & $\begin{array}{l}\text { Width } \\
\text { [min] }\end{array}$ & $\begin{array}{c}\text { Area } \\
{\left[\mathrm{mAU}^{\star} \mathrm{S}\right]}\end{array}$ & $\begin{array}{l}\text { Height } \\
{[\mathrm{mAU}]}\end{array}$ & $\begin{array}{c}\text { Area } \\
\frac{\circ}{0}\end{array}$ \\
\hline & & & & & & \\
\hline 2 & & & 156 & 1956.60266 & $\begin{array}{l}8641 \\
2950\end{array}$ & 0067 \\
\hline
\end{tabular}

Totals : $\quad 3912.68042 \quad 297.81596$

\section{Enantioenriched compound}

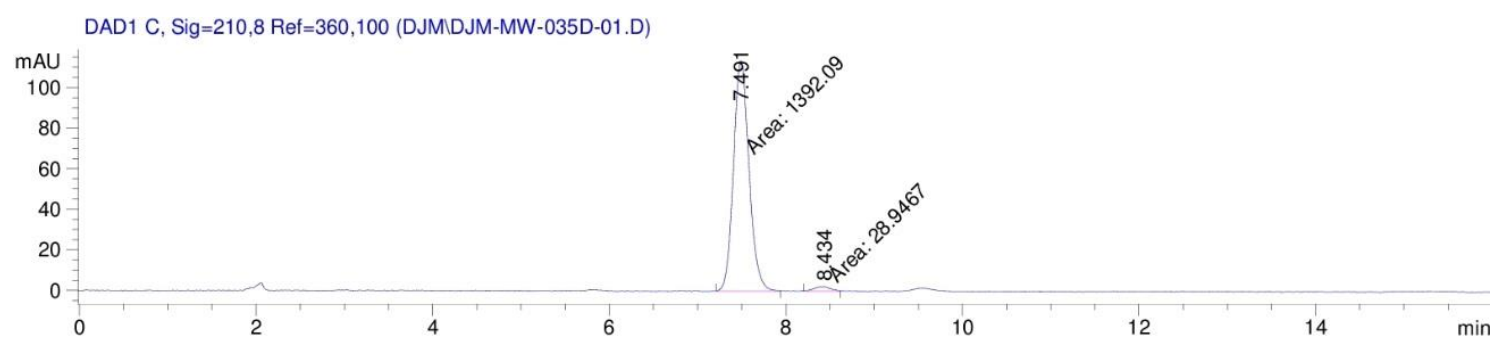

Signal 3: DAD1 C, Sig=210,8 Ref=360,100

\begin{tabular}{|c|c|c|c|c|c|c|}
\hline $\begin{array}{c}\text { Peak } \\
\#\end{array}$ & $\begin{array}{c}\text { RetTime } \\
\text { [min] }\end{array}$ & Type & $\begin{array}{l}\text { Width } \\
\text { [min] }\end{array}$ & $\begin{array}{c}\text { Area } \\
{\left[\mathrm{mAU}^{*} \mathrm{~s}\right]}\end{array}$ & $\begin{array}{l}\text { Height } \\
{[\mathrm{mAU}]}\end{array}$ & $\begin{array}{c}\text { Area } \\
\frac{\circ}{0}\end{array}$ \\
\hline 1 & & & & 139 & & \\
\hline 2 & 8.434 & $\mathrm{MM}$ & .2069 & 28.94671 & 2.33124 & 2.0370 \\
\hline
\end{tabular}

Totals : $\quad 1421.03716 \quad 115.98144$ 
(R)-5-(4-(tert-Butyl)phenyl)-3H-spiro[benzofuran-2,2'-inden]-1'(3'H)-one (2j)<smiles>CC(C)(C)c1ccc(-c2ccc3c(c2)C[C@]2(Cc4ccccc4C2=O)O3)cc1</smiles>

2j

\section{Racemic compound}

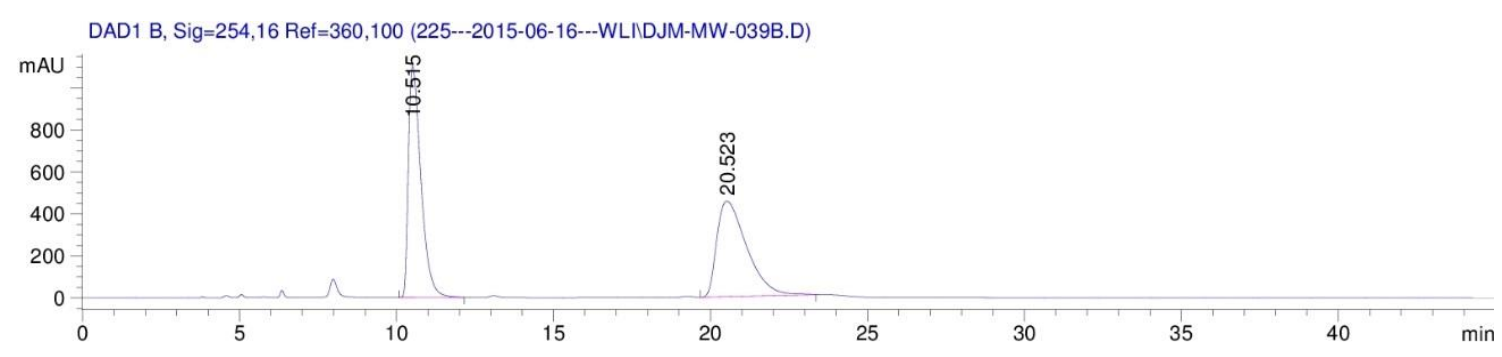

Signal 2: DAD1 B, Sig=254,16 Ref=360,100

\begin{tabular}{|c|c|c|c|c|c|c|}
\hline $\begin{array}{c}\text { Peak } \\
\#\end{array}$ & $\begin{array}{c}\text { RetTime } \\
\text { [min] }\end{array}$ & Type & $\begin{array}{l}\text { Width } \\
\text { [min] }\end{array}$ & $\begin{array}{c}\text { Area } \\
{\left[\mathrm{mAU}^{*} \mathrm{~S}\right]}\end{array}$ & $\begin{array}{l}\text { Height } \\
{[\mathrm{mAU}]}\end{array}$ & $\begin{array}{c}\text { Area } \\
\quad \%\end{array}$ \\
\hline 1 & & & & & 11 & 50 \\
\hline 2 & 20.5 & $V B$ & 0005 & $3.01950 \mathrm{e} 4$ & 456.02942 & 49.6540 \\
\hline
\end{tabular}

Totals : $\quad 6.08108 \mathrm{e} 4 \quad 1561.52222$

\section{Enantioenriched compound}

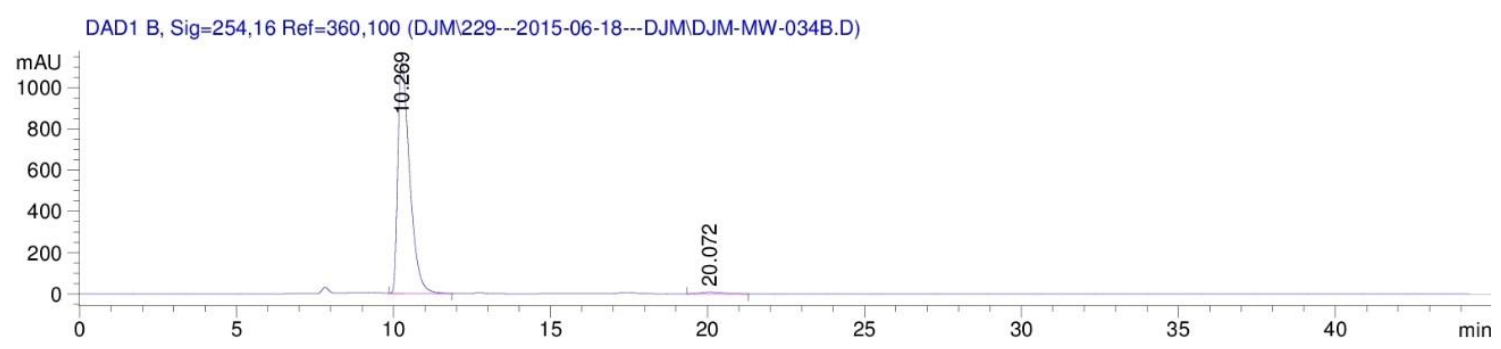

Signal 2: DAD1 B, Sig=254,16 Ref=360,100

\begin{tabular}{|c|c|c|c|c|c|c|}
\hline $\begin{array}{c}\text { Peak } \\
\#\end{array}$ & $\begin{array}{c}\text { RetTime } \\
\text { [min] }\end{array}$ & Type & $\begin{array}{l}\text { Width } \\
\text { [min] }\end{array}$ & $\begin{array}{c}\text { Area } \\
{\left[\mathrm{mAU}^{\star} \mathrm{s}\right]}\end{array}$ & $\begin{array}{l}\text { Height } \\
{[\mathrm{mAU}]}\end{array}$ & $\begin{array}{c}\text { Area } \\
\quad \%\end{array}$ \\
\hline & & & & & & \\
\hline 1 & 10.2 & VB & 4011 & $2.95056 \mathrm{e} 4$ & 1117.35876 & 5599 \\
\hline 2 & 20.072 & $\mathrm{BB}$ & 0.6011 & 431.10681 & 8.46612 & 1.4401 \\
\hline
\end{tabular}
Totals :
$2.99367 e 4 \quad 1125.82488$ 
(R)-5-Phenyl-3H-spiro[benzofuran-2,2'-inden]-1'(3'H)-one (2k)<smiles>O=C1c2ccccc2C[C@]12Cc1cc(-c3ccccc3)ccc1O2</smiles>

\section{Racemic compound}

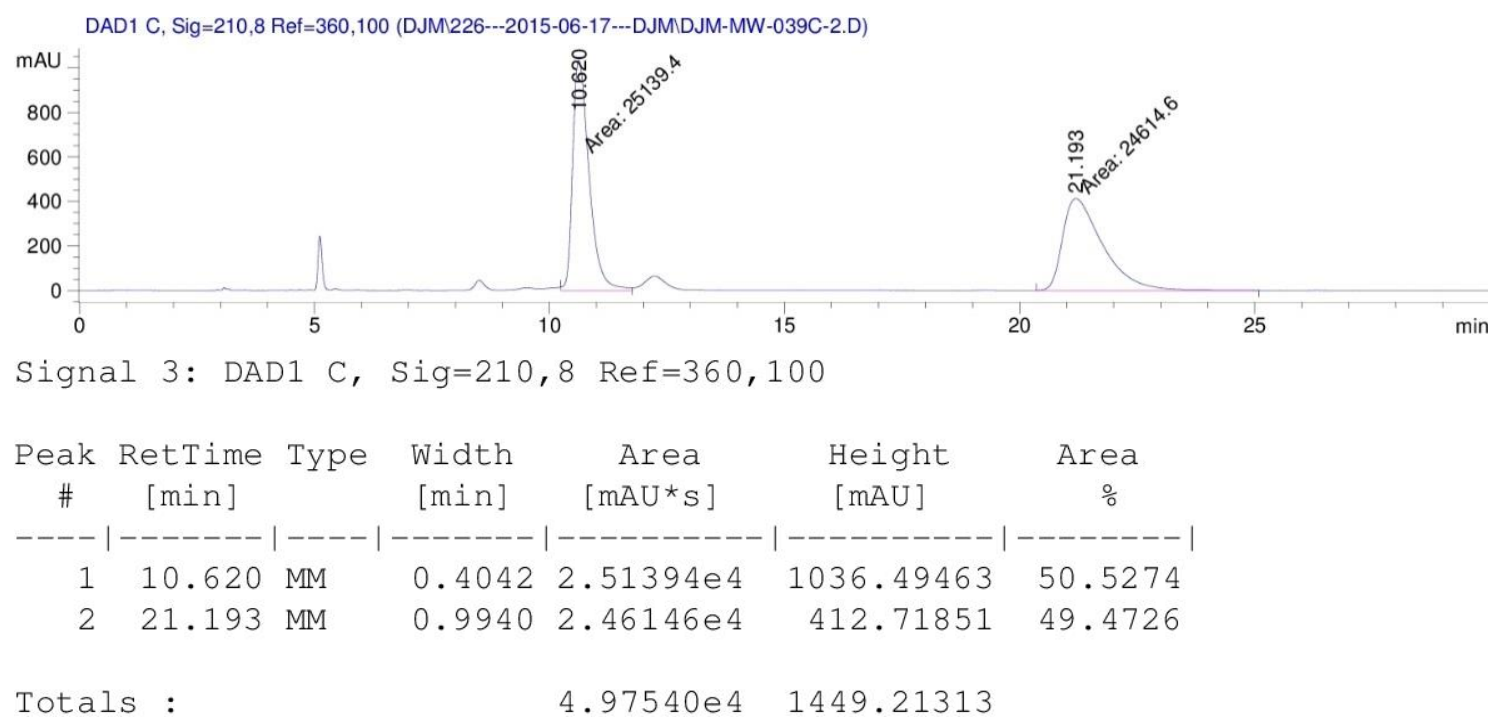

\section{Enantioenriched compound}

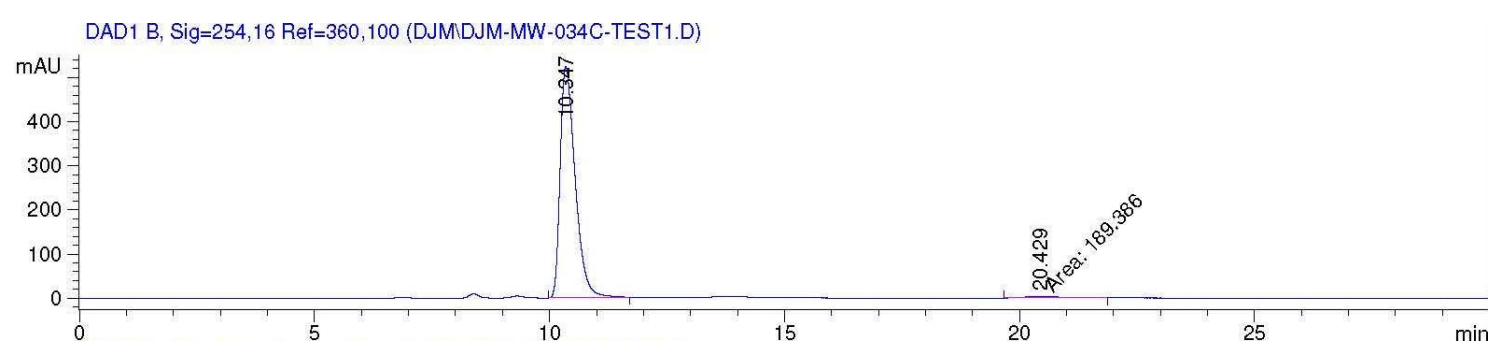

\begin{tabular}{|c|c|c|c|c|c|c|}
\hline $\begin{array}{c}\text { Peak } \\
\#\end{array}$ & $\begin{array}{c}\text { RetTime } \\
\text { [min] }\end{array}$ & Type & $\begin{array}{l}\text { Width } \\
\text { [min] }\end{array}$ & $\begin{array}{c}\text { Area } \\
{\left[\mathrm{mAU}^{*} \mathrm{~s}\right]}\end{array}$ & $\begin{array}{l}\text { Height } \\
{[\mathrm{mAU}]}\end{array}$ & $\begin{array}{c}\text { Area } \\
\%\end{array}$ \\
\hline & & & ------- & $\mid----------$ & $----------\mid$ & -------- \\
\hline 1 & 10.347 & $\mathrm{BB}$ & 0.3391 & $1.16370 \mathrm{e} 4$ & 523.91907 & 98.3986 \\
\hline 2 & 20.429 & $\mathrm{MM}$ & 0.8986 & 189.38576 & 3.51273 & 1.6014 \\
\hline
\end{tabular}

Totals : $\quad 1.18264$ e4 527.43180 
(R)-5-(4-(Hydroxymethyl)phenyl)-3H-spiro[benzofuran-2,2'-inden]-1'(3'H)-one (2I)<smiles>O=C1c2ccccc2C[C@]12Cc1cc(-c3ccc(CO)cc3)ccc1O2</smiles>

2I

\section{Racemic compound}

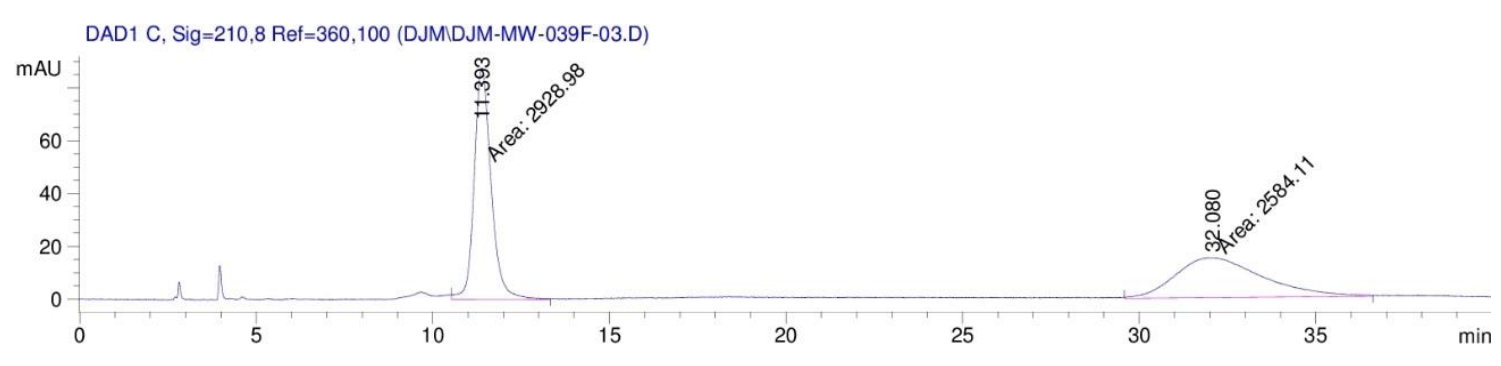

Signal 3: DAD1 C, Sig=210,8 Ref=360,100

\begin{tabular}{|c|c|c|c|c|c|c|}
\hline $\begin{array}{c}\text { Peak } \\
\#\end{array}$ & $\begin{array}{c}\text { RetTime } \\
\text { [min] }\end{array}$ & Type & $\begin{array}{l}\text { Width } \\
\text { [min] }\end{array}$ & $\begin{array}{c}\text { Area } \\
{\left[\mathrm{mAU}^{*} \mathrm{~S}\right]}\end{array}$ & $\begin{array}{l}\text { Height } \\
\text { [mAU] }\end{array}$ & $\begin{array}{c}\text { Area } \\
\quad \%\end{array}$ \\
\hline & & & & & & \\
\hline 1 & 11.393 & MM & 5569 & 2928.97827 & 65136 & 1277 \\
\hline 2 & 32.080 & MM & 2.8479 & 2584.11084 & 15.12287 & 46.8723 \\
\hline
\end{tabular}

Totals :

$5513.08911 \quad 102.77423$

\section{Enantioenriched compound}

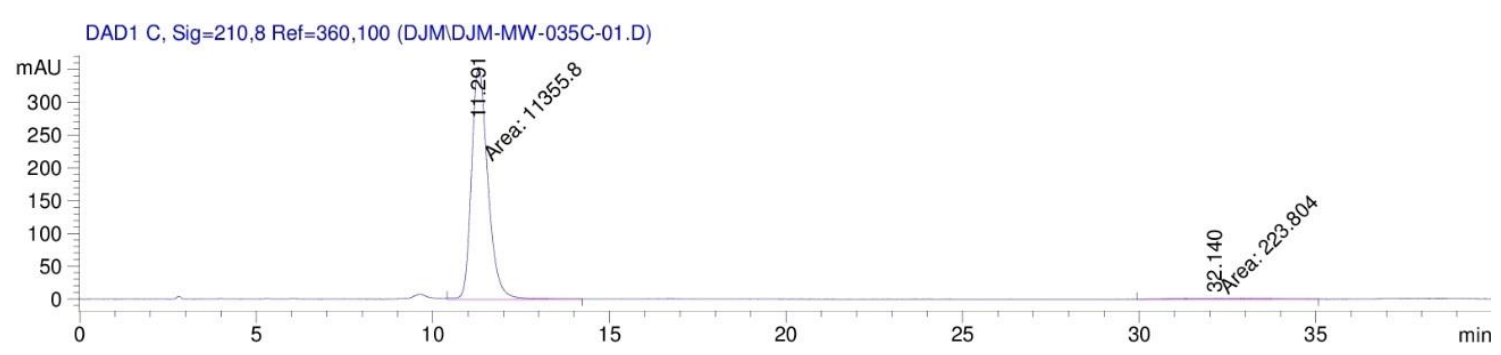

\begin{tabular}{|c|c|c|c|c|c|c|}
\hline $\begin{array}{c}\text { Peak } \\
\#\end{array}$ & $\begin{array}{c}\text { RetTime } \\
\text { [min] }\end{array}$ & Type & $\begin{array}{l}\text { Width } \\
\text { [min] }\end{array}$ & $\begin{array}{c}\text { Area } \\
{\left[\mathrm{mAU}^{*} \mathrm{~S}\right]}\end{array}$ & $\begin{array}{l}\text { Height } \\
{[\mathrm{mAU}]}\end{array}$ & $\begin{array}{c}\text { Area } \\
\frac{\circ}{\circ}\end{array}$ \\
\hline-- & & & - & |----- & --1 & --------- \\
\hline 1 & 11.291 & $\mathrm{MM}$ & 0.5350 & $1.13558 \mathrm{e} 4$ & 353.77740 & 98.0672 \\
\hline 2 & 140 & MM & 2.4509 & 223.80432 & 1.52190 & 1.9328 \\
\hline
\end{tabular}

Totals :

$1.15796 \mathrm{e} \quad 355.29931$ 
<smiles>O=C1c2ccccc2C[C@]12Cc1cc(-c3ccc(OC(F)(F)F)cc3)ccc1O2</smiles>

\section{Racemic compound}

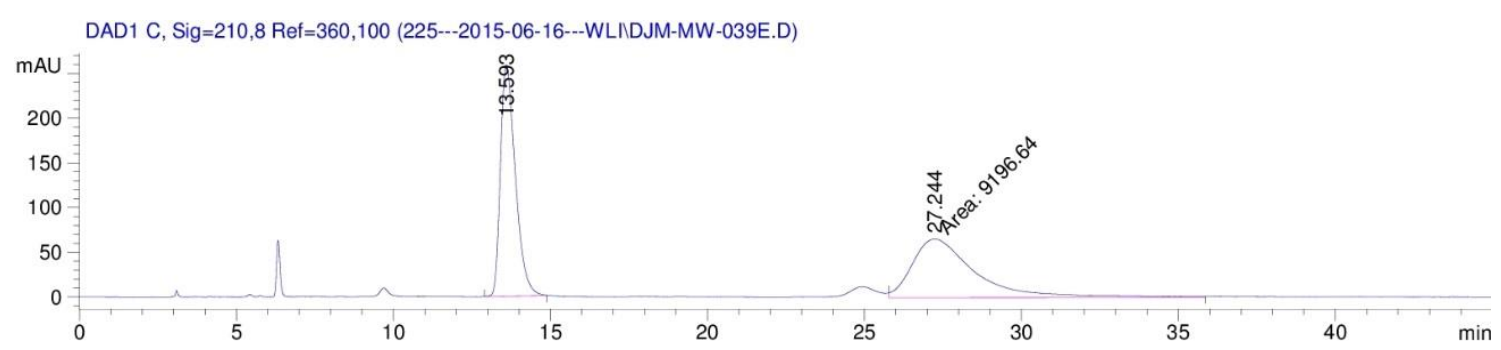

Signal 3: DAD1 C, Sig=210,8 Ref=360,100

\begin{tabular}{|c|c|c|c|c|c|c|}
\hline $\begin{array}{c}\text { Peak } \\
\#\end{array}$ & $\begin{array}{c}\text { RetTime } \\
\text { [min] }\end{array}$ & Type & $\begin{array}{l}\text { Width } \\
\text { [min] }\end{array}$ & $\begin{array}{c}\text { Area } \\
{\left[\mathrm{mAU}^{*} \mathrm{~S}\right]}\end{array}$ & $\begin{array}{l}\text { Height } \\
{[\mathrm{mAU}]}\end{array}$ & $\begin{array}{c}\text { Area } \\
\frac{\circ}{\circ}\end{array}$ \\
\hline & & & & & & \\
\hline 2 & 27.244 & MM & .3334 & 9196.63965 & 65.68928 & 51.7926 \\
\hline
\end{tabular}

Totals : $\quad 1.77567 e 4 \quad 324.60255$

\section{Enantioenriched compound}

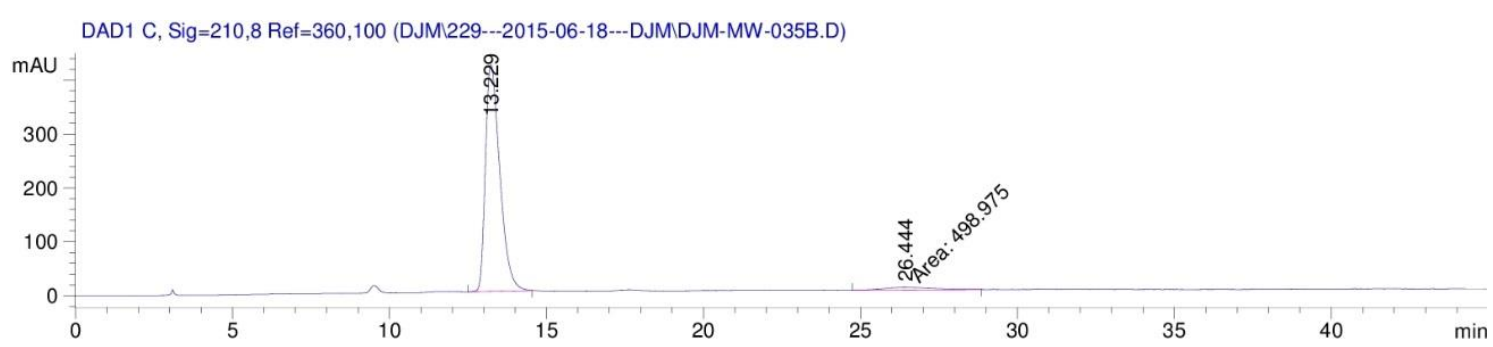

Signal 3: DAD1 C, Sig=210,8 Ref=360,100

\begin{tabular}{|c|c|c|c|c|c|c|}
\hline $\begin{array}{c}\text { Peak } \\
\#\end{array}$ & $\begin{array}{c}\text { RetTime } \\
\text { [min] }\end{array}$ & Type & $\begin{array}{l}\text { Width } \\
\text { [min] }\end{array}$ & $\begin{array}{c}\text { Area } \\
{\left[\mathrm{mAU}{ }^{*} \mathrm{~S}\right]}\end{array}$ & $\begin{array}{l}\text { Height } \\
{[\mathrm{mAU}]}\end{array}$ & $\begin{array}{c}\text { Area } \\
\frac{\circ}{0}\end{array}$ \\
\hline 1 & & & & 1.3 & 42 & 96 \\
\hline 2 & 26.444 & $\mathrm{MM}$ & 5949 & 498.97504 & 4.90675 & 3.5909 \\
\hline
\end{tabular}

Totals : $\quad 1.38954 \mathrm{e} 4 \quad 425.89961$ 
(R)-5-(4-Chlorophenyl)-3H-spiro[benzofuran-2,2'-inden]-1'(3'H)-one (2n)<smiles>O=C1c2ccccc2C[C@]12Cc1cc(-c3ccc(Cl)cc3)ccc1O2</smiles>

\section{Racemic compound}

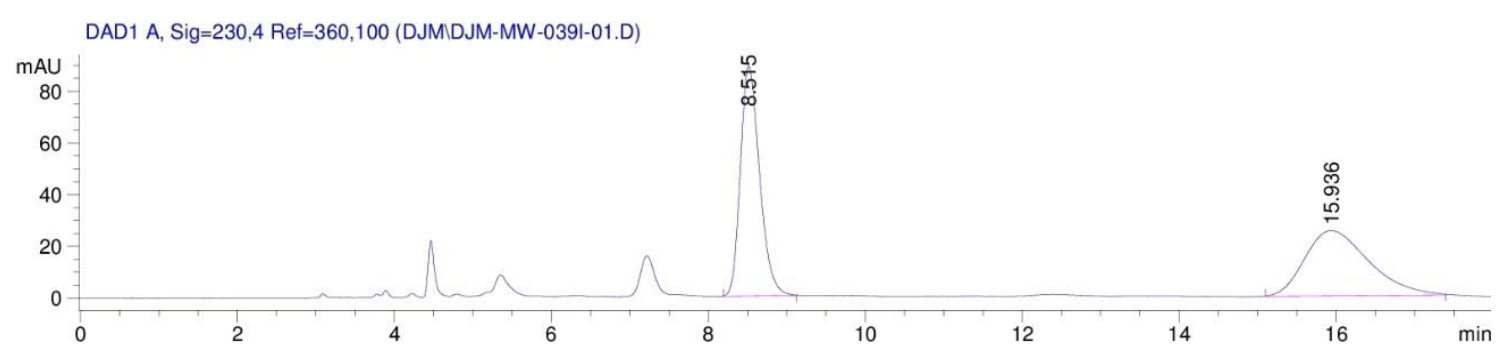

Signal 1: DAD1 A, Sig=230,4 Ref=360,100

\begin{tabular}{|c|c|c|c|c|c|c|}
\hline $\begin{array}{c}\text { Peak } \\
\#\end{array}$ & $\begin{array}{c}\text { RetTime } \\
\text { [min] }\end{array}$ & Type & $\begin{array}{l}\text { Width } \\
\text { [min] }\end{array}$ & $\begin{array}{c}\text { Area } \\
{\left[\mathrm{mAU}^{*} \mathrm{~S}\right]}\end{array}$ & $\begin{array}{l}\text { Height } \\
{[\mathrm{mAU}]}\end{array}$ & $\begin{array}{c}\text { Area } \\
\frac{\circ}{0}\end{array}$ \\
\hline 1 & & $\mathrm{BB}$ & & 15 & & 10 \\
\hline 2 & 15 & $\mathrm{BB}$ & 0.6795 & 1435.03906 & .27223 & 48.6390 \\
\hline
\end{tabular}

Totals :

\section{Enantioenriched compound}

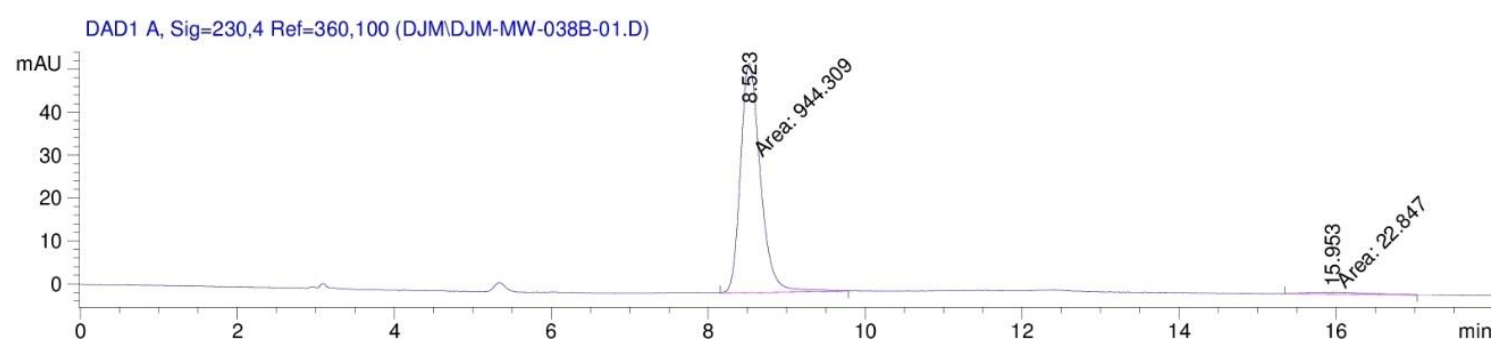

Signal 1: DAD1 A, Sig=230,4 Ref=360,100

\begin{tabular}{|c|c|c|c|c|c|c|}
\hline $\begin{array}{c}\text { Peak } \\
\#\end{array}$ & $\begin{array}{c}\text { RetTime } \\
\text { [min] }\end{array}$ & Type & $\begin{array}{l}\text { Width } \\
\text { [min] }\end{array}$ & $\begin{array}{c}\text { Area } \\
{\left[\mathrm{mAU}^{*} \mathrm{~S}\right]}\end{array}$ & $\begin{array}{l}\text { Height } \\
{[\mathrm{mAU}]}\end{array}$ & $\begin{array}{c}\text { Area } \\
\frac{\circ}{0}\end{array}$ \\
\hline 1 & & 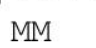 & & 944 & 50 & 77 \\
\hline$?$ & 15.953 & $M$ & 332 & 22.84696 & $4.57002 e-1$ & 2.3623 \\
\hline
\end{tabular}

Totals :

$$
967.15555 \quad 53.91160
$$


(R)-5-(4-(Trifluoromethyl)phenyl)-3H-spiro[benzofuran-2,2'-inden]-1'(3'H)-one (2o)<smiles>O=C1c2ccccc2C[C@]12Cc1cc(-c3ccc(C(F)(F)F)cc3)ccc1O2</smiles>

\section{Racemic compound}

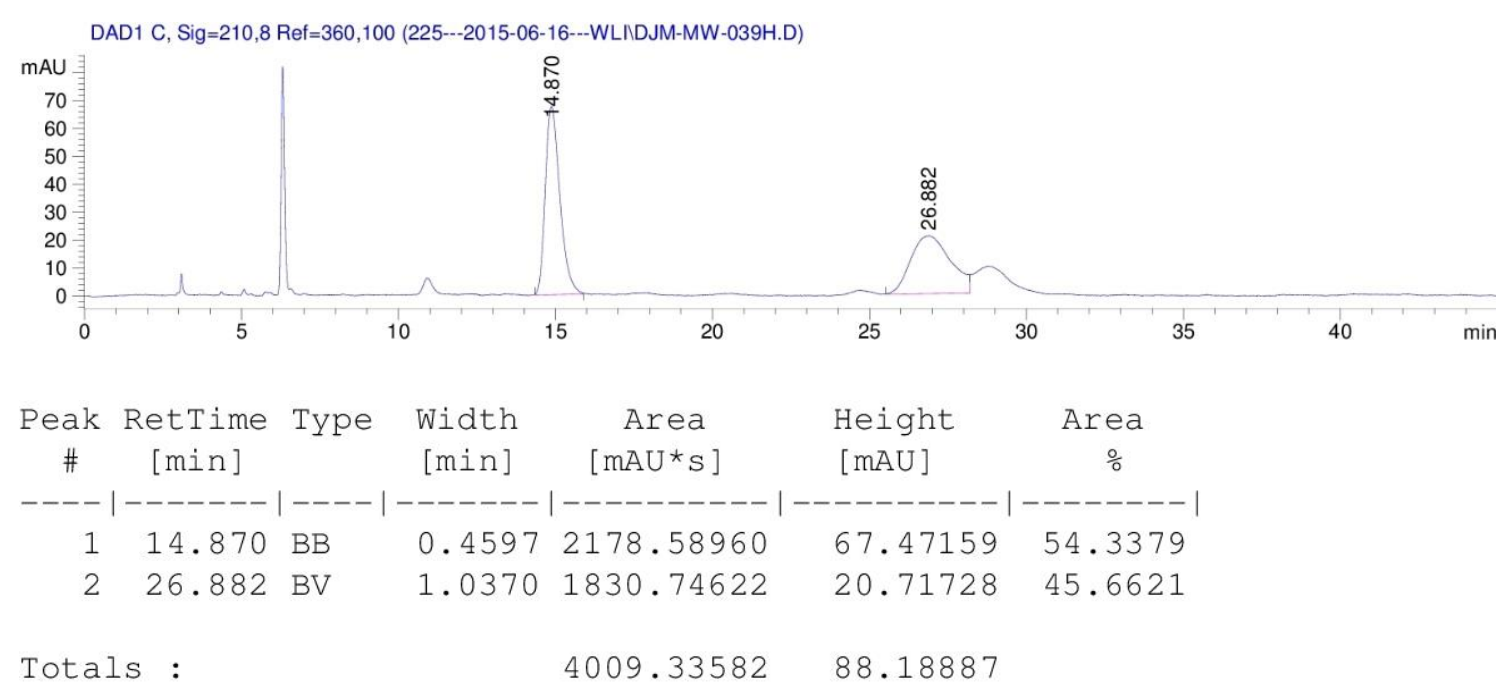

\section{Enantioenriched compound}

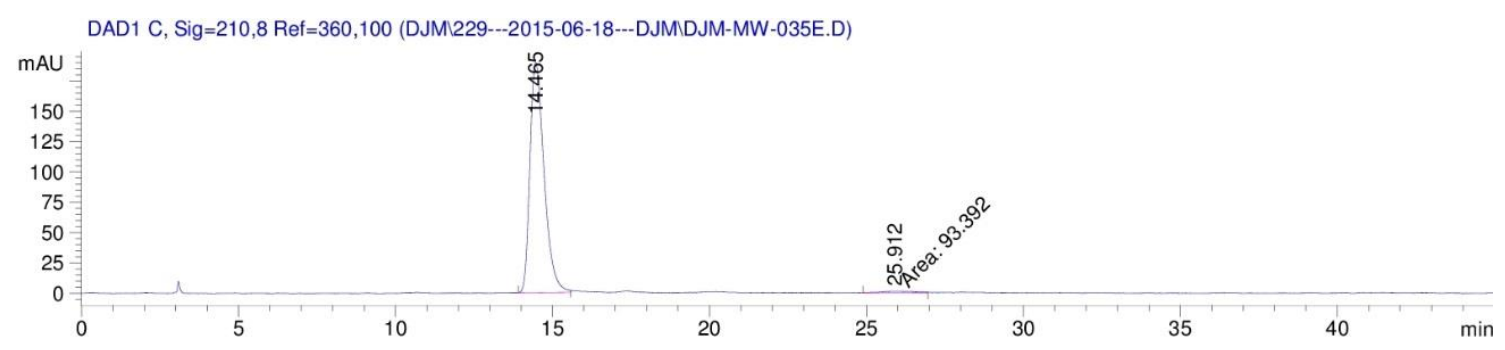

Signal 3: DAD1 C, Sig=210,8 Ref=360,100

\begin{tabular}{|c|c|c|c|c|c|c|}
\hline $\begin{array}{c}\text { Peak } \\
\#\end{array}$ & $\begin{array}{c}\text { RetTime } \\
\text { [min] }\end{array}$ & Type & $\begin{array}{l}\text { Width } \\
\text { [min] }\end{array}$ & $\begin{array}{c}\text { Area } \\
{\left[\mathrm{mAU}^{*} \mathrm{~s}\right]}\end{array}$ & $\begin{array}{l}\text { Height } \\
{[\mathrm{mAU}]}\end{array}$ & $\begin{array}{c}\text { Area } \\
\frac{\circ}{0}\end{array}$ \\
\hline & & & & & & - \\
\hline 1 & & & & 5953. & 7 & 5 \\
\hline 2 & 12 & $M$ & & 93.39204 & 728 & 5445 \\
\hline
\end{tabular}

Totals : $\quad 6046.65327 \quad 191.07345$ 
(R)-5-(4-Acetylphenyl)-3H-spiro[benzofuran-2,2'-inden]-1'(3'H)-one (2p)<smiles>CC(C)(C)c1ccc(-c2ccc3c(c2)C[C@]2(Cc4ccccc4C2=O)O3)cc1</smiles>

\section{Racemic compound}

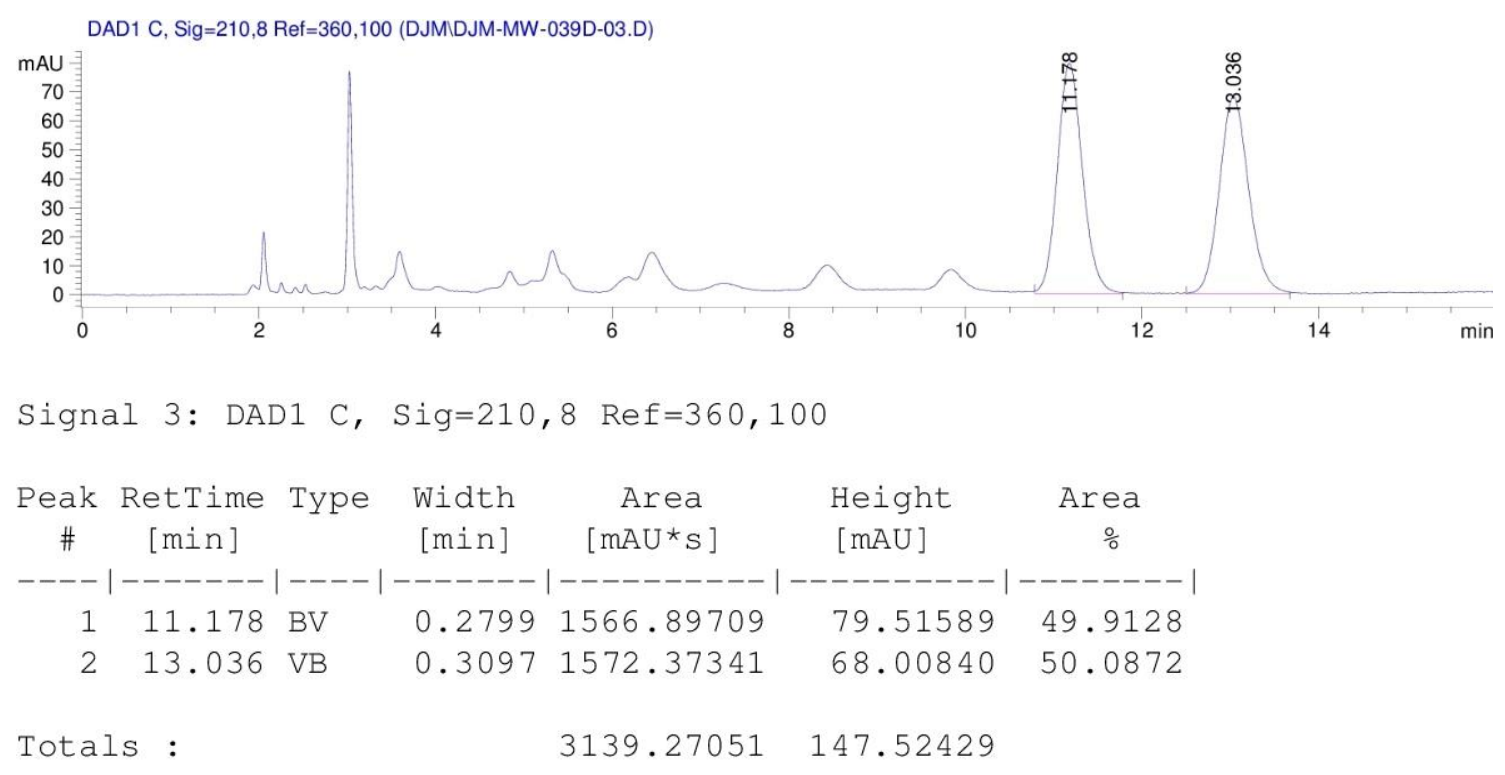

\section{Enantioenriched compound}

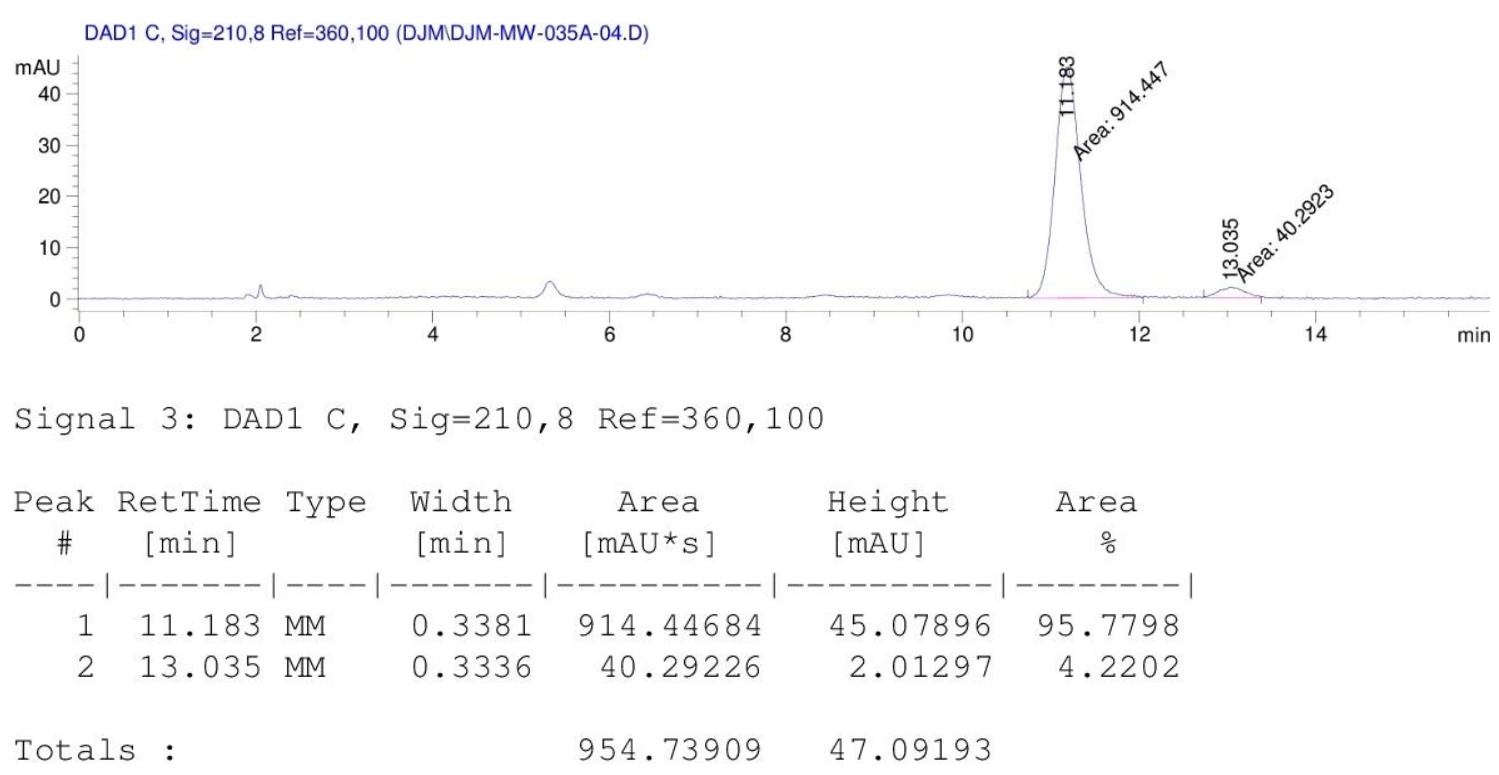


(R)-4-(1'-Oxo-1',3'-dihydro-3H-spiro[benzofuran-2,2'-inden]-5-yl)benzonitrile (2q)<smiles>N#Cc1ccc(-c2ccc3c(c2)C[C@]2(Cc4ccccc4C2=O)O3)cc1</smiles>

$2 q$

\section{Racemic compound}

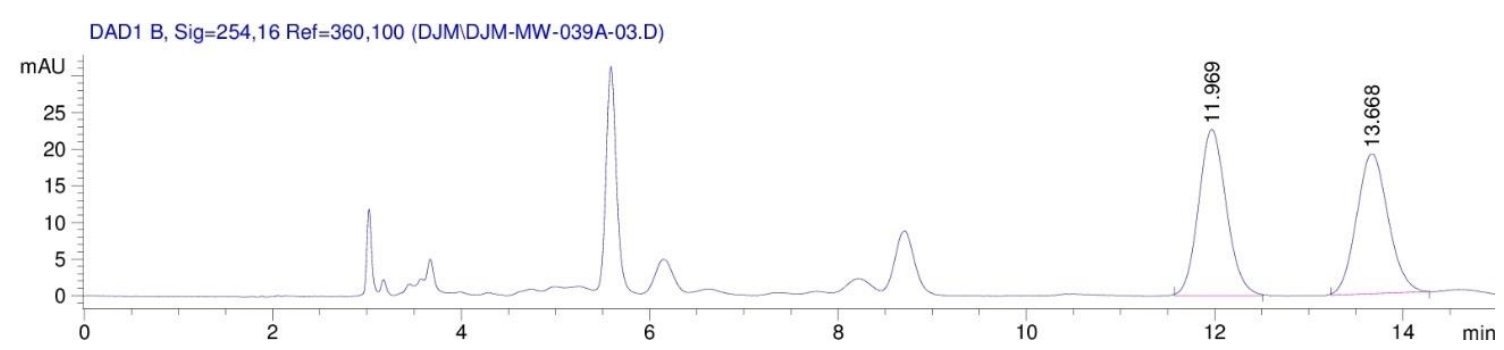

Signal 2: DAD1 B, Sig=254,16 Ref=360,100

\begin{tabular}{|c|c|c|c|c|c|c|}
\hline $\begin{array}{c}\text { Peak } \\
\#\end{array}$ & $\begin{array}{c}\text { RetTime } \\
\text { [min] }\end{array}$ & Type & $\begin{array}{l}\text { Width } \\
\text { [min] }\end{array}$ & $\begin{array}{c}\text { Area } \\
{\left[\mathrm{mAU}^{*} \mathrm{~s}\right]}\end{array}$ & $\begin{array}{l}\text { Height } \\
{[\mathrm{mAU}]}\end{array}$ & $\begin{array}{c}\text { Area } \\
\frac{\circ}{0}\end{array}$ \\
\hline 1 & & $\mathrm{BB}$ & & 467 & 99 & 51 \\
\hline . & 13.668 & D & 421 & 444.86200 & 19.14670 & 48.784 \\
\hline
\end{tabular}

Totals : $\quad 911.90216 \quad 41.84669$

\section{Enantioenriched compound}

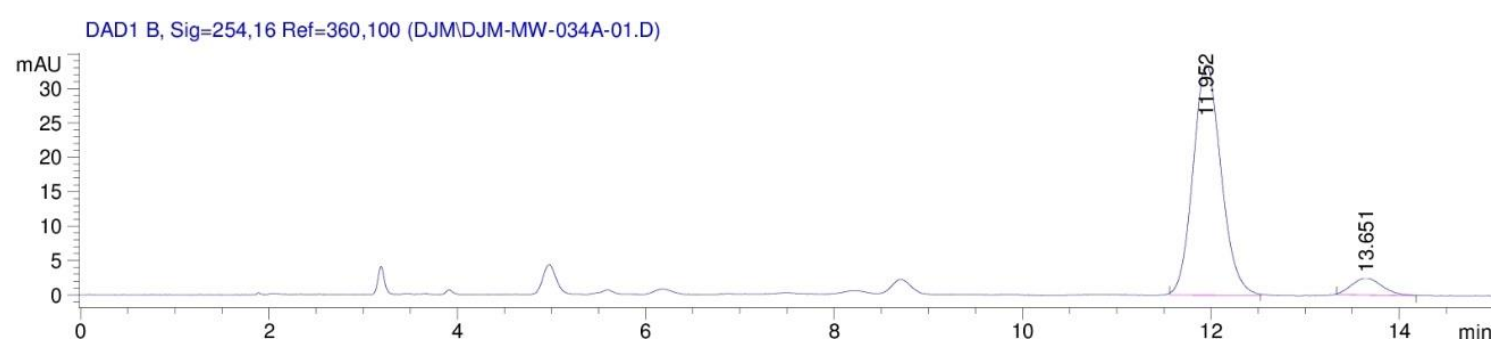

Signal 2: DAD1 B, Sig=254,16 Ref=360,100

\begin{tabular}{|c|c|c|c|c|c|c|}
\hline $\begin{array}{c}\text { Peak } \\
\#\end{array}$ & $\begin{array}{c}\text { RetTime } \\
\text { [min] }\end{array}$ & Type & $\begin{array}{l}\text { Width } \\
\text { [min] }\end{array}$ & $\begin{array}{c}\text { Area } \\
{\left[\mathrm{mAU}{ }^{*} \mathrm{~S}\right]}\end{array}$ & $\begin{array}{l}\text { Height } \\
{[\mathrm{mAU}]}\end{array}$ & $\begin{array}{c}\text { Area } \\
\frac{\circ}{0}\end{array}$ \\
\hline 1 & 11.95 & & 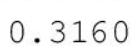 & 692 & 40 & 90 \\
\hline 2 & 13.651 & 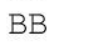 & 595 & 55.66621 & 2.48336 & 7.441 \\
\hline
\end{tabular}

$\begin{array}{lll}\text { Totals : } & 748.09718 \quad 35.99876\end{array}$ 


\section{(R)-3H-spiro[benzo[b]thiophene-2,2'-inden]-1'(3'H)-one (2r)}<smiles>[Z7]C[C@]12Cc3ccccc3C(=O)[C@]1(Cc1ccccc1)C2</smiles>

\section{Racemic compound}

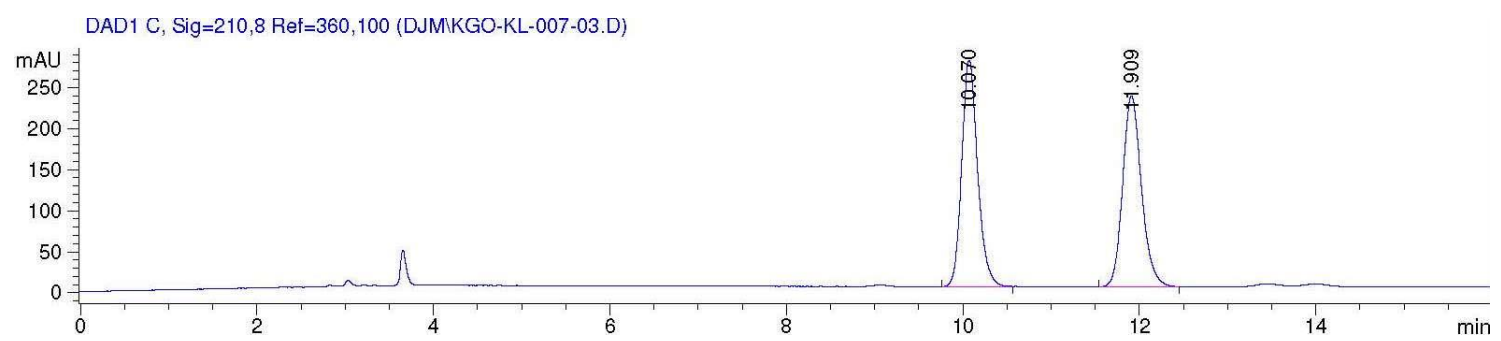

Signal 3: DAD1 C, Sig=210,8 $\operatorname{Re} f=360,100$

\begin{tabular}{|c|c|c|c|c|c|c|}
\hline $\begin{array}{c}\text { Peak } \\
\#\end{array}$ & $\begin{array}{c}\text { RetTime } \\
\text { [min] }\end{array}$ & Type & $\begin{array}{l}\text { Width } \\
\text { [min] }\end{array}$ & $\begin{array}{c}\text { Area } \\
{\left[\mathrm{mAU}^{*} \mathrm{~S}\right]}\end{array}$ & $\begin{array}{l}\text { Height } \\
\text { [mAU] }\end{array}$ & $\begin{array}{c}\text { Area } \\
\%\end{array}$ \\
\hline 1 & & $\mathrm{~V}$ & 0 - & 345 & & \\
\hline 2 & 11.909 & $\mathrm{D}$ & 0.22 & 3448.92529 & 232.39845 & 49.9449 \\
\hline
\end{tabular}

Totals : $\quad 6905.45508 \quad 508.21950$

\section{Enantioenriched compound}

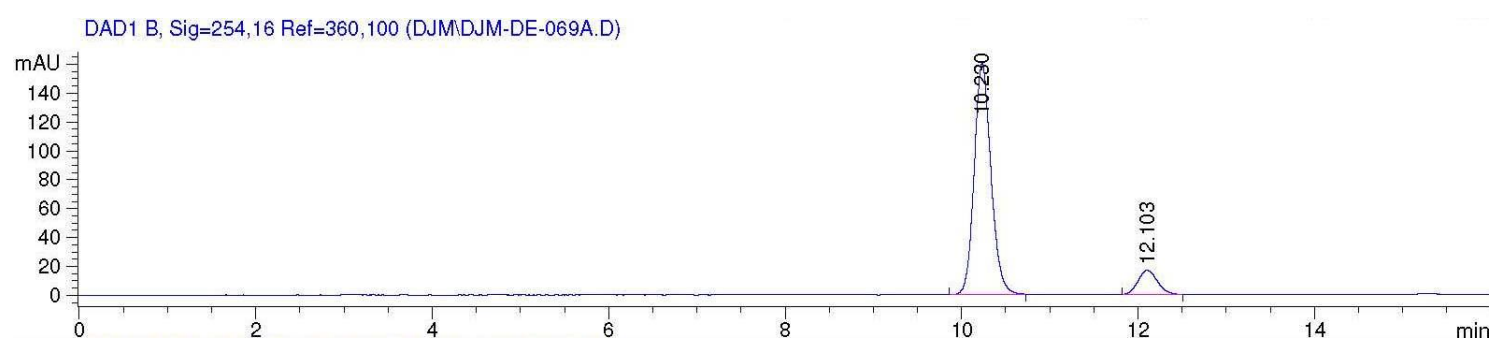

Signal 3: DAD1 C, $\operatorname{Sig}=210,8$ Ref $=360,100$

\begin{tabular}{|c|c|c|c|c|c|c|}
\hline $\begin{array}{c}\text { Peak } \\
\#\end{array}$ & $\begin{array}{c}\text { RetTime } \\
\text { [min] }\end{array}$ & Type & $\begin{array}{l}\text { Width } \\
\text { [min] }\end{array}$ & $\begin{array}{c}\text { Area } \\
{\left[\mathrm{mAU}^{*} \mathrm{~s}\right]}\end{array}$ & $\begin{array}{l}\text { Height } \\
\text { [mAU] }\end{array}$ & $\begin{array}{c}\text { Area } \\
\frac{\%}{\sigma}\end{array}$ \\
\hline 1 & 10.230 & VB & 0.2030 & 4858.50830 & 363.44409 & 88.5685 \\
\hline 2 & 12.100 & $\mathrm{VB}$ & 0.2334 & 627.08734 & 39.24952 & 11.4315 \\
\hline Tota & . & & & 5485.59564 & 402.69361 & \\
\hline
\end{tabular}


(R)-5-Bromo-3H-spiro[benzo[b]thiophene-2,2'-inden]-1'(3'H)-one (2s)<smiles>O=C1c2ccccc2C[C@]12Cc1cc(Br)ccc1S2</smiles>

2s

\section{Racemic compound}

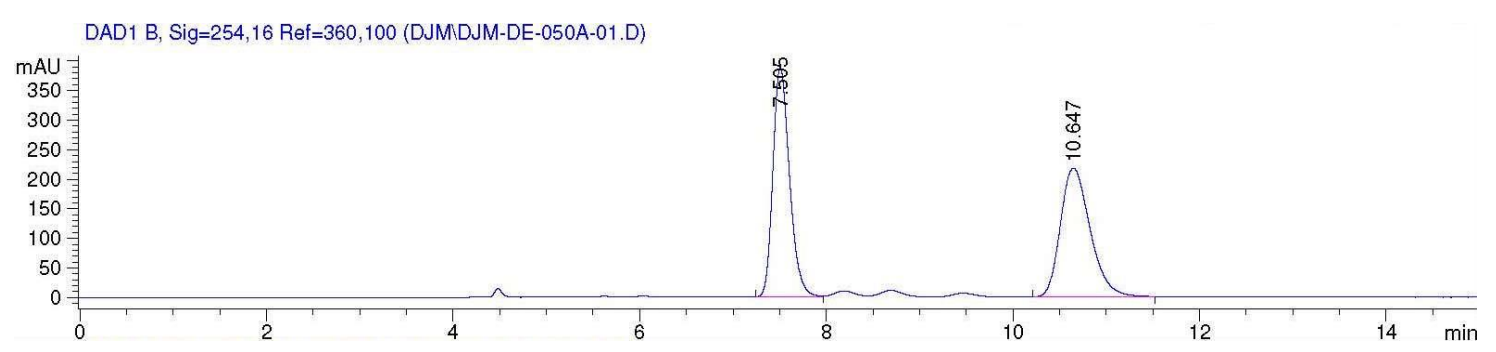

\begin{tabular}{|c|c|c|c|c|c|c|}
\hline $\begin{array}{c}\text { Peak } \\
\#\end{array}$ & $\begin{array}{c}\text { RetTime } \\
\text { [min] }\end{array}$ & Type & $\begin{array}{l}\text { Width } \\
\text { [min] }\end{array}$ & $\begin{array}{c}\text { Area } \\
{\left[\mathrm{mAU} U^{*} \mathrm{~s}\right]}\end{array}$ & $\begin{array}{l}\text { Height } \\
\text { [mAU] }\end{array}$ & $\begin{array}{c}\text { Area } \\
\frac{\circ}{0}\end{array}$ \\
\hline 1 & 7.505 & $\mathrm{BV}$ & 0.1919 & 4820.04736 & 388.16632 & 50.0329 \\
\hline 2 & 10.647 & $\mathrm{BB}$ & 0.3415 & 4813.70215 & 218.08931 & 49.9671 \\
\hline btal & & & & 9633.74951 & 606.25563 & \\
\hline
\end{tabular}

\section{Enantioenriched compound}

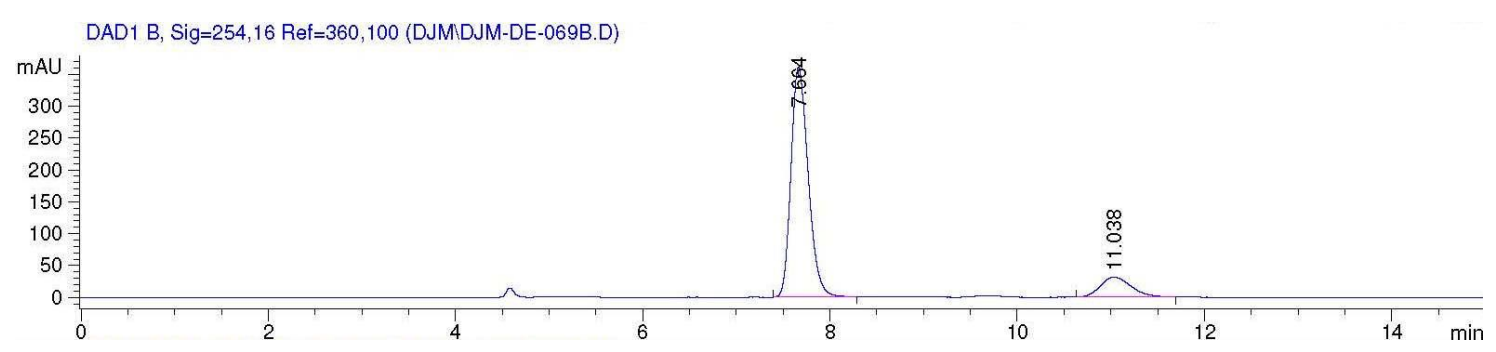

Signal 2: DAD1 B, Sig=254,16 Ref $=360,100$

\begin{tabular}{|c|c|c|c|c|c|c|}
\hline $\begin{array}{c}\text { Peak } \\
\#\end{array}$ & $\begin{array}{c}\text { RetTime } \\
\text { [min] }\end{array}$ & Type & $\begin{array}{l}\text { Width } \\
\text { [min] }\end{array}$ & $\begin{array}{c}\text { Area } \\
{\left[\mathrm{mAU}^{*} \mathrm{~s}\right]}\end{array}$ & $\begin{array}{c}\text { Height } \\
\text { [mAU] }\end{array}$ & $\begin{array}{c}\text { Area } \\
\%\end{array}$ \\
\hline 1 & 7.664 & $\mathrm{BB}$ & 0.1961 & 4573.24023 & 360.26767 & 86.3878 \\
\hline 2 & 11.038 & $\mathrm{BB}$ & 0.3502 & 720.61127 & 31.35009 & 13.6122 \\
\hline Tot & : & & & 5293.85150 & 391.61776 & \\
\hline
\end{tabular}


3' $H$-Spiro[indene-2,2'-thieno[3,2-c]pyridin]-1(3H)-one (2t)<smiles>O=C1c2ccccc2C[C@]12Cc1cnccc1S2</smiles>

2t

\section{Racemic compound}

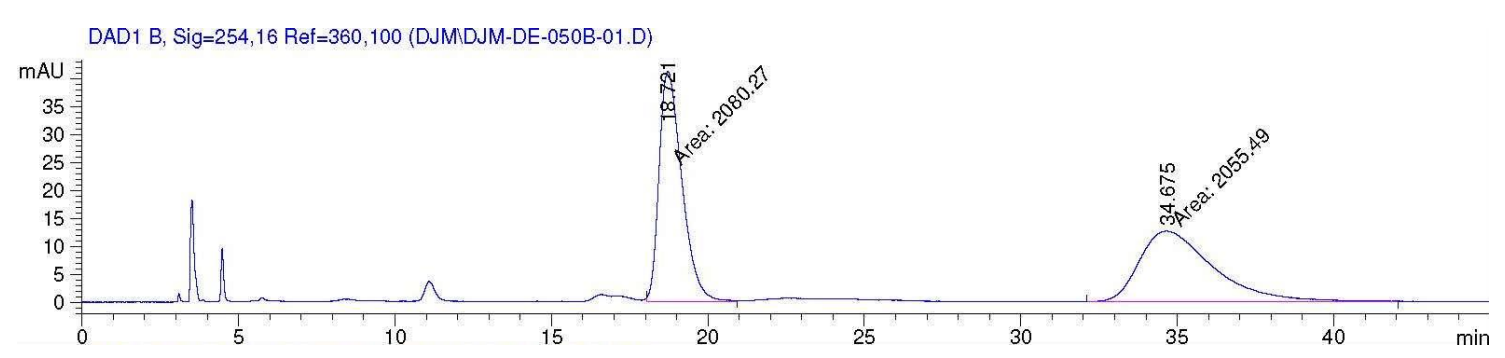

Signal 2: DAD1 B, Sig=254,16 Ref $=360,100$

\begin{tabular}{|c|c|c|c|c|c|c|}
\hline $\begin{array}{c}\text { Peak } \\
\#\end{array}$ & $\begin{array}{c}\text { RetTime } \\
\text { [min] }\end{array}$ & Type & $\begin{array}{l}\text { Width } \\
\text { [min] }\end{array}$ & $\begin{array}{c}\text { Area } \\
{\left[\mathrm{mAU}^{*} \mathrm{~s}\right]}\end{array}$ & $\begin{array}{l}\text { Height } \\
\text { [mAU] }\end{array}$ & $\begin{array}{c}\text { Area } \\
\quad \%\end{array}$ \\
\hline 1 & 18.721 & MM & 0.8433 & 2080.26733 & 41.11317 & 50.2995 \\
\hline 2 & 34.675 & MM & 2.7123 & 2055.49219 & 12.63068 & 49.7005 \\
\hline Total & : & & & 4135.75952 & 4385 & \\
\hline
\end{tabular}

\section{Enantioenriched compound}

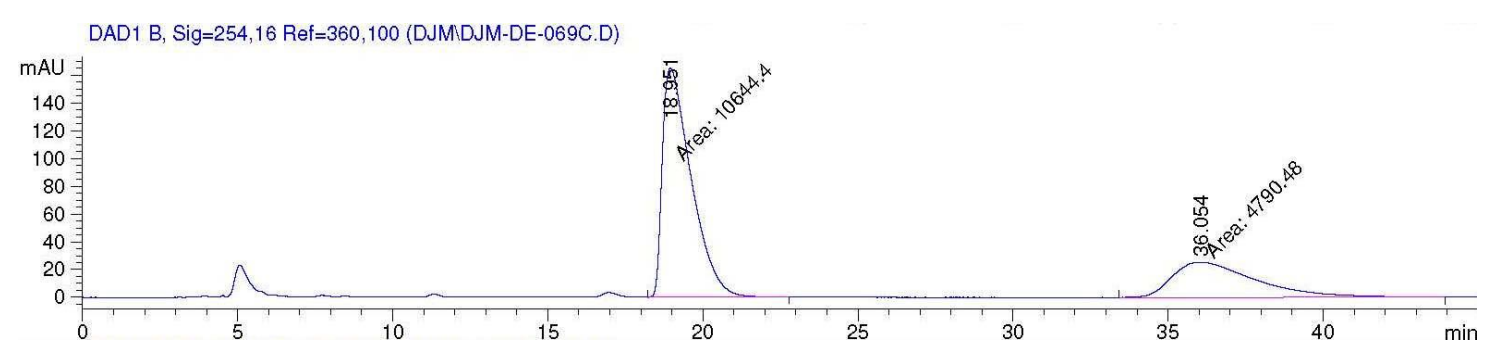

Signal 2: DAD1 B, Sig $=254,16$ Ref $=360,100$

\begin{tabular}{|c|c|c|c|c|c|c|}
\hline $\begin{array}{c}\text { Peak } \\
\#\end{array}$ & $\begin{array}{c}\text { RetTime } \\
\text { [min] }\end{array}$ & Type & $\begin{array}{l}\text { Width } \\
\text { [min] }\end{array}$ & $\begin{array}{c}\text { Area } \\
{\left[\mathrm{mAU}^{*} \mathrm{~s}\right]}\end{array}$ & $\begin{array}{l}\text { Height } \\
\text { [mAU] }\end{array}$ & $\begin{array}{c}\text { Area } \\
\%\end{array}$ \\
\hline 1 & 18.951 & MM & 1.0740 & $1.06444 \mathrm{e} 4$ & 165.18335 & 68.9632 \\
\hline 2 & 36.054 & MM & 3.1276 & 4790.48145 & 25.52798 & 31.0368 \\
\hline Tota & : & & & $1.54348 e 4$ & 190.71133 & \\
\hline
\end{tabular}


<smiles>COc1ccc(-c2ccc3c(c2)C[C@]2(Cc4ccccc4C2=O)S3)cc1</smiles>

\section{Racemic compound}

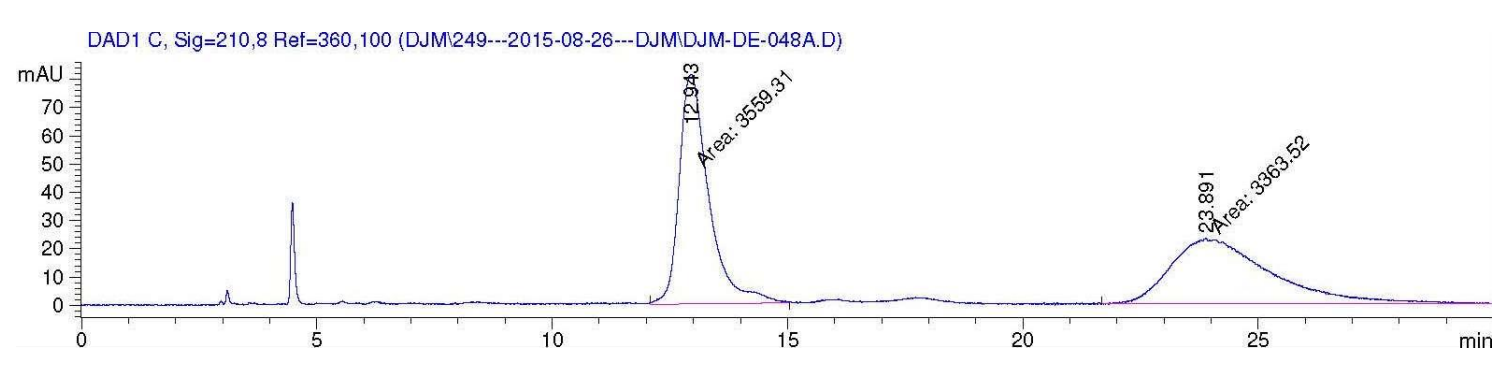

Signal 3: DAD1 C, $\operatorname{Sig}=210,8$ Ref $=360,100$

\begin{tabular}{|c|c|c|c|c|c|c|}
\hline $\begin{array}{c}\text { Peak } \\
\#\end{array}$ & $\begin{array}{c}\text { RetTime } \\
\text { [min] }\end{array}$ & Type & $\begin{array}{l}\text { Width } \\
\text { [min] }\end{array}$ & $\begin{array}{c}\text { Area } \\
{\left[\mathrm{mAU}^{*} \mathrm{~s}\right]}\end{array}$ & $\begin{array}{l}\text { Height } \\
\text { [mAU] }\end{array}$ & $\begin{array}{c}\text { Area } \\
\%\end{array}$ \\
\hline 1 & 12. & $\mathrm{MM}$ & . & 3559. & 903 & 41 \\
\hline 2 & 23.891 & MM & 2.4258 & 3363.52051 & 23.10981 & 48.5859 \\
\hline
\end{tabular}

Totals : $\quad 6922.83203 \quad 104.57884$

\section{Enantioenriched compound}

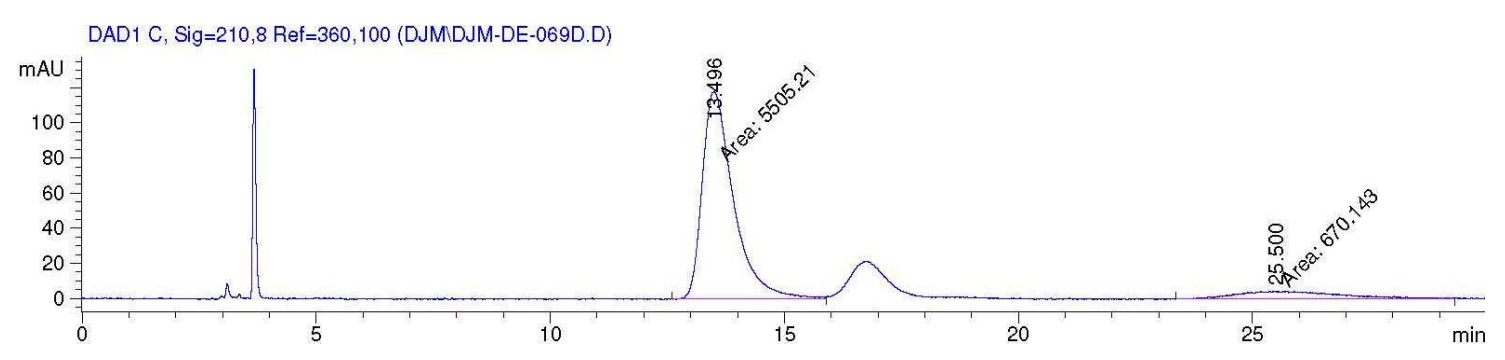

Signal 3: DAD1 C, $\operatorname{Sig}=210,8$ Ref $=360,100$

\begin{tabular}{|c|c|c|c|c|c|c|}
\hline $\begin{array}{c}\text { Peak } \\
\#\end{array}$ & $\begin{array}{c}\text { RetTime } \\
\text { [min] }\end{array}$ & Type & $\begin{array}{l}\text { Width } \\
\text { [min] }\end{array}$ & $\begin{array}{c}\text { Area } \\
{\left[\mathrm{mAU}^{*} \mathrm{~s}\right]}\end{array}$ & $\begin{array}{l}\text { Height } \\
{[\mathrm{mAU}]}\end{array}$ & $\begin{array}{c}\text { Area } \\
\%\end{array}$ \\
\hline & & & & & & \\
\hline & & $\mathrm{MM}$ & & & & \\
\hline
\end{tabular}

Totals :

$6175.35754 \quad 122.86153$ 


\section{(R)-5-(p-Tolyl)-3H-spiro[benzo[b]thiophene-2,2'-inden]-1'(3'H)-one (2v)}<smiles>Cc1ccc(-c2ccc3c(c2)C[C@]2(Cc4ccccc4C2=O)S3)cc1</smiles>

\section{Racemic compound}

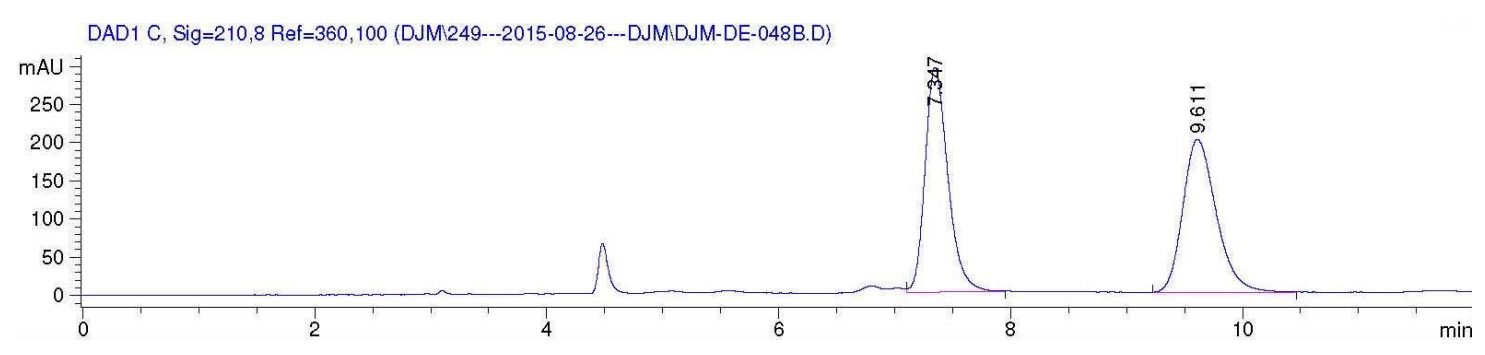

Signal 3: DAD1 C, $\operatorname{Sig}=210,8$ Ref $=360,100$

\begin{tabular}{|c|c|c|c|c|c|c|}
\hline $\begin{array}{c}\text { Peak } \\
\quad \#\end{array}$ & $\begin{array}{c}\text { RetTime } \\
\text { [min] }\end{array}$ & Type & $\begin{array}{l}\text { Width } \\
\text { [min] }\end{array}$ & $\begin{array}{c}\text { Area } \\
{\left[\mathrm{mAU}^{*} \mathrm{~s}\right]}\end{array}$ & $\begin{array}{l}\text { Height } \\
{[\mathrm{mAU}]}\end{array}$ & $\begin{array}{c}\text { Area } \\
\frac{0}{\circ}\end{array}$ \\
\hline 1 & & & & & & \\
\hline 2 & 9.611 & $\mathrm{BB}$ & 0.3083 & 4004.01587 & 200.19933 & 49.7989 \\
\hline
\end{tabular}

Totals : $\quad 8040.37573 \quad 494.68153$

\section{Enantioenriched compound}

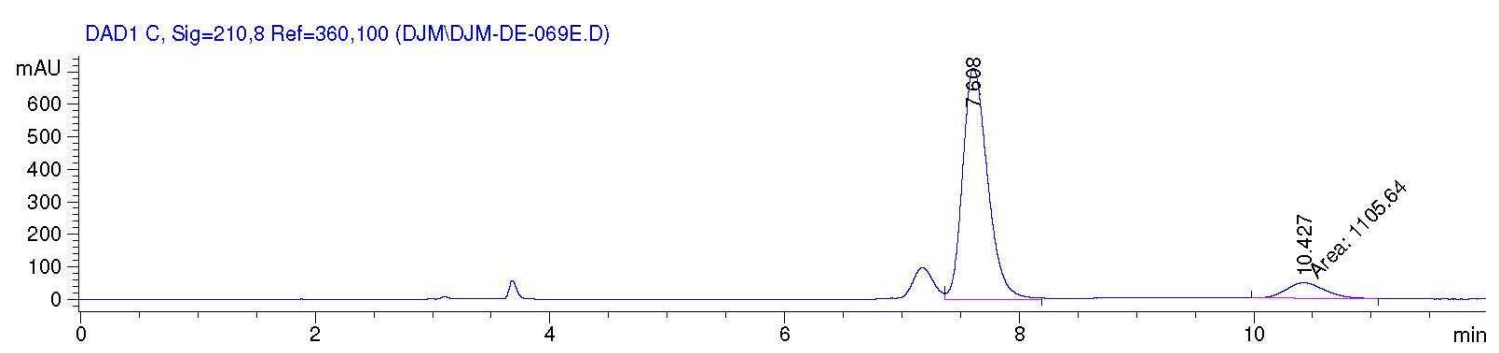

Signal 3: DAD1 C, $\operatorname{Sig}=210,8$ Ref $=360,100$

\begin{tabular}{|c|c|c|c|c|c|c|}
\hline $\begin{array}{c}\text { Peak } \\
\#\end{array}$ & $\begin{array}{c}\text { RetTime } \\
\text { [min] }\end{array}$ & Type & $\begin{array}{l}\text { Width } \\
\text { [min] }\end{array}$ & $\begin{array}{c}\text { Area } \\
{\left[\mathrm{mAU}^{*} \mathrm{~s}\right]}\end{array}$ & $\begin{array}{l}\text { Height } \\
{[\mathrm{mAU}]}\end{array}$ & $\begin{array}{c}\text { Area } \\
\%\end{array}$ \\
\hline & & & & 1.0 & & \\
\hline 2 & 10.427 & & 1 & 1105.63684 & 47.60791 & 9.536 \\
\hline
\end{tabular}

Totals : $\quad 1.15940 \mathrm{e} 4 \quad 759.96662$ 
(R)-5-Phenyl-3H-spiro[benzo[b]thiophene-2,2'-inden]-1'( $\left.3^{\prime} H\right)$-one (2w)<smiles>O=C1c2ccccc2C[C@]12Cc1cc(-c3ccccc3)ccc1S2</smiles>

2w

\section{Racemic compound}

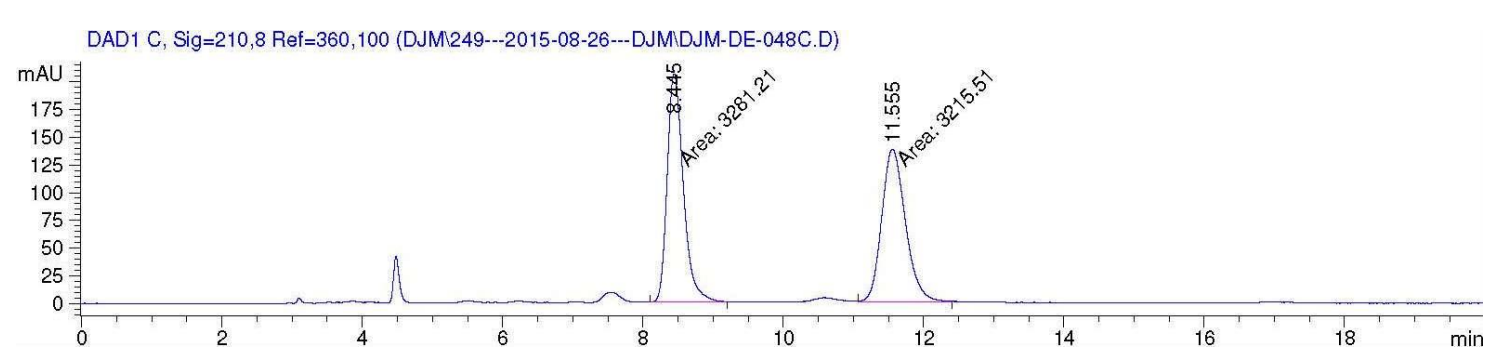

Signal 3: DAD1 C, Sig $=210,8$ Ref $=360,100$

\begin{tabular}{|c|c|c|c|c|c|c|}
\hline $\begin{array}{c}\text { Peak } \\
\#\end{array}$ & $\begin{array}{c}\text { RetTime } \\
\text { [min] }\end{array}$ & Type & $\begin{array}{l}\text { Width } \\
\text { [min] }\end{array}$ & $\begin{array}{c}\text { Area } \\
{[m A U * s]}\end{array}$ & $\begin{array}{l}\text { Height } \\
{[\mathrm{mAU}]}\end{array}$ & $\begin{array}{c}\text { Area } \\
\frac{\%}{\%}\end{array}$ \\
\hline 1 & 8.445 & MM & 0.2654 & 3281.20508 & 206.01927 & 50.5056 \\
\hline 2 & 11.555 & $\mathrm{MM}$ & 0.3902 & 3215.51221 & 137.33586 & 49.4944 \\
\hline
\end{tabular}

Totals : $\quad 6496.71729 \quad 343.35513$

\section{Enantioenriched compound}

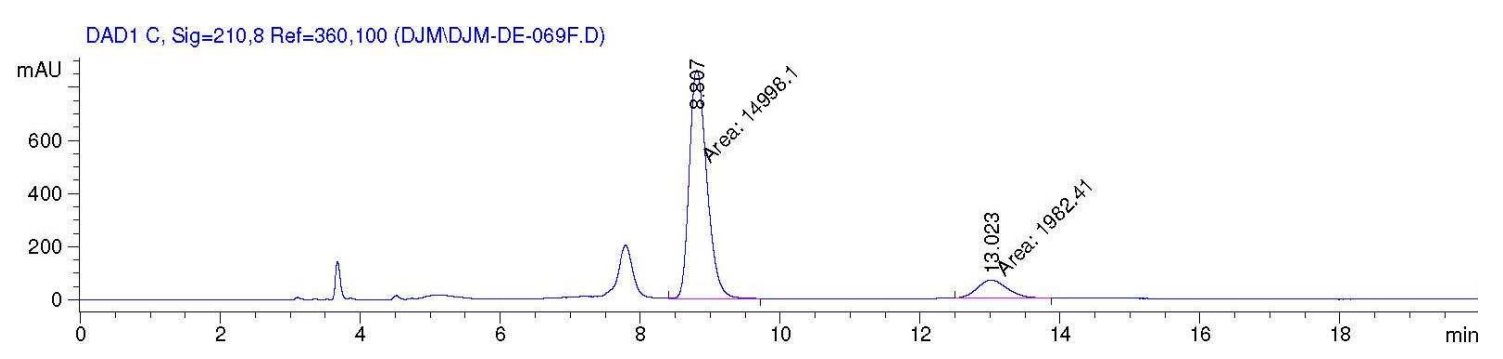

Signal 3: DAD1 C, $\operatorname{Sig}=210,8$ Ref $=360,100$

\begin{tabular}{|c|c|c|c|c|c|c|}
\hline $\begin{array}{c}\text { Peak } \\
\#\end{array}$ & $\begin{array}{c}\text { RetTime } \\
\text { [min] }\end{array}$ & Type & $\begin{array}{l}\text { Width } \\
\text { [min] }\end{array}$ & $\begin{array}{c}\text { Area } \\
{\left[\mathrm{mAU}^{*} \mathrm{~s}\right]}\end{array}$ & $\begin{array}{l}\text { Height } \\
{[\mathrm{mAU}]}\end{array}$ & $\begin{array}{c}\text { Area } \\
\%\end{array}$ \\
\hline 1 & 8.807 & $\mathrm{MM}$ & 0.2901 & $1.49981 \mathrm{e} 4$ & 861.65155 & 88.3253 \\
\hline 2 & 13.023 & $\mathrm{MM}$ & 0.4885 & 1982.41370 & 67.64130 & 11.6747 \\
\hline
\end{tabular}

Totals : $\quad 1.69805 e 4 \quad 929.29285$ 
(R)-5-(4-(Trifluoromethoxy)phenyl)-3H-spiro[benzo[b]thiophene-2,2'-inden]-1'(3'H)-one (2x)<smiles></smiles>

\section{Racemic compound}

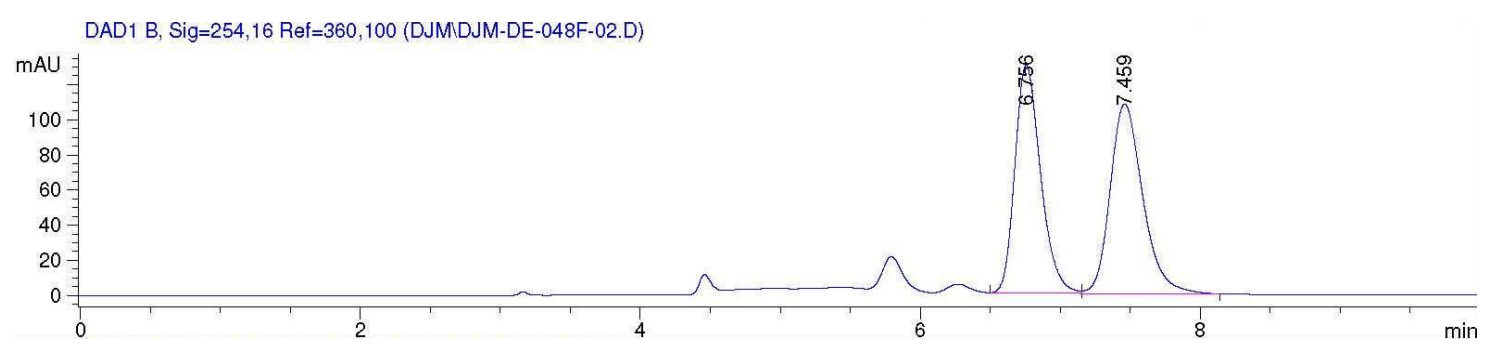

Signal 2: DAD1 B, Sig=254,16 Ref=360,100

\begin{tabular}{|c|c|c|c|c|c|c|}
\hline $\begin{array}{c}\text { Peak } \\
\#\end{array}$ & $\begin{array}{c}\text { RetTime } \\
\text { [min] }\end{array}$ & Type & $\begin{array}{c}\text { Width } \\
\text { [min] }\end{array}$ & $\begin{array}{c}\text { Area } \\
{\left[\mathrm{mAU}^{*} \mathrm{~s}\right]}\end{array}$ & $\begin{array}{l}\text { Height } \\
{[\mathrm{mAU}]}\end{array}$ & $\begin{array}{c}\text { Area } \\
\%\end{array}$ \\
\hline 1 & 6.756 & VV & 0.1980 & 1667.81592 & 129.68294 & 48.8583 \\
\hline 2 & 7.459 & VB & 0.2465 & 1745.75806 & 108.07205 & 51.1417 \\
\hline Total & Ls : & & & 3413.57397 & 237.75499 & \\
\hline
\end{tabular}

\section{Enantioenriched compound}

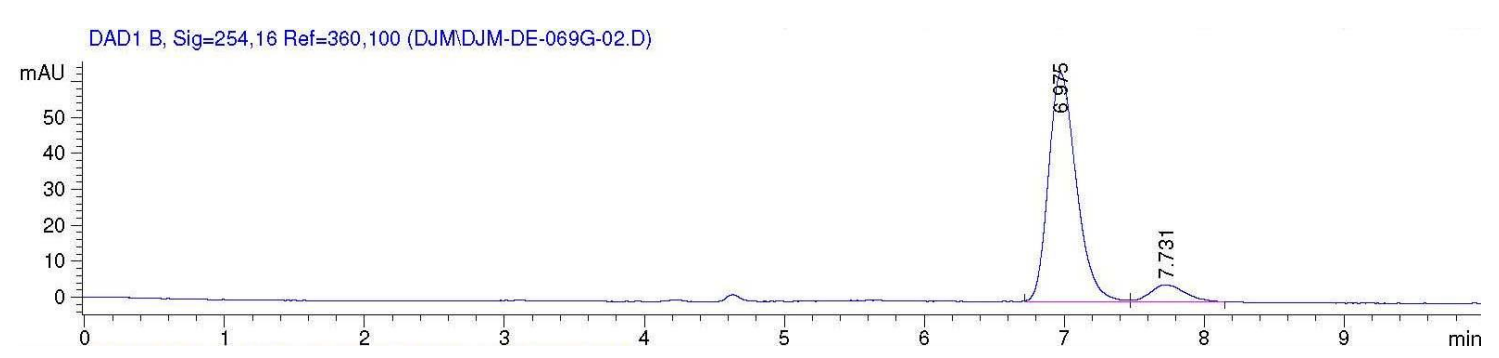

Signal 2: DAD1 B, Sig=254,16 Ref=360,100

\begin{tabular}{|c|c|c|c|c|c|c|}
\hline $\begin{array}{c}\text { Peak } \\
\#\end{array}$ & $\begin{array}{c}\text { RetTime } \\
\text { [min] }\end{array}$ & Type & $\begin{array}{l}\text { Width } \\
\text { [min] }\end{array}$ & $\begin{array}{c}\text { Area } \\
{\left[\mathrm{mAU}^{\star} \mathrm{S}\right]}\end{array}$ & $\begin{array}{l}\text { Height } \\
{[\mathrm{mAU}]}\end{array}$ & $\begin{array}{c}\text { Area } \\
\%\end{array}$ \\
\hline & & & & 876 & 6 & 91 \\
\hline 2 & 7.731 & $\mathrm{~B}$ & .2 & 83.47498 & 4.80031 & 8.6981 \\
\hline
\end{tabular}

Totals : $\quad 959.68939 \quad 68.31767$ 
<smiles>O=C1c2ccccc2C[C@]12Cc1cc(-c3ccc(C(F)(F)F)cc3)ccc1S2</smiles>

\section{Racemic compound}

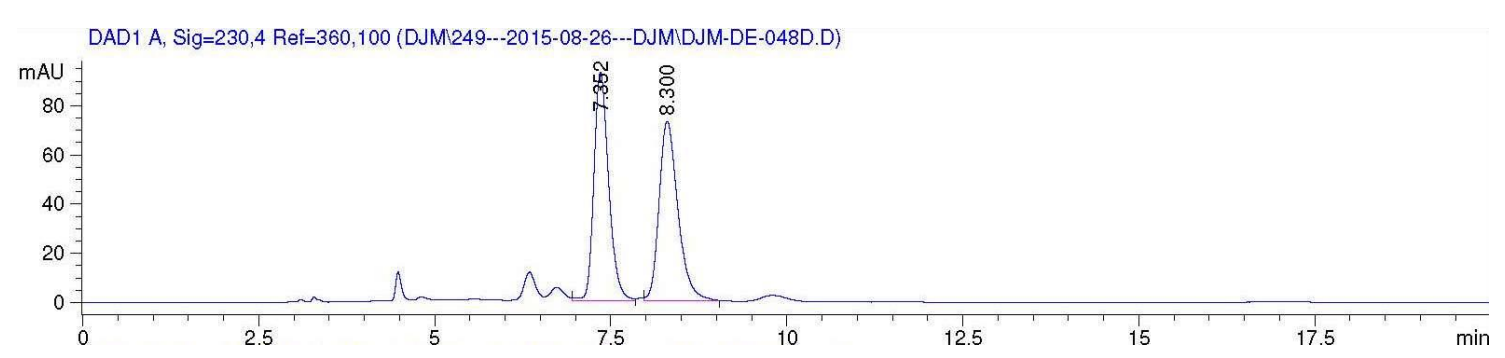

Signal 1: DAD1 A, $\operatorname{Sig}=230,4$ Ref $=360,100$

\begin{tabular}{|c|c|c|c|c|c|c|}
\hline $\begin{array}{c}\text { Peak } \\
\#\end{array}$ & $\begin{array}{c}\text { RetTime } \\
\text { [min] }\end{array}$ & Type & $\begin{array}{l}\text { Width } \\
\text { [min] }\end{array}$ & $\begin{array}{c}\text { Area } \\
{\left[\mathrm{mAU}^{*} \mathrm{~S}\right]}\end{array}$ & $\begin{array}{l}\text { Height } \\
{[\mathrm{mAU}]}\end{array}$ & $\begin{array}{c}\text { Area } \\
\frac{\%}{\circ}\end{array}$ \\
\hline 1 & 7 & VB & & & 49 & 49 \\
\hline 2 & 8.300 & $\mathrm{BB}$ & 0.2839 & 1370.06323 & 73.02206 & 50.1341 \\
\hline
\end{tabular}

Totals : $\quad 2732.79883 \quad 165.96655$

\section{Enantioenriched compound}

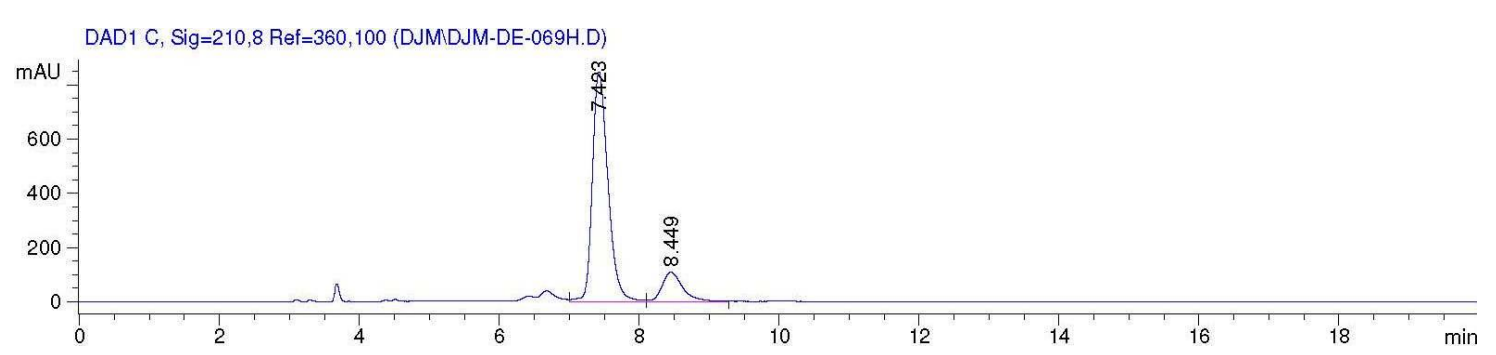

Signal 3: DAD1 C, $\operatorname{Sig}=210,8$ Ref $=360,100$

\begin{tabular}{|c|c|c|c|c|c|c|}
\hline $\begin{array}{c}\text { Peak } \\
\#\end{array}$ & $\begin{array}{c}\text { RetTime } \\
\text { [min] }\end{array}$ & Type & $\begin{array}{l}\text { Width } \\
\text { [min] }\end{array}$ & $\begin{array}{c}\text { Area } \\
{\left[\mathrm{mAU}^{*} \mathrm{~s}\right]}\end{array}$ & $\begin{array}{l}\text { Height } \\
{[\mathrm{mAU}]}\end{array}$ & $\begin{array}{c}\text { Area } \\
\frac{\circ}{\circ}\end{array}$ \\
\hline 1 & 7.423 & VV & 0.2364 & $1.31922 \mathrm{e} 4$ & 848.53961 & 85.0959 \\
\hline 2 & 8.449 & VB & 0.3135 & 2310.54126 & 109.35867 & 14.9041 \\
\hline Total & Is : & & & $1.55027 \mathrm{e} 4$ & 957.89828 & \\
\hline
\end{tabular}


<smiles></smiles>

\section{Racemic compound}

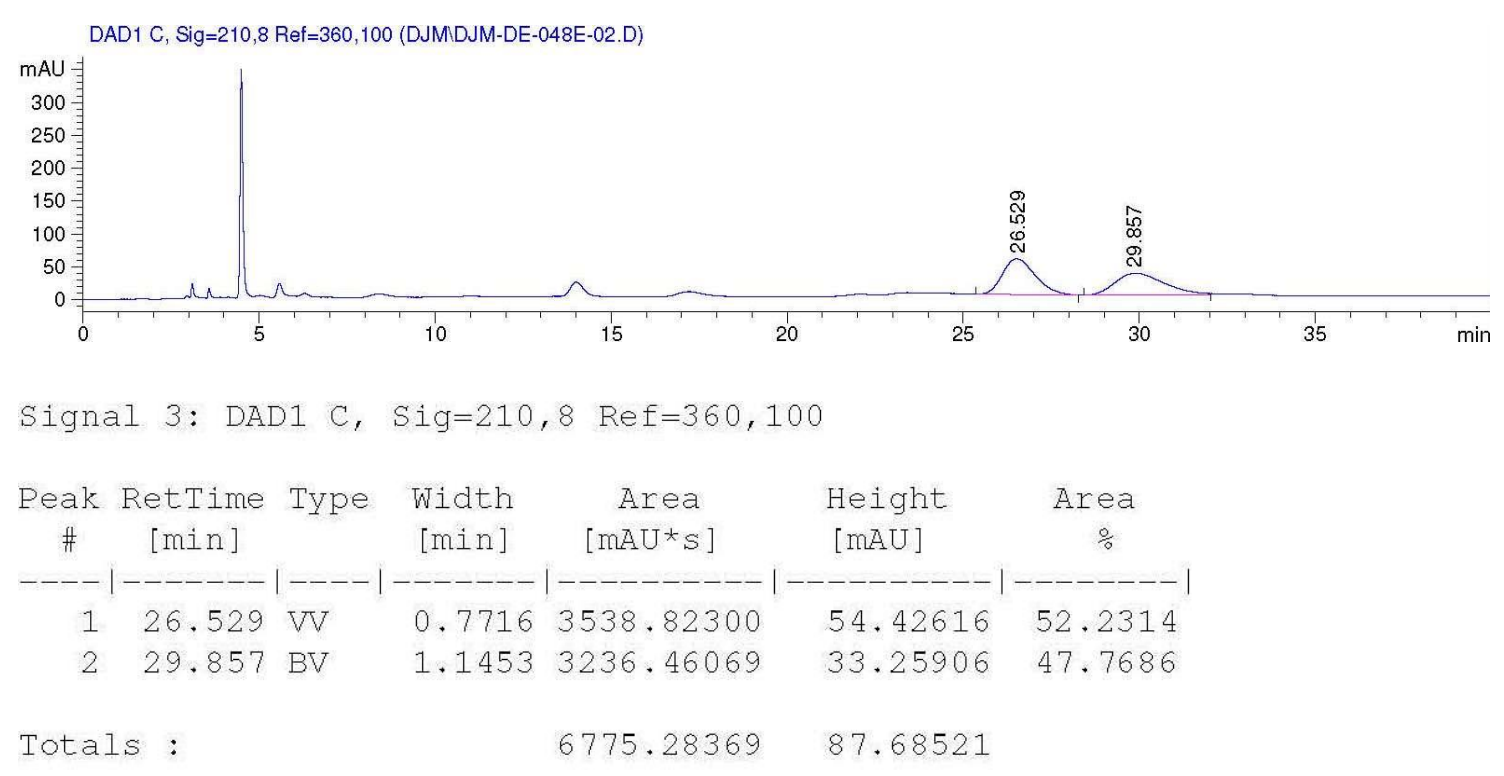

\section{Enantioenriched compound}

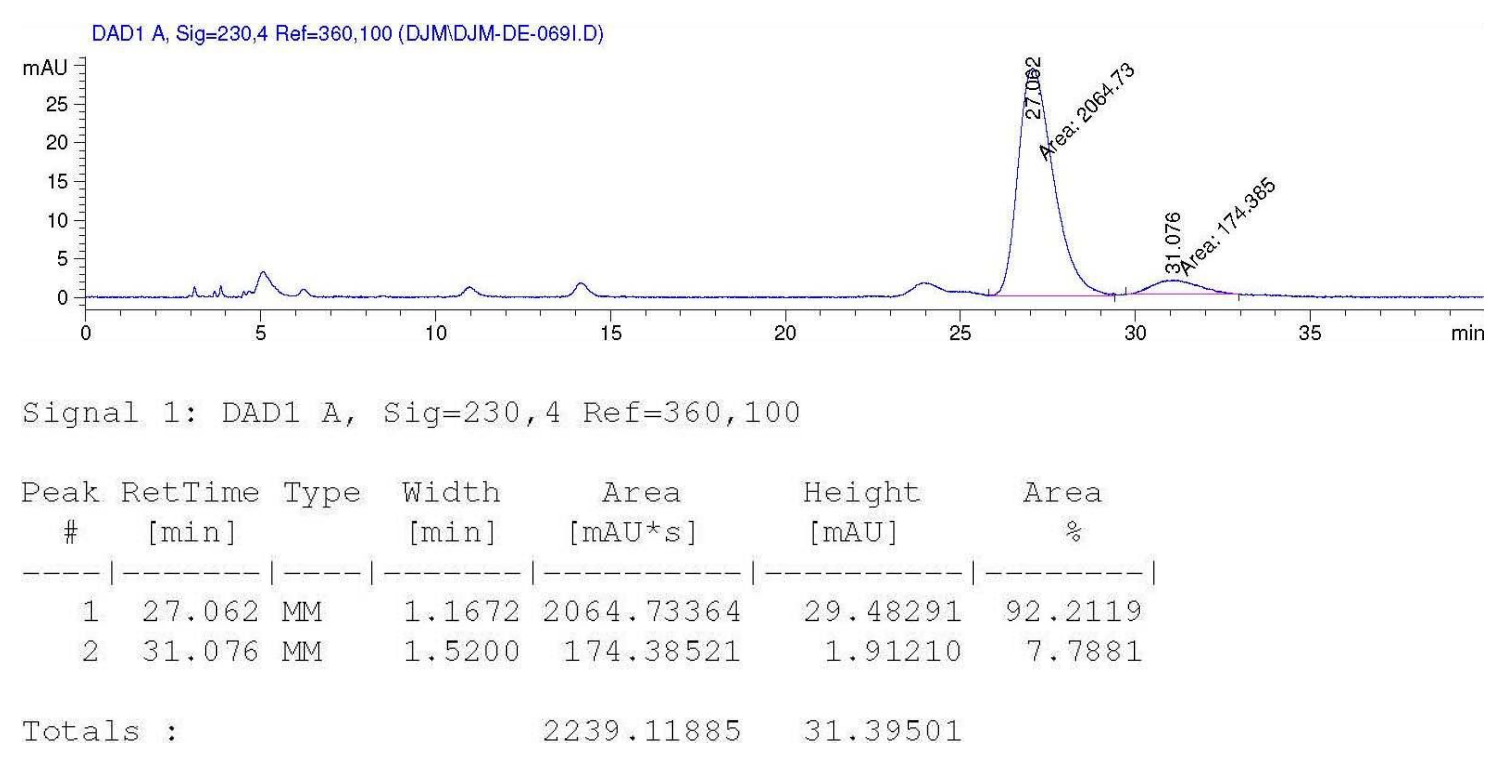




\section{(R)-3H-3H-Spiro[benzofuran-2,2'-inden]-1'(3'H)-one oxime (7)}<smiles>O=C1c2ccccc2C[C@]12Cc1ccccc1O2</smiles>

7

Racemic compound ( $E / Z$ mixture)

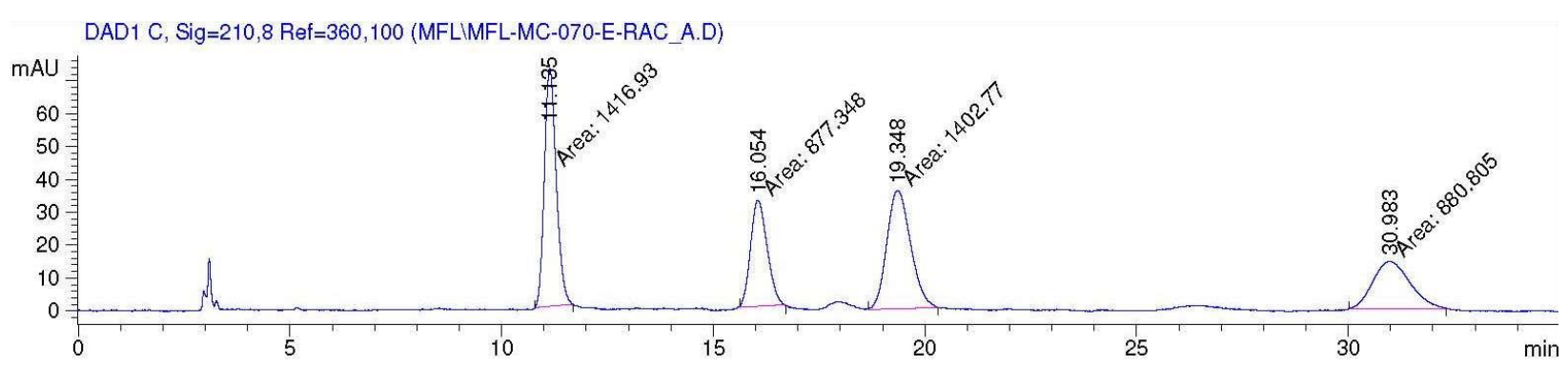

Signal 3: DAD1 C, Sig=210,8 Ref $=360,100$

\begin{tabular}{|c|c|c|c|c|c|c|}
\hline $\begin{array}{c}\text { Peak } \\
\#\end{array}$ & $\begin{array}{c}\text { RetTime } \\
\text { [min] }\end{array}$ & Type & $\begin{array}{l}\text { Width } \\
\text { [min] }\end{array}$ & $\begin{array}{c}\text { Area } \\
{\left[\mathrm{mAU}^{*} \mathrm{~s}\right]}\end{array}$ & $\begin{array}{l}\text { Height } \\
\text { [mAU] }\end{array}$ & $\begin{array}{c}\text { Area } \\
\%\end{array}$ \\
\hline 1 & 11. & MM & & 141 & & \\
\hline 2 & 16.054 & MM & 0.4532 & 877.34833 & 32.26 & 19.1650 \\
\hline 3 & 19.348 & $\mathrm{MM}$ & 0.6495 & 1402.77478 & 35.99 & 30.6426 \\
\hline 1 & 30.983 & MM & 1.0175 & 880.80469 & 14.42751 & 19.2405 \\
\hline
\end{tabular}

Totals :

$4577.86151 \quad 155.09630$

\section{Enantioenriched compounds}

\section{E-Isomer}

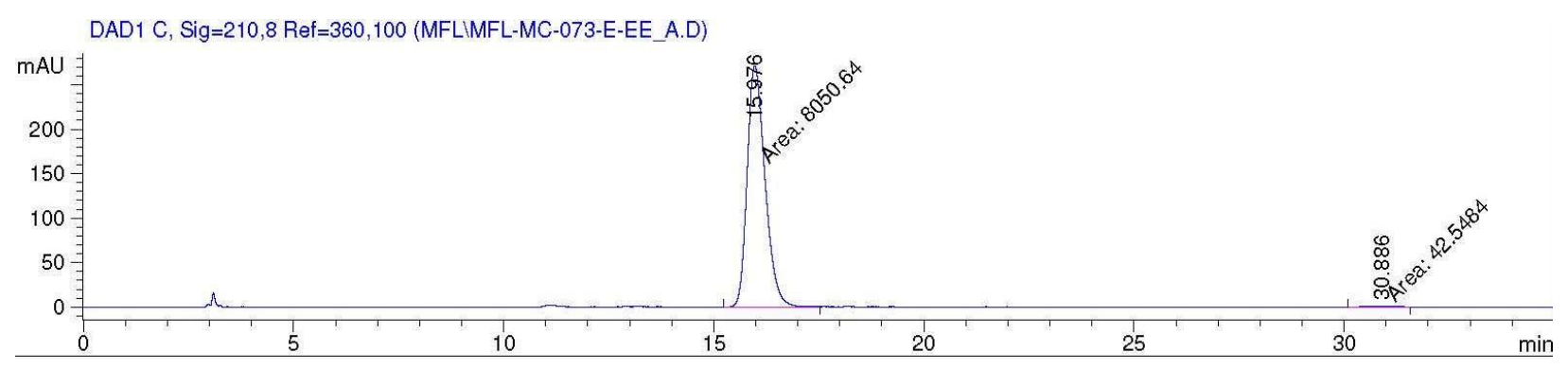

Signal 3: DAD1 C, $\operatorname{Sig}=210,8$ Ref $=360,100$

\begin{tabular}{|c|c|c|c|c|c|c|}
\hline $\begin{array}{c}\text { Peak } \\
\#\end{array}$ & $\begin{array}{c}\text { RetTime } \\
\text { [min] }\end{array}$ & Type & $\begin{array}{l}\text { Width } \\
\text { [min] }\end{array}$ & $\begin{array}{c}\text { Area } \\
{\left[\mathrm{mAU}^{*} \mathrm{~s}\right]}\end{array}$ & $\begin{array}{l}\text { Height } \\
{[\mathrm{mAU}]}\end{array}$ & $\begin{array}{c}\text { Area } \\
\%\end{array}$ \\
\hline 1 & 15.976 & MM & 0.4944 & 8050.64209 & 271.41077 & 99.4743 \\
\hline 2 & 30.886 & MM & 0.7804 & 42.54839 & $9.08738 e-1$ & 0.5257 \\
\hline
\end{tabular}




\section{Z-Isomer:}

DAD1 B, Sig=254,16 Ref=360,100 (MFLIMFL-MC-073-Z-EE_A.D)

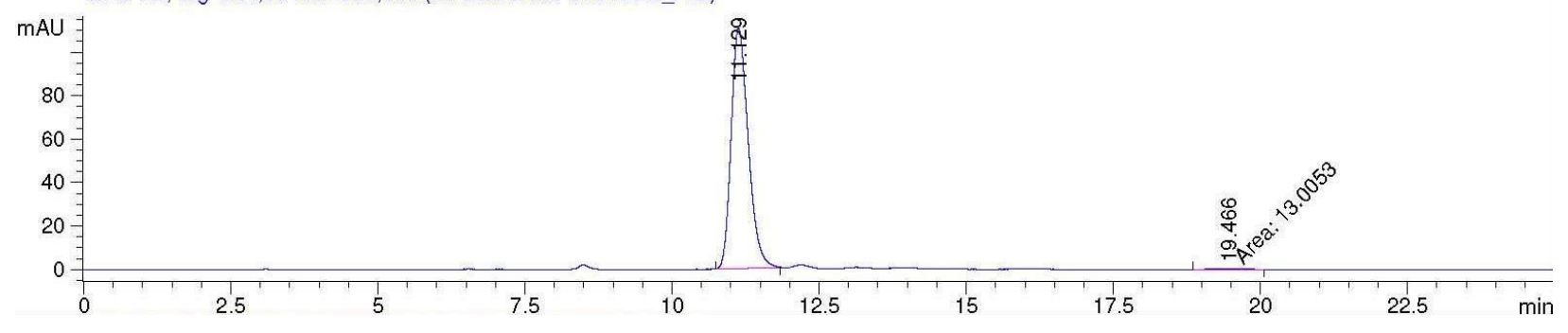

Signal 2: DAD1 B, Sig=254,16 Ref=360,100

\begin{tabular}{|c|c|c|c|c|c|c|}
\hline $\begin{array}{c}\text { Peak } \\
\#\end{array}$ & $\begin{array}{c}\text { RetTime } \\
\text { [min] }\end{array}$ & Type & $\begin{array}{l}\text { Width } \\
\text { [min] }\end{array}$ & $\begin{array}{c}\text { Area } \\
{\left[\mathrm{mAU}^{*} \mathrm{~s}\right]}\end{array}$ & $\begin{array}{l}\text { Height } \\
{[\mathrm{mAU}]}\end{array}$ & $\begin{array}{c}\text { Area } \\
\%\end{array}$ \\
\hline 1 & 11.129 & $\mathrm{BB}$ & 0.3124 & 2229.95044 & 110.51981 & 99.4202 \\
\hline 2 & 19.466 & $\mathrm{MM}$ & 0.5788 & 13.00525 & $3.74481 e-1$ & 0.5798 \\
\hline
\end{tabular}

Totals :

$2242.95569 \quad 110.89429$ 


\section{(2R)-1',3'-Dihydro-3H-spiro[benzofuran-2,2'-inden]-1'-ol (8)}<smiles>O[C@H]1c2ccccc2C[C@]12Cc1ccccc1O2</smiles>

8

\section{Racemic compound}

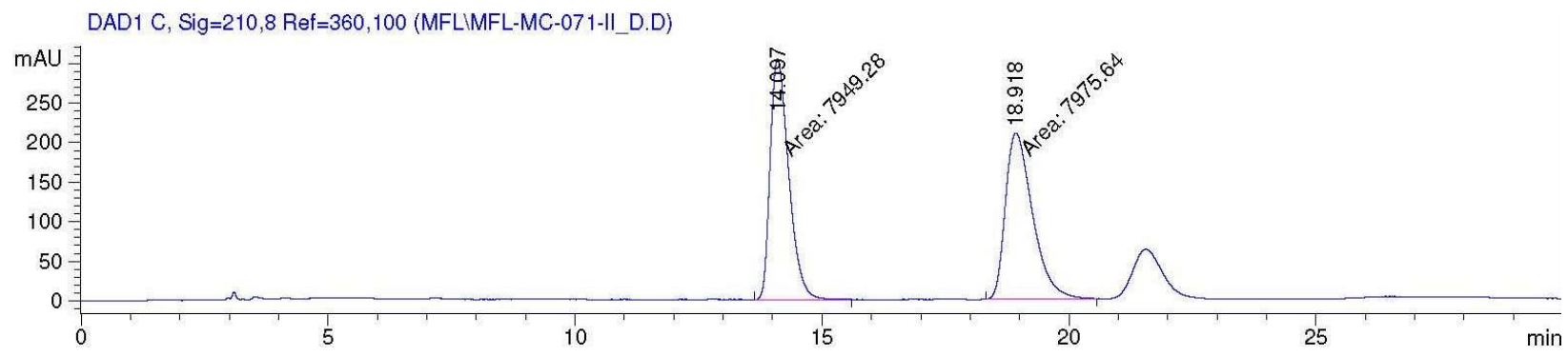

Signal 3: DAD1 C, $\operatorname{Sig}=210,8$ Ref $=360,100$

\begin{tabular}{|c|c|c|c|c|c|c|}
\hline $\begin{array}{c}\text { Peak } \\
\#\end{array}$ & $\begin{array}{c}\text { RetTime } \\
\text { [min] }\end{array}$ & Type & $\begin{array}{l}\text { Width } \\
\text { [min] }\end{array}$ & $\begin{array}{c}\text { Area } \\
{\left[\mathrm{mAU}^{*} \mathrm{~s}\right]}\end{array}$ & $\begin{array}{l}\text { Height } \\
\text { [mAU] }\end{array}$ & $\begin{array}{c}\text { Area } \\
\%\end{array}$ \\
\hline & & & & & & A \\
\hline 2 & 18.918 & MM & .6354 & 7975.64063 & 209.20584 & 50.0828 \\
\hline
\end{tabular}

Totals :

$1.59249 e 4 \quad 513.90836$

\section{Enantioenriched compound}

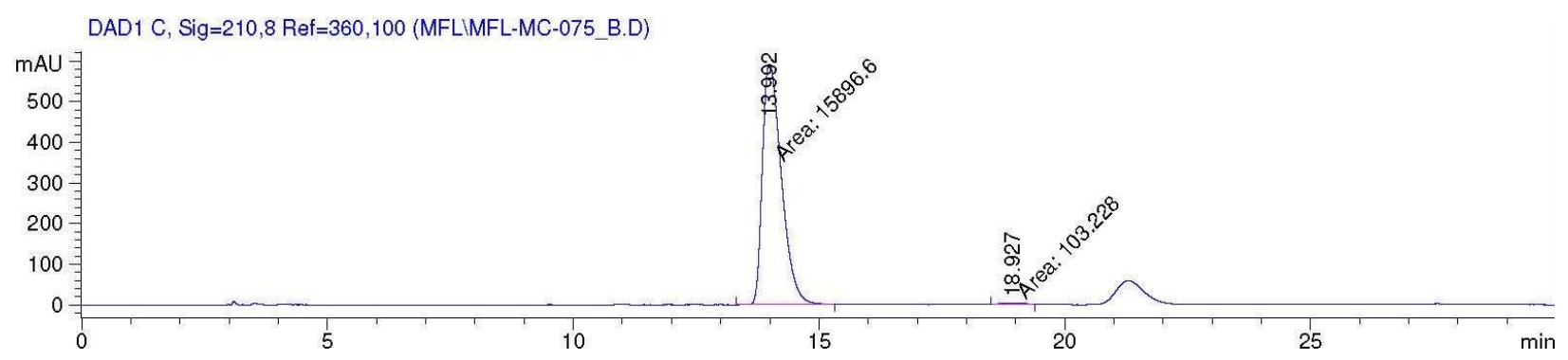

Signal 3: DAD1 C, Sig=210,8 Ref=360,100

\begin{tabular}{|c|c|c|c|c|c|c|}
\hline $\begin{array}{c}\text { Peak } \\
\#\end{array}$ & $\begin{array}{c}\text { RetTime } \\
\text { [min] }\end{array}$ & Type & $\begin{array}{l}\text { Width } \\
\text { [min] }\end{array}$ & $\begin{array}{c}\text { Area } \\
{\left[\mathrm{mAU}^{*} \mathrm{~s}\right]}\end{array}$ & $\begin{array}{l}\text { Height } \\
{[\mathrm{mAU}]}\end{array}$ & $\begin{array}{c}\text { Area } \\
\%\end{array}$ \\
\hline 1 & & 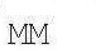 & 3 & 1.5 & 97 & 99.1 \\
\hline 2 & 18.927 & $M$ & 0.4986 & 103.22849 & 3.45041 & 0.6452 \\
\hline
\end{tabular}

Totals : $\quad 1.59998 \mathrm{e} 4 \quad 595.74338$ 


\section{(S)-3H-Spiro[benzofuran-2,3'-isochroman]-1'-one (9)}<smiles>O=C1Oc2ccccc2C[C@]2(Cc3ccccc3O2)O1</smiles>

\section{Racemic compound}

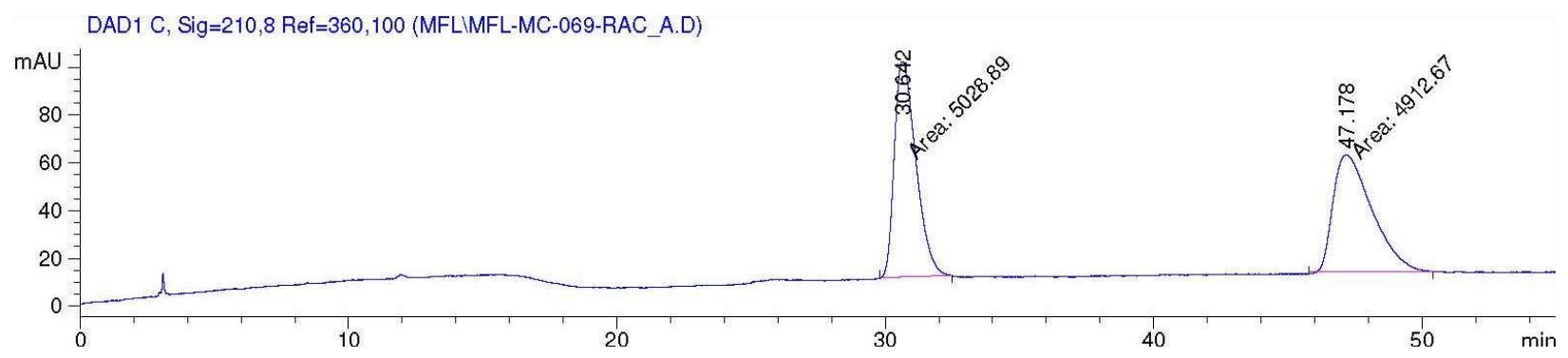

Signal 3: DAD1 C, $\operatorname{Sig}=210,8$ Ref $=360,100$

\begin{tabular}{|c|c|c|c|c|c|c|}
\hline $\begin{array}{c}\text { Peak } \\
\#\end{array}$ & $\begin{array}{c}\text { RetTime } \\
\text { [min] }\end{array}$ & Type & $\begin{array}{l}\text { Width } \\
\text { [min] }\end{array}$ & $\begin{array}{c}\text { Area } \\
{\left[\mathrm{mAU}^{*} \mathrm{~s}\right]}\end{array}$ & $\begin{array}{l}\text { Height } \\
{[\mathrm{mAU}]}\end{array}$ & $\begin{array}{c}\text { Area } \\
\%\end{array}$ \\
\hline 1 & 30.642 & MM & 0.9282 & 5028.88672 & 90.30029 & 50.5845 \\
\hline 2 & 47.178 & MM & 1.6725 & 4912.66846 & 48.95638 & 49.4155 \\
\hline
\end{tabular}

Totals : $\quad 9941.55518 \quad 139.25666$

\section{Enantioenriched compound}

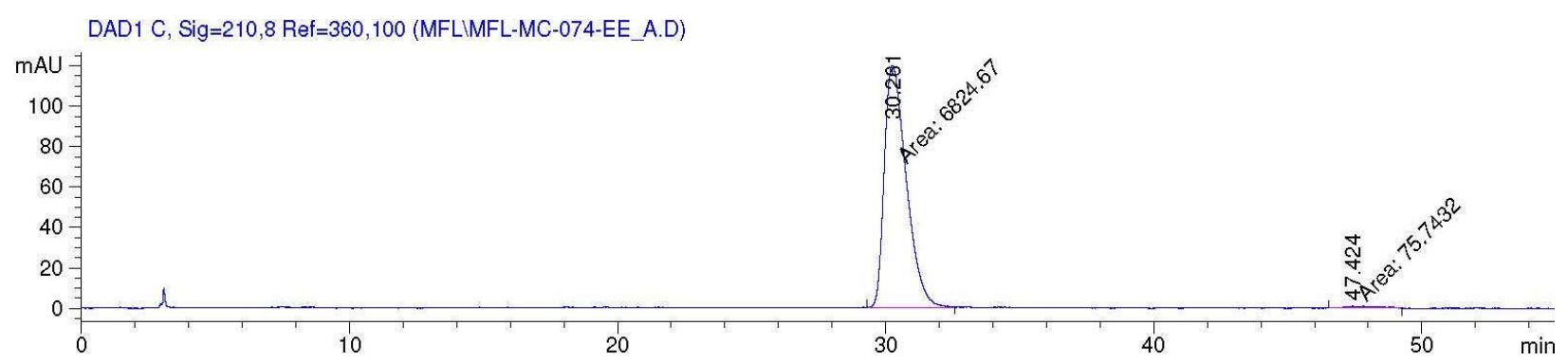

Signal 3: DAD1 C, Sig=210,8 Ref=360, 100

\begin{tabular}{|c|c|c|c|c|c|c|}
\hline $\begin{array}{c}\text { Peak } \\
\#\end{array}$ & $\begin{array}{c}\text { RetTime } \\
\text { [min] }\end{array}$ & Type & $\begin{array}{l}\text { Width } \\
\text { [min] }\end{array}$ & $\begin{array}{c}\text { Area } \\
{\left[\mathrm{mAU}^{*} \mathrm{~s}\right]}\end{array}$ & $\begin{array}{l}\text { Height } \\
\text { [mAU] }\end{array}$ & $\begin{array}{c}\text { Area } \\
\%\end{array}$ \\
\hline 1 & & $\mathbb{M}$ & & 6824 & & \\
\hline 2 & 47.424 & $\mathrm{M}$ & 1.3581 & 75.74318 & $9.29501 e-1$ & 1.0977 \\
\hline
\end{tabular}

Totals : $\quad 6900.41457 \quad 120.87042$ 


\section{Calculated geometries}

\subsection{Optimized Geometries for presented reaction pathways}

\subsection{Reactants}

$\begin{array}{lrrr}47 & & & \\ 3 \mathrm{a} & & & \\ \mathrm{C} & -0.63816 & -2.35816 & -2.00105 \\ \mathrm{C} & 1.66221 & -1.88138 & -2.14616 \\ \mathrm{C} & -0.90167 & -1.61993 & -0.68673 \\ \mathrm{H} & 2.63897 & -2.35399 & -2.02625 \\ \mathrm{H} & -0.77473 & -1.67000 & -2.85245 \\ \mathrm{H} & -1.33247 & -3.19320 & -2.10666 \\ \mathrm{H} & -0.91697 & -2.35111 & 0.13016 \\ \mathrm{H} & 1.61137 & -1.42523 & -3.14885 \\ \mathrm{O} & 0.68023 & -2.91517 & -2.03258 \\ \mathrm{C} & 1.44330 & -0.83756 & -1.09515 \\ \mathrm{~N} & 2.29241 & 0.05565 & -0.67218 \\ \mathrm{~N} & 1.52747 & 0.75194 & 0.28135 \\ \mathrm{C} & 0.25929 & 0.32064 & 0.46275 \\ \mathrm{~N} & 0.22622 & -0.70674 & -0.45466 \\ \mathrm{C} & 2.13751 & 1.83184 & 0.97844 \\ \mathrm{C} & 2.11860 & 3.11881 & 0.42249 \\ \mathrm{C} & 2.75926 & 1.60219 & 2.21415 \\ \mathrm{C} & 2.71526 & 4.18449 & 1.10930 \\ \mathrm{C} & 3.35372 & 2.66754 & 2.90384 \\ \mathrm{C} & 3.32169 & 3.94007 & 2.33904 \\ \mathrm{H} & 2.70845 & 5.18568 & 0.69647 \\ \mathrm{H} & 3.83554 & 2.51002 & 3.86085 \\ \mathrm{H} & 3.78367 & 4.76584 & 2.87276 \\ \mathrm{O} & 2.73426 & 0.31597 & 2.65906 \\ \mathrm{O} & 1.49533 & 3.23263 & -0.78327 \\ \mathrm{C} & 1.44460 & 4.53358 & -1.37342 \\ \mathrm{H} & 0.90796 & 4.40294 & -2.31342 \\ \mathrm{H} & 0.90171 & 5.23627 & -0.72952 \\ \mathrm{H} & 2.45382 & 4.91528 & -1.57108 \\ \mathrm{C} & 3.33203 & 0.05007 & 3.93006 \\ \mathrm{H} & 2.83400 & 0.61920 & 4.72447 \\ \mathrm{H} & 3.19174 & -1.01820 & 4.09619 \\ \mathrm{H} & 4.40296 & 0.28753 & 3.91880 \\ \mathrm{C} & -2.23089 & -0.84560 & -0.70013 \\ \mathrm{H} & -2.26079 & -0.23965 & 0.21063 \\ \mathrm{H} & -2.22689 & -0.15314 & -1.54980 \\ & & & \end{array}$




\begin{tabular}{|c|c|c|c|}
\hline $\mathrm{C}$ & -3.41709 & -1.77576 & -0.78176 \\
\hline $\mathrm{C}$ & -3.80713 & -2.52762 & 0.33481 \\
\hline $\mathrm{C}$ & -4.13371 & -1.93289 & -1.97359 \\
\hline $\mathrm{C}$ & -4.87913 & -3.41511 & 0.25999 \\
\hline $\mathrm{H}$ & -3.26919 & -2.40819 & 1.27270 \\
\hline $\mathrm{C}$ & -5.20909 & -2.81855 & -2.05288 \\
\hline $\mathrm{H}$ & -3.84781 & -1.35074 & -2.84691 \\
\hline $\mathrm{C}$ & -5.58338 & -3.56506 & -0.93604 \\
\hline $\mathrm{H}$ & -5.16907 & -3.98682 & 1.13726 \\
\hline $\mathrm{H}$ & -5.75493 & -2.92360 & -2.98648 \\
\hline $\mathrm{H}$ & -6.42051 & -4.25477 & -0.99457 \\
\hline \multicolumn{4}{|c|}{46} \\
\hline \multicolumn{4}{|c|}{$3 d$} \\
\hline $\mathrm{C}$ & -0.18569 & -2.71914 & -1.04257 \\
\hline $\mathrm{C}$ & 0.28175 & -2.89761 & 0.43173 \\
\hline $\mathrm{C}$ & -0.87763 & -1.33086 & -1.11510 \\
\hline $\mathrm{H}$ & -1.95052 & -1.42544 & -0.90363 \\
\hline $\mathrm{C}$ & 0.41266 & -1.47651 & 0.87427 \\
\hline $\mathrm{N}$ & 0.94665 & -0.81943 & 1.86227 \\
\hline $\mathrm{N}$ & 0.56746 & 0.50909 & 1.53686 \\
\hline $\mathrm{C}$ & -0.16447 & 0.67652 & 0.40307 \\
\hline $\mathrm{N}$ & -0.23807 & -0.63143 & 0.00441 \\
\hline $\mathrm{C}$ & 0.99988 & 1.55678 & 2.39526 \\
\hline $\mathrm{C}$ & 0.21997 & 1.91966 & 3.50271 \\
\hline $\mathrm{C}$ & 2.20746 & 2.22014 & 2.13349 \\
\hline $\mathrm{C}$ & 0.64211 & 2.95712 & 4.34508 \\
\hline $\mathrm{C}$ & 2.63084 & 3.25886 & 2.97352 \\
\hline $\mathrm{C}$ & 1.84031 & 3.60939 & 4.06517 \\
\hline $\mathrm{H}$ & 0.05022 & 3.25301 & 5.20243 \\
\hline $\mathrm{H}$ & 3.55888 & 3.78399 & 2.78414 \\
\hline $\mathrm{H}$ & 2.16805 & 4.41402 & 4.71741 \\
\hline $\mathrm{O}$ & 2.89733 & 1.78472 & 1.04297 \\
\hline $\mathrm{O}$ & -0.92989 & 1.21030 & 3.67431 \\
\hline $\mathrm{C}$ & -1.75774 & 1.56358 & 4.78422 \\
\hline $\mathrm{H}$ & -1.22594 & 1.42094 & 5.73309 \\
\hline $\mathrm{H}$ & -2.10061 & 2.60251 & 4.70414 \\
\hline $\mathrm{H}$ & -2.61164 & 0.88754 & 4.73360 \\
\hline $\mathrm{C}$ & 4.11801 & 2.45783 & 0.72865 \\
\hline $\mathrm{H}$ & 4.84687 & 2.35262 & 1.54190 \\
\hline $\mathrm{H}$ & 4.49384 & 1.96839 & -0.17018 \\
\hline $\mathrm{H}$ & 3.93961 & 3.52106 & 0.52628 \\
\hline $\mathrm{C}$ & -0.69303 & -0.58162 & -2.44120 \\
\hline $\mathrm{H}$ & -1.07778 & 0.43504 & -2.30743 \\
\hline $\mathrm{H}$ & 0.38005 & -0.49988 & -2.64799 \\
\hline $\mathrm{C}$ & -1.39915 & -1.28373 & -3.57556 \\
\hline $\mathrm{C}$ & -2.79070 & -1.19008 & -3.71314 \\
\hline $\mathrm{C}$ & -0.69174 & -2.07374 & -4.48887 \\
\hline
\end{tabular}




$\begin{array}{crrr}\mathrm{C} & -3.45649 & -1.87064 & -4.73118 \\ \mathrm{H} & -3.35480 & -0.56997 & -3.01968 \\ \mathrm{C} & -1.35327 & -2.75546 & -5.51093 \\ \mathrm{H} & 0.38977 & -2.14926 & -4.40115 \\ \mathrm{C} & -2.73910 & -2.65800 & -5.63355 \\ \mathrm{H} & -4.53545 & -1.78203 & -4.82450 \\ \mathrm{H} & -0.78539 & -3.35979 & -6.21309 \\ \mathrm{H} & -3.25625 & -3.18573 & -6.42980 \\ \mathrm{H} & -0.47676 & -3.41383 & 1.03304 \\ \mathrm{H} & 1.21337 & -3.46174 & 0.51537 \\ \mathrm{H} & 0.69121 & -2.70602 & -1.69928 \\ \mathrm{H} & -0.84417 & -3.52116 & -1.38055 \\ & & & \\ 30 & & & \\ \mathrm{la} & & & \\ \mathrm{C} & 1.17983 & -2.72761 & -1.30753 \\ \mathrm{O} & 2.01109 & -2.60501 & -0.42201 \\ \mathrm{C} & 0.53316 & -1.65107 & -2.08474 \\ \mathrm{C} & 0.79695 & -0.26999 & -1.92480 \\ \mathrm{C} & -0.38976 & -2.07541 & -3.05531 \\ \mathrm{C} & 0.12106 & 0.62942 & -2.75092 \\ \mathrm{C} & -1.05930 & -1.16556 & -3.86421 \\ \mathrm{H} & -0.57214 & -3.14275 & -3.16387 \\ \mathrm{C} & -0.79719 & 0.19553 & -3.70817 \\ \mathrm{H} & 0.31363 & 1.69258 & -2.63413 \\ \mathrm{H} & -1.77255 & -1.50894 & -4.60718 \\ \mathrm{H} & -1.30756 & 0.92406 & -4.33197 \\ \mathrm{C} & 1.74747 & 0.27226 & -0.86986 \\ \mathrm{H} & 2.12015 & 1.24748 & -1.20496 \\ \mathrm{H} & 2.59066 & -0.40322 & -0.72732 \\ \mathrm{C} & 1.06131 & 0.44056 & 0.44572 \\ \mathrm{C} & 1.11103 & -0.24132 & 1.62160 \\ \mathrm{O} & 0.14776 & 1.48842 & 0.50720 \\ \mathrm{C} & 0.17589 & 0.39677 & 2.51233 \\ \mathrm{H} & 1.73173 & -1.10404 & 1.81313 \\ \mathrm{C} & -0.39040 & 1.45506 & 1.77368 \\ \mathrm{C} & -1.34676 & 2.32497 & 2.27553 \\ \mathrm{C} & -0.24662 & 0.19973 & 3.83555 \\ \mathrm{C} & -1.75034 & 2.10690 & 3.59379 \\ \mathrm{H} & -2.49836 & 2.75877 & 4.03516 \\ \mathrm{C} & -1.20761 & 1.06009 & 4.35958 \\ \mathrm{H} & -1.54761 & 0.92314 & 5.38207 \\ \mathrm{H} & 0.16550 & -0.60522 & 4.43722 \\ \mathrm{H} & -1.75744 & 3.12838 & 1.67287 \\ \mathrm{H} & 0.83638 & -3.74399 & -1.60846 \\ & & & \\ 30 & & & \\ 1 \mathrm{l} & & & \\ & & & \end{array}$




$\begin{array}{lrrr}\mathrm{C} & 0.87431 & -2.71916 & -1.13109 \\ \mathrm{O} & 1.66909 & -2.59937 & -0.21292 \\ \mathrm{C} & 0.43705 & -1.67453 & -2.07946 \\ \mathrm{C} & 0.86385 & -0.32554 & -2.04727 \\ \mathrm{C} & -0.44039 & -2.10310 & -3.08909 \\ \mathrm{C} & 0.40088 & 0.53321 & -3.04606 \\ \mathrm{C} & -0.90183 & -1.23035 & -4.06712 \\ \mathrm{H} & -0.75266 & -3.14557 & -3.09409 \\ \mathrm{C} & -0.47127 & 0.09584 & -4.04409 \\ \mathrm{H} & 0.72741 & 1.57035 & -3.03746 \\ \mathrm{H} & -1.58221 & -1.57688 & -4.83886 \\ \mathrm{H} & -0.81386 & 0.79416 & -4.80252 \\ \mathrm{C} & 1.75090 & 0.23543 & -0.95137 \\ \mathrm{H} & 2.24857 & 1.13299 & -1.33819 \\ \mathrm{H} & 2.51293 & -0.49023 & -0.66401 \\ \mathrm{C} & 0.95927 & 0.58512 & 0.28207 \\ \mathrm{C} & 1.03721 & 0.01636 & 1.51887 \\ \mathrm{~S} & -0.24914 & 1.85457 & 0.21597 \\ \mathrm{C} & 0.12858 & 0.58852 & 2.46995 \\ \mathrm{H} & 1.71390 & -0.80144 & 1.73792 \\ \mathrm{C} & -0.65507 & 1.62504 & 1.89574 \\ \mathrm{C} & -1.60872 & 2.32478 & 2.63990 \\ \mathrm{C} & -0.07197 & 0.26474 & 3.82427 \\ \mathrm{C} & -1.78303 & 1.98303 & 3.97651 \\ \mathrm{H} & -2.51895 & 2.51481 & 4.57258 \\ \mathrm{C} & -1.01944 & 0.95867 & 4.56371 \\ \mathrm{H} & -1.17516 & 0.70958 & 5.60942 \\ \mathrm{H} & 0.51566 & -0.52664 & 4.28174 \\ \mathrm{H} & -2.19993 & 3.11487 & 2.18676 \\ \mathrm{H} & 0.40401 & -3.70924 & -1.33183\end{array}$




\subsection{3a-(R)-a-path}

77

3a-(R)-4-a

$\begin{array}{llll}\mathrm{C} & 1.61206 & 0.49916 & 2.64091\end{array}$

$\begin{array}{llll}\mathrm{H} & 2.04844 & 0.82532 & 0.29562\end{array}$

$\begin{array}{llll}\mathrm{O} & 1.64848 & 0.57448 & -0.56176\end{array}$

$\begin{array}{llll}\text { C } & -2.31268 & 2.76239 & -0.73297\end{array}$

$\begin{array}{llll}\text { C } & -3.76364 & 0.95137 & -0.75346\end{array}$

$\begin{array}{llll}\text { C } & -1.00401 & 1.93808 & -0.79933\end{array}$

$\begin{array}{llll}\mathrm{H} & -4.60356 & 0.49315 & -0.23084\end{array}$

$\mathrm{H} \quad-2.65225 \quad 2.99000 \quad-1.75693$

$\begin{array}{llll}\mathrm{H} & -2.10820 & 3.70089 & -0.21667\end{array}$

$\begin{array}{llll}\mathrm{H} & -0.41548 & 2.12242 & 0.10298\end{array}$

$\begin{array}{llll}\mathrm{H} & -4.07663 & 1.23763 & -1.77142\end{array}$

$\begin{array}{llll}\mathrm{O} & -3.37458 & 2.12367 & -0.02688\end{array}$

$\begin{array}{llll}\text { C } & -2.60873 & 0.00890 & -0.77392\end{array}$

$\begin{array}{llll}\mathrm{N} & -2.66785 & -1.27681 & -0.63293\end{array}$

$\begin{array}{llll}\mathrm{N} & -1.31897 & -1.67326 & -0.56691\end{array}$

$\begin{array}{llll}\text { C } & -0.45439 & -0.57034 & -0.57142\end{array}$

$\begin{array}{llll}\mathrm{N} & -1.32056 & 0.50744 & -0.83017\end{array}$

$\begin{array}{llll}\text { C } & -0.98561 & -3.03244 & -0.72222\end{array}$

$\begin{array}{llll}\text { C } & -1.47106 & -3.99078 & 0.18917\end{array}$

$\begin{array}{llll}\text { C } & -0.17232 & -3.45311 & -1.79270\end{array}$

$\begin{array}{llll}\text { C } & -1.12393 & -5.33785 & 0.04986\end{array}$

$\begin{array}{llll}\text { C } & 0.20173 & -4.79539 & -1.91091\end{array}$

$\begin{array}{llll}\text { C } & -0.27888 & -5.72058 & -0.98906\end{array}$

$\begin{array}{llll}\mathrm{H} & -1.48920 & -6.07711 & 0.75199\end{array}$

$\mathrm{H} \quad 0.84378 \quad-5.11911 \quad-2.72056$

$\begin{array}{llll}\mathrm{H} & 0.00729 & -6.76379 & -1.08457\end{array}$

$\begin{array}{llll}\text { O } & 0.20177 & -2.47658 & -2.66686\end{array}$

$\begin{array}{llll}\mathrm{O} & -2.26158 & -3.50767 & 1.19157\end{array}$

$\begin{array}{llll}\text { C } & -2.74722 & -4.44224 & 2.15618\end{array}$

$\begin{array}{llll}\mathrm{H} & -3.33465 & -3.85099 & 2.85906\end{array}$

$\begin{array}{llll}\mathrm{H} & -3.38484 & -5.19852 & 1.68260\end{array}$

$\begin{array}{llll}\mathrm{H} & -1.91874 & -4.93388 & 2.68180\end{array}$

$\begin{array}{llll}\text { C } & 1.07178 & -2.85123 & -3.73693\end{array}$

$\begin{array}{llll}\mathrm{H} & 0.58662 & -3.57749 & -4.40081\end{array}$

$\mathrm{H} \quad 1.27384 \quad-1.92783 \quad-4.28028$

$\begin{array}{llll}\mathrm{H} & 2.00807 & -3.26924 & -3.34930\end{array}$

$\begin{array}{llll}\text { C } & -0.20049 & 2.39545 & -2.03479\end{array}$

$\begin{array}{llll}\mathrm{H} & 0.68071 & 1.75833 & -2.12580\end{array}$

$\begin{array}{llll}\mathrm{H} & -0.82110 & 2.26418 & -2.92906\end{array}$

$\begin{array}{llll}\text { C } & 0.21260 & 3.84258 & -1.89390\end{array}$

$\begin{array}{llll}\text { C } & 1.03311 & 4.24207 & -0.82919\end{array}$

$\begin{array}{llll}\text { C } & -0.23308 & 4.81669 & -2.79402\end{array}$

$\begin{array}{llll}\mathrm{C} & 1.39543 & 5.57770 & -0.67147\end{array}$

$\begin{array}{llll}\mathrm{H} & 1.38459 & 3.50378 & -0.11369\end{array}$ 


\begin{tabular}{|c|c|c|c|}
\hline $\mathrm{C}$ & 0.13179 & 6.15518 & -2.64198 \\
\hline $\mathrm{H}$ & -0.87232 & 4.52138 & -3.62333 \\
\hline $\mathrm{C}$ & 0.94649 & 6.54036 & -1.57767 \\
\hline $\mathrm{H}$ & 2.02850 & 5.86643 & 0.16291 \\
\hline $\mathrm{H}$ & -0.22328 & 6.89622 & -3.35307 \\
\hline $\mathrm{H}$ & 1.22859 & 7.58216 & -1.45404 \\
\hline $\mathrm{C}$ & 0.88241 & -0.57734 & -0.29745 \\
\hline $\mathrm{C}$ & 1.63894 & -1.74759 & 0.16358 \\
\hline $\mathrm{C}$ & 1.33444 & -2.35574 & 1.40227 \\
\hline $\mathrm{C}$ & 2.74101 & -2.21877 & -0.56732 \\
\hline $\mathrm{C}$ & 2.06658 & -3.46792 & 1.82093 \\
\hline $\mathrm{C}$ & 3.47492 & -3.31795 & -0.13180 \\
\hline $\mathrm{H}$ & 3.00269 & -1.71159 & -1.49147 \\
\hline $\mathrm{C}$ & 3.12188 & -3.96337 & 1.05510 \\
\hline $\mathrm{H}$ & 1.82396 & -3.93480 & 2.77320 \\
\hline $\mathrm{H}$ & 4.31412 & -3.67913 & -0.72055 \\
\hline $\mathrm{H}$ & 3.68233 & -4.82877 & 1.39711 \\
\hline $\mathrm{C}$ & 0.27371 & -1.73981 & 2.28955 \\
\hline $\mathrm{H}$ & 0.28214 & -2.24256 & 3.26614 \\
\hline $\mathrm{H}$ & -0.73008 & -1.88070 & 1.87346 \\
\hline $\mathrm{C}$ & 0.49369 & -0.27111 & 2.46710 \\
\hline $\mathrm{C}$ & 1.16591 & 1.86356 & 2.76829 \\
\hline $\mathrm{H}$ & 2.62671 & 0.12685 & 2.67865 \\
\hline $\mathrm{C}$ & -0.24114 & 1.81099 & 2.67897 \\
\hline $\mathrm{C}$ & -1.06684 & 2.92378 & 2.73416 \\
\hline $\mathrm{C}$ & 1.77754 & 3.11769 & 2.92977 \\
\hline $\mathrm{C}$ & -0.43206 & 4.15815 & 2.88864 \\
\hline $\mathrm{H}$ & -1.03033 & 5.06330 & 2.92680 \\
\hline $\mathrm{C}$ & 0.96698 & 4.24911 & 2.98752 \\
\hline $\mathrm{H}$ & 1.42415 & 5.22710 & 3.10799 \\
\hline $\mathrm{H}$ & 2.85782 & 3.20554 & 3.00467 \\
\hline $\mathrm{H}$ & -2.14380 & 2.83204 & 2.64122 \\
\hline $\mathrm{O}$ & -0.65352 & 0.50789 & 2.51068 \\
\hline \multicolumn{4}{|c|}{77} \\
\hline \multicolumn{4}{|c|}{$3 a-(R)-t s 1-a$} \\
\hline $\mathrm{C}$ & -2.68473 & 0.63132 & 0.60756 \\
\hline $\mathrm{H}$ & -2.20783 & -0.16229 & -0.35092 \\
\hline $\mathrm{O}$ & -1.27616 & -0.59143 & -1.15640 \\
\hline $\mathrm{C}$ & 0.87258 & -2.75078 & 2.20154 \\
\hline $\mathrm{C}$ & 2.63057 & -1.26523 & 2.55248 \\
\hline $\mathrm{C}$ & 0.27689 & -2.03298 & 0.96560 \\
\hline $\mathrm{H}$ & 3.09846 & -0.62365 & 3.30022 \\
\hline $\mathrm{H}$ & 1.61848 & -3.49164 & 1.87030 \\
\hline $\mathrm{H}$ & 0.07098 & -3.26909 & 2.72775 \\
\hline $\mathrm{H}$ & -0.74968 & -1.72865 & 1.17552 \\
\hline $\mathrm{H}$ & 3.34820 & -2.04263 & 2.24113 \\
\hline $\mathrm{O}$ & 1.48465 & -1.87469 & 3.14931 \\
\hline
\end{tabular}




\begin{tabular}{|c|c|c|c|}
\hline $\mathrm{C}$ & 2.18048 & -0.44397 & 1.38970 \\
\hline $\mathrm{N}$ & 2.69120 & 0.67678 & 0.98110 \\
\hline $\mathrm{N}$ & 1.82350 & 1.08615 & -0.04535 \\
\hline $\mathrm{C}$ & 0.75536 & 0.21523 & -0.19438 \\
\hline $\mathrm{N}$ & 1.04817 & -0.81236 & 0.69510 \\
\hline $\mathrm{C}$ & 2.26818 & 2.07120 & -0.95860 \\
\hline $\mathrm{C}$ & 2.47579 & 3.39395 & -0.53073 \\
\hline $\mathrm{C}$ & 2.54120 & 1.71922 & -2.29243 \\
\hline $\mathrm{C}$ & 2.93056 & 4.35925 & -1.43503 \\
\hline $\mathrm{C}$ & 2.97510 & 2.68939 & -3.20107 \\
\hline $\mathrm{C}$ & 3.16520 & 3.99521 & -2.75845 \\
\hline $\mathrm{H}$ & 3.08649 & 5.38289 & -1.11833 \\
\hline $\mathrm{H}$ & 3.16965 & 2.43132 & -4.23455 \\
\hline $\mathrm{H}$ & 3.50646 & 4.74861 & -3.46225 \\
\hline $\mathrm{O}$ & 2.34780 & 0.40701 & -2.60442 \\
\hline $\mathrm{O}$ & 2.19572 & 3.64520 & 0.77908 \\
\hline $\mathrm{C}$ & 2.34535 & 4.99112 & 1.23827 \\
\hline $\mathrm{H}$ & 2.04787 & 4.97130 & 2.28678 \\
\hline $\mathrm{H}$ & 3.38803 & 5.31943 & 1.15320 \\
\hline $\mathrm{H}$ & 1.69399 & 5.67201 & 0.67659 \\
\hline $\mathrm{C}$ & 2.56535 & 0.01306 & -3.96200 \\
\hline $\mathrm{H}$ & 3.61217 & 0.16684 & -4.25079 \\
\hline $\mathrm{H}$ & 2.32113 & -1.04878 & -3.99568 \\
\hline $\mathrm{H}$ & 1.90683 & 0.56932 & -4.63894 \\
\hline $\mathrm{C}$ & 0.27552 & -2.99376 & -0.24113 \\
\hline $\mathrm{H}$ & -0.05196 & -2.42421 & -1.11416 \\
\hline $\mathrm{H}$ & 1.29506 & -3.36148 & -0.40878 \\
\hline $\mathrm{C}$ & -0.66969 & -4.14363 & 0.01467 \\
\hline $\mathrm{C}$ & -2.04872 & -3.90204 & 0.09121 \\
\hline $\mathrm{C}$ & -0.20405 & -5.44648 & 0.22082 \\
\hline $\mathrm{C}$ & -2.93588 & -4.93764 & 0.37404 \\
\hline $\mathrm{H}$ & -2.41887 & -2.89447 & -0.07560 \\
\hline $\mathrm{C}$ & -1.09156 & -6.48739 & 0.49899 \\
\hline $\mathrm{H}$ & 0.86350 & -5.64754 & 0.16049 \\
\hline $\mathrm{C}$ & -2.46061 & -6.23460 & 0.58000 \\
\hline $\mathrm{H}$ & -4.00127 & -4.73238 & 0.43365 \\
\hline $\mathrm{H}$ & -0.71282 & -7.49411 & 0.65393 \\
\hline $\mathrm{H}$ & -3.15305 & -7.04236 & 0.79985 \\
\hline $\mathrm{C}$ & -0.39244 & 0.38749 & -0.97595 \\
\hline $\mathrm{C}$ & -0.62904 & 1.65423 & -1.72118 \\
\hline $\mathrm{C}$ & -0.95224 & 2.81711 & -0.99845 \\
\hline $\mathrm{C}$ & -0.66797 & 1.68369 & -3.11960 \\
\hline $\mathrm{C}$ & -1.24112 & 3.99905 & -1.68328 \\
\hline $\mathrm{C}$ & -0.95486 & 2.86594 & -3.79720 \\
\hline $\mathrm{H}$ & -0.47277 & 0.76568 & -3.66609 \\
\hline $\mathrm{C}$ & -1.22744 & 4.03115 & -3.07760 \\
\hline $\mathrm{H}$ & -1.49853 & 4.89512 & -1.12269 \\
\hline $\mathrm{H}$ & -0.96977 & 2.88114 & -4.88381 \\
\hline
\end{tabular}




$\begin{array}{lrrr}\mathrm{H} & -1.45477 & 4.95567 & -3.60112 \\ \mathrm{C} & -1.00386 & 2.71832 & 0.50828 \\ \mathrm{H} & -1.63175 & 3.52591 & 0.91673 \\ \mathrm{H} & -0.00219 & 2.85214 & 0.93753 \\ \mathrm{C} & -1.49979 & 1.37751 & 0.94414 \\ \mathrm{C} & -2.92206 & -0.22722 & 1.78225 \\ \mathrm{H} & -3.50275 & 1.12339 & 0.08267 \\ \mathrm{C} & -1.84355 & -0.02871 & 2.65615 \\ \mathrm{C} & -1.65160 & -0.74734 & 3.82589 \\ \mathrm{C} & -3.87612 & -1.19365 & 2.10721 \\ \mathrm{C} & -2.62191 & -1.70654 & 4.13332 \\ \mathrm{H} & -2.51833 & -2.29467 & 5.04036 \\ \mathrm{C} & -3.71932 & -1.92212 & 3.28933 \\ \mathrm{H} & -4.45379 & -2.67721 & 3.55386 \\ \mathrm{H} & -4.72051 & -1.38320 & 1.45015 \\ \mathrm{H} & -0.78360 & -0.58304 & 4.45427 \\ \mathrm{O} & -0.99753 & 0.95457 & 2.17594\end{array}$

77

3a-(R)-6-a

$\begin{array}{lrrr}\mathrm{C} & 0.15045 & 0.84166 & 2.76680 \\ \mathrm{H} & 1.17725 & 1.08128 & 2.45802 \\ \mathrm{O} & 1.53533 & 0.66895 & 0.38591 \\ \mathrm{C} & -1.40061 & 2.62598 & -2.12361 \\ \mathrm{C} & -1.77664 & 0.86560 & -3.65959 \\ \mathrm{C} & -0.25440 & 1.95150 & -1.37332 \\ \mathrm{H} & -2.86773 & 0.75510 & -3.54528 \\ \mathrm{H} & -1.25260 & 3.70672 & -2.11795 \\ \mathrm{H} & -2.37043 & 2.38738 & -1.66484 \\ \mathrm{H} & -0.31440 & 2.15196 & -0.30547 \\ \mathrm{H} & -1.50342 & 0.56319 & -4.67326 \\ \mathrm{O} & -1.39419 & 2.22538 & -3.50749 \\ \mathrm{C} & -1.10853 & -0.02151 & -2.64812 \\ \mathrm{~N} & -1.15307 & -1.32802 & -2.61347 \\ \mathrm{~N} & -0.51049 & -1.63900 & -1.42165 \\ \mathrm{C} & -0.08916 & -0.54462 & -0.75592 \\ \mathrm{~N} & -0.45815 & 0.49340 & -1.55502 \\ \mathrm{C} & -0.42379 & -3.01202 & -1.04484 \\ \mathrm{C} & -1.53518 & -3.62991 & -0.45455 \\ \mathrm{C} & 0.73651 & -3.74056 & -1.34047 \\ \mathrm{C} & -1.45710 & -4.97603 & -0.08453 \\ \mathrm{C} & 0.81497 & -5.08529 & -0.96068 \\ \mathrm{C} & -0.27839 & -5.67601 & -0.33473 \\ \mathrm{H} & -2.29475 & -5.47034 & 0.39129 \\ \mathrm{H} & 1.71360 & -5.65970 & -1.14532 \\ \mathrm{H} & -0.21294 & -6.71818 & -0.03630 \\ \mathrm{O} & 1.70928 & -3.05733 & -1.99253 \\ \mathrm{O} & -2.62653 & -2.83534 & -0.27768\end{array}$




\begin{tabular}{|c|c|c|c|}
\hline $\mathrm{C}$ & -3.77252 & -3.41934 & 0.35271 \\
\hline $\mathrm{H}$ & -4.51136 & -2.61980 & 0.39908 \\
\hline $\mathrm{H}$ & -4.15879 & -4.25577 & -0.24107 \\
\hline $\mathrm{H}$ & -3.52845 & -3.76092 & 1.36568 \\
\hline $\mathrm{C}$ & 2.93230 & -3.75398 & -2.26517 \\
\hline $\mathrm{H}$ & 2.75129 & -4.60347 & -2.93398 \\
\hline $\mathrm{H}$ & 3.57472 & -3.02256 & -2.75472 \\
\hline $\mathrm{H}$ & 3.39377 & -4.09556 & -1.33265 \\
\hline $\mathrm{C}$ & 1.12358 & 2.40075 & -1.89966 \\
\hline $\mathrm{H}$ & 1.85009 & 1.67682 & -1.52274 \\
\hline $\mathrm{H}$ & 1.11184 & 2.40206 & -2.99472 \\
\hline $\mathrm{C}$ & 1.43019 & 3.77652 & -1.36002 \\
\hline $\mathrm{C}$ & 1.69133 & 3.92992 & 0.00972 \\
\hline $\mathrm{C}$ & 1.41275 & 4.91026 & -2.17881 \\
\hline $\mathrm{C}$ & 1.92552 & 5.19476 & 0.54348 \\
\hline $\mathrm{H}$ & 1.70726 & 3.03874 & 0.63417 \\
\hline $\mathrm{C}$ & 1.65422 & 6.17782 & -1.64443 \\
\hline $\mathrm{H}$ & 1.20966 & 4.79893 & -3.24209 \\
\hline $\mathrm{C}$ & 1.90783 & 6.32284 & -0.28081 \\
\hline $\mathrm{H}$ & 2.12228 & 5.30224 & 1.60695 \\
\hline $\mathrm{H}$ & 1.64129 & 7.05004 & -2.29278 \\
\hline $\mathrm{H}$ & 2.09210 & 7.30869 & 0.13751 \\
\hline $\mathrm{C}$ & 0.69384 & -0.34657 & 0.56296 \\
\hline $\mathrm{C}$ & 1.33811 & -1.64691 & 1.06489 \\
\hline $\mathrm{C}$ & 0.56620 & -2.30691 & 2.02275 \\
\hline $\mathrm{C}$ & 2.62688 & -2.07133 & 0.76404 \\
\hline $\mathrm{C}$ & 1.05469 & -3.45390 & 2.64711 \\
\hline $\mathrm{C}$ & 3.11781 & -3.22087 & 1.38554 \\
\hline $\mathrm{H}$ & 3.22997 & -1.48602 & 0.07597 \\
\hline $\mathrm{C}$ & 2.32946 & -3.91590 & 2.31053 \\
\hline $\mathrm{H}$ & 0.46564 & -3.97211 & 3.40049 \\
\hline $\mathrm{H}$ & 4.12609 & -3.56870 & 1.17300 \\
\hline $\mathrm{H}$ & 2.72548 & -4.80462 & 2.79497 \\
\hline $\mathrm{C}$ & -0.72654 & -1.56725 & 2.27235 \\
\hline $\mathrm{H}$ & -1.02283 & -1.56496 & 3.32775 \\
\hline $\mathrm{H}$ & -1.55535 & -1.99193 & 1.69634 \\
\hline $\mathrm{C}$ & -0.42772 & -0.15809 & 1.74443 \\
\hline $\mathrm{C}$ & -0.75932 & 2.03168 & 2.62856 \\
\hline $\mathrm{H}$ & 0.15531 & 0.43075 & 3.78292 \\
\hline $\mathrm{C}$ & -1.77275 & 1.71341 & 1.72321 \\
\hline $\mathrm{C}$ & -2.77360 & 2.61337 & 1.37258 \\
\hline $\mathrm{C}$ & -0.72541 & 3.29824 & 3.19731 \\
\hline $\mathrm{C}$ & -2.72587 & 3.88551 & 1.95493 \\
\hline $\mathrm{H}$ & -3.48850 & 4.61526 & 1.69753 \\
\hline $\mathrm{C}$ & -1.71425 & 4.22988 & 2.85361 \\
\hline $\mathrm{H}$ & -1.69194 & 5.22602 & 3.28484 \\
\hline $\mathrm{H}$ & 0.06326 & 3.56852 & 3.89485 \\
\hline $\mathrm{H}$ & -3.55956 & 2.33538 & 0.67705 \\
\hline
\end{tabular}




\begin{tabular}{|c|c|c|c|}
\hline $\mathrm{O}$ & -1.66489 & 0.44481 & 1.21844 \\
\hline \multicolumn{4}{|c|}{77} \\
\hline \multicolumn{4}{|c|}{$3 a-(R)-t s 3-a$} \\
\hline $\mathrm{C}$ & 2.00338 & 0.63148 & 2.40619 \\
\hline $\mathrm{H}$ & 2.85800 & 0.72217 & 1.73102 \\
\hline ) & 1.55084 & 0.84822 & -0.43887 \\
\hline y & -2.58902 & 2.53111 & -0.79181 \\
\hline C & -3.71100 & 0.78847 & -1.94098 \\
\hline $\mathrm{C}$ & -1.20180 & 1.89418 & -0.84498 \\
\hline $\mathrm{H}$ & -4.55895 & 0.62295 & -1.25634 \\
\hline $\mathrm{H}$ & -2.49737 & 3.61782 & -0.81770 \\
\hline $\mathrm{H}$ & -3.12676 & 2.22880 & 0.11929 \\
\hline $\mathrm{H}$ & -0.65954 & 2.07364 & 0.08113 \\
\hline $\mathrm{H}$ & -4.02972 & 0.52913 & -2.95345 \\
\hline $\mathrm{O}$ & -3.34837 & 2.16478 & -1.95783 \\
\hline $\mathrm{C}$ & -2.57175 & -0.08614 & -1.50250 \\
\hline $\mathrm{N}$ & -2.56042 & -1.39206 & -1.48237 \\
\hline $\mathrm{N}$ & -1.34500 & -1.67751 & -0.85835 \\
\hline $\mathrm{C}$ & -0.64180 & -0.58688 & -0.50348 \\
\hline $\mathrm{N}$ & -1.42940 & 0.43596 & -0.93883 \\
\hline $\mathrm{C}$ & -0.98597 & -3.04578 & -0.68620 \\
\hline $\mathrm{C}$ & -1.49362 & -3.76700 & 0.40269 \\
\hline $\mathrm{C}$ & -0.18539 & -3.67116 & -1.65253 \\
\hline $\mathrm{C}$ & -1.15332 & -5.11503 & 0.55713 \\
\hline $\mathrm{C}$ & 0.15517 & -5.01997 & -1.49591 \\
\hline $\mathrm{C}$ & -0.33070 & -5.71586 & -0.39362 \\
\hline $\mathrm{H}$ & -1.52214 & -5.68824 & 1.39850 \\
\hline $\mathrm{H}$ & 0.79304 & -5.51621 & -2.21602 \\
\hline $\mathrm{H}$ & -0.06116 & -6.76077 & -0.27047 \\
\hline $\mathrm{O}$ & 0.19382 & -2.89037 & -2.69560 \\
\hline $\mathrm{O}$ & -2.29771 & -3.06737 & 1.25244 \\
\hline $\mathrm{C}$ & -2.81110 & -3.76316 & 2.39377 \\
\hline $\mathrm{H}$ & -3.40892 & -3.02827 & 2.93245 \\
\hline $\mathrm{H}$ & -3.44075 & -4.60576 & 2.08524 \\
\hline $\mathrm{H}$ & -1.99487 & -4.12114 & 3.03258 \\
\hline $\mathrm{C}$ & 1.02320 & -3.48975 & -3.69797 \\
\hline $\mathrm{H}$ & 0.49970 & -4.31944 & -4.18757 \\
\hline $\mathrm{H}$ & 1.22105 & -2.69582 & -4.41778 \\
\hline $\mathrm{H}$ & 1.96322 & -3.84088 & -3.25928 \\
\hline $\mathrm{C}$ & -0.37188 & 2.42503 & -2.03113 \\
\hline $\mathrm{H}$ & 0.50057 & 1.77545 & -2.13172 \\
\hline $\mathrm{H}$ & -0.97619 & 2.38386 & -2.94332 \\
\hline $\mathrm{C}$ & 0.06103 & 3.84228 & -1.73851 \\
\hline $\mathrm{C}$ & 0.98646 & 4.08465 & -0.71264 \\
\hline $\mathrm{C}$ & -0.48045 & 4.92942 & -2.43276 \\
\hline $\mathrm{C}$ & 1.35506 & 5.38875 & -0.39052 \\
\hline$H$ & 1.41040 & 3.23665 & -0.17986 \\
\hline
\end{tabular}




$\begin{array}{lrrc}\mathrm{C} & -0.10565 & 6.23645 & -2.11469 \\ \mathrm{H} & -1.20199 & 4.74960 & -3.22716 \\ \mathrm{C} & 0.81124 & 6.46891 & -1.08976 \\ \mathrm{H} & 2.06656 & 5.56114 & 0.41220 \\ \mathrm{H} & -0.53195 & 7.07087 & -2.66546 \\ \mathrm{H} & 1.10099 & 7.48550 & -0.83759 \\ \mathrm{C} & 1.15029 & -0.18299 & 0.16692 \\ \mathrm{C} & 1.84173 & -1.52469 & 0.03494 \\ \mathrm{C} & 1.60407 & -2.31981 & 1.16089 \\ \mathrm{C} & 2.70904 & -1.93444 & -0.96998 \\ \mathrm{C} & 2.21223 & -3.56714 & 1.27591 \\ \mathrm{C} & 3.32377 & -3.18322 & -0.85289 \\ \mathrm{H} & 2.90587 & -1.27493 & -1.81086 \\ \mathrm{C} & 3.06849 & -3.99582 & 0.25766 \\ \mathrm{H} & 2.03823 & -4.19553 & 2.14616 \\ \mathrm{H} & 4.01919 & -3.52346 & -1.61654 \\ \mathrm{H} & 3.55533 & -4.96419 & 0.33736 \\ \mathrm{C} & 0.69488 & -1.60460 & 2.13677 \\ \mathrm{H} & 0.96533 & -1.77755 & 3.18422 \\ \mathrm{H} & -0.35033 & -1.90656 & 2.00380 \\ \mathrm{C} & 0.84301 & -0.14002 & 1.71856 \\ \mathrm{C} & 1.36833 & 1.96139 & 2.71444 \\ \mathrm{H} & 2.33079 & 0.11998 & 3.32069 \\ \mathrm{C} & -0.00344 & 1.85259 & 2.48432 \\ \mathrm{C} & -0.88233 & 2.90870 & 2.69747 \\ \mathrm{C} & 1.89613 & 3.16533 & 3.15917 \\ \mathrm{C} & -0.33616 & 4.11778 & 3.14396 \\ \mathrm{H} & -0.99325 & 4.96737 & 3.30643 \\ \mathrm{C} & 1.03460 & 4.25128 & 3.36989 \\ \mathrm{H} & 1.43563 & 5.20256 & 3.70628 \\ \mathrm{H} & 2.96370 & 3.26961 & 3.33530 \\ \mathrm{H} & -1.94714 & 2.79894 & 2.51792 \\ \mathrm{O} & -0.37710 & 0.61669 & 2.02216 \\ & & & \end{array}$

77

3a-(R)- [2a+3a]

$\begin{array}{llll}\mathrm{C} & -0.08622 & 0.76574 & 3.58693 \\ \mathrm{H} & 0.96468 & 0.68206 & 3.87942 \\ \mathrm{O} & 1.71017 & 1.00829 & 1.07339 \\ \mathrm{C} & -1.46280 & 2.38641 & -2.64720 \\ \mathrm{C} & -1.63388 & 0.56464 & -4.14525 \\ \mathrm{C} & -0.47668 & 1.73001 & -1.68144 \\ \mathrm{H} & -2.73400 & 0.49217 & -4.16409 \\ \mathrm{H} & -1.31232 & 3.46702 & -2.66417 \\ \mathrm{H} & -2.49960 & 2.16688 & -2.34792 \\ \mathrm{H} & -0.75862 & 1.94163 & -0.64843 \\ \mathrm{H} & -1.24779 & 0.22843 & -5.11008 \\ \mathrm{O} & -1.23525 & 1.92694 & -3.99022\end{array}$




\begin{tabular}{|c|c|c|c|}
\hline $\mathrm{C}$ & -1.10056 & -0.28391 & -3.02986 \\
\hline $\mathrm{N}$ & -1.09374 & -1.58686 & -2.96051 \\
\hline $\mathrm{N}$ & -0.56539 & -1.80475 & -1.67692 \\
\hline $\mathrm{C}$ & -0.25381 & -0.69637 & -0.97181 \\
\hline $\mathrm{N}$ & -0.61100 & 0.27835 & -1.86903 \\
\hline $\mathrm{C}$ & -0.40122 & -3.14631 & -1.23400 \\
\hline $\mathrm{C}$ & -1.47368 & -3.81768 & -0.63090 \\
\hline $\mathrm{C}$ & 0.82497 & -3.79816 & -1.42709 \\
\hline $\mathrm{C}$ & -1.32570 & -5.15036 & -0.22660 \\
\hline $\mathrm{C}$ & 0.97423 & -5.13186 & -1.02438 \\
\hline $\mathrm{C}$ & -0.10402 & -5.78604 & -0.43627 \\
\hline $\mathrm{H}$ & -2.14421 & -5.68488 & 0.23947 \\
\hline $\mathrm{H}$ & 1.91476 & -5.65020 & -1.16292 \\
\hline $\mathrm{H}$ & 0.01222 & -6.82039 & -0.12513 \\
\hline $\mathrm{O}$ & 1.80114 & -3.05569 & -2.01207 \\
\hline $\mathrm{O}$ & -2.61386 & -3.08641 & -0.47346 \\
\hline $\mathrm{C}$ & -3.73018 & -3.73639 & 0.14007 \\
\hline $\mathrm{H}$ & -4.52176 & -2.98740 & 0.16449 \\
\hline $\mathrm{H}$ & -4.05320 & -4.60189 & -0.45067 \\
\hline $\mathrm{H}$ & -3.48633 & -4.05427 & 1.16135 \\
\hline $\mathrm{C}$ & 3.07953 & -3.67259 & -2.18779 \\
\hline $\mathrm{H}$ & 3.00796 & -4.53924 & -2.85626 \\
\hline $\mathrm{H}$ & 3.70846 & -2.90575 & -2.63994 \\
\hline $\mathrm{H}$ & 3.49841 & -3.97780 & -1.22203 \\
\hline $\mathrm{C}$ & 0.98070 & 2.18128 & -1.91473 \\
\hline $\mathrm{H}$ & 1.61890 & 1.56617 & -1.27421 \\
\hline $\mathrm{H}$ & 1.24409 & 1.99314 & -2.96096 \\
\hline $\mathrm{C}$ & 1.14761 & 3.64358 & -1.57784 \\
\hline $\mathrm{C}$ & 1.20298 & 4.05417 & -0.23869 \\
\hline $\mathrm{C}$ & 1.19107 & 4.61711 & -2.58343 \\
\hline $\mathrm{C}$ & 1.29088 & 5.40735 & 0.08376 \\
\hline $\mathrm{H}$ & 1.18528 & 3.30268 & 0.54557 \\
\hline $\mathrm{C}$ & 1.28525 & 5.97209 & -2.26218 \\
\hline $\mathrm{H}$ & 1.14915 & 4.30810 & -3.62581 \\
\hline $\mathrm{C}$ & 1.33112 & 6.37096 & -0.92587 \\
\hline $\mathrm{H}$ & 1.32612 & 5.70723 & 1.12757 \\
\hline $\mathrm{H}$ & 1.32204 & 6.71470 & -3.05495 \\
\hline $\mathrm{H}$ & 1.40199 & 7.42558 & -0.67356 \\
\hline $\mathrm{C}$ & 1.04434 & 0.06028 & 1.45773 \\
\hline $\mathrm{C}$ & 1.44737 & -1.35445 & 1.59545 \\
\hline $\mathrm{C}$ & 0.36160 & -2.10927 & 2.06087 \\
\hline $\mathrm{C}$ & 2.70179 & -1.92719 & 1.38588 \\
\hline $\mathrm{C}$ & 0.52607 & -3.46408 & 2.33258 \\
\hline $\mathrm{C}$ & 2.86122 & -3.28308 & 1.65823 \\
\hline $\mathrm{H}$ & 3.52460 & -1.31539 & 1.02728 \\
\hline $\mathrm{C}$ & 1.78024 & -4.04275 & 2.12734 \\
\hline $\mathrm{H}$ & -0.30399 & -4.06908 & 2.68655 \\
\hline $\mathrm{H}$ & 3.82831 & -3.75875 & 1.51801 \\
\hline
\end{tabular}




$\begin{array}{lrrl}\mathrm{H} & 1.92029 & -5.10063 & 2.33072 \\ \mathrm{C} & -0.88572 & -1.26299 & 2.15574 \\ \mathrm{H} & -1.50128 & -1.49002 & 3.03256 \\ \mathrm{H} & -1.48862 & -1.39532 & 1.24987 \\ \mathrm{C} & -0.33602 & 0.16606 & 2.16490 \\ \mathrm{C} & -0.54109 & 2.19105 & 3.40825 \\ \mathrm{H} & -0.68772 & 0.23975 & 4.33967 \\ \mathrm{C} & -1.20675 & 2.28348 & 2.18635 \\ \mathrm{C} & -1.78583 & 3.46440 & 1.73932 \\ \mathrm{C} & -0.42827 & 3.31258 & 4.21822 \\ \mathrm{C} & -1.66411 & 4.58825 & 2.56362 \\ \mathrm{H} & -2.09051 & 5.53181 & 2.23546 \\ \mathrm{C} & -0.99159 & 4.52115 & 3.78559 \\ \mathrm{H} & -0.90358 & 5.40980 & 4.40316 \\ \mathrm{H} & 0.09196 & 3.25933 & 5.17112 \\ \mathrm{H} & -2.29457 & 3.51625 & 0.78249 \\ \mathrm{O} & -1.22602 & 1.10131 & 1.48969\end{array}$




\subsection{3d-(R)-a-path}

76

3d-(R)-4-a

$\begin{array}{llll}\text { C } & -2.47549 & -0.38064 & -1.79815\end{array}$

$\begin{array}{llll}\mathrm{H} & -0.11772 & -0.81157 & -2.08307\end{array}$

$\begin{array}{llll}\mathrm{O} & 0.72277 & -0.53817 & -1.66268\end{array}$

$\begin{array}{llll}\text { C } & 0.60679 & -2.43918 & 2.51928\end{array}$

$\begin{array}{llll}\text { C } & 1.18864 & -1.30653 & 3.41063\end{array}$

$\begin{array}{llll}\text { C } & 0.80593 & -1.98201 & 1.04544\end{array}$

$\begin{array}{llll}\mathrm{C} & 1.02306 & -0.12015 & 2.51794\end{array}$

$\begin{array}{llll}\mathrm{N} & 0.91463 & 1.15977 & 2.67929\end{array}$

$\begin{array}{llll}\mathrm{N} & 0.69255 & 1.62963 & 1.35697\end{array}$

$\begin{array}{llll}\mathrm{C} & 0.65434 & 0.58383 & 0.42015\end{array}$

$\begin{array}{llll}\mathrm{N} & 0.90426 & -0.53064 & 1.21212\end{array}$

$\begin{array}{llll}\text { C } & 0.73560 & 3.01109 & 1.09069\end{array}$

$\begin{array}{llll}\text { C } & -0.22691 & 3.87139 & 1.65303\end{array}$

$\begin{array}{llll}\text { C } & 1.73573 & 3.54762 & 0.25645\end{array}$

$\begin{array}{llll}\text { C } & -0.21417 & 5.23748 & 1.35504\end{array}$

$\begin{array}{llll}\text { C } & 1.72606 & 4.90779 & -0.07055\end{array}$

$\begin{array}{llll}\text { C } & 0.75262 & 5.73380 & 0.48338\end{array}$

$\begin{array}{llll}\mathrm{H} & -0.95694 & 5.90307 & 1.77730\end{array}$

$\begin{array}{llll}\mathrm{H} & 2.47446 & 5.32095 & -0.73512\end{array}$

$\begin{array}{llll}\mathrm{H} & 0.75086 & 6.79127 & 0.23586\end{array}$

$\begin{array}{llll}\mathrm{O} & 2.67267 & 2.66248 & -0.18203\end{array}$

$\begin{array}{llll}\mathrm{O} & -1.15169 & 3.27443 & 2.46036\end{array}$

$\begin{array}{llll}\text { C } & -2.16840 & 4.10575 & 3.02195\end{array}$

$\mathrm{H} \quad-2.79511 \quad 3.43583 \quad 3.61110$

$\begin{array}{llll}\mathrm{H} & -1.73191 & 4.87406 & 3.67145\end{array}$

$\begin{array}{llll}\mathrm{H} & -2.76736 & 4.58300 & 2.23587\end{array}$

$\begin{array}{llll}\text { C } & 3.67716 & 3.15698 & -1.07079\end{array}$

$\mathrm{H} \quad 4.29234 \quad 3.92087 \quad-0.57925$

$\mathrm{H} \quad 4.28999 \quad 2.29105 \quad-1.32227$

$\begin{array}{llll}\mathrm{H} & 3.22297 & 3.57001 & -1.97870\end{array}$

$\begin{array}{llll}\text { C } & 2.04939 & -2.59121 & 0.36858\end{array}$

$\begin{array}{llll}\mathrm{H} & 2.21755 & -2.04439 & -0.56357\end{array}$

$\begin{array}{llll}\mathrm{H} & 2.92502 & -2.44880 & 1.01303\end{array}$

$\begin{array}{llll}\text { C } & 1.83394 & -4.05889 & 0.09025\end{array}$

$\begin{array}{llll}\text { C } & 0.83931 & -4.46397 & -0.81125\end{array}$

$\begin{array}{llll}\text { C } & 2.57843 & -5.04406 & 0.74792\end{array}$

$\begin{array}{llll}\text { C } & 0.59733 & -5.81531 & -1.04626\end{array}$

$\begin{array}{llll}\mathrm{H} & 0.24575 & -3.71490 & -1.32928\end{array}$

$\begin{array}{llll}\text { C } & 2.34282 & -6.39954 & 0.51152\end{array}$

$\begin{array}{llll}\mathrm{H} & 3.35418 & -4.74456 & 1.44963\end{array}$

$\begin{array}{llll}\text { C } & 1.34911 & -6.78927 & -0.38587\end{array}$

$\begin{array}{llll}\mathrm{H} & -0.18137 & -6.10749 & -1.74529\end{array}$

$\begin{array}{llll}\mathrm{H} & 2.93465 & -7.14956 & 1.02933\end{array}$

$\begin{array}{llll}\mathrm{H} & 1.16167 & -7.84340 & -0.57015\end{array}$ 


\begin{tabular}{|c|c|c|c|}
\hline $\mathrm{C}$ & 0.42141 & 0.62588 & -0.92454 \\
\hline $\mathrm{C}$ & -0.02478 & 1.79534 & -1.67938 \\
\hline $\mathrm{C}$ & -1.25200 & 2.42083 & -1.35706 \\
\hline $\mathrm{C}$ & 0.70341 & 2.26911 & -2.78275 \\
\hline $\mathrm{C}$ & -1.65228 & 3.55800 & -2.05966 \\
\hline $\mathrm{C}$ & 0.28275 & 3.39075 & -3.49129 \\
\hline $\mathrm{H}$ & 1.61589 & 1.74963 & -3.06128 \\
\hline $\mathrm{C}$ & -0.88446 & 4.05807 & -3.11174 \\
\hline $\mathrm{H}$ & -2.59254 & 4.03972 & -1.80009 \\
\hline $\mathrm{H}$ & 0.86929 & 3.75390 & -4.33140 \\
\hline $\mathrm{H}$ & -1.21131 & 4.94249 & -3.65112 \\
\hline $\mathrm{C}$ & -2.15421 & 1.77589 & -0.32616 \\
\hline $\mathrm{H}$ & -3.12148 & 2.29698 & -0.31281 \\
\hline $\mathrm{H}$ & -1.74508 & 1.85106 & 0.68780 \\
\hline $\mathrm{C}$ & -2.35781 & 0.32494 & -0.63140 \\
\hline $\mathrm{C}$ & -2.67269 & -1.76106 & -1.43762 \\
\hline $\mathrm{H}$ & -2.42150 & 0.04421 & -2.79093 \\
\hline $\mathrm{C}$ & -2.68733 & -1.78485 & -0.02695 \\
\hline $\mathrm{C}$ & -2.84996 & -2.93741 & 0.72655 \\
\hline $\mathrm{C}$ & -2.82507 & -2.97474 & -2.12780 \\
\hline $\mathrm{C}$ & -2.99329 & -4.13153 & 0.01618 \\
\hline $\mathrm{H}$ & -3.11030 & -5.06382 & 0.55967 \\
\hline $\mathrm{C}$ & -2.98046 & -4.14523 & -1.38854 \\
\hline $\mathrm{H}$ & -3.09542 & -5.09259 & -1.90753 \\
\hline $\mathrm{H}$ & -2.82102 & -3.00188 & -3.21381 \\
\hline $\mathrm{H}$ & -2.86473 & -2.90916 & 1.81118 \\
\hline $\mathrm{O}$ & -2.50733 & -0.51186 & 0.46776 \\
\hline $\mathrm{H}$ & -0.07516 & -2.21760 & 0.44497 \\
\hline $\mathrm{H}$ & 0.66022 & -1.19386 & 4.35889 \\
\hline $\mathrm{H}$ & 2.25008 & -1.47081 & 3.63238 \\
\hline $\mathrm{H}$ & 1.07549 & -3.40883 & 2.70083 \\
\hline $\mathrm{H}$ & -0.46381 & -2.53948 & 2.71739 \\
\hline \multicolumn{4}{|l|}{76} \\
\hline \multicolumn{4}{|c|}{$3 d-(R)-t s 1-a$} \\
\hline $\mathrm{C}$ & -1.71343 & -0.67862 & -2.12475 \\
\hline $\mathrm{H}$ & -0.38388 & -0.87976 & -2.05330 \\
\hline $\mathrm{O}$ & 0.78452 & -0.60480 & -1.58958 \\
\hline $\mathrm{C}$ & -0.09028 & -2.54856 & 2.43123 \\
\hline $\mathrm{C}$ & 0.19273 & -1.40266 & 3.44458 \\
\hline $\mathrm{C}$ & 0.41005 & -2.05447 & 1.04529 \\
\hline $\mathrm{C}$ & 0.24128 & -0.20852 & 2.54779 \\
\hline $\mathrm{N}$ & 0.21832 & 1.08197 & 2.69455 \\
\hline $\mathrm{N}$ & 0.33183 & 1.55106 & 1.36773 \\
\hline $\mathrm{C}$ & 0.41794 & 0.52147 & 0.44673 \\
\hline $\mathrm{N}$ & 0.36087 & -0.59942 & 1.23930 \\
\hline $\mathrm{C}$ & 0.48711 & 2.93725 & 1.13637 \\
\hline $\mathrm{C}$ & -0.57395 & 3.82106 & 1.39797 \\
\hline
\end{tabular}




\begin{tabular}{|c|c|c|c|}
\hline $\mathrm{C}$ & 1.71723 & 3.43551 & 0.67388 \\
\hline $\mathrm{C}$ & -0.41640 & 5.19105 & 1.16369 \\
\hline $\mathrm{C}$ & 1.86585 & 4.80238 & 0.41568 \\
\hline $\mathrm{C}$ & 0.79714 & 5.65832 & 0.66455 \\
\hline $\mathrm{H}$ & -1.22883 & 5.88206 & 1.35145 \\
\hline $\mathrm{H}$ & 2.79932 & 5.19507 & 0.03290 \\
\hline $\mathrm{H}$ & 0.91356 & 6.71996 & 0.46723 \\
\hline $\mathrm{O}$ & 2.70134 & 2.50971 & 0.50975 \\
\hline $\mathrm{O}$ & -1.72136 & 3.24784 & 1.85836 \\
\hline $\mathrm{C}$ & -2.82653 & 4.11309 & 2.13288 \\
\hline $\mathrm{H}$ & -3.62339 & 3.45708 & 2.48314 \\
\hline $\mathrm{H}$ & -2.56915 & 4.83918 & 2.91304 \\
\hline $\mathrm{H}$ & -3.14823 & 4.64036 & 1.22617 \\
\hline $\mathrm{C}$ & 3.96296 & 2.97499 & 0.01992 \\
\hline $\mathrm{H}$ & 4.41310 & 3.68792 & 0.72119 \\
\hline $\mathrm{H}$ & 4.58675 & 2.08452 & -0.05849 \\
\hline $\mathrm{H}$ & 3.84861 & 3.43976 & -0.96576 \\
\hline $\mathrm{C}$ & 1.82447 & -2.53496 & 0.66922 \\
\hline $\mathrm{H}$ & 2.14716 & -1.93668 & -0.18818 \\
\hline $\mathrm{H}$ & 2.51113 & -2.35431 & 1.50523 \\
\hline $\mathrm{C}$ & 1.79914 & -3.99936 & 0.30893 \\
\hline $\mathrm{C}$ & 1.14721 & -4.41512 & -0.86077 \\
\hline $\mathrm{C}$ & 2.37207 & -4.96961 & 1.13754 \\
\hline $\mathrm{C}$ & 1.06941 & -5.76646 & -1.18866 \\
\hline $\mathrm{H}$ & 0.70176 & -3.66886 & -1.51372 \\
\hline $\mathrm{C}$ & 2.29971 & -6.32476 & 0.80970 \\
\hline $\mathrm{H}$ & 2.88537 & -4.65966 & 2.04568 \\
\hline $\mathrm{C}$ & 1.64493 & -6.72693 & -0.35380 \\
\hline $\mathrm{H}$ & 0.55931 & -6.07174 & -2.09832 \\
\hline $\mathrm{H}$ & 2.75409 & -7.06456 & 1.46328 \\
\hline $\mathrm{H}$ & 1.58532 & -7.78086 & -0.61062 \\
\hline $\mathrm{C}$ & 0.48540 & 0.53199 & -0.95532 \\
\hline $\mathrm{C}$ & 0.45624 & 1.78264 & -1.75817 \\
\hline $\mathrm{C}$ & -0.76725 & 2.45337 & -1.92333 \\
\hline $\mathrm{C}$ & 1.58497 & 2.23342 & -2.45224 \\
\hline $\mathrm{C}$ & -0.82473 & 3.60733 & -2.70818 \\
\hline $\mathrm{C}$ & 1.52215 & 3.38186 & -3.23733 \\
\hline $\mathrm{H}$ & 2.50848 & 1.66849 & -2.36749 \\
\hline $\mathrm{C}$ & 0.31825 & 4.08178 & -3.35044 \\
\hline $\mathrm{H}$ & -1.77284 & 4.12492 & -2.83614 \\
\hline $\mathrm{H}$ & 2.40569 & 3.73021 & -3.76614 \\
\hline $\mathrm{H}$ & 0.26386 & 4.97860 & -3.96152 \\
\hline $\mathrm{C}$ & -1.98470 & 1.85098 & -1.26168 \\
\hline $\mathrm{H}$ & -2.89212 & 2.12691 & -1.82180 \\
\hline $\mathrm{H}$ & -2.11111 & 2.24294 & -0.24501 \\
\hline $\mathrm{C}$ & -1.85307 & 0.36265 & -1.13548 \\
\hline $\mathrm{C}$ & -2.35219 & -1.85670 & -1.51480 \\
\hline $\mathrm{H}$ & -1.83787 & -0.44616 & -3.18174 \\
\hline
\end{tabular}




\begin{tabular}{|c|c|c|c|}
\hline $\mathrm{C}$ & -2.73668 & -1.48691 & -0.21652 \\
\hline $\mathrm{C}$ & -3.30509 & -2.36076 & 0.69796 \\
\hline $\mathrm{C}$ & -2.54465 & -3.18035 & -1.91550 \\
\hline $\mathrm{C}$ & -3.49327 & -3.68108 & 0.27317 \\
\hline $\mathrm{H}$ & -3.93769 & -4.40141 & 0.95356 \\
\hline $\mathrm{C}$ & -3.11769 & -4.08316 & -1.01436 \\
\hline $\mathrm{H}$ & -3.27058 & -5.11543 & -1.31534 \\
\hline $\mathrm{H}$ & -2.24947 & -3.50721 & -2.90891 \\
\hline $\mathrm{H}$ & -3.59910 & -2.02839 & 1.68863 \\
\hline $\mathrm{O}$ & -2.47212 & -0.15400 & 0.01587 \\
\hline $\mathrm{H}$ & -0.28456 & -2.33498 & 0.25291 \\
\hline $\mathrm{H}$ & -0.57790 & -1.31666 & 4.21345 \\
\hline $\mathrm{H}$ & 1.15655 & -1.53077 & 3.95103 \\
\hline $\mathrm{H}$ & 0.39319 & -3.48708 & 2.70989 \\
\hline $\mathrm{H}$ & -1.16626 & -2.72791 & 2.37197 \\
\hline \multicolumn{4}{|l|}{76} \\
\hline \multicolumn{4}{|c|}{$3 d-(R)-6-a$} \\
\hline $\mathrm{C}$ & -0.72030 & -0.68375 & -2.78419 \\
\hline $\mathrm{H}$ & 0.33779 & -0.96788 & -2.83941 \\
\hline $\mathrm{O}$ & 1.27268 & -0.70687 & -0.92686 \\
\hline $\mathrm{C}$ & -0.81046 & -2.56617 & 2.49141 \\
\hline $\mathrm{C}$ & -0.68870 & -1.44109 & 3.56164 \\
\hline $\mathrm{C}$ & 0.03877 & -2.09638 & 1.28181 \\
\hline $\mathrm{C}$ & -0.45761 & -0.24544 & 2.69537 \\
\hline $\mathrm{N}$ & -0.51955 & 1.05540 & 2.81191 \\
\hline $\mathrm{N}$ & -0.18028 & 1.48979 & 1.52593 \\
\hline $\mathrm{C}$ & 0.07603 & 0.47094 & 0.67295 \\
\hline $\mathrm{N}$ & -0.09063 & -0.62949 & 1.43446 \\
\hline $\mathrm{C}$ & -0.19642 & 2.88537 & 1.23877 \\
\hline $\mathrm{C}$ & -1.40913 & 3.50271 & 0.89917 \\
\hline $\mathrm{C}$ & 0.98954 & 3.62662 & 1.33839 \\
\hline $\mathrm{C}$ & -1.42096 & 4.86468 & 0.57964 \\
\hline $\mathrm{C}$ & 0.97576 & 4.98741 & 1.01168 \\
\hline $\mathrm{C}$ & -0.22526 & 5.57859 & 0.63055 \\
\hline $\mathrm{H}$ & -2.34153 & 5.36017 & 0.29725 \\
\hline $\mathrm{H}$ & 1.88513 & 5.57352 & 1.04528 \\
\hline $\mathrm{H}$ & -0.23181 & 6.63310 & 0.37058 \\
\hline $\mathrm{O}$ & 2.08099 & 2.93770 & 1.75535 \\
\hline $\mathrm{O}$ & -2.50048 & 2.69301 & 0.90270 \\
\hline $\mathrm{C}$ & -3.74509 & 3.25537 & 0.47443 \\
\hline $\mathrm{H}$ & -4.45587 & 2.43015 & 0.50334 \\
\hline $\mathrm{H}$ & -4.06560 & 4.05494 & 1.15270 \\
\hline $\mathrm{H}$ & -3.66685 & 3.64257 & -0.54857 \\
\hline $\mathrm{C}$ & 3.32158 & 3.65237 & 1.82375 \\
\hline $\mathrm{H}$ & 3.25761 & 4.46691 & 2.55466 \\
\hline $\mathrm{H}$ & 4.05778 & 2.91699 & 2.14748 \\
\hline $\mathrm{H}$ & 3.59155 & 4.04530 & 0.83798 \\
\hline
\end{tabular}




\begin{tabular}{|c|c|c|c|}
\hline $\mathrm{C}$ & 1.51401 & -2.53739 & 1.34587 \\
\hline $\mathrm{H}$ & 2.06908 & -1.83147 & 0.72024 \\
\hline $\mathrm{H}$ & 1.87563 & -2.49071 & 2.38096 \\
\hline $\mathrm{C}$ & 1.64491 & -3.93338 & 0.79188 \\
\hline $\mathrm{C}$ & 1.47789 & -4.12896 & -0.58714 \\
\hline $\mathrm{C}$ & 1.88418 & -5.04191 & 1.60921 \\
\hline $\mathrm{C}$ & 1.54644 & -5.40932 & -1.12991 \\
\hline $\mathrm{H}$ & 1.29872 & -3.25862 & -1.21505 \\
\hline $\mathrm{C}$ & 1.95716 & -6.32619 & 1.06488 \\
\hline $\mathrm{H}$ & 2.02235 & -4.89920 & 2.67949 \\
\hline $\mathrm{C}$ & 1.78497 & -6.51268 & -0.30610 \\
\hline $\mathrm{H}$ & 1.41593 & -5.54896 & -2.19989 \\
\hline $\mathrm{H}$ & 2.14762 & -7.17860 & 1.71166 \\
\hline $\mathrm{H}$ & 1.83979 & -7.51098 & -0.73165 \\
\hline $\mathrm{C}$ & 0.51493 & 0.38205 & -0.80550 \\
\hline $\mathrm{C}$ & 1.16057 & 1.68072 & -1.30620 \\
\hline $\mathrm{C}$ & 0.30094 & 2.46267 & -2.07795 \\
\hline $\mathrm{C}$ & 2.50448 & 2.01426 & -1.17256 \\
\hline $\mathrm{C}$ & 0.76556 & 3.63105 & -2.68215 \\
\hline $\mathrm{C}$ & 2.97229 & 3.18309 & -1.77425 \\
\hline $\mathrm{H}$ & 3.16640 & 1.34782 & -0.62700 \\
\hline $\mathrm{C}$ & 2.10323 & 3.99399 & -2.51486 \\
\hline $\mathrm{H}$ & 0.10399 & 4.24359 & -3.29077 \\
\hline $\mathrm{H}$ & 4.02138 & 3.45765 & -1.69113 \\
\hline $\mathrm{H}$ & 2.48027 & 4.89747 & -2.98705 \\
\hline $\mathrm{C}$ & -1.07165 & 1.84222 & -2.17097 \\
\hline $\mathrm{H}$ & -1.49152 & 1.89500 & -3.18250 \\
\hline $\mathrm{H}$ & -1.77977 & 2.33760 & -1.49853 \\
\hline $\mathrm{C}$ & -0.86931 & 0.39689 & -1.69888 \\
\hline $\mathrm{C}$ & -1.57160 & -1.80372 & -2.24636 \\
\hline $\mathrm{H}$ & -1.07164 & -0.33344 & -3.76281 \\
\hline $\mathrm{C}$ & -2.29826 & -1.31712 & -1.15667 \\
\hline $\mathrm{C}$ & -3.19032 & -2.10567 & -0.43814 \\
\hline $\mathrm{C}$ & -1.71517 & -3.13170 & -2.62314 \\
\hline $\mathrm{C}$ & -3.32470 & -3.44328 & -0.83199 \\
\hline $\mathrm{H}$ & -4.00456 & -4.09145 & -0.28573 \\
\hline $\mathrm{C}$ & -2.59347 & -3.95633 & -1.90481 \\
\hline $\mathrm{H}$ & -2.70182 & -5.00061 & -2.18126 \\
\hline $\mathrm{H}$ & -1.14568 & -3.53393 & -3.45705 \\
\hline $\mathrm{H}$ & -3.75008 & -1.69736 & 0.39799 \\
\hline $\mathrm{O}$ & -2.02868 & -0.00586 & -0.87761 \\
\hline $\mathrm{H}$ & -0.38935 & -2.38608 & 0.32461 \\
\hline $\mathrm{H}$ & -1.58026 & -1.33997 & 4.18359 \\
\hline $\mathrm{H}$ & 0.16910 & -1.59823 & 4.22585 \\
\hline $\mathrm{H}$ & -0.46768 & -3.53612 & 2.85654 \\
\hline $\mathrm{H}$ & -1.85497 & -2.66339 & 2.18269 \\
\hline
\end{tabular}

76 


\begin{tabular}{|c|c|c|c|}
\hline \multicolumn{4}{|c|}{$3 d-(R)-t s 3-a$} \\
\hline $\mathrm{C}$ & -3.09924 & -0.72518 & -0.51460 \\
\hline $\mathrm{H}$ & -2.97517 & -0.76905 & -1.59951 \\
\hline $\mathrm{O}$ & -0.40203 & -0.82677 & -1.57739 \\
\hline $\mathrm{C}$ & 2.40859 & -2.24737 & 1.78290 \\
\hline $\mathrm{C}$ & 3.40062 & -1.05545 & 1.92997 \\
\hline $\mathrm{C}$ & 1.48478 & -1.87685 & 0.59405 \\
\hline $\mathrm{C}$ & 2.56531 & 0.07273 & 1.41730 \\
\hline $\mathrm{N}$ & 2.55483 & 1.37548 & 1.49401 \\
\hline $\mathrm{N}$ & 1.38205 & 1.69308 & 0.78383 \\
\hline $\mathrm{C}$ & 0.71034 & 0.62249 & 0.30546 \\
\hline $\mathrm{N}$ & 1.49325 & -0.40467 & 0.70552 \\
\hline $\mathrm{C}$ & 1.01912 & 3.06193 & 0.63959 \\
\hline $\mathrm{C}$ & 0.30772 & 3.70773 & 1.66007 \\
\hline $\mathrm{C}$ & 1.41333 & 3.75882 & -0.51177 \\
\hline $\mathrm{C}$ & -0.04920 & 5.05233 & 1.51107 \\
\hline $\mathrm{C}$ & 1.05429 & 5.10383 & -0.66052 \\
\hline $\mathrm{C}$ & 0.32804 & 5.72498 & 0.35054 \\
\hline $\mathrm{H}$ & -0.60859 & 5.56766 & 2.28189 \\
\hline $\mathrm{H}$ & 1.32809 & 5.65424 & -1.55146 \\
\hline $\mathrm{H}$ & 0.04567 & 6.76705 & 0.23153 \\
\hline $\mathrm{O}$ & 2.13889 & 3.04606 & -1.41076 \\
\hline $\mathrm{O}$ & 0.00417 & 2.94065 & 2.74492 \\
\hline $\mathrm{C}$ & -0.75042 & 3.55542 & 3.79465 \\
\hline $\mathrm{H}$ & -0.88876 & 2.77522 & 4.54291 \\
\hline $\mathrm{H}$ & -0.19959 & 4.39803 & 4.22868 \\
\hline $\mathrm{H}$ & -1.72541 & 3.89683 & 3.42667 \\
\hline $\mathrm{C}$ & 2.54209 & 3.71785 & -2.60940 \\
\hline $\mathrm{H}$ & 3.20162 & 4.56256 & -2.37709 \\
\hline $\mathrm{H}$ & 3.08356 & 2.96994 & -3.18837 \\
\hline $\mathrm{H}$ & 1.66680 & 4.06356 & -3.16961 \\
\hline $\mathrm{C}$ & 2.02575 & -2.35667 & -0.76753 \\
\hline $\mathrm{H}$ & 1.53855 & -1.75567 & -1.53969 \\
\hline $\mathrm{H}$ & 3.11072 & -2.19982 & -0.81456 \\
\hline $\mathrm{C}$ & 1.68818 & -3.81567 & -0.95424 \\
\hline $\mathrm{C}$ & 0.34844 & -4.18751 & -1.14137 \\
\hline $\mathrm{C}$ & 2.66557 & -4.81417 & -0.90038 \\
\hline $\mathrm{C}$ & 0.00038 & -5.53010 & -1.26598 \\
\hline $\mathrm{H}$ & -0.41072 & -3.41040 & -1.19292 \\
\hline $\mathrm{C}$ & 2.31840 & -6.16067 & -1.03093 \\
\hline $\mathrm{H}$ & 3.70870 & -4.53536 & -0.76228 \\
\hline $\mathrm{C}$ & 0.98350 & -6.52142 & -1.21056 \\
\hline $\mathrm{H}$ & -1.04229 & -5.80184 & -1.40417 \\
\hline $\mathrm{H}$ & 3.08996 & -6.92516 & -0.99135 \\
\hline $\mathrm{H}$ & 0.71041 & -7.56852 & -1.30965 \\
\hline $\mathrm{C}$ & -0.77337 & 0.18066 & -0.92082 \\
\hline $\mathrm{C}$ & -1.04531 & 1.53260 & -1.54458 \\
\hline $\mathrm{C}$ & -1.90450 & 2.28392 & -0.73519 \\
\hline
\end{tabular}




\begin{tabular}{|c|c|c|c|}
\hline $\mathrm{C}$ & -0.62472 & 2.00117 & -2.78301 \\
\hline $\mathrm{C}$ & -2.33018 & 3.54321 & -1.14888 \\
\hline $\mathrm{C}$ & -1.05668 & 3.26215 & -3.20142 \\
\hline $\mathrm{H}$ & 0.01371 & 1.37936 & -3.40494 \\
\hline $\mathrm{C}$ & -1.89557 & 4.02941 & -2.38543 \\
\hline $\mathrm{H}$ & -2.99769 & 4.13841 & -0.53024 \\
\hline $\mathrm{H}$ & -0.75569 & 3.64761 & -4.17270 \\
\hline $\mathrm{H}$ & -2.22527 & 5.00813 & -2.72384 \\
\hline $\mathrm{C}$ & -2.26234 & 1.51836 & 0.52063 \\
\hline $\mathrm{H}$ & -3.30546 & 1.65496 & 0.82644 \\
\hline $\mathrm{H}$ & -1.62207 & 1.81269 & 1.36037 \\
\hline $\mathrm{C}$ & -1.93721 & 0.07258 & 0.13946 \\
\hline $\mathrm{C}$ & -2.96811 & -2.07590 & 0.13868 \\
\hline $\mathrm{H}$ & -4.07013 & -0.26365 & -0.29148 \\
\hline $\mathrm{C}$ & -2.06374 & -1.96046 & 1.19536 \\
\hline $\mathrm{C}$ & -1.74195 & -3.03105 & 2.02244 \\
\hline $\mathrm{C}$ & -3.56579 & -3.30057 & -0.12266 \\
\hline $\mathrm{C}$ & -2.35124 & -4.26093 & 1.74673 \\
\hline $\mathrm{H}$ & -2.10933 & -5.12149 & 2.36391 \\
\hline $\mathrm{C}$ & -3.24866 & -4.40100 & 0.68704 \\
\hline $\mathrm{H}$ & -3.70122 & -5.36766 & 0.48740 \\
\hline $\mathrm{H}$ & -4.26562 & -3.40982 & -0.94721 \\
\hline $\mathrm{H}$ & -1.04039 & -2.91606 & 2.84280 \\
\hline $\mathrm{O}$ & -1.53573 & -0.70271 & 1.31865 \\
\hline $\mathrm{H}$ & 0.46576 & -2.23411 & 0.72402 \\
\hline $\mathrm{H}$ & 3.73346 & -0.89804 & 2.95774 \\
\hline $\mathrm{H}$ & 4.29088 & -1.18774 & 1.30413 \\
\hline $\mathrm{H}$ & 2.91100 & -3.20116 & 1.61018 \\
\hline $\mathrm{H}$ & 1.81294 & -2.33298 & 2.69697 \\
\hline \multicolumn{4}{|l|}{76} \\
\hline \multicolumn{4}{|c|}{$3 \mathrm{~d}-(\mathrm{R})-[3 \mathrm{~d}+2 \mathrm{a}]$} \\
\hline $\mathrm{C}$ & -3.50676 & -0.80913 & -0.37643 \\
\hline $\mathrm{H}$ & -3.64218 & -0.76932 & -1.46134 \\
\hline $\mathrm{O}$ & -0.77693 & -1.02665 & -1.79357 \\
\hline $\mathrm{C}$ & 2.72943 & -2.09772 & 1.77418 \\
\hline $\mathrm{C}$ & 3.69713 & -0.87853 & 1.85816 \\
\hline $\mathrm{C}$ & 1.68043 & -1.73711 & 0.68815 \\
\hline $\mathrm{C}$ & 2.80831 & 0.23157 & 1.39762 \\
\hline $\mathrm{N}$ & 2.79355 & 1.53466 & 1.42597 \\
\hline $\mathrm{N}$ & 1.57074 & 1.80431 & 0.76383 \\
\hline $\mathrm{C}$ & 0.86316 & 0.72415 & 0.35066 \\
\hline $\mathrm{N}$ & 1.69081 & -0.27167 & 0.77369 \\
\hline $\mathrm{C}$ & 1.16139 & 3.15585 & 0.60101 \\
\hline $\mathrm{C}$ & 0.40900 & 3.78424 & 1.60332 \\
\hline $\mathrm{C}$ & 1.50606 & 3.85194 & -0.56649 \\
\hline $\mathrm{C}$ & 0.00398 & 5.11543 & 1.44371 \\
\hline $\mathrm{C}$ & 1.10419 & 5.18458 & -0.72688 \\
\hline
\end{tabular}




\begin{tabular}{|c|c|c|c|}
\hline $\mathrm{C}$ & 0.36327 & 5.79387 & 0.28127 \\
\hline $\mathrm{H}$ & -0.57676 & 5.61626 & 2.20851 \\
\hline $\mathrm{H}$ & 1.35764 & 5.73571 & -1.62377 \\
\hline $\mathrm{H}$ & 0.05131 & 6.82694 & 0.15606 \\
\hline $\mathrm{O}$ & 2.22465 & 3.14912 & -1.48145 \\
\hline $\mathrm{O}$ & 0.11224 & 3.01357 & 2.68832 \\
\hline $\mathrm{C}$ & -0.67281 & 3.61156 & 3.72308 \\
\hline $\mathrm{H}$ & -0.79764 & 2.83315 & 4.47588 \\
\hline $\mathrm{H}$ & -0.15496 & 4.47387 & 4.15977 \\
\hline $\mathrm{H}$ & -1.65359 & 3.92179 & 3.34176 \\
\hline $\mathrm{C}$ & 2.55181 & 3.81023 & -2.70652 \\
\hline $\mathrm{H}$ & 3.19155 & 4.68217 & -2.52300 \\
\hline $\mathrm{H}$ & 3.09333 & 3.07055 & -3.29630 \\
\hline $\mathrm{H}$ & 1.64262 & 4.11721 & -3.23609 \\
\hline $\mathrm{C}$ & 2.05253 & -2.21993 & -0.72935 \\
\hline $\mathrm{H}$ & 1.42629 & -1.67204 & -1.43907 \\
\hline $\mathrm{H}$ & 3.10246 & -1.97659 & -0.93433 \\
\hline $\mathrm{C}$ & 1.81075 & -3.70322 & -0.86247 \\
\hline $\mathrm{C}$ & 0.49878 & -4.17886 & -0.99874 \\
\hline $\mathrm{C}$ & 2.85870 & -4.62820 & -0.79944 \\
\hline $\mathrm{C}$ & 0.24409 & -5.54732 & -1.05794 \\
\hline $\mathrm{H}$ & -0.31748 & -3.46479 & -1.06590 \\
\hline $\mathrm{C}$ & 2.60658 & -5.99979 & -0.86440 \\
\hline $\mathrm{H}$ & 3.88284 & -4.27108 & -0.70767 \\
\hline $\mathrm{C}$ & 1.29666 & -6.46274 & -0.98870 \\
\hline $\mathrm{H}$ & -0.77967 & -5.89729 & -1.15801 \\
\hline $\mathrm{H}$ & 3.43245 & -6.70483 & -0.81861 \\
\hline $\mathrm{H}$ & 1.09788 & -7.52990 & -1.03767 \\
\hline $\mathrm{C}$ & -1.25625 & -0.06654 & -1.20874 \\
\hline $\mathrm{C}$ & -1.36396 & 1.33593 & -1.66747 \\
\hline $\mathrm{C}$ & -1.99680 & 2.10595 & -0.68146 \\
\hline $\mathrm{C}$ & -0.97703 & 1.88624 & -2.88875 \\
\hline $\mathrm{C}$ & -2.25343 & 3.45354 & -0.91345 \\
\hline $\mathrm{C}$ & -1.23574 & 3.23567 & -3.11667 \\
\hline $\mathrm{H}$ & -0.49094 & 1.26401 & -3.63482 \\
\hline $\mathrm{C}$ & -1.86746 & 4.01107 & -2.13469 \\
\hline $\mathrm{H}$ & -2.73222 & 4.07015 & -0.15772 \\
\hline $\mathrm{H}$ & -0.95687 & 3.69295 & -4.06245 \\
\hline $\mathrm{H}$ & -2.05687 & 5.06371 & -2.32549 \\
\hline $\mathrm{C}$ & -2.26447 & 1.28633 & 0.55846 \\
\hline $\mathrm{H}$ & -3.23169 & 1.50425 & 1.02390 \\
\hline $\mathrm{H}$ & -1.46737 & 1.45729 & 1.29121 \\
\hline $\mathrm{C}$ & -2.15423 & -0.15592 & 0.05677 \\
\hline $\mathrm{C}$ & -3.35950 & -2.21261 & 0.15163 \\
\hline $\mathrm{H}$ & -4.35472 & -0.28905 & 0.08785 \\
\hline $\mathrm{C}$ & -2.24509 & -2.24465 & 0.99018 \\
\hline $\mathrm{C}$ & -1.85998 & -3.39147 & 1.67405 \\
\hline $\mathrm{C}$ & -4.11552 & -3.36141 & -0.03510 \\
\hline
\end{tabular}




$\begin{array}{llll}\mathrm{C} & -2.62928 & -4.54338 & 1.47675 \\ \mathrm{H} & -2.34490 & -5.46099 & 1.98381 \\ \mathrm{C} & -3.74019 & -4.53680 & 0.63067 \\ \mathrm{H} & -4.31603 & -5.44615 & 0.48792 \\ \mathrm{H} & -4.98299 & -3.35463 & -0.69009 \\ \mathrm{H} & -0.98895 & -3.39583 & 2.32097 \\ \mathrm{O} & -1.58804 & -1.04429 & 1.06462 \\ \mathrm{H} & 0.68271 & -2.09673 & 0.93677 \\ \mathrm{H} & 4.09139 & -0.71909 & 2.86409 \\ \mathrm{H} & 4.55083 & -0.99066 & 1.17912 \\ \mathrm{H} & 3.24304 & -3.03241 & 1.53998 \\ \mathrm{H} & 2.22471 & -2.22050 & 2.73737\end{array}$




\subsection{3a-(R)-r-path}

77

3a-(R)-4-r

$\begin{array}{llll}\text { C } & -1.91112 & -0.52858 & 2.28373\end{array}$

$\begin{array}{llll}\mathrm{H} & -1.94660 & -0.84517 & -0.05349\end{array}$

$\begin{array}{llll}\mathrm{O} & -1.43575 & -0.56139 & -0.83931\end{array}$

$\begin{array}{llll}\text { C } & 2.50588 & -2.69804 & -0.38680\end{array}$

$\begin{array}{llll}\text { C } & 3.92121 & -0.87741 & -0.13196\end{array}$

$\begin{array}{llll}\text { C } & 1.21992 & -1.88115 & -0.66463\end{array}$

$\begin{array}{llll}\mathrm{H} & 4.65709 & -0.42360 & 0.53219\end{array}$

$\begin{array}{llll}\mathrm{H} & 3.01628 & -2.90774 & -1.34142\end{array}$

$\begin{array}{llll}\mathrm{H} & 2.22524 & -3.64668 & 0.07248\end{array}$

$\mathrm{H} \quad 0.48435 \quad-2.08675 \quad 0.11703$

$\begin{array}{llll}\mathrm{H} & 4.40314 & -1.14261 & -1.08779\end{array}$

$\begin{array}{llll}\mathrm{O} & 3.42716 & -2.06556 & 0.49865\end{array}$

$\begin{array}{llll}\mathrm{C} & 2.77705 & 0.05812 & -0.32857\end{array}$

$\begin{array}{llll}\mathrm{N} & 2.79748 & 1.33961 & -0.14523\end{array}$

$\begin{array}{llll}\mathrm{N} & 1.45174 & 1.72576 & -0.29179\end{array}$

$\begin{array}{llll}\text { C } & 0.61227 & 0.61843 & -0.47845\end{array}$

$\begin{array}{llll}\mathrm{N} & 1.52177 & -0.44708 & -0.61134\end{array}$

$\begin{array}{llll}\text { C } & 1.13038 & 3.08768 & -0.44493\end{array}$

$\begin{array}{llll}\mathrm{C} & 1.45069 & 4.01108 & 0.56983\end{array}$

$\begin{array}{llll}\text { C } & 0.49349 & 3.54726 & -1.61459\end{array}$

$\begin{array}{llll}\mathrm{C} & 1.10713 & 5.35917 & 0.43330\end{array}$

$\begin{array}{llll}\text { C } & 0.11892 & 4.88927 & -1.73431\end{array}$

$\begin{array}{llll}\text { C } & 0.43165 & 5.77840 & -0.71048\end{array}$

$\begin{array}{llll}\mathrm{H} & 1.34316 & 6.07057 & 1.21503\end{array}$

$\begin{array}{llll}\mathrm{H} & -0.39434 & 5.24089 & -2.62058\end{array}$

$\begin{array}{llll}\mathrm{H} & 0.14878 & 6.82230 & -0.80813\end{array}$

$\begin{array}{llll}\mathrm{O} & 0.28559 & 2.60766 & -2.57943\end{array}$

$\begin{array}{llll}\mathrm{O} & 2.08044 & 3.49281 & 1.66523\end{array}$

$\begin{array}{llll}\text { C } & 2.38948 & 4.38967 & 2.73319\end{array}$

$\begin{array}{llll}\mathrm{H} & 2.87090 & 3.77526 & 3.49443\end{array}$

$\begin{array}{llll}\mathrm{H} & 3.07822 & 5.17459 & 2.39852\end{array}$

$\mathrm{H} \quad 1.47884 \quad 4.84614 \quad 3.14162$

$\begin{array}{llll}\text { C } & -0.39870 & 3.02261 & -3.76359\end{array}$

$\begin{array}{llll}\mathrm{H} & 0.17697 & 3.78599 & -4.30147\end{array}$

$\mathrm{H} \quad-0.48700 \quad 2.12402 \quad-4.37466$

$\begin{array}{llll}\mathrm{H} & -1.39546 & 3.40945 & -3.52184\end{array}$

$\begin{array}{llll}\text { C } & 0.65003 & -2.31972 & -2.02989\end{array}$

$\begin{array}{llll}\mathrm{H} & -0.20605 & -1.68523 & -2.26453\end{array}$

$\begin{array}{llll}\mathrm{H} & 1.41772 & -2.17107 & -2.79848\end{array}$

$\begin{array}{llll}\text { C } & 0.22921 & -3.77084 & -1.98621\end{array}$

$\begin{array}{llll}\text { C } & -0.76689 & -4.19070 & -1.09276\end{array}$

$\begin{array}{llll}\text { C } & 0.83823 & -4.72905 & -2.80404\end{array}$

$\begin{array}{llll}\text { C } & -1.14024 & -5.53067 & -1.02091\end{array}$

$\begin{array}{llll}\mathrm{H} & -1.25049 & -3.46506 & -0.44452\end{array}$ 


\begin{tabular}{|c|c|c|c|}
\hline $\mathrm{C}$ & 0.46318 & -6.07177 & -2.73838 \\
\hline $\mathrm{H}$ & 1.61399 & -4.41780 & -3.50029 \\
\hline $\mathrm{C}$ & -0.52720 & -6.47716 & -1.84415 \\
\hline $\mathrm{H}$ & -1.91032 & -5.83446 & -0.31769 \\
\hline $\mathrm{H}$ & 0.94680 & -6.80013 & -3.38374 \\
\hline $\mathrm{H}$ & -0.81871 & -7.52223 & -1.78801 \\
\hline $\mathrm{C}$ & -0.75080 & 0.60386 & -0.44525 \\
\hline $\mathrm{C}$ & -1.60816 & 1.74272 & -0.09571 \\
\hline $\mathrm{C}$ & -1.55363 & 2.32751 & 1.18963 \\
\hline $\mathrm{C}$ & -2.57780 & 2.19876 & -1.00389 \\
\hline $\mathrm{C}$ & -2.39176 & 3.40404 & 1.48953 \\
\hline $\mathrm{C}$ & -3.41819 & 3.26159 & -0.68804 \\
\hline $\mathrm{H}$ & -2.64956 & 1.70742 & -1.96988 \\
\hline $\mathrm{C}$ & -3.30975 & 3.88528 & 0.55682 \\
\hline $\mathrm{H}$ & -2.34155 & 3.85355 & 2.47902 \\
\hline $\mathrm{H}$ & -4.14983 & 3.61046 & -1.41213 \\
\hline $\mathrm{H}$ & -3.95463 & 4.72189 & 0.81029 \\
\hline $\mathrm{C}$ & -0.64217 & 1.74316 & 2.24677 \\
\hline $\mathrm{H}$ & -0.86896 & 2.21389 & 3.21279 \\
\hline $\mathrm{H}$ & 0.40586 & 1.97828 & 2.02841 \\
\hline $\mathrm{C}$ & -0.78318 & 0.24670 & 2.36665 \\
\hline $\mathrm{C}$ & -1.65128 & -1.93189 & 2.44020 \\
\hline $\mathrm{H}$ & -2.89607 & -0.10978 & 2.10168 \\
\hline $\mathrm{C}$ & -0.27132 & -2.19136 & 2.67461 \\
\hline $\mathrm{C}$ & 0.21891 & -3.49121 & 2.82748 \\
\hline $\mathrm{C}$ & -2.53518 & -3.02674 & 2.37401 \\
\hline $\mathrm{C}$ & -0.67822 & -4.55126 & 2.74932 \\
\hline $\mathrm{H}$ & -0.31537 & -5.56922 & 2.85398 \\
\hline $\mathrm{C}$ & -2.04656 & -4.31714 & 2.52825 \\
\hline $\mathrm{H}$ & -2.73019 & -5.15972 & 2.47462 \\
\hline $\mathrm{H}$ & -3.59452 & -2.85569 & 2.20034 \\
\hline $\mathrm{H}$ & 1.27713 & -3.66743 & 2.99557 \\
\hline $\mathrm{S}$ & 0.64130 & -0.70675 & 2.70808 \\
\hline \multicolumn{4}{|l|}{77} \\
\hline \multicolumn{4}{|c|}{$3 a-(R)-t s 1-r$} \\
\hline $\mathrm{C}$ & -2.61436 & 0.57804 & 0.57179 \\
\hline $\mathrm{H}$ & -2.09818 & -0.22293 & -0.35400 \\
\hline $\mathrm{O}$ & -1.16347 & -0.66065 & -1.14138 \\
\hline $\mathrm{C}$ & 0.92112 & -2.71320 & 2.23329 \\
\hline $\mathrm{C}$ & 2.68960 & -1.24020 & 2.56911 \\
\hline $\mathrm{C}$ & 0.36580 & -2.03064 & 0.95760 \\
\hline $\mathrm{H}$ & 3.15832 & -0.58796 & 3.30696 \\
\hline $\mathrm{H}$ & 1.66185 & -3.47746 & 1.94656 \\
\hline $\mathrm{H}$ & 0.09858 & -3.20037 & 2.75701 \\
\hline $\mathrm{H}$ & -0.66760 & -1.72554 & 1.12216 \\
\hline $\mathrm{H}$ & 3.39691 & -2.03748 & 2.28599 \\
\hline $\mathrm{O}$ & 1.52484 & -1.81515 & 3.16497 \\
\hline
\end{tabular}




\begin{tabular}{|c|c|c|c|}
\hline $\mathrm{C}$ & 2.26248 & -0.43667 & 1.38527 \\
\hline $\mathrm{N}$ & 2.76285 & 0.69073 & 0.98374 \\
\hline $\mathrm{N}$ & 1.89914 & 1.09414 & -0.04407 \\
\hline $\mathrm{C}$ & 0.84872 & 0.20838 & -0.21919 \\
\hline $\mathrm{N}$ & 1.13636 & -0.80871 & 0.68219 \\
\hline $\mathrm{C}$ & 2.25575 & 2.20755 & -0.83762 \\
\hline $\mathrm{C}$ & 2.34651 & 3.48566 & -0.25836 \\
\hline $\mathrm{C}$ & 2.56543 & 2.03297 & -2.19753 \\
\hline $\mathrm{C}$ & 2.70908 & 4.58507 & -1.04271 \\
\hline $\mathrm{C}$ & 2.90249 & 3.13711 & -2.98696 \\
\hline $\mathrm{C}$ & 2.96974 & 4.39619 & -2.39791 \\
\hline $\mathrm{H}$ & 2.77297 & 5.57585 & -0.61027 \\
\hline $\mathrm{H}$ & 3.11748 & 3.01801 & -4.04140 \\
\hline $\mathrm{H}$ & 3.23573 & 5.25301 & -3.00986 \\
\hline $\mathrm{O}$ & 2.51109 & 0.75017 & -2.65163 \\
\hline $\mathrm{O}$ & 2.04519 & 3.55755 & 1.06962 \\
\hline $\mathrm{C}$ & 2.11182 & 4.84310 & 1.69337 \\
\hline $\mathrm{H}$ & 1.82906 & 4.67201 & 2.73207 \\
\hline $\mathrm{H}$ & 3.12950 & 5.24777 & 1.64445 \\
\hline $\mathrm{H}$ & 1.40991 & 5.54440 & 1.22535 \\
\hline $\mathrm{C}$ & 2.79492 & 0.53306 & -4.03723 \\
\hline $\mathrm{H}$ & 3.82492 & 0.82584 & -4.27392 \\
\hline $\mathrm{H}$ & 2.66669 & -0.53827 & -4.19243 \\
\hline $\mathrm{H}$ & 2.09341 & 1.08928 & -4.66921 \\
\hline $\mathrm{C}$ & 0.41561 & -3.02296 & -0.22150 \\
\hline $\mathrm{H}$ & 0.09881 & -2.48681 & -1.11906 \\
\hline $\mathrm{H}$ & 1.44664 & -3.37271 & -0.35384 \\
\hline $\mathrm{C}$ & -0.50836 & -4.18789 & 0.04511 \\
\hline $\mathrm{C}$ & -1.89210 & -3.97373 & 0.11683 \\
\hline $\mathrm{C}$ & -0.01663 & -5.47869 & 0.26607 \\
\hline $\mathrm{C}$ & -2.75834 & -5.02414 & 0.40942 \\
\hline $\mathrm{H}$ & -2.28382 & -2.97603 & -0.05893 \\
\hline $\mathrm{C}$ & -0.88318 & -6.53464 & 0.55339 \\
\hline $\mathrm{H}$ & 1.05501 & -5.65824 & 0.21032 \\
\hline $\mathrm{C}$ & -2.25726 & -6.30899 & 0.62939 \\
\hline $\mathrm{H}$ & -3.82750 & -4.83905 & 0.46664 \\
\hline $\mathrm{H}$ & -0.48415 & -7.53165 & 0.71976 \\
\hline $\mathrm{H}$ & -2.93345 & -7.12832 & 0.85695 \\
\hline $\mathrm{C}$ & -0.29467 & 0.33888 & -1.01737 \\
\hline $\mathrm{C}$ & -0.56210 & 1.54518 & -1.84717 \\
\hline $\mathrm{C}$ & -1.00501 & 2.72691 & -1.23001 \\
\hline $\mathrm{C}$ & -0.52481 & 1.47502 & -3.24528 \\
\hline $\mathrm{C}$ & -1.32695 & 3.83649 & -2.01685 \\
\hline $\mathrm{C}$ & -0.84823 & 2.58270 & -4.02377 \\
\hline $\mathrm{H}$ & -0.24159 & 0.53570 & -3.71065 \\
\hline $\mathrm{C}$ & -1.23503 & 3.77420 & -3.40609 \\
\hline $\mathrm{H}$ & -1.67176 & 4.75022 & -1.53765 \\
\hline $\mathrm{H}$ & -0.80669 & 2.51878 & -5.10804 \\
\hline
\end{tabular}




$\begin{array}{llll}\mathrm{H} & -1.49096 & 4.64305 & -4.00626 \\ \mathrm{C} & -1.13844 & 2.75488 & 0.27665 \\ \mathrm{H} & -1.90062 & 3.49733 & 0.55959 \\ \mathrm{H} & -0.19663 & 3.09619 & 0.72321 \\ \mathrm{C} & -1.47274 & 1.40779 & 0.86427 \\ \mathrm{C} & -2.92110 & -0.30836 & 1.70320 \\ \mathrm{H} & -3.43617 & 1.01706 & 0.00206 \\ \mathrm{C} & -1.97153 & -0.22163 & 2.74676 \\ \mathrm{C} & -2.00816 & -1.07507 & 3.85215 \\ \mathrm{C} & -3.94480 & -1.25799 & 1.81129 \\ \mathrm{C} & -3.03598 & -2.01241 & 3.93283 \\ \mathrm{H} & -3.08513 & -2.68202 & 4.78653 \\ \mathrm{C} & -4.00323 & -2.09614 & 2.92253 \\ \mathrm{H} & -4.79802 & -2.83245 & 2.99983 \\ \mathrm{H} & -4.68353 & -1.34637 & 1.01847 \\ \mathrm{H} & -1.24971 & -1.01084 & 4.62612 \\ \mathrm{~S} & -0.79234 & 1.03605 & 2.43727\end{array}$

77

3a-(R)-5-r

$\begin{array}{llll}\mathrm{C} & -1.79705 & -0.73548 & 1.98692 \\ \mathrm{H} & -2.34149 & -0.89142 & 1.03689 \\ \mathrm{O} & -1.50393 & -0.58370 & -0.81178 \\ \mathrm{C} & 2.27993 & -2.83115 & -0.32444 \\ \mathrm{C} & 3.79307 & -1.05483 & -0.30019 \\ \mathrm{C} & 1.03041 & -1.96574 & -0.65327 \\ \mathrm{H} & 4.60066 & -0.60342 & 0.27726 \\ \mathrm{H} & 2.73098 & -3.18686 & -1.26413 \\ \mathrm{H} & 1.96527 & -3.69103 & 0.26596 \\ \mathrm{H} & 0.24862 & -2.12497 & 0.08837 \\ \mathrm{H} & 4.18403 & -1.39318 & -1.27391 \\ \mathrm{O} & 3.28682 & -2.16509 & 0.44081 \\ \mathrm{C} & 2.67897 & -0.07534 & -0.45884 \\ \mathrm{~N} & 2.73467 & 1.22058 & -0.35311 \\ \mathrm{~N} & 1.39607 & 1.60817 & -0.38098 \\ \mathrm{C} & 0.54928 & 0.54598 & -0.47891 \\ \mathrm{~N} & 1.38572 & -0.53470 & -0.57195 \\ \mathrm{C} & 1.08792 & 2.99462 & -0.36716 \\ \mathrm{C} & 1.36888 & 3.75749 & 0.77771 \\ \mathrm{C} & 0.55623 & 3.60017 & -1.51598 \\ \mathrm{C} & 1.06081 & 5.12158 & 0.79145 \\ \mathrm{C} & 0.23236 & 4.96078 & -1.49290 \\ \mathrm{C} & 0.48605 & 5.69705 & -0.33945 \\ \mathrm{H} & 1.25576 & 5.72353 & 1.67023 \\ \mathrm{H} & -0.20997 & 5.43831 & -2.35771 \\ \mathrm{H} & 0.23257 & 6.75293 & -0.32241 \\ \mathrm{O} & 0.39755 & 2.78442 & -2.59162 \\ \mathrm{O} & 1.91997 & 3.07895 & 1.82003\end{array}$




\begin{tabular}{|c|c|c|c|}
\hline $\mathrm{C}$ & 2.18941 & 3.80918 & 3.02154 \\
\hline $\mathrm{H}$ & 2.60012 & 3.07483 & 3.71380 \\
\hline $\mathrm{H}$ & 2.92019 & 4.60509 & 2.83707 \\
\hline $\mathrm{H}$ & 1.26771 & 4.23668 & 3.43478 \\
\hline $\mathrm{C}$ & -0.17182 & 3.36140 & -3.77325 \\
\hline $\mathrm{H}$ & 0.47353 & 4.15683 & -4.16426 \\
\hline $\mathrm{H}$ & -0.23284 & 2.54321 & -4.49065 \\
\hline $\mathrm{H}$ & -1.17295 & 3.75367 & -3.56523 \\
\hline $\mathrm{C}$ & 0.48293 & -2.34162 & -2.04441 \\
\hline $\mathrm{H}$ & -0.29329 & -1.61359 & -2.29173 \\
\hline $\mathrm{H}$ & 1.29384 & -2.29151 & -2.78165 \\
\hline $\mathrm{C}$ & -0.11045 & -3.72978 & -1.99379 \\
\hline $\mathrm{C}$ & -1.30051 & -3.93556 & -1.28118 \\
\hline $\mathrm{C}$ & 0.51224 & -4.82470 & -2.60109 \\
\hline $\mathrm{C}$ & -1.84752 & -5.21192 & -1.17636 \\
\hline $\mathrm{H}$ & -1.78561 & -3.07900 & -0.81995 \\
\hline $\mathrm{C}$ & -0.03697 & -6.10431 & -2.49880 \\
\hline $\mathrm{H}$ & 1.43249 & -4.67462 & -3.16211 \\
\hline $\mathrm{C}$ & -1.21714 & -6.30120 & -1.78255 \\
\hline $\mathrm{H}$ & -2.77026 & -5.35853 & -0.62119 \\
\hline $\mathrm{H}$ & 0.45704 & -6.94524 & -2.97816 \\
\hline $\mathrm{H}$ & -1.64543 & -7.29633 & -1.69994 \\
\hline $\mathrm{C}$ & -0.91529 & 0.45647 & -0.33964 \\
\hline $\mathrm{C}$ & -1.72167 & 1.73226 & -0.31237 \\
\hline $\mathrm{C}$ & -1.89511 & 2.40606 & 0.89853 \\
\hline $\mathrm{C}$ & -2.41581 & 2.15360 & -1.45087 \\
\hline $\mathrm{C}$ & -2.70206 & 3.54624 & 0.94974 \\
\hline $\mathrm{C}$ & -3.21687 & 3.29103 & -1.39953 \\
\hline $\mathrm{H}$ & -2.32667 & 1.56985 & -2.36223 \\
\hline $\mathrm{C}$ & -3.34870 & 3.99738 & -0.19916 \\
\hline $\mathrm{H}$ & -2.84035 & 4.06958 & 1.89338 \\
\hline $\mathrm{H}$ & -3.75405 & 3.62142 & -2.28520 \\
\hline $\mathrm{H}$ & -3.97930 & 4.88136 & -0.15473 \\
\hline $\mathrm{C}$ & -1.19670 & 1.80641 & 2.09549 \\
\hline $\mathrm{H}$ & -1.85046 & 1.84719 & 2.98105 \\
\hline $\mathrm{H}$ & -0.30763 & 2.39578 & 2.34237 \\
\hline $\mathrm{C}$ & -0.78617 & 0.37747 & 1.78116 \\
\hline $\mathrm{C}$ & -1.05450 & -1.98869 & 2.36360 \\
\hline $\mathrm{H}$ & -2.53388 & -0.46482 & 2.76002 \\
\hline $\mathrm{C}$ & 0.29846 & -1.78267 & 2.68351 \\
\hline $\mathrm{C}$ & 1.13531 & -2.84592 & 3.02934 \\
\hline $\mathrm{C}$ & -1.56434 & -3.28461 & 2.38750 \\
\hline $\mathrm{C}$ & 0.60247 & -4.13606 & 3.05370 \\
\hline $\mathrm{H}$ & 1.24397 & -4.97380 & 3.31329 \\
\hline $\mathrm{C}$ & -0.73851 & -4.35845 & 2.73185 \\
\hline $\mathrm{H}$ & -1.13701 & -5.36857 & 2.73460 \\
\hline $\mathrm{H}$ & -2.60410 & -3.45956 & 2.12221 \\
\hline $\mathrm{H}$ & 2.18442 & -2.67537 & 3.24870 \\
\hline
\end{tabular}




\begin{tabular}{|c|c|c|c|}
\hline$S$ & 0.76969 & -0.09418 & 2.55591 \\
\hline \multicolumn{4}{|c|}{77} \\
\hline \multicolumn{4}{|c|}{$3 a-(R)-t s 2-r$} \\
\hline $\mathrm{C}$ & -1.82226 & -0.71738 & 2.01588 \\
\hline $\mathrm{H}$ & -2.39558 & -0.87309 & 1.08535 \\
\hline $\mathrm{O}$ & -1.49000 & -0.60596 & -0.76475 \\
\hline $\mathrm{C}$ & 2.29487 & -2.83706 & -0.33992 \\
\hline $\mathrm{C}$ & 3.80570 & -1.05855 & -0.33010 \\
\hline $\mathrm{C}$ & 1.03990 & -1.97318 & -0.65146 \\
\hline $\mathrm{H}$ & 4.61975 & -0.60706 & 0.23824 \\
\hline H & 2.73541 & -3.19019 & -1.28546 \\
\hline $\mathrm{H}$ & 1.98890 & -3.69849 & 0.25274 \\
\hline H & 0.26480 & -2.13686 & 0.09568 \\
\hline $\mathrm{H}$ & 4.18504 & -1.39373 & -1.30944 \\
\hline $\mathrm{O}$ & 3.30975 & -2.17041 & 0.41481 \\
\hline $\mathrm{C}$ & 2.68796 & -0.08051 & -0.47239 \\
\hline $\mathrm{N}$ & 2.74218 & 1.21645 & -0.36777 \\
\hline $\mathrm{N}$ & 1.40374 & 1.59941 & -0.37476 \\
\hline $\mathrm{C}$ & 0.55958 & 0.53812 & -0.46213 \\
\hline $\mathrm{N}$ & 1.39368 & -0.54047 & -0.56564 \\
\hline $\mathrm{C}$ & 1.09154 & 2.98614 & -0.34666 \\
\hline $\mathrm{C}$ & 1.35954 & 3.73452 & 0.81035 \\
\hline $\mathrm{C}$ & 0.57119 & 3.60328 & -1.49419 \\
\hline $\mathrm{C}$ & 1.04527 & 5.09701 & 0.83845 \\
\hline $\mathrm{C}$ & 0.24236 & 4.96244 & -1.45719 \\
\hline $\mathrm{C}$ & 0.48014 & 5.68425 & -0.29131 \\
\hline $\mathrm{H}$ & 1.22924 & 5.68863 & 1.72656 \\
\hline $\mathrm{H}$ & -0.19294 & 5.44934 & -2.32029 \\
\hline $\mathrm{H}$ & 0.22186 & 6.73874 & -0.26340 \\
\hline $\mathrm{O}$ & 0.42845 & 2.80060 & -2.58126 \\
\hline $\mathrm{O}$ & 1.90535 & 3.04454 & 1.84735 \\
\hline $\mathrm{C}$ & 2.15373 & 3.75703 & 3.06418 \\
\hline $\mathrm{H}$ & 2.55648 & 3.01332 & 3.75101 \\
\hline $\mathrm{H}$ & 2.88390 & 4.55851 & 2.90295 \\
\hline $\mathrm{H}$ & 1.22402 & 4.17429 & 3.46977 \\
\hline $\mathrm{C}$ & -0.13321 & 3.38901 & -3.76122 \\
\hline $\mathrm{H}$ & 0.51172 & 4.19225 & -4.13656 \\
\hline $\mathrm{H}$ & -0.18341 & 2.57923 & -4.48889 \\
\hline $\mathrm{H}$ & -1.13817 & 3.77333 & -3.55721 \\
\hline $\mathrm{C}$ & 0.47971 & -2.33880 & -2.04011 \\
\hline $\mathrm{H}$ & -0.29905 & -1.60844 & -2.27286 \\
\hline $\mathrm{H}$ & 1.28352 & -2.28409 & -2.78486 \\
\hline $\mathrm{C}$ & -0.11447 & -3.72667 & -1.99310 \\
\hline $\mathrm{C}$ & -1.30036 & -3.93399 & -1.27393 \\
\hline $\mathrm{C}$ & 0.50311 & -4.81919 & -2.60984 \\
\hline $\mathrm{C}$ & -1.84834 & -5.21020 & -1.17233 \\
\hline $\mathrm{H}$ & -1.78001 & -3.07828 & -0.80527 \\
\hline
\end{tabular}




$\begin{array}{lrrr}\mathrm{C} & -0.04732 & -6.09854 & -2.51066 \\ \mathrm{H} & 1.41988 & -4.66749 & -3.17616 \\ \mathrm{C} & -1.22324 & -6.29729 & -1.78795 \\ \mathrm{H} & -2.76777 & -5.35850 & -0.61211 \\ \mathrm{H} & 0.44248 & -6.93778 & -2.99727 \\ \mathrm{H} & -1.65252 & -7.29222 & -1.70789 \\ \mathrm{C} & -0.91042 & 0.44092 & -0.28450 \\ \mathrm{C} & -1.71531 & 1.72169 & -0.31587 \\ \mathrm{C} & -1.91399 & 2.41763 & 0.87772 \\ \mathrm{C} & -2.38082 & 2.12196 & -1.47795 \\ \mathrm{C} & -2.71842 & 3.56039 & 0.89201 \\ \mathrm{C} & -3.18046 & 3.26186 & -1.46419 \\ \mathrm{H} & -2.27311 & 1.52084 & -2.37598 \\ \mathrm{C} & -3.33791 & 3.99045 & -0.27995 \\ \mathrm{H} & -2.87650 & 4.10181 & 1.82223 \\ \mathrm{H} & -3.69817 & 3.57707 & -2.36692 \\ \mathrm{H} & -3.96790 & 4.87591 & -0.26656 \\ \mathrm{C} & -1.24003 & 1.82586 & 2.09235 \\ \mathrm{H} & -1.91235 & 1.85888 & 2.96341 \\ \mathrm{H} & -0.36041 & 2.42047 & 2.35957 \\ \mathrm{C} & -0.81744 & 0.39771 & 1.77567 \\ \mathrm{C} & -1.06573 & -1.96891 & 2.36990 \\ \mathrm{H} & -2.52838 & -0.44607 & 2.81661 \\ \mathrm{C} & 0.28719 & -1.75364 & 2.68352 \\ \mathrm{C} & 1.13491 & -2.81257 & 3.01514 \\ \mathrm{C} & -1.56358 & -3.26930 & 2.38228 \\ \mathrm{C} & 0.61405 & -4.10810 & 3.02852 \\ \mathrm{H} & 1.26473 & -4.94255 & 3.27563 \\ \mathrm{C} & -0.72600 & -4.33952 & 2.71031 \\ \mathrm{H} & -1.11492 & -5.35333 & 2.70277 \\ \mathrm{H} & -2.60302 & -3.45087 & 2.12029 \\ \mathrm{H} & 2.18381 & -2.63510 & 3.23021 \\ \mathrm{~S} & 0.74408 & -0.05955 & 2.56681\end{array}$

77

3a-(R)-6-r

$\begin{array}{llll}\mathrm{C} & -2.66028 & -0.57460 & 1.14805 \\ \mathrm{H} & -3.01208 & -0.65583 & 0.11537 \\ \mathrm{O} & -0.98093 & -0.72995 & -0.99022 \\ \mathrm{C} & 2.43646 & -2.64938 & 0.68287 \\ \mathrm{C} & 4.04501 & -0.92923 & 0.43583 \\ \mathrm{C} & 1.33494 & -1.96652 & -0.12172 \\ \mathrm{H} & 4.34385 & -0.80257 & 1.48943 \\ \mathrm{H} & 2.35381 & -3.73120 & 0.56990 \\ \mathrm{H} & 2.36645 & -2.38516 & 1.74775 \\ \mathrm{H} & 0.35484 & -2.13110 & 0.32213 \\ \mathrm{H} & 4.89270 & -0.65925 & -0.19849 \\ \mathrm{O} & 3.73251 & -2.28858 & 0.16530\end{array}$




\begin{tabular}{|c|c|c|c|}
\hline $\mathrm{C}$ & 2.87846 & -0.02059 & 0.17129 \\
\hline $\mathrm{N}$ & 2.88889 & 1.28713 & 0.21383 \\
\hline $\mathrm{N}$ & 1.55123 & 1.62183 & 0.05161 \\
\hline $\mathrm{C}$ & 0.75502 & 0.54231 & -0.07764 \\
\hline $\mathrm{N}$ & 1.61239 & -0.51128 & -0.02674 \\
\hline $\mathrm{C}$ & 1.18651 & 3.00008 & 0.07994 \\
\hline $\mathrm{C}$ & 0.98688 & 3.62884 & 1.31658 \\
\hline $\mathrm{C}$ & 1.10270 & 3.71894 & -1.12068 \\
\hline $\mathrm{C}$ & 0.61487 & 4.97642 & 1.34981 \\
\hline $\mathrm{C}$ & 0.72302 & 5.06549 & -1.08487 \\
\hline $\mathrm{C}$ & 0.47828 & 5.66630 & 0.14641 \\
\hline $\mathrm{H}$ & 0.43551 & 5.47953 & 2.29176 \\
\hline $\mathrm{H}$ & 0.61358 & 5.63320 & -1.99986 \\
\hline $\mathrm{H}$ & 0.17780 & 6.70968 & 0.17075 \\
\hline $\mathrm{O}$ & 1.40945 & 3.02508 & -2.24416 \\
\hline $\mathrm{O}$ & 1.16746 & 2.84063 & 2.41127 \\
\hline $\mathrm{C}$ & 0.91370 & 3.42134 & 3.69651 \\
\hline $\mathrm{H}$ & 1.07798 & 2.61496 & 4.41029 \\
\hline $\mathrm{H}$ & 1.60558 & 4.24878 & 3.89111 \\
\hline $\mathrm{H}$ & -0.12215 & 3.77394 & 3.76662 \\
\hline $\mathrm{C}$ & 1.28945 & 3.71443 & -3.49562 \\
\hline $\mathrm{H}$ & 1.98450 & 4.56107 & -3.53711 \\
\hline $\mathrm{H}$ & 1.55223 & 2.97752 & -4.25412 \\
\hline $\mathrm{H}$ & 0.26042 & 4.05738 & -3.64538 \\
\hline $\mathrm{C}$ & 1.31344 & -2.42181 & -1.59429 \\
\hline $\mathrm{H}$ & 0.69802 & -1.69584 & -2.13101 \\
\hline $\mathrm{H}$ & 2.33526 & -2.42336 & -1.98853 \\
\hline $\mathrm{C}$ & 0.69435 & -3.79397 & -1.69348 \\
\hline $\mathrm{C}$ & -0.68673 & -3.93611 & -1.49726 \\
\hline $\mathrm{C}$ & 1.46770 & -4.93394 & -1.93752 \\
\hline $\mathrm{C}$ & -1.27613 & -5.19711 & -1.54853 \\
\hline $\mathrm{H}$ & -1.27527 & -3.04255 & -1.30064 \\
\hline $\mathrm{C}$ & 0.87565 & -6.19740 & -1.99048 \\
\hline $\mathrm{H}$ & 2.54015 & -4.82996 & -2.09014 \\
\hline $\mathrm{C}$ & -0.49889 & -6.33130 & -1.79503 \\
\hline $\mathrm{H}$ & -2.34745 & -5.29682 & -1.39447 \\
\hline $\mathrm{H}$ & 1.48733 & -7.07455 & -2.18481 \\
\hline $\mathrm{H}$ & -0.96212 & -7.31351 & -1.83635 \\
\hline $\mathrm{C}$ & -0.77466 & 0.37753 & -0.29202 \\
\hline $\mathrm{C}$ & -1.40118 & 1.63929 & -0.90869 \\
\hline $\mathrm{C}$ & -2.06884 & 2.44239 & 0.01430 \\
\hline $\mathrm{C}$ & -1.46988 & 1.90741 & -2.27215 \\
\hline $\mathrm{C}$ & -2.77276 & 3.56888 & -0.41191 \\
\hline $\mathrm{C}$ & -2.17111 & 3.03442 & -2.70267 \\
\hline $\mathrm{H}$ & -1.00134 & 1.22287 & -2.97348 \\
\hline $\mathrm{C}$ & -2.80941 & 3.86752 & -1.77513 \\
\hline $\mathrm{H}$ & -3.30184 & 4.19702 & 0.30129 \\
\hline $\mathrm{H}$ & -2.24597 & 3.25772 & -3.76442 \\
\hline
\end{tabular}




$\begin{array}{llll}\mathrm{H} & -3.36190 & 4.73673 & -2.12246 \\ \mathrm{C} & -1.94308 & 1.89135 & 1.41253 \\ \mathrm{H} & -2.88128 & 1.94816 & 1.97688 \\ \mathrm{H} & -1.19492 & 2.44894 & 1.98483 \\ \mathrm{C} & -1.49441 & 0.43010 & 1.20189 \\ \mathrm{C} & -2.16432 & -1.89881 & 1.66132 \\ \mathrm{H} & -3.48038 & -0.21626 & 1.78691 \\ \mathrm{C} & -1.02131 & -1.79980 & 2.47049 \\ \mathrm{C} & -0.45420 & -2.92623 & 3.06736 \\ \mathrm{C} & -2.72805 & -3.15029 & 1.43813 \\ \mathrm{C} & -1.03493 & -4.17564 & 2.83239 \\ \mathrm{H} & -0.59421 & -5.06289 & 3.27830 \\ \mathrm{C} & -2.16134 & -4.29018 & 2.01816 \\ \mathrm{H} & -2.59115 & -5.26778 & 1.82244 \\ \mathrm{H} & -3.60165 & -3.24106 & 0.79752 \\ \mathrm{H} & 0.42899 & -2.83800 & 3.69378 \\ \mathrm{~S} & -0.42123 & -0.14477 & 2.59913\end{array}$

77

3a-(R)-ts3-r

$\begin{array}{llll}\mathrm{C} & -1.04218 & 0.28629 & -0.27367\end{array}$

$\begin{array}{llll}\text { C } & 0.87775 & 0.54274 & -0.00289\end{array}$

$\begin{array}{llll}\text { C } & -2.94868 & -0.30505 & 1.26536\end{array}$

$\mathrm{H} \quad-3.47124 \quad-0.18762 \quad 0.31176$

$\begin{array}{llll}\mathrm{O} & -1.17994 & -0.79381 & -0.90805\end{array}$

$\begin{array}{llll}\mathrm{C} & 2.46565 & -2.72388 & 0.60030\end{array}$

$\begin{array}{llll}\text { C } & 4.14178 & -1.09246 & 0.20613\end{array}$

$\begin{array}{llll}\text { C } & 1.34215 & -1.97719 & -0.11389\end{array}$

$\mathrm{H} \quad 4.55352 \quad-0.98542 \quad 1.22296$

$\begin{array}{llll}\mathrm{H} & 2.31752 & -3.80000 & 0.49917\end{array}$

$\mathrm{H} \quad 2.49796 \quad-2.45419 \quad 1.66652$

$\begin{array}{llll}\mathrm{H} & 0.39647 & -2.09619 & 0.41435\end{array}$

$\mathrm{H} \quad 4.93601 \quad-0.87420 \quad-0.51197$

$\begin{array}{llll}\mathrm{O} & 3.73001 & -2.43538 & -0.02436\end{array}$

$\begin{array}{llll}\mathrm{C} & 3.00528 & -0.12254 & 0.05396\end{array}$

$\begin{array}{llll}\mathrm{N} & 3.08427 & 1.18021 & 0.11840\end{array}$

$\begin{array}{llll}\mathrm{N} & 1.74429 & 1.56861 & 0.07244\end{array}$

$\begin{array}{llll}\mathrm{N} & 1.69931 & -0.54393 & -0.04204\end{array}$

$\begin{array}{llll}\mathrm{C} & 1.44312 & 2.96024 & 0.09923\end{array}$

$\begin{array}{llll}\mathrm{C} & 1.33053 & 3.62214 & 1.32899\end{array}$

$\begin{array}{llll}\text { C } & 1.32565 & 3.66099 & -1.10943\end{array}$

$\begin{array}{lllr}\text { C } & 1.03688 & 4.98972 & 1.35290\end{array}$

$\begin{array}{llll}\text { C } & 1.03428 & 5.03015 & -1.08437\end{array}$

$\begin{array}{llll}\mathrm{C} & 0.88975 & 5.66769 & 0.14390\end{array}$

$\begin{array}{llll}\mathrm{H} & 0.93027 & 5.51895 & 2.29161\end{array}$

$\mathrm{H} \quad 0.91296 \quad 5.58670 \quad-2.00479$

$\begin{array}{llll}\mathrm{H} & 0.65692 & 6.72845 & 0.16155\end{array}$

$\begin{array}{llll}\mathrm{O} & 1.51847 & 2.92782 & -2.23461\end{array}$ 


\begin{tabular}{|c|c|c|c|}
\hline $\mathrm{O}$ & 1.51043 & 2.84480 & 2.43425 \\
\hline $\mathrm{C}$ & 1.36191 & 3.47213 & 3.71342 \\
\hline $\mathrm{H}$ & 1.52390 & 2.67792 & 4.44166 \\
\hline $\mathrm{H}$ & 2.10754 & 4.26443 & 3.84688 \\
\hline $\mathrm{H}$ & 0.35273 & 3.88477 & 3.83068 \\
\hline $\mathrm{C}$ & 1.40285 & 3.60803 & -3.49000 \\
\hline $\mathrm{H}$ & 2.15690 & 4.39980 & -3.57134 \\
\hline $\mathrm{H}$ & 1.58045 & 2.84408 & -4.24665 \\
\hline $\mathrm{H}$ & 0.39787 & 4.02634 & -3.60912 \\
\hline $\mathrm{C}$ & 1.17725 & -2.43643 & -1.57576 \\
\hline $\mathrm{H}$ & 0.49706 & -1.73024 & -2.05770 \\
\hline $\mathrm{H}$ & 2.15244 & -2.41111 & -2.07325 \\
\hline $\mathrm{C}$ & 0.59701 & -3.82953 & -1.61610 \\
\hline $\mathrm{C}$ & -0.72744 & -4.04331 & -1.20773 \\
\hline $\mathrm{C}$ & 1.36828 & -4.92610 & -2.01707 \\
\hline $\mathrm{C}$ & -1.26367 & -5.32926 & -1.20846 \\
\hline $\mathrm{H}$ & -1.32501 & -3.19049 & -0.89517 \\
\hline $\mathrm{C}$ & 0.82985 & -6.21416 & -2.01964 \\
\hline $\mathrm{H}$ & 2.39862 & -4.76753 & -2.32872 \\
\hline $\mathrm{C}$ & -0.48912 & -6.41831 & -1.61448 \\
\hline $\mathrm{H}$ & -2.29039 & -5.48041 & -0.88767 \\
\hline $\mathrm{H}$ & 1.44015 & -7.05553 & -2.33730 \\
\hline $\mathrm{H}$ & -0.91115 & -7.41977 & -1.61534 \\
\hline $\mathrm{C}$ & -1.45340 & 1.61671 & -0.87462 \\
\hline $\mathrm{C}$ & -1.83133 & 2.52677 & 0.11458 \\
\hline $\mathrm{C}$ & -1.57414 & 1.92656 & -2.22437 \\
\hline $\mathrm{C}$ & -2.31160 & 3.78603 & -0.23743 \\
\hline $\mathrm{C}$ & -2.05967 & 3.18658 & -2.57993 \\
\hline $\mathrm{H}$ & -1.30891 & 1.18441 & -2.97245 \\
\hline $\mathrm{C}$ & -2.41799 & 4.11160 & -1.59199 \\
\hline $\mathrm{H}$ & -2.61026 & 4.50257 & 0.52401 \\
\hline $\mathrm{H}$ & -2.17894 & 3.44893 & -3.62844 \\
\hline $\mathrm{H}$ & -2.79926 & 5.08709 & -1.88240 \\
\hline $\mathrm{C}$ & -1.66689 & 1.92625 & 1.49206 \\
\hline $\mathrm{H}$ & -2.49104 & 2.17556 & 2.16948 \\
\hline $\mathrm{H}$ & -0.74264 & 2.28066 & 1.95969 \\
\hline $\mathrm{C}$ & -1.58001 & 0.41378 & 1.22026 \\
\hline $\mathrm{C}$ & -2.69108 & -1.74964 & 1.60046 \\
\hline $\mathrm{H}$ & -3.56580 & 0.14786 & 2.05527 \\
\hline $\mathrm{C}$ & -1.47984 & -1.95366 & 2.28038 \\
\hline $\mathrm{C}$ & -1.09618 & -3.22232 & 2.71403 \\
\hline $\mathrm{C}$ & -3.52094 & -2.83649 & 1.35089 \\
\hline $\mathrm{C}$ & -1.94399 & -4.30351 & 2.45908 \\
\hline $\mathrm{H}$ & -1.65014 & -5.29958 & 2.77744 \\
\hline $\mathrm{C}$ & -3.14815 & -4.11507 & 1.78147 \\
\hline $\mathrm{H}$ & -3.79382 & -4.96419 & 1.57744 \\
\hline $\mathrm{H}$ & -4.45502 & -2.69200 & 0.81380 \\
\hline $\mathrm{H}$ & -0.15149 & -3.37229 & 3.22882 \\
\hline
\end{tabular}




\begin{tabular}{|c|c|c|c|}
\hline S & -0.55470 & -0.46157 & 2.47762 \\
\hline \multicolumn{4}{|c|}{77} \\
\hline \multicolumn{4}{|c|}{$3 a-(R)-[2 r+3 a]$} \\
\hline $\mathrm{C}$ & -1.40351 & -0.14575 & -0.98081 \\
\hline $\mathrm{C}$ & 0.99073 & 0.69350 & 0.47565 \\
\hline $\mathrm{C}$ & -3.62892 & -0.64371 & 0.04440 \\
\hline $\mathrm{H}$ & -3.93797 & -0.41755 & -0.98195 \\
\hline $\mathrm{O}$ & -0.99926 & -1.17461 & -1.49529 \\
\hline $\mathrm{C}$ & 2.91880 & -2.28052 & 1.59403 \\
\hline $\mathrm{C}$ & 4.31690 & -0.37635 & 1.65146 \\
\hline $\mathrm{C}$ & 1.85497 & -1.69080 & 0.66900 \\
\hline $\mathrm{H}$ & 4.40217 & -0.28970 & 2.74743 \\
\hline $\mathrm{H}$ & 2.98658 & -3.35997 & 1.44975 \\
\hline $\mathrm{H}$ & 2.67709 & -2.06780 & 2.64697 \\
\hline $\mathrm{H}$ & 0.85451 & -1.95883 & 1.02028 \\
\hline $\mathrm{H}$ & 5.23331 & 0.01128 & 1.20090 \\
\hline $\mathrm{O}$ & 4.21629 & -1.74932 & 1.27477 \\
\hline $\mathrm{C}$ & 3.12236 & 0.40119 & 1.18513 \\
\hline $\mathrm{N}$ & 2.98261 & 1.69796 & 1.17103 \\
\hline $\mathrm{N}$ & 1.65768 & 1.84148 & 0.72854 \\
\hline $\mathrm{N}$ & 1.96403 & -0.22864 & 0.77916 \\
\hline $\mathrm{C}$ & 1.14879 & 3.15990 & 0.56963 \\
\hline $\mathrm{C}$ & 0.42320 & 3.76164 & 1.60704 \\
\hline $\mathrm{C}$ & 1.41335 & 3.86612 & -0.61270 \\
\hline $\mathrm{C}$ & -0.04741 & 5.07292 & 1.46139 \\
\hline $\mathrm{C}$ & 0.95623 & 5.18290 & -0.75408 \\
\hline $\mathrm{C}$ & 0.23485 & 5.76362 & 0.28474 \\
\hline $\mathrm{H}$ & -0.61107 & 5.55247 & 2.25213 \\
\hline $\mathrm{H}$ & 1.15059 & 5.74245 & -1.66045 \\
\hline $\mathrm{H}$ & -0.12301 & 6.78329 & 0.17342 \\
\hline $\mathrm{O}$ & 2.11603 & 3.18839 & -1.55751 \\
\hline $\mathrm{O}$ & 0.22337 & 2.98960 & 2.71321 \\
\hline $\mathrm{C}$ & -0.52611 & 3.56247 & 3.78891 \\
\hline $\mathrm{H}$ & -0.57336 & 2.78525 & 4.55155 \\
\hline $\mathrm{H}$ & -0.02151 & 4.45014 & 4.18845 \\
\hline $\mathrm{H}$ & -1.53990 & 3.82601 & 3.46328 \\
\hline $\mathrm{C}$ & 2.39396 & 3.86929 & -2.78420 \\
\hline $\mathrm{H}$ & 3.01283 & 4.75724 & -2.60690 \\
\hline $\mathrm{H}$ & 2.94167 & 3.15053 & -3.39366 \\
\hline $\mathrm{H}$ & 1.46392 & 4.15436 & -3.28910 \\
\hline $\mathrm{C}$ & 2.01827 & -2.15115 & -0.79440 \\
\hline $\mathrm{H}$ & 1.31766 & -1.57148 & -1.40159 \\
\hline $\mathrm{H}$ & 3.03914 & -1.92896 & -1.12327 \\
\hline $\mathrm{C}$ & 1.72687 & -3.62722 & -0.92173 \\
\hline $\mathrm{C}$ & 0.40519 & -4.09068 & -0.85258 \\
\hline $\mathrm{C}$ & 2.76165 & -4.56041 & -1.05688 \\
\hline $\mathrm{C}$ & 0.13007 & -5.45565 & -0.91404 \\
\hline
\end{tabular}




$\begin{array}{lrrr}\mathrm{H} & -0.40455 & -3.37171 & -0.76691 \\ \mathrm{C} & 2.48712 & -5.92754 & -1.12244 \\ \mathrm{H} & 3.79051 & -4.21049 & -1.11021 \\ \mathrm{C} & 1.16922 & -6.37899 & -1.04811 \\ \mathrm{H} & -0.90076 & -5.79537 & -0.86046 \\ \mathrm{H} & 3.30215 & -6.63823 & -1.23163 \\ \mathrm{H} & 0.95324 & -7.44281 & -1.09916 \\ \mathrm{C} & -1.43582 & 1.20904 & -1.57143 \\ \mathrm{C} & -1.97841 & 2.11645 & -0.65336 \\ \mathrm{C} & -1.06525 & 1.60068 & -2.85815 \\ \mathrm{C} & -2.17143 & 3.44443 & -1.02139 \\ \mathrm{C} & -1.25873 & 2.93028 & -3.22304 \\ \mathrm{H} & -0.64739 & 0.87319 & -3.54810 \\ \mathrm{C} & -1.80924 & 3.84153 & -2.31030 \\ \mathrm{H} & -2.58117 & 4.16600 & -0.32058 \\ \mathrm{H} & -0.99407 & 3.26589 & -4.22222 \\ \mathrm{H} & -1.95351 & 4.87583 & -2.61035 \\ \mathrm{C} & -2.24245 & 1.44774 & 0.67348 \\ \mathrm{H} & -3.18504 & 1.75743 & 1.13690 \\ \mathrm{H} & -1.42246 & 1.67984 & 1.36232 \\ \mathrm{C} & -2.21977 & -0.05423 & 0.33850 \\ \mathrm{C} & -3.56639 & -2.12256 & 0.32128 \\ \mathrm{H} & -4.35439 & -0.17084 & 0.72177 \\ \mathrm{C} & -2.54822 & -2.47102 & 1.22194 \\ \mathrm{C} & -2.37938 & -3.78892 & 1.64437 \\ \mathrm{C} & -4.41058 & -3.10951 & -0.17390 \\ \mathrm{C} & -3.24160 & -4.76880 & 1.14605 \\ \mathrm{H} & -3.11118 & -5.80124 & 1.45827 \\ \mathrm{C} & -4.24772 & -4.43727 & 0.23796 \\ \mathrm{H} & -4.90182 & -5.20999 & -0.15476 \\ \mathrm{H} & -5.19277 & -2.85027 & -0.88331 \\ \mathrm{H} & -1.57823 & -4.05600 & 2.32669 \\ \mathrm{~S} & -1.54357 & -1.09747 & 1.68918\end{array}$




\subsection{3d-(R)-r-path}

76

3d-(R)-4-r

$\begin{array}{llll}\mathrm{C} & 1.47156 & 0.46127 & 2.55288\end{array}$

$\begin{array}{llll}\mathrm{H} & 1.97089 & 0.81482 & 0.24589\end{array}$

$\begin{array}{llll}\mathrm{O} & 1.63015 & 0.50019 & -0.61647\end{array}$

$\begin{array}{llll}\text { C } & -2.52050 & 2.38678 & -0.82626\end{array}$

$\begin{array}{llll}\text { C } & -3.38768 & 1.23761 & -1.41353\end{array}$

$\begin{array}{llll}\text { C } & -1.04189 & 1.91969 & -0.94271\end{array}$

$\begin{array}{llll}\mathrm{H} & -0.47864 & 2.18891 & -0.04723\end{array}$

$\begin{array}{llll}\text { C } & -2.51034 & 0.05435 & -1.16209\end{array}$

$\begin{array}{llll}\mathrm{N} & -2.68313 & -1.22030 & -1.01404\end{array}$

$\begin{array}{llll}\mathrm{N} & -1.37676 & -1.68406 & -0.69614\end{array}$

$\begin{array}{llll}\text { C } & -0.43946 & -0.63886 & -0.65025\end{array}$

$\begin{array}{llll}\mathrm{N} & -1.21171 & 0.46587 & -0.98704\end{array}$

$\begin{array}{llll}\text { C } & -1.10961 & -3.06580 & -0.68025\end{array}$

$\begin{array}{llll}\text { C } & -1.73680 & -3.89787 & 0.26712\end{array}$

$\begin{array}{llll}\text { C } & -0.21035 & -3.63222 & -1.60520\end{array}$

$\begin{array}{llll}\text { C } & -1.43925 & -5.26307 & 0.31672\end{array}$

$\begin{array}{llll}\text { C } & 0.11493 & -4.99082 & -1.53105\end{array}$

$\begin{array}{llll}\text { C } & -0.50418 & -5.78772 & -0.57290\end{array}$

$\begin{array}{llll}\mathrm{H} & -1.91198 & -5.90621 & 1.04879\end{array}$

$\begin{array}{llll}\mathrm{H} & 0.82784 & -5.42562 & -2.22040\end{array}$

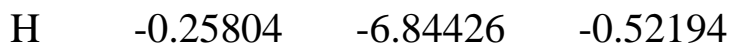

$\begin{array}{llll}\mathrm{O} & 0.28923 & -2.77703 & -2.53919\end{array}$

$\begin{array}{llll}\mathrm{O} & -2.60518 & -3.27431 & 1.11684\end{array}$

$\begin{array}{llll}\text { C } & -3.23360 & -4.07518 & 2.11912\end{array}$

$\begin{array}{llll}\mathrm{H} & -3.86428 & -3.38763 & 2.68321\end{array}$

$\begin{array}{llll}\mathrm{H} & -3.85157 & -4.85837 & 1.66393\end{array}$

$\begin{array}{llll}\mathrm{H} & -2.48934 & -4.53118 & 2.78437\end{array}$

$\begin{array}{llll}\text { C } & 1.24876 & -3.29919 & -3.46132\end{array}$

$\mathrm{H} \quad 0.80445 \quad-4.08461 \quad-4.08521$

$\begin{array}{llll}\mathrm{H} & 1.54116 & -2.45199 & -4.08209\end{array}$

$\begin{array}{llll}\mathrm{H} & 2.12296 & -3.69297 & -2.93033\end{array}$

$\begin{array}{llll}\text { C } & -0.30806 & 2.47221 & -2.18031\end{array}$

$\begin{array}{llll}\mathrm{H} & 0.62324 & 1.90909 & -2.28822\end{array}$

$\begin{array}{llll}\mathrm{H} & -0.91745 & 2.30372 & -3.07612\end{array}$

$\begin{array}{llll}\text { C } & -0.01852 & 3.94351 & -2.00923\end{array}$

$\begin{array}{llll}\text { C } & 0.85816 & 4.37395 & -1.00309\end{array}$

$\begin{array}{llll}\text { C } & -0.64185 & 4.90851 & -2.80778\end{array}$

$\begin{array}{llll}\text { C } & 1.10295 & 5.73038 & -0.80295\end{array}$

$\begin{array}{llll}\mathrm{H} & 1.35041 & 3.64056 & -0.36917\end{array}$

$\begin{array}{llll}\mathrm{C} & -0.39615 & 6.26886 & -2.61347\end{array}$

$\begin{array}{llll}\mathrm{H} & -1.32339 & 4.58909 & -3.59350\end{array}$

$\begin{array}{llll}\text { C } & 0.47677 & 6.68392 & -1.60823\end{array}$

$\begin{array}{llll}\mathrm{H} & 1.78261 & 6.04202 & -0.01484\end{array}$

$\begin{array}{llll}\mathrm{H} & -0.88739 & 7.00291 & -3.24655\end{array}$ 


\begin{tabular}{|c|c|c|c|}
\hline $\mathrm{H}$ & 0.66875 & 7.74199 & -1.45343 \\
\hline $\mathrm{C}$ & 0.88783 & -0.66575 & -0.33652 \\
\hline $\mathrm{C}$ & 1.62667 & -1.80094 & 0.21237 \\
\hline $\mathrm{C}$ & 1.24280 & -2.38003 & 1.44461 \\
\hline $\mathrm{C}$ & 2.78430 & -2.27874 & -0.42468 \\
\hline $\mathrm{C}$ & 1.95016 & -3.47786 & 1.93864 \\
\hline $\mathrm{C}$ & 3.49329 & -3.36044 & 0.08834 \\
\hline $\mathrm{H}$ & 3.10589 & -1.79271 & -1.34144 \\
\hline $\mathrm{C}$ & 3.06018 & -3.98274 & 1.26187 \\
\hline $\mathrm{H}$ & 1.64456 & -3.92397 & 2.88268 \\
\hline $\mathrm{H}$ & 4.37619 & -3.72643 & -0.42949 \\
\hline $\mathrm{H}$ & 3.60077 & -4.83516 & 1.66335 \\
\hline $\mathrm{C}$ & 0.12923 & -1.74888 & 2.25175 \\
\hline $\mathrm{H}$ & 0.09473 & -2.22023 & 3.24331 \\
\hline $\mathrm{H}$ & -0.84876 & -1.93029 & 1.79074 \\
\hline $\mathrm{C}$ & 0.31499 & -0.25926 & 2.40394 \\
\hline $\mathrm{C}$ & 1.25683 & 1.87580 & 2.66591 \\
\hline $\mathrm{H}$ & 2.45136 & -0.00588 & 2.55912 \\
\hline $\mathrm{C}$ & -0.12732 & 2.20583 & 2.63509 \\
\hline $\mathrm{C}$ & -0.57093 & 3.52873 & 2.70710 \\
\hline $\mathrm{C}$ & 2.19127 & 2.92426 & 2.77525 \\
\hline $\mathrm{C}$ & 0.37656 & 4.54228 & 2.80800 \\
\hline $\mathrm{H}$ & 0.05119 & 5.57713 & 2.85118 \\
\hline $\mathrm{C}$ & 1.74833 & 4.23849 & 2.84300 \\
\hline $\mathrm{H}$ & 2.47167 & 5.04510 & 2.92286 \\
\hline $\mathrm{H}$ & 3.25424 & 2.69884 & 2.80288 \\
\hline $\mathrm{H}$ & -1.63099 & 3.76272 & 2.67811 \\
\hline $\mathrm{S}$ & -1.10120 & 0.76749 & 2.47506 \\
\hline $\mathrm{H}$ & -4.36143 & 1.14614 & -0.92922 \\
\hline $\mathrm{H}$ & -3.55564 & 1.36653 & -2.48961 \\
\hline $\mathrm{H}$ & -2.67758 & 3.34197 & -1.33199 \\
\hline $\mathrm{H}$ & -2.76693 & 2.51710 & 0.23146 \\
\hline \multicolumn{4}{|c|}{76} \\
\hline \multicolumn{4}{|c|}{$3 d-(R)-t s 1-r$} \\
\hline $\mathrm{C}$ & 1.67860 & 0.75935 & 1.97717 \\
\hline $\mathrm{H}$ & 1.84839 & 0.88817 & 0.65133 \\
\hline $\mathrm{O}$ & 1.63933 & 0.55290 & -0.56681 \\
\hline $\mathrm{C}$ & -2.49247 & 2.40540 & -0.53044 \\
\hline $\mathrm{C}$ & -3.40450 & 1.25038 & -1.03461 \\
\hline $\mathrm{C}$ & -1.02760 & 1.94415 & -0.76137 \\
\hline $\mathrm{H}$ & -0.38740 & 2.23533 & 0.07103 \\
\hline $\mathrm{C}$ & -2.50027 & 0.07125 & -0.88252 \\
\hline $\mathrm{N}$ & -2.63087 & -1.21940 & -0.82518 \\
\hline $\mathrm{N}$ & -1.30444 & -1.66133 & -0.63883 \\
\hline $\mathrm{C}$ & -0.39772 & -0.61716 & -0.58983 \\
\hline $\mathrm{N}$ & -1.19918 & 0.48496 & -0.74804 \\
\hline $\mathrm{C}$ & -1.04356 & -3.04583 & -0.52454 \\
\hline
\end{tabular}




\begin{tabular}{|c|c|c|c|}
\hline $\mathrm{C}$ & -1.48371 & -3.74872 & 0.61007 \\
\hline $\mathrm{C}$ & -0.36586 & -3.72164 & -1.55255 \\
\hline $\mathrm{C}$ & -1.21539 & -5.11587 & 0.73257 \\
\hline $\mathrm{C}$ & -0.07651 & -5.08430 & -1.41883 \\
\hline $\mathrm{C}$ & -0.50529 & -5.75851 & -0.27934 \\
\hline $\mathrm{H}$ & -1.53978 & -5.66895 & 1.60537 \\
\hline $\mathrm{H}$ & 0.47034 & -5.61267 & -2.18944 \\
\hline $\mathrm{H}$ & -0.28230 & -6.81657 & -0.17729 \\
\hline $\mathrm{O}$ & -0.03338 & -2.96526 & -2.63327 \\
\hline $\mathrm{O}$ & -2.14146 & -3.00610 & 1.54473 \\
\hline $\mathrm{C}$ & -2.60686 & -3.68047 & 2.71742 \\
\hline $\mathrm{H}$ & -3.09055 & -2.90961 & 3.31709 \\
\hline $\mathrm{H}$ & -3.32928 & -4.46258 & 2.45622 \\
\hline $\mathrm{H}$ & -1.77112 & -4.11822 & 3.27747 \\
\hline $\mathrm{C}$ & 0.67559 & -3.61582 & -3.69316 \\
\hline $\mathrm{H}$ & 0.07079 & -4.42044 & -4.12824 \\
\hline $\mathrm{H}$ & 0.85419 & -2.84001 & -4.43779 \\
\hline $\mathrm{H}$ & 1.62973 & -4.01557 & -3.33222 \\
\hline $\mathrm{C}$ & -0.40119 & 2.44106 & -2.07721 \\
\hline $\mathrm{H}$ & 0.52425 & 1.87603 & -2.22446 \\
\hline $\mathrm{H}$ & -1.07815 & 2.23156 & -2.91425 \\
\hline $\mathrm{C}$ & -0.11046 & 3.91876 & -1.98757 \\
\hline $\mathrm{C}$ & 0.86520 & 4.38255 & -1.09407 \\
\hline $\mathrm{C}$ & -0.82690 & 4.85384 & -2.74174 \\
\hline $\mathrm{C}$ & 1.11291 & 5.74615 & -0.95750 \\
\hline $\mathrm{H}$ & 1.42869 & 3.66661 & -0.50146 \\
\hline $\mathrm{C}$ & -0.57692 & 6.22119 & -2.61185 \\
\hline $\mathrm{H}$ & -1.58477 & 4.50659 & -3.44116 \\
\hline $\mathrm{C}$ & 0.39256 & 6.67118 & -1.71626 \\
\hline $\mathrm{H}$ & 1.86901 & 6.08759 & -0.25583 \\
\hline $\mathrm{H}$ & -1.14039 & 6.93324 & -3.20889 \\
\hline $\mathrm{H}$ & 0.58688 & 7.73485 & -1.61062 \\
\hline $\mathrm{C}$ & 0.98757 & -0.59275 & -0.36977 \\
\hline $\mathrm{C}$ & 1.80539 & -1.81344 & -0.13660 \\
\hline $\mathrm{C}$ & 1.82714 & -2.39569 & 1.14055 \\
\hline $\mathrm{C}$ & 2.65127 & -2.31474 & -1.13391 \\
\hline $\mathrm{C}$ & 2.63376 & -3.51296 & 1.37674 \\
\hline $\mathrm{C}$ & 3.45648 & -3.42450 & -0.89217 \\
\hline $\mathrm{H}$ & 2.66635 & -1.81986 & -2.10040 \\
\hline $\mathrm{C}$ & 3.43484 & -4.03620 & 0.36379 \\
\hline $\mathrm{H}$ & 2.64904 & -3.96384 & 2.36660 \\
\hline $\mathrm{H}$ & 4.10608 & -3.80882 & -1.67452 \\
\hline $\mathrm{H}$ & 4.06078 & -4.90244 & 0.56021 \\
\hline $\mathrm{C}$ & 0.98272 & -1.77377 & 2.23110 \\
\hline $\mathrm{H}$ & 1.47877 & -1.91990 & 3.20304 \\
\hline $\mathrm{H}$ & 0.02272 & -2.29791 & 2.29523 \\
\hline $\mathrm{C}$ & 0.70737 & -0.30796 & 1.99368 \\
\hline $\mathrm{C}$ & 1.08536 & 2.02709 & 2.41692 \\
\hline
\end{tabular}




\begin{tabular}{|c|c|c|c|}
\hline $\mathrm{H}$ & 2.70222 & 0.52381 & 2.27583 \\
\hline $\mathrm{C}$ & -0.30578 & 1.93621 & 2.65315 \\
\hline $\mathrm{C}$ & -1.07228 & 3.05825 & 2.97746 \\
\hline $\mathrm{C}$ & 1.70081 & 3.27937 & 2.54030 \\
\hline $\mathrm{C}$ & -0.43529 & 4.29276 & 3.09128 \\
\hline $\mathrm{H}$ & -1.01500 & 5.17437 & 3.34835 \\
\hline $\mathrm{C}$ & 0.94491 & 4.39971 & 2.87962 \\
\hline $\mathrm{H}$ & 1.42888 & 5.36785 & 2.97127 \\
\hline $\mathrm{H}$ & 2.76877 & 3.37369 & 2.35938 \\
\hline $\mathrm{H}$ & -2.14079 & 2.96947 & 3.15250 \\
\hline$S$ & -0.88213 & 0.29074 & 2.46946 \\
\hline $\mathrm{H}$ & -4.32315 & 1.14842 & -0.45360 \\
\hline $\mathrm{H}$ & -3.68489 & 1.38069 & -2.08654 \\
\hline $\mathrm{H}$ & -2.69144 & 3.35366 & -1.03378 \\
\hline $\mathrm{H}$ & -2.64868 & 2.55063 & 0.54154 \\
\hline \multicolumn{4}{|l|}{76} \\
\hline \multicolumn{4}{|c|}{$3 d-(R)-6-r$} \\
\hline $\mathrm{C}$ & 1.58647 & 0.53615 & 2.48978 \\
\hline $\mathrm{H}$ & 2.47237 & 0.57479 & 1.84945 \\
\hline $\mathrm{O}$ & 1.35464 & 0.73438 & -0.22328 \\
\hline $\mathrm{C}$ & -2.59206 & 2.38449 & -1.17012 \\
\hline $\mathrm{C}$ & -3.24050 & 1.21689 & -1.97233 \\
\hline $\mathrm{C}$ & -1.11247 & 1.97532 & -0.94983 \\
\hline $\mathrm{H}$ & -0.71222 & 2.30112 & 0.00738 \\
\hline $\mathrm{C}$ & -2.41149 & 0.06218 & -1.51083 \\
\hline $\mathrm{N}$ & -2.50125 & -1.24245 & -1.48956 \\
\hline $\mathrm{N}$ & -1.32618 & -1.61345 & -0.82697 \\
\hline $\mathrm{C}$ & -0.56520 & -0.55426 & -0.46108 \\
\hline $\mathrm{N}$ & -1.25882 & 0.50412 & -0.92389 \\
\hline $\mathrm{C}$ & -1.08553 & -2.99480 & -0.57647 \\
\hline $\mathrm{C}$ & -1.65331 & -3.59690 & 0.55503 \\
\hline $\mathrm{C}$ & -0.32893 & -3.74165 & -1.49086 \\
\hline $\mathrm{C}$ & -1.39895 & -4.94704 & 0.81657 \\
\hline $\mathrm{C}$ & -0.06978 & -5.09077 & -1.22314 \\
\hline $\mathrm{C}$ & -0.60216 & -5.66581 & -0.07297 \\
\hline $\mathrm{H}$ & -1.81162 & -5.42998 & 1.69356 \\
\hline $\mathrm{H}$ & 0.54130 & -5.68025 & -1.89445 \\
\hline $\mathrm{H}$ & -0.39487 & -6.71143 & 0.13526 \\
\hline $\mathrm{O}$ & 0.09620 & -3.07033 & -2.58940 \\
\hline $\mathrm{O}$ & -2.41632 & -2.78109 & 1.33240 \\
\hline $\mathrm{C}$ & -2.96040 & -3.33164 & 2.53819 \\
\hline $\mathrm{H}$ & -3.48731 & -2.50611 & 3.01529 \\
\hline $\mathrm{H}$ & -3.65686 & -4.14732 & 2.31226 \\
\hline $\mathrm{H}$ & -2.16127 & -3.69236 & 3.19664 \\
\hline $\mathrm{C}$ & 0.91460 & -3.78818 & -3.52204 \\
\hline $\mathrm{H}$ & 0.36028 & -4.62997 & -3.95311 \\
\hline $\mathrm{H}$ & 1.16181 & -3.06620 & -4.29996 \\
\hline
\end{tabular}




\begin{tabular}{|c|c|c|c|}
\hline $\mathrm{H}$ & 1.82883 & -4.14211 & -3.03442 \\
\hline $\mathrm{C}$ & -0.16883 & 2.41261 & -2.08611 \\
\hline $\mathrm{H}$ & 0.70760 & 1.76086 & -2.01551 \\
\hline $\mathrm{H}$ & -0.66223 & 2.27520 & -3.05677 \\
\hline $\mathrm{C}$ & 0.23538 & 3.85123 & -1.88969 \\
\hline $\mathrm{C}$ & 1.09168 & 4.17406 & -0.82717 \\
\hline $\mathrm{C}$ & -0.24365 & 4.87731 & -2.71005 \\
\hline $\mathrm{C}$ & 1.45650 & 5.49761 & -0.59524 \\
\hline $\mathrm{H}$ & 1.46213 & 3.37155 & -0.19326 \\
\hline $\mathrm{C}$ & 0.12494 & 6.20461 & -2.48021 \\
\hline $\mathrm{H}$ & -0.90364 & 4.63510 & -3.54119 \\
\hline $\mathrm{C}$ & 0.97519 & 6.51749 & -1.42015 \\
\hline $\mathrm{H}$ & 2.11890 & 5.73482 & 0.23268 \\
\hline $\mathrm{H}$ & -0.25150 & 6.99155 & -3.12850 \\
\hline $\mathrm{H}$ & 1.26323 & 7.54942 & -1.23866 \\
\hline $\mathrm{C}$ & 0.81132 & -0.38146 & 0.24117 \\
\hline $\mathrm{C}$ & 1.67179 & -1.64867 & 0.09809 \\
\hline $\mathrm{C}$ & 1.65234 & -2.46087 & 1.23154 \\
\hline $\mathrm{C}$ & 2.51533 & -1.93096 & -0.97070 \\
\hline $\mathrm{C}$ & 2.44480 & -3.60703 & 1.28960 \\
\hline $\mathrm{C}$ & 3.30995 & -3.07746 & -0.91682 \\
\hline $\mathrm{H}$ & 2.55804 & -1.24408 & -1.81141 \\
\hline $\mathrm{C}$ & 3.26558 & -3.91690 & 0.20319 \\
\hline $\mathrm{H}$ & 2.44029 & -4.24296 & 2.17206 \\
\hline $\mathrm{H}$ & 3.98522 & -3.31219 & -1.73645 \\
\hline $\mathrm{H}$ & 3.89613 & -4.80158 & 0.23856 \\
\hline $\mathrm{C}$ & 0.74228 & -1.89358 & 2.29377 \\
\hline $\mathrm{H}$ & 1.16396 & -1.97356 & 3.30246 \\
\hline $\mathrm{H}$ & -0.21715 & -2.42047 & 2.30715 \\
\hline $\mathrm{C}$ & 0.55414 & -0.42378 & 1.86726 \\
\hline $\mathrm{C}$ & 0.94906 & 1.89213 & 2.62062 \\
\hline $\mathrm{H}$ & 1.87268 & 0.16637 & 3.48509 \\
\hline $\mathrm{C}$ & -0.45430 & 1.86113 & 2.60960 \\
\hline $\mathrm{C}$ & -1.20747 & 3.02625 & 2.75885 \\
\hline $\mathrm{C}$ & 1.59761 & 3.11164 & 2.78206 \\
\hline $\mathrm{C}$ & -0.53912 & 4.24338 & 2.91829 \\
\hline $\mathrm{H}$ & -1.11340 & 5.15972 & 3.02180 \\
\hline $\mathrm{C}$ & 0.85466 & 4.28835 & 2.93006 \\
\hline $\mathrm{H}$ & 1.36477 & 5.24044 & 3.04273 \\
\hline $\mathrm{H}$ & 2.68417 & 3.14871 & 2.78006 \\
\hline $\mathrm{H}$ & -2.29325 & 2.99159 & 2.74249 \\
\hline $\mathrm{S}$ & -1.09661 & 0.23176 & 2.38820 \\
\hline $\mathrm{H}$ & -4.30158 & 1.08108 & -1.75465 \\
\hline $\mathrm{H}$ & -3.13277 & 1.35446 & -3.05448 \\
\hline $\mathrm{H}$ & -2.66178 & 3.34372 & -1.68651 \\
\hline $\mathrm{H}$ & -3.09137 & 2.47604 & -0.20095 \\
\hline
\end{tabular}

76 


\begin{tabular}{|c|c|c|c|}
\hline \multicolumn{4}{|c|}{$3 d-(R)-t s 3-r$} \\
\hline $\mathrm{C}$ & 0.46075 & 0.58534 & 3.15575 \\
\hline $\mathrm{H}$ & 1.55106 & 0.50481 & 3.11527 \\
\hline $\mathrm{O}$ & 1.31807 & 0.92791 & 0.44244 \\
\hline $\mathrm{C}$ & -1.97802 & 2.18936 & -2.35513 \\
\hline $\mathrm{C}$ & -2.12878 & 0.99581 & -3.34530 \\
\hline $\mathrm{C}$ & -0.75628 & 1.84123 & -1.46667 \\
\hline $\mathrm{H}$ & -0.85784 & 2.19084 & -0.44047 \\
\hline $\mathrm{C}$ & -1.57664 & -0.12316 & -2.52278 \\
\hline $\mathrm{N}$ & -1.64700 & -1.42673 & -2.49898 \\
\hline $\mathrm{N}$ & -0.90867 & -1.72949 & -1.34007 \\
\hline $\mathrm{C}$ & -0.42641 & -0.64989 & -0.68525 \\
\hline $\mathrm{N}$ & -0.84134 & 0.36721 & -1.47412 \\
\hline $\mathrm{C}$ & -0.74827 & -3.09162 & -0.96182 \\
\hline $\mathrm{C}$ & -1.71767 & -3.71032 & -0.16068 \\
\hline $\mathrm{C}$ & 0.36782 & -3.80636 & -1.42015 \\
\hline $\mathrm{C}$ & -1.54792 & -5.04436 & 0.22569 \\
\hline $\mathrm{C}$ & 0.53589 & -5.14207 & -1.03508 \\
\hline $\mathrm{C}$ & -0.42079 & -5.73475 & -0.21682 \\
\hline $\mathrm{H}$ & -2.27836 & -5.53847 & 0.85425 \\
\hline $\mathrm{H}$ & 1.40148 & -5.70634 & -1.35767 \\
\hline $\mathrm{H}$ & -0.28565 & -6.76900 & 0.08636 \\
\hline $\mathrm{O}$ & 1.21548 & -3.11921 & -2.22687 \\
\hline $\mathrm{O}$ & -2.77163 & -2.92477 & 0.19900 \\
\hline $\mathrm{C}$ & -3.76073 & -3.50048 & 1.05984 \\
\hline $\mathrm{H}$ & -4.48335 & -2.70439 & 1.23729 \\
\hline $\mathrm{H}$ & -4.25023 & -4.35303 & 0.57484 \\
\hline $\mathrm{H}$ & -3.31441 & -3.81682 & 2.01025 \\
\hline $\mathrm{C}$ & 2.37766 & -3.81025 & -2.69902 \\
\hline $\mathrm{H}$ & 2.09254 & -4.67060 & -3.31610 \\
\hline $\mathrm{H}$ & 2.91832 & -3.08141 & -3.30259 \\
\hline $\mathrm{H}$ & 3.00011 & -4.13463 & -1.85847 \\
\hline $\mathrm{C}$ & 0.58110 & 2.33240 & -2.05453 \\
\hline $\mathrm{H}$ & 1.37486 & 1.75682 & -1.57164 \\
\hline $\mathrm{H}$ & 0.60254 & 2.14347 & -3.13532 \\
\hline $\mathrm{C}$ & 0.76113 & 3.80285 & -1.76974 \\
\hline $\mathrm{C}$ & 0.98912 & 4.22276 & -0.45097 \\
\hline $\mathrm{C}$ & 0.67313 & 4.76534 & -2.78066 \\
\hline $\mathrm{C}$ & 1.12572 & 5.57754 & -0.15793 \\
\hline $\mathrm{H}$ & 1.06234 & 3.47475 & 0.33487 \\
\hline $\mathrm{C}$ & 0.81240 & 6.12358 & -2.48790 \\
\hline $\mathrm{H}$ & 0.50264 & 4.44813 & -3.80791 \\
\hline $\mathrm{C}$ & 1.03776 & 6.53247 & -1.17391 \\
\hline $\mathrm{H}$ & 1.30044 & 5.88698 & 0.86844 \\
\hline $\mathrm{H}$ & 0.74590 & 6.85933 & -3.28510 \\
\hline $\mathrm{H}$ & 1.14687 & 7.58864 & -0.94269 \\
\hline $\mathrm{C}$ & 0.79871 & -0.16706 & 0.77876 \\
\hline $\mathrm{C}$ & 1.60126 & -1.44505 & 0.91799 \\
\hline
\end{tabular}




\begin{tabular}{|c|c|c|c|}
\hline $\mathrm{C}$ & 0.99071 & -2.32579 & 1.81399 \\
\hline $\mathrm{C}$ & 2.82864 & -1.74163 & 0.33707 \\
\hline $\mathrm{C}$ & 1.59430 & -3.54237 & 2.12205 \\
\hline $\mathrm{C}$ & 3.43865 & -2.95855 & 0.64944 \\
\hline $\mathrm{H}$ & 3.29608 & -1.02345 & -0.33120 \\
\hline $\mathrm{C}$ & 2.82104 & -3.85470 & 1.52981 \\
\hline $\mathrm{H}$ & 1.12844 & -4.23668 & 2.81730 \\
\hline $\mathrm{H}$ & 4.40631 & -3.20870 & 0.22107 \\
\hline $\mathrm{H}$ & 3.30872 & -4.79655 & 1.76740 \\
\hline $\mathrm{C}$ & -0.30278 & -1.74676 & 2.34189 \\
\hline $\mathrm{H}$ & -0.45169 & -1.93111 & 3.41147 \\
\hline $\mathrm{H}$ & -1.15940 & -2.17762 & 1.81287 \\
\hline $\mathrm{C}$ & -0.18369 & -0.24666 & 2.02209 \\
\hline $\mathrm{C}$ & -0.01406 & 2.00531 & 2.99933 \\
\hline $\mathrm{H}$ & 0.12304 & 0.19252 & 4.12614 \\
\hline $\mathrm{C}$ & -1.23297 & 2.10877 & 2.31003 \\
\hline $\mathrm{C}$ & -1.85388 & 3.34238 & 2.11730 \\
\hline $\mathrm{C}$ & 0.59013 & 3.15768 & 3.48782 \\
\hline $\mathrm{C}$ & -1.23714 & 4.49067 & 2.62126 \\
\hline $\mathrm{H}$ & -1.70265 & 5.45945 & 2.46383 \\
\hline $\mathrm{C}$ & -0.02166 & 4.40280 & 3.29935 \\
\hline $\mathrm{H}$ & 0.45625 & 5.30266 & 3.67572 \\
\hline $\mathrm{H}$ & 1.54156 & 3.09057 & 4.00953 \\
\hline $\mathrm{H}$ & -2.79110 & 3.41445 & 1.57302 \\
\hline S & -1.82275 & 0.54593 & 1.73769 \\
\hline $\mathrm{H}$ & -3.16169 & 0.81963 & -3.65166 \\
\hline $\mathrm{H}$ & -1.52829 & 1.14007 & -4.25105 \\
\hline $\mathrm{H}$ & -1.84004 & 3.14731 & -2.86056 \\
\hline $\mathrm{H}$ & -2.87518 & 2.25305 & -1.73172 \\
\hline \multicolumn{4}{|c|}{76} \\
\hline \multicolumn{4}{|c|}{$3 \mathrm{~d}-(\mathrm{R})-[2 \mathrm{r}+3 \mathrm{~d}]$} \\
\hline $\mathrm{C}$ & 0.39907 & 0.72292 & 3.55095 \\
\hline $\mathrm{H}$ & 1.46171 & 0.52879 & 3.73300 \\
\hline $\mathrm{O}$ & 1.59644 & 1.19502 & 0.73837 \\
\hline $\mathrm{C}$ & -1.91714 & 1.92999 & -3.00406 \\
\hline $\mathrm{C}$ & -1.89498 & 0.65487 & -3.89996 \\
\hline $\mathrm{C}$ & -0.90760 & 1.66299 & -1.85590 \\
\hline $\mathrm{H}$ & -1.24231 & 2.07012 & -0.90080 \\
\hline $\mathrm{C}$ & -1.47751 & -0.38709 & -2.91317 \\
\hline $\mathrm{N}$ & -1.47584 & -1.68705 & -2.82373 \\
\hline $\mathrm{N}$ & -0.90493 & -1.86845 & -1.54100 \\
\hline $\mathrm{C}$ & -0.57385 & -0.73856 & -0.86475 \\
\hline $\mathrm{N}$ & -0.95444 & 0.19657 & -1.78320 \\
\hline $\mathrm{C}$ & -0.73480 & -3.19376 & -1.05728 \\
\hline $\mathrm{C}$ & -1.68698 & -3.75396 & -0.19381 \\
\hline $\mathrm{C}$ & 0.38590 & -3.93843 & -1.45293 \\
\hline $\mathrm{C}$ & -1.51743 & -5.06080 & 0.28136 \\
\hline
\end{tabular}




\begin{tabular}{|c|c|c|c|}
\hline $\mathrm{C}$ & 0.54966 & -5.25085 & -0.99078 \\
\hline $\mathrm{C}$ & -0.40407 & -5.78931 & -0.13196 \\
\hline $\mathrm{H}$ & -2.24274 & -5.50794 & 0.94996 \\
\hline $\mathrm{H}$ & 1.41001 & -5.83882 & -1.28482 \\
\hline $\mathrm{H}$ & -0.27497 & -6.80548 & 0.22997 \\
\hline $\mathrm{O}$ & 1.25701 & -3.29800 & -2.27635 \\
\hline $\mathrm{O}$ & -2.73699 & -2.94705 & 0.13122 \\
\hline $\mathrm{C}$ & -3.71368 & -3.46974 & 1.03618 \\
\hline $\mathrm{H}$ & -4.43946 & -2.66759 & 1.16958 \\
\hline $\mathrm{H}$ & -4.20564 & -4.35427 & 0.61415 \\
\hline $\mathrm{H}$ & -3.25768 & -3.72302 & 2.00113 \\
\hline $\mathrm{C}$ & 2.42628 & -4.01519 & -2.68000 \\
\hline $\mathrm{H}$ & 2.15785 & -4.90835 & -3.25723 \\
\hline $\mathrm{H}$ & 2.98587 & -3.32199 & -3.30813 \\
\hline $\mathrm{H}$ & 3.02846 & -4.29813 & -1.80899 \\
\hline $\mathrm{C}$ & 0.52051 & 2.16835 & -2.14627 \\
\hline $\mathrm{H}$ & 1.19899 & 1.66239 & -1.45385 \\
\hline $\mathrm{H}$ & 0.80450 & 1.89544 & -3.17030 \\
\hline $\mathrm{C}$ & 0.60337 & 3.66156 & -1.94734 \\
\hline $\mathrm{C}$ & 0.63411 & 4.18216 & -0.64541 \\
\hline $\mathrm{C}$ & 0.60286 & 4.55031 & -3.02772 \\
\hline $\mathrm{C}$ & 0.65583 & 5.55903 & -0.43406 \\
\hline $\mathrm{H}$ & 0.65563 & 3.49697 & 0.19784 \\
\hline $\mathrm{C}$ & 0.62767 & 5.93055 & -2.81825 \\
\hline $\mathrm{H}$ & 0.59268 & 4.15833 & -4.04314 \\
\hline $\mathrm{C}$ & 0.65042 & 6.43835 & -1.51947 \\
\hline $\mathrm{H}$ & 0.68194 & 5.94321 & 0.58201 \\
\hline $\mathrm{H}$ & 0.63126 & 6.60715 & -3.66879 \\
\hline $\mathrm{H}$ & 0.67072 & 7.51216 & -1.35412 \\
\hline $\mathrm{C}$ & 1.14966 & 0.17000 & 1.22728 \\
\hline $\mathrm{C}$ & 1.75536 & -1.17917 & 1.21325 \\
\hline $\mathrm{C}$ & 0.92311 & -2.08172 & 1.88667 \\
\hline $\mathrm{C}$ & 2.98310 & -1.57177 & 0.67993 \\
\hline $\mathrm{C}$ & 1.31832 & -3.40683 & 2.04202 \\
\hline $\mathrm{C}$ & 3.37603 & -2.89830 & 0.83764 \\
\hline $\mathrm{H}$ & 3.60712 & -0.84830 & 0.16311 \\
\hline $\mathrm{C}$ & 2.54838 & -3.80573 & 1.51432 \\
\hline $\mathrm{H}$ & 0.68043 & -4.12581 & 2.54824 \\
\hline $\mathrm{H}$ & 4.33163 & -3.23459 & 0.44410 \\
\hline $\mathrm{H}$ & 2.86811 & -4.83813 & 1.62708 \\
\hline $\mathrm{C}$ & -0.36270 & -1.41637 & 2.31126 \\
\hline $\mathrm{H}$ & -0.69092 & -1.70482 & 3.31540 \\
\hline $\mathrm{H}$ & -1.15146 & -1.67573 & 1.59620 \\
\hline $\mathrm{C}$ & -0.05428 & 0.08811 & 2.20529 \\
\hline $\mathrm{C}$ & 0.07841 & 2.19302 & 3.49180 \\
\hline $\mathrm{H}$ & -0.16818 & 0.25429 & 4.36776 \\
\hline $\mathrm{C}$ & -0.94602 & 2.49722 & 2.58204 \\
\hline $\mathrm{C}$ & -1.42114 & 3.80111 & 2.44291 \\
\hline
\end{tabular}




$\begin{array}{lccc}\mathrm{C} & 0.64393 & 3.20942 & 4.25291 \\ \mathrm{C} & -0.85025 & 4.81081 & 3.22158 \\ \mathrm{H} & -1.20498 & 5.83203 & 3.11258 \\ \mathrm{C} & 0.18060 & 4.52315 & 4.11699 \\ \mathrm{H} & 0.62803 & 5.31884 & 4.70510 \\ \mathrm{H} & 1.44758 & 2.98372 & 4.94975 \\ \mathrm{H} & -2.20210 & 4.03449 & 1.72561 \\ \mathrm{~S} & -1.49080 & 1.09465 & 1.66142 \\ \mathrm{H} & -2.86272 & 0.44259 & -4.35949 \\ \mathrm{H} & -1.15429 & 0.73662 & -4.70452 \\ \mathrm{H} & -1.66628 & 2.83965 & -3.55356 \\ \mathrm{H} & -2.91940 & 2.05411 & -2.58281\end{array}$




\subsection{3a-(S)-a-path}

77

3a-(S)-4-a

$\begin{array}{llll}\text { C } & -0.07339 & -0.97298 & -2.57932\end{array}$

$\begin{array}{llll}\mathrm{H} & -2.40991 & -0.69013 & -0.44301\end{array}$

$\begin{array}{llll}\mathrm{O} & -2.26669 & -0.22646 & 0.40079\end{array}$

$\begin{array}{llll}\text { C } & 0.30164 & -2.22318 & 3.41514\end{array}$

$\begin{array}{llll}\text { C } & 1.33071 & -0.31882 & 4.27171\end{array}$

$\begin{array}{llll}\text { C } & -0.37971 & -1.56051 & 2.20166\end{array}$

$\begin{array}{llll}\mathrm{H} & 1.34476 & 0.34993 & 5.13442\end{array}$

$\mathrm{H} \quad 1.31536 \quad-2.55335 \quad 3.13405$

$\begin{array}{llll}\mathrm{H} & -0.27492 & -3.09514 & 3.72682\end{array}$

$\begin{array}{llll}\mathrm{H} & -1.46636 & -1.62860 & 2.29007\end{array}$

$\begin{array}{llll}\mathrm{H} & 2.33717 & -0.74855 & 4.13718\end{array}$

$\begin{array}{llll}\mathrm{O} & 0.38968 & -1.35561 & 4.54702\end{array}$

$\begin{array}{llll}\mathrm{C} & 0.90504 & 0.42750 & 3.04318\end{array}$

$\begin{array}{llll}\mathrm{N} & 1.28211 & 1.61436 & 2.68641\end{array}$

$\begin{array}{llll}\mathrm{N} & 0.56899 & 1.85588 & 1.49253\end{array}$

$\begin{array}{llll}\mathrm{C} & -0.28513 & 0.79453 & 1.17892\end{array}$

$\begin{array}{llll}\mathrm{N} & -0.01795 & -0.13780 & 2.19095\end{array}$

$\begin{array}{llll}\mathrm{C} & 0.50056 & 3.17255 & 0.98884\end{array}$

$\begin{array}{llll}\mathrm{C} & -0.70058 & 3.90500 & 1.04941\end{array}$

$\begin{array}{llll}\text { C } & 1.64155 & 3.75960 & 0.41153\end{array}$

$\begin{array}{llll}\text { C } & -0.78212 & 5.16893 & 0.45490\end{array}$

$\begin{array}{llll}\text { C } & 1.56798 & 5.03678 & -0.15368\end{array}$

$\begin{array}{llll}\text { C } & 0.35117 & 5.71498 & -0.14003\end{array}$

$\begin{array}{llll}\mathrm{H} & -1.71102 & 5.72514 & 0.46660\end{array}$

$\begin{array}{llll}\mathrm{H} & 2.43816 & 5.49144 & -0.61087\end{array}$

$\begin{array}{llll}\mathrm{H} & 0.28794 & 6.69865 & -0.59598\end{array}$

$\begin{array}{llll}\mathrm{O} & 2.77124 & 2.99495 & 0.43136\end{array}$

$\begin{array}{llll}\mathrm{O} & -1.72534 & 3.30287 & 1.71017\end{array}$

$\begin{array}{llll}\text { C } & -2.98297 & 3.98366 & 1.73617\end{array}$

$\begin{array}{llll}\mathrm{H} & -3.66013 & 3.31399 & 2.26657\end{array}$

$\begin{array}{llll}\mathrm{H} & -2.89997 & 4.93595 & 2.27425\end{array}$

$\begin{array}{llll}\mathrm{H} & -3.34921 & 4.15754 & 0.71816\end{array}$

$\begin{array}{llll}\text { C } & 3.93537 & 3.52504 & -0.20578\end{array}$

$\begin{array}{llll}\mathrm{H} & 4.26652 & 4.44698 & 0.28710\end{array}$

$\begin{array}{llll}\mathrm{H} & 4.69817 & 2.75354 & -0.09936\end{array}$

$\begin{array}{llll}\mathrm{H} & 3.74792 & 3.72069 & -1.26907\end{array}$

$\begin{array}{llll}\text { C } & 0.08093 & -2.24547 & 0.89921\end{array}$

$\begin{array}{llll}\mathrm{H} & -0.25198 & -1.63464 & 0.05554\end{array}$

$\begin{array}{llll}\mathrm{H} & 1.17616 & -2.25075 & 0.87683\end{array}$

$\begin{array}{llll}\text { C } & -0.44610 & -3.65135 & 0.76843\end{array}$

$\begin{array}{llll}\text { C } & 0.41648 & -4.75093 & 0.80823\end{array}$

$\begin{array}{llll}\text { C } & -1.81838 & -3.88494 & 0.60126\end{array}$

$\begin{array}{llll}\text { C } & -0.07231 & -6.05066 & 0.67702\end{array}$

$\begin{array}{llll}\mathrm{H} & 1.48425 & -4.58309 & 0.92124\end{array}$ 


\begin{tabular}{|c|c|c|c|}
\hline $\mathrm{C}$ & -2.31159 & -5.18140 & 0.47234 \\
\hline $\mathrm{H}$ & -2.50250 & -3.04021 & 0.57075 \\
\hline $\mathrm{C}$ & -1.43840 & -6.27066 & 0.50754 \\
\hline $\mathrm{H}$ & 0.61632 & -6.89090 & 0.69958 \\
\hline $\mathrm{H}$ & -3.37797 & -5.34297 & 0.33924 \\
\hline $\mathrm{H}$ & -1.82132 & -7.28180 & 0.40203 \\
\hline $\mathrm{C}$ & -1.21738 & 0.69883 & 0.17917 \\
\hline $\mathrm{C}$ & -1.38125 & 1.60682 & -0.94394 \\
\hline $\mathrm{C}$ & -0.30581 & 1.95244 & -1.80220 \\
\hline $\mathrm{C}$ & -2.66175 & 2.11733 & -1.24228 \\
\hline $\mathrm{C}$ & -0.51877 & 2.86872 & -2.83261 \\
\hline $\mathrm{C}$ & -2.85992 & 3.01023 & -2.28934 \\
\hline $\mathrm{H}$ & -3.49352 & 1.81642 & -0.61188 \\
\hline $\mathrm{C}$ & -1.77877 & 3.41584 & -3.07526 \\
\hline $\mathrm{H}$ & 0.31657 & 3.13387 & -3.47721 \\
\hline $\mathrm{H}$ & -3.85473 & 3.40316 & -2.48374 \\
\hline $\mathrm{H}$ & -1.91977 & 4.12471 & -3.88581 \\
\hline $\mathrm{C}$ & 1.02500 & 1.24628 & -1.69283 \\
\hline $\mathrm{H}$ & 1.47616 & 1.34038 & -0.69884 \\
\hline $\mathrm{H}$ & 1.73587 & 1.70676 & -2.39294 \\
\hline $\mathrm{C}$ & 0.90238 & -0.20827 & -2.01208 \\
\hline $\mathrm{O}$ & 2.01483 & -0.97798 & -1.69286 \\
\hline $\mathrm{C}$ & 0.42165 & -2.32575 & -2.62017 \\
\hline $\mathrm{H}$ & -1.02842 & -0.60445 & -2.92620 \\
\hline $\mathrm{C}$ & 1.71729 & -2.27031 & -2.06889 \\
\hline $\mathrm{C}$ & 2.54481 & -3.37268 & -1.92181 \\
\hline $\mathrm{C}$ & -0.07745 & -3.57006 & -3.03302 \\
\hline $\mathrm{C}$ & 0.73406 & -4.69196 & -2.88948 \\
\hline $\mathrm{H}$ & 0.35905 & -5.66463 & -3.19351 \\
\hline $\mathrm{C}$ & 2.02681 & -4.59734 & -2.34806 \\
\hline $\mathrm{H}$ & 2.63105 & -5.49408 & -2.24859 \\
\hline $\mathrm{H}$ & -1.07684 & -3.65953 & -3.44880 \\
\hline $\mathrm{H}$ & 3.53864 & -3.28433 & -1.49487 \\
\hline \multicolumn{4}{|l|}{77} \\
\hline \multicolumn{4}{|c|}{$3 a-(S)-t s 1-a$} \\
\hline $\mathrm{C}$ & -0.97537 & -0.37781 & -2.44150 \\
\hline $\mathrm{H}$ & -1.79348 & -0.52580 & -1.16753 \\
\hline $\mathrm{O}$ & -1.85286 & -0.32664 & 0.21696 \\
\hline $\mathrm{C}$ & 0.78544 & -2.92332 & 2.70652 \\
\hline $\mathrm{C}$ & 2.34705 & -1.34924 & 3.40352 \\
\hline $\mathrm{C}$ & 0.03782 & -2.01414 & 1.70598 \\
\hline $\mathrm{H}$ & 2.75354 & -0.84642 & 4.28201 \\
\hline $\mathrm{H}$ & 1.61255 & -3.43357 & 2.18641 \\
\hline $\mathrm{H}$ & 0.09686 & -3.67847 & 3.08761 \\
\hline $\mathrm{H}$ & -1.00710 & -1.88367 & 1.99741 \\
\hline $\mathrm{H}$ & 3.14822 & -1.92645 & 2.91242 \\
\hline $\mathrm{O}$ & 1.31123 & -2.23162 & 3.84062 \\
\hline
\end{tabular}




\begin{tabular}{|c|c|c|c|}
\hline $\mathrm{C}$ & 1.75119 & -0.34252 & 2.47406 \\
\hline $\mathrm{N}$ & 2.09227 & 0.90362 & 2.36055 \\
\hline $\mathrm{N}$ & 1.16091 & 1.42901 & 1.44969 \\
\hline $\mathrm{C}$ & 0.22034 & 0.47874 & 1.06206 \\
\hline $\mathrm{N}$ & 0.65730 & -0.67811 & 1.71063 \\
\hline $\mathrm{C}$ & 1.07848 & 2.83893 & 1.34151 \\
\hline $\mathrm{C}$ & 0.03241 & 3.53597 & 1.97102 \\
\hline $\mathrm{C}$ & 2.07321 & 3.54881 & 0.64825 \\
\hline $\mathrm{C}$ & -0.07210 & 4.92320 & 1.82408 \\
\hline $\mathrm{C}$ & 1.98342 & 4.93897 & 0.52348 \\
\hline $\mathrm{C}$ & 0.90276 & 5.60258 & 1.09977 \\
\hline $\mathrm{H}$ & -0.89404 & 5.46654 & 2.27285 \\
\hline $\mathrm{H}$ & 2.73465 & 5.49626 & -0.02225 \\
\hline $\mathrm{H}$ & 0.82328 & 6.67994 & 0.98778 \\
\hline $\mathrm{O}$ & 3.07717 & 2.79422 & 0.11533 \\
\hline $\mathrm{O}$ & -0.82485 & 2.77211 & 2.70014 \\
\hline $\mathrm{C}$ & -1.91639 & 3.44036 & 3.34041 \\
\hline $\mathrm{H}$ & -2.47918 & 2.65388 & 3.84320 \\
\hline $\mathrm{H}$ & -1.54894 & 4.16748 & 4.07442 \\
\hline $\mathrm{H}$ & -2.55102 & 3.94088 & 2.60064 \\
\hline $\mathrm{C}$ & 4.08375 & 3.47634 & -0.63754 \\
\hline $\mathrm{H}$ & 4.62216 & 4.19525 & -0.00871 \\
\hline $\mathrm{H}$ & 4.76572 & 2.69692 & -0.97751 \\
\hline $\mathrm{H}$ & 3.64625 & 3.99282 & -1.50089 \\
\hline $\mathrm{C}$ & 0.10856 & -2.62435 & 0.29921 \\
\hline $\mathrm{H}$ & -0.30119 & -1.88335 & -0.38392 \\
\hline $\mathrm{H}$ & 1.16010 & -2.78763 & 0.04045 \\
\hline $\mathrm{C}$ & -0.67251 & -3.90531 & 0.16226 \\
\hline $\mathrm{C}$ & -0.02880 & -5.13474 & -0.00644 \\
\hline $\mathrm{C}$ & -2.07380 & -3.87276 & 0.17383 \\
\hline $\mathrm{C}$ & -0.76335 & -6.31076 & -0.16065 \\
\hline $\mathrm{H}$ & 1.05789 & -5.16607 & -0.03876 \\
\hline $\mathrm{C}$ & -2.80996 & -5.04592 & 0.02158 \\
\hline $\mathrm{H}$ & -2.57733 & -2.91549 & 0.28463 \\
\hline $\mathrm{C}$ & -2.15718 & -6.26923 & -0.14670 \\
\hline $\mathrm{H}$ & -0.24728 & -7.25717 & -0.29860 \\
\hline $\mathrm{H}$ & -3.89597 & -5.00580 & 0.02433 \\
\hline $\mathrm{H}$ & -2.73199 & -7.18281 & -0.27099 \\
\hline $\mathrm{C}$ & -0.93535 & 0.64366 & 0.29529 \\
\hline $\mathrm{C}$ & -1.31503 & 1.94608 & -0.30596 \\
\hline $\mathrm{C}$ & -0.54592 & 2.49900 & -1.34567 \\
\hline $\mathrm{C}$ & -2.51551 & 2.56894 & 0.05797 \\
\hline $\mathrm{C}$ & -0.93308 & 3.70525 & -1.93113 \\
\hline $\mathrm{C}$ & -2.89982 & 3.77038 & -0.53196 \\
\hline $\mathrm{H}$ & -3.13734 & 2.09458 & 0.81112 \\
\hline $\mathrm{C}$ & -2.09526 & 4.35372 & -1.51382 \\
\hline $\mathrm{H}$ & -0.33562 & 4.12632 & -2.73644 \\
\hline $\mathrm{H}$ & -3.82704 & 4.25192 & -0.23173 \\
\hline
\end{tabular}




\begin{tabular}{|c|c|c|c|}
\hline $\mathrm{H}$ & -2.38914 & 5.29202 & -1.97592 \\
\hline $\mathrm{C}$ & 0.61718 & 1.68597 & -1.84882 \\
\hline $\mathrm{H}$ & 1.48423 & 1.73751 & -1.17791 \\
\hline $\mathrm{H}$ & 0.95476 & 2.07278 & -2.82408 \\
\hline $\mathrm{C}$ & 0.21792 & 0.24966 & -1.97157 \\
\hline $\mathrm{O}$ & 1.29380 & -0.62785 & -2.06268 \\
\hline $\mathrm{C}$ & -0.54216 & -1.69875 & -2.91983 \\
\hline $\mathrm{H}$ & -1.73805 & 0.20622 & -2.95273 \\
\hline $\mathrm{C}$ & 0.82776 & -1.79831 & -2.63894 \\
\hline $\mathrm{C}$ & 1.59008 & -2.92841 & -2.88625 \\
\hline $\mathrm{C}$ & -1.18936 & -2.79918 & -3.48591 \\
\hline $\mathrm{C}$ & -0.44416 & -3.94821 & -3.75790 \\
\hline $\mathrm{H}$ & -0.93523 & -4.81310 & -4.19354 \\
\hline $\mathrm{C}$ & 0.92395 & -4.01345 & -3.46344 \\
\hline $\mathrm{H}$ & 1.47637 & -4.92304 & -3.67939 \\
\hline $\mathrm{H}$ & -2.25371 & -2.76837 & -3.69969 \\
\hline $\mathrm{H}$ & 2.64756 & -2.96348 & -2.64495 \\
\hline \multicolumn{4}{|l|}{77} \\
\hline \multicolumn{4}{|c|}{$3 a-(S)-6-a$} \\
\hline $\mathrm{C}$ & -0.35800 & -1.14798 & -2.45011 \\
\hline $\mathrm{H}$ & -1.19696 & -1.61000 & -1.90557 \\
\hline $\mathrm{O}$ & -1.77627 & -0.66716 & -0.10924 \\
\hline $\mathrm{C}$ & -0.07423 & -2.29731 & 3.41685 \\
\hline $\mathrm{C}$ & 0.71759 & -0.32973 & 4.36188 \\
\hline $\mathrm{C}$ & -0.43030 & -1.73880 & 2.02996 \\
\hline $\mathrm{H}$ & 0.54486 & 0.39468 & 5.15944 \\
\hline $\mathrm{H}$ & 0.97149 & -2.64648 & 3.42560 \\
\hline $\mathrm{H}$ & -0.72667 & -3.14607 & 3.62484 \\
\hline $\mathrm{H}$ & -1.50778 & -1.68092 & 1.86929 \\
\hline $\mathrm{H}$ & 1.73078 & -0.75075 & 4.47108 \\
\hline $\mathrm{O}$ & -0.26004 & -1.35780 & 4.48073 \\
\hline $\mathrm{C}$ & 0.56185 & 0.34179 & 3.03456 \\
\hline $\mathrm{N}$ & 0.78814 & 1.60010 & 2.76965 \\
\hline $\mathrm{N}$ & 0.38104 & 1.72690 & 1.45061 \\
\hline $\mathrm{C}$ & -0.09691 & 0.57564 & 0.93428 \\
\hline $\mathrm{N}$ & 0.04121 & -0.32740 & 1.95006 \\
\hline $\mathrm{C}$ & 0.53705 & 2.99421 & 0.81494 \\
\hline $\mathrm{C}$ & -0.43664 & 3.98641 & 1.00047 \\
\hline $\mathrm{C}$ & 1.70775 & 3.25484 & 0.08858 \\
\hline $\mathrm{C}$ & -0.28828 & 5.21719 & 0.35133 \\
\hline $\mathrm{C}$ & 1.85246 & 4.48609 & -0.56014 \\
\hline $\mathrm{C}$ & 0.84434 & 5.43907 & -0.42712 \\
\hline $\mathrm{H}$ & -1.04616 & 5.98452 & 0.44224 \\
\hline $\mathrm{H}$ & 2.73576 & 4.70198 & -1.14837 \\
\hline $\mathrm{H}$ & 0.95200 & 6.39190 & -0.93721 \\
\hline $\mathrm{O}$ & 2.62665 & 2.25456 & 0.08100 \\
\hline $\mathrm{O}$ & -1.46671 & 3.65552 & 1.81644 \\
\hline
\end{tabular}




\begin{tabular}{|c|c|c|c|}
\hline $\mathrm{C}$ & -2.50008 & 4.63173 & 1.99897 \\
\hline $\mathrm{H}$ & -3.22406 & 4.16074 & 2.66345 \\
\hline $\mathrm{H}$ & -2.09566 & 5.53763 & 2.46521 \\
\hline $\mathrm{H}$ & -2.97169 & 4.87317 & 1.04071 \\
\hline $\mathrm{C}$ & 3.77121 & 2.40871 & -0.76482 \\
\hline $\mathrm{H}$ & 4.39448 & 3.24584 & -0.42885 \\
\hline $\mathrm{H}$ & 4.31970 & 1.47160 & -0.67693 \\
\hline $\mathrm{H}$ & 3.46689 & 2.56242 & -1.80675 \\
\hline $\mathrm{C}$ & 0.18473 & -2.62652 & 0.94075 \\
\hline $\mathrm{H}$ & 0.04997 & -2.10826 & -0.00444 \\
\hline $\mathrm{H}$ & 1.25663 & -2.76403 & 1.12248 \\
\hline $\mathrm{C}$ & -0.52775 & -3.95462 & 0.86584 \\
\hline $\mathrm{C}$ & 0.09778 & -5.14931 & 1.23308 \\
\hline $\mathrm{C}$ & -1.85318 & -3.99099 & 0.40860 \\
\hline $\mathrm{C}$ & -0.58575 & -6.36397 & 1.15242 \\
\hline $\mathrm{H}$ & 1.13112 & -5.12972 & 1.57303 \\
\hline $\mathrm{C}$ & -2.53560 & -5.20320 & 0.32929 \\
\hline $\mathrm{H}$ & -2.32471 & -3.05766 & 0.10675 \\
\hline $\mathrm{C}$ & -1.90543 & -6.39298 & 0.70273 \\
\hline $\mathrm{H}$ & -0.08615 & -7.28611 & 1.43717 \\
\hline $\mathrm{H}$ & -3.56066 & -5.22193 & -0.03133 \\
\hline $\mathrm{H}$ & -2.43879 & -7.33741 & 0.63786 \\
\hline $\mathrm{C}$ & -0.90476 & 0.30088 & -0.37696 \\
\hline $\mathrm{C}$ & -1.56464 & 1.58906 & -0.89974 \\
\hline $\mathrm{C}$ & -0.92138 & 2.13004 & -2.01302 \\
\hline $\mathrm{C}$ & -2.78507 & 2.09377 & -0.46304 \\
\hline $\mathrm{C}$ & -1.47045 & 3.22517 & -2.67973 \\
\hline $\mathrm{C}$ & -3.33628 & 3.19043 & -1.12682 \\
\hline $\mathrm{H}$ & -3.29904 & 1.60835 & 0.36139 \\
\hline $\mathrm{C}$ & -2.67582 & 3.76074 & -2.22235 \\
\hline $\mathrm{H}$ & -0.97843 & 3.64789 & -3.55275 \\
\hline $\mathrm{H}$ & -4.29647 & 3.59161 & -0.81110 \\
\hline $\mathrm{H}$ & -3.12043 & 4.60788 & -2.73824 \\
\hline $\mathrm{C}$ & 0.32385 & 1.35373 & -2.35124 \\
\hline $\mathrm{H}$ & 1.22032 & 1.86593 & -1.98802 \\
\hline $\mathrm{H}$ & 0.45224 & 1.20050 & -3.42913 \\
\hline $\mathrm{C}$ & 0.15695 & 0.02411 & -1.59251 \\
\hline $\mathrm{O}$ & 1.47071 & -0.39675 & -1.06751 \\
\hline $\mathrm{C}$ & 0.83801 & -2.05634 & -2.54009 \\
\hline $\mathrm{H}$ & -0.72490 & -0.80871 & -3.42397 \\
\hline $\mathrm{C}$ & 1.83357 & -1.55598 & -1.69854 \\
\hline $\mathrm{C}$ & 3.04904 & -2.20286 & -1.50730 \\
\hline $\mathrm{C}$ & 1.05788 & -3.24460 & -3.22574 \\
\hline $\mathrm{C}$ & 2.27745 & -3.91439 & -3.05862 \\
\hline $\mathrm{H}$ & 2.45985 & -4.84441 & -3.58853 \\
\hline $\mathrm{C}$ & 3.25696 & -3.39693 & -2.20769 \\
\hline $\mathrm{H}$ & 4.19734 & -3.92724 & -2.08474 \\
\hline $\mathrm{H}$ & 0.29092 & -3.65679 & -3.87633 \\
\hline
\end{tabular}




\begin{tabular}{|c|c|c|c|}
\hline $\mathrm{H}$ & 3.80149 & -1.79527 & -0.83897 \\
\hline \multicolumn{4}{|c|}{77} \\
\hline \multicolumn{4}{|c|}{$3 a-(S)-t s 3-a$} \\
\hline $\mathrm{C}$ & -0.44327 & -1.05302 & -2.79161 \\
\hline $\mathrm{H}$ & -1.27505 & -1.64143 & -2.38497 \\
\hline $\mathrm{O}$ & -1.84344 & -0.85416 & -0.30621 \\
\hline $\mathrm{C}$ & -0.59539 & -2.23518 & 3.55536 \\
\hline $\mathrm{C}$ & -0.20320 & -0.19578 & 4.60874 \\
\hline $\mathrm{C}$ & -0.64732 & -1.72117 & 2.10979 \\
\hline $\mathrm{H}$ & -0.64228 & 0.53169 & 5.29375 \\
\hline $\mathrm{H}$ & 0.44213 & -2.49460 & 3.82510 \\
\hline $\mathrm{H}$ & -1.21111 & -3.13191 & 3.63460 \\
\hline $\mathrm{H}$ & -1.66401 & -1.74620 & 1.71315 \\
\hline $\mathrm{H}$ & 0.76344 & -0.53386 & 5.01784 \\
\hline $\mathrm{O}$ & -1.10503 & -1.29444 & 4.50617 \\
\hline $\mathrm{C}$ & -0.01835 & 0.42372 & 3.25906 \\
\hline $\mathrm{N}$ & 0.28974 & 1.66462 & 3.00133 \\
\hline $\mathrm{N}$ & 0.23747 & 1.70945 & 1.60893 \\
\hline $\mathrm{C}$ & -0.09400 & 0.53878 & 1.02957 \\
\hline $\mathrm{N}$ & -0.24002 & -0.29550 & 2.10378 \\
\hline $\mathrm{C}$ & 0.58154 & 2.92796 & 0.95982 \\
\hline $\mathrm{C}$ & -0.28385 & 4.02931 & 1.04140 \\
\hline $\mathrm{C}$ & 1.81523 & 3.03064 & 0.29981 \\
\hline $\mathrm{C}$ & 0.06103 & 5.22320 & 0.39640 \\
\hline $\mathrm{C}$ & 2.14972 & 4.22007 & -0.35820 \\
\hline $\mathrm{C}$ & 1.26315 & 5.29387 & -0.30146 \\
\hline $\mathrm{H}$ & -0.60386 & 6.07699 & 0.42331 \\
\hline $\mathrm{H}$ & 3.08858 & 4.31354 & -0.88990 \\
\hline $\mathrm{H}$ & 1.52254 & 6.21754 & -0.81081 \\
\hline $\mathrm{O}$ & 2.60680 & 1.92658 & 0.35708 \\
\hline $\mathrm{O}$ & -1.41905 & 3.83729 & 1.75731 \\
\hline $\mathrm{C}$ & -2.32581 & 4.93993 & 1.86180 \\
\hline $\mathrm{H}$ & -3.15676 & 4.57086 & 2.46275 \\
\hline $\mathrm{H}$ & -1.84668 & 5.78802 & 2.36508 \\
\hline $\mathrm{H}$ & -2.68579 & 5.23947 & 0.87176 \\
\hline $\mathrm{C}$ & 3.80153 & 1.92398 & -0.43100 \\
\hline $\mathrm{H}$ & 4.50607 & 2.68596 & -0.07660 \\
\hline $\mathrm{H}$ & 4.22702 & 0.92946 & -0.30182 \\
\hline $\mathrm{H}$ & 3.56974 & 2.09092 & -1.48939 \\
\hline $\mathrm{C}$ & 0.26584 & -2.56625 & 1.20920 \\
\hline $\mathrm{H}$ & 0.34713 & -2.05407 & 0.25213 \\
\hline $\mathrm{H}$ & 1.26881 & -2.61395 & 1.65170 \\
\hline $\mathrm{C}$ & -0.29143 & -3.95494 & 1.00967 \\
\hline $\mathrm{C}$ & 0.32186 & -5.07682 & 1.57596 \\
\hline $\mathrm{C}$ & -1.45463 & -4.13059 & 0.24639 \\
\hline $\mathrm{C}$ & -0.21170 & -6.35304 & 1.38814 \\
\hline $\mathrm{H}$ & 1.23011 & -4.95083 & 2.16194 \\
\hline
\end{tabular}




\begin{tabular}{|c|c|c|c|}
\hline $\mathrm{C}$ & -1.98852 & -5.40408 & 0.05991 \\
\hline $\mathrm{H}$ & -1.92262 & -3.25552 & -0.19889 \\
\hline $\mathrm{C}$ & -1.37033 & -6.51934 & 0.63036 \\
\hline $\mathrm{H}$ & 0.27913 & -7.21551 & 1.83115 \\
\hline $\mathrm{H}$ & -2.88768 & -5.52853 & -0.53775 \\
\hline $\mathrm{H}$ & -1.78720 & -7.51158 & 0.48136 \\
\hline $\mathrm{C}$ & -1.09412 & 0.11758 & -0.60921 \\
\hline $\mathrm{C}$ & -1.68808 & 1.47274 & -0.93548 \\
\hline $\mathrm{C}$ & -0.92932 & 2.14652 & -1.89563 \\
\hline $\mathrm{C}$ & -2.90253 & 1.98204 & -0.48733 \\
\hline $\mathrm{C}$ & -1.37226 & 3.36360 & -2.40950 \\
\hline $\mathrm{C}$ & -3.34853 & 3.19882 & -1.00355 \\
\hline $\mathrm{H}$ & -3.49047 & 1.41924 & 0.23233 \\
\hline $\mathrm{C}$ & -2.58353 & 3.88827 & -1.95258 \\
\hline $\mathrm{H}$ & -0.79160 & 3.89531 & -3.15942 \\
\hline $\mathrm{H}$ & -4.30082 & 3.61080 & -0.67909 \\
\hline $\mathrm{H}$ & -2.94572 & 4.83220 & -2.35191 \\
\hline $\mathrm{C}$ & 0.31024 & 1.36586 & -2.25566 \\
\hline $\mathrm{H}$ & 1.18944 & 1.77714 & -1.75245 \\
\hline $\mathrm{H}$ & 0.51696 & 1.36522 & -3.33175 \\
\hline $\mathrm{C}$ & 0.03481 & -0.04849 & -1.71159 \\
\hline $\mathrm{O}$ & 1.27522 & -0.61445 & -1.16684 \\
\hline $\mathrm{C}$ & 0.77420 & -1.91151 & -3.00935 \\
\hline $\mathrm{H}$ & -0.79118 & -0.53827 & -3.69246 \\
\hline $\mathrm{C}$ & 1.69630 & -1.61236 & -2.00593 \\
\hline $\mathrm{C}$ & 2.90367 & -2.28795 & -1.87224 \\
\hline $\mathrm{C}$ & 1.06393 & -2.90946 & -3.93071 \\
\hline $\mathrm{C}$ & 2.28004 & -3.59874 & -3.82835 \\
\hline $\mathrm{H}$ & 2.51949 & -4.38095 & -4.54236 \\
\hline $\mathrm{C}$ & 3.18222 & -3.29149 & -2.80727 \\
\hline $\mathrm{H}$ & 4.11835 & -3.83821 & -2.73498 \\
\hline $\mathrm{H}$ & 0.35603 & -3.16022 & -4.71660 \\
\hline $\mathrm{H}$ & 3.59699 & -2.04478 & -1.07312 \\
\hline \multicolumn{4}{|c|}{77} \\
\hline \multicolumn{4}{|c|}{$3 a-(S)-[2 a+3 a]$} \\
\hline $\mathrm{C}$ & -1.53195 & -0.84596 & -3.23860 \\
\hline $\mathrm{H}$ & -2.60032 & -0.73383 & -3.02903 \\
\hline $\mathrm{O}$ & -2.04626 & -1.03343 & -0.11727 \\
\hline $\mathrm{C}$ & 0.48365 & -1.91754 & 3.74498 \\
\hline $\mathrm{C}$ & 0.80116 & 0.19539 & 4.70430 \\
\hline $\mathrm{C}$ & 0.19125 & -1.45066 & 2.31509 \\
\hline $\mathrm{H}$ & 0.34970 & 0.90223 & 5.40303 \\
\hline $\mathrm{H}$ & 1.56915 & -2.06536 & 3.87530 \\
\hline $\mathrm{H}$ & -0.02390 & -2.86493 & 3.93193 \\
\hline $\mathrm{H}$ & -0.87698 & -1.54658 & 2.09958 \\
\hline $\mathrm{H}$ & 1.82734 & -0.02957 & 5.04030 \\
\hline $\mathrm{O}$ & 0.00850 & -0.99244 & 4.72997 \\
\hline
\end{tabular}




\begin{tabular}{|c|c|c|c|}
\hline $\mathrm{C}$ & 0.81639 & 0.76512 & 3.32015 \\
\hline $\mathrm{N}$ & 1.05574 & 1.99751 & 2.97104 \\
\hline $\mathrm{N}$ & 0.88920 & 1.94129 & 1.57709 \\
\hline $\mathrm{C}$ & 0.56098 & 0.72974 & 1.06862 \\
\hline $\mathrm{N}$ & 0.53354 & -0.02004 & 2.22002 \\
\hline $\mathrm{C}$ & 1.06177 & 3.13663 & 0.82992 \\
\hline $\mathrm{C}$ & 0.22540 & 4.23843 & 1.07422 \\
\hline $\mathrm{C}$ & 2.07234 & 3.22325 & -0.14135 \\
\hline $\mathrm{C}$ & 0.41018 & 5.42870 & 0.35954 \\
\hline $\mathrm{C}$ & 2.23428 & 4.40411 & -0.87832 \\
\hline $\mathrm{C}$ & 1.40712 & 5.49150 & -0.60946 \\
\hline $\mathrm{H}$ & -0.22637 & 6.28564 & 0.54091 \\
\hline $\mathrm{H}$ & 3.00738 & 4.48276 & -1.63257 \\
\hline $\mathrm{H}$ & 1.54278 & 6.41111 & -1.17162 \\
\hline $\mathrm{O}$ & 2.85181 & 2.11651 & -0.29538 \\
\hline $\mathrm{O}$ & -0.74725 & 4.05004 & 2.00420 \\
\hline $\mathrm{C}$ & -1.58114 & 5.16593 & 2.32043 \\
\hline $\mathrm{H}$ & -2.26960 & 4.80270 & 3.08350 \\
\hline $\mathrm{H}$ & -0.98644 & 5.99705 & 2.71840 \\
\hline $\mathrm{H}$ & -2.14409 & 5.49794 & 1.44005 \\
\hline $\mathrm{C}$ & 3.87243 & 2.16095 & -1.29591 \\
\hline $\mathrm{H}$ & 4.60633 & 2.94591 & -1.07573 \\
\hline $\mathrm{H}$ & 4.35101 & 1.18237 & -1.25962 \\
\hline $\mathrm{H}$ & 3.43954 & 2.32579 & -2.29017 \\
\hline $\mathrm{C}$ & 0.99809 & -2.25787 & 1.28653 \\
\hline $\mathrm{H}$ & 0.85913 & -1.77837 & 0.31453 \\
\hline $\mathrm{H}$ & 2.06242 & -2.18839 & 1.54443 \\
\hline $\mathrm{C}$ & 0.56822 & -3.70420 & 1.22813 \\
\hline $\mathrm{C}$ & 1.41213 & -4.72878 & 1.67078 \\
\hline $\mathrm{C}$ & -0.69076 & -4.04437 & 0.71250 \\
\hline $\mathrm{C}$ & 1.01484 & -6.06527 & 1.60001 \\
\hline $\mathrm{H}$ & 2.39388 & -4.47696 & 2.06705 \\
\hline $\mathrm{C}$ & -1.08961 & -5.37730 & 0.64350 \\
\hline $\mathrm{H}$ & -1.34268 & -3.25695 & 0.34321 \\
\hline $\mathrm{C}$ & -0.23967 & -6.39288 & 1.08642 \\
\hline $\mathrm{H}$ & 1.68555 & -6.84820 & 1.94407 \\
\hline $\mathrm{H}$ & -2.06275 & -5.62499 & 0.22833 \\
\hline $\mathrm{H}$ & -0.55111 & -7.43216 & 1.02707 \\
\hline $\mathrm{C}$ & -1.59142 & -0.14562 & -0.82019 \\
\hline $\mathrm{C}$ & -1.93773 & 1.29110 & -0.83490 \\
\hline $\mathrm{C}$ & -1.08275 & 1.96679 & -1.72017 \\
\hline $\mathrm{C}$ & -2.97627 & 1.94737 & -0.17447 \\
\hline $\mathrm{C}$ & -1.27490 & 3.32120 & -1.96984 \\
\hline $\mathrm{C}$ & -3.16662 & 3.30270 & -0.43027 \\
\hline $\mathrm{H}$ & -3.62041 & 1.39980 & 0.50745 \\
\hline $\mathrm{C}$ & -2.32353 & 3.97952 & -1.32249 \\
\hline $\mathrm{H}$ & -0.61624 & 3.86569 & -2.64028 \\
\hline $\mathrm{H}$ & -3.97576 & 3.84028 & 0.05641 \\
\hline
\end{tabular}




$\begin{array}{lrrr}\mathrm{H} & -2.48491 & 5.03752 & -1.51122 \\ \mathrm{C} & -0.02277 & 1.03528 & -2.25407 \\ \mathrm{H} & 0.86767 & 1.09544 & -1.61699 \\ \mathrm{H} & 0.25165 & 1.22835 & -3.29585 \\ \mathrm{C} & -0.65348 & -0.34495 & -2.04210 \\ \mathrm{O} & 0.34524 & -1.37113 & -1.81191 \\ \mathrm{C} & -1.10515 & -2.28554 & -3.36006 \\ \mathrm{H} & -1.30910 & -0.27203 & -4.14695 \\ \mathrm{C} & -0.01431 & -2.49289 & -2.51798 \\ \mathrm{C} & 0.63318 & -3.71776 & -2.41992 \\ \mathrm{C} & -1.58639 & -3.33263 & -4.13544 \\ \mathrm{C} & -0.95815 & -4.58199 & -4.05060 \\ \mathrm{H} & -1.32772 & -5.41363 & -4.64282 \\ \mathrm{C} & 0.13798 & -4.76442 & -3.20374 \\ \mathrm{H} & 0.61018 & -5.74041 & -3.13794 \\ \mathrm{H} & -2.43896 & -3.18946 & -4.79444 \\ \mathrm{H} & 1.46833 & -3.86018 & -1.74333\end{array}$




\subsection{3d-(S)-a-path}

76

3d-(S)-4-a

$\begin{array}{llll}\text { C } & -1.80976 & -0.66860 & -2.01512\end{array}$

$\begin{array}{llll}\mathrm{H} & -2.35226 & -0.54622 & 0.90664\end{array}$

$\begin{array}{llll}\text { O } & -1.77276 & -0.14821 & 1.58046\end{array}$

$\begin{array}{llll}\text { C } & 2.05357 & -2.15892 & 2.78352\end{array}$

$\begin{array}{llll}\text { C } & 3.17237 & -1.16929 & 2.34736\end{array}$

$\begin{array}{llll}\text { C } & 0.77889 & -1.72624 & 2.01438\end{array}$

$\begin{array}{llll}\mathrm{H} & 3.86260 & -0.92774 & 3.15925\end{array}$

$\mathrm{H} \quad 2.30754 \quad-3.20116 \quad 2.57858$

$\mathrm{H} \quad 1.87415 \quad-2.05954 \quad 3.85774$

$\begin{array}{llll}\mathrm{H} & -0.12895 & -1.88287 & 2.59867\end{array}$

$\begin{array}{llll}\mathrm{H} & 3.76606 & -1.56390 & 1.51535\end{array}$

$\begin{array}{llll}\text { C } & 2.38078 & 0.01390 & 1.88295\end{array}$

$\begin{array}{llll}\mathrm{N} & 2.66410 & 1.18068 & 1.39809\end{array}$

$\begin{array}{llll}\mathrm{N} & 1.39355 & 1.67200 & 0.98141\end{array}$

$\begin{array}{llll}\mathrm{C} & 0.37130 & 0.73507 & 1.20360\end{array}$

$\begin{array}{llll}\mathrm{N} & 1.03756 & -0.29125 & 1.86487\end{array}$

$\begin{array}{llll}\text { C } & 1.23200 & 3.03568 & 0.67194\end{array}$

$\begin{array}{llll}\text { C } & 0.38793 & 3.85309 & 1.45009\end{array}$

$\begin{array}{llll}\text { C } & 1.89609 & 3.59228 & -0.43855\end{array}$

$\begin{array}{llll}\text { C } & 0.14548 & 5.17787 & 1.07201\end{array}$

$\begin{array}{llll}\text { C } & 1.68107 & 4.92741 & -0.79443\end{array}$

$\begin{array}{llll}\text { C } & 0.79437 & 5.69689 & -0.04425\end{array}$

$\begin{array}{lllr}\mathrm{H} & -0.52746 & 5.80109 & 1.64754\end{array}$

$\mathrm{H} \quad 2.18103 \quad 5.35813 \quad-1.65324$

$\begin{array}{llll}\mathrm{H} & 0.61178 & 6.72797 & -0.33234\end{array}$

$\begin{array}{llll}\mathrm{O} & 2.71138 & 2.74042 & -1.12473\end{array}$

$\begin{array}{llll}\text { O } & -0.14585 & 3.26496 & 2.55538\end{array}$

$\begin{array}{llll}\text { C } & -1.07092 & 4.03397 & 3.32802\end{array}$

$\mathrm{H} \quad-1.40549 \quad 3.36646 \quad 4.12242$

$\begin{array}{llll}\mathrm{H} & -0.58121 & 4.91501 & 3.76102\end{array}$

$\begin{array}{llll}\mathrm{H} & -1.92475 & 4.34247 & 2.71418\end{array}$

$\begin{array}{llll}\text { C } & 3.35371 & 3.24531 & -2.29636\end{array}$

$\begin{array}{llll}\mathrm{H} & 4.03021 & 4.07188 & -2.04747\end{array}$

$\mathrm{H} \quad 3.92500 \quad 2.40682 \quad-2.69524$

$\begin{array}{llll}\mathrm{H} & 2.61673 & 3.58117 & -3.03683\end{array}$

$\begin{array}{llll}\text { C } & 0.61886 & -2.37286 & 0.62299\end{array}$

$\begin{array}{llll}\mathrm{H} & -0.15239 & -1.80500 & 0.09307\end{array}$

$\begin{array}{llll}\mathrm{H} & 1.55216 & -2.24703 & 0.06228\end{array}$

$\begin{array}{llll}\text { C } & 0.23818 & -3.82649 & 0.68604\end{array}$

$\begin{array}{llll}\text { C } & 1.18256 & -4.83400 & 0.45995\end{array}$

$\begin{array}{llll}\text { C } & -1.07835 & -4.20124 & 0.98176\end{array}$

$\begin{array}{llll}\text { C } & 0.82482 & -6.18040 & 0.52400\end{array}$

$\begin{array}{llll}\mathrm{H} & 2.20459 & -4.55745 & 0.20956\end{array}$

$\begin{array}{llll}\text { C } & -1.44129 & -5.54452 & 1.04916\end{array}$ 


\begin{tabular}{|c|c|c|c|}
\hline $\mathrm{H}$ & -1.82691 & -3.42906 & 1.14261 \\
\hline $\mathrm{C}$ & -0.49015 & -6.53991 & 0.81951 \\
\hline $\mathrm{H}$ & 1.57063 & -6.94813 & 0.33615 \\
\hline $\mathrm{H}$ & -2.47019 & -5.81586 & 1.26942 \\
\hline $\mathrm{H}$ & -0.77344 & -7.58768 & 0.86529 \\
\hline $\mathrm{C}$ & -0.95903 & 0.79016 & 0.90130 \\
\hline $\mathrm{C}$ & -1.63810 & 1.82618 & 0.13601 \\
\hline $\mathrm{C}$ & -1.21924 & 2.18754 & -1.16897 \\
\hline $\mathrm{C}$ & -2.78189 & 2.45226 & 0.66910 \\
\hline $\mathrm{C}$ & -1.87288 & 3.22517 & -1.83542 \\
\hline $\mathrm{C}$ & -3.43640 & 3.46829 & -0.01890 \\
\hline $\mathrm{H}$ & -3.12888 & 2.13869 & 1.64960 \\
\hline $\mathrm{C}$ & -2.96452 & 3.88151 & -1.26727 \\
\hline $\mathrm{H}$ & -1.53623 & 3.50239 & -2.83209 \\
\hline $\mathrm{H}$ & -4.30508 & 3.94945 & 0.42347 \\
\hline $\mathrm{H}$ & -3.45906 & 4.68467 & -1.80570 \\
\hline $\mathrm{C}$ & -0.16318 & 1.37782 & -1.88453 \\
\hline $\mathrm{H}$ & 0.79204 & 1.36019 & -1.34817 \\
\hline $\mathrm{H}$ & 0.04469 & 1.83707 & -2.86091 \\
\hline $\mathrm{C}$ & -0.60399 & -0.03545 & -2.08998 \\
\hline $\mathrm{O}$ & 0.40091 & -0.92253 & -2.45632 \\
\hline $\mathrm{C}$ & -1.57793 & -2.05567 & -2.33017 \\
\hline $\mathrm{H}$ & -2.74733 & -0.19133 & -1.76678 \\
\hline $\mathrm{C}$ & -0.19784 & -2.15640 & -2.59828 \\
\hline $\mathrm{C}$ & 0.44510 & -3.34246 & -2.91426 \\
\hline $\mathrm{C}$ & -2.35721 & -3.22081 & -2.38641 \\
\hline $\mathrm{C}$ & -1.73050 & -4.42477 & -2.69827 \\
\hline $\mathrm{H}$ & -2.31551 & -5.33911 & -2.73084 \\
\hline $\mathrm{C}$ & -0.35215 & -4.48785 & -2.95875 \\
\hline $\mathrm{H}$ & 0.10545 & -5.44604 & -3.18397 \\
\hline $\mathrm{H}$ & -3.42352 & -3.18851 & -2.18085 \\
\hline $\mathrm{H}$ & 1.51336 & -3.37741 & -3.10133 \\
\hline \multicolumn{4}{|c|}{76} \\
\hline \multicolumn{4}{|c|}{$3 d-(S)-t s 1-a$} \\
\hline $\mathrm{C}$ & -0.30527 & -0.93180 & -2.55699 \\
\hline $\mathrm{H}$ & -1.22594 & -1.03192 & -1.55430 \\
\hline $\mathrm{O}$ & -1.75577 & -0.59893 & -0.48505 \\
\hline $\mathrm{C}$ & -0.02769 & -1.95780 & 3.38412 \\
\hline $\mathrm{C}$ & 1.21069 & -1.04121 & 3.59840 \\
\hline $\mathrm{C}$ & -0.44069 & -1.77724 & 1.89709 \\
\hline $\mathrm{H}$ & 1.28934 & -0.65437 & 4.61590 \\
\hline $\mathrm{H}$ & 0.17530 & -3.00625 & 3.61157 \\
\hline $\mathrm{H}$ & -0.84167 & -1.61901 & 4.03199 \\
\hline $\mathrm{H}$ & -1.52094 & -1.77947 & 1.75462 \\
\hline $\mathrm{H}$ & 2.14557 & -1.56243 & 3.36040 \\
\hline $\mathrm{C}$ & 0.94675 & 0.03215 & 2.59404 \\
\hline $\mathrm{N}$ & 1.28740 & 1.27540 & 2.42462 \\
\hline
\end{tabular}




\begin{tabular}{|c|c|c|c|}
\hline $\mathrm{N}$ & 0.56940 & 1.64259 & 1.27404 \\
\hline $\mathrm{C}$ & -0.19986 & 0.61488 & 0.77296 \\
\hline $\mathrm{N}$ & 0.06261 & -0.41212 & 1.64355 \\
\hline $\mathrm{C}$ & 0.63887 & 2.97826 & 0.80686 \\
\hline $\mathrm{C}$ & -0.39648 & 3.87862 & 1.10502 \\
\hline $\mathrm{C}$ & 1.76062 & 3.40110 & 0.07529 \\
\hline $\mathrm{C}$ & -0.35262 & 5.18029 & 0.59304 \\
\hline $\mathrm{C}$ & 1.80970 & 4.70706 & -0.42633 \\
\hline $\mathrm{C}$ & 0.74520 & 5.56863 & -0.16978 \\
\hline $\mathrm{H}$ & -1.15872 & 5.87797 & 0.78099 \\
\hline $\mathrm{H}$ & 2.65853 & 5.04663 & -1.00700 \\
\hline $\mathrm{H}$ & 0.77684 & 6.57794 & -0.56999 \\
\hline $\mathrm{O}$ & 2.73096 & 2.46673 & -0.10048 \\
\hline $\mathrm{O}$ & -1.39100 & 3.39142 & 1.89319 \\
\hline $\mathrm{C}$ & -2.47193 & 4.27774 & 2.20455 \\
\hline $\mathrm{H}$ & -3.15087 & 3.69490 & 2.82710 \\
\hline $\mathrm{H}$ & -2.10896 & 5.14984 & 2.76124 \\
\hline $\mathrm{H}$ & -2.98351 & 4.59767 & 1.29036 \\
\hline $\mathrm{C}$ & 3.84564 & 2.81452 & -0.92623 \\
\hline $\mathrm{H}$ & 4.41996 & 3.63663 & -0.48246 \\
\hline $\mathrm{H}$ & 4.45650 & 1.91372 & -0.97584 \\
\hline $\mathrm{H}$ & 3.51449 & 3.09291 & -1.93425 \\
\hline $\mathrm{C}$ & 0.20387 & -2.80246 & 0.95184 \\
\hline $\mathrm{H}$ & 0.07877 & -2.43582 & -0.06749 \\
\hline $\mathrm{H}$ & 1.27899 & -2.86784 & 1.15075 \\
\hline $\mathrm{C}$ & -0.44260 & -4.15668 & 1.08059 \\
\hline $\mathrm{C}$ & 0.21691 & -5.22904 & 1.68900 \\
\hline $\mathrm{C}$ & -1.74058 & -4.35590 & 0.58981 \\
\hline $\mathrm{C}$ & -0.40205 & -6.47433 & 1.80951 \\
\hline $\mathrm{H}$ & 1.23134 & -5.09042 & 2.05714 \\
\hline $\mathrm{C}$ & -2.36208 & -5.59715 & 0.70974 \\
\hline $\mathrm{H}$ & -2.25494 & -3.52998 & 0.10351 \\
\hline $\mathrm{C}$ & -1.69509 & -6.66090 & 1.32174 \\
\hline $\mathrm{H}$ & 0.12719 & -7.29857 & 2.28020 \\
\hline $\mathrm{H}$ & -3.36661 & -5.73783 & 0.31977 \\
\hline $\mathrm{H}$ & -2.17857 & -7.62960 & 1.41199 \\
\hline $\mathrm{C}$ & -1.04799 & 0.53979 & -0.35745 \\
\hline $\mathrm{C}$ & -1.60894 & 1.76429 & -1.01018 \\
\hline $\mathrm{C}$ & -0.94249 & 2.31659 & -2.11281 \\
\hline $\mathrm{C}$ & -2.84251 & 2.29709 & -0.61747 \\
\hline $\mathrm{C}$ & -1.47918 & 3.42743 & -2.76899 \\
\hline $\mathrm{C}$ & -3.37950 & 3.40024 & -1.27738 \\
\hline $\mathrm{H}$ & -3.37490 & 1.83389 & 0.20808 \\
\hline $\mathrm{C}$ & -2.68835 & 3.97615 & -2.34709 \\
\hline $\mathrm{H}$ & -0.95703 & 3.85432 & -3.62230 \\
\hline $\mathrm{H}$ & -4.33994 & 3.80609 & -0.96907 \\
\hline $\mathrm{H}$ & -3.10395 & 4.83647 & -2.86486 \\
\hline $\mathrm{C}$ & 0.33872 & 1.64769 & -2.54472 \\
\hline
\end{tabular}




\begin{tabular}{|c|c|c|c|}
\hline $\mathrm{H}$ & 1.20200 & 2.17740 & -2.12862 \\
\hline $\mathrm{H}$ & 0.43826 & 1.69360 & -3.63977 \\
\hline $\mathrm{C}$ & 0.40193 & 0.22669 & -2.05792 \\
\hline $\mathrm{O}$ & 1.68841 & -0.15497 & -1.62095 \\
\hline $\mathrm{C}$ & 0.65546 & -2.03787 & -2.42926 \\
\hline $\mathrm{H}$ & -0.94944 & -0.83778 & -3.43080 \\
\hline $\mathrm{C}$ & 1.80262 & -1.51465 & -1.80906 \\
\hline $\mathrm{C}$ & 2.88923 & -2.28432 & -1.42444 \\
\hline $\mathrm{C}$ & 0.60469 & -3.40960 & -2.68721 \\
\hline $\mathrm{C}$ & 1.69027 & -4.20871 & -2.31576 \\
\hline $\mathrm{H}$ & 1.65635 & -5.27782 & -2.50412 \\
\hline $\mathrm{C}$ & 2.81568 & -3.65636 & -1.69174 \\
\hline $\mathrm{H}$ & 3.64390 & -4.29988 & -1.40933 \\
\hline $\mathrm{H}$ & -0.27193 & -3.85467 & -3.14971 \\
\hline $\mathrm{H}$ & 3.75159 & -1.83964 & -0.93744 \\
\hline \multicolumn{4}{|l|}{76} \\
\hline \multicolumn{4}{|c|}{$3 d-(S)-6-a$} \\
\hline $\mathrm{C}$ & -1.83768 & -0.83475 & -1.93322 \\
\hline $\mathrm{H}$ & -2.30745 & -1.27824 & -1.04052 \\
\hline $\mathrm{O}$ & -1.64730 & -0.60408 & 0.80819 \\
\hline $\mathrm{C}$ & 1.61687 & -2.13418 & 3.01829 \\
\hline $\mathrm{C}$ & 2.81081 & -1.16213 & 2.78135 \\
\hline $\mathrm{C}$ & 0.59026 & -1.87053 & 1.88076 \\
\hline $\mathrm{H}$ & 3.26585 & -0.80664 & 3.70785 \\
\hline $\mathrm{H}$ & 1.92608 & -3.18123 & 3.03162 \\
\hline $\mathrm{H}$ & 1.15455 & -1.90673 & 3.98295 \\
\hline $\mathrm{H}$ & -0.44490 & -1.83963 & 2.21618 \\
\hline $\mathrm{H}$ & 3.59943 & -1.62270 & 2.17496 \\
\hline $\mathrm{C}$ & 2.15078 & -0.06722 & 2.01031 \\
\hline $\mathrm{N}$ & 2.39824 & 1.18101 & 1.71095 \\
\hline $\mathrm{N}$ & 1.27754 & 1.53864 & 0.95665 \\
\hline $\mathrm{C}$ & 0.38786 & 0.52649 & 0.81294 \\
\hline $\mathrm{N}$ & 0.95276 & -0.48650 & 1.50082 \\
\hline $\mathrm{C}$ & 1.17133 & 2.87563 & 0.47563 \\
\hline $\mathrm{C}$ & 0.54099 & 3.84423 & 1.27094 \\
\hline $\mathrm{C}$ & 1.74636 & 3.21446 & -0.75760 \\
\hline $\mathrm{C}$ & 0.39909 & 5.14676 & 0.77950 \\
\hline $\mathrm{C}$ & 1.60316 & 4.51814 & -1.24611 \\
\hline $\mathrm{C}$ & 0.92161 & 5.45621 & -0.47311 \\
\hline $\mathrm{H}$ & -0.11728 & 5.90276 & 1.35684 \\
\hline $\mathrm{H}$ & 2.01840 & 4.79861 & -2.20615 \\
\hline $\mathrm{H}$ & 0.80308 & 6.46560 & -0.85630 \\
\hline $\mathrm{O}$ & 2.40664 & 2.21186 & -1.39192 \\
\hline $\mathrm{O}$ & 0.11264 & 3.42039 & 2.48572 \\
\hline $\mathrm{C}$ & -0.56995 & 4.37356 & 3.31034 \\
\hline $\mathrm{H}$ & -0.84059 & 3.82783 & 4.21401 \\
\hline $\mathrm{H}$ & 0.09271 & 5.21011 & 3.56106 \\
\hline
\end{tabular}




\begin{tabular}{|c|c|c|c|}
\hline $\mathrm{H}$ & -1.47222 & 4.73714 & 2.80805 \\
\hline $\mathrm{C}$ & 2.87726 & 2.46224 & -2.72045 \\
\hline $\mathrm{H}$ & 3.65238 & 3.23777 & -2.72061 \\
\hline $\mathrm{H}$ & 3.29401 & 1.51427 & -3.05906 \\
\hline $\mathrm{H}$ & 2.05031 & 2.75898 & -3.37633 \\
\hline $\mathrm{C}$ & 0.69810 & -2.82236 & 0.68122 \\
\hline $\mathrm{H}$ & 0.16408 & -2.35542 & -0.14720 \\
\hline $\mathrm{H}$ & 1.74615 & -2.94923 & 0.38435 \\
\hline $\mathrm{C}$ & 0.06042 & -4.15114 & 0.99637 \\
\hline $\mathrm{C}$ & 0.81736 & -5.31324 & 1.16817 \\
\hline $\mathrm{C}$ & -1.33342 & -4.22074 & 1.13464 \\
\hline $\mathrm{C}$ & 0.19901 & -6.52776 & 1.47280 \\
\hline $\mathrm{H}$ & 1.89827 & -5.27037 & 1.04914 \\
\hline $\mathrm{C}$ & -1.95129 & -5.43138 & 1.43923 \\
\hline $\mathrm{H}$ & -1.91952 & -3.31419 & 0.99500 \\
\hline $\mathrm{C}$ & -1.18704 & -6.58868 & 1.61033 \\
\hline $\mathrm{H}$ & 0.79962 & -7.42466 & 1.59872 \\
\hline $\mathrm{H}$ & -3.03256 & -5.47497 & 1.53880 \\
\hline $\mathrm{H}$ & -1.67083 & -7.53284 & 1.84490 \\
\hline $\mathrm{C}$ & -1.03112 & 0.39518 & 0.18541 \\
\hline $\mathrm{C}$ & -1.77358 & 1.74174 & 0.20743 \\
\hline $\mathrm{C}$ & -1.77426 & 2.39071 & -1.02806 \\
\hline $\mathrm{C}$ & -2.51121 & 2.23793 & 1.27677 \\
\hline $\mathrm{C}$ & -2.48444 & 3.57917 & -1.19722 \\
\hline $\mathrm{C}$ & -3.22137 & 3.42808 & 1.11134 \\
\hline $\mathrm{H}$ & -2.54376 & 1.67843 & 2.20747 \\
\hline $\mathrm{C}$ & -3.19864 & 4.10082 & -0.11685 \\
\hline $\mathrm{H}$ & -2.49650 & 4.08740 & -2.15887 \\
\hline $\mathrm{H}$ & -3.81526 & 3.82688 & 1.93054 \\
\hline $\mathrm{H}$ & -3.76373 & 5.02152 & -0.23733 \\
\hline $\mathrm{C}$ & -0.98030 & 1.61857 & -2.05124 \\
\hline $\mathrm{H}$ & 0.01286 & 2.05549 & -2.19458 \\
\hline $\mathrm{H}$ & -1.46670 & 1.58790 & -3.03335 \\
\hline $\mathrm{C}$ & -0.83202 & 0.21882 & -1.43071 \\
\hline $\mathrm{O}$ & 0.51009 & -0.31187 & -1.73471 \\
\hline $\mathrm{C}$ & -0.96752 & -1.82129 & -2.66180 \\
\hline $\mathrm{H}$ & -2.61588 & -0.39041 & -2.56219 \\
\hline $\mathrm{C}$ & 0.36635 & -1.45258 & -2.47667 \\
\hline $\mathrm{C}$ & 1.42770 & -2.19660 & -2.97986 \\
\hline $\mathrm{C}$ & -1.26237 & -2.97389 & -3.37968 \\
\hline $\mathrm{C}$ & -0.21292 & -3.74114 & -3.90322 \\
\hline $\mathrm{H}$ & -0.43187 & -4.64544 & -4.46304 \\
\hline $\mathrm{C}$ & 1.11401 & -3.35413 & -3.70141 \\
\hline $\mathrm{H}$ & 1.91882 & -3.95958 & -4.10933 \\
\hline $\mathrm{H}$ & -2.29386 & -3.28337 & -3.52747 \\
\hline $\mathrm{H}$ & 2.45555 & -1.88945 & -2.81237 \\
\hline
\end{tabular}

76 


\begin{tabular}{|c|c|c|c|}
\hline \multicolumn{4}{|c|}{$3 d-(S)-t s 3-a$} \\
\hline $\mathrm{C}$ & -1.58584 & -0.82632 & -2.54590 \\
\hline $\mathrm{H}$ & -2.50950 & -1.00178 & -1.98572 \\
\hline $\mathrm{O}$ & -1.89606 & -0.65714 & 0.33205 \\
\hline $\mathrm{C}$ & 0.39273 & -1.76759 & 3.64961 \\
\hline $\mathrm{C}$ & 1.47036 & -0.68868 & 3.97277 \\
\hline $\mathrm{C}$ & 0.13474 & -1.67699 & 2.12329 \\
\hline $\mathrm{H}$ & 1.34920 & -0.24858 & 4.96458 \\
\hline $\mathrm{H}$ & 0.70371 & -2.77385 & 3.93858 \\
\hline $\mathrm{H}$ & -0.52827 & -1.52734 & 4.18863 \\
\hline $\mathrm{H}$ & -0.91140 & -1.80597 & 1.84819 \\
\hline $\mathrm{H}$ & 2.48717 & -1.09355 & 3.90798 \\
\hline $\mathrm{C}$ & 1.23585 & 0.29589 & 2.87380 \\
\hline $\mathrm{N}$ & 1.53561 & 1.53951 & 2.61479 \\
\hline $\mathrm{N}$ & 0.92188 & 1.72701 & 1.36358 \\
\hline $\mathrm{C}$ & 0.28061 & 0.63528 & 0.88231 \\
\hline $\mathrm{N}$ & 0.48994 & -0.26588 & 1.87035 \\
\hline $\mathrm{C}$ & 1.01361 & 3.00662 & 0.74898 \\
\hline $\mathrm{C}$ & 0.18611 & 4.04716 & 1.19690 \\
\hline $\mathrm{C}$ & 1.95827 & 3.22754 & -0.26318 \\
\hline $\mathrm{C}$ & 0.26940 & 5.30509 & 0.58795 \\
\hline $\mathrm{C}$ & 2.03223 & 4.48204 & -0.87904 \\
\hline $\mathrm{C}$ & 1.18326 & 5.49776 & -0.44350 \\
\hline $\mathrm{H}$ & -0.37649 & 6.11520 & 0.90158 \\
\hline $\mathrm{H}$ & 2.74349 & 4.66930 & -1.67387 \\
\hline $\mathrm{H}$ & 1.23950 & 6.47128 & -0.92202 \\
\hline $\mathrm{O}$ & 2.74821 & 2.16247 & -0.57608 \\
\hline $\mathrm{O}$ & -0.65219 & 3.73473 & 2.21674 \\
\hline $\mathrm{C}$ & -1.51351 & 4.77094 & 2.69973 \\
\hline $\mathrm{H}$ & -2.09799 & 4.30976 & 3.49574 \\
\hline $\mathrm{H}$ & -0.92700 & 5.60608 & 3.10088 \\
\hline $\mathrm{H}$ & -2.17877 & 5.12218 & 1.90370 \\
\hline $\mathrm{C}$ & 3.68002 & 2.32530 & -1.65080 \\
\hline $\mathrm{H}$ & 4.41646 & 3.10258 & -1.41478 \\
\hline $\mathrm{H}$ & 4.17384 & 1.35901 & -1.74979 \\
\hline $\mathrm{H}$ & 3.15993 & 2.57445 & -2.58349 \\
\hline $\mathrm{C}$ & 1.01860 & -2.61071 & 1.27747 \\
\hline $\mathrm{H}$ & 1.10377 & -2.15982 & 0.28634 \\
\hline $\mathrm{H}$ & 2.02724 & -2.66092 & 1.70715 \\
\hline $\mathrm{C}$ & 0.42597 & -3.99217 & 1.15586 \\
\hline $\mathrm{C}$ & 1.06095 & -5.11329 & 1.69708 \\
\hline $\mathrm{C}$ & -0.78460 & -4.16387 & 0.46919 \\
\hline $\mathrm{C}$ & 0.50678 & -6.38707 & 1.55181 \\
\hline $\mathrm{H}$ & 2.00379 & -4.99030 & 2.22681 \\
\hline $\mathrm{C}$ & -1.33650 & -5.43314 & 0.32055 \\
\hline $\mathrm{H}$ & -1.28404 & -3.29492 & 0.04638 \\
\hline $\mathrm{C}$ & -0.69309 & -6.54945 & 0.86099 \\
\hline $\mathrm{H}$ & 1.01436 & -7.24986 & 1.97501 \\
\hline
\end{tabular}




\begin{tabular}{|c|c|c|c|}
\hline $\mathrm{H}$ & -2.26735 & -5.55282 & -0.22672 \\
\hline $\mathrm{H}$ & -1.12390 & -7.53973 & 0.74117 \\
\hline $\mathrm{C}$ & -1.25334 & 0.24754 & -0.26890 \\
\hline $\mathrm{C}$ & -1.80552 & 1.64673 & -0.46309 \\
\hline $\mathrm{C}$ & -1.23043 & 2.25741 & -1.58292 \\
\hline $\mathrm{C}$ & -2.82053 & 2.26590 & 0.25605 \\
\hline $\mathrm{C}$ & -1.65371 & 3.52144 & -1.98429 \\
\hline $\mathrm{C}$ & -3.25074 & 3.53104 & -0.15056 \\
\hline $\mathrm{H}$ & -3.27178 & 1.75322 & 1.10112 \\
\hline $\mathrm{C}$ & -2.66458 & 4.15661 & -1.25690 \\
\hline $\mathrm{H}$ & -1.21549 & 4.00657 & -2.85318 \\
\hline $\mathrm{H}$ & -4.05618 & 4.02964 & 0.38325 \\
\hline $\mathrm{H}$ & -3.01150 & 5.13971 & -1.56442 \\
\hline $\mathrm{C}$ & -0.20213 & 1.34962 & -2.21623 \\
\hline $\mathrm{H}$ & 0.80778 & 1.59659 & -1.87386 \\
\hline $\mathrm{H}$ & -0.20737 & 1.38641 & -3.31119 \\
\hline $\mathrm{C}$ & -0.57509 & -0.03220 & -1.66586 \\
\hline $\mathrm{O}$ & 0.62102 & -0.87105 & -1.55262 \\
\hline $\mathrm{C}$ & -0.85442 & -2.11203 & -2.82464 \\
\hline $\mathrm{H}$ & -1.83187 & -0.27732 & -3.46217 \\
\hline $\mathrm{C}$ & 0.40095 & -2.04547 & -2.22242 \\
\hline $\mathrm{C}$ & 1.31755 & -3.08938 & -2.28838 \\
\hline $\mathrm{C}$ & -1.22743 & -3.26218 & -3.50796 \\
\hline $\mathrm{C}$ & -0.32875 & -4.33500 & -3.57894 \\
\hline $\mathrm{H}$ & -0.61149 & -5.24392 & -4.10131 \\
\hline $\mathrm{C}$ & 0.92756 & -4.24267 & -2.97658 \\
\hline $\mathrm{H}$ & 1.61370 & -5.08299 & -3.03177 \\
\hline $\mathrm{H}$ & -2.20623 & -3.33449 & -3.97559 \\
\hline $\mathrm{H}$ & 2.28892 & -3.01520 & -1.81029 \\
\hline \multicolumn{4}{|l|}{76} \\
\hline \multicolumn{4}{|c|}{$3 d-(S)-[3 d+2 a]$} \\
\hline $\mathrm{C}$ & -0.73665 & -0.94006 & -3.40177 \\
\hline $\mathrm{H}$ & -1.82968 & -0.89750 & -3.44318 \\
\hline $\mathrm{O}$ & -2.02792 & -0.95777 & -0.54948 \\
\hline $\mathrm{C}$ & -0.69500 & -1.46558 & 3.74708 \\
\hline $\mathrm{C}$ & 0.15364 & -0.32406 & 4.38679 \\
\hline $\mathrm{C}$ & -0.42701 & -1.41297 & 2.21748 \\
\hline $\mathrm{H}$ & -0.34401 & 0.14292 & 5.23961 \\
\hline $\mathrm{H}$ & -0.45500 & -2.44898 & 4.15758 \\
\hline $\mathrm{H}$ & -1.75532 & -1.27341 & 3.93418 \\
\hline $\mathrm{H}$ & -1.33469 & -1.57678 & 1.63567 \\
\hline $\mathrm{H}$ & 1.13031 & -0.68571 & 4.72973 \\
\hline $\mathrm{C}$ & 0.32533 & 0.60707 & 3.23083 \\
\hline $\mathrm{N}$ & 0.71785 & 1.83336 & 3.02875 \\
\hline $\mathrm{N}$ & 0.61464 & 1.92094 & 1.61921 \\
\hline $\mathrm{C}$ & 0.18655 & 0.80264 & 0.97885 \\
\hline $\mathrm{N}$ & -0.00189 & -0.01720 & 2.05023 \\
\hline
\end{tabular}




\begin{tabular}{|c|c|c|c|}
\hline $\mathrm{C}$ & 0.90971 & 3.15627 & 0.98320 \\
\hline $\mathrm{C}$ & 0.01251 & 4.22896 & 1.09888 \\
\hline $\mathrm{C}$ & 2.08126 & 3.29488 & 0.22430 \\
\hline $\mathrm{C}$ & 0.29325 & 5.44607 & 0.46499 \\
\hline $\mathrm{C}$ & 2.35195 & 4.50481 & -0.42855 \\
\hline $\mathrm{C}$ & 1.45623 & 5.56266 & -0.29102 \\
\hline $\mathrm{H}$ & -0.38972 & 6.28259 & 0.54444 \\
\hline $\mathrm{H}$ & 3.25080 & 4.62691 & -1.02020 \\
\hline $\mathrm{H}$ & 1.67057 & 6.50384 & -0.78950 \\
\hline $\mathrm{O}$ & 2.89074 & 2.19942 & 0.17579 \\
\hline $\mathrm{O}$ & -1.10702 & 3.98377 & 1.82970 \\
\hline $\mathrm{C}$ & -2.04774 & 5.05072 & 1.96777 \\
\hline $\mathrm{H}$ & -2.85831 & 4.64283 & 2.57188 \\
\hline $\mathrm{H}$ & -1.59464 & 5.90757 & 2.48107 \\
\hline $\mathrm{H}$ & -2.43258 & 5.36082 & 0.98935 \\
\hline $\mathrm{C}$ & 4.07290 & 2.28878 & -0.62402 \\
\hline $\mathrm{H}$ & 4.74502 & 3.06888 & -0.24648 \\
\hline $\mathrm{H}$ & 4.55049 & 1.31269 & -0.53950 \\
\hline $\mathrm{H}$ & 3.82190 & 2.49023 & -1.67262 \\
\hline $\mathrm{C}$ & 0.67056 & -2.37143 & 1.72297 \\
\hline $\mathrm{H}$ & 0.96540 & -2.02521 & 0.72756 \\
\hline $\mathrm{H}$ & 1.54683 & -2.29554 & 2.37901 \\
\hline $\mathrm{C}$ & 0.18336 & -3.79750 & 1.65718 \\
\hline $\mathrm{C}$ & 0.70149 & -4.78499 & 2.50133 \\
\hline $\mathrm{C}$ & -0.81335 & -4.15278 & 0.73632 \\
\hline $\mathrm{C}$ & 0.24352 & -6.10212 & 2.42841 \\
\hline $\mathrm{H}$ & 1.47865 & -4.52131 & 3.21615 \\
\hline $\mathrm{C}$ & -1.27109 & -5.46608 & 0.66150 \\
\hline $\mathrm{H}$ & -1.22000 & -3.39497 & 0.07099 \\
\hline $\mathrm{C}$ & -0.74478 & -6.44570 & 1.50676 \\
\hline $\mathrm{H}$ & 0.66041 & -6.85762 & 3.08913 \\
\hline $\mathrm{H}$ & -2.03488 & -5.72745 & -0.06574 \\
\hline $\mathrm{H}$ & -1.10097 & -7.47034 & 1.44463 \\
\hline $\mathrm{C}$ & -1.40058 & -0.08418 & -1.13014 \\
\hline $\mathrm{C}$ & -1.74534 & 1.34525 & -1.30314 \\
\hline $\mathrm{C}$ & -0.71003 & 1.99549 & -1.99247 \\
\hline $\mathrm{C}$ & -2.90643 & 2.01749 & -0.92394 \\
\hline $\mathrm{C}$ & -0.83254 & 3.34245 & -2.31614 \\
\hline $\mathrm{C}$ & -3.02583 & 3.36593 & -1.25249 \\
\hline $\mathrm{H}$ & -3.69196 & 1.48911 & -0.39125 \\
\hline $\mathrm{C}$ & -1.99599 & 4.02025 & -1.94206 \\
\hline $\mathrm{H}$ & -0.03462 & 3.86790 & -2.83350 \\
\hline $\mathrm{H}$ & -3.92311 & 3.91538 & -0.98041 \\
\hline $\mathrm{H}$ & -2.10213 & 5.07357 & -2.18747 \\
\hline $\mathrm{C}$ & 0.44910 & 1.05925 & -2.22943 \\
\hline $\mathrm{H}$ & 1.18510 & 1.18149 & -1.42659 \\
\hline $\mathrm{H}$ & 0.93570 & 1.19851 & -3.20034 \\
\hline $\mathrm{C}$ & -0.18848 & -0.32446 & -2.06875 \\
\hline
\end{tabular}




$\begin{array}{lrrr}\mathrm{O} & 0.74538 & -1.29590 & -1.52233 \\ \mathrm{C} & -0.21232 & -2.35060 & -3.34188 \\ \mathrm{H} & -0.34882 & -0.39195 & -4.26929 \\ \mathrm{C} & 0.63884 & -2.45723 & -2.24325 \\ \mathrm{C} & 1.30586 & -3.63518 & -1.92812 \\ \mathrm{C} & -0.42423 & -3.45236 & -4.16047 \\ \mathrm{C} & 0.22788 & -4.65557 & -3.86034 \\ \mathrm{H} & 0.06621 & -5.52854 & -4.48540 \\ \mathrm{C} & 1.08212 & -4.73807 & -2.75805 \\ \mathrm{H} & 1.57581 & -5.67825 & -2.52945 \\ \mathrm{H} & -1.08827 & -3.38633 & -5.01859 \\ \mathrm{H} & 1.95509 & -3.70002 & -1.06193\end{array}$




\subsection{3a-(S)-r-path}

77

3a-(S)-4-r

$\begin{array}{llll}\text { C } & -0.31185 & -0.89162 & -2.49446\end{array}$

$\begin{array}{llll}\mathrm{H} & -2.36151 & -0.72164 & 0.00836\end{array}$

$\begin{array}{llll}\mathrm{O} & -2.06053 & -0.25770 & 0.80919\end{array}$

$\begin{array}{llll}\text { C } & 1.04624 & -2.23184 & 3.26727\end{array}$

$\begin{array}{llll}\text { C } & 2.21145 & -0.32310 & 3.91128\end{array}$

$\begin{array}{llll}\text { C } & 0.13418 & -1.57136 & 2.21570\end{array}$

$\begin{array}{llll}\mathrm{H} & 2.39559 & 0.34185 & 4.75708\end{array}$

$\begin{array}{llll}\mathrm{H} & 1.98666 & -2.55332 & 2.78976\end{array}$

$\begin{array}{llll}\mathrm{H} & 0.54773 & -3.10898 & 3.68217\end{array}$

$\begin{array}{llll}\mathrm{H} & -0.91112 & -1.63302 & 2.52668\end{array}$

$\begin{array}{llll}\mathrm{H} & 3.17217 & -0.74609 & 3.57376\end{array}$

$\begin{array}{llll}\mathrm{O} & 1.35053 & -1.36753 & 4.36363\end{array}$

$\begin{array}{llll}\text { C } & 1.54346 & 0.42689 & 2.79865\end{array}$

$\begin{array}{llll}\mathrm{N} & 1.82105 & 1.62756 & 2.39878\end{array}$

$\begin{array}{llll}\mathrm{N} & 0.88956 & 1.86808 & 1.36487\end{array}$

$\begin{array}{llll}\mathrm{C} & 0.01641 & 0.78894 & 1.19818\end{array}$

$\begin{array}{llll}\mathrm{N} & 0.49055 & -0.14992 & 2.12466\end{array}$

$\begin{array}{llll}\text { C } & 0.67821 & 3.19564 & 0.93237\end{array}$

$\begin{array}{llll}\mathrm{C} & -0.51920 & 3.87204 & 1.23600\end{array}$

$\begin{array}{llll}\text { C } & 1.67297 & 3.85244 & 0.18456\end{array}$

$\begin{array}{llll}\text { C } & -0.75647 & 5.14753 & 0.71193\end{array}$

$\begin{array}{llll}\text { C } & 1.44822 & 5.14034 & -0.31155\end{array}$

$\begin{array}{llll}\text { C } & 0.22798 & 5.76196 & -0.05578\end{array}$

$\mathrm{H} \quad-1.68896 \quad 5.66040 \quad 0.91113$

$\begin{array}{llll}\mathrm{H} & 2.20342 & 5.64710 & -0.89956\end{array}$

$\begin{array}{llll}\mathrm{H} & 0.04485 & 6.75474 & -0.45621\end{array}$

$\begin{array}{llll}\mathrm{O} & 2.81878 & 3.14106 & -0.02421\end{array}$

$\begin{array}{llll}\text { O } & -1.38232 & 3.20831 & 2.05047\end{array}$

$\begin{array}{llll}\text { C } & -2.64279 & 3.82789 & 2.32115\end{array}$

$\begin{array}{llll}\mathrm{H} & -3.18428 & 3.11482 & 2.94291\end{array}$

$\begin{array}{llll}\mathrm{H} & -2.50559 & 4.77065 & 2.86485\end{array}$

$\begin{array}{llll}\mathrm{H} & -3.19285 & 4.00679 & 1.39046\end{array}$

$\begin{array}{llll}\text { C } & 3.82618 & 3.74143 & -0.84089\end{array}$

$\begin{array}{llll}\mathrm{H} & 4.20126 & 4.66539 & -0.38483\end{array}$

$\begin{array}{llll}\mathrm{H} & 4.62777 & 3.00464 & -0.89721\end{array}$

$\begin{array}{llll}\mathrm{H} & 3.44273 & 3.95426 & -1.84668\end{array}$

$\begin{array}{llll}\text { C } & 0.31590 & -2.26753 & 0.85244\end{array}$

$\begin{array}{llll}\mathrm{H} & -0.14515 & -1.64148 & 0.08357\end{array}$

$\begin{array}{llll}\mathrm{H} & 1.38548 & -2.30971 & 0.62222\end{array}$

$\begin{array}{llll}\text { C } & -0.27312 & -3.65475 & 0.82144\end{array}$

$\begin{array}{llll}\text { C } & 0.54099 & -4.77582 & 0.63368\end{array}$

$\begin{array}{llll}\text { C } & -1.65367 & -3.85026 & 0.97022\end{array}$

$\begin{array}{llll}\text { C } & -0.00416 & -6.05875 & 0.58645\end{array}$

$\begin{array}{llll}\mathrm{H} & 1.61085 & -4.63711 & 0.50200\end{array}$ 


\begin{tabular}{|c|c|c|c|}
\hline $\mathrm{C}$ & -2.20245 & -5.13003 & 0.92641 \\
\hline $\mathrm{H}$ & -2.30084 & -2.98945 & 1.12030 \\
\hline $\mathrm{C}$ & -1.37860 & -6.24061 & 0.73171 \\
\hline $\mathrm{H}$ & 0.64541 & -6.91554 & 0.42920 \\
\hline $\mathrm{H}$ & -3.27525 & -5.26164 & 1.03953 \\
\hline $\mathrm{H}$ & -1.80626 & -7.23849 & 0.69305 \\
\hline $\mathrm{C}$ & -1.08751 & 0.68456 & 0.39243 \\
\hline $\mathrm{C}$ & -1.48310 & 1.58815 & -0.67304 \\
\hline $\mathrm{C}$ & -0.59843 & 1.98914 & -1.70863 \\
\hline $\mathrm{C}$ & -2.82165 & 2.03321 & -0.73327 \\
\hline $\mathrm{C}$ & -1.04507 & 2.88798 & -2.67859 \\
\hline $\mathrm{C}$ & -3.25270 & 2.91038 & -1.72147 \\
\hline $\mathrm{H}$ & -3.50944 & 1.69285 & 0.03537 \\
\hline $\mathrm{C}$ & -2.35495 & 3.36684 & -2.68926 \\
\hline $\mathrm{H}$ & -0.35414 & 3.19465 & -3.46110 \\
\hline $\mathrm{H}$ & -4.28539 & 3.25008 & -1.72937 \\
\hline $\mathrm{H}$ & -2.67677 & 4.06252 & -3.45856 \\
\hline $\mathrm{C}$ & 0.77609 & 1.38086 & -1.84424 \\
\hline $\mathrm{H}$ & 1.38845 & 1.54194 & -0.95054 \\
\hline $\mathrm{H}$ & 1.30334 & 1.88687 & -2.66415 \\
\hline $\mathrm{C}$ & 0.73717 & -0.09894 & -2.12804 \\
\hline $\mathrm{S}$ & 2.22914 & -1.01027 & -2.00871 \\
\hline $\mathrm{C}$ & 0.04172 & -2.27209 & -2.66973 \\
\hline $\mathrm{H}$ & -1.31526 & -0.50049 & -2.62632 \\
\hline $\mathrm{C}$ & 1.42706 & -2.49580 & -2.44702 \\
\hline $\mathrm{C}$ & 1.99485 & -3.76698 & -2.56009 \\
\hline $\mathrm{C}$ & -0.77177 & -3.37350 & -2.99201 \\
\hline $\mathrm{C}$ & -0.21015 & -4.63839 & -3.09361 \\
\hline $\mathrm{H}$ & -0.84111 & -5.48989 & -3.33011 \\
\hline $\mathrm{C}$ & 1.16525 & -4.83481 & -2.88549 \\
\hline $\mathrm{H}$ & 1.58496 & -5.83307 & -2.96760 \\
\hline $\mathrm{H}$ & -1.83690 & -3.22927 & -3.15265 \\
\hline $\mathrm{H}$ & 3.05674 & -3.92001 & -2.39088 \\
\hline \multicolumn{4}{|l|}{77} \\
\hline \multicolumn{4}{|c|}{$3 a-(S)-t s 1-r$} \\
\hline $\mathrm{C}$ & -0.86084 & -0.36867 & -2.51294 \\
\hline $\mathrm{H}$ & -1.67895 & -0.51666 & -1.23898 \\
\hline $\mathrm{O}$ & -1.73833 & -0.31750 & 0.14551 \\
\hline $\mathrm{C}$ & 0.95290 & -2.89801 & 2.59193 \\
\hline $\mathrm{C}$ & 2.68931 & -1.41172 & 2.98833 \\
\hline $\mathrm{C}$ & 0.15947 & -1.99532 & 1.61714 \\
\hline $\mathrm{H}$ & 3.25867 & -0.91366 & 3.77371 \\
\hline $\mathrm{H}$ & 1.66809 & -3.50745 & 2.01608 \\
\hline $\mathrm{H}$ & 0.25580 & -3.56729 & 3.09777 \\
\hline $\mathrm{H}$ & -0.82615 & -1.75707 & 2.02482 \\
\hline $\mathrm{H}$ & 3.35947 & -2.06650 & 2.40710 \\
\hline $\mathrm{O}$ & 1.66541 & -2.19502 & 3.60952 \\
\hline
\end{tabular}




\begin{tabular}{|c|c|c|c|}
\hline $\mathrm{C}$ & 2.02603 & -0.39294 & 2.12568 \\
\hline $\mathrm{N}$ & 2.35455 & 0.85681 & 2.02579 \\
\hline $\mathrm{N}$ & 1.34331 & 1.41326 & 1.23271 \\
\hline $\mathrm{C}$ & 0.36770 & 0.47893 & 0.90506 \\
\hline $\mathrm{N}$ & 0.86487 & -0.70661 & 1.44923 \\
\hline $\mathrm{C}$ & 1.27403 & 2.82674 & 1.16368 \\
\hline $\mathrm{C}$ & 0.30260 & 3.52346 & 1.90311 \\
\hline $\mathrm{C}$ & 2.22329 & 3.53822 & 0.41091 \\
\hline $\mathrm{C}$ & 0.21562 & 4.91635 & 1.80505 \\
\hline $\mathrm{C}$ & 2.15258 & 4.93273 & 0.33327 \\
\hline $\mathrm{C}$ & 1.13890 & 5.59871 & 1.01861 \\
\hline $\mathrm{H}$ & -0.55237 & 5.46150 & 2.33900 \\
\hline $\mathrm{H}$ & 2.86770 & 5.49191 & -0.25718 \\
\hline $\mathrm{H}$ & 1.07325 & 6.68026 & 0.94466 \\
\hline $\mathrm{O}$ & 3.16463 & 2.78054 & -0.22317 \\
\hline $\mathrm{O}$ & -0.50026 & 2.75489 & 2.68704 \\
\hline $\mathrm{C}$ & -1.50606 & 3.42673 & 3.45244 \\
\hline $\mathrm{H}$ & -2.03192 & 2.63800 & 3.99039 \\
\hline $\mathrm{H}$ & -1.04924 & 4.12576 & 4.16315 \\
\hline $\mathrm{H}$ & -2.20137 & 3.95975 & 2.79447 \\
\hline $\mathrm{C}$ & 4.12480 & 3.46511 & -1.03345 \\
\hline $\mathrm{H}$ & 4.72751 & 4.15176 & -0.42759 \\
\hline $\mathrm{H}$ & 4.75857 & 2.68220 & -1.44984 \\
\hline $\mathrm{H}$ & 3.63261 & 4.01767 & -1.84324 \\
\hline $\mathrm{C}$ & -0.00554 & -2.73921 & 0.28415 \\
\hline $\mathrm{H}$ & -0.41353 & -2.03438 & -0.43662 \\
\hline $\mathrm{H}$ & 0.97991 & -3.05885 & -0.06892 \\
\hline $\mathrm{C}$ & -0.93371 & -3.92150 & 0.40277 \\
\hline $\mathrm{C}$ & -0.46291 & -5.23220 & 0.28040 \\
\hline $\mathrm{C}$ & -2.30202 & -3.71321 & 0.62614 \\
\hline $\mathrm{C}$ & -1.33459 & -6.31698 & 0.38339 \\
\hline $\mathrm{H}$ & 0.59397 & -5.40162 & 0.08717 \\
\hline $\mathrm{C}$ & -3.17416 & -4.79493 & 0.73080 \\
\hline $\mathrm{H}$ & -2.67415 & -2.69413 & 0.69862 \\
\hline $\mathrm{C}$ & -2.69331 & -6.10090 & 0.61156 \\
\hline $\mathrm{H}$ & -0.95311 & -7.32944 & 0.28124 \\
\hline $\mathrm{H}$ & -4.23361 & -4.61951 & 0.89795 \\
\hline $\mathrm{H}$ & -3.37469 & -6.94346 & 0.69046 \\
\hline $\mathrm{C}$ & -0.84271 & 0.66574 & 0.22957 \\
\hline $\mathrm{C}$ & -1.28481 & 1.98850 & -0.28330 \\
\hline $\mathrm{C}$ & -0.64087 & 2.57656 & -1.38460 \\
\hline $\mathrm{C}$ & -2.43473 & 2.59383 & 0.23903 \\
\hline $\mathrm{C}$ & -1.10133 & 3.79693 & -1.88501 \\
\hline $\mathrm{C}$ & -2.89154 & 3.80857 & -0.26516 \\
\hline $\mathrm{H}$ & -2.96100 & 2.09530 & 1.04749 \\
\hline $\mathrm{C}$ & -2.21108 & 4.42294 & -1.31931 \\
\hline $\mathrm{H}$ & -0.59999 & 4.24857 & -2.73801 \\
\hline $\mathrm{H}$ & -3.77905 & 4.27415 & 0.15573 \\
\hline
\end{tabular}




\begin{tabular}{|c|c|c|c|}
\hline $\mathrm{H}$ & -2.56174 & 5.37073 & -1.71829 \\
\hline $\mathrm{C}$ & 0.50529 & 1.82850 & -2.01655 \\
\hline $\mathrm{H}$ & 1.44097 & 2.03194 & -1.48372 \\
\hline $\mathrm{H}$ & 0.65524 & 2.18613 & -3.04712 \\
\hline $\mathrm{C}$ & 0.27927 & 0.33781 & -2.01355 \\
\hline $\mathrm{S}$ & 1.71229 & -0.67519 & -2.01003 \\
\hline $\mathrm{C}$ & -0.50061 & -1.71879 & -2.95830 \\
\hline $\mathrm{H}$ & -1.62339 & 0.19263 & -3.05425 \\
\hline $\mathrm{C}$ & 0.85799 & -2.02996 & -2.72578 \\
\hline $\mathrm{C}$ & 1.38406 & -3.29227 & -3.00436 \\
\hline $\mathrm{C}$ & -1.32759 & -2.70847 & -3.50385 \\
\hline $\mathrm{C}$ & -0.80612 & -3.96496 & -3.80036 \\
\hline $\mathrm{H}$ & -1.45314 & -4.73051 & -4.21823 \\
\hline $\mathrm{C}$ & 0.54096 & -4.25747 & -3.55054 \\
\hline $\mathrm{H}$ & 0.93267 & -5.24403 & -3.78033 \\
\hline $\mathrm{H}$ & -2.37918 & -2.49620 & -3.67747 \\
\hline $\mathrm{H}$ & 2.42816 & -3.51593 & -2.80515 \\
\hline \multicolumn{4}{|l|}{77} \\
\hline \multicolumn{4}{|c|}{$3 a-(S)-6-r$} \\
\hline $\mathrm{C}$ & -0.65764 & -0.90506 & -2.52701 \\
\hline $\mathrm{H}$ & -1.58959 & -1.13789 & -1.99436 \\
\hline $\mathrm{O}$ & -1.54087 & -0.68102 & 0.06102 \\
\hline $\mathrm{C}$ & 0.59380 & -2.24943 & 3.36627 \\
\hline $\mathrm{C}$ & 1.49181 & -0.27304 & 4.18629 \\
\hline $\mathrm{C}$ & 0.06496 & -1.70306 & 2.03008 \\
\hline $\mathrm{H}$ & 1.42840 & 0.45276 & 4.99858 \\
\hline $\mathrm{H}$ & 1.63316 & -2.59611 & 3.24507 \\
\hline $\mathrm{H}$ & -0.02469 & -3.09832 & 3.66053 \\
\hline $\mathrm{H}$ & -1.02484 & -1.66721 & 1.99687 \\
\hline $\mathrm{H}$ & 2.51166 & -0.69082 & 4.15468 \\
\hline $\mathrm{O}$ & 0.54292 & -1.30371 & 4.44002 \\
\hline $\mathrm{C}$ & 1.14905 & 0.39497 & 2.89283 \\
\hline $\mathrm{N}$ & 1.31456 & 1.65774 & 2.60454 \\
\hline $\mathrm{N}$ & 0.72900 & 1.77979 & 1.35487 \\
\hline $\mathrm{C}$ & 0.20854 & 0.61864 & 0.90016 \\
\hline $\mathrm{N}$ & 0.49536 & -0.28169 & 1.88900 \\
\hline $\mathrm{C}$ & 0.70470 & 3.07282 & 0.75239 \\
\hline $\mathrm{C}$ & -0.26534 & 3.99937 & 1.16492 \\
\hline $\mathrm{C}$ & 1.70314 & 3.43246 & -0.16266 \\
\hline $\mathrm{C}$ & -0.30010 & 5.26407 & 0.56771 \\
\hline $\mathrm{C}$ & 1.66704 & 4.69853 & -0.75689 \\
\hline $\mathrm{C}$ & 0.65732 & 5.58599 & -0.39005 \\
\hline $\mathrm{H}$ & -1.06444 & 5.98137 & 0.83747 \\
\hline $\mathrm{H}$ & 2.41349 & 4.98986 & -1.48514 \\
\hline $\mathrm{H}$ & 0.62322 & 6.56530 & -0.85841 \\
\hline $\mathrm{O}$ & 2.64890 & 2.48553 & -0.40229 \\
\hline $\mathrm{O}$ & -1.10834 & 3.57472 & 2.13679 \\
\hline
\end{tabular}




\begin{tabular}{|c|c|c|c|}
\hline $\mathrm{C}$ & -2.13790 & 4.47931 & 2.55601 \\
\hline $\mathrm{H}$ & -2.70159 & 3.93835 & 3.31571 \\
\hline $\mathrm{H}$ & -1.70029 & 5.38684 & 2.98776 \\
\hline $\mathrm{H}$ & -2.79028 & 4.73256 & 1.71388 \\
\hline $\mathrm{C}$ & 3.64864 & 2.77904 & -1.38714 \\
\hline $\mathrm{H}$ & 4.25718 & 3.63545 & -1.07477 \\
\hline $\mathrm{H}$ & 4.25924 & 1.87959 & -1.44884 \\
\hline $\mathrm{H}$ & 3.18691 & 2.97787 & -2.36108 \\
\hline $\mathrm{C}$ & 0.56860 & -2.57401 & 0.87514 \\
\hline $\mathrm{H}$ & 0.34003 & -2.03804 & -0.04194 \\
\hline $\mathrm{H}$ & 1.65468 & -2.70384 & 0.94595 \\
\hline $\mathrm{C}$ & -0.13474 & -3.90702 & 0.83233 \\
\hline $\mathrm{C}$ & 0.53558 & -5.10318 & 1.10213 \\
\hline $\mathrm{C}$ & -1.49195 & -3.94964 & 0.48191 \\
\hline $\mathrm{C}$ & -0.13345 & -6.32611 & 1.02821 \\
\hline $\mathrm{H}$ & 1.59317 & -5.07792 & 1.35653 \\
\hline $\mathrm{C}$ & -2.16023 & -5.16989 & 0.41040 \\
\hline $\mathrm{H}$ & -1.99596 & -3.01390 & 0.24876 \\
\hline $\mathrm{C}$ & -1.48436 & -6.36178 & 0.68388 \\
\hline $\mathrm{H}$ & 0.40134 & -7.24941 & 1.23494 \\
\hline $\mathrm{H}$ & -3.21053 & -5.19350 & 0.13165 \\
\hline $\mathrm{H}$ & -2.00682 & -7.31268 & 0.62375 \\
\hline $\mathrm{C}$ & -0.77711 & 0.34663 & -0.28361 \\
\hline $\mathrm{C}$ & -1.59313 & & -0.60620 \\
\hline $\mathrm{C}$ & -1.20369 & 2.25010 & -1.78257 \\
\hline $\mathrm{C}$ & -2.73987 & 2.00942 & 0.07524 \\
\hline $\mathrm{C}$ & -1.93214 & 3.33505 & -2.27072 \\
\hline $\mathrm{C}$ & -3.47080 & 3.09447 & -0.40913 \\
\hline $\mathrm{H}$ & -3.06115 & 1.45056 & 0.94935 \\
\hline $\mathrm{C}$ & -3.06176 & 3.76171 & -1.57077 \\
\hline $\mathrm{H}$ & -1.63699 & 3.83250 & -3.19183 \\
\hline $\mathrm{H}$ & -4.37754 & 3.41126 & 0.10086 \\
\hline $\mathrm{H}$ & -3.64540 & 4.59861 & -1.94555 \\
\hline $\mathrm{C}$ & 0.00238 & 1.58553 & -2.39007 \\
\hline $\mathrm{H}$ & 0.90826 & 2.16216 & -2.17777 \\
\hline $\mathrm{H}$ & -0.06363 & 1.49633 & -3.48052 \\
\hline $\mathrm{C}$ & 0.06332 & 0.19558 & -1.71393 \\
\hline S & 1.82793 & -0.33937 & -1.53292 \\
\hline $\mathrm{C}$ & 0.21086 & -2.12970 & -2.61975 \\
\hline $\mathrm{H}$ & -0.90365 & -0.53314 & -3.52978 \\
\hline $\mathrm{C}$ & 1.52590 & -1.95278 & -2.16750 \\
\hline $\mathrm{C}$ & 2.44637 & -3.00239 & -2.18184 \\
\hline $\mathrm{C}$ & -0.18296 & -3.38438 & -3.07898 \\
\hline $\mathrm{C}$ & 0.72624 & -4.44542 & -3.09496 \\
\hline $\mathrm{H}$ & 0.40855 & -5.42557 & -3.43746 \\
\hline $\mathrm{C}$ & 2.03560 & -4.25077 & -2.65256 \\
\hline $\mathrm{H}$ & 2.73935 & -5.07853 & -2.65775 \\
\hline $\mathrm{H}$ & -1.20702 & -3.53966 & -3.40914 \\
\hline
\end{tabular}




\begin{tabular}{|c|c|c|c|}
\hline $\mathrm{H}$ & 3.45960 & -2.85590 & -1.81861 \\
\hline \multicolumn{4}{|c|}{77} \\
\hline \multicolumn{4}{|c|}{$3 a-(S)-t s 3-r$} \\
\hline $\mathrm{C}$ & -0.82532 & -0.51925 & -2.93143 \\
\hline $\mathrm{H}$ & -1.89720 & -0.53553 & -2.71640 \\
\hline $\mathrm{O}$ & -1.49176 & -0.88264 & -0.22393 \\
\hline $\mathrm{C}$ & 0.22842 & -2.29585 & 3.38528 \\
\hline $\mathrm{C}$ & 0.74646 & -0.28569 & 4.42945 \\
\hline $\mathrm{C}$ & -0.03463 & -1.74214 & 1.97734 \\
\hline $\mathrm{H}$ & 0.41344 & 0.41384 & 5.19823 \\
\hline $\mathrm{H}$ & 1.29631 & -2.54778 & 3.49669 \\
\hline $\mathrm{H}$ & -0.36005 & -3.20404 & 3.52093 \\
\hline $\mathrm{H}$ & -1.09251 & -1.79175 & 1.71466 \\
\hline $\mathrm{H}$ & 1.76892 & -0.62152 & 4.66996 \\
\hline $\mathrm{O}$ & -0.14870 & -1.39348 & 4.43065 \\
\hline $\mathrm{C}$ & 0.70901 & 0.38080 & 3.08999 \\
\hline $\mathrm{N}$ & 0.94663 & 1.63817 & 2.83911 \\
\hline $\mathrm{N}$ & 0.67718 & 1.73734 & 1.47671 \\
\hline $\mathrm{C}$ & 0.28182 & 0.57797 & 0.90575 \\
\hline $\mathrm{N}$ & 0.32289 & -0.30092 & 1.95749 \\
\hline $\mathrm{C}$ & 0.88092 & 3.00664 & 0.86428 \\
\hline $\mathrm{C}$ & 0.00328 & 4.06297 & 1.15475 \\
\hline $\mathrm{C}$ & 2.01152 & 3.21553 & 0.05987 \\
\hline $\mathrm{C}$ & 0.22582 & 5.32074 & 0.58044 \\
\hline $\mathrm{C}$ & 2.22536 & 4.47078 & -0.52186 \\
\hline $\mathrm{C}$ & 1.32451 & 5.49994 & -0.25455 \\
\hline $\mathrm{H}$ & -0.45319 & 6.14188 & 0.77155 \\
\hline $\mathrm{H}$ & 3.08148 & 4.64578 & -1.16128 \\
\hline $\mathrm{H}$ & 1.49050 & 6.47433 & -0.70501 \\
\hline $\mathrm{O}$ & 2.82932 & 2.14105 & -0.09741 \\
\hline $\mathrm{O}$ & -1.01854 & 3.76791 & 1.99277 \\
\hline $\mathrm{C}$ & -1.92463 & 4.82450 & 2.32934 \\
\hline $\mathrm{H}$ & -2.65618 & 4.37054 & 2.99726 \\
\hline $\mathrm{H}$ & -1.39647 & 5.63527 & 2.84484 \\
\hline $\mathrm{H}$ & -2.42647 & 5.20409 & 1.43335 \\
\hline $\mathrm{C}$ & 3.95106 & 2.28321 & -0.97815 \\
\hline $\mathrm{H}$ & 4.64365 & 3.04624 & -0.60391 \\
\hline $\mathrm{H}$ & 4.43250 & 1.30629 & -0.98484 \\
\hline $\mathrm{H}$ & 3.61899 & 2.53682 & -1.99107 \\
\hline $\mathrm{C}$ & 0.77902 & -2.52398 & 0.93935 \\
\hline $\mathrm{H}$ & 0.71559 & -1.97183 & 0.00248 \\
\hline $\mathrm{H}$ & 1.83207 & -2.55205 & 1.24709 \\
\hline $\mathrm{C}$ & 0.24740 & -3.92073 & 0.73720 \\
\hline $\mathrm{C}$ & 0.97412 & -5.04475 & 1.14250 \\
\hline $\mathrm{C}$ & -0.99389 & -4.10301 & 0.11233 \\
\hline $\mathrm{C}$ & 0.47605 & -6.33122 & 0.92965 \\
\hline $\mathrm{H}$ & 1.94395 & -4.91153 & 1.61808 \\
\hline
\end{tabular}




\begin{tabular}{|c|c|c|c|}
\hline $\mathrm{C}$ & -1.49216 & -5.38656 & -0.09773 \\
\hline $\mathrm{H}$ & -1.53937 & -3.22598 & -0.22614 \\
\hline $\mathrm{C}$ & -0.76120 & -6.50479 & 0.30975 \\
\hline $\mathrm{H}$ & 1.05466 & -7.19540 & 1.24537 \\
\hline $\mathrm{H}$ & -2.45009 & -5.51613 & -0.59447 \\
\hline $\mathrm{H}$ & -1.15102 & -7.50492 & 0.14054 \\
\hline $\mathrm{C}$ & -0.91898 & 0.21288 & -0.51423 \\
\hline $\mathrm{C}$ & -1.74424 & 1.49340 & -0.54324 \\
\hline $\mathrm{C}$ & -1.29571 & 2.37322 & -1.52840 \\
\hline $\mathrm{C}$ & -2.90124 & 1.75194 & 0.18497 \\
\hline $\mathrm{C}$ & -1.99529 & 3.55132 & -1.78586 \\
\hline $\mathrm{C}$ & -3.60503 & 2.92795 & -0.07309 \\
\hline $\mathrm{H}$ & -3.24955 & 1.02834 & 0.91706 \\
\hline $\mathrm{C}$ & -3.15019 & 3.82437 & -1.04906 \\
\hline $\mathrm{H}$ & -1.65878 & 4.24172 & -2.55572 \\
\hline $\mathrm{H}$ & -4.51687 & 3.14907 & 0.47602 \\
\hline $\mathrm{H}$ & -3.71440 & 4.73176 & -1.24994 \\
\hline $\mathrm{C}$ & -0.07036 & 1.83083 & -2.22425 \\
\hline $\mathrm{H}$ & 0.83290 & 2.32055 & -1.84896 \\
\hline $\mathrm{H}$ & -0.09196 & 1.98904 & -3.30799 \\
\hline $\mathrm{C}$ & -0.08200 & 0.32978 & -1.86936 \\
\hline $\mathrm{S}$ & 1.61663 & -0.37620 & -1.79760 \\
\hline $\mathrm{C}$ & -0.22626 & -1.89861 & -2.93305 \\
\hline $\mathrm{H}$ & -0.67783 & -0.06022 & -3.91957 \\
\hline $\mathrm{C}$ & 1.07959 & -1.93830 & -2.42273 \\
\hline $\mathrm{C}$ & 1.81087 & -3.12556 & -2.39341 \\
\hline $\mathrm{C}$ & -0.80446 & -3.06927 & -3.41139 \\
\hline $\mathrm{C}$ & -0.08401 & -4.26797 & -3.38274 \\
\hline $\mathrm{H}$ & -0.54531 & -5.18549 & -3.73529 \\
\hline $\mathrm{C}$ & 1.21645 & -4.29205 & -2.87986 \\
\hline $\mathrm{H}$ & 1.76535 & -5.22833 & -2.83886 \\
\hline $\mathrm{H}$ & -1.82194 & -3.05266 & -3.79424 \\
\hline $\mathrm{H}$ & 2.81318 & -3.15064 & -1.97606 \\
\hline \multicolumn{4}{|c|}{77} \\
\hline \multicolumn{4}{|c|}{$3 a-(S)-[2 r+3 a]$} \\
\hline $\mathrm{C}$ & -0.95904 & -0.76335 & -3.40951 \\
\hline $\mathrm{H}$ & -2.04178 & -0.59717 & -3.42482 \\
\hline $\mathrm{O}$ & -1.67086 & -1.24711 & -0.44019 \\
\hline $\mathrm{C}$ & -0.10287 & -1.69859 & 3.80895 \\
\hline $\mathrm{C}$ & 0.07305 & 0.47474 & 4.65332 \\
\hline $\mathrm{C}$ & -0.10215 & -1.34474 & 2.31674 \\
\hline $\mathrm{H}$ & -0.48147 & 1.23635 & 5.20492 \\
\hline $\mathrm{H}$ & 0.93342 & -1.83995 & 4.15993 \\
\hline $\mathrm{H}$ & -0.65388 & -2.62698 & 3.96457 \\
\hline $\mathrm{H}$ & -1.09839 & -1.48819 & 1.88961 \\
\hline $\mathrm{H}$ & 1.01353 & 0.26243 & 5.18872 \\
\hline $\mathrm{O}$ & -0.74029 & -0.69832 & 4.61200 \\
\hline
\end{tabular}




\begin{tabular}{|c|c|c|c|}
\hline $\mathrm{C}$ & 0.35326 & 0.94156 & 3.25891 \\
\hline $\mathrm{N}$ & 0.68069 & 2.14319 & 2.87515 \\
\hline $\mathrm{N}$ & 0.74870 & 1.99381 & 1.48065 \\
\hline $\mathrm{C}$ & 0.47647 & 0.75583 & 1.00499 \\
\hline $\mathrm{N}$ & 0.23831 & 0.08490 & 2.18162 \\
\hline $\mathrm{C}$ & 1.10615 & 3.11849 & 0.69143 \\
\hline $\mathrm{C}$ & 0.30851 & 4.27463 & 0.71416 \\
\hline $\mathrm{C}$ & 2.25917 & 3.07296 & -0.11149 \\
\hline $\mathrm{C}$ & 0.66553 & 5.38451 & -0.06280 \\
\hline $\mathrm{C}$ & 2.59832 & 4.17446 & -0.90840 \\
\hline $\mathrm{C}$ & 1.79949 & 5.31442 & -0.86727 \\
\hline $\mathrm{H}$ & 0.05909 & 6.28169 & -0.05408 \\
\hline $\mathrm{H}$ & 3.48013 & 4.14979 & -1.53655 \\
\hline $\mathrm{H}$ & 2.07029 & 6.17202 & -1.47657 \\
\hline $\mathrm{O}$ & 2.98211 & 1.92177 & -0.05421 \\
\hline $\mathrm{O}$ & -0.79880 & 4.21595 & 1.49869 \\
\hline $\mathrm{C}$ & -1.57347 & 5.40819 & 1.63660 \\
\hline $\mathrm{H}$ & -2.38264 & 5.14710 & 2.31863 \\
\hline $\mathrm{H}$ & -0.96849 & 6.21729 & 2.06327 \\
\hline $\mathrm{H}$ & -1.99033 & 5.72243 & 0.67272 \\
\hline $\mathrm{C}$ & 4.09373 & 1.79041 & -0.94529 \\
\hline $\mathrm{H}$ & 4.86679 & 2.53670 & -0.72443 \\
\hline $\mathrm{H}$ & 4.48185 & 0.78732 & -0.77021 \\
\hline $\mathrm{H}$ & 3.76969 & 1.88386 & -1.98848 \\
\hline $\mathrm{C}$ & 0.91711 & -2.19785 & 1.54286 \\
\hline $\mathrm{H}$ & 0.96403 & -1.79871 & 0.52552 \\
\hline $\mathrm{H}$ & 1.90419 & -2.05726 & 2.00166 \\
\hline $\mathrm{C}$ & 0.55755 & -3.66383 & 1.51457 \\
\hline $\mathrm{C}$ & 1.21567 & -4.58850 & 2.33435 \\
\hline $\mathrm{C}$ & -0.44737 & -4.12428 & 0.65218 \\
\hline $\mathrm{C}$ & 0.87953 & -5.94264 & 2.30042 \\
\hline $\mathrm{H}$ & 2.00611 & -4.24516 & 2.99892 \\
\hline $\mathrm{C}$ & -0.78300 & -5.47639 & 0.61610 \\
\hline $\mathrm{H}$ & -0.95048 & -3.41440 & 0.00215 \\
\hline $\mathrm{C}$ & -0.12336 & -6.39030 & 1.44028 \\
\hline $\mathrm{H}$ & 1.40416 & -6.64691 & 2.94076 \\
\hline $\mathrm{H}$ & -1.55544 & -5.81798 & -0.06771 \\
\hline $\mathrm{H}$ & -0.38411 & -7.44471 & 1.40799 \\
\hline $\mathrm{C}$ & -1.34130 & -0.21492 & -1.00207 \\
\hline $\mathrm{C}$ & -1.99522 & 1.10860 & -0.90186 \\
\hline $\mathrm{C}$ & -1.32668 & 2.03292 & -1.71314 \\
\hline $\mathrm{C}$ & -3.14814 & 1.45201 & -0.19372 \\
\hline $\mathrm{C}$ & -1.82176 & 3.32745 & -1.84403 \\
\hline $\mathrm{C}$ & -3.64007 & 2.74709 & -0.32529 \\
\hline $\mathrm{H}$ & -3.64383 & 0.71340 & 0.42965 \\
\hline $\mathrm{C}$ & -2.98547 & 3.67104 & -1.15262 \\
\hline $\mathrm{H}$ & -1.31464 & 4.05920 & -2.46692 \\
\hline $\mathrm{H}$ & -4.54090 & 3.04517 & 0.20380 \\
\hline
\end{tabular}




$\begin{array}{lccc}\mathrm{H} & -3.39381 & 4.67295 & -1.25810 \\ \mathrm{C} & -0.09208 & 1.41845 & -2.32445 \\ \mathrm{H} & 0.78368 & 1.72733 & -1.74437 \\ \mathrm{H} & 0.06783 & 1.70744 & -3.36791 \\ \mathrm{C} & -0.31318 & -0.09576 & -2.16146 \\ \mathrm{~S} & 1.23975 & -1.03046 & -1.87858 \\ \mathrm{C} & -0.59284 & -2.22362 & -3.37370 \\ \mathrm{H} & -0.53726 & -0.29625 & -4.31103 \\ \mathrm{C} & 0.58284 & -2.47594 & -2.65009 \\ \mathrm{C} & 1.10671 & -3.76373 & -2.55180 \\ \mathrm{C} & -1.25743 & -3.27668 & -3.99102 \\ \mathrm{C} & -0.74415 & -4.57552 & -3.89495 \\ \mathrm{H} & -1.26822 & -5.40082 & -4.36775 \\ \mathrm{C} & 0.43277 & -4.81140 & -3.18376 \\ \mathrm{H} & 0.82085 & -5.82229 & -3.09692 \\ \mathrm{H} & -2.17650 & -3.09193 & -4.54200 \\ \mathrm{H} & 2.00064 & -3.95719 & -1.96722\end{array}$




\section{$12.103 d-(S)-r-p a t h$}

76

3d-(S)-4-r

$\begin{array}{llll}\text { C } & -1.17784 & -0.73022 & -2.29158\end{array}$

$\begin{array}{llll}\mathrm{H} & -2.39675 & -0.57878 & 0.42605\end{array}$

$\begin{array}{llll}\mathrm{O} & -1.96602 & -0.14252 & 1.18220\end{array}$

$\begin{array}{llll}\text { C } & 1.56766 & -1.99334 & 3.20571\end{array}$

$\begin{array}{llll}\text { C } & 2.73398 & -0.99431 & 2.95488\end{array}$

$\begin{array}{llll}\text { C } & 0.46613 & -1.62827 & 2.17735\end{array}$

$\begin{array}{llll}\mathrm{H} & 3.24763 & -0.70413 & 3.87453\end{array}$

$\mathrm{H} \quad 1.87570 \quad-3.03633 \quad 3.10478$

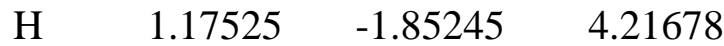

$\begin{array}{llll}\mathrm{H} & -0.53808 & -1.78027 & 2.57546\end{array}$

$\begin{array}{llll}\mathrm{H} & 3.48535 & -1.40670 & 2.27230\end{array}$

$\begin{array}{llll}\mathrm{C} & 2.02950 & 0.14992 & 2.29401\end{array}$

$\begin{array}{llll}\mathrm{N} & 2.37906 & 1.31124 & 1.83989\end{array}$

$\begin{array}{llll}\mathrm{N} & 1.20892 & 1.75345 & 1.15594\end{array}$

$\begin{array}{llll}\mathrm{C} & 0.18480 & 0.79305 & 1.19748\end{array}$

$\begin{array}{llll}\mathrm{N} & 0.72711 & -0.19530 & 2.01187\end{array}$

$\begin{array}{llll}\mathrm{C} & 1.07347 & 3.10890 & 0.79969\end{array}$

$\begin{array}{llll}\mathrm{C} & 0.06257 & 3.90660 & 1.37285\end{array}$

$\begin{array}{llll}\mathrm{C} & 1.93812 & 3.67725 & -0.15627\end{array}$

$\begin{array}{llll}\mathrm{C} & -0.13513 & 5.21905 & 0.93066\end{array}$

$\begin{array}{llll}\text { C } & 1.76255 & 5.00099 & -0.57078\end{array}$

$\begin{array}{llll}\text { C } & 0.71633 & 5.74849 & -0.03415\end{array}$

$\begin{array}{lllr}\mathrm{H} & -0.93100 & 5.82577 & 1.34420\end{array}$

$\mathrm{H} \quad 2.41787 \quad 5.43941 \quad-1.31334$

$\begin{array}{llll}\mathrm{H} & 0.56722 & 6.77030 & -0.37046\end{array}$

$\begin{array}{llll}\mathrm{O} & 2.90406 & 2.84565 & -0.64405\end{array}$

$\begin{array}{llll}\mathrm{O} & -0.67010 & 3.31441 & 2.35499\end{array}$

$\begin{array}{llll}\text { C } & -1.76370 & 4.05730 & 2.89960\end{array}$

$\mathrm{H} \quad-2.23586 \quad 3.38700 \quad 3.61818$

$\begin{array}{llll}\mathrm{H} & -1.40740 & 4.96109 & 3.40929\end{array}$

$\begin{array}{llll}\mathrm{H} & -2.47808 & 4.32596 & 2.11321\end{array}$

$\begin{array}{llll}\text { C } & 3.75874 & 3.35891 & -1.66718\end{array}$

$\begin{array}{llll}\mathrm{H} & 4.34245 & 4.21142 & -1.29961\end{array}$

$\mathrm{H} \quad 4.42677 \quad 2.53634 \quad-1.92352$

$\begin{array}{llll}\mathrm{H} & 3.18049 & 3.65992 & -2.55005\end{array}$

$\begin{array}{llll}\text { C } & 0.60328 & -2.34204 & 0.81724\end{array}$

$\begin{array}{llll}\mathrm{H} & -0.03533 & -1.80737 & 0.10628\end{array}$

$\begin{array}{llll}\mathrm{H} & 1.63399 & -2.23223 & 0.46276\end{array}$

$\begin{array}{llll}\text { C } & 0.22048 & -3.79567 & 0.86133\end{array}$

$\begin{array}{llll}\text { C } & 1.19359 & -4.80136 & 0.85144\end{array}$

$\begin{array}{llll}\text { C } & -1.12733 & -4.17292 & 0.91284\end{array}$

$\begin{array}{llll}\text { C } & 0.83399 & -6.14846 & 0.88800\end{array}$

$\begin{array}{llll}\mathrm{H} & 2.24395 & -4.52321 & 0.79441\end{array}$

$\begin{array}{llll}\text { C } & -1.49241 & -5.51662 & 0.95158\end{array}$ 


\begin{tabular}{|c|c|c|c|}
\hline $\mathrm{H}$ & -1.89512 & -3.40313 & 0.90317 \\
\hline $\mathrm{C}$ & -0.51219 & -6.51030 & 0.93828 \\
\hline $\mathrm{H}$ & 1.60412 & -6.91501 & 0.87078 \\
\hline $\mathrm{H}$ & -2.54367 & -5.79010 & 0.97982 \\
\hline $\mathrm{H}$ & -0.79612 & -7.55863 & 0.96170 \\
\hline $\mathrm{C}$ & -1.06054 & 0.80124 & 0.63845 \\
\hline $\mathrm{C}$ & -1.61070 & 1.78644 & -0.28020 \\
\hline $\mathrm{C}$ & -0.95837 & 2.14474 & -1.48729 \\
\hline $\mathrm{C}$ & -2.87022 & 2.35765 & -0.00673 \\
\hline $\mathrm{C}$ & -1.52377 & 3.12573 & -2.30421 \\
\hline $\mathrm{C}$ & -3.42826 & 3.31740 & -0.84360 \\
\hline $\mathrm{H}$ & -3.38925 & 2.04589 & 0.89522 \\
\hline $\mathrm{C}$ & -2.74086 & 3.72837 & -1.98822 \\
\hline $\mathrm{H}$ & -1.00976 & 3.40002 & -3.22300 \\
\hline $\mathrm{H}$ & -4.39207 & 3.75590 & -0.59734 \\
\hline $\mathrm{H}$ & -3.16080 & 4.48705 & -2.64217 \\
\hline $\mathrm{C}$ & 0.27121 & 1.40404 & -1.95530 \\
\hline $\mathrm{H}$ & 1.09646 & 1.48705 & -1.23987 \\
\hline $\mathrm{H}$ & 0.62919 & 1.86701 & -2.88455 \\
\hline $\mathrm{C}$ & 0.00873 & -0.06051 & -2.20080 \\
\hline $\mathrm{S}$ & 1.37685 & -1.13284 & -2.41892 \\
\hline $\mathrm{C}$ & -1.02926 & -2.14235 & -2.50230 \\
\hline $\mathrm{H}$ & -2.13507 & -0.22793 & -2.19738 \\
\hline $\mathrm{C}$ & 0.33697 & -2.52246 & -2.59423 \\
\hline $\mathrm{C}$ & 0.72001 & -3.85553 & -2.75600 \\
\hline $\mathrm{C}$ & -2.01174 & -3.14563 & -2.58883 \\
\hline $\mathrm{C}$ & -1.63015 & -4.47088 & -2.74662 \\
\hline $\mathrm{H}$ & -2.38821 & -5.24694 & -2.79770 \\
\hline $\mathrm{C}$ & -0.27356 & -4.82594 & -2.82736 \\
\hline $\mathrm{H}$ & 0.00479 & -5.87001 & -2.93217 \\
\hline $\mathrm{H}$ & -3.06346 & -2.87873 & -2.52174 \\
\hline $\mathrm{H}$ & 1.76886 & -4.13152 & -2.80766 \\
\hline \multicolumn{4}{|c|}{76} \\
\hline \multicolumn{4}{|c|}{$3 d-(S)-t s 1-r$} \\
\hline $\mathrm{C}$ & -1.07601 & -0.85554 & -2.23911 \\
\hline $\mathrm{H}$ & -1.58611 & -0.95990 & -0.98867 \\
\hline $\mathrm{O}$ & -1.74910 & -0.55341 & 0.21376 \\
\hline $\mathrm{C}$ & 1.36859 & -1.91298 & 3.13047 \\
\hline $\mathrm{C}$ & 2.57542 & -0.96988 & 2.85864 \\
\hline $\mathrm{C}$ & 0.39505 & -1.71949 & 1.93446 \\
\hline $\mathrm{H}$ & 3.04373 & -0.59607 & 3.77078 \\
\hline $\mathrm{H}$ & 1.66226 & -2.95965 & 3.23358 \\
\hline $\mathrm{H}$ & 0.87348 & -1.60514 & 4.05628 \\
\hline $\mathrm{H}$ & -0.65183 & -1.73388 & 2.23863 \\
\hline $\mathrm{H}$ & 3.34783 & -1.46525 & 2.25827 \\
\hline $\mathrm{C}$ & 1.91971 & 0.11028 & 2.06329 \\
\hline $\mathrm{N}$ & 2.14452 & 1.36271 & 1.80364 \\
\hline
\end{tabular}




\begin{tabular}{|c|c|c|c|}
\hline $\mathrm{N}$ & 1.03041 & 1.72663 & 1.02310 \\
\hline $\mathrm{C}$ & 0.13980 & 0.68369 & 0.84538 \\
\hline $\mathrm{N}$ & 0.74632 & -0.34818 & 1.51814 \\
\hline $\mathrm{C}$ & 0.87782 & 3.07473 & 0.62144 \\
\hline $\mathrm{C}$ & -0.04330 & 3.90457 & 1.28159 \\
\hline $\mathrm{C}$ & 1.67222 & 3.58642 & -0.41761 \\
\hline $\mathrm{C}$ & -0.22734 & 5.22252 & 0.84851 \\
\hline $\mathrm{C}$ & 1.50231 & 4.90999 & -0.83782 \\
\hline $\mathrm{C}$ & 0.54508 & 5.70106 & -0.20579 \\
\hline $\mathrm{H}$ & -0.95755 & 5.86547 & 1.32327 \\
\hline $\mathrm{H}$ & 2.09695 & 5.31611 & -1.64674 \\
\hline $\mathrm{H}$ & 0.40169 & 6.72391 & -0.54184 \\
\hline $\mathrm{O}$ & 2.56047 & 2.71057 & -0.96356 \\
\hline $\mathrm{O}$ & -0.70344 & 3.33590 & 2.32544 \\
\hline $\mathrm{C}$ & -1.66326 & 4.14708 & 3.01174 \\
\hline $\mathrm{H}$ & -2.07684 & 3.50595 & 3.79031 \\
\hline $\mathrm{H}$ & -1.17918 & 5.02081 & 3.46401 \\
\hline $\mathrm{H}$ & -2.45763 & 4.46642 & 2.32831 \\
\hline $\mathrm{C}$ & 3.36324 & 3.17400 & -2.05403 \\
\hline $\mathrm{H}$ & 4.00854 & 4.00193 & -1.73800 \\
\hline $\mathrm{H}$ & 3.96809 & 2.31644 & -2.34720 \\
\hline $\mathrm{H}$ & 2.73555 & 3.49060 & -2.89619 \\
\hline $\mathrm{C}$ & 0.60966 & -2.72185 & 0.79203 \\
\hline $\mathrm{H}$ & 0.07316 & -2.34014 & -0.07645 \\
\hline $\mathrm{H}$ & 1.67306 & -2.76251 & 0.53230 \\
\hline $\mathrm{C}$ & 0.09270 & -4.09344 & 1.13751 \\
\hline $\mathrm{C}$ & 0.95946 & -5.15625 & 1.40959 \\
\hline $\mathrm{C}$ & -1.28925 & -4.32121 & 1.19236 \\
\hline $\mathrm{C}$ & 0.46268 & -6.42018 & 1.73189 \\
\hline $\mathrm{H}$ & 2.03398 & -4.99535 & 1.35108 \\
\hline $\mathrm{C}$ & -1.78893 & -5.58087 & 1.51570 \\
\hline $\mathrm{H}$ & -1.96963 & -3.50329 & 0.96586 \\
\hline $\mathrm{C}$ & -0.91401 & -6.63509 & 1.78780 \\
\hline $\mathrm{H}$ & 1.15050 & -7.23689 & 1.93409 \\
\hline $\mathrm{H}$ & -2.86288 & -5.74382 & 1.54812 \\
\hline $\mathrm{H}$ & -1.30377 & -7.61852 & 2.03533 \\
\hline $\mathrm{C}$ & -1.07306 & 0.59826 & 0.14090 \\
\hline $\mathrm{C}$ & -1.80664 & 1.79203 & -0.36947 \\
\hline $\mathrm{C}$ & -1.48950 & 2.31970 & -1.62953 \\
\hline $\mathrm{C}$ & -2.88805 & 2.32451 & 0.34384 \\
\hline $\mathrm{C}$ & -2.21011 & 3.40955 & -2.12712 \\
\hline $\mathrm{C}$ & -3.60757 & 3.40612 & -0.15784 \\
\hline $\mathrm{H}$ & -3.15526 & 1.87755 & 1.29696 \\
\hline $\mathrm{C}$ & -3.25728 & 3.96035 & -1.39184 \\
\hline $\mathrm{H}$ & -1.95955 & 3.81785 & -3.10365 \\
\hline $\mathrm{H}$ & -4.44543 & 3.81195 & 0.40368 \\
\hline $\mathrm{H}$ & -3.81470 & 4.80424 & -1.78936 \\
\hline $\mathrm{C}$ & -0.37461 & 1.66811 & -2.41174 \\
\hline
\end{tabular}




\begin{tabular}{|c|c|c|c|}
\hline $\mathrm{H}$ & 0.55571 & 2.22420 & -2.25832 \\
\hline $\mathrm{H}$ & -0.59504 & 1.73183 & -3.48851 \\
\hline $\mathrm{C}$ & -0.15184 & 0.22808 & -2.01180 \\
\hline S & 1.51513 & -0.35618 & -2.02491 \\
\hline $\mathrm{C}$ & -0.37169 & -2.10922 & -2.53540 \\
\hline $\mathrm{H}$ & -1.99408 & -0.62945 & -2.78586 \\
\hline $\mathrm{C}$ & 1.03183 & -1.99696 & -2.41534 \\
\hline $\mathrm{C}$ & 1.87266 & -3.10313 & -2.54572 \\
\hline $\mathrm{C}$ & -0.91778 & -3.36759 & -2.81756 \\
\hline $\mathrm{C}$ & -0.08344 & -4.47368 & -2.96602 \\
\hline $\mathrm{H}$ & -0.51413 & -5.44804 & -3.17751 \\
\hline $\mathrm{C}$ & 1.30408 & -4.34434 & -2.82755 \\
\hline $\mathrm{H}$ & 1.94358 & -5.21522 & -2.93873 \\
\hline $\mathrm{H}$ & -1.99577 & -3.48009 & -2.90118 \\
\hline $\mathrm{H}$ & 2.94834 & -2.99942 & -2.43412 \\
\hline \multicolumn{4}{|c|}{10} \\
\hline \multicolumn{4}{|c|}{$3 d-(S)-6-r$} \\
\hline $\mathrm{C}$ & -1.33485 & -0.67467 & -2.43901 \\
\hline $\mathrm{H}$ & -2.24832 & -0.75056 & -1.84083 \\
\hline $\mathrm{O}$ & -1.48477 & -0.70373 & 0.29311 \\
\hline $\mathrm{C}$ & 1.08605 & -1.84863 & 3.41323 \\
\hline $\mathrm{C}$ & 2.25278 & -0.81734 & 3.44743 \\
\hline $\mathrm{C}$ & 0.43431 & -1.72909 & 2.00855 \\
\hline $\mathrm{H}$ & 2.41774 & -0.38526 & 4.43627 \\
\hline $\mathrm{H}$ & 1.42310 & -2.86982 & 3.60273 \\
\hline $\mathrm{H}$ & 0.35193 & -1.58492 & 4.18026 \\
\hline $\mathrm{H}$ & -0.65290 & -1.77569 & 2.01467 \\
\hline $\mathrm{H}$ & 3.19829 & -1.25819 & 3.11044 \\
\hline $\mathrm{C}$ & 1.77758 & 0.19062 & 2.45369 \\
\hline $\mathrm{N}$ & 2.02677 & 1.43956 & 2.15900 \\
\hline $\mathrm{N}$ & 1.14096 & 1.68403 & 1.10703 \\
\hline $\mathrm{C}$ & 0.38678 & 0.60286 & 0.77951 \\
\hline $\mathrm{N}$ & 0.79543 & -0.33456 & 1.66084 \\
\hline $\mathrm{C}$ & 1.07319 & 3.00584 & 0.57911 \\
\hline $\mathrm{C}$ & 0.25361 & 3.95264 & 1.21265 \\
\hline $\mathrm{C}$ & 1.87892 & 3.36263 & -0.51086 \\
\hline $\mathrm{C}$ & 0.17206 & 5.24776 & 0.68871 \\
\hline $\mathrm{C}$ & 1.79703 & 4.65886 & -1.03170 \\
\hline $\mathrm{C}$ & 0.93622 & 5.57381 & -0.42807 \\
\hline $\mathrm{H}$ & -0.48103 & 5.98547 & 1.13680 \\
\hline $\mathrm{H}$ & 2.39408 & 4.95133 & -1.88646 \\
\hline $\mathrm{H}$ & 0.86644 & 6.57752 & -0.83725 \\
\hline $\mathrm{O}$ & 2.68635 & 2.37952 & -0.98957 \\
\hline $\mathrm{O}$ & -0.40529 & 3.51717 & 2.31399 \\
\hline $\mathrm{C}$ & -1.28820 & 4.44248 & 2.96109 \\
\hline $\mathrm{H}$ & -1.72263 & 3.88864 & 3.79299 \\
\hline $\mathrm{H}$ & -0.73054 & 5.30827 & 3.33671 \\
\hline
\end{tabular}




\begin{tabular}{|c|c|c|c|}
\hline $\mathrm{H}$ & -2.07738 & 4.76293 & 2.27322 \\
\hline $\mathrm{C}$ & 3.44768 & 2.66071 & -2.17128 \\
\hline $\mathrm{H}$ & 4.17269 & 3.46102 & -1.98305 \\
\hline $\mathrm{H}$ & 3.96216 & 1.72988 & -2.40569 \\
\hline $\mathrm{H}$ & 2.78698 & 2.93698 & -3.00109 \\
\hline $\mathrm{C}$ & 0.98078 & -2.70964 & 0.96178 \\
\hline $\mathrm{H}$ & 0.82911 & -2.24793 & -0.01469 \\
\hline $\mathrm{H}$ & 2.05946 & -2.85582 & 1.10128 \\
\hline $\mathrm{C}$ & 0.24953 & -4.02687 & 0.99884 \\
\hline $\mathrm{C}$ & 0.88664 & -5.21779 & 1.35650 \\
\hline $\mathrm{C}$ & -1.10722 & -4.05670 & 0.64592 \\
\hline $\mathrm{C}$ & 0.18628 & -6.42610 & 1.36049 \\
\hline $\mathrm{H}$ & 1.94236 & -5.20237 & 1.62111 \\
\hline $\mathrm{C}$ & -1.80543 & -5.26129 & 0.65055 \\
\hline $\mathrm{H}$ & -1.58967 & -3.12548 & 0.35324 \\
\hline $\mathrm{C}$ & -1.16241 & -6.44955 & 1.00752 \\
\hline $\mathrm{H}$ & 0.69470 & -7.34639 & 1.63592 \\
\hline $\mathrm{H}$ & -2.85414 & -5.27635 & 0.36549 \\
\hline $\mathrm{H}$ & -1.70952 & -7.38848 & 1.00748 \\
\hline $\mathrm{C}$ & -0.84959 & 0.36514 & -0.15969 \\
\hline $\mathrm{C}$ & -1.69525 & 1.65411 & -0.22393 \\
\hline $\mathrm{C}$ & -1.50172 & 2.38202 & -1.39786 \\
\hline $\mathrm{C}$ & -2.66839 & 2.02521 & 0.69742 \\
\hline $\mathrm{C}$ & -2.25975 & 3.52538 & -1.64946 \\
\hline $\mathrm{C}$ & -3.42840 & 3.16972 & 0.44993 \\
\hline $\mathrm{H}$ & -2.83785 & 1.40538 & 1.57367 \\
\hline $\mathrm{C}$ & -3.21735 & 3.92128 & -0.71320 \\
\hline $\mathrm{H}$ & -2.12105 & 4.09404 & -2.56624 \\
\hline $\mathrm{H}$ & -4.20233 & 3.47227 & 1.15152 \\
\hline $\mathrm{H}$ & -3.82311 & 4.80409 & -0.90115 \\
\hline $\mathrm{C}$ & -0.45961 & 1.73436 & -2.27484 \\
\hline $\mathrm{H}$ & 0.49856 & 2.25526 & -2.18912 \\
\hline $\mathrm{H}$ & -0.73062 & 1.74383 & -3.33679 \\
\hline $\mathrm{C}$ & -0.36358 & 0.29445 & -1.72597 \\
\hline $\mathrm{S}$ & 1.33071 & -0.40911 & -1.96044 \\
\hline $\mathrm{C}$ & -0.67674 & -2.01842 & -2.58385 \\
\hline $\mathrm{H}$ & -1.58547 & -0.27681 & -3.43211 \\
\hline $\mathrm{C}$ & 0.71370 & -2.00036 & -2.40680 \\
\hline $\mathrm{C}$ & 1.47804 & -3.16066 & -2.53996 \\
\hline $\mathrm{C}$ & -1.30426 & -3.22289 & -2.88750 \\
\hline $\mathrm{C}$ & -0.55264 & -4.39504 & -3.01466 \\
\hline $\mathrm{H}$ & -1.04948 & -5.33665 & -3.22786 \\
\hline $\mathrm{C}$ & 0.83144 & -4.36030 & -2.84496 \\
\hline $\mathrm{H}$ & 1.41246 & -5.27416 & -2.93058 \\
\hline $\mathrm{H}$ & -2.38435 & -3.25191 & -3.00855 \\
\hline $\mathrm{H}$ & 2.55297 & -3.13755 & -2.38566 \\
\hline
\end{tabular}

76 


\begin{tabular}{|c|c|c|c|}
\hline \multicolumn{4}{|c|}{$3 d-(S)-t s 3-r$} \\
\hline $\mathrm{C}$ & -0.49181 & -0.70902 & -3.02731 \\
\hline $\mathrm{H}$ & -1.58437 & -0.66026 & -3.00587 \\
\hline $\mathrm{O}$ & -1.51328 & -0.89046 & -0.37314 \\
\hline $\mathrm{C}$ & -0.43556 & -1.60345 & 3.64029 \\
\hline $\mathrm{C}$ & 0.52926 & -0.49996 & 4.16601 \\
\hline $\mathrm{C}$ & -0.33319 & -1.57183 & 2.09305 \\
\hline $\mathrm{H}$ & 0.17444 & -0.02240 & 5.08157 \\
\hline $\mathrm{H}$ & -0.19511 & -2.59495 & 4.02992 \\
\hline $\mathrm{H}$ & -1.45836 & -1.35947 & 3.94200 \\
\hline $\mathrm{H}$ & -1.28737 & -1.72330 & 1.59241 \\
\hline $\mathrm{H}$ & 1.53369 & -0.89356 & 4.36143 \\
\hline $\mathrm{C}$ & 0.56347 & 0.43677 & 3.00370 \\
\hline $\mathrm{N}$ & 0.93085 & 1.66817 & 2.77691 \\
\hline $\mathrm{N}$ & 0.64731 & 1.80452 & 1.40769 \\
\hline $\mathrm{C}$ & 0.13974 & 0.69080 & 0.82533 \\
\hline $\mathrm{N}$ & 0.07760 & -0.16709 & 1.87246 \\
\hline $\mathrm{C}$ & 0.90262 & 3.06222 & 0.79352 \\
\hline $\mathrm{C}$ & -0.01959 & 4.10752 & 0.95032 \\
\hline $\mathrm{C}$ & 2.09382 & 3.25734 & 0.07935 \\
\hline $\mathrm{C}$ & 0.23585 & 5.34780 & 0.35153 \\
\hline $\mathrm{C}$ & 2.34358 & 4.49430 & -0.52667 \\
\hline $\mathrm{C}$ & 1.40733 & 5.51667 & -0.38062 \\
\hline $\mathrm{H}$ & -0.47135 & 6.16228 & 0.44346 \\
\hline $\mathrm{H}$ & 3.25059 & 4.66088 & -1.09428 \\
\hline $\mathrm{H}$ & 1.60084 & 6.47719 & -0.84964 \\
\hline $\mathrm{O}$ & 2.92495 & 2.18238 & 0.01987 \\
\hline $\mathrm{O}$ & -1.11760 & 3.81746 & 1.69050 \\
\hline $\mathrm{C}$ & -2.08555 & 4.85755 & 1.87010 \\
\hline $\mathrm{H}$ & -2.87692 & 4.41031 & 2.47117 \\
\hline $\mathrm{H}$ & -1.64418 & 5.70807 & 2.40313 \\
\hline $\mathrm{H}$ & -2.49021 & 5.17939 & 0.90510 \\
\hline $\mathrm{C}$ & 4.10192 & 2.29761 & -0.78893 \\
\hline $\mathrm{H}$ & 4.77218 & 3.06934 & -0.39249 \\
\hline $\mathrm{H}$ & 4.57970 & 1.32009 & -0.73703 \\
\hline $\mathrm{H}$ & 3.83784 & 2.52498 & -1.82806 \\
\hline $\mathrm{C}$ & 0.70958 & -2.53667 & 1.50535 \\
\hline $\mathrm{H}$ & 0.97532 & -2.15552 & 0.51578 \\
\hline $\mathrm{H}$ & 1.61725 & -2.52922 & 2.12258 \\
\hline $\mathrm{C}$ & 0.15864 & -3.93364 & 1.38024 \\
\hline $\mathrm{C}$ & 0.62047 & -4.98311 & 2.18003 \\
\hline $\mathrm{C}$ & -0.84509 & -4.18901 & 0.43521 \\
\hline $\mathrm{C}$ & 0.09424 & -6.26942 & 2.04225 \\
\hline $\mathrm{H}$ & 1.40677 & -4.79508 & 2.90898 \\
\hline $\mathrm{C}$ & -1.37024 & -5.47107 & 0.29768 \\
\hline $\mathrm{H}$ & -1.19280 & -3.37407 & -0.19565 \\
\hline $\mathrm{C}$ & -0.90412 & -6.51543 & 1.10034 \\
\hline $\mathrm{H}$ & 0.46587 & -7.07682 & 2.66774 \\
\hline
\end{tabular}




\begin{tabular}{|c|c|c|c|}
\hline $\mathrm{H}$ & -2.13861 & -5.65893 & -0.44739 \\
\hline $\mathrm{H}$ & -1.31344 & -7.51582 & 0.98844 \\
\hline $\mathrm{C}$ & -0.93169 & 0.17836 & -0.70874 \\
\hline $\mathrm{C}$ & -1.70457 & 1.46519 & -0.93981 \\
\hline $\mathrm{C}$ & -1.05735 & 2.28270 & -1.86783 \\
\hline $\mathrm{C}$ & -2.94188 & 1.81278 & -0.40825 \\
\hline $\mathrm{C}$ & -1.63754 & 3.48570 & -2.26508 \\
\hline $\mathrm{C}$ & -3.52692 & 3.01494 & -0.80789 \\
\hline $\mathrm{H}$ & -3.43738 & 1.14051 & 0.28714 \\
\hline $\mathrm{C}$ & -2.87507 & 3.84769 & -1.72633 \\
\hline $\mathrm{H}$ & -1.14450 & 4.13033 & -2.98899 \\
\hline $\mathrm{H}$ & -4.49766 & 3.30611 & -0.41426 \\
\hline $\mathrm{H}$ & -3.34587 & 4.77703 & -2.03725 \\
\hline $\mathrm{C}$ & 0.24002 & 1.65830 & -2.32890 \\
\hline $\mathrm{H}$ & 1.08926 & 2.13119 & -1.82719 \\
\hline $\mathrm{H}$ & 0.40432 & 1.76267 & -3.40705 \\
\hline $\mathrm{C}$ & 0.10462 & 0.18189 & -1.90957 \\
\hline $\mathrm{S}$ & 1.72401 & -0.58830 & -1.49388 \\
\hline $\mathrm{C}$ & 0.01913 & -2.10995 & -2.82902 \\
\hline $\mathrm{H}$ & -0.15126 & -0.33378 & -4.00332 \\
\hline $\mathrm{C}$ & 1.21171 & -2.16798 & -2.09220 \\
\hline $\mathrm{C}$ & 1.86414 & -3.37955 & -1.86373 \\
\hline $\mathrm{C}$ & -0.52890 & -3.28616 & -3.32859 \\
\hline $\mathrm{C}$ & 0.11215 & -4.50905 & -3.10085 \\
\hline $\mathrm{H}$ & -0.32575 & -5.42856 & -3.47783 \\
\hline $\mathrm{C}$ & 1.30332 & -4.55131 & -2.37675 \\
\hline $\mathrm{H}$ & 1.78872 & -5.50381 & -2.18494 \\
\hline $\mathrm{H}$ & -1.46017 & -3.25373 & -3.88887 \\
\hline $\mathrm{H}$ & 2.77815 & -3.41726 & -1.27838 \\
\hline \multicolumn{4}{|l|}{76} \\
\hline \multicolumn{4}{|c|}{$3 d-(S)-[2 r+3 d]$} \\
\hline $\mathrm{C}$ & -0.47934 & -0.73221 & -3.46988 \\
\hline $\mathrm{H}$ & -1.54551 & -0.57067 & -3.66333 \\
\hline $\mathrm{O}$ & -1.69998 & -1.18495 & -0.66279 \\
\hline $\mathrm{C}$ & -0.75785 & -1.40632 & 3.69835 \\
\hline $\mathrm{C}$ & 0.06435 & -0.25957 & 4.36073 \\
\hline $\mathrm{C}$ & -0.36516 & -1.40924 & 2.19679 \\
\hline $\mathrm{H}$ & -0.47394 & 0.23075 & 5.17481 \\
\hline $\mathrm{H}$ & -0.57384 & -2.37948 & 4.15890 \\
\hline $\mathrm{H}$ & -1.82492 & -1.18404 & 3.79291 \\
\hline $\mathrm{H}$ & -1.21266 & -1.62242 & 1.54518 \\
\hline $\mathrm{H}$ & 1.01764 & -0.62235 & 4.76329 \\
\hline $\mathrm{C}$ & 0.30518 & 0.64336 & 3.19465 \\
\hline $\mathrm{N}$ & 0.70550 & 1.86610 & 2.98860 \\
\hline $\mathrm{N}$ & 0.67473 & 1.92598 & 1.57523 \\
\hline $\mathrm{C}$ & 0.28281 & 0.79406 & 0.93474 \\
\hline $\mathrm{N}$ & 0.04328 & -0.00809 & 2.01158 \\
\hline
\end{tabular}




\begin{tabular}{|c|c|c|c|}
\hline $\mathrm{C}$ & 1.02242 & 3.14203 & 0.93013 \\
\hline $\mathrm{C}$ & 0.15987 & 4.24619 & 1.00989 \\
\hline $\mathrm{C}$ & 2.21335 & 3.22636 & 0.19184 \\
\hline $\mathrm{C}$ & 0.49487 & 5.44149 & 0.36035 \\
\hline $\mathrm{C}$ & 2.53795 & 4.41474 & -0.47582 \\
\hline $\mathrm{C}$ & 1.67593 & 5.50442 & -0.37463 \\
\hline $\mathrm{H}$ & -0.15986 & 6.30250 & 0.41273 \\
\hline $\mathrm{H}$ & 3.45120 & 4.49429 & -1.05248 \\
\hline $\mathrm{H}$ & 1.93264 & 6.42920 & -0.88365 \\
\hline $\mathrm{O}$ & 2.97843 & 2.10017 & 0.17143 \\
\hline $\mathrm{O}$ & -0.98259 & 4.05050 & 1.71906 \\
\hline $\mathrm{C}$ & -1.87356 & 5.16003 & 1.85137 \\
\hline $\mathrm{H}$ & -2.70734 & 4.78905 & 2.44752 \\
\hline $\mathrm{H}$ & -1.38423 & 5.99340 & 2.36994 \\
\hline $\mathrm{H}$ & -2.23696 & 5.48976 & 0.87136 \\
\hline $\mathrm{C}$ & 4.14397 & 2.10637 & -0.65841 \\
\hline $\mathrm{H}$ & 4.87000 & 2.85146 & -0.31099 \\
\hline $\mathrm{H}$ & 4.56345 & 1.10467 & -0.56889 \\
\hline $\mathrm{H}$ & 3.87720 & 2.30340 & -1.70355 \\
\hline $\mathrm{C}$ & 0.79670 & -2.35914 & 1.85003 \\
\hline $\mathrm{H}$ & 1.19623 & -2.03084 & 0.88474 \\
\hline $\mathrm{H}$ & 1.59264 & -2.25387 & 2.59794 \\
\hline $\mathrm{C}$ & 0.34307 & -3.79415 & 1.75565 \\
\hline $\mathrm{C}$ & 0.71352 & -4.74492 & 2.71249 \\
\hline $\mathrm{C}$ & -0.47605 & -4.19286 & 0.68907 \\
\hline $\mathrm{C}$ & 0.28199 & -6.06890 & 2.60971 \\
\hline $\mathrm{H}$ & 1.35515 & -4.44790 & 3.53996 \\
\hline $\mathrm{C}$ & -0.90517 & -5.51390 & 0.58360 \\
\hline $\mathrm{H}$ & -0.76870 & -3.46159 & -0.06016 \\
\hline $\mathrm{C}$ & -0.52889 & -6.45655 & 1.54339 \\
\hline $\mathrm{H}$ & 0.58247 & -6.79603 & 3.35949 \\
\hline $\mathrm{H}$ & -1.52922 & -5.80876 & -0.25568 \\
\hline $\mathrm{H}$ & -0.86320 & -7.48698 & 1.45863 \\
\hline $\mathrm{C}$ & -1.26484 & -0.15875 & -1.16294 \\
\hline $\mathrm{C}$ & -1.90246 & 1.17801 & -1.17712 \\
\hline $\mathrm{C}$ & -1.08076 & 2.08943 & -1.85070 \\
\hline $\mathrm{C}$ & -3.14183 & 1.55305 & -0.65763 \\
\hline $\mathrm{C}$ & -1.49680 & 3.40703 & -2.01833 \\
\hline $\mathrm{C}$ & -3.55703 & 2.87100 & -0.82907 \\
\hline $\mathrm{H}$ & -3.75753 & 0.82341 & -0.13941 \\
\hline $\mathrm{C}$ & -2.74075 & 3.78661 & -1.50839 \\
\hline $\mathrm{H}$ & -0.86611 & 4.13194 & -2.52584 \\
\hline $\mathrm{H}$ & -4.51973 & 3.19459 & -0.44281 \\
\hline $\mathrm{H}$ & -3.08325 & 4.80980 & -1.64142 \\
\hline $\mathrm{C}$ & 0.21961 & 1.44406 & -2.25877 \\
\hline $\mathrm{H}$ & 0.99725 & 1.73296 & -1.54472 \\
\hline $\mathrm{H}$ & 0.54840 & 1.72520 & -3.26456 \\
\hline $\mathrm{C}$ & -0.05395 & -0.06495 & -2.13038 \\
\hline
\end{tabular}




$\begin{array}{lrrr}\mathrm{S} & 1.40486 & -1.02103 & -1.55926 \\ \mathrm{C} & -0.11794 & -2.19145 & -3.38545 \\ \mathrm{H} & 0.08124 & -0.25933 & -4.28880 \\ \mathrm{C} & 0.90994 & -2.45130 & -2.46604 \\ \mathrm{C} & 1.41786 & -3.73938 & -2.29950 \\ \mathrm{C} & -0.65301 & -3.23632 & -4.13000 \\ \mathrm{C} & -0.15643 & -4.53462 & -3.96607 \\ \mathrm{H} & -0.58059 & -5.35314 & -4.54006 \\ \mathrm{C} & 0.87628 & -4.77829 & -3.05969 \\ \mathrm{H} & 1.25221 & -5.78846 & -2.92411 \\ \mathrm{H} & -1.45904 & -3.04490 & -4.83444 \\ \mathrm{H} & 2.19682 & -3.93969 & -1.57030\end{array}$




\section{Literature}

[S1] Liu, F.; Bugaut, X.; Schedler, M.; Fröhlich, R.; Glorius, F. Angew. Chem., Int. Ed. 2011, 50, 12626-12630.

[S2] Piel, I.; Steinmetz, M.; Hirano, K.; Fröhlich, R.; Grimme, S.; Glorius, F. Angew. Chem., Int. Ed. 2011, 50, 4983-4987.

[S3] Wurz, N. E.; Daniliuc, C. G.; Glorius, F.; Chem. Eur. J. 2012, 18, 16297-16301.

[S4] Schedler, M.; Fröhlich, R.; Daniliuc, C. G.; Glorius, F. Eur. J. Org. Chem. 2012, 41644171.

[S5] Romanov-Michailidis, F.; Besnard, C.; Alexakis, A. Org. Lett. 2012, 14, 4906-4609.

[S6] Lebeuf, R.; Hirano, K.; Glorius, F. Org. Lett. 2008, 10, 4243-4246.

[S7] Kerr, M. S.; Read de Alaniz, J.; Rovis, T. J. Org. Chem. 2005, 70, 5725-5728.

[S8] Enders, D.; Breuer, K.; Runsink, J.; Teles, J. H. Liebigs Ann. 1996, 2019-2028.

[S9] Aljaar, N.; Conrad, J.; Beifuss, U. J. Org. Chem. 2013, 78, 1045-1053.

[S10] Zhang, L.; Ang, G. Y.; Chiba, S. Org. Lett. 2011, 13, 1622-1625.

[S11] Barker, P.; Finke, P.; Thompson, K. Synth. Commun. 1989, 19, 257-565.

[S12] Ashfield, L.; Barnard, C. F. J. Org. Process Res. Dev. 2007, 11, 39-43.

[S13] Zhang, X.-X.; Lippard, S. J. J. Org. Chem. 2000, 65, 5298-5305.

[S14] Urban, S.; Beiring, B.; Ortega, N.; Paul, D.; Glorius, F. J. Am. Chem. Soc. 2012, 134, 15241-15244.

[S15] Vautravers, N. R.; Regent, D. D.; Breit, B. Chem. Commun. 2011, 47, 6635-6637.

[S16] Graulich, A.; Liegeois, J.-F. Synthesis 2004, 12, 1935-1937.

[S17] Barrett, S.; Boys, M.; Chen, H.; Kramer, J. Therapeutic Pyrazolyl Thienopyridines. US 20080090861 A1, April 17, 2008.

[S18] Sahoo, B.; Li, J.-L.; Glorius, F. Angew. Chem., Int. Ed. 2015, 54, 11577-11580.

[S19] Bruker (2013). APEX2, SAINT and SADABS Bruker AXS Inc., Madison, Wisconsin, USA.

[S20] SHELXT and SHELXL: Sheldrick, G. M. Acta Cryst. A 2008, 64, 112-122.

[S21] Tang, D.-T. D.; Collins, K.; Glorius, F. J. Am. Chem. Soc. 2013, 135, 7450-7453. 
[S22] Domingo, L.; Saéz, J. A.; Arnó, M. RSC Adv. 2012, 2, 7127-7134.

[S23] Berkessel, A.; Yatham, V. R.; Elfert, S.; Neudörfl, J.-M. Angew. Chem., Int. Ed. 2013, 52, $11158-11162$.

[S24] Hawkes K. J.; Yates, B. F. Eur. J. Org. Chem. 2008, 5563-5570.

[S25] TURBOMOLE V6.5 2013, a development of University of Karlsruhe and Forschungszentrum Karlsruhe GmbH, 1989-2007, TURBOMOLE GmbH, since 2007.

[S26] Tao, J.; Perdew, J. P.; Staroverov, V. N.; Scuseria, G. E. Phys. Rev. Lett. 2003, 91, 146401.

[S27] Grimme, S. J. Chem. Phys. 2010, 132, 154104.

[S28] Grimme, S.; Ehrlich, S.; Goerigk, L. J. Comput. Chem. 2011, 32, 1456-1465.

[S29] Ahlrichs, R. Phys. Chem. Chem. Phys. 2004, 6, 5119-5121.

[S30] Eichkorn, K.; Weigend, F.; Treutler, O.; Ahlrichs, R. Theor. Chem. Acc. 1997, 97, 119124.

[S31] Schäfer, A.; Horn, H.; Ahlrichs, R. J. Chem. Phys. 1992, 97, 2571-2577.

[S32] Neese, F. WIREs Comput. Mol. Sci. 2012, 2, 73-78.

[S33] Weigend, F.; Ahlrichs, R. Phys. Chem. Chem. Phys. 2005, 7, 3297-3305.

[S34] Deglmann, P.; May, K.; Furche, F.; Ahlrichs, R. Chem. Phys. Lett. 2004, 384, 103-107.

[S35] Klamt, A.; Schüürmann, G. J. Chem. Soc. Perk. Trans. 2, 1993, 799-805.

[S36] Schäfer, A.; Klamt, A.; Sattel, D.; Lohrenz J. C. W.; Eckert, F. Phys. Chem. Chem. Phys. 2000, 2, 2187-2193.

[S37] Zhao, Y.; Truhlar, D. G. Theor. Chem. Acc. 2008, 120, 215-241.

[S38] TURBOMOLE V7.0 2015, a development of University of Karlsruhe and Forschungszentrum Karlsruhe GmbH, 1989-2007, TURBOMOLE GmbH, since 2007.

[S39] Humphrey, W.; Dalke, A.; Schulten, K. J. Molec. Graphics 1996, 14.1, 33-38. 